NUREG/CR--5270

TI89 009560

\title{
ASSESSMENT OF SEISMIC MARGIN CALCULATION METHODS
}

Manuscript Completed: November 1988

Date Published: March 1989

Prepared by

R.P. Kennedy

RPK/Structural Mechanics Consulting

R.C. Murray

Lawrence Livermore National Laboratory

M.K. Ravindra

EQE Engineering; Inc.

J.W. Reed

Jack R. Benjamin \& Associates, Inc.

J.D. Stevenson

Stevenson \& Associates

Lawrence Livermore National Laboratory

7000 East Avenue

Livermore, CA 94550

Prepared for

Division of Engineering

Office of Nuclear Regulatory Research

U.S. Nuclear Regulatory Commission

Washington, D.C. 20555

NRC FIN No. A0398
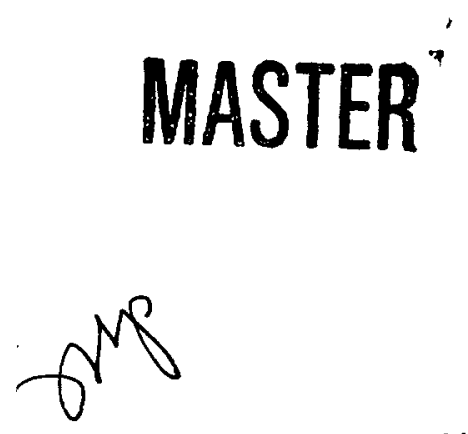

DISTRIBUTION OF THIS DOCUMENT IS UNLIMITED 


\section{DISCLAIMER}

This report was prepared as an account of work sponsored by an agency of the United States Government. Neither the United States Government nor any agency Thereof, nor any of their employees, makes any warranty, express or implied, or assumes any legal liability or responsibility for the accuracy, completeness, or usefulness of any information, apparatus, product, or process disclosed, or represents that its use would not infringe privately owned rights. Reference herein to any specific commercial product, process, or service by trade name, trademark, manufacturer, or otherwise does not necessarily constitute or imply its endorsement, recommendation, or favoring by the United States Government or any agency thereof. The views and opinions of authors expressed herein do not necessarily state or reflect those of the United States Government or any agency thereof. 


\section{DISCLAIMER}

Portions of this document may be illegible in electronic image products. Images are produced from the best available original document. 


\begin{abstract}
Seismic margin review of nuclear power plants requires that the High Confidence of Low Probability of Failure (HCLPF) capacity be calculated for certain components. The candidate methods for calculating the HCLPF capacity as recommended by the Expert Panel on Quantification of Seismic Margins are the Conservative Deterministic Failure Margin (CDFM) method and the Fragility Analysis (FA) method. The present study evaluated these two methods using some representative components in order to provide further guidance in conducting seismic margin reviews. It is concluded that either of the two methods could be used for calculating HCLPF capacities.
\end{abstract}




\section{CONTENTS}

Page

ABSTRACT iii

PREFACE ix $x i$

1. INTRODUCTION $1-1$

1.1 Background on Seismic Margin Methodology $1-1$

1.2 Definition of Seismic Margin $1-2$

1.3 Objectives of Present Study $1-2$

1.4 Organization of this Study $1-3$

2. METHODS OF CALCULATING HCLPF CAPACITIES 2-1

2.1 Evolution of Methods $2-1$

2.2 Description of CDFM Method $2-4$

2.3 Description of Fragility Analysis Method $2-4$

3. HCLPF CAPACITY CALCULATIONS PERFORMED BY

THE STUDY GROUP 3-1

3.1 Components Selected for Study 3-1

3.2 Ground Rules for Capacity Calculations 3-2

3.3 Ground Motion Aspects 3-3

3.4 Floor Spectrum Aspects 3-3

3.5 Discussion of Failure Modes 3.4

3.6 Results of First Round of Calculations 3-4

3.6.1 Comparison of CDFM and FA HCLPF Capacities 3-5

3.6.2 Comparison of HCLPF and Median Capacities 3-5

3.6.3 Overall Conclusions from the First Round Calculations 3-6

3.7 Results of Second Round of Calculations 3-6 


\section{CONTENTS (Continued)}

Page

4. SUMMARY AND CONCLUSIONS .................................................................................. 4-1

4.1 Summary ........................................................................................................ 4-1

4.2 Conclusions from this Study ................................................................................ 4-2

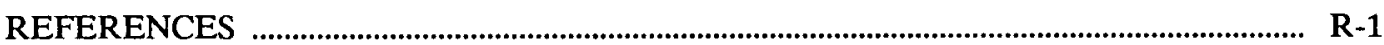

\section{APPENDICES}

\section{CALCULATIONS BY STUDY GROUP MEMBERS}

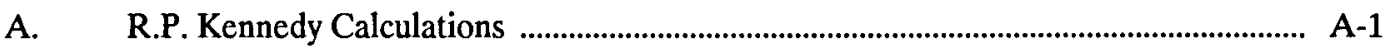

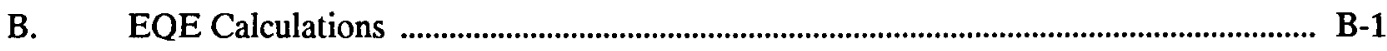

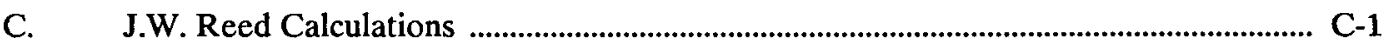

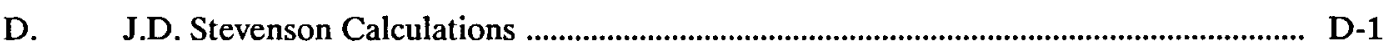

\section{LIST OF TABLES}

2-1 Summary of Conservative Deterministic Failure

Margin Approach ........................................................................................................... 2-6

3-1 Comparison of HCLPF Computations for Representative

Components (First Round Calculations) ........................................................................ 3-7

3-2 Comparison of EPRI CDFM and FA HCLPF (Kennedy and

Ravindra Comparable Computations) …................................................................... 3-9

3-3 Comparison of HCLPF and Median Capacities (First

Round Calculations) ................................................................................................. 3-10

3-4 Comparison of HCLPF Capacity Computations for

Representative Components (Second Round Calculations) ........................................... 3-11

3-5 Comparison of HCLPF and Median Capacities (Second

Round Calculations) …....................................................................................................... 3-13 


\section{LIST OF FIGURES}

Page

2-1 Typical Fragility Curves for a Component …........................................... 2-7

3-1 Flat Bottom Vertical Water Storage Tank ........................................................ 3-14

3-2 Diesel Generator Room Starting Air Tank Supports ................................. 3-15

3-3 Component Cooling Heat Exchanger Supports ......................................... 3-16

3-4 Cantilever Reinforced Block Wall ............................................................... 3-17

3-5 Comparison of GERS with Failure Data: Function

During and After for MCC (ANCO 1987) .................................................... 3-18

3-6 Median Ground Response Spectra Anchored to 0.18 pga. ....................... 3-19

3-7 Horizontal Floor Spectra ........................................................................................ 3-20

3-8 Vertical Floor Spectra ................................................................................ 3-21 


\section{PREFACE}

Seismic margin review, of nuclear power plants require that the High Confidence of Low Probability of Failure (HCLPF) capacity be calculated for certain components. Two candidate methods for calculating the HCLPF capacity have been recommended by the Expert Panel on Quantification of Seismic Margins. The present study evaluated these two methods: the Conservative Deterministic Failure Margin method and the Fragility Analysis method using some representative components in order to provide further guidance in conducting the seismic margin reviews. The following persons have participated in this project.

\section{STUDY GROUP:}

R.P. Kennedy (Chairman),

R.C. Murray,

M.K. Ravindra,

J.W. Reed,

J.D. Stevenson,

\section{OTHERS:}

D.J. Guzy, Project Manager, R.D. Campbell,

P.S. Hashimoto,
R.P.K. Structural Mechanics Consulting Lawrence Livermore National Laboratory EQE Engineering, Inc.

Jack R. Ben jamin and Associates, Inc.

Stevenson \& Associates

U.S. Nuclear Regulatory Commission

EQE Engineering, Inc.

EQE Engineering, Inc. 


\section{EXECUTIVE SUMMARY}

Seismic margin reviews of nuclear power plants require that the High Confidence of Low Probability of Failure (HCLPF) capacity be calculated for certain components. The candidate methods for calculating the HCLPF capacity as recommended by the Expert Panel on Quantification of Seismic Margins are the Conservative Deterministic Failure Margin (CDFM) method and the Fragility Analysis (FA) method. The HCLPF Study Group consisted of a Lawrence Livermore National Laboratory Project Manager and four independent consultants who evaluated these methods by performing the HCLPF capacity calculations on a representative set of components. The components selected were: flat-bottom vertical storage tank, auxiliary contactor for motor starter in an older motor control center, starting air tank, component cooling heat exchanger, and cantilevered reinforced block wall. Two different locations in the building (at grade and at a floor high in the building) were studied for the motor control center. First, the Study Group members calculated the median and HCLPF capacities of these components using either the CDFM or FA method or both. The results of the first round of calculations were reviewed and the sources of differences in the capacities calculated by the members were identified. Afterwards, each investigator was allowed to revise his calculations to determine if closer agreement could be obtained.

The four consultants differed from each other by a median ratio of the high/low values of 1.39 to 1.55 in their final estimates of the HCLPF capacity, the median capacity, and the ratio of median/HCLPF capacity. These differences are mainly due to the differences in models, parameters, and assumptions used by the investigators.

Based on these calculations, the HCLPF Study Group has concluded that any future effort in reducing the differences in calculated HCLPF capacities should be spent in reducing the differences between the assumptions and judgment of different analysts rather than in trying to reconcile the smaller differences between the CDFM and FA methods. It is concluded that either of the two methods could be used for calculating the HCLPF capacities in seismic margin reviews.

The Study Group recommended that HCLPF and median capacity estimates be independently performed for the selected components by a number of representatives from architect-engineering firms. Six Architect-Engineering firms have been selected to make independent calculations on the same five components. This would give an indication of any increased variability in the estimates to be expected in practice in future seismic margin reviews. This effort is expected to be completed in 1989 and a report will be issued summarizing the results and conclusions. 


\section{CHAPTER 1}

INTRODUCTION

\subsection{Background on Seismic Margin Methodology}

In recent years there has been increasing interest in assessing the capability of nuclear power plants in the United States to withstand earthquakes beyond their original design bases. This interest has developed because of the following concerns:

a. The perception of seismic hazard in the plant vicinity has changed and in most cases increased since the design of the plant, and

b. The seismic design criteria have been revised substantially.

To resolve these concerns, a seismic margin study can be performed to estimate the seismic capacity of the plant. Seismic margin study methodology has evolved over the years, beginning with the Systematic Evaluation Program (SEP). More recently, the NRC formed an expert panel to develop a seismic margin review methodology and guidelines for application (Budnitz et al., 1985, Prassinos et al., 1986). A parallel effort was initiated by the Electric Power Research Institute (EPRI 1987). A discussion of the evolution of the seismic margin review methodology is given in Section 2.1 of this report.

A seismic margin review studies the question of whether the capacity of the plant exceeds target earthquake input selected for review. It is assumed that the regulatory agency and the plant owner jointly select the review earthquake level. The objectives are then to show that the plant can withstand the effects of this review earthquake level with high confidence and to identify seismic vulnerabilities. This is accomplished using the results and insights obtained from past seismic Probabilistic Risk Assessments (PRA), the data on actual performance of structures and equipment in recorded earthquakes, and analytical qualification and test data.

Although a seismic PRA would provide answers regarding the seismic capacities of components, systems, and the plant, the large uncertainties in the seismic hazard curves make decisions regarding seismic adequacy difficult. The large number of systems and components to be considered in a PRA limit the attention paid to the more critical components and systems in the plant. The seismic margin review, on the other hand, focuses on the few components and systems in the plant whose failure would lead to severe core damage. The output of a seismic margin review is an estimate of the plant seismic capacity, whereas the seismic PRA provides estimates of seismic risks of core damage and adverse public health effects. 


\subsection{Definition of Seismic Margin}

The concept of a high confidence of low probability of failure (HCLPF) capacity is used in the seismic margin reviews to quantify the seismic margin of a nuclear power plant. This is a conservative capacity, and in simple terms it corresponds to the earthquake level at which, with high confidence, it is extremely unlikely that failure of the component, system, or plant will occur. The use of the term component refers to mechanical and electrical equipment, piping, structural elements, etc. When the component capacity is described in terms of probability distributions, the HCLPF capacity is equal to approximately a 95 percent confidence (subjective probability) of not exceeding approximately 5 percent probability of failure. The concept of HCLPF capacities of components is used in the seismic margin studies in (1) screening out certain components as having capacities generically higher than the review earthquake level and (2) evaluating the capacities of certain critical components in order to assess the seismic capacity of the plant.

Estimating the HCLPF of a component requires estimating the response of the component, conditional on the occurrence of the seismic margin earthquake, and estimating the capacity of the component. Two candidate methods for calculating the HCLPF capacities for components have been recommended: the Conservative Deterministic Failure Margin (CDFM) method and the Fragility Analysis (FA) method (Kennedy, 1984; Prassinos et al., 1986).

The fragility analysis method was used in the Maine Yankee seismic margin study (Ravindra et al., 1987). This method requires evaluation of parameters such as the median, $\beta_{R}$ and $\beta_{U}$ using considerable judgment.

The CDFM method prescribes the parameter values and procedures to be used in calculating the HCLPF capacities and requires less subjective judgment than the FA method, although, some subjective decisions were made in formulating the procedures used in the CDFM method.

\subsection{Obiectives of the Present Study}

The original objectives of this study were to:

- perform a comparison of the HCLPF capacities obtained by using the CDFM and FA methods to study a representative set of

$a$ components;

- modify the details of the CDFM method as given in the EPRI report based on the results of calculations, and

- compare the methods and provide additional guidance for use in seismic margin reviews. 


\subsection{Organization of this Study}

A five-member HCLPF Calculation Study Group was assembled to perform this study. Members of the Group were:

\author{
R.P. Kennedy (Chairman) \\ R.C. Murray \\ M.K. Ravindra \\ J.W. Reed \\ J.D. Stevenson
}

RPK/Structural Mechanics Consulting Lawrence Livermore National Laboratory EQE Engineering Inc. Jack R. Benjamin and Associates, Inc. Stevenson \& Associates

The Study Group laid out the approach followed in this study and performed the capacity evaluation. EQE prepared the draft reports for review and modification by the Study Group.

The Study Group met three times. In the first meeting, the ground rules for the study were established, and a set of five components were selected; the members of the Group agreed to independently calculate the median and HCLPF capacities of these components using either the CDFM or FA method or both. In the second meeting, the results of the first round of calculations were reviewed, and the sources of differences in the capacities calculated by different investigators were identified. Afterwards, each investigator was allowed to revise his calculations to determine if closer agreement could be obtained. A draft report was prepared summarizing the results of the two sets of calculations and the conclusions of the Group. In the third meeting the draft report was reviewed by the Group, and the final report was prepared. This report represents a consensus of all Study Group members. 


\section{CHAPTER 2 \\ METHODS OF CALCULATING HCLPF CAPACITIES}

\subsection{Evolution of Methods}

Kennedy (1984) and Prassinos, et al., (1986) have both recommended two possible approaches for estimating the High-Confidence-Low-Probability-of-Failure (HCLPF) seismic capacity of a component. These approaches are the Conservative Deterministic Failure Margin (CDFM) and the Fragility Analysis (FA) methods. This section gives a brief historical background on both approaches.

Traditionally, the seismic capacity of nuclear power plant components has been very conservatively evaluated by using design evaluation procedures which have gradually changed over the years (generally with increasing conservatism). Current seismic criteria used in the seismic evaluation of more recent plants are contained in the Standard Review Plan (NRC, 1981). At the same time, the perceived ground motion levels to which these plants might be subjected by some future earthquake have also been increasing over the years. Changed perception of ground motion levels and changes in seismic design evaluation procedures have led to questions concerning the seismic resisting capability of components, particularly in the older nuclear power plants. The need for studying the seismic capability of the oldest U.S. nuclear power plants because of these changes was first implemented in the U.S. Nuclear Regulatory Commission (NRC) Systematic Evaluation Program (SEP).

At its inception, NRC senior management recognized that seismic evaluations in the SEP should use evaluation criteria which were less conservative than the seismic design criteria contained in the Standard Review Plan. In 1978 a Senior Seismic Review Team (SSRT) ${ }^{1}$ was retained to provide guidance and assist NRC during seismic evaluation of older plants. A set of more rational but still quite conservative seismic evaluation criteria came out of these reviews. These criteria are documented in the plant-specific reviews of Oyster Creek (Murray, et al., 1981), Ginna (Murray, et al., 1980), Dresden \#2 (Newmark, et al., 1980), Millstone \#1 (Nelson, et al., 1981a), and Palisades (Nelson, et al., 1981b) conducted by the SSRT in 1979 and 1980.

In 1982, as part of the Seismic Safety Margins Research Program, Lawrence Livermore National Laboratory published a Fragilities Development Report (Bohn, et al., 1982) which included an expert opinion survey of fragilities including HCLPF and median estimate for a large number of mechanical and electrical components found in nuclear power plants. An expert opinion or Delphi procedure coupled with plant walkdowns also have been used to evaluate seismic capabilities of DOE facilities at Hanford and Savannah River sites (Becker and Stevenson, 1984).

1 Senior Seismic Review Team consisted of chairman: N.M. Newmark; members: W.J. Hall, R.P. Kennedy, R.C. Murray, and J.D. Stevenson 
In 1983, an extensive Seismic Margin Review was initiated on the Midland Nuclear Power Plant. The SEP seismic evaluation criteria were expanded, slightly modified in conjunction with discussions with the NRC, and documented by Wesley et al. (1983).

During this same period (1978 through 1984), a number of plants were undergoing Seismic Probabilistic Risk Assessments (SPRAs), which necessitated the development of seismic fragility curves for components to define their failure probability estimates. The methodology used in developing these seismic fragility curves was first presented by Kennedy et al. (1980) and expanded upon and updated by Kennedy and Ravindra (1984). This methodology was heavily based upon earlier work of Ang and Newmark (1977) and Cornell and Newmark (1978). The methodology presented in Kennedy et al. (1980) and Kennedy and Ravindra (1984) has become known as the Fragility Analysis (FA) method, and it is called such in Prassinos et al. (1986).

One aspect of the FA method is that it presents for each component a suite of curves (corresponding to different confidence levels) of probabilities of failure versus ground motion levels. This complexity is necessary for use in SPRAs, but it leads to great difficulty in making decisions as to whether an adequate seismic margin exits. Such decisions are easier when only a single conservative but realistic capacity is reported for each component. In order to discuss the adequacy of seismic margins with the NRC staff and the Advisory Committee on Reactor Safeguards (ACRS), it was found useful to convert the information displayed in the seismic fragility curves into a single seismic margin descriptor. The descriptor chosen was the High-Confidence-Low-Probability-of-Failure (HCLPF) capacity, which corresponds to about $95 \%$ confidence of less than about a $5 \%$ probability of failure. Such a descriptor is conservative because there is very little chance of failure below the HCLPF capacity; and yet it is realistic because it is an attempt to describe failure. These HCLPF capacities derived from the FA method fragility curves were first used to interpret the Limerick SPRA results in a Seismic Margin context, and they were subsequently used for the same purpose with the Millstone 3 SPRA results as described in Ravindra et al. (1984). In both cases, these HCLPF capacities were very useful in defining a conservative but adequately high seismic margin capability for each plant.

Although HCLPF capacities obtained from fragility curves using the FA.method proved to be a useful descriptor of seismic margin, several potential deficiencies were identified in the method:

1. The method requires an excessive number of judgments and calculations because a median capacity, a randomness variability factor, and an uncertainty variability factor must each be estimated before the HCLPF capacity can be calculated. When a SPRA is already being performed on a plant, this condition is no deficiency since median, randomness, and uncertainty estimates are required for development of fragility curves to use in the SPRA. However, if one only needs the HCLPF capacity and does not need the entire fragility curve, there should be a more direct way to compute the HCLPF capacity. 
2. There are a very limited number of practitioners making seismic fragility estimates. On the other hand, a large number of qualified engineers have substantial experience in making and reviewing deterministic seismic margin evaluations by using criteria similar to that used in the SEP.

3. Because of the requirement for a significant use of judgment in the estimation of median capacities, randomness, and uncertainty factors, and because of the dependence of the HCLPF capacity on all three, there was a lack of consistency in the estimated HCLPF capacities for different plants or different components in the same plant even when made by the same team of people. This situation is illustrated by the variation in HCLPF capacities reported for similar components in different nuclear plants in Appendix B of Budnitz et al. (1985). Each of these estimates was made by the same team of people following the methodology given in Kennedy et al. (1980) and Kennedy and Ravindra (1984), but the estimates were made at different times from 1978 through 1984, and they are of ten not consistent with each other.

4. At present time there is no consensus methodology available to develop randomness and uncertainty factors in a consistent manner.

Because of the considerations described above, Kennedy (1984) recommended that the HCLPF capacity be directly computed by using deterministic approaches similar to those used in the SEP and the Midland Seismic Margin Review (Wesley et al., 1983). Realistic HCLPF capacities being calculated from fragility curves using the FA method were somewhat more liberal (Kennedy, 1984) than seismic margin capacities obtained when using the deterministic SEP and Midland Seismic Margin Methodologies (Wesley et al., 1983). Therefore, Kennedy (1984) recommended using a Conservative Deterministic Failure Margin (CDFM) methodology to directly obtain HCLPF capacities. CDFM methodology was heavily based upon the SEP and Midland Seismic Margin Methodologies, which Kennedy (1984) called a Code Margin (CM) methodology. The CDFM method was to use the same building and component response criteria as the $C M$ method, which had been laid out and accepted by the NRC for the Midland Seismic Margin Review. However, in lieu of using code seismic capacities, the CDFM method more liberally allowed the use of approximately $84 \%$ exceedance capacities based on actual test data, where such data existed. Furthermore, the CDFM method more liberally allowed the explicit incorporation of an inelastic energy absorption capacity increase factor for ductile failure modes. Otherwise, it was identical to the conventional $\mathrm{CM}$ method which had already had considerable use.

Only a philosophical and sketchy outline of the CDFM method is contained in Kennedy (1984), and a considerable number of details were left open to judgment and interpretation. Subsequently, in an Electric Power Research Institute (EPRI) Seismic Margin Methodology Project (EPRI, 1987), the CDFM method was slightly modified and the details of the approach were expanded. The slight modification consisted of changing the recommendation for structure and equipment damping to be used in response evaluations. Kennedy (1984) recommended such damping be at 
the $84 \%$ exceedance probability; EPRI (1987) recommends that a conservative estimate of the median damping be used. However, the specific example damping values contained in the two references are essentially identical to each other, and this change is therefore primarily a clarification to avoid the introduction of potentially excessive conservative damping values. Even though EPRI (1987) expands on the details of the CDFM method, the method is still not prescriptive in many details and is open to considerable interpretation and judgment, which leads to differences in HCLPF values calculated by different analysts.

Although the CDFM method in its current form (EPRI, 1987) requires fewer computations, is more prescriptive, and requires less subjective judgment than does the FA method in its current form (Kennedy, 1980; Kennedy and Ravindra, 1984; Prassinos et al., 1986), there is debate concerning the degree of reduction in computational effort and the requirement for individual judgments that has resulted. There is also debate about how prescriptive the CDFM and FA methods should become in the future. Undoubtedly, both methods will continue to evolve, and there will be pressure to make them more prescriptive in order to reduce the variability of HCLPF capacity estimates.

\subsection{Description of CDFM Method}

In the CDFM method, a set of deterministic guidelines (e.g., ground response spectra, damping, material strength, and ductility) have been recommended. The HCLPF capacity of the component is determined using these guidelines. The procedure is similar to that used in the Systematic Evaluation Program, although the choice of some of the parameter values (e.g., damping) may be more liberal in the CDFM method. The method is appealing because it is very similar to the design procedures followed in the industry, except that some of the parameter values have been liberalized.

The details of the CDFM method are given in Chapter 2 of the EPRI Seismic Margin Report (EPRI, 1987). The basic approach is to judiciously select the parameter values of different variables (e.g., strength, damping, ductility, load combination, and response analysis methods), taking into account the margins and uncertainties. The object is to obtain a conservative yet somewhat realistic assessment of the capacity. Table 2-1, reproduced from Kennedy (1984), gives the highlights of the CDFM method.

\subsection{Description of Fragility Analysis Method}

In many seismic PRAs, the fragility of a component has been represented by a double lognormal model using three parameters: (1) median ground acceleration capacity $A_{m}$, logarithmic standard deviations $\beta_{R}$ and $\beta_{U}$ representing, respectively, (2) randomness in the capacity and (3) uncertainty in the median value. Using the double lognormal model, the fragility curves as shown in Figure 2-1 are developed. The median capacity, $\beta_{R}$ and $\beta_{U}$, are estimated using designanalysis information, test data, earthquake experience data, and engineering judgment (Kennedy et al., 1980; Kennedy and Ravindra, 1984). The median capacity can be estimated as a product of an overall median safety factor times the SSE peak ground acceleration for the plant. The overall safety factor is a product 
of a number of factors representing the conservatisms at different stages of analysis and design. When the linear scaling of response is not appropriate (e.g., soil sites), the median capacity is evaluated directly using median structural and equipment response parameters, median material properties and ductility factors, median static capacity predictions, and realistic structural modeling and method of analysis.

The HCLPF capacity is calculated using this fragility model as:

$$
\text { HCLPF capacity }=A_{m} \exp \left[-1.65\left(\beta_{R}+\beta_{U}\right)\right]
$$

Further details on the development of $A_{m}, \beta_{R}$, and $\beta_{U}$ values for a given component may be obtained from the cited references. 
Table 2-1

\section{SUMMARY OF CONSER VATIVE DETERMINISTIC FAILURE MARGIN APPROACH}

Load Combination:

Ground Response Spectrum:

Damping:

Structural Model:

Soil-Structure-Interaction:

Material Strength:

Static Capacity Equations:

Inelastic Energy Absorption: (ductility)

In-structure (Floor) Spectra Generation:
Normal + Seismic Margin Earthquake

Conservatively specified (preferably $84 \%$ NonExceedance Probability Site-Specific Spectrum, if available)

Conservative estimate of median damping

Best Estimate (Median) + Uncertainty Variation in frequency

Best Estimate (Median) + Parameter Variation

Code Specified minimum strength or $95 \%$ exceedance actual strength if test data are available.

Code ultimate strength (ACI), maximum strength (AISC), Service Level D (ASME), or functional limits. If test data are available to demonstrate excessive conservatism of code equations, then use $84 \%$ exceedance of test data for capacity equation.

For non-brittle failure modes and linear analysis, use $80 \%$ of computed seismic stress in capacity evaluation to account for ductility benefits, or perform nonlinear analysis and go to $95 \%$ exceedance ductility levels.

Use frequency shifting rather than peak broadening to account for uncertainty plus use median damping. 


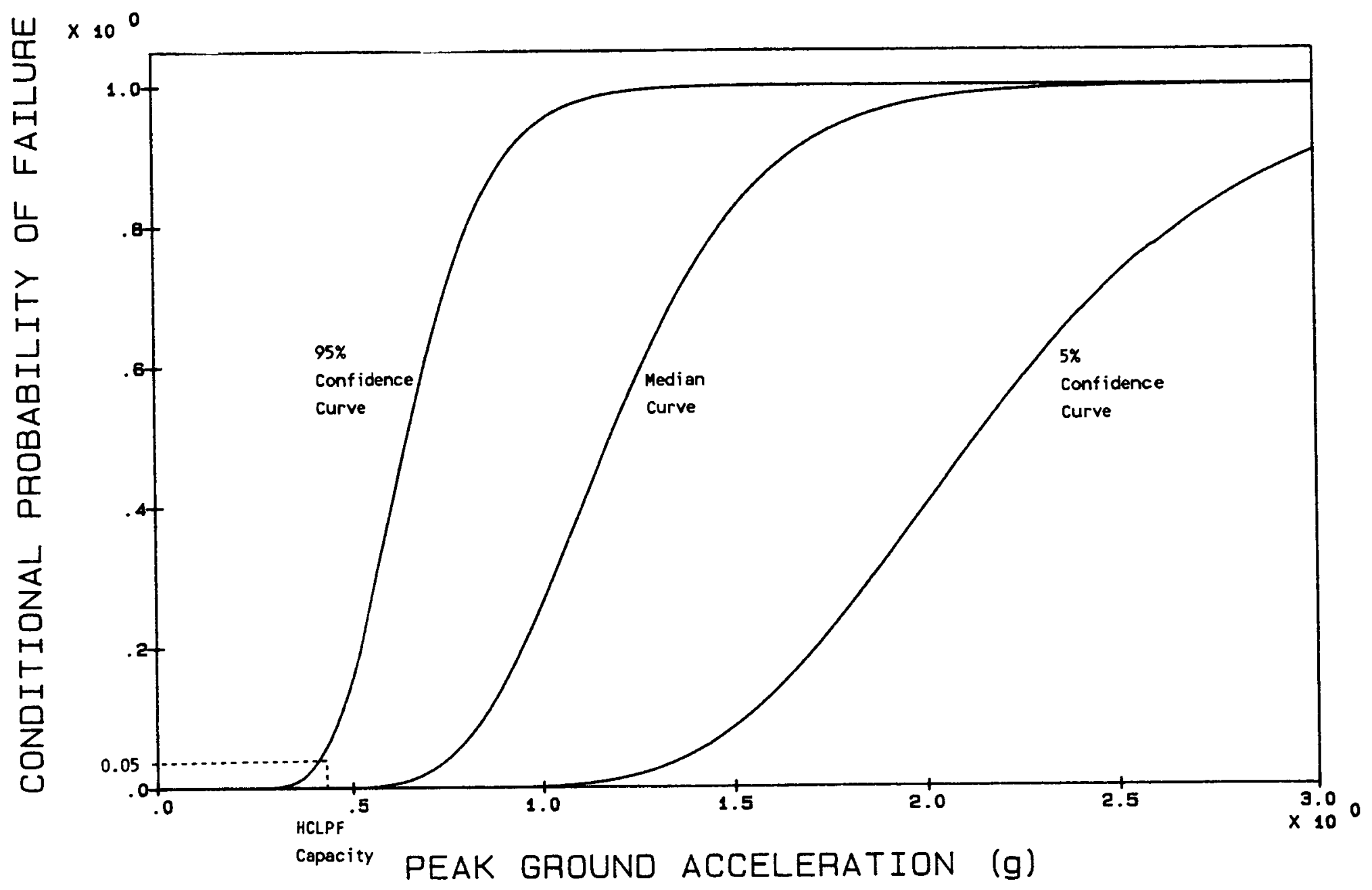

Figure 2-1: Typical Fragility Curves for a Component 
CHAPTER 3

HCLPF CAPACITY CALCULATIONS PER FORMED BY THE STUDY GROUP

The calculations performed by the Study Group are discussed in this chapter. The basis for selection of components, description of components, and ground rules agreed upon by the Group for calculating the capacities are described. The results of the first round of calculations and the underlying reasons for differences in the capacities calculated by the investigators are discussed. The results of the second round of calculations are presented along with an analysis of the differences in the HCLPF capacities and median capacities as reported by the Study Group members.

\subsection{Components Selected for Study}

The objectives of the study were to compare the HCLPF capacity calculations performed by different analysts using either the CDFM method or the FA method. The comparison was performed by selecting a set of representative components. Also, the purpose of the comparison study was to modify the HCLPF capacity determination procedures if needed.

Components for which HCLPF capacity estimates are likely to be made in future seismic margin reviews include:

$\begin{array}{ll}\text { o } & \text { Block walls } \\ \text { o } & \text { Heat exchangers } \\ \text { o } & \text { Tanks } \\ \text { o } & \text { Active electrical equipment } \\ \text { o } & \text { HVAC fans and cooler units }\end{array}$

Based on a review of these components, the Study Group selected the following components for performing seismic capacity calculations.

1. Flat-Bottom Vertical Water Storage Tank at grade. The failure mode to be considered is the combined bolt yielding and shell buckling. Water hold down force and the fluid stabilization effect should be taken into account.

2. Auxiliary Contactor Chatter for Motor Starter in an older Motor Control Center (MCC). This example will focus on equipment qualified using Generic Equipment Ruggedness Spectrum (GERS). The failure mode to be investigated is the auxiliary contactor chatter. No qualification test data exists since the component is assumed to be in an older plant. The cabinet is well anchored and calculations were performed for cabinets mounted at grade and high up in the building. It is assumed to have an estimated frequency of $6.5 \mathrm{~Hz}$. It was agreed that the seismic HCLPF capacity would be based upon the "Function-during GERS." 
3. Diesel Generator Room Starting Air Tank mounted high in the structure. This is an example of a vertical skirt-mounted pressure vessel on a concrete floor high up in the building. The failure mode to be investigated is the anchorage or support failure and not skirt buckling.

4. Component Cooling Heat Exchanger mounted high in the structure. This is an example of a component governed by ASME rules. It is a horizontal heat exchanger fixed on one saddle support and free longitudinally at the other support. It is assumed to be bolted to a rigid support frame. The area of concern is the saddle and anchorage.

5. Cantilevered Reinforced Block Wall mounted high in the structure. This example is selected to avoid the case of a wall where arching action may be present. If arching action is present, the wall would have higher capacity; therefore a cantilevered wall with reinforcing steel is chosen.

Three tanks or vessels (air receiver tank, heat exchanger, and flat-bottom vertical tank), one structural component (reinforced block wall) and one electrical component (auxiliary contactor in motor starter) are included in this set. The analysis includes two different locations in the building (at grade and at a floor high in the building). This set covers typical features of components that may require seismic margin evaluation.

Figures 3-1 through 3-4 schematically illustrate the important seismic capacity aspects of the storage tank, starting air receiver tank supports, heat exchanger supports, and block wall, respectively. Figure 3-5 shows the "Function-during" GERS as reproduced from the ANCO Report (1987).

The question of the adequacy of this set for comparing the CDFM and FA methods was discussed. The Study Group judged that substantial understanding of the applicability of the methods could be obtained by focusing on this limited set of components. Another important feature being investigated is how different investigators would apply the CDFM and FA methods to identical components and how much variation could be expected between the capacity predictions by different investigators.

\subsection{Ground Rules for Capacity Calculations}

The following ground rules were established for performing the seismic capacity calculations:

- Each consultant agreed to perform seismic capacity calculations on the 5 components listed above using methods he would use in production type Seismic Margin Review computations.

- Each consultant agreed to use the same structural properties for each component. These are illustrated in Figures 3-1 through 3-4. 
- Both median and HCLPF capacities of the components were evaluated. In the fragility analysis, the median capacity, and $\beta_{R}$ and $\beta_{U}$ were estimated.

- In the first round of calculations, Kennedy and Ravindra agreed to use both CDFM and FA methods. Reed agreed to calculate the capacity using the FA method. Stevenson calculated the HCLPF capacity using the EPRI (1987) method with a probabilistic procedure used to determine median values (Stevenson 1985). Thus comparisons exist between different investigators using the same method and between the same investigators using the CDFM and FA methods.

- As a separate study, Reed took the results from the FA approach and determined the CDFM input necessary to derive the same HCLPF capacity; the purpose was to investigate the distribution of conservatisms in different parts of the CDFM method. The results of this study are included in Reed's calculations in the Appendix.

\subsection{Ground Motion Aspects}

For all HCLPF capacity computations, it was agreed to assume that the ground motion for the largest horizontal component was given by a uniform hazard spectrum defined at the $84 \%$ non-exceedance probability (NEP) at all frequencies. Furthermore, this uniform hazard spectrum shape was to be defined by the NUREG/CR-0098 (Newmark and Hall, 1978) median spectrum shape for rock sites (Figure 3-6). The $84 \%$ vertical response spectrum was then defined to be equal to $2 / 3$ of the $84 \%$ NEP largest horizontal response spectrum.

The result of using the $84 \%$ NEP largest horizontal component response spectrum to determine the HCLPF capacity is that this capacity is conditional on this response spectrum not being exceeded at more than $16 \%$ of the frequencies in the frequency range and directions that dominate the component capacity.

The above ground motion information is sufficient to enable HCLPF capacity computations to be made using the CDFM method. However, the Fragility Analysis method requires additional description of the random variability of the ground motion response spectrum. Variability associated with peaks and valleys of actual response spectrum and directional response variability should be included. Each consultant using the Fragility Analysis method was expected to make his own estimate of these sources of response spectrum variability.

\subsection{Floor Spectrum Aspects}

It was agreed that the floor spectra shown in Figures 3-7 and 3-8 would be used to represent floor spectra obtained high in a structure from a median centered building response model subjected to median NUREG/CR-0098 response spectra (Figure 3-6) anchored to a horizontal peak ground acceleration (PGA) of $0.18 \mathrm{~g}$ (this was assumed to be the $84 \%$ NEP ground response spectrum as in Section 3.3). 
Figures 3-7 and 3-8 represent horizontal and vertical floor response spectrum respectively. Since these floor spectra are assumed to represent unbroadened median response to an $84 \%$ NEP ground motion input, they may be used directly without scaling in the CDFM method or the Fragility Analysis method for a review earthquake of $0.18 \mathrm{~g}$ PGA. Linear scaling is used for other earthquake levels.

\subsection{Discussion of Failure Modes}

In the evaluation of seismic margin or seismic fragilities, it is important to define what is meant by failure of the component. In the following, the failure modes as identified by the Study Group members are discussed for the components analyzed.

Flat Bottom Tank. Failure of the vertical storage tank is defined to be gross loss of fluid contents. Horizontal seismic load initiates uplift of the tank shell from its foundation. This uplift is resisted by the anchor bolts, the tank bottom plate, and the tank weight. The anchor bolts are permitted to yield, so long as their behavior is ductile, since yielding does not directly result in loss of fluid contents. Shell compressive stresses progressively increase until buckling occurs.

Motor Control Centers. A functional failure mode associated with the motor control centers was assumed to be governed by chatter of auxiliary contactors for purposes of this study. This may result in spurious signals and may adversely affect any equipment controlled by auxiliary contactors.

Starting Air Receiver Tank. The starting air receiver tank is a vertical, skirtsupported cylindrical tank which is anchored to the building floor by three angles welded to the tank skirt and bolted to the floor. The leg of the angle was found to be much weaker in bending than the anchor bolts. The angle leg is very ductile in bending, and the failure mode is tearing the mounting angles. When this occurs, the air tank is assumed to fail through failure of attached piping.

Horizontal Heat Exchanger. The failure mode governing the median capacity of the horizontal heat exchanger was combined tension and shear induced failure of the anchor bolts. Tension results from overturning of the heat exchanger in the lateral direction, while shear results from inertial loads in both horizontal directions. When this anchorage failure occurs, the heat exchanger is assumed to fail through failure of the nozzles and attached piping.

Block Wall. The block wall is represented as a vertical cantilever fixed at its base. Failure is collapse or excessive lateral distortion of the wall.

\subsection{Results of First Round of Calculations}

The four investigators independently estimated the HCLPF and median seismic capacities of the components described in Section 3.1. The results of the calculations are summarized in Table 3-1, and the complete set of calculations are included in the Appendix. Kennedy and Stevenson initially performed the calculations using deterministic methods; Ravindra and Reed initially performed the calculations using the Fragility Analysis method. Kennedy also calculated the HCLPF capacities of components using the Fragility Analysis method; Ravindra 
also independently calculated the HCLPF capacities using the deterministic method. Kennedy, Ravindra and Stevenson performed the deterministic HCLPF calculations using their individual interpretations of the CDFM method as described in the EPRI Seismic Margin Methodology report (EPRI, 1987). Therefore, their results are directly comparable for the EPRI CDFM method. Kennedy, Reed and Ravindra each performed fragility analysis computations using their independent interpretations of the parameters involved, but they used a common methodology so that all HCLPF capacities computed by the FA method are comparable.

Failure modes for different components identified by the investigators are also included in Table 3-1. In general, the same failure mode was identified for each component type; however, there were differences in the estimation of the seismic capacity of the component for the identified failure mode.

\subsubsection{Comparison of CDFM and FA HCLPF Capacities}

Since Kennedy and Ravindra performed both CDFM and FA calculations following identical approaches (although making different judgments in their application), Table 3-2 compares the range of their two sets of results by both methods. This comparison was judged to be the most appropriate for HCLPF capacities by the CDFM and FA methods.

In all of the example cases, the CDFM method produced less difference in the HCLPF capacity estimates between Kennedy and Ravindra than did the FA estimate. This is to be expected since the parameters of the CDFM method are more specified than the parameters used in the $F A$ method. The ratio of High/Low estimates by the CDFM method ranged from 1.00 to 1.29 with a median value of 1.11. For the FA method, this ratio ranged from 1.11 to 1.57 with a median of 1.30 .

In all of Kennedy's computations the FA method produced a higher HCLPF capacity than did the CDFM method. In all of Ravindra's computations the FA method produced a lower or equal HCLPF capacity than did the CDFM method. Therefore, there is no conclusion as to whether the current CDFM method is more or less conservative than the FA method for estimating the HCLPF capacities.

\subsubsection{Comparison of HCLPF and Median Capacities}

The range of HCLPF capacities from the four investigators was larger than expected. The ratio of high to low HCLPF capacity estimates ranged from 1.23 to 1.82, with a median of 1.48 (Table 3-3). The differences in estimated HCLPF capacities between the four investigators was much larger than the differences in results between the CDFM and FA methods, and this was due primarily to differences in the subjective judgment and personal experience of the investigators. The differences were due to the relative degree of conservatism introduced into each investigator's computations.

Each investigator also independently estimated the median capacities. The high to low ratio of median capacity estimates ranged from 1.23 to 2.13 , with a median of 
1.47. Thus, there was no closer agreement in the estimates of the HCLPF capacities than there was in the estimates of the medians. This conclusion was surprising and contradictory to the judgment of the NRC Expert Panel on Seismic Margins, which believed that greater agreement would exist in the computations of HCLPF capacities than for median capacities.

There was also essentially as large a difference in the estimates of the ratio of median/HCLPF capacity. For the two components mounted at grade, the median of this ratio was 2.39 , but the range for the four investigators was 1.96 to 4.05 . For the four components high in the structure, the median ratio was 3.07 but ranged for the four investigators from 2.23 to 5.14. The ratio of the High/Low prediction of the ratio of median/HCLPF capacity for all components ranged from 1.25 to 1.80 , with a median of 1.65 . Therefore, even if the four investigators had agreed on estimates of either the median or HCLPF capacities, they would have had equally substantial differences in converting medians to HCLPF capacities, or vice versa.

\subsubsection{Overall Conclusions from the First Round Calculations}

The results of the first round calculations indicate that differences in the HCLPF capacity calculations using the CDFM method and the FA method are indeed small relative to the differences in capacity calculated by four independent investigators. The four investigators differ from each other by a median ratio of about 1.5 to 1.7 for estimates of the HCLPF capacity, the median capacity, and the ratio of median/HCLPF capacity. Thus, all three quantities are nearly equally uncertain. Hence, it was concluded that further comparisons of the HCLPF capacity calculations by the CDFM method versus the FA method is not beneficial. Further study should be directed at achieving greater agreement between investigators as opposed to refining differences between the CDFM and FA methods. The major differences are in the selection of models and parameter values.

\subsection{Results of Second Round of Calculations}

Based on a review of the first round calculations and subsequent discussions by the Study Group, the investigators were allowed to revise their calculations and state the HCLPF and median capacity for each component. The results of this round of calculations are summarized in Table 3-4. Further details are included in the Appendix. A review of the two sets of calculations (Tables 3-1 and 3-4) has indicated that the investigators converged in those cases where the differences were not initially large (flat bottom storage tank, starting air tank, and heat exchanger). In those cases where the differences were initially large, the second round calculations did not bring the capacities closer; in fact they moved farther apart in some cases (cabinet high up, and blockwall).

The four investigators differ from each other by a median of the high/low ratios of 1.39 to 1.55 for estimates of the HCLPF capacity, the median capacity, and the ratio of median/HCLPF capacity, (See Table 3-5). As before, these differences are mainly due to the differences in models, parameters, and assumptions used by the investigators. 
Comparison of HCLPF Computations for

Representative Components (First Round Calculations)

\begin{tabular}{|c|c|c|c|c|}
\hline \multirow{2}{*}{ Component } & \multicolumn{2}{|c|}{ HCLPF Cepacity (g) } & \multirow{2}{*}{$\begin{array}{l}\text { Median } \\
\text { Capacity } \\
\text { (g) }\end{array}$} & \multirow{2}{*}{ Failure Mode } \\
\hline & $\operatorname{CDFM}$ & FA & & \\
\hline \multicolumn{5}{|c|}{$\begin{array}{l}\text { Flat Botton storage Tank } \\
\text { (At Grade) }\end{array}$} \\
\hline RPK & 0.29 & 0.31 & $0.67 \mid$ & Combination of shell buckling \\
\hline MKR/PSH & 0.29 & 0.26 & 0.54 & and anchor bolt yields \\
\hline JWR & $\cdots$ & 0.27 & $0.53)$ & \\
\hline JDS & 0.32 & $\cdots$ & 1.13 & rield of anchor bolts \\
\hline
\end{tabular}

Auxilliary Contector Chatter

(Function during GERS lock-in

circuit potential)

a) Cobinet at Grado

$\begin{array}{lllll}\text { RPK } & 0.54 & 0.59 & 1.26 & \text { Contactor chatter } \\ \text { MKR/RDC } & 0.47 & 0.39 & 1.58 & \text { Contactor chatter } \\ \text { JWR } & \ldots . . & 0.48 & 1.20 & \text { Contactor Chatter } \\ \text { JDS } & 0.71 & \ldots . . & 1.88 & \text { Contactor Chatter }\end{array}$

b) Cobinet High-up

$\begin{array}{lllll}\text { RPK } & 0.10 & 0.11 & 0.30 & \text { Contactor Chatter } \\ \text { MKR/RDC } & 0.09 & 0.07 & 0.36 & \text { Contactor Chatter } \\ \text { JWR } & \cdots . . & 0.11 & 0.43 & \text { Contactor Chatter } \\ \text { JDS } & 0.12 & \cdots & 0.43 & \text { Contactor Chatter }\end{array}$

RPK Did calculations by CDFM first, then FA.

MKR/ROC/PSM Did calculation by FA first, then CDFM.

JWh Tabulated values are from HCLPFs calculations using input spectro as 84X MEP maximum horizontal direction.

JDS Did Calculations by CDFM. 


\begin{tabular}{|c|c|c|c|c|c|}
\hline \multirow{2}{*}{ Component } & HCLPF & Copacity (g) & \multirow{2}{*}{$\begin{array}{l}\text { Median } \\
\text { Capecity } \\
\text { (g) }\end{array}$} & \multirow{2}{*}{ Failure Mode } & \multirow{2}{*}{ Comments/Remarks/Assumptions } \\
\hline & CDFM & $\mathbf{F A}$ & & & \\
\hline
\end{tabular}

Starting Air Tank

(High-up)

$\begin{array}{llll}\text { RPK } & 0.48 & 0.50 & 1.07 \\ \text { MKR/ROC } & 0.53 & 0.44 & 1.55 \\ \text { JWR } & \cdots .- & 0.43 & 1.40 \\ \text { JDS } & 0.39 & \cdots & 1.04\end{array}$

Heat Exchanger CHigh-

\section{up, Bolted to Rigid}

Support Frame)

RPK

MKR/RDC

JWR

Jos

Block Hall (High-up)

RPK

MKR/PSH

JWR

JDS
0.40

0.42

1.18

$0.64 \quad 0.38$

… $\quad 0.39$

1.08

1.00

0.30

0.96
Plestic Bending of Mounting Angles

Plestic Bending of Mounting Angles

Plastic Bending of Mounting Angles

Plastic Bending of Mounting Angles

RPK Did calculations by COFM first, then FA.

MKR/RDC/PSH Did calculations by FA first, then CDFM.

JWR Tobulated values ore from HCLPF capacity calculations using input spectra as $84 \%$ NEP maximum horizontal direction.

JoS Did Calculations by COFM.

Anchor Bolt Shear Follure:

failure through the threads

Anchor Bolt Shear a Tension Faflure

Anchor Bolt Shear Failure

failure through the threads

Anchor Bolt Shear Failure

$\begin{array}{llll}0.62 & 0.67 & 1.94 & \text { out-of-Plane Bending } \\ 0.48 & 0.48 & 1.55 & \text { Out-of-Plane Bending } \\ \cdots . . & 0.38 & 1.41 & \text { out-of-Plane Bending } \\ 0.51 & \cdots . . & 1.34 & \text { out-of-Plane Bending }\end{array}$


Table 3-2

Comparison of EPRI CDFM and FA HCLPF

(Kennedy and Ravindra Comparable Computations)

\begin{tabular}{|c|c|c|c|c|}
\hline & \multicolumn{2}{|c|}{ HCLPF Range (g) } & \multicolumn{2}{|c|}{ High/Low Ratio } \\
\hline & CDFM & FA & CDFM & FA \\
\hline Flat Bottom Tank & 0.29 & $0.26-0.31$ & 1.00 & 1.19 \\
\hline Cabinet at Grade & $0.47 \cdot 0.54$ & $0.39-0.59$ & 1.15 & 1.51 \\
\hline Cabinet on Floor & $0.09-0.10$ & $0.07-0.11$ & 1.11 & 1.57 \\
\hline Air Tank & $0.48-0.53$ & $0.44-0.50$ & 1.10 & 1.14 \\
\hline Heat Exchanger & $0.40-0.44$ & $0.38-0.42$ & 1.10 & 1.11 \\
\hline \multirow[t]{3}{*}{ Block Walls } & $0.48-0.62$ & $\underline{0.48-0.67}$ & 1.29 & 1.40 \\
\hline & & Mean & 1.13 & 1.32 \\
\hline & & Median & 1.11 & 1.30 \\
\hline
\end{tabular}


Table 3-3

Comparison of HCLPF and Median Capacities

First Round Calculations

\begin{tabular}{|c|c|c|c|c|c|c|c|}
\hline \multirow[b]{2}{*}{ Component } & \multicolumn{2}{|c|}{ Range $(g)$} & \multicolumn{2}{|c|}{ High/Low Ratio } & \multicolumn{3}{|c|}{ Median/HCLPF Ratio } \\
\hline & HCLPF & Median & HCLPF & Median & Median & Range & High/Low Ratio \\
\hline Flat Bottom Tank & $0.26-0.32$ & $0.53-1.13$ & 1.23 & 2.13 & 2.20 & $1.96-3.53$ & 1.80 \\
\hline Cabinet ot Grade & $0.39-0.71$ & $1.20-1.88$ & 1.82 & 1.57 & 2.58 & $2.33-4.05$ & 1.74 \\
\hline Cabinet on floor & $0.07-0.12$ & $0.30-0.43$ & 1.71 & 1.43 & $3 . \pi$ & $3.00-5.14$ & 1.71 \\
\hline Air Tank & $0.39-0.48$ & $1.04-1.55$ & 1.23 & 1.49 & 2.96 & $2.23-3.52$ & 1.58 \\
\hline Heat Exchanger & $0.30-0.40$ & $0.96-1.18$ & 1.33 & 1.23 & 2.90 & $2.56-3.20$ & 1.25 \\
\hline \multirow[t]{3}{*}{ Block Walls } & $0.38-0.62$ & $1.34-1.94$ & 1.63 & 1.45 & 3.18 & $2.63-3.71$ & 1.41 \\
\hline & & Mean & 1.49 & 1.55 & & & 1.58 \\
\hline & & Median & 1.48 & 1.47 & & & 1.65 \\
\hline
\end{tabular}


Table 3-4

Comparison of HCLPF Capacity Computations for

Representative Components (Second Round Calculations)

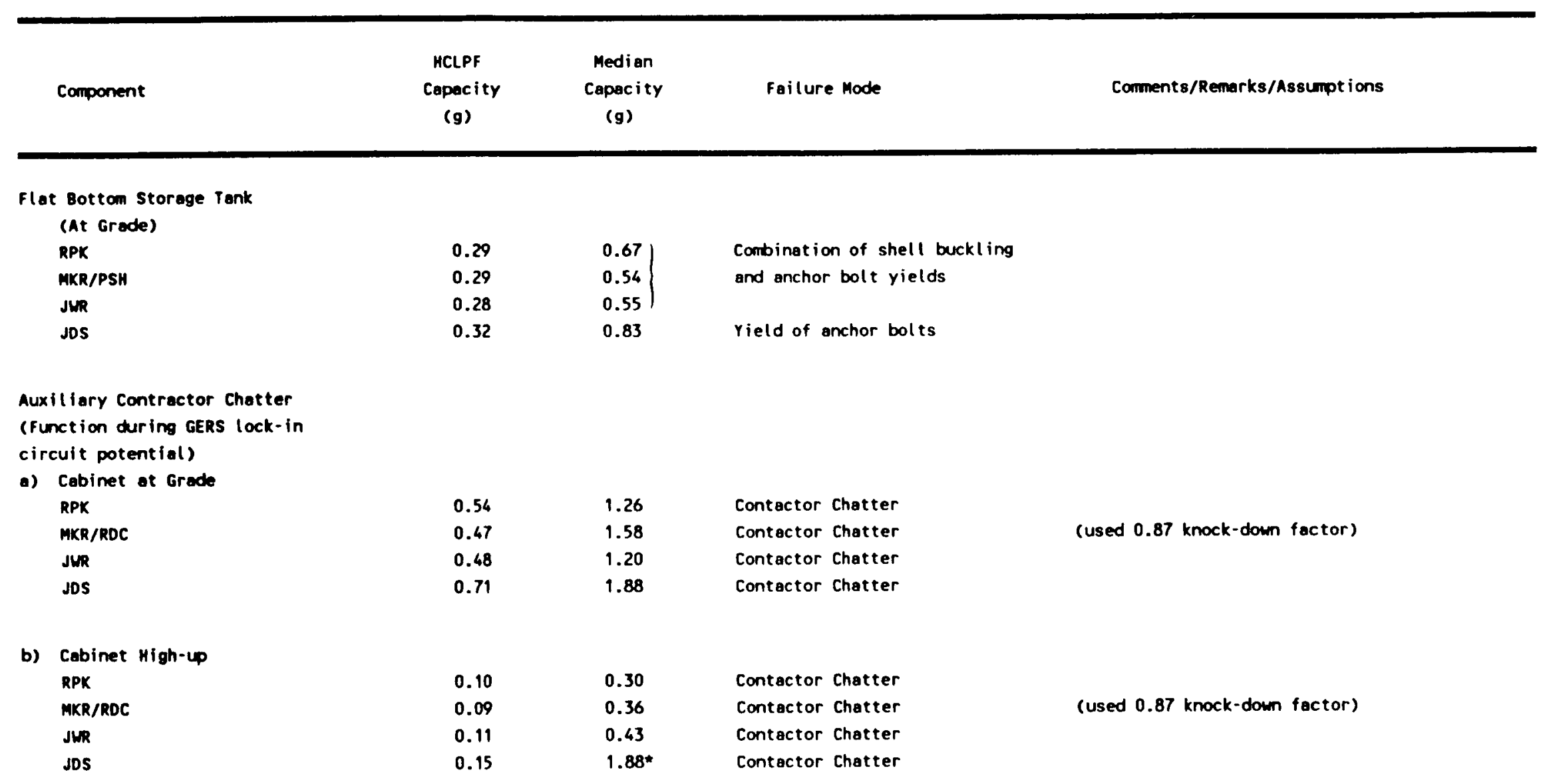

- See calculations (Appendix) for further explanation. 
Table 3-4 (Cont inued)

Comparison of HCLPF Capacity Computations for

Representative Components (Second Round Calculations)

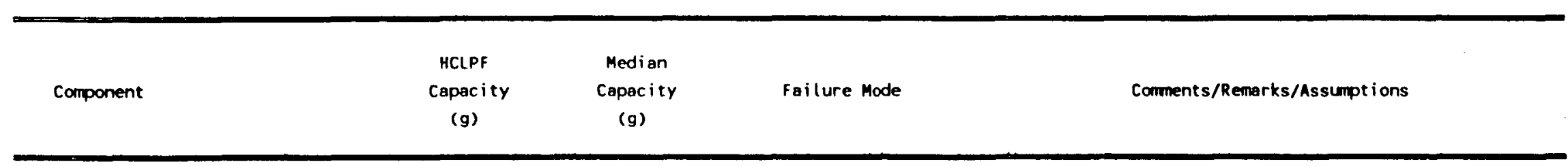

Starting Air Tank

(High-up)

RPK

MKR/ROC

JWR

$0.48 \quad 1.07$

1.07

$0.53 \quad 1.55$

$0.43 \quad 1.40$

JDS

0.42

1.10

Plastic Bending of Mounting Angles

Plastic Bending of Mounting Angles

Plastic Bending of Mounting Angles

Plastic Bending of Mounting Angles

Heat Exchanger (HIgh-up,

Bolted to Rigid

Support Frame)

RPK

0.40

1.18

Anchor Bolt Shear failure;

MKR/RDC

0.44

1.08

failure through the threads

Anchor Bolt Shear \& Tension Failure

Anchor Bolt Shear Failure:

failure through the threads

JDS

0.44

Anchor Bolt Shear Failure

Block Hall (High-up)

RPK

MKR/PSH

JWR

JDS
0.62

0.63

0.52

0.32
1.94

2.10

1.96

1.30
Out-of-Plane Bending

Out- of-Plane Bending

Out-of-Plane Bending

Out-of-Plane Bending 
Table 3-5

Comparison of HCLPF and Median Capacities

Second Round Calculations

\begin{tabular}{|c|c|c|c|c|c|c|c|}
\hline \multirow[b]{2}{*}{ Component } & \multicolumn{2}{|c|}{ Renge $(g)$} & \multicolumn{2}{|c|}{ High/Low Ratio } & \multicolumn{3}{|c|}{ Median/HCLPF Ratio } \\
\hline & HCLPF & Median & HCLPF & Median & Median & Range & High/Low Ratio \\
\hline Flat Botton Tank & $0.28-0.32$ & $0.54-0.83$ & 1.14 & 1.54 & 2.13 & $1.86-2.59$ & 1.39 \\
\hline Cabinet at Grade & $0.47-0.71$ & $1.20-1.88$ & 1.51 & 1.57 & 2.58 & $2.33-3.36$ & 1.44 \\
\hline Cabinet on floor & $0.09-0.15$ & $0.30-1.88$ & 1.67 & 6.27 & 3.96 & $3.00-12.53$ & 4.18 \\
\hline Air Tank & $0.42 \cdot 0.53$ & $1.07-1.55$ & 1.26 & 1.45 & 2.77 & $2.23 \cdot 3.26$ & 1.46 \\
\hline Heat Exchanger & $0.39-0.44$ & $1.00-1.18$ & 1.13 & 1.18 & 2.59 & $2.45-2.95$ & 1.20 \\
\hline \multirow[t]{3}{*}{ Block Walls } & $0.32-0.63$ & $1.30-2.10$ & 1.97 & 1.62 & 3.55 & $3.13-4.06$ & 1.30 \\
\hline & & Mean & 1.45 & 2.27 & & & 1.83 \\
\hline & & Median & 1.39 & 1.55 & & & 1.42 \\
\hline
\end{tabular}


MATERIALS.

SHELL S CHAIAS: SA24O. TYPE 304 SST BOLTS: A $307 \cdot 2 " \varnothing$

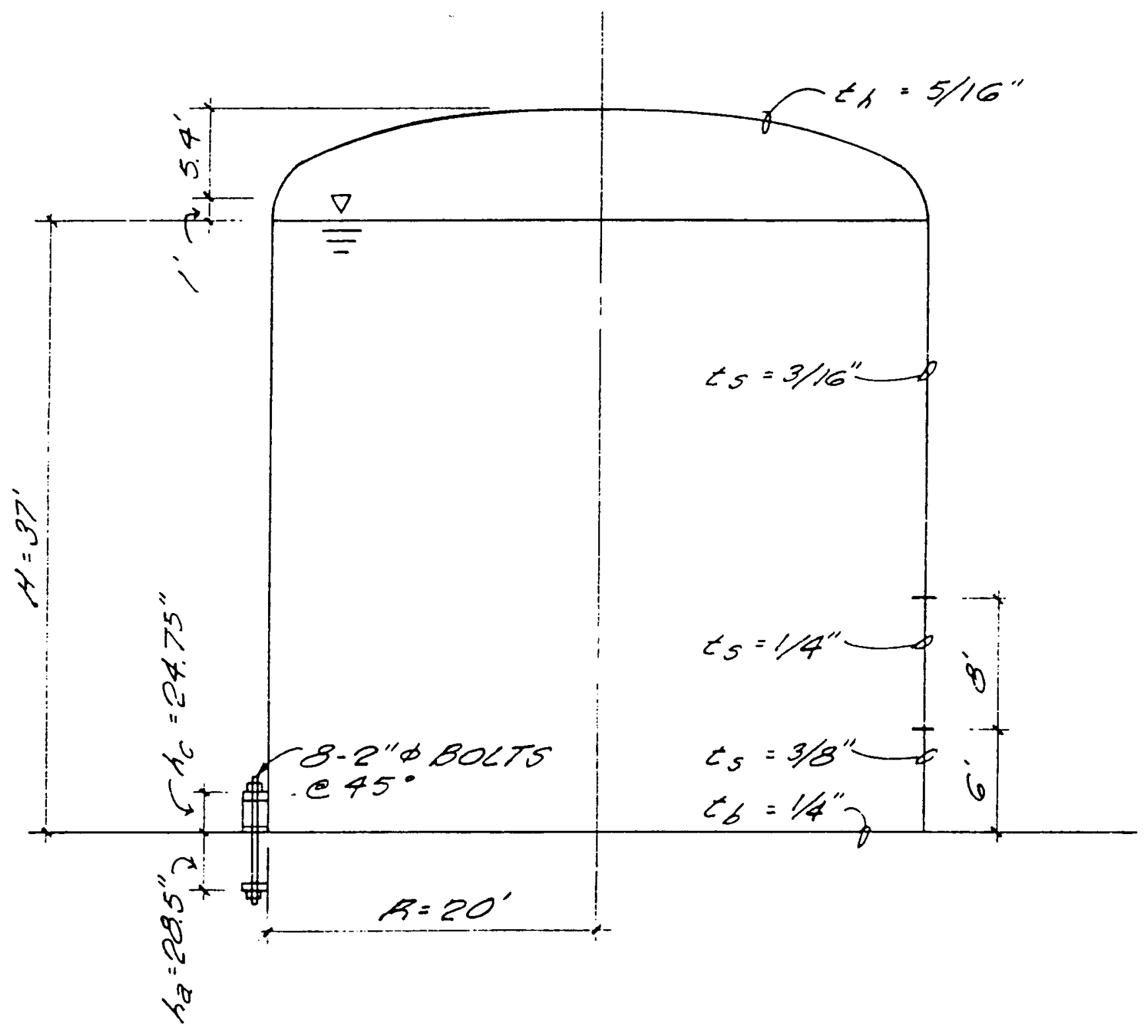

Figure 3-1: Flat Bottom Vertical Water Storage Tank. 


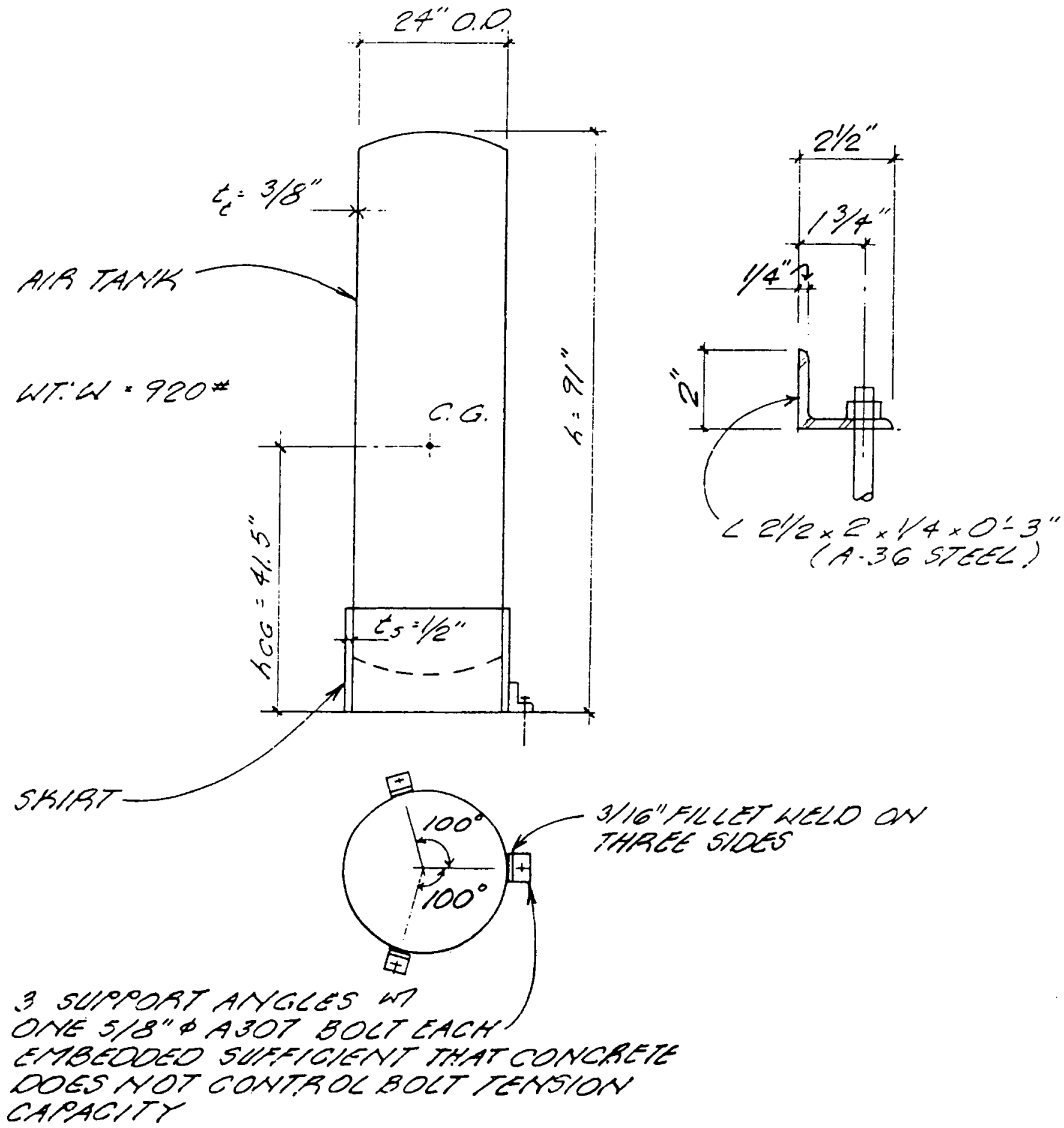

Figure 3-2: Diesel Generator Room Starting Air Tank Supports. 

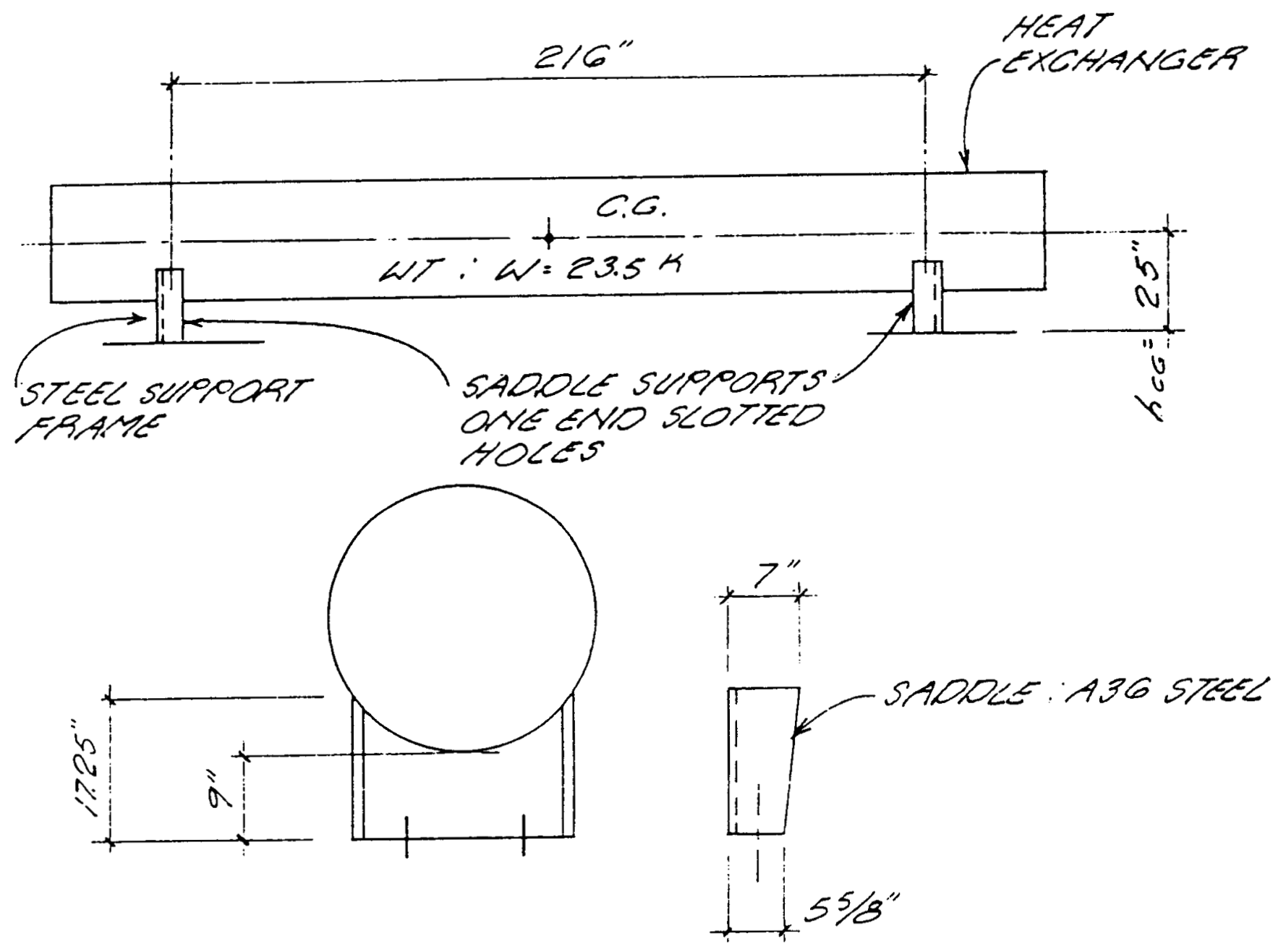

2. $7 / 8 " \phi A 307$

$80<55$

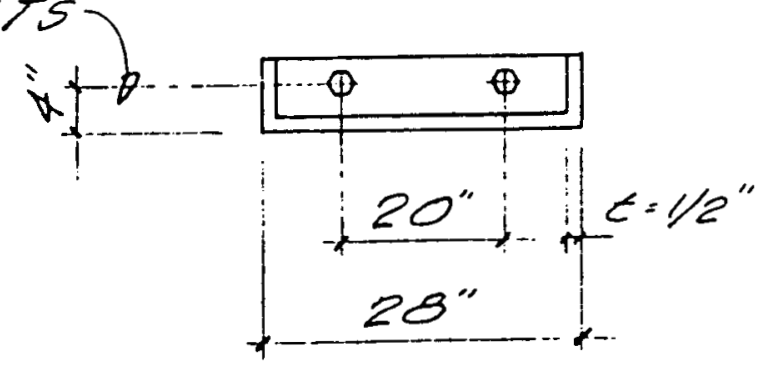

Figure 3-3: Component Cooling Heat Exchanger Supports. 

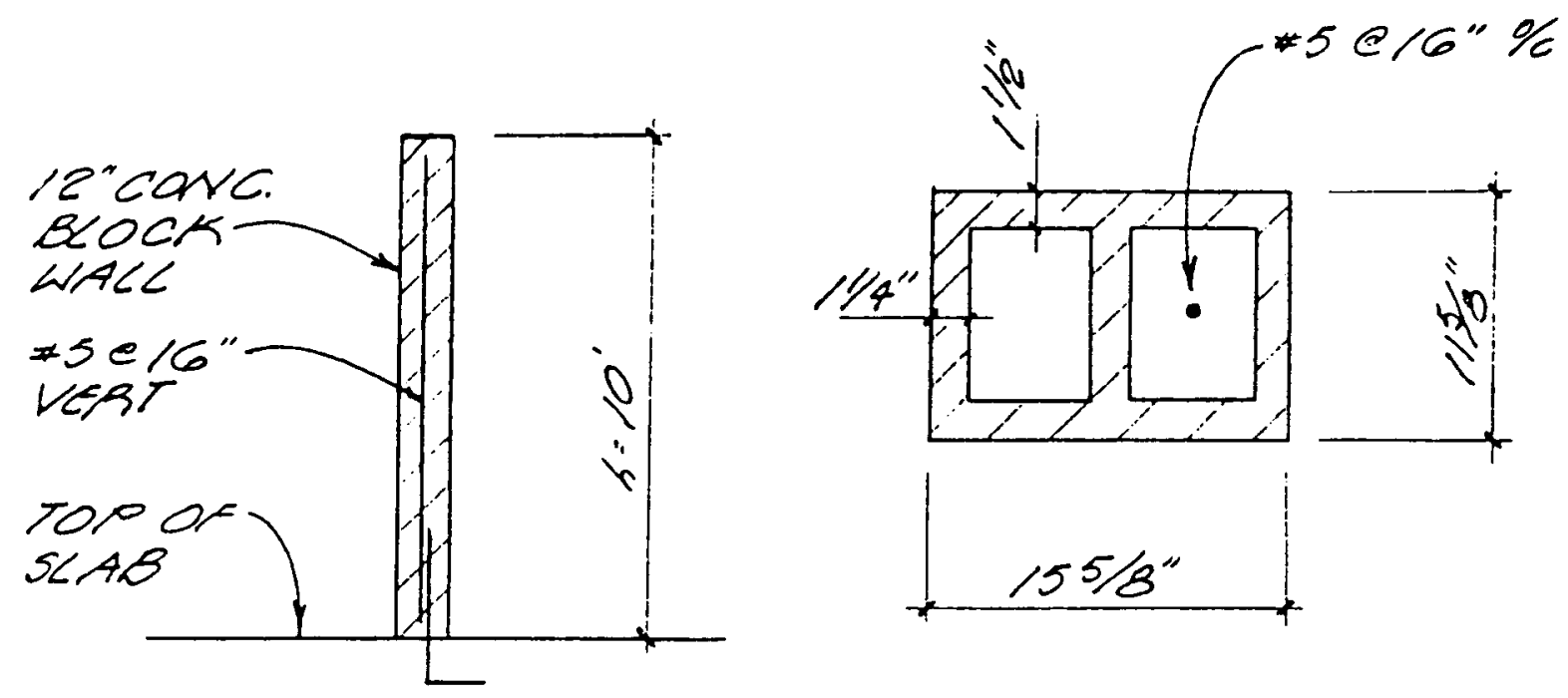

SPECIAL INSPECTION BEQUIREMENT, ARE MET.

WALL IS CONSTRUCTED IN BUNWING BOND CLLLS WD REINF GROUTED SOLID

BLOCH: 12 "HOLLOW UNITS, MOMMAL WEIGHT. ASTM C9O GAAOEN $F_{C}^{\prime}=3000 \mathrm{PSI}$ MORTAR: ASTM CPTO TYPES

VEAT. AEINF: \# $516 " \%$ GAADE 60

HORIZ. REINF: EXTSA HEAVY, 3 ROD DUROWAL $16 " \%$ WALL LONG COMPARED TO HEIGHT: ACTS AS VEATICAL GANTILEVEA

WALC WT: $W=$ III DSF

Figure 3-4: Cantilever Reinforced Block Wall. 


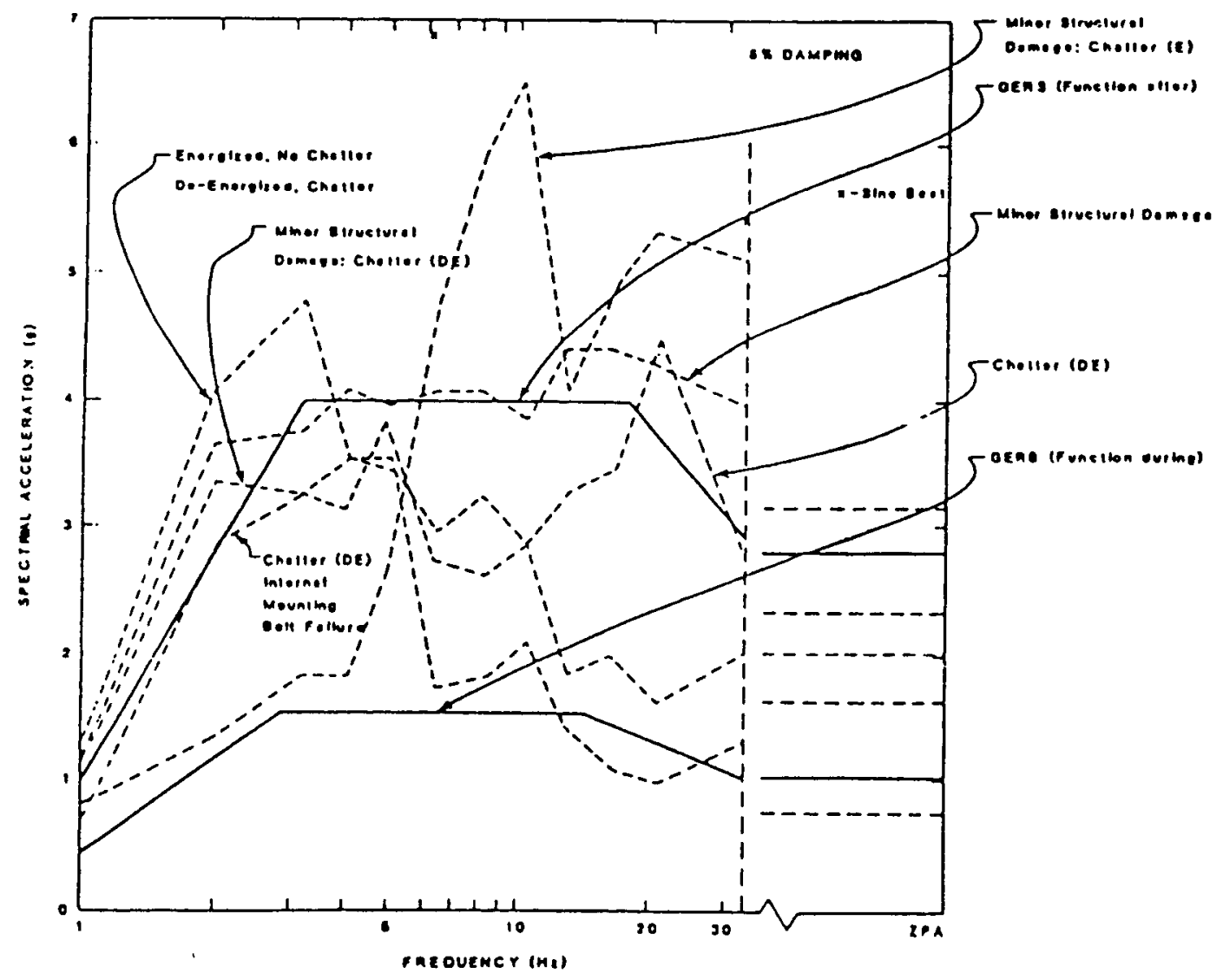

Figure 3-5: Comparison of GERS with Failure Data: Function During and After for MCC (ANCO 1987) 


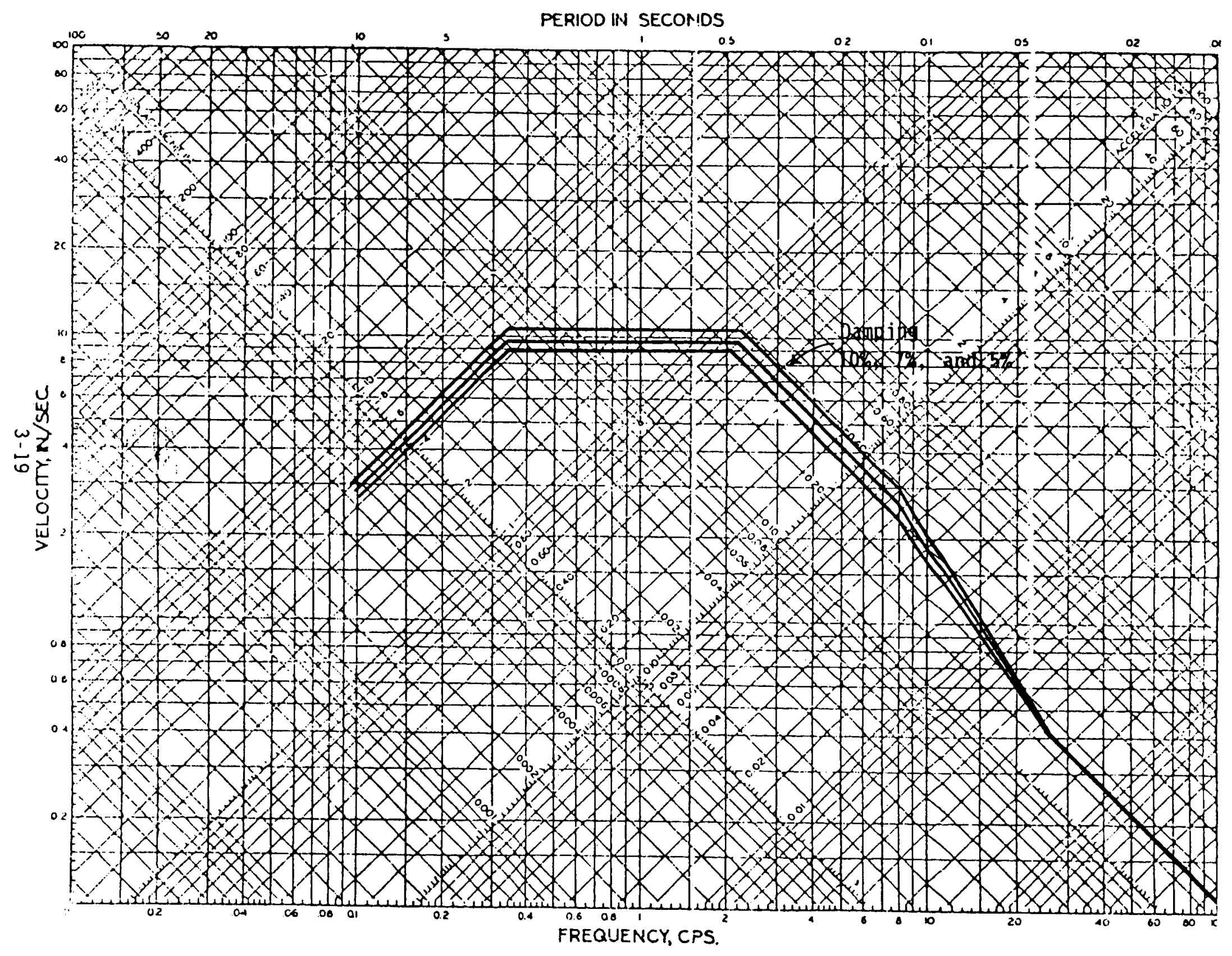

Figure 3-6: Median Ground Response Spectra Anchored to 0.18 pga. 


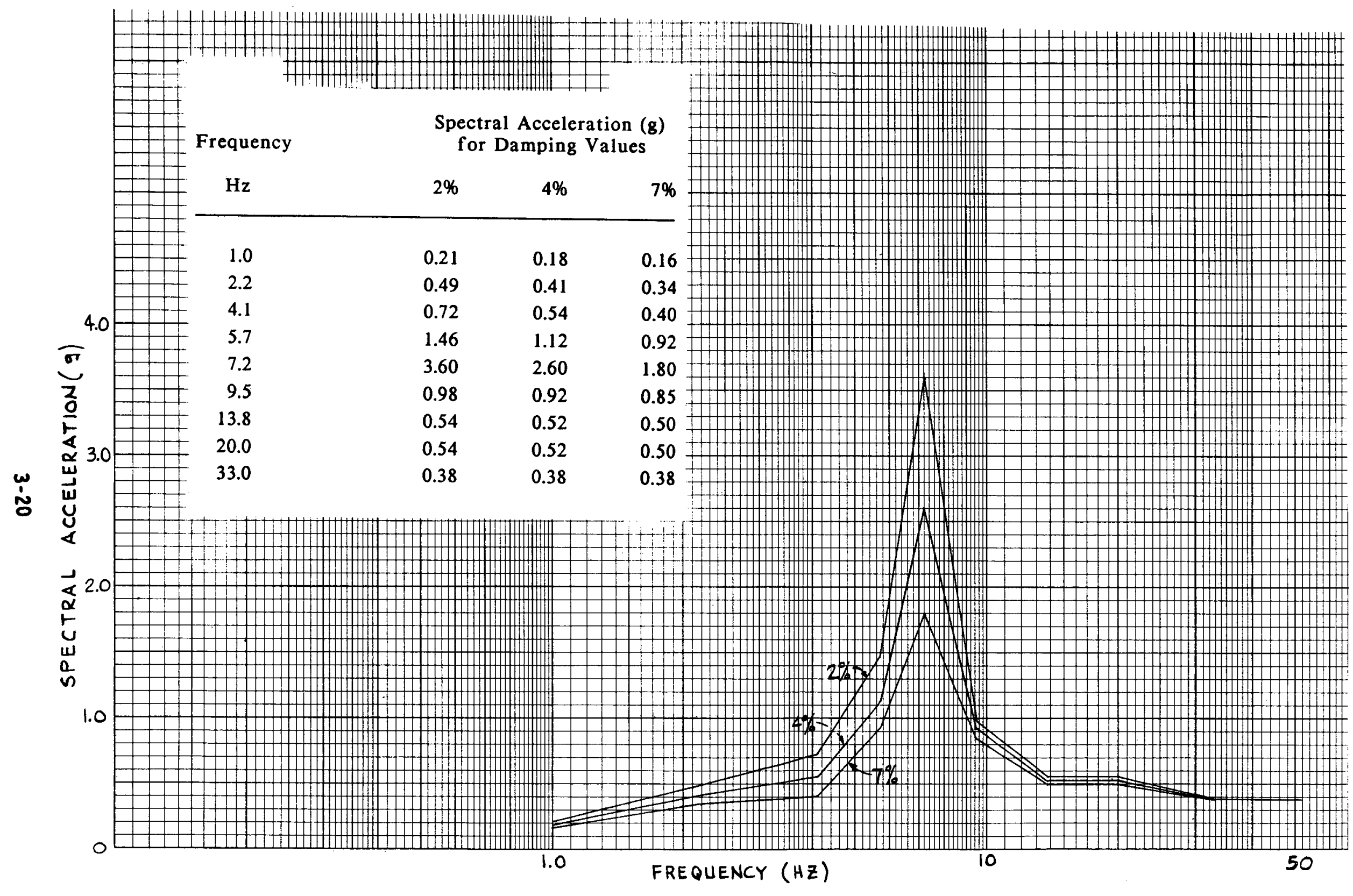

Figure 3-7: Horizontal Floor Spectra 


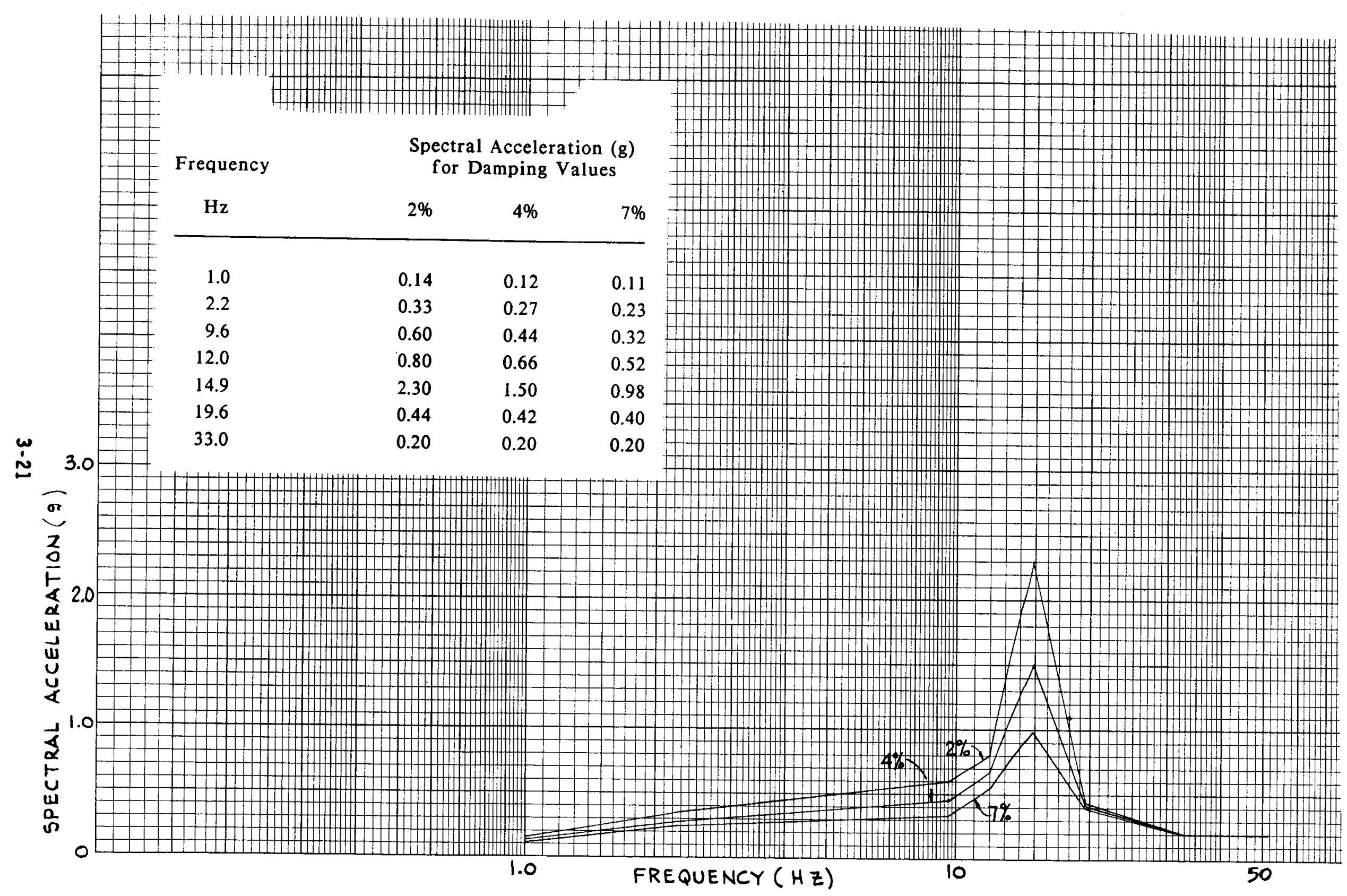

Figure 3-8: Vertical floor Spectra 


\section{CHAPTER 4 \\ SUMMARY AND CONCLUSIONS}

\subsection{Summary}

This study evaluated the CDFM and FA methods for calculating the HCLPF capacities of components. This was accomplished by performing the HCLPF capacity calculations on a representative set of components using the two methods by four investigators. The components selected were: flat-bottom vertical storage tank, auxiliary contactor for motor starter in an older MCC, starting air tank, component cooling heat exchanger, and cantilevered reinforced block wall. Two different locations in the building (at grade and at a floor high in the building) were studied for the MCC. The details of the components, ground response, and floor response spectra were provided.

In the first round of calculations, Kennedy and Ravindra used both the CDFM and FA methods to obtain the HCLPF capacities. They used the EPRI CDFM method (as discussed in Section 2.2). Reed calculated the capacity using the FA method. Stevenson calculated the HCLPF capacity using a modified SEP deterministic method.

Since Kennedy and Ravindra performed both CDFM and FA calculations following identical approaches, the comparison of their capacity estimates was deemed most appropriate. The ratio of high/low estimates of HCLPF capacities by the CDFM method ranged from 1.00 to 1.29 , with a median value of 1.11 . For the FA method, this ratio ranged from 1.11 to 1.57 , with a median of 1.30 .

The range of HCLPF capacities from the four investigators was larger than expected. The ratio of high to low HCLPF capacity estimates ranged from 1.23 to 1.82, with a median of 1.48. The differences in estimated HCLPF capacities between the four investigators was much larger than the differences in results between the CDFM method and FA method. This was primarily due to differences in the models, parameter values, and assumptions used by the investigators.

The differences in median capacity estimates ranged from 1.23 to 2.13 , with a median of 1.47. Thus, there was only slightly closer agreement in the estimates of the HCLPF capacities than there was in the estimates of the medians.

Based on a review of the first round calculations and subsequent discussions by the Study Group, the investigators were allowed to revise their calculations and resubmit the HCLPF and median capacity for each component. The four investigators differed from each other by a median ratio of 1.39 to 1.55 in their final estimates of the HCLPF capacity, the median capacity, and the ratio of median/HCLPF capacity.

As before, these differences are mainly due to the differences in models, parameters, and assumptions used by the investigators. Their estimates converged in those cases where the differences were not initially large; they did not converge (and in some cases diverged further) in those cases where the differences were initially large. 


\subsection{Conclusions from this Study}

1. The differences in estimated HCLPF capacities between the four investigators (median high-to-low ratio equals 1.48 ) are much larger than the differences in results between the CDFM methodology and the FA method.

2. These differences are primarily due to differences in the assumptions and judgments of the four investigators.

3. The differences between the investigators estimating the HCLPF capacities are similar in size to the differences in estimating the median capacities. This is contradictory to the judgment of the NRC Expert Panel on Seismic Margins. The Panel had stated that the median capacity of a component is difficult to calculate and could vary widely between the analysts. The HCLPF capacity was thought to be a more robust quantity which most engineers would agree upon. This has not been borne out by the calculations done by the Study Group. The four investigators differ from each other by a median of the ratio of high-to-low values of about 1.5 to 1.7 for estimates of the HCLPF capacity, the median capacity, and the ratio of median/HCLPF capacities.

4. The differences between the investigators' estimates of the median/HCLPF capacity ratios (median high-to-low ratio equals 1.5) are of the same size as estimates of either HCLPF capacities or median capacities individually. This indicates that differences in estimates of $\beta_{R}$ and $\beta_{U}$ are also relatively large.

5. Any future effort in reducing the differences in calculated HCLPF capacities should be spent in reducing the differences between the assumptions and judgment of different investigators rather than in trying to reconcile the much smaller differences between the CDFM and FA methods.

6. Although there was as much dispersion in the computed HCLPF capacities as there was in the computed median capacities, there was unanimous agreement that for each of the components all four investigators' HCLPF capacities represented a conservative estimate of the component capacity. Differences were over the degree of conservatism represented and not over whether conservatism existed or not. Thus, for each component, the highest HCLPF is still a conservative capacity estimate. Obviously, the same unanimity cannot exist at the median capacity since each separate estimate cannot represent the $50 \%$ failure probability, although all median estimates are within each investigator's $90 \%$ confidence band on the median. Thus, unanimity exists that all median estimates are credible medians for each component, and investigator differences simply reflect uncertainty about the median. In the face of uncertainties in both the median and HCLPF capacities, this Study Group 
recommends that future seismic margin studies continue to concentrate on estimating the HCLPF capacities since all computed HCLPF estimate were judged to be conservative capacity estimates. In this context, the HCLPF capacity estimate is generally considered to be a more useful capacity estimate for seismic margin reviews than is the median capacity, even though there appears to be as much dispersion in HCLPF capacity estimates as there is in median estimates.

7. The HCLPF Study Group does not recommend any revision to the Expert Panel recommendation that either of the two methods (CDFM or FA) could be used in a seismic margin review for calculating the HCLPF capacities.

8. This study has further confirmed that the estimated median capacity is at least twice the HCLPF capacity of a component. Hence there is no "proverbial cliff" in the seismic capacity in that seismic failure is not imminent if the ground acceleration exceeds the HCLPF capacity of the component.

9. The Study Group believes that the classes of components examined herein are representative and are adequate for the purposes of this comparison study. The differences identified in this study are likely to be larger if (a) a larger group of competent engineers were to make the capacity estimates; (b) investigators are required to make building response calculations independently; (c) the definition of what constitutes failure and (d) what constitutes the limiting failure mode are independently assessed or determined by the investigators.

10. The large differences in the HCLPF capacities estimated by the investigators in this study stem from the differences in failure modes assumed, capacity equations associated with any potential failure mode, different assumed as-built material properties, different estimates of inelastic energy absorption, different damping, and different frequency estimates. Even when the same models are adopted, such differences between the analysts are expected to persist because of variations in parameters. Hence a need exists for peer review of seismic margin studies. The Study Group endorses the recommendations of the Expert Panel on Seismic Margins that a peer review be an integral part of the seismic margin study.

11. It is suggested that pretest predictions of failure modes and levels should be made by independent investigators prior to conducting fragility tests. These predictions should be published prior to the tests. The purpose of this recommendation is to improve the capability of engineers to predict component capacity and to decrease the differences between investigators in predicting component capacities. 


\section{REFERENCES}

ANCO Engineers (May 1987), Generic Seismic Ruggedness of Power Plant Equipment, Prepared for the Electric Power Research Institute, Palo Alto, CA, EPRI-NP-5223.

Ang, A. H.-S., and N.M. Newmark (November 1977), A Probabilistic Seismic Assessment of the Diablo Canyon Nuclear Power Plant Report to U.S. Nuclear Regulatory Commission: N. M. Newmark Consulting Engineering Services, Urbana, IL.

Becker, D.L. and J.D. Stevenson (August 1984), Use of the Delphi Approach in Seismic Qualification of Existing Structures and Equipment in Industrial Facilities, Presented at the ASCE Energy 84 Specialty Conference, Pasadena, CA.

Bohn, M.P. et al., (December 1982), Seismic Safety Margins Research Program Phase 1 Final Report - Fragilities Development (Project VI), NUREG/CR-2015, Vol. 7, UCRL-53021, Vol. 7.

Budnitz, R. J., P.J. Amico, C.A. Cornell, W.J. Hall, R.P. Kennedy, J.W. Reed, and M. Shinozuka (July 1985), An Approach to the Quantification of Seismic Margins in Nuclear Power Plants, Lawrence Livermore National Laboratory, CA, NUREG/CR-4334, UCID-20444.

Cornell, C. A., and N.M. Newmark (May 1978), "On the Seismic Reliability of Nuclear Power Plants," ANS Topical Meeting on Probabilistic Reactor Safety Newport Beach, CA.

EPRI (August 1987), Evaluation of Nuclear Power Plant Seismic Margin (draft), Technical Report No. 1551.05, Electric Power Research Institute.

Kennedy, R.P. et al., (August 1980), "Probabilistic Seismic Safety Study of an Existing Nuclear Power Plant," Nuclear Engineering and Design, Vol. 59, No. 2, pp 315-338.

Kennedy, R.P. and M.K. Ravindra (May 1984), "Seismic Fragilities for Nuclear Power Plant Risk Studies," Nuclear Engineering and Design, Vol. 79, No. 1, pp 47-68.

Kennedy, R.P., "Various Types of Reported Seismic Margins and Their Uses," Section 2. Proceedings of EPRI/NRC Workshop on Nuclear Power Plant Reevaluation for Earthquakes Larger Than SSE. Palo Alto CA, October 16-18, 1984.

Murray, R.C. et al., (April 1981), Seismic Review of the Oyster Creek Nuclear Power Plants as Part of the Systematic Evaluation Program, NUREG/CR-1981, UCRL-530118.

Murray, R.C. et al., (November 1980), Seismic Review of the Robert E. Ginna Nuclear Power Plant as Part of the Systematic Evaluation Program, NUREG/CR1821, UCRL-53014. 
Murray, R.C., P.G. Prassinos, M.K. Ravindra and D. Moore (August 1987), "Seismic Margins Review of Nuclear Power Plants- NRC Program Overview," Presented at the 9th International Conference on Structural Mechanics in Reactor Technologv, Lausanne, Switzerland, 1987, Lawrence Livermore National Laboratory, Livermore, CA, UCRL- 94867.

Newmark, N.M, and W.J. Hall, (May 1987) Development of Criteria for Seimsje Review of Selected Nuclear Power Plants, NUREG/CR-0098.

Nelson, T.A. et al., (July 1981), Seismic Review of the Millstone 1 Nuclear Power Plant as Part of the Systematic Evaluation Program, NUREG/CR-2024, UCRL53022.

Nelson, T.A. et al., (January 1981), Seismic Review of the Palisades Nuclear Power Plant as Part of the Systematic Evaluation Program, NUREG/CR-1833, UCRL53015 .

Newmark, N.M., W.J. Hall, R.P. Kennedy, J.D. Stevenson and F.J. Tokarz (April 1980), Seismic Review of Dresden Nuclear Power Station-Unit 2 for the Systematic Evaluation Program, NUREG/CR-0891.

Prassinos, P.G., M.K. Ravindra, and J.B. Savy (March 1986), Recommendations to the Nuclear Regulatory Commission on Trial Guidelines for Seismic Margin Reviews of Nuclear Power Plants, Lawrence Livermore National Laboratory, Livermore, CA, UCID-20579, NUREG/CR-4482.

Prassinos, P.G., R.C. Murray, and G.E. Cummings (March 1987), Seismic Margin Review of the Maine Yankee Atomic Power Station-Summary Report, Lawrence Livermore National Laboratory, Livermore, CA, UCID-20948, NUREG/CR-4826, Vol.1.

Ravindra, M.K., G.S. Hardy, P.S. Hashimoto and M.J. Griffin (March 1987), Seismic Margin Review of the Maine Yankee Atomic Power Station - Fragility Analysis, Lawrence Livermore National Laboratory, Livermore, CA, UCID-20948, NUREG/CR-4826, Vol. 3.

Ravindra, M.K., R.H. Sues, R.P. Kennedy and D.A. Wesley (November 1984), A Program to Determine the Capability of the Millstone 3 Nuclear Power Plant to Withstand Seismic Excitation Above the Design SSE, Prepared for Northeast Utilities, NTS/SMA 20601.01-R2.

Stevenson, J.D. (Feb. 1985), Seismic Evaluation of Mechanical and Electrical Components for the L-Reactor at the Savannah River Plant, Prepared for Dupont.

U.S. Nuclear Regulatory Commission (July 1981), Standard Review Plan for the Review of Safety Analysis Reports for Nuclear Power Plants, Office of Nuclear Reactor Regulations, NRC, Washington, D.C., NUREG-0800, LWR Edition.

Wesley, D.A. et al., (February 1983), Seismic Margin Review: Midland Energy Center Project--Methodology and Criteria, SMA 13701.05R003, Vol. 1. 
APPENDIX A

ROBERT P. KENNEDY

$A-1$ 


\section{H I GH-CONF I DENCE-LOW-PROBAB IL I TY-OF-FA ILURE COMPUTATIONS FOR SELECTED COMPONENTS}

\section{Introductory Remarks}

Both a Conservative-Deterministic-Failure-Margin (CDFM) and a Fragility Method have been suggested in Ref. (1) and (2) for estimating the HighConfidence-Low-Probability-of-Failure (HCLPF) capacity of components subjected to seismic input. The CDFM method is expanded upon in Ref. (3) and is favored by this writer because it directly leads to a HCLPF capacity estimate using straightforward deterministic computations. The alternate Fragility Method first requires an estimate of the median (50\% probability of failure) capacity estimate for the component, and this is often difficult to make. Next one must estimate both the uncertainty in the median estimate and the random variability about this estimate. This uncertainty and random variability are often defined in terms of the logarithmic standard deviations, $\beta_{U}$ and $\beta_{R}$, respectively. Then the HCLPF capacity is arbitrarily estimated from:

$$
H C L P F=\check{A} e^{-1.65\left(\beta_{R}+\beta_{U}\right)}
$$

where $\check{A}$ is the median capacity estimate. Thus, by the Fragility Method, the HCLPF capacity is highly dependent upon the judgmental and often highly uncertain estimates of $\beta_{R}$ and $\beta_{U}$. 
The attached set of calculations compares the HCLPF Seismic Margin Earthquake (SME) capacity of 5 components estimated by the writer using both the CDFM method defined in Ref. (3) and the Fragility Method. Consistent assumptions were made in both methods. The 5 components selected were:

1. Flat-Bottom Vertical Water Storage Tank at grade.

2. Auxiliary Contactor Chatter for Motor Starter in an older MotorControl-Center (MCC) which has a fundamental frequency of about $6.5 \mathrm{~Hz}$. This estimate is made for both the cabinet high in the structure and on the base slab at grade.

3. Diesel Generator Room Starting Air Tank Supports mounted high in the structure.

4. Component Cooling Heat Exchanger Supports mounted high in the structure.

5. Cantilever Reinforced Block Wall mounted high in the structure.

Figures 1 through 4 schematically illustrate the important seismic capacity aspects of the Storage Tank, Starting Air Tank Supports, Heat Exchanger Supports, and Block Wall, respectively. The attached calculations were made as part of a project for four independent consultants to perform HCLPF SME capacity computations on the same 5 components using the simplified methods each would suggest to be used for production type Seismic Margin Review computations. Each consultant agreed to use the same structural properties for each component; these are illustrated in Figures 1 through 4 . For the MCC Auxiliary Contactor Chatter cases, it was agreed to assume that no component-specific seismic qualification test data was available, that the $6.5 \mathrm{~Hz}$ frequency estimate was approximate, and that the seismic capacity would be based upon the "Function-during GERS (Generic Equipment Ruggedness Spectrum)" presented in Figure 5 as reproduced from Ref. (ㄴ) .

\section{Ground Motion Aspects}

For a 11 HCLPF SME capacity computations, it was agreed to assume that the ground motion for the largest horizontal component is given by a uniform hazard spectrum defined at the $84 \%$ non-exceedance probability (NEP) at all frequencies. Furthermore, this uniform hazard spectrum shape was to be defined by the NUREG/CR-0098 (Ref. 5 ) Median spectrum shape for Rock sites. 
The $84 \%$ NEP vertical response spectrum was then defined to be equal to $2 / 3$ of the $84 \%$ NEP largest horizontal response spectrum.

The above ground motion information is sufficient to enable HCLPF SME capacity computations to be made using the Ref. (3) described CDFM method. However, the Fragility Method requires a further description of the random variability of the ground motion response spectrum. Variability associated with peaks and valleys of actual response spectrum and directional response variability should be included. Each consultant using the Fragility Method was expected to make his own estimate of these sources of response spectrum variability. The lognormal parameters used to define random response spectrum variability for this writer's HCLPF computations using the Fragility Method are given in Table 1. The first four parameter values are the basic assumed parameter values. The remaining parameter values have been derived from these four basic parameter values.

The fact that the Fragility Method requires the incorporation of ground motion response spectrum random variability parameters is one of its weaknesses, since it requires the component capacity evaluator to make an assumption on ground motion variability. However, so long as the SME ground motion is defined at the 84\% NEP largest horizontal component levels, this is not a major weakness. The resultant HCLPF SME capacity will be rather insensitive to the assumed parameter variabilities. However, if the SME ground motion is defined at the 50\% NEP for the average horizontal component, the resultant HCLPF SME capacity is very sensitive to the assumed response spectrum parameter variabilities. For this reason, this writer strongly recommends that $84 \%$ NEP response spectrum be used as the basic ground motion definition for HCLPF SME computations by either the CDFM or Fragility Methods. This recommendation removes the sensitivity of the results to the assumed variability of ground motion response spectra and the capacity evaluator is not the right person to be estimating ground motion response spectra variability. The result of using the $84 \%$ NEP largest horizontal component response spectrum to determine the HCLPF SME capacity is that this capacity is conditional on this response spectrum not being exceeded at more than $16 \%$ of the frequencies in the frequency range and directions that dominate the component capacity. 
The above-described ground response spectrum properties are used for computing the HCLPF SME capacity of the ground mounted Flat-Bottom Storage Tank and the ground mounted MCC Auxiliary Contactor Chatter Case. For the other sample components, a floor spectrum is used as discussed in the next section.

\section{Floor Spectrum Aspects}

It was agreed that the floor spectra shown in Figures 6 and 7 would be used to represent floor spectra obtained high in a structure from a mediancentered building response model subjected to 84\% NEP ground response spectra anchored to an SME largest horizontal Peak Ground Acceleration (PGA) of $0.18 \mathrm{~g}$. Figures 6 and 7 represent horizontal and vertical floor response, respectively. Since these spectra are assumed to represent unbroadened median response to an $84 \%$ NEP ground motion input, they may be used directly without scaling in the CDFM Method defined by Ref. (3) or the Fragility Method for an SME PGA of $0.18 \mathrm{~g}$. Linear scaling is used for other SME levels.

Actualiy, in this writer's opinion, the floor spectra shown in Figures 6 and 7 are not realistic median-centered floor spectra. They have too much resonant amplification to be representative of floor spectra from mediancentered response analyses even high in a structure. Their use results in a conservative underestimation of the HCLPF SME capacity for components mounted high in the structure. However, so long as all calculations assume those spectra came from a median-centered response analysis, the above-described problem will not influence the relative comparisons between HCLPF capacities obtained by the CDFM Method versus the Fragility Method or comparisons in capacities obtained from the four independent consultants.

\section{Results}

Table 2 compares the HCLPF SME PGA capacities obtained by the CDFM Method versus the Fragility Method for each of the five sample components. Table 2 also presents this writer's estimate of the median capacity and the summation $\left(B_{R}+B_{U}\right)$ used in the Fragility Method. Lastly, Table 2 presents the summation $\left(\beta_{R}^{\prime}+\beta_{U}^{\prime}\right)$ necessary so that both the CDFM and Fragility methods produce the identical HCLPF capacity for each component and the ratio between those two summations. Several conclusions are reached: 
1. For each case, excellent agreement exists between the HCLPF capacities obtained from the CDFM and Fragility methods.

2. The estimated median capacity is at least a factor of 2 greater than the HCLPF capacity in each case.

3. Considering all of the assumptions which must be made in the Fragility Method to estimate both $\beta_{R}$ and $\beta_{U}$, it requires only a negligible change in the summation $\left(B_{R}+\beta_{U}\right)$ as denoted by the ratio $\left(\beta_{R}^{\prime}+\beta_{U}^{\prime}\right) /\left(\beta_{R}+\beta_{U}\right)$ to get HCLPF capacity from the Fragility Method to agree with that from the CDFM Method. Such changes are easily within the uncertainty range on $\beta_{R}$ and $\beta_{U}$. Since these estimates are uncertain and the HCLPF capacity from the Fragility Method is sensitive to the estimates of $\beta_{R}$ and $\beta_{U}$, the CDFM Method provides a more stable estimate of the HCLPF capacity than does the Fragility Method.

\section{Interpretation of Results}

Each of the capacity estimates presented in Table 2 is intended to represent component failure capacity estimates. However, the definition of component failure can be somewhat judgmental and therefore warrants further discussion for each component.

The flat-bottom vertical water storage tank capacity estimates are intended to correspond to the onset of development of a significant throughwall crack in the tank shell, thus resulting in a loss of tank contents over a period of less than about 24 hours. This crack would be expected to occur either at the weld between the tank side wall and base-plate or within the lower few feet of the tank side wall.

The auxiliary contactor chatter capacity estimates are intended to correspond to the onset of at least 2 milliseconds of auxiliary contactor chatter. The potential consequences of such chatter would depend upon the electrical circuitry involved and is not addressed.

The diesel generator room starting air tank support capacity is intended to correspond to the loss of lateral constraint for at least one of support angles or tie-down bolts. Prior to such loss of lateral constraint, the top of the air tank would be expected to move laterally less than 4 inches, which would be expected to have no adverse consequences on attached air lines so 
long as they have flexibility. After loss of lateral constraint, the functional performance of the air tank would be uncertain.

The component cooling heat exchanger support capacity is intended to correspond to breaking of anchor bolts on one of the supports, thus allowing the heat exchanger to slide. With such sliding, the functional performance of the heat exchanger would be uncertain.

The cantilever reinforced block wall capacity is intended to correspond to essentially unconstrained lateral deformation of this wall, such that it would come down unless it hit some support and was held up by that support prior to falling. Prior to reaching this condition, deformations of the top of the wall will be less than 10 inches, so that the Table 2 capacity estimates are judged appropriate unless a component which $c$ an be damaged by wall impact exists within 10 inches of the wall.

Of course, capacities of components are influenced by characteristics of ground motion not defined by the response spectral amplitudes. Most important of these are the duration of strong ground motion and the number of strong nonlinear response cycles to which the component is subjected by the ground motion. Since neither were defined, for this exercise I have assumed that the duration of strong ground motion as defined in accordance with Ref. (6) is 3 to 10 seconds, subjecting each component to 3 strong nonlinear response cycles also as defined by Ref. (6) . In my judgment, such ground motion characteristics correspond to the ground motion levels listed in Table 2 having come from an earthquake with a local magnitude of about 6.5. For ground motions from earthquakes with local magnitudes less than about 6.0, the capacities given in Table 2 are too conservative. Conversely, for earthquakes with local magnitudes exceeding about 7.0, these capacities may be too liberal.

Several capacities were checked for each component. Only calculations for the controlling capacity for each component are shown in the attached calculation package.

\section{Reconciliation of Results}

Each of the four consultants who participated in this project independently produced his calculations prior to October 1987. During November 1987, these consultants met to compare results. After that meeting, each consultant was allowed to revise his calculations to see whether such revised 
calculations would come closer together. All the attached calculations and summary results in Table 2 are from the independently produced work prior to October 1987. This section contains my discussion on reconciliation of my results with those from the other consultants (Reed, Stevenson, and EQE).

\section{$6.1 \quad$ Flat Bottom Tank}

Reed and EQE have HCLPF estimates of 0.27 and $0.26 \mathrm{~g}$, respectively, which are very close to my estimate of $0.29 \mathrm{~g}$, whereas Stevenson has a HCLPF estimate much higher than our three estimates. Similarly, Reed and EQE have median estimates of 0.53 and $0.54 \mathrm{~g}$, compared to my estimate of $0.67 \mathrm{~g}$, whereas Stevenson has a median estimate much higher than mine. Thus, one might think that it might be easy for me to reconcile on an estimate between that of Reed, EQE, and myself for both the HCLPF and median capacities, with Stevenson being an outlier. However, this is not the case.

As discussed in Ref. (7), a copy of which is included in the attached calculations, my capacity estimates are conservatively biased in two areas. First, I have ignored any benefit from inelastic energy absorption. Second, I have conservatively underestimated the benefits from fluid-holddown forces on the base plate. These conservatisms were intentionally introduced, because I believed that producing defensible estimates of the inelastic energy absorption capacity increase and/or less conservative estimates of the benefits of fluid-holddown would require more sophisticated and much more costly analyses than I considered to be warranted. Therefore, I chose to ignore these additional capacity enhancement benefits. However, these additional factors are real, and ignoring them results in a conservative bias to my calculations.

Based on approximate computations (not attached), I estimate that inclusion of a realistic (but difficult to defend without more sophisticated analyses) estimate of the inelastic energy absorption capacity increase factor would increase my HCLPF capacity by a factor of 1.25 to $0.36 \mathrm{~g}$, and my median capacity by a factor of 1.75 to $1.17 \mathrm{~g}$. Furthermore, a nonlinear, largedeflection theory base plate uplift analysis to better account for fluidholddown effects would increase these capacities by an unknown further amount.

Based on these considerations, it is impossible for me to compromise with Reed or EQE on any HCLPF capacity estimate less than $0.29 \mathrm{~g}$ or median estimate less than $0.67 \mathrm{~g}$. On the other hand, it would be very easy for me to reach 
reconciliation with Stevenson on any HCLPF capacity between $0.29 \mathrm{~g}$ and $0.40 \mathrm{~g}$ and any median capacity between $0.67 \mathrm{~g}$ and $1.3 \mathrm{~g}$.

\subsection{Auxiliary Contact Chatter}

When component-specific fragility or qualification test data is unavailable, Ref. (3) suggests that the Generic Equipment Ruggedness Spectra (GERS) from Ref. (4) be used to make HCLPF capacity estimates. However, when this is done, I have recommended in Ref. (3) that the GERS be divided by 1.3 before being compared with Required Response Spectra (RRS) being input at the base of the component. This recommended reduction factor of 1.3 was based upon two considerations:

1. The GERS were based on only a limited amount of test data and cover broad generic component categories. As such, they do not represent HCLPF capacity estimates. Based upon material presented in Ref. (4) plus material presented to the Senior Seismic Review and Advisory Panel (SSRAP), I have judged that the typical ratio between GERS and HCLPF capacities is about 1.2 in the case of a broad-frequency input RRS.

2. It is my judgment that an RRS developed following the recommendation of Ref. (3) for HCLPF computations lies in the $84 \%$ to $90 \%$ nonexceedance probability range. As such, additional conservatism of about a factor of 1.1 is necessary to account for variability in the input spectrum when GERS are compared to such RRS to achieve an overall HCLPF capacity estimate.

For cases where chatter of auxiliary contacts in motor control centers is of concern, Ref. (4) has suggested that the "Function During" GERS shown in Figure 5 be factored by 0.87 . No basis is given for this recommendation, nor was any basis ever discussed with the SSRAP. I believe this factor is simply an additional conservatism factor in recognition that the GERS do not represent the HCLPF capacity. Since that consideration is already covered by the 1.3 reduction factor recommended in Ref. (3) for HCLPF capacity computations, I do not believe that both the 0.87 factor and the 1.3 factor should be combined as was done by EQE when computing the HCLPF capacity by the CDFM method of Ref. ( $\left.{ }^{3}\right)$. If this 0.87 factor was removed from the EQE HCLPF 
capacity computations by the CDFM method, their CDFM HCLPF capacities would be identical to mine.

My judgment that the 0.87 factor from Ref. (4) and the 1.3 factor from Ref. (3) should not both be included, is supported by the fragility and qualification test data presented in Ref. () for auxiliary contact chatter in motor control centers. Ref. ()ㅡㄹ presents results from 51 fragility tests in which auxiliary contacts chattered and 10 qualification tests in which no auxiliary contact chatter occurred. For broad frequency input, the $2 \%$ damped spectral acceleration fragility levels ranged from $2.1 \mathrm{~g}$ to $7.4 \mathrm{~g}$ for the 51 fragility tests with auxiliary contact chatter, while the 10 qualification tests without auxiliary contact chatter ranged from $2.0 \mathrm{~g}$ to $5.6 \mathrm{~g}$. Assuming the data fits a lognormal distribution, Ref. () reports a $2 \%$ damped spectral acceleration HCLPF capacity of $1.7 \mathrm{~g}$. However, the data does not fit a lognormal distribution; and making the erroneous assumption of a lognormal distribution results in the highest fragility test capacity of $7.4 \mathrm{~g}$ and the highest qualification test capacity of $5.6 \mathrm{~g}$, actually driving down the HCLPF capacity estimate because these high test results increase the estimates of logarithmic standard deviation. If instead one performs a distribution-free one-sided tolerance limit check (which is generally very conservative because it is a distribution-free check which makes no assumptions on the data distribution) on the data, one determines a $2 \%$ damped spectral acceleration HCLPF (95\% confidence of less than $5 \%$ failure probability) capacity of $2.1 \mathrm{~g}$. Since the GERS of Ref. (4) are based upon 5\% damped spectra, this $2 \%$ damped HCLPF capacity of $2.1 \mathrm{~g}$ must be converted to a $5 \%$ damped HCLPF capacity before being compared with the GERS "Function During" level of $1.5 \mathrm{~g}$ shown in Figure 5 . To convert from $2 \%$ damped to $5 \%$ damped spectra, the $2 \%$ damped HCLPF capacity must be divided by about 1.5, which leads to a $5 \%$ damped HCLPF capacity of $1.4 \mathrm{~g}$. Thus, the ratio of the GERS capacity from Ref. (4) to the HCLPF capacity from the data of Ref. (ㅇ) is $1.5 / 1.4=1.07$, which is less than the 1.2 ratio of GERS to HCLPF upon which the 1.3 reduction factor recommended in Ref. (3) is based. Thus, it would be very inappropriate to both multiply the GERS by 0.87 and also divide by 1.3 to obtain a HCLPF capacity for auxiliary contact chatter. In fact, the 1.3 reduction factor by itself appears to be too high for this case.

For the case of the motor control center cabinet at grade, the HCLPF estimates from the four consultants range from a low value of $0.39 \mathrm{~g}$ by $E Q E$ to 
a high value of $0.71 \mathrm{~g}$ by Stevenson, with my estimate of $0.54 \mathrm{~g}$ being about midway. Because of the above-discussed considerations, I consider my HCLPF estimate to be somewhat conservatively biased. For this reason, I could accept a consensus HCLPF estimate ranging between $0.54 \mathrm{~g}$ and $0.64 \mathrm{~g}$. Therefore, it is likely to be easier for me to reach a compromise HCLPF estimate with Stevenson than it is with the low HCLPF estimates of EQE.

For the motor control center cabinet high in the structure, the HCLPF estimates range from a low value of $0.07 \mathrm{~g}$ by EQE to $0.12 \mathrm{~g}$ by Stevenson, with my estimate of $0.10 \mathrm{~g}$ being about midway. For the reasons discussed above, my estimate might be slightly conservative so that I could concur with a compromise HCLPF ranging between $0.10 \mathrm{~g}$ and $0.12 \mathrm{~g}$. However, an estimate as low as $0.07 \mathrm{~g}$ by EQE is totally unacceptable to me.

\subsection{Starting Air Tank}

For the Starting Air Tank, the HCLPF estimates for the four consultants ranged from $0.39 \mathrm{~g}$ by Stevenson to $0.53 \mathrm{~g}$ by EQE using the CDFM, with my estimate of $0.48 \mathrm{~g}$ being midway. For the median capacity, the estimates of Reed, Stevenson, and myself were tightiy bunched from $1.01 \mathrm{~g}$ to $1.07 \mathrm{~g}$, with the median estimate from EQE being much higher at $1.55 \mathrm{~g}$.

An important difference in the capacity computations is that neither Stevenson nor I took any credit for an inelastic energy absorption factor increase. Both EQE and Reed did take credit for such an increase using procedures which are really only appropriate when the input consists of broad frequency ground spectra with 5\% damped amplified spectral accelerations 2 to 3 times the zero period acceleration. The floor spectrum shown in Figure 6 which is input to this component certainly does not fit into this category. Its frequency content is very narrow, centered at about $7.5 \mathrm{~Hz}$, and the $5 \%$ spectral accelerations are amplified by more than a factor of 5 . Using an inappropriate approach in my judgment, EQE increased their median capacity by an inelastic energy absorption factor of 2.08 and their CDFM HCLPF capacity by 1.25. Reed increased his median capacity by a much more moderate factor of 1.35 and made essentially no increase in his HCLPF capacity for inelastic energy absorption.

By my calculations, the natural frequency of this starting air tank is about $16 \mathrm{~Hz}$. Considering both the uncertainty at which the floor spectra 
peaks occur and the uncertainty in the component natural frequency, I recommend that this frequency be shifted down to about $12.8 \mathrm{~Hz}$ when entering the unbroadened floor spectrum of Figure 6 . The nonlinear analyses of Ref. ( $\underline{6})$ clearly demonstrate that the shape of the input spectrum to the lower frequency side of the elastic frequency plays a very dominant role on the inelastic energy absorption increase factor as the structure softens due to ducti1ity. In Figure 6 , as the component frequency shifts downward from $12.8 \mathrm{~Hz}$, the spectral accelerations rapidly increase. With such a rapid increase in spectral accelerations as the frequency is reduced due to inelasticity, there is essentially no benefit from inelastic behavior since such behavior forces the component right into the power of the input. Unless one performs a series of nonlinear time history analyses, I would not recommend taking any credit for an inelastic energy absorption increase factor for this case even though the failure mode is very ductile. Even with nonlinear time history analyses, it is difficult for me to conceive that this factor could exceed 1.35 for the median capacity or 1.1 for the HCLPF capacity with such a spiked spectrum as shown in Figure 6 lying immediately below the component natural frequency. For this reason, I believe that the median inelastic energy absorption factor of 2.08 used by $E Q E$ to get their high median capacity to be totally unrealistic.

For the starting air tank, I can accept a consensus HCLPF capacity anywhere in the range from $0.45 \mathrm{~g}$ to $0.53 \mathrm{~g}$ and a median capacity in the range from $1.0 \mathrm{~g}$ to $1.35 \mathrm{~g}$.

\subsection{Heat Exchanger}

For the heat exchanger, the HCLPF capacity estimates ranged from a low of 0.30 by Stevenson to a high of 0.44 by EQE using the CDFM method, with my estimate of $0.40 \mathrm{~g}$ being between. The differences result from a number of factors, and no clear cause is apparent to me. I can accept a consensus HCLPF capacity for this heat exchanger anywhere in the range from $0.37 \mathrm{~g}$ to $0.44 \mathrm{~g}$, which covers all but Stevenson's existing estimate.

\subsection{Block Wall}

My HCLPF capacity estimate for the block wall is $0.62 \mathrm{~g}$. The other three consultants had lower HCLPF capacities ranging from a low of $0.38 \mathrm{~g}$ by Reed to $0.51 \mathrm{~g}$ by Stevenson. My estimate of the median capacity of $1.94 \mathrm{~g}$ is similarly 
high compared to the range of $1.34 \mathrm{~g}$ to $1.55 \mathrm{~g}$ estimated by the other consultants. The difference between my results and those of the other consultants primarily lies in my treatment of the inelastic response of this block wall.

Since the block wall is reinforced, its behavior prior to failure will be highly ductile. Therefore, a realistic computation of its capacity requires a realistic assessment of its inelastic behavior. This wall is subjected to the very narrow frequency, highly amplified input spectrum shown in Figure 6 . In my judgment, simplified approaches based on broad frequency, less amplified ground spectra, cannot be used to realistically estimate the inelastic response of highly ductile components subjected to a narrow frequency input spectrum such as Figure 6 . Based on a large number of non linear time-history analyses, Ref. (6) recommends an approach for estimating inelastic response which is appropriate for narrow frequency input spectra such as that in Figure 6. This approach requires the estimation of an effective frequency and effective damping. The spectral acceleration at this effective frequency and damping are then used to estimate inelastic response rather than the spectral acceleration at the elastic frequency and damping. I have used the approach of Ref. (6) to estimate the inelastic response of this block wall. This approach leads to much greater capacity estimates for this ductile wall subjected to the floor spectrum of Figure 6 due to inelastic response than would be obtained using ductility factor correction approaches which are appropriate for broader frequency content ground spectra.

Accounting for uncertainty in the natural frequency of this block wall and uncertainty in the frequency content of the Figure 6 input spectrum, I would conservatively estimate the "so-called elastic" response of this wall using the $5.5 \mathrm{~Hz}$, 7\% damped spectral acceleration from Figure 6 of $0.87 \mathrm{~g}$. This spectral acceleration value lies high up on the narrow frequency amplified spectrum of Figure 6 , and softening of the component due to inelastic behavior will dramatically reduce the input spectral acceleration. In such a case there will be very large benefits from inelastic response. Following the recommendations of Ref. (6), even for the HCLPF capacity I can conservatively estimate that the effective frequency of this wall will not exceed $2.9 \mathrm{~Hz}$ with an effective damping of $10 \%$. Using these values, the spectral acceleration from Figure 6 is $0.33 \mathrm{~g}$, which is on $1 y 38 \%$ of the "elastic" spectral acceleration of $0.87 \mathrm{~g}$. Conservatively limiting the inelastic energy absorption correction factor to 1.25 as recommended by Ref. (3) rather than using the higher 
value developed by the procedures of Ref. ( $\underline{6})$, I obtained an effective spectral acceleration for HCLPF capacity of $0.33 / 1.25=0.26 \mathrm{~g}$. In my judgment, this effective spectral acceleration of $0.26 \mathrm{~g}$ very conservatively represents the inelastic response of this block wall prior to failure when subjected to the input of Figure 6 . For comparison, the effective spectral acceleration used by EQE in their CDFM HCLPF capacity computations was $0.36 \mathrm{~g}$. This difference of $0.36 \mathrm{~g}$ versus $0.26 \mathrm{~g}$ for the effective spectral acceleration more than accounts for the differences in our HCLPF capacities of $0.48 \mathrm{~g}$ versus $0.62 \mathrm{~g}$. Similarly, at the median level, the differences in effective spectral accelerations used fully accounts for the resultant differences in median capacities.

Because I strongly believe that I have used a more realistic approach based on Ref. (6) to estimate the inelastic response of this block wall, I cannot support any consensus HCLPF or median capacity estimate significantly less than those I provided in Table 2, even though my estimates are higher than those of any of the other consultants. I could not support a consensus HCLPF capacity estimate less than $0.57 \mathrm{~g}$ or a median capacity estimate less than $1.75 \mathrm{~g}$ for this block wall.

\section{References}

1. R. P. Kennedy, "Various Types of Reported Seismic Margins and Their Use," Proceedings: EPRI/NRC Workshop on Nuclear Power Plant Reevaluation to Quantify Seismic Margins, EPRI NP-4104-SR, Electric Power Research Institute, August 1985.

2. P. G. Prassinos, et al., Recommendations to the Nuclear Regulatory Commission on Trial Guidelines for Seismic Margin Reviews of Nuclear Power Plants, NUREG/CR-4482, Lawrence Livermore National Laboratory, prepared for U. S. Nuclear Regulatory Commission, March 1986.

3. Evaluation of Nuclear Power Plant Seismic Margin, NTS Engineering, prepared for Electric Power Research Institute, August 1987 (draft).

4. Generic Seismic Ruggedness of Power Plant Equipment, NP-5223, Electric Power Research Institute, May 1987.

5. N. M. Newmark and W. J. Hall, Development of Criteria for Seismic Review of Selected Nuclear Power Plants, NUREG/CR-0098, Nuclear Regulatory Commission, May 1978. 
6. R. P. Kennedy, et al., Engineering Characterization of Ground Motion-Task 1, NUREG/CR-3805, Vol. 1, Nuclear Regulatory Commission, May 1984.

7. R. P. Kennedy, "Flat-Bottom Vertical Water Storage Tank," Appendix $H$ to Ref. ()ㅜ).

8. Seismic Fragility of Nuclear Power Plant Components--Phase II Study of MCC, Switchboard, Panelboard, and Power Supply, Brookhaven National Laboratory, October 1987 (final draft). 
TABLE 1

GROUND MOTION RANDOM VARIABILITY RESPONSE PARAMETERS

\begin{tabular}{|c|c|c|c|c|}
\hline & Parameter & Median & $\beta_{R}$ & Comments \\
\hline $\begin{array}{l}\text { (1) } \\
\text { (2) } \\
\text { (3) } \\
\text { (4) }\end{array}$ & $\begin{array}{l}\text { F}_{1}: \text { Peak \& Valley Variability Factor } \\
F_{2}: \text { Horizontal Direction Variability } \\
\text { Factor } \\
84 \% \text { NEP Vertical / } 84 \% \text { NEP Largest } \\
\text { Horizontal } \\
\text { Vertical / Median Average Horizontal }\end{array}$ & $\begin{array}{l}1.00 \\
1.00 \\
0.67\end{array}$ & $\begin{array}{l}0.20 \\
0.15 \\
0.34\end{array}$ & $\begin{array}{c}\text { Basic } \\
\text { Assumed } \\
\text { Parameter } \\
\text { Values }\end{array}$ \\
\hline & & & & $\begin{array}{l}\text { Derived } \\
\text { Parameter Values }\end{array}$ \\
\hline$(5)$ & $\begin{array}{l}\text { Largest Horizontal / Median Average } \\
\text { Horizontal }\end{array}$ & 1.13 & 0.22 & From (1) \& (2) \\
\hline (6) & $\begin{array}{l}\text { F6: 84\% NEP Largest Horizontal / } \\
\text { Median Average Horizontal }\end{array}$ & 1.41 & $-\cdots$ & From (5) \\
\hline (7) & $\begin{array}{l}\text { F7: 84\% NEP Largest Horizontal / } \\
\text { Median Largest Horizontal }\end{array}$ & 1.22 & --- & From (1) \\
\hline (8) & $\begin{array}{l}\text { F8: Vertical / Median Average } \\
\text { Horizontal }\end{array}$ & 0.67 & 0.34 & $\begin{array}{l}\text { From } \\
\&(6)\end{array}$ \\
\hline (9) & $\begin{array}{l}\text { Fg: Vertical / } 84 \% \text { NEP Largest } \\
\text { Horizontal }\end{array}$ & 0.48 & 0.34 & From (6) \& (8) \\
\hline
\end{tabular}


TABLE 2

COMPARISON OF CAPACITY RESULTS FROM CDFM AND FRAGILITY METHODS

\begin{tabular}{|c|c|c|c|c|c|c|}
\hline \multirow[b]{2}{*}{ Component } & \multirow{2}{*}{$\begin{array}{c}\text { CDFM } \\
\text { HCLPF } \\
\text { SME }\end{array}$} & \multicolumn{3}{|c|}{ Fragility Method } & \multicolumn{2}{|c|}{ Equal HCLPF Capacities } \\
\hline & & $\begin{array}{l}\text { HCLPF } \\
\text { SME }\end{array}$ & $\begin{array}{l}\text { Median } \\
\text { SME }\end{array}$ & $\left(\beta_{R}+\beta_{U}\right)$ & $\left(\beta_{R}^{\prime}+\beta_{U}^{\prime}\right)$ & $\frac{\left(\beta_{R}^{\prime}+\beta_{U}^{\prime}\right)}{\left(\beta_{R}+\beta_{U}\right)}$ \\
\hline Flat Bottom Storage Tank & $0.29 \mathrm{~g}$ & $0.31 \mathrm{~g}$ & $0.67 \mathrm{~g}$ & 0.47 & 0.51 & 1.08 \\
\hline Auxiliary Contactor Chatter & & & & & & \\
\hline (a) Cabinet At Grade & $0.54 \mathrm{~g}$ & $0.59 \mathrm{~g}$ & $1.26 \mathrm{~g}$ & 0.46 & 0.51 & 1.12 \\
\hline (b) Cabinet $\mathrm{High}$ in Structure & $0.10 \mathrm{~g}$ & $0.11 \mathrm{~g}$ & $0.30 \mathrm{~g}$ & 0.60 & 0.67 & 1.11 \\
\hline Starting Air Tank & $0.48 \mathrm{~g}$ & $0.50 \mathrm{~g}$ & $1.07 \mathrm{~g}$ & 0.46 & 0.49 & 1.06 \\
\hline Heat Exchanger & $0.40 \mathrm{~g}$ & $0.42 \mathrm{~g}$ & $1.18 \mathrm{~g}$ & 0.62 & 0.66 & 1.06 \\
\hline Block Wall & $0.62 \mathrm{~g}$ & $0.67 \mathrm{~g}$ & $1.94 \mathrm{~g}$ & 0.64 & 0.69 & 1.08 \\
\hline
\end{tabular}


Materials: Shell \& Chairs: SA240 - Type 304 SST

Bolts: $A 307$ - 2"

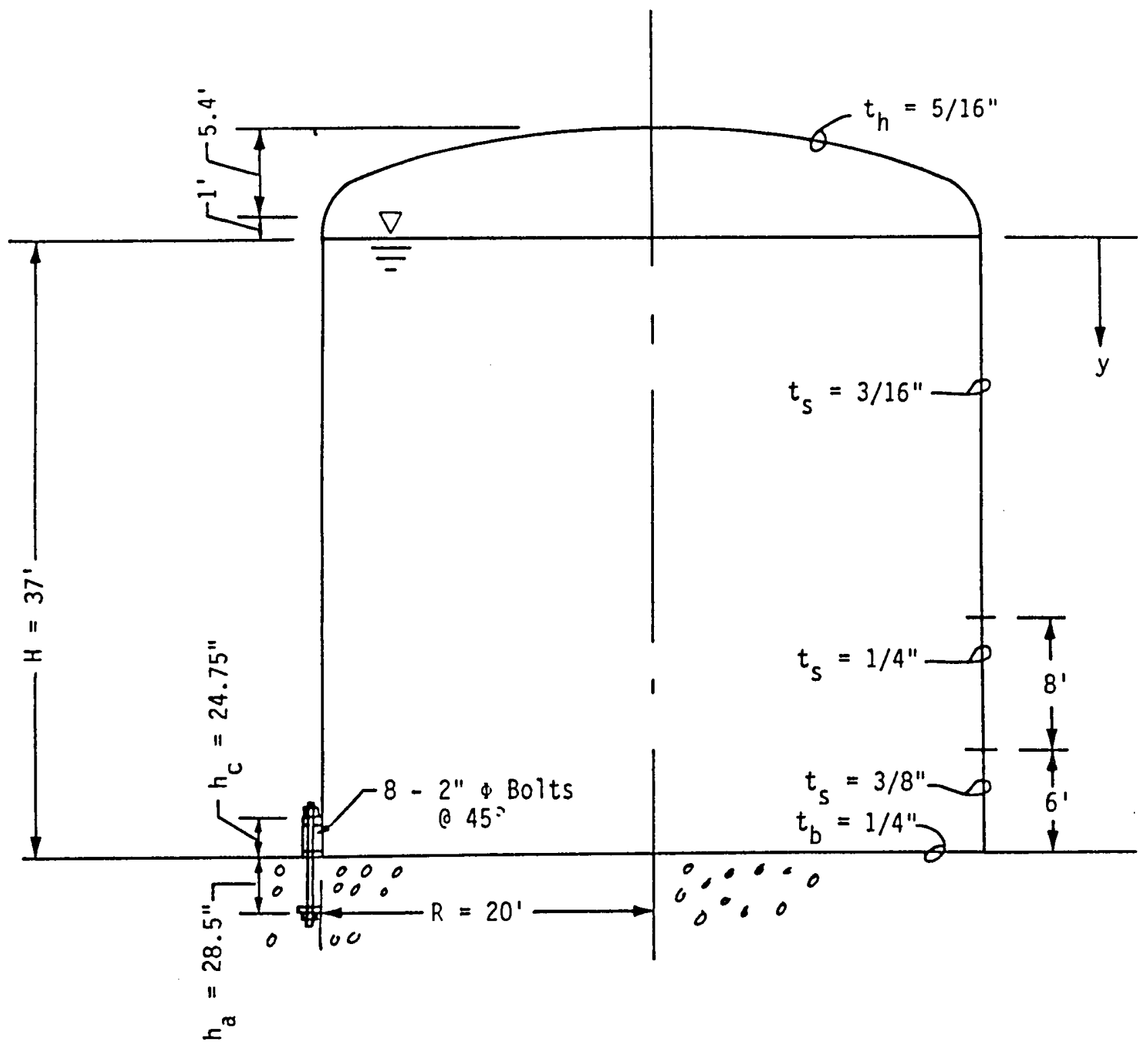

Figure 1. Flat Bottom Vertical Water Storage Tank 


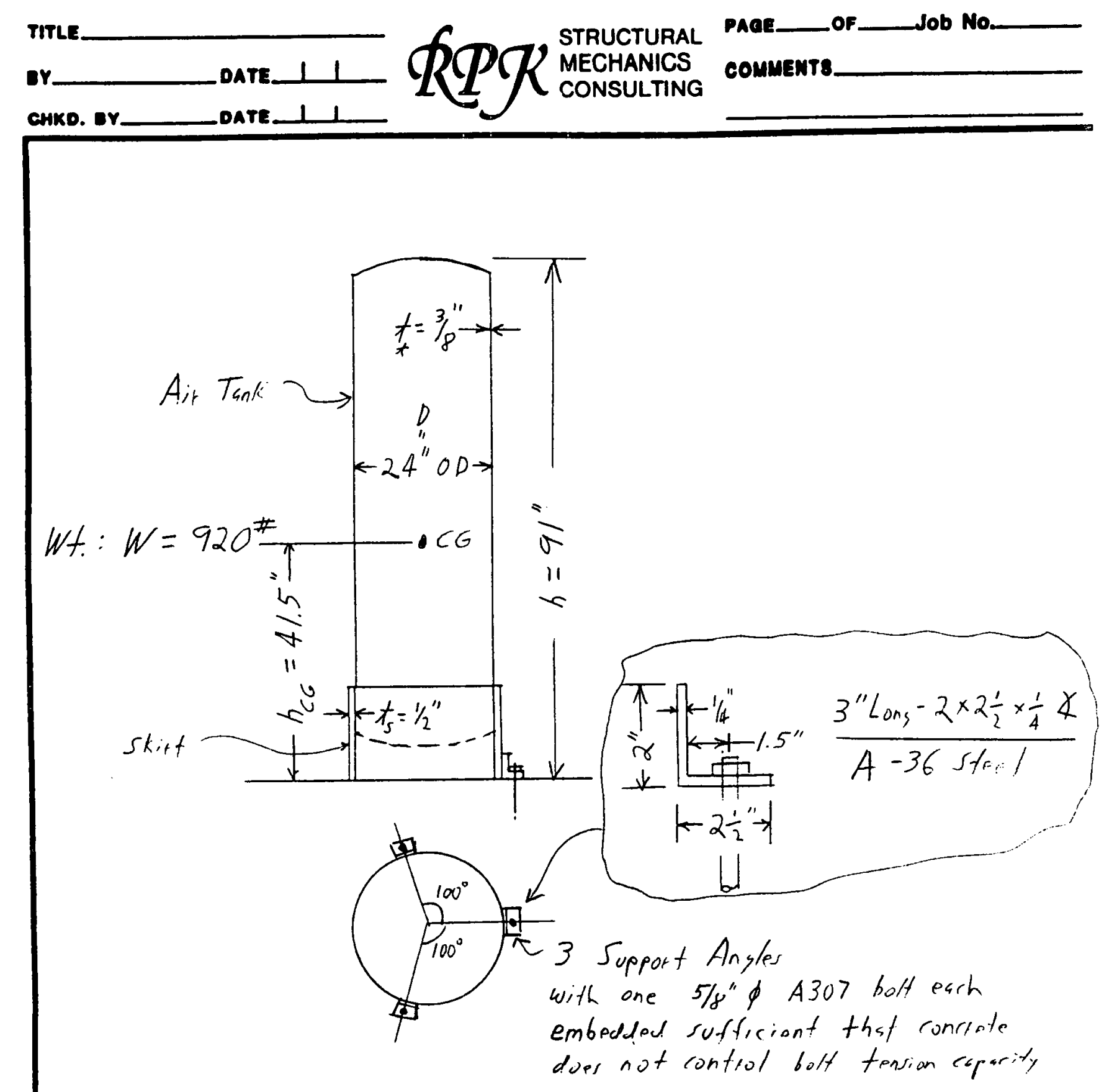

Fig 2: Diesel/ Generator Room Starting Air Tank Supports

A-19 


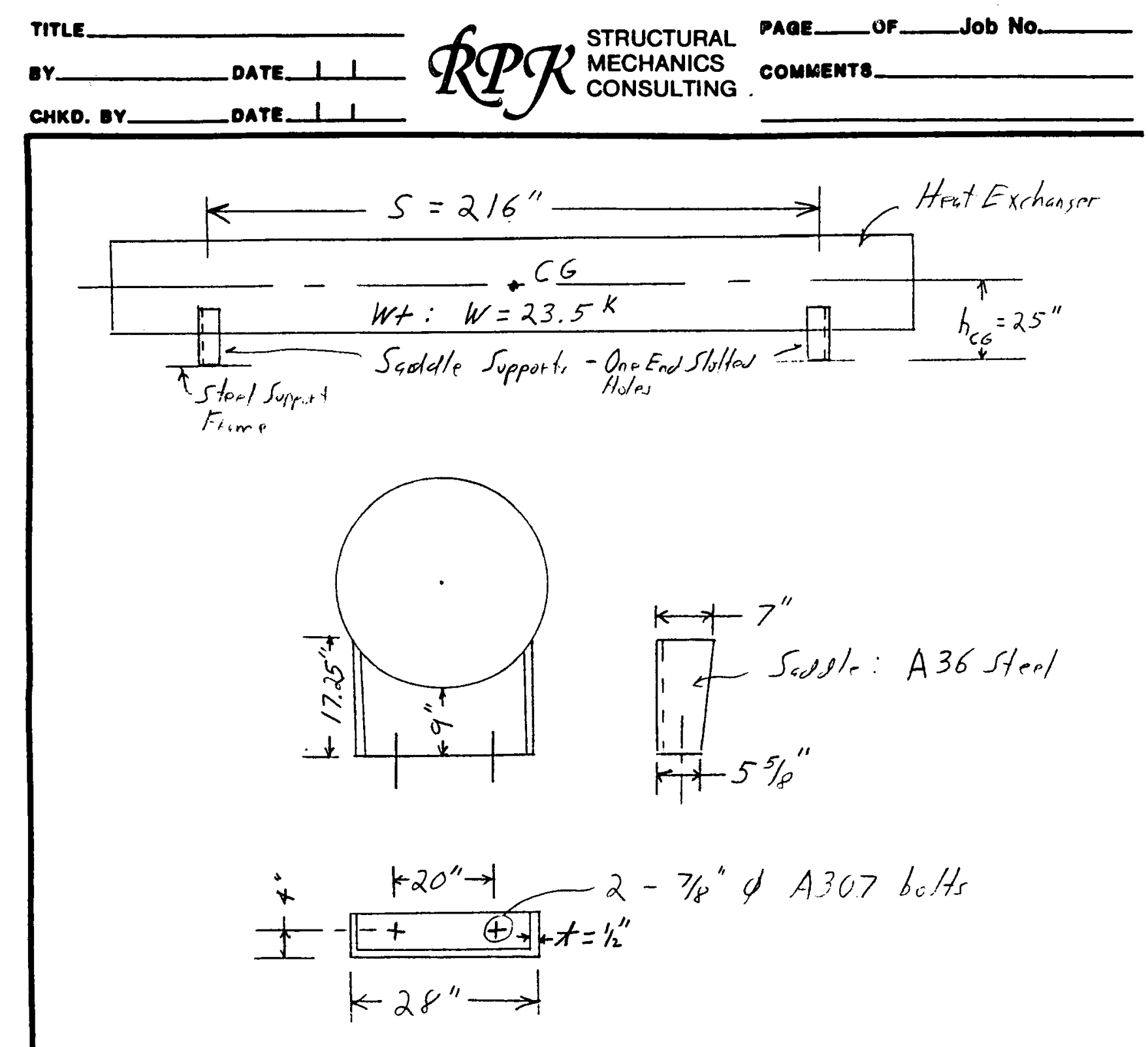

Fig 3: Componant Cooling Heot Exathon Suppute

$A-20$ 


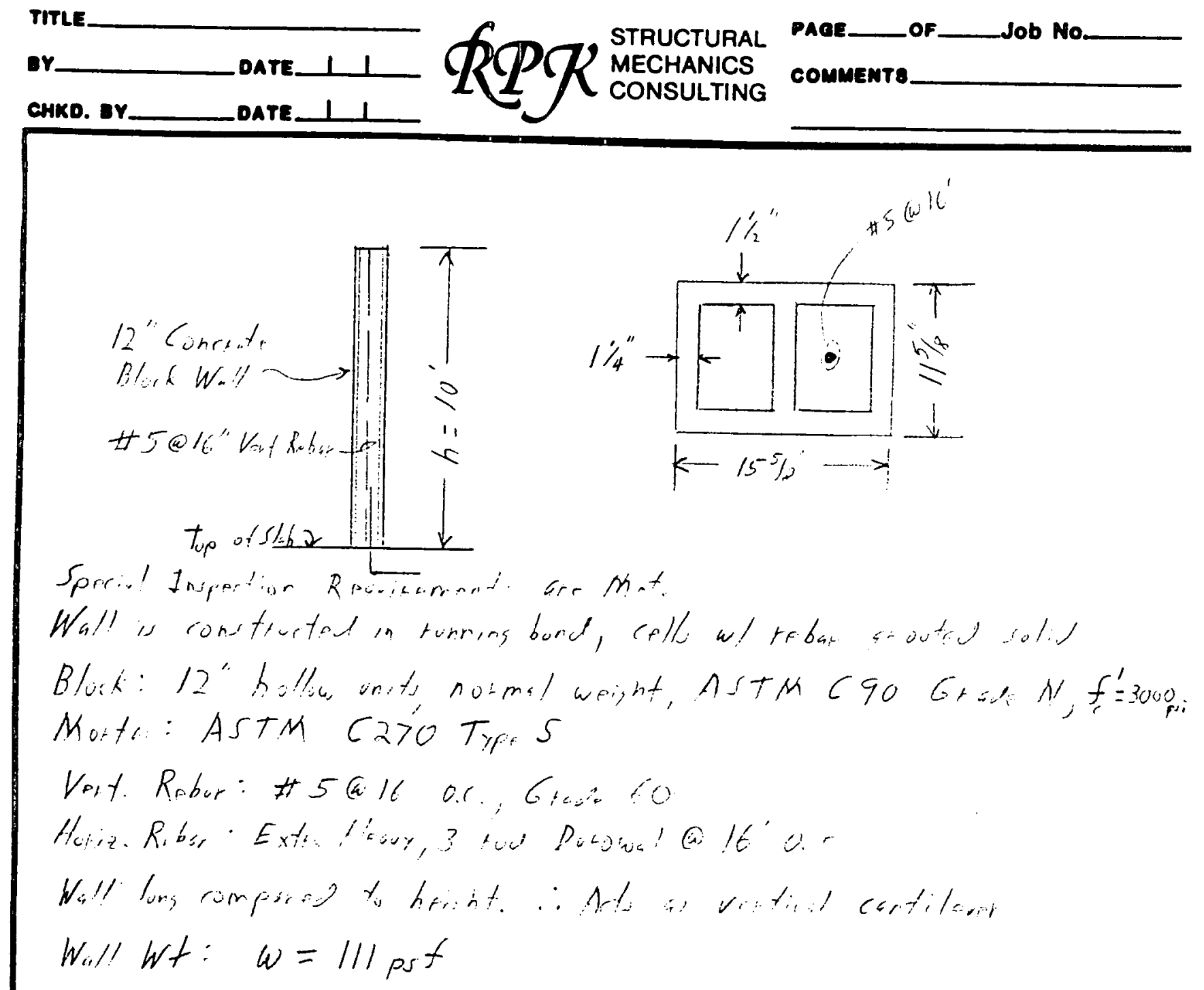

Fig 4: Cantilarer Reinfoureu Blout Well

$A-21$ 


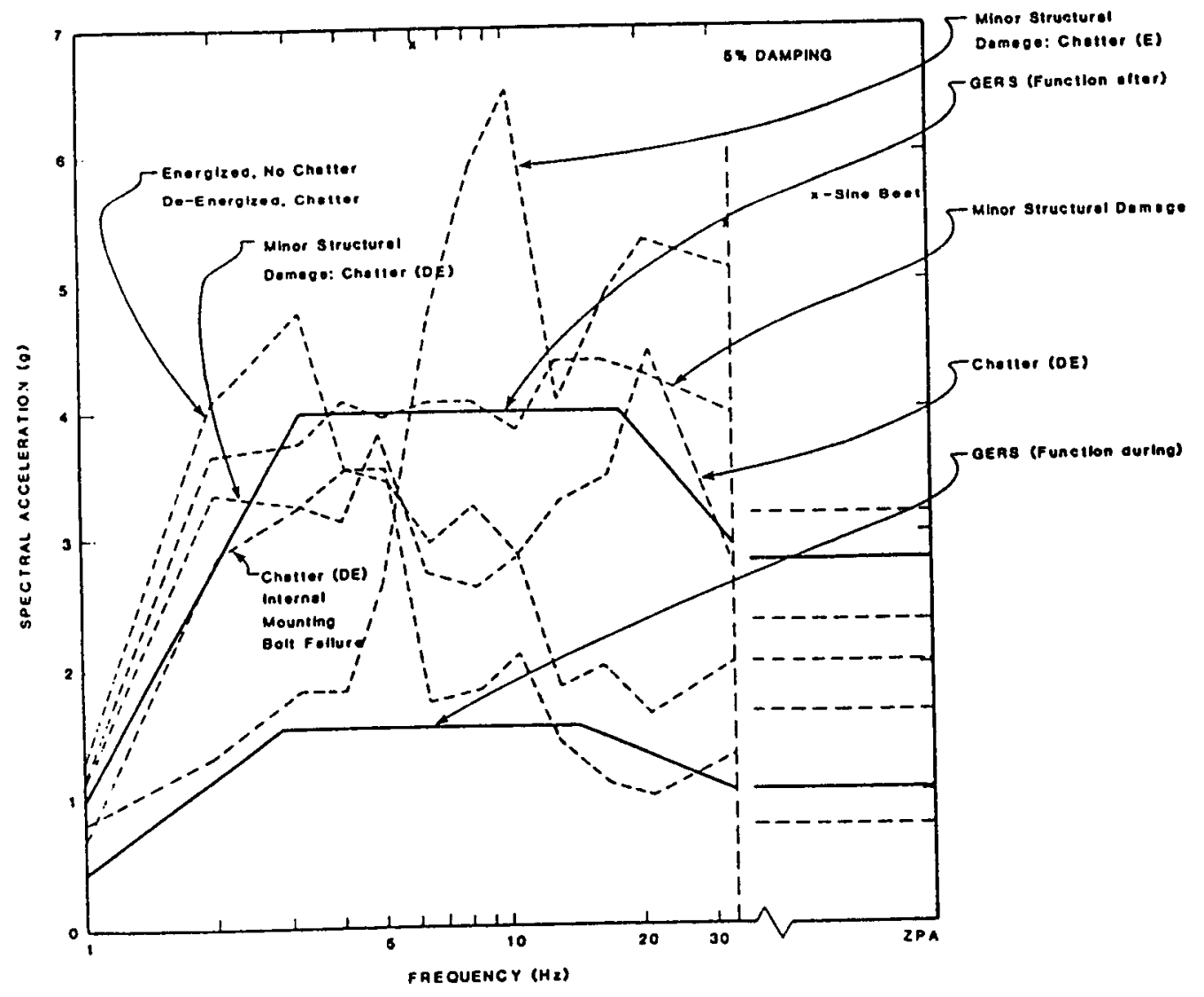

Figure 5. Comparison of GERS with failure data: function during and after for MCC (from Ref. 4 ). 


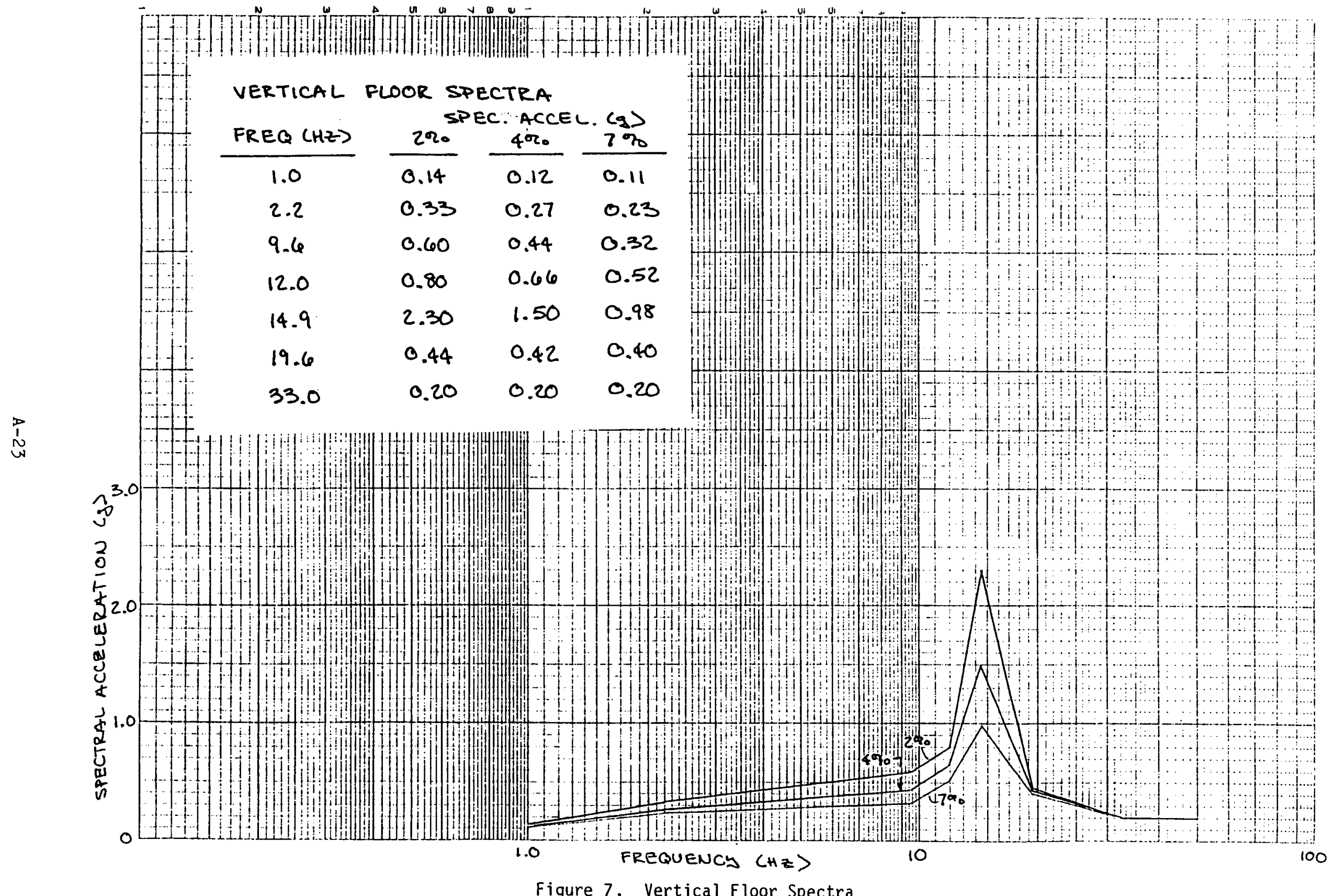




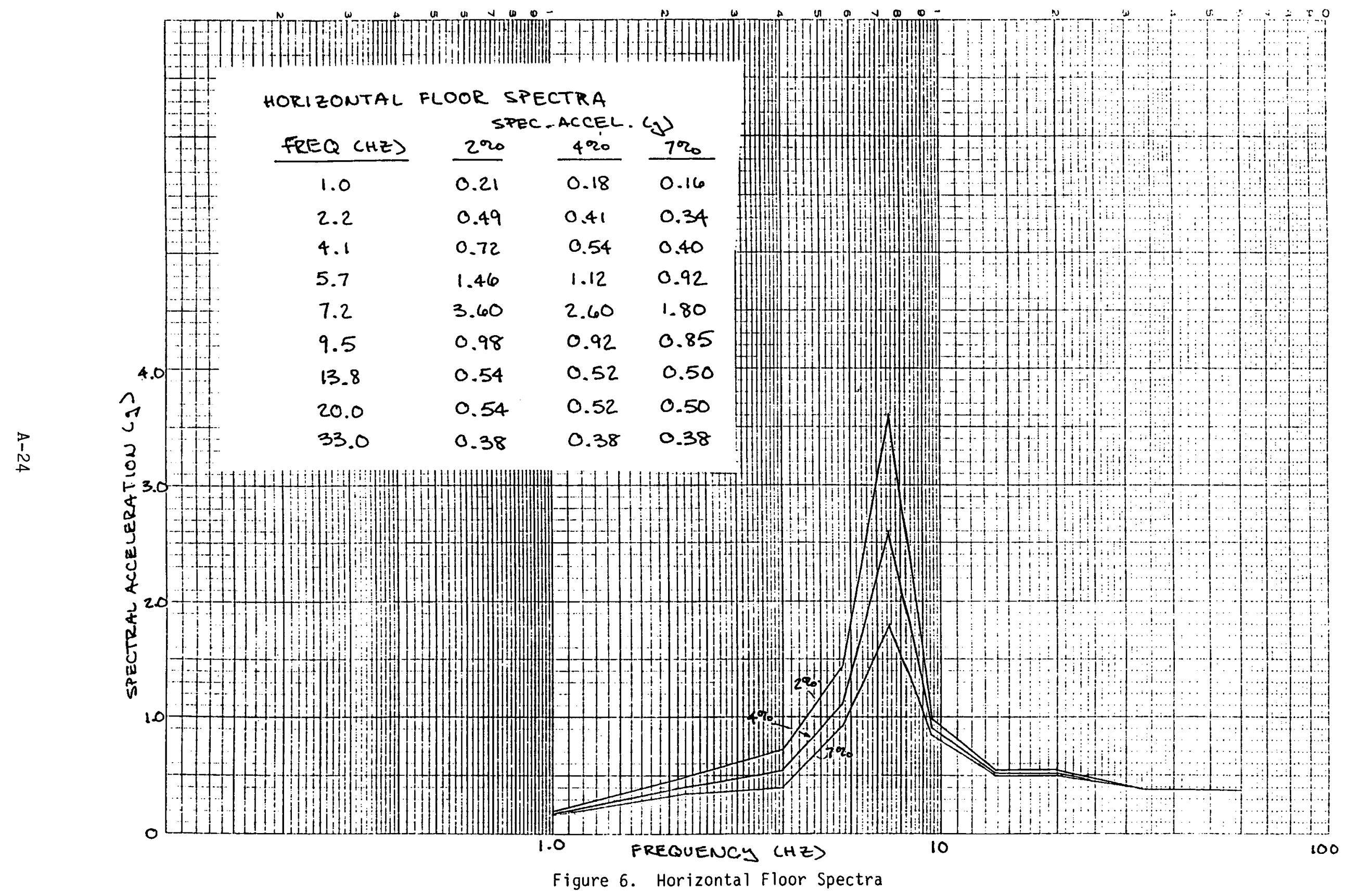




\section{FLAT-BOTTOM VERTICAL WATER STORAGE TANK}

\section{CDFM METHOD*}

By

R. P. Kennedy

* Reproduced from Appendix H, Ref. (3), "Flat-Bottom Vertical Water Storage Tank" by R. P. Kennedy, August 1987. 


\author{
Appendix H \\ FLAT-BOTTOM VERTICAL FLUID STORAGE TANKS \\ by \\ R.P. Kennedy
}

\title{
H.1 Introduction
}

Flat-bottom vertical fluid storage tanks of the type illustrated in Figure $\mathrm{H}-1$ should be evaluated during a Seismic Margin Assessment (SMA) if they are needed in the success paths being considered. First, examples of failures with loss of contents during strong earthquake shaking exist for such tanks when they have minimal or no anchorage. Secondly, even though such tanks at nuclear power plants have been designed for seismic effects, past designs (those predating about 1977) tend to have less seismic margin than exists for most other seismic designed components.

Many of these tanks were designed using the seismic evaluation procedure of TID7024 (1). The major problem is that direct application of this method is consistent with the assumption that the combined fivid-tank system in the horizontal impulsive mode is sufficiently rigid to justify the assumption of a rigid tank. For the case of flat bottomed tanks mounted directly on the ir base, or tanks with very stiff skirt supports, this assumption leads to the usage of a spectral acceleration equal to the zero-period base acceleration. This assumption is unconservative for tanks mounted on the ground or low in structures when the spectral acceleration does not return to the zero period base acceleration at frequencies below about $20 \mathrm{~Hz}$. More recent evaluation techniques (2) ( $\underline{3})(\underline{4})(\underline{5})$ have shown that for typical tank designs, the modal frequency for this fundamental horizontal impulsive mode of the tank shell and contained fluid is generally between 2 and $20 \mathrm{~Hz}$. Within this regime, the spectral acceleration is typically significantly greater than the zero period acceleration.

The above described problem with such tanks in the nuclear industry was publicized during the U.S. Nuclear Regulatory Commission Task Action Plan A-40 (2) which provided the basis for Unresolved Safety Issue A-40. One way to resolve this issue is through showing an adequate seismic margin of such tanks by a seismic evaluation as a part of a SMA. This appendix is intended to summarize a reasonable procedure to evaluate the High-Confidence-Low-Probability-of-Failure 
(HCLPF) seismic capacity of such tanks following the general ConservativeDeterministic-Failure-Marg in (CDFM) approach summarized in Section 2.

The seismic evaluation of these tanks consists of two parts: a seismic response evaluation, and a seismic capacity assessment. The topic of response evaluation has been extensively described in the literature in the last 10 years ((2) through $(10))$. Therefore, response evaluation will only be sumarized herein as it applies to the example tank, illustrated in Figure $\mathrm{H}-1$. The general approach followed will be that given in $(\underline{2})(\underline{5})(\underline{10})$ and the reader is referred to any of these readily available references for further details. Herein, it will be assumed that the example tank is founded on a rock site so that the topic of soilstructure-interaction (SSI) is not germane to this example. When flat-bottomed tanks are founded on soil, the resultant SSI can substantially modify the tank response (5) and such modifications should be considered.

The seismic capacity assessment of minimally anchored tanks such as that illustrated in Figure $H-1$ has not received as thorough of a treatment in the literature and so will be discussed herein in greater detail. The example tank shown in Figure $\mathrm{H}-1$ will be used as the vehicle for this discussion.

The example tank is representative of tanks often found at low seismic (SSE<0.15g) nuclear power plant sites. The tank radius, $R$, is 20 feet, while the water height, $\mathrm{H}$, when full, is 37 feet, with the overall tank height to the top of its dome roof being 43.4 feet. Thus, this tank is about twice as high as its radius. Because this tank was designed for a low SSE using the TID-7024 (1) response evaluation approach, this tank was built with only a minimal number of hold-down anchor bolts consisting of eight 2 inch diameter A307 bolts around its circumference. These bolts provide hold-down forces to the tank shell through the top plate of well-designed bolt chairs at a height, $h_{c}$, of 24.75 inches above the tank bottom. The bolts are anchored into the concrete foundation through an anchor plate at a depth, $h_{a}$, of 28.5 inches. The bolt chairs, their attachment to the tank, and the bolt anchorage are sufficient to develop the full capacity of the bolts. Because this is an atmospheric storage tank (no internal pressure) with a low design SSE, the tank head, side wall, and base plate thicknesses are thin which is typical for these tanks. The tanks shell is SA240-Type 304 stainless steel.

Although this tank had an unconservative TID-7024 (1) seismic response analysis for a low SSE during design, the design capacity assessment was performed very 
conservatively as is typical of nuclear plant designs and the detailing was good. Therefore, simply from a review of design calculations and a walkdown it is impossible for experienced seismic engineers to assess whether this tank has a sufficiently high seismic margin (HCLPF > $0.30 \mathrm{~g}$ ) such that an evaluation is unnecessary or such a low seismic margin (HCLPF $<0.15 \mathrm{~g}$ ) so that the seismic capability of this tank should be dismissed and an alternate success path selected. A detailed seismic evaluation is warranted.

As noted in Section 2, the first step in a SMA is the selection of a Seismic Margin Earthquake (SME) response spectrum shape anchored to a SME Peak Ground Acceleration (PGA) level. For this example problem, the fourth alternative approach described in Step 1 of Section 2 will be used for this selection. In this alternative, a standard median spectrum shape which approximates a uniform hazard spectrum shape will simply be specified. For this example, the median NUREG/CR-0098(11) spectrum shape for rock sites will be specified for the horizontal ground motion and the vertical ground motion will be specified as twothirds of the horizontal motion. When specified in this way, the resultant HCLPF SME statement is conditional on this standard spectrum anchored to the SME level PGA not being exceeded by a future ground motion at more than $16 \%$ of the natural frequencies in the frequency range and direction of interest. The resultant SME PGA level will then be determined by the HCLPF seismic evaluation of the tank.

Because capacities to withstand horizontal responses are slightly influenced by vertical responses, a small amount of nonlinearity develops when computing the SME capacity of tanks. Therefore, it is preferable to estimate an SME capacity of the tank, SME $E_{e}$, and to compute the seismic response, SEISMIC $e$, for this $S_{2} E_{e}$. Then the actual SME capacity can be estimated from:

$$
\text { SME }=\frac{\text { CAPACITY }- \text { STATIC }}{k \cdot S E I S M I C}\left(S_{e} E_{e}\right)
$$

where CAPACITY is the HCLPF capacity of the tank, STATIC is the portion of this capacity used up by static loads and $k$ is the inelastic energy absorption effective seismic stress correction factor described in Section 2 and discussed in the capacity assessment section of this appendix. If the resultant SME differs substantially from SME then iteration of the procedure is necessary because of the slight nonlinearities. So long as SME and SME are close, no iteration is necessary. For this example tank, the estimated SME e will be taken to be 0.27 g. Thus, using (11) for rock sites, the ground motion estimates to be used for the response evaluation will be: 


$$
\begin{array}{ll}
\text { Horizontal PGA: } & A_{H}=S M E_{e}=0.27 \mathrm{~g} \\
\text { Vertical PGA: } & A_{V}=(2 / 3) A_{H}=0.18 \mathrm{~g}
\end{array}
$$

Horizontal Velocity: $V_{H}=36 \mathrm{in} / \mathrm{sec}-g\left(A_{H}\right)=9.7 \mathrm{in} / \mathrm{sec}$

Vertical Velocity: $\quad V_{V}=(2 / 3) V_{H}=6.5 \mathrm{in} / \mathrm{sec}$

Horizontal Displacement: $D_{H}=\left(6 V_{H}^{2} / A_{H}\right)=5.4$ inches

Using the response evaluation and capacity assessment procedures recommended here in together with the above definition for the SME response spectrum shape, the example tank defined by Figure H.I will be shown to have a HCLPF SME PGA capacity of $0.29 \mathrm{~g}$. It should be noted that the HCLPF capacity level is a conservative, essentially lower-bound estimate of the failure capacity. The actual failure capacity is likely to be much higher. In fact, a median failure capacity estimate (50\% probability of failure) for this tank is in excess of $0.6 \mathrm{~g}$. Therefore, despite this tank having an unconservative TID-7024 (1) response analysis for a low SSE during design, this tank has a substantial seismic margin capability.

\section{H.2 Response Evaluation}

The seismic response evaluation should provide estimates of each of the following:

1. The overturning moment in the tank shell immediately above the base plate of the tank. This moment is then compared with base moment capacity as governed by a combination of shell buckling and anchor bolt yielding or failure and generally governs the SME capacity of the tank.

2. The overturning moment applied to the tank foundation through a combination of the tank shell and the base plate. This moment is only needed for tanks founded on soil sites where a foundation failure mode should be investigated and is generally obtained as part of the SSI evaluation. It seldom governs the SME capacity.

3. The base shear beneath the tank base plate. This base shear is compared to the horizontal sliding capacity of the tank. For atmospheric tanks with a radius greater than 15 feet it seldom controls the SME capacity.

4. The combination of the hydrostatic plus hydrodynamic pressures on the tank side wall. It is common design practice to compare these combined 
pressures with the membrane hoop capacity of the tank side walls at one-foot above the base and each wall thickness change. Thus, for the example tank these combined pressures are needed at 22-feet, 30-feet, and 36-feet below the top of the water. These combined pressures essentially never govern the SME capacity of a properly designed tank.

5. The average hydrostatic minus hydrodynamic pressure on the base plate of the tank. This pressure is used when evaluating the sliding capacity of the tank. If hold-down forces due to fluid on the base plate are included in the overturning moment capacity estimate, then the minimum value of the hydrostatic minus hydrodynamic pressure near the tank side wall should also be estimated.

6. The fluid slosh height. This slosh height is compared with the freeboard above the top of the fluid to estimate whether roof damage is likely. However, roof damage seldom interferes with the safety function of the tank immediately after an earthquake and is generally not of concern in a SMA.

In estimating each of these response quantities at least two horizontal modes of combined fluid-tank vibration and one vertical mode of fluid vibration should be considered. The two horizontal response modes should include at least one impulsive mode in which the response of the tank shell and roof are coupled together with the portion of the fluid contents which move in unison with the shell and at least the fundamental sloshing (convective) mode of the fluid. As noted previously, the response evaluation will only be summarized here in as it applies to the example tank and will generally follow the approach given in (2) (ㅁ) $(\underline{10})$.

The first step of a response evaluation is to make a weight takeoff and to determine the hydrostatic fluid pressure, $P_{S T}$, at capacity evaluation locations along the tankshell and the base. For the example tank, the component weights, $W$, and their center of gravity heights, $X$, above the tank base are:

$$
\begin{array}{lll}
\text { Head: } & W_{H}=17.2 \mathrm{kips} & x_{H}=42-\mathrm{ft} \\
\text { She 11: } & W_{S}=44.9 \mathrm{kips} & x_{S}=16.4-\mathrm{ft} \\
\text { Bottom: } & W_{B}=12.8 \mathrm{kips} & X_{B}=0 \\
\text { Water: } & W_{W}=2900 \mathrm{kips} & x_{W}=18.5-\mathrm{ft}
\end{array}
$$


It should be noted that the water weight totally dominates over the tank weight being nearly 40 times the total tank weight. As a first order estimate, one could base the entire computed seismic response on the water weight ignoring tank weights. This approach will not be done herein, but tank weight responses will be only approximately computed.

The hydrostatic fluid pressures, $P_{S T}$, at capacity evaluation locations are given in Table $\mathrm{H}-1$.

\section{H.2.1 Horizontal Impulsive Mode Response}

One must first estimate the horizontal impulsive mode natural frequency, $f_{I}$. One approach is to use:

$$
f_{I}=\frac{C_{L I}}{2 \pi H} \sqrt{E_{S} / \rho_{S}} \text { where } C_{L I}=C_{W I} \sqrt{\frac{0.127 \rho_{S}}{\rho_{L}}}
$$

where $E_{S}$ is the modulus of elasticity for the tank wall material, og is its mass density, $o_{L}$ is the fluid mass density, and $C_{W I}$ is a horizontal impulsive frequency coefficient for water in a steel tank. This coefficient is a function of the ratio $(H / R)$ and the tank wall thickness, $t$. For the case of roofless tanks with a uniform wall thickness, $t$, the value of $C_{W I}$ may be obtained from ( $\underline{5}$ ) or ( $\left.\underline{8}\right)$. For variable wall thickness tanks, the variable wall thickness can be approximated by an average value where in the averaging should be done so as to emphasize the section of the tank for which modal displacements are the largest. For water in steel tanks $\left(\rho_{L} / \rho_{S}\right)=0.127$ so that $C_{L I}=C_{W I}$ and $\sqrt{E_{S} / \rho_{S}}=16,200 \mathrm{ft} . / \mathrm{sec}$.

For the example tank, the approximate average thickness is estimated to be 0.22 inch and $(H / R)=1.85$. Using Table 7.4 of $(\underline{5})$ and these properties, $C_{W I}$ is estimated at 0.085 . Thus, $f_{I}$ is $6.0 \mathrm{~Hz}$ with an expected accuracy range of about $5.5 \mathrm{~Hz}$ to $6.6 \mathrm{~Hz}$.

Next, one must estimate the horizontal impulsive mode spectral acceleration, $S_{A_{I}}$, for frequencies in the range of 5.5 to $6.6 \mathrm{~Hz}$. For such an estimate, one needs an estimate of energy dissipation as expressed by equivalent viscous damping. For CDFM capacity evaluations, Section 2 recommends using a conservative estimate of median damping. For tanks similar to the example tank in which inelastic bolt stretching, some nonlinear tank uplift, and slight "elephant-foot" buckling of the tank shell is expected to occur prior to failure, $5 \%$ of critical damping represents a conservative estimate of median damping for this mode. Using the 
median NUREG/CR-0098 (11) spectrum shape to define the SME spectrum shape, the 5\% damped spectral acceleration for frequencies in the range of 5.5 to $6.6 \mathrm{~Hz}$ and a $S M E_{e}$ of $0.27 g$ can be given by:

$$
S_{A_{I}}=2.12(0.27 g)=0.57 g
$$

Reierence (2) () (10) then recommend that the impulsive mode base shear, $V_{1}$, and moment at the base of the tank shell, $M_{I}$, be given by:

$$
\begin{aligned}
& v_{I}=S_{A_{I}}\left[w_{H}+w_{S}+w_{I}\right] \\
& M_{I}=S_{A_{I}}\left[w_{H} x_{H}+w_{S} x_{S}+w_{I} x_{I}\right]
\end{aligned}
$$

where $W_{I}$ is the effective impulsive weight of the contained fluid and $x_{I}$ is its effective height above the tank base. Only this effective impulsive fluid weight is important and the head weight, $W_{H}$, and side wall weight, $W_{S}$, and their effective heights could be ignored with less than a $5 \%$ error.

For tanks with $(H / R)$ ratios greater than 1.5 such as for this example tank, $(\underline{2})(10)$ suggest that $W_{I}$ and $X_{I}$ can be estimated from:

$H / R \geq 1.5$

$$
\begin{aligned}
& \frac{W_{I}}{W_{W}}=1.0-0.436(R / H) \\
& \frac{X_{I}}{H}=0.50-0.188(R / H)
\end{aligned}
$$

For $(H / R)$ less than 1.5, see (2) or (10) for corresponding equations. Thus, for this example tank with $(H / R)=1.85$,

$$
\begin{aligned}
& W_{I}=0.764 W_{W} \\
& W_{I} X_{I}=0.304 W_{W} H
\end{aligned}
$$

Thus, the impulsive mode base shear and moments from Equations (H.3) and (H.4) are:

$$
v_{I}=1310 \text { kips } \quad M_{I}=19,500 \mathrm{kip}-\mathrm{ft}
$$


Veletsos ( 2 ) provides a slightly different formulation for $W_{1}$ and $X_{I}$ which leads to the following for this example tank:

$$
W_{I}=0.724 W_{W} \quad W_{I} X_{I}=0.304 W_{W} H
$$

which will lead to a slightly lower base shear, $V_{I}$, and the identical base moment, $M_{I}$, as that given above in Equation $(H-7)$. Haroun and Housner (8) provide a slightly different formulation for computing $V_{I}$ and $M_{I}$ which leads to the following valües for this example tank:

$$
V_{I}=1150 \text { kips } \quad M_{I}=20,800 \mathrm{kip}-\mathrm{ft}
$$

The differences in impulsive response results from each of these three approaches are small and within the underlying accuracy of the computations. Any of these three approaches may be used. For this example, the results given in Equation (H.7) will be used.

Next, the impulsive mode hydrodynamic pressures, $P_{I}$, on the tank should be approximately estimated. Reference (2) and (10) suggest that for depths y from the top of the fluid greater than $0.15 \mathrm{H}$, this pressure $P_{I}$ can be approximated by:

$y / H \geq 0.15$

$$
P_{I}=\frac{W_{I} X_{I} S_{A_{I}}}{1.36 R H^{2}}
$$

with the impulsive pressure varying approximately linearly from zero at the top of the fluid $(y=0)$ to the value for Equation ( $H .8)$ at $y=0.15 \mathrm{H}$. For the example tank, at depths greater than 5.6-ft, the impulsive pressure is estimated from Equation $(\mathrm{H}-8)$ to be:

$$
\underline{y \geq 5.6 \mathrm{ft}}: P_{I}=3.5 \mathrm{psi}
$$

Both Veletsos ( $\underline{5}$ ) and Haroun and Housner ( 1 ) provide alternate formulations for estimating the impulsive pressure. For this example tank, by () the maximum impulsive pressure is estimated to be 3.4 psi, whereas by ( $\underline{8}$ ) the average impulsive pressure over the tank height is estimated to be 3.4 psi. Since hydrodynamic pressures seldom govern the determination of the SME capacity, any of these approaches are adequate for estimating the impulsive pressure. The impulsive pressure given by Equation $(\mathrm{H}-9)$ has been used in Table $\mathrm{H}-1$ for this example tank. 


\section{H.2.2 Horizontal Corrective (Sloshing) Mode Response}

The fundamental convective mode frequency, $f_{c}$, can be estimated from:

$$
f_{C}=\sqrt{\frac{1.50 \mathrm{ft} / \mathrm{sec}^{2}}{R} \tanh (1.835(\mathrm{H} / \mathrm{R}))}
$$

Thus, for the example tank, $f_{C}=0.274 \mathrm{~Hz}$. This convective mode is very lightly damped and it is suggested that a damping ratio of 0.5 percent of critical damping be used when estimating the convective mode spectral acceleration, $S_{A_{C}}$. For the SME spectrum shape anchored to the estimated $S M E_{e}$ of $0.27 \mathrm{~g}$, at $f_{C}=0.274 \mathrm{~Hz}$ and 0.5 percent damping the convective mode spectral acceleration is:

$$
S_{A_{C}}=0.084 g
$$

The convective mode base shear and moment are then given by:

$$
\begin{aligned}
& V_{C}=S_{A_{C}} W_{C} \\
& M_{C}=S_{A_{C}} W_{C} x_{C}
\end{aligned}
$$

where $W_{c}$ is the effective convective mode fluid weight and $x_{c}$ is its effective height of application above the base.

From (1), (2) and (10), these effective convective mode weights and heights may be estimated from:

$$
\begin{aligned}
& \frac{W_{C}}{W_{W}}=0.46(R / H) \tanh (1.835(H / R)) \\
& \frac{X_{C}}{H}=1.0-\frac{\cosh (1.835(H / R))-1.0}{1.835(H / R) \sinh (1.835(H / R))}
\end{aligned}
$$

For the example tank with $(H / R)=1.85$, these equations lead to:

$$
W_{C}=0.248 W_{W} \quad W_{C} X_{C}=0.180 W_{w} H
$$

which result in a convective mode base shear and moment of : 


$$
V_{c}=60 \mathrm{kips} \quad M_{C}=1600 \mathrm{kip}-\mathrm{ft}
$$

Note how low these convective mode shears and moments are relative to the impulsive mode values (Eqn $(H-7)$.

The hydrodynamic convective pressure can be estimated from $(\underline{1}),(\underline{2})$ and (10):

$$
P_{C}=\frac{0.267 W_{W} S_{A_{C}}}{R H} \quad \frac{\cosh \left(1.835\left(\frac{H-y}{R}\right)\right)}{\cosh (1.835(H / R))}
$$

Such pressures are generally negligible compared to either the hydrodynamic impulsive pressure, $P_{I}$, or the hydrostatic pressure, $P_{S T}$, except at shallow depths below the fluid surface. For instance, at the first critical section ( $y=22 \mathrm{ft}$ ) listed in Table $\mathrm{H}-1$ for the example tank, the convective pressure, $P_{C}$, is only 0.1 ps $i$ and is less at greater depths.

Lastly, the fundamental mode fluid slosh height, $h_{5}$, can be approximated by (1), $(\underline{2}),(\underline{5})$, and $(\underline{10})$ :

$$
h_{S}=0.837 R\left(S_{A_{C}} / g\right)
$$

which leads to the following slosh height for the example tank:

$$
h_{s}=1.41 \mathrm{ft}
$$

\section{H.2.3 Vertical Fluid Mode Response}

Hydrodynamic pressures due to the fundamental vertical fluid mode should be estimated at critical locations on the tank shell. The fundamental frequency of this vertical response mode is heavily influenced by the breathing flexibility of the tank shell and is typically only slightly greater than the horizontal impulsive mode frequency. Thus, the vertical fluid response mode typically lies in the highly amplified spectral acceleration response regime so that the use of the vertical PGA to compute the hydrodynamic pressures due to vertical response as recomended by (2) is generally inappropriate and unconservative.

One approach to compute the fundamental frequency of the vertical fluid response mode is to use:

$$
f_{V}=\frac{C_{L V}}{2 \pi H} \sqrt{E_{s / \rho_{s}}} \text { where } C_{L V}=C_{W V} \sqrt{\frac{0.127 \rho_{s}}{\rho_{L}}}
$$


which is the same equation form as that used to compute the horizontal impulsive mode response. The coefficient $C_{W V}$ for water in a rigidly supported steel tank of uniform thickness, $t$, can be obtained from Table 1 of ( $(\underline{6})$ as a function of the $(t / R)$ and $(H / R)$ ratios. For variable wall thickness tanks, an effective uniform wall thickness must be estimated similarly as was done for the horizontal impulsive modal frequency. Assuming a ( $t / R)$ ratio of 0.00092 and $(H / R)=1.85$ for the example tank, $C_{W V}$ is estimated to be 0.091 for which Equation $(H-19)$ provides a frequency $f_{v}$ of $6.4 \mathrm{~Hz}$ with an estimate accuracy range of about 5.9 to $7.0 \mathrm{~Hz}$. The frequency of the vertical fluid response mode can also be estimated using Eqn C3500-13 of (10) which for the above example tank properties leads to a frequency $f_{v}$ estimate of $6.6 \mathrm{~Hz}$ which is in the same range.

The hydrodynamic vertical fluid response mode pressure for a tank on a rigid foundation can be estimated from ( 7 ):

$$
P_{V}=0.8 \rho_{L} H\left(S_{A_{V}}\right) \cos \left(\frac{\pi}{2} \frac{H-y}{H}\right)
$$

which is more accurate than the linear varying pressure defined by Eqn 3500-7 of $(10)$. An effective damping value together with the vertical mode frequency $f_{V}$ must be used to estimate the vertical spectral acceleration $S_{A_{v}}$. A flexible foundation greatly reduces the vertical fluid mode hydrodynamic pressures below that computed for a rigid foundation ( $\underline{5})(2)$. One way to approximate this influence is through the use of increased damping. Even for tanks on a rock site there will be some foundation flexibility. To partially account for this effect, it is recommended that for a CDFM evaluation $5 \%$ of critical damping be used when estimating the vertical spectral acceleration $S_{A_{V}}$ for tanks on rock sites.

For the previously defined SME spectrum shape anchored to a SME horizontal PGA of $0.27 \mathrm{~g}$ (corresponding vertical PGA of $0.18 \mathrm{~g}$ ), the $5 \%$ damped $S_{A_{V}}$ for a frequency range of 5.9 to $7.0 \mathrm{~Hz}$ is:

$$
S_{A_{V}}=2.12(0.18 g)=0.38 g
$$

Using this spectral accelerations, the hydrodynamic vertical fluid response mode pressures, $P_{V}$, computed by Equation $(H-20)$ for the various capacity evaluation locations are presented in Table $\mathrm{H}-1$ for the example tank. Note that the hydrodynamic pressures due to the vertical fluid response mode, $P_{v}$, exceed those due to the horizontal response mode at all locations. 


\section{H.2.4 Combined Responses}

The combined horizontal seismic responses for base shear ( $V_{S H}$ ) base moment ( $M_{S H}$ ), and horizontal seismic hydrodynamic pressures $\left(P_{S H}\right)$ can be obtained by the SRSS combination the corresponding horizontal impulsive and convective responses (2). $(\underline{5})(10)$. Thus, for the example tank the impulsive responses (Eqn $(H-7)$ ) and the convective responses (Eqn $(H-15)$ ) produce the following combined horizontal seismic responses:

$$
V_{S H}=1310 \text { kips } \quad M_{S H}=19,600 \mathrm{kip} \mathrm{ft}
$$

Table $H-1$ presents the combined horizontal seismic hydrodynamic pressures, $P_{S H}$, at the capacity evaluation locations. Note that for this example tank, the combined horizontal seismic responses are essentially equal to the impulsive mode responses and the influence of the convective mode is negligible.

For the purposes of the membrane hoip stress capacity check, it is necessary to have an estimate of the maximum seismic hydrodynamic pressures, $P_{S M}$, which can be obtained by the SRSS combination of the horizontal seismic pressures, $P_{S H}$, and the vertical fluid response hydrodynamic pressures $P_{V}$. For the example tank, Table $H-$ 1 presents the maximum seismic hydrodynamic pressures, $P_{S M}$, at capacity evaluation locations.

For the purposes of estimating the compressive buckling capacity of the tank shell, it is necessary to have an estimate of the expected maximum and minimum fluid pressures acting against the tank shell near its base at the location of maximum axial compression during the time of maximum base moment. Those expected maximum and minimum compression zone pressures, $P_{C_{+}}$and $P_{C_{-}}$, at the time of maximum base moment can be estimated from:

$$
\begin{aligned}
& P_{C_{+}}=P_{S T}+P_{S H}+0.4 P_{V} \\
& P_{C-}=P_{S T}+P_{S H}-0.4 P_{V}
\end{aligned}
$$

where the 0.4 factor on $P_{v}$ is to account for the probable vertical mode hydrodynamic vertical pressure at the time of maximum base moment. 
Similarly, for the purposes of estimating the expected minimum fluid hold-down forces in the zone of maximum tank wall axial tension, one needs an estimate of the minimum tension zone fluid pressure, $\mathrm{P}_{\mathrm{T}_{-}}$, at the time of maximum moment as given by:

$$
P_{T-}=P_{S T}-P_{S H}-0.4 P_{V}
$$

For the sliding capacity evaluation one needs the expected minimum average fluid pressure on the base plate, $P_{a}$, at the time of maximum base shear as given by:

$$
P_{a}=P_{S T}-0.4 P_{v}
$$

Using the base plate hydrostatic and hydrodynamic pressures given in Table $\mathrm{H}-1$, the following combined pressures are computed for the base plate from Eqns (H-22) through $(H-24)$ :

\section{Base Plate}

$$
\begin{aligned}
& P_{C_{+}}=16.0+3.5+(0.4)(4.9)=21.5 \mathrm{psi} \\
& P_{C_{-}}=16.0+3.5-(0.4)(4.9)=17.5 \mathrm{psi} \\
& P_{T_{-}}=16.0-3.5-(0.4)(4.9)=10.5 \mathrm{psi} \\
& P_{a}=16.0-(0.4)(4.9)=14.0 \mathrm{psi}
\end{aligned}
$$

Lastiy, one also needs an estimate of the expected minimum total effective weight, $W_{T e}$, of the tank shell acting on the base at the time of maximum moment and base shear:

$$
W_{T e}=\left(W_{H}+W_{S}\right)\left(1-0.4\left(A_{V} / g\right)\right)
$$

which for the example tank problem is:

$$
W_{T e}=57.6 \mathrm{kips}
$$


Generally the SME capacity of a minimally anchored flat bottom tank such as that shown in Figure $\mathrm{H}-1$ is governed by the seismic overturning moment capacity at its base, MSC, compared to the applied overturning moment seismic response, $M_{S H}$. In turn, this moment capacity depends upon the axial compressive buckling capacity of the tank shell $\left(C_{m}\right)$, the tensile hold-down capacity of the anchor bolts including their anchorage and attachment to the tank $\left(T_{B C}\right)$, and the hold-down capacity of fluid pressure acting on the tank base plate $\left(T_{e}\right)$. Thus, each of these capacities must be estimated prior to estimating the overturning moment capacity.

Although unlikely for larger radius tanks, the SME capacity is sometimes governed by the sliding shear capacity at the tank base, $V_{S C}$, compared to the seismic base shear response, $V_{S H}$. Ever though it does not appear that any butt welded steel tank has ever failed due to seismic induced membrane hoop stresses due to combined hydrostatic plus hydrodynamic fluid pressures, the SME capacity of this failure mode should also be checked. Such a check requires the computation of the pressure capacity, $P_{C A}$, of the tank shell for comparison with the combined hydrostatic, $P_{S T}$, and maximum seismic hydrodynamic, $P_{S M}$, pressures.

Some assessment of the possibility and consequences of fluid sloshing against the tank roof should be made. For soil sites, foundation failure modes should also be checked. Lastly, the possibility of failure of piping or their attachment to the tank should be assessed.

Each of these topics will be further discussed in this subsection.

\section{H.3.1 Compressive Buckling Capacity of the Tank Shell}

The most likely way for tank shells to buckle is in "elephant-foot" buckling near the base of the tank shell. The tank shell is subjected to a biaxial stress state consisting of hoop tension and vertical (axial) compression. In addition, radial deformations under internal pressure which are prevented at the base due to membrane tension in the base plate introduce eccentricity and bending stresses in the axial plane which further induce the tendency to "elephant-foot" buckle. The onset of such "elephant-foot" buckles can be estimated using elastic-plastic collapse theory $(\underline{12})(\underline{13})(\underline{14})$. However, it should be noted that the initiating of "elephant-foot" buckles does not directly correspond to failure of a tank. Many tanks have continued to perform their function of containing fluid even after 
developing substantial "elephant-foot" buckles. However, no simple capability to predict tank performance after the development of "elephant-foot" buckles exists. Therefore, for a CDFM approach to estimating the HCLPF SME capacity of tanks, the onset of "elephant-foot" buckling will be judged to represent the limit to the compressive buckling capacity of the tank she $11, C_{m}$. However, because such buckling is not failure, no significant conservatism needs to be intentionally introduced when estimating $C_{m}$.

The "elephant-foot" buckling axial stress capacity, "o, of the tank shell can be accurately (no intentional conservatism) estimated by $(\underline{12})(\underline{13})(\underline{14})$ :

$$
\sigma_{p}=\frac{0.6 E_{S}}{\left(R / t_{s}\right)}\left[1-\left(\frac{P}{\sigma_{y e} t_{s}}\right)^{2}\right]\left[1-\frac{1}{1.12+S_{1}^{1.5}}\right]\left[\frac{s_{1}+\left(\sigma_{y e} / 36 k s i\right)}{S_{1}+1}\right]
$$

where $\left.S_{1}=\left(R / t_{s}\right) / 400\right)$

and $t_{s}$ is the sidewall thickness near the shell base, $P$ is the tank internal pressure near its base, and $\sigma_{y e}$ is the effective yield stress of the tank shell material. For HCLPF capacity computations it is suggested that a slight conservatism be introduced by specifying $C_{m}$ in terms of $0.9 \sigma_{p}$. Thus:

$$
c_{m}=0.9 \sigma_{p} t_{s}
$$

Furthermore, $P$ should be set equal to $P_{C+}$ which represents the maximum combined pressure against the tank wall at the time of maximum moment. Lastly, for a tank shell material such as SA 240-Type 304 stainless steel with no specific yield point, it is uncertain what stress to use for $\sigma_{y e}$. This material shows no flat yield plateau and continues to show increasing stress with increased strain until its minimum ultimate stress capacity of $75 \mathrm{ksi}$ is reached. For a CDFM capacity evaluation it seems reasonable to set $\sigma_{y e}$ at the ASME Code (15) seismic design limit for primary local membrane plus primary bending which is $2.4 S_{M}$ or $45 \mathrm{ksi}$ for this material. The potential uncertainty range for $\sigma_{y e}$ is estimated to be from 30 ksi to $60 \mathrm{ksi}$ with it likely to exceed $45 \mathrm{ksi}$.

For the example tank, $P=P_{C_{+}}=21.5 \mathrm{psi}, t_{s}=0.375$ inch, $E=27.7 \times 10^{3} \mathrm{ksi}$, $\left(R / t_{s}\right)=640, s_{1}=1.6,\left(P / \sigma_{y e}\right)=0.48 \times 10^{-3}$, and $\left(\sigma_{y e} / 36 \mathrm{ksi}\right)=1.25$. Thus, from Eqn $(H-27), \sigma_{p}=17.6 \mathrm{ksi}$. When one considers the potential range on $\sigma_{y e}$ of 30 to $60 \mathrm{ksi}$, then the resultant range on $\sigma_{p}$ is $13.1 \mathrm{ksi}$ to $21.1 \mathrm{ksi}$. The influence of this uncertainty range on the SME capacity will be subsequently assessed. Using Ean $(H-28)$, the compressive capacity of the shell is: 


$$
C_{m}=0.9(17.6 \mathrm{ksi})(0.375 \mathrm{in})=5.92 \mathrm{kip} / \mathrm{in}
$$

Although unlikely to govern for overall seismic response of fluid containing tanks, one should also check the buckling capacity of supported cylindrical shelis under combined axial bending and internal pressure. The axial bending induced buckling stress, ${ }^{\circ} C B$, for such a case can be conservatively (essentially lower bound) estimated from (16):

$$
\sigma_{C B}=\left(0 . \sigma_{Y}+\Delta Y\right) \frac{E_{S}}{\left(R / t_{S}\right)}
$$

where $\Phi=\frac{1}{16} \sqrt{R / t_{s}}$

$$
Y=1-0.73\left(1-e^{-\phi}\right)
$$

and $\Delta y$ is an increase factor for internal pressure as given by figure 6 of (16). The minimum compression zone pressure at the base of the tank shell, $P_{C_{-}}$, corresponding to the time of maximum moment should be used with figure 6 of ( 16 ) when estimating $\Delta y$. Equation $(H-30)$ is appropriate for ${ }^{\circ} C B$ so long as ${ }^{\circ} C B$ is less than the yield stress, $\sigma_{Y}$. Otherwise, see $(\underline{16})$. Since ${ }^{\circ} \mathrm{CB}$ is conservatively estimated, it may be directly used with no reduction for estimating $C_{m}={ }^{\circ}{ }_{C B} t_{s}$.

For the example tank, $P_{C-}=17.5 \mathrm{psi}, y=0.419$, and $\Delta_{Y}=0.15$. Thus, $\sigma_{C B}=17.4$ ksi which exceeds $0.9 \sigma_{p}$ so this buckling mode does not govern.

\section{H.3.2 Bolt Hold-Down Capacity}

The bolt hold-down capacity, $T_{B C}$, is governed by the weakest of the following elements:

1. Bolt tensile capacity

2. Anchorage of bolt into concrete foundation

3. Capacity of the top plate of bolt chairs to transfer bolt loads to the vertical chair gussets.

4. Attachment of the top plate and vertical chair gussets to the tank shell.

5. Capability of tank shell to withstand concentrated loads imposed on it by bolt chairs. 
To simplify this already long tank evaluation example, it will be assumed that the bolt tensile capacity is the weakest bolt hold-down link for the example tank. However, in an actual application, each of these five capacity elements need to be checked.

Type A307 anchor bolts are the most common low strength anchor bolt material used to anchor tanks and other heavy equipment. For a CDFM capacity evaluation, their capacity can be estimated based upon the Part 2 provisions of the AISC Code (17). The example tank has 2 -inch diameter bolts which have a nominal crosssectional area, $A_{\text {nom }}$, of 3.14 square inches. Based upon Part 2 of the AISC Code for $A 307$ bolts the tension capacity, $T_{B C}$, is:

$$
T_{B C}=1.7(20 \mathrm{ksi})\left(3.14 \mathrm{in}^{2}\right)=107 \mathrm{kips}
$$

\section{H.3.3 Fluid Hold-Down Forces}

For tanks with minimum anchorage, hold-down forces resulting from fluid pressure acting on the tank bottom will contribute significantly to the overturning moment capacity, MSC, of the tank. The situation in the region of axial tension in the tank shell is illustrated in Figure $\mathrm{H}-2$ for small uplift displacements, $\delta e^{\text {. At }}$ point "O" away from the tank sidewall, the tank bottom is in full contact with the foundation and the displacements, rotation, and moment in the tank bottom is zero. However, at the intersection of the tank bottom and side wall at point "1", the tank bottom has uplifted $\delta_{e}$ and rotated $\alpha_{e}$. The length of the uplift zone is $\ell$ and the fluid pressure, $P$, on the tank bottom and side wall resists this uplift. This uplift is accompanied by the development of a tension, $T_{e}$, and moment, $M_{e}$, in the side shell at the intersection with the tank bottom. This tension, $T_{e}$, acts as a fluid hold-down force on the tank shell. For a given uplift height, $\delta e$, the hold-down tension, $T_{e}$, that develops is both a function of the bending stiffness of the tank shell which is a function of its thickness, $t_{s}$, and radius, $R$, and the bending stiffness of the base plate which is a function of its thickness, $t_{b}$.

For a tank she 11 restrained against radial displacement at point " 1 " by the base plate, the relationship between $M_{e}$ and $a_{e}$ can be obtained from pages 276 through 278 of Flugge (18) to be:

$$
M_{e}=K_{S} a_{e}+M_{F}
$$


where $K_{S}=\frac{2 K K}{R}$

$$
\begin{aligned}
& \frac{M_{F}}{P}=\frac{R t_{s}}{\sqrt{12\left(1-u^{2}\right)}}\left[1-\frac{R}{H K}\right] \\
& K=\frac{E_{S} t_{s}^{3}}{12\left(1-u^{2}\right)} \\
& K=\left[\left(R / t_{s}\right) \sqrt{3\left(1-u^{2}\right)}\right]^{\frac{1}{2}}
\end{aligned}
$$

One can show for the base plate that:

$$
\begin{aligned}
& a_{e}=\frac{P_{\ell}{ }^{3}}{12 E_{s} I_{b}}-\frac{M_{e} e^{2}}{2 E_{s} I_{b}} \\
& \delta_{e}=\frac{P_{l}{ }^{4}}{24 E_{s} I_{b}}-\frac{M_{e} e^{2}}{6 E_{s} I_{b}} \\
& T_{e}=\frac{P_{l}}{2}+\frac{M e}{2}
\end{aligned}
$$

where $\quad I_{b}=\frac{t_{b}{ }^{3}}{12\left(1-v^{2}\right)}$

Combining Equations ( $\mathrm{H}-32)$ and $(\mathrm{H}-33)$, one obtains:

$$
\begin{aligned}
& \frac{E_{s} I_{b} b_{e}}{P}=\left[\frac{e^{4}}{24}-\left(\frac{1}{F}\right)\left(\frac{K_{s} e^{5}}{72 E_{s} I_{b}}+\frac{M_{F}}{P} \frac{e^{2}}{6}\right)\right] \\
& \frac{T e}{P}=\left[\frac{\ell}{2}+\left(\frac{1}{F}\right)\left(\frac{K_{s} \ell^{2}}{12 E_{s} I_{b}}+\frac{M_{F}}{P_{l}}\right)\right] \\
& \frac{M_{e}}{P}=\left(\frac{1}{F}\right)\left(\frac{K_{s} e^{3}}{12 E_{s} I_{b}}+\frac{M_{F}}{P}\right) \\
& \frac{M_{+}}{P}=\frac{\ell^{2}}{8}-\frac{\left(M_{e / P}\right)}{2}+{\frac{\left(M_{e / P}\right.}{2 q^{2}}}^{2}
\end{aligned}
$$

where $F=\left[1+\frac{K_{s}^{\ell}}{2 E_{s} I_{b}}\right]$ 
Using Equations $(H-34)$ through $(H-37)$, one can determine the uplift height $\left(\delta_{e}\right)$, tank shell hold-down tension $\left(T_{e}\right)$, end moment $\left(M_{e}\right)$, and maximum positive moment $\left(M_{+}\right)$in the base plate as a function of the uplift length, $l$, and fluid pressure, $P$. From this information the relationship between $\delta_{e}$ and $T_{e}$ is obtained. This small displacement theory solution is only strictly applicable under the following conditions:

1. $(\ell / R) \leq 0.15$. The solution ignores the stiffening of the base plate from hoop behavior and thus conservatively overpredicts the displacement $\delta_{e}$ corresponding to a given $T_{e}$ as the ratio $(2 / R)$ becomes larger.

2. $\left(\delta_{e} / t_{b}\right) \leq 0.6$. This solution is based upon small displacement theory and conservatively ignores the beneficial influence of large displacement membrane theory together with membrane tensions in the base plate to reduce $\delta_{e}$ corresponding to a given $T_{e}$. For unanchored tanks it has been shown $(\underline{19})(\underline{20)}$ that large displacement membrane theory greatly increase the fluid hold-down forces, $T_{e}$. Thus, for unanchored tanks, ignoring large displacement membrane theory is likely to lead to excessive conservatism. For anchored tanks the uplift heights $\delta$ are not expected to be so great and only moderate conservatism is expected to result from ignoring large displacement membrane effects. Unfortunately no simple solution exists for considering such membrane effects and so one must either accept this source of conservatism for anchored tanks at this time or make judgmental corrections to the completed fluid hold-down forces following guidance from (19) and (20).

3. $\left(M_{e} / M_{P_{p}}\right) \leq 0.9 ;\left(M_{e} / M_{p}\right) \leq 0.9$; and $\left(M_{+} / M_{p_{b}}\right) \leq 0.9$ where $M_{p_{b}}$ and $M_{p_{s}}$ are the plastic moment capacity of the base plate and shell sidewalls, respectively. The previous solution is an elastic solution and becomes unconservative if these conditions are not met. An alternate solution with plastic hinges at locations where these conditions are not met is easily formulated following the same approach as was used herein but is judged to be unwarranted because violation of these conditions is nighly unlikely in a CDFM evaluation.

Only this third condition leads to unconservative estimates of the hold-down force $T_{e}$ corresponding to a given uplift displacement $\delta_{e}$. The first two conditions can be violated so long as one is willing to accept the resulting conservative 
uncerestimation of the fluid hold-down force $T_{e}$ for a given uplift displacement is

Since the hold-down force $T_{e}$ increases with increasing fluid pressure, $P$, one should conservatively substitute for $P$ the minimum tension zone fluid pressure $P_{T-}$, expected at the time of maximum moment. For the example tank problem, $P=$ $P_{T_{-}}=10.5 \mathrm{psi}$. Using this fluid pressure and Equations $(\mathrm{H}-34)$ through $(\mathrm{H}-37)$, the relationship between $T_{e}, \delta_{e}$, and 2 has been estimated for the example tank. Figure H-3 shows the relationship between $T_{e}$ and $\delta_{e}$ while figure $H-4$ relates $T_{\varepsilon}$ and $\varepsilon$. Note that even with zero uplift there is a hold-down tension $T_{e}$ of 58 ibs/inch resulting from base rotation of the side wall due to the outward acting fluid pressure thereon. At an uplift $\delta_{e}$ of 0.15 inches which corresponds to $0.6 t_{b}$ or the approximate limit of small displacement theory, the hold-down tension has incraased to 98-lbs/inch. Beyond this point, small displacement theory will become increasingly conservative. The relationship shown in Figure $H-3$ between $T_{e}$ and $\delta_{e}$ can be reasonably and slightly conservatively approximated by the linear expression:

$$
T e=T_{e 0}+T_{e 1} \delta_{e}
$$

where for the example tank: $T_{e o}=60 \mathrm{lbs} / \mathrm{inch}$, and $T_{e l}=270 \mathrm{lbs} / \mathrm{inch}^{2}$. This approximation will be used in the overturning moment capacity evaluation. To partially account for memorane tension effects, this equation for $T_{e}$ will be linearly extrapolated beyond the displacement $\delta_{e}$ of 0.15 inch for which it is directly applicable.

\section{H.3.4 Overturning Moment Capacity}

With an estimate of the compressive capacity of the tank she $11\left(C_{m}\right)$, the anchor bolt hold-down capacity $\left(T_{B C}\right)$, and the relationship between fluid hold-down force and uplift displacement, it is possible to estimate the overturning moment capacity $\left(M_{S C}\right)$ of the tank making several conservative, but reasonable as sumptions:

1. The bottom of the tank shell is assumed to rigidly rotate vertically (plane sections remain plane).

2. The cross-section of the tank at the top of the top plate of the bolt cha ihs ( $h_{c}$ above the base in figure H.1) is assumed to remain 
horizontal so that all vertical tank distortions needed to result in base uplift and mobilization of the anchor bolts must be accormodated over the height, $h_{c}$.

3. The tank she 11 remains linear elastic until the compressive buckling capacity is reached at the point of maximum compression and reaching this limit defines the overturning moment capacity. The maximum compressive shortening of the tank shell which takes place over the length $h_{c}$ between the tank base and the top plate of the bolt chains is conservatively underestimated to be:

$$
\delta_{c}=\frac{c_{m} h_{c}}{E_{s} t_{s}}
$$

Figure H-5 schematically illustrates the vertical loadings applied to the tank shell and its vertical rotational distortion resulting from these assumptions. At any angle $a$, the vertical uplift, $\delta_{e} ;$ is then:

$$
\delta_{e}=\delta_{C}\left(\frac{\cos \alpha-\cos \beta}{1+\cos \beta}\right)
$$

Then, if the anchor bolts are assumed to be anchored at a depth $h_{a}$ below the tank base as is shown in Figure $\mathrm{H}-1$, and chair distortions are considered negligible, then the anchor bolt tension. $T_{B}$, in anchor bolt " $i$ " corresponding to a maximum tank shell compressive distortion, $\varepsilon_{C}$, below the top of the bolt chair is:

$$
T_{B_{i}}=T_{B P}+d_{e_{i}}\left(\frac{A_{B} E_{B}}{h_{a}+h_{C}}\right) \leq T_{B C}
$$

or by combining with Equation $\mathrm{H}-40$ :

$$
T_{B_{i}}=T_{B P}+K_{B}\left(\frac{\cos a_{i}-\cos B}{1+\cos B}\right) \leq T_{B C}
$$

where $K_{B}=\frac{\delta_{C} A_{B} E_{B}}{\left(h_{a}+h_{C}\right)}$

and $a_{j}$ is the angle a at bolt " $i ", T_{B P}$ is any bolt pretension, $A_{B}$ is the bolt area, and $E_{B}$ is the bolt modulus of elasticity. The fluid hold-down force, $T_{e_{\alpha}}$, at angle a obtained by combining Equations $H-38$ and $H-40$ is:

$$
T_{e}=T_{e o}+\Delta T_{e}\left(\frac{\cos a-\cos B}{1+\cos \beta}\right)
$$

where $\Delta T_{e}=T_{e l}{ }^{\delta} \mathrm{C}$ 
For the force distribution shown in Figure $\mathrm{H}-5$, the tank maximum shell compression, $C_{m}$ ', and overturning moment capacity, $M_{S C}$ can be obtained by setting the sum of all vertical forces to $W_{T e}$ and summing moments about the centerline of the circular tank cross-section. Thus:

$$
\begin{aligned}
& C_{m}^{\prime}=\left(\frac{1 W_{T e}+\sum_{i=1}^{n} T_{B i}}{2 R}+T_{e o} s\right) C_{1}+\Delta T_{e} C_{3} \\
& M_{S C}=C_{m}^{\prime} C_{2} R^{2}+\sum_{i=1}^{n}\left(T_{B} R \cos a_{i}\right)+T_{e 0} R^{2}(2 \sin 3)+\Delta T_{e} C_{4} R^{2}
\end{aligned}
$$

where

$$
\begin{aligned}
& C_{1}=\frac{1+\cos B}{\sin B+(\pi-B) \cos B} \\
& C_{2}=\frac{\sin B \cos B+\pi-B}{1+\cos B} \\
& C_{3}=\frac{\sin B-B \cos B}{\sin B+(\pi-B) \cos B} \\
& C_{4}=\frac{B-\sin B \cos B}{1+\cos B}
\end{aligned}
$$

First, a trial angle $B$ is selected and Equation $H-46$ is used to obtain $C_{m}{ }^{\prime}$ which is compared to the shell compressive capacity $C_{m}$ from Equation $H-28$. The angle $B$ is varied until $C_{m}{ }^{\prime}=C_{m}$. Then Equation $H-47$ is used with this $B$ to determine the tank overturning moment capacity.

The example tank problem has the following properties:

$$
\begin{aligned}
& C_{m}=5.92 \mathrm{kips} / \text { in } \\
& T_{\text {edo }}=0.060 \mathrm{kips} / \mathrm{in} \\
& W_{T e}=57.6 \mathrm{kips} \\
& R=20 \mathrm{ft}=240 \text { inches } \\
& t_{s}=0.375 \text { inch }
\end{aligned}
$$

$$
\begin{array}{ll}
T_{B C}=107 \mathrm{kips} & \\
T_{e 1}=0.270 \mathrm{kips} / \mathrm{in}^{2} & \\
A_{B}=3.14 \mathrm{inch}^{2} & E_{B}=29 \times 10^{3} \mathrm{ksi} \\
h_{C}=24.75 \text { inches } & h_{a}=28.5 \text { inches } \\
E_{s}=27.7 \times 10^{3} \mathrm{ksi} &
\end{array}
$$

Then, from Equation $(H-39) \delta_{C}=0.0141$ inch; from Equation $(H-43) K_{B}=24.5$ kips; and from Equation $(H-45) \Delta T_{e}=0.0038 \mathrm{kipps} /$ inch. Since any bolt pretension, $T_{B P}$, is unreliable after a number of years, it will be conservatively assumed that $T_{B P}$ is zero. Now it is possible to compute the overturning moment capacity, $M_{S C}$, using Equations $(H-47)$ and $(H-48)$ as shown in Table $H-2$.

A -47 
Thus,

$$
\begin{aligned}
& M_{S C}=20,800 \mathrm{kip}-f \mathrm{t} \\
& \Sigma T_{B}=635.7 \mathrm{kips} \\
& B \quad=2.70
\end{aligned}
$$

One should check whether the largest bolt elongation ( $a=0$ ) is acceptable using Equation $(\mathrm{H}-40)$ to determine the elongation. For this solution:

$$
\delta_{\text {eo }}=0.28 \text { inches }
$$

Certainly these bolts which have an overall length of 53.25 inches can accommodate a 0.28 inch or $0.53 \%$ elongation. The ability of the connection between the bottom plate and the tank side wall to withstand the distortions associated with this uplift height, $\delta_{e o}$, should also be considered. However, a well-designed detail at this location should be capable of easily withstanding more than 0.3 inch uplift. If the elongation in considered too great, then neither $\delta_{c}$ nor the compressive buckling capacity can develop in the compressive zone. Then, a maximum $\delta_{e o}$ should be defined and the quantities $\delta_{c}$ and $C_{m}$ should be back calculated using Equations $(H-40)$ and $(H-39)$, respectively, for any given trial B. These back calculated $\delta_{C}$ and $C_{m}$ values should then be used to solve for Msc. However, seldom will the resultant $\delta_{\text {eo }}$ be excessive so that this additional refinement is seldom necessary.

The previous solution was for the case when $a=0$ is aligned with one of the bolts. This case will nearly always govern. However, one should also check the case where $a=0$ lies midway between bolts (i.e., for the example problem rotate the $a=0$ line by 22.5 degrees). For the example problem, $M_{S C}=21,000 \mathrm{kip}-\mathrm{ft}$ for this alternate case and thus does not govern.

As noted in Subsection H.3.1, the HCLPF buckling capacity of the tank shell has some uncertainty. This capacity could possibly (but highly unlikely) range as low as $4.42 \mathrm{kips} / \mathrm{in}$, rather than the $5.92 \mathrm{kips} / \mathrm{in}$ used in the above calculations. With $C_{m}$ of $4.42 \mathrm{kips} / \mathrm{in}$, the overturning capacity $M_{S C}$ would be reduced to 19500 kip-ft or $94 \%$ of that for $C_{m}=5.92 \mathrm{kips} / \mathrm{in}$. Thus, $M_{S C}$ is rather insensitive to the estimate of $c_{m}$ for this tank and the uncertainty issue previously raised for estimating $C_{m}$ turns out to be relatively unimportant. 
Given the estimate of $M_{S C}=20,800 \mathrm{kip}-f t$ and the previously estimated overturning response $M_{S H}=19,600$ kip-ft for an $S M E_{e}$ of $027 \mathrm{~g}$, it is now possible to estimate the SME level from Equation $H-1$. However for this estimate, one must have an estimate of the inelastic energy absorption reduction factor, $k$, to apply to linear computed seismic response. Certainly, this combined bolt yielding and tank shell buckling failure mode for overturning moment is not brittle so that a $k$ value less than unity should be appropriate in a HCLPF capacity evaluation. However, within the existing state of knowledge it is very difficult to make an appropriate estimate of $k$ for this failure mode. Therefore, it is conservatively reconmended that $k$ be taken as unity at this time for this failure mode. Future research into tank failure capacities will likely lend to a less conservative recommendation in the future. With this recommendation, from Equation $\mathrm{H}-1$ :

$$
\text { SME }_{M}=\frac{20,800 \mathrm{kip}-\mathrm{ft}}{19,600 \mathrm{kip}-\mathrm{ft}}(0.27 \mathrm{~g})=0.29 \mathrm{~g}
$$

based upon the overturning moment capacity.

\section{H.3.5 Sliding Capacity}

As noted previously, one should also check the sliding shear capacity, $V_{S C}$, with the seismic base shear response, $V_{S H}$. Since the base shear, $V_{S H}$ and the base overturning moment, $M_{S H}$, are primarily due to the fluid horizontal impulsive mode of response, they both are maximum at the same time. Thus, the sliding shear capacity is:

$$
V_{S C}=(C O F)\left[H_{V_{e}}+\left(\Sigma T_{B}\right)\right]
$$

where $W_{V_{e}}=W_{T}+P_{a}\left(\pi R^{2}\right)$

and (COF) is the coefficient of friction between the tank base and its

foundation. It should be noted that the effective tank shell weight, $W_{T}$, used for the overturning moment calculations did not include the effective weight of the tank base plate. Neither does the seismic base shear response, $V_{S H}$, include the response forces associated with this base plate. One could add the effective base plate weight to $W_{T_{e}}$ in Equation $H-52$, but then would also have to add its response contribution to $V_{S H}$. For any tank where the base plate weight is small compared to the fluid weight, this refinement is unnecessary in that the SME for base shear capacity will be uneffected. 
Most large diameter flat bottomed tanks such as the example tank have a slight cone to their bottom plate so that contained fluid will always drain away from the center and toward the drain pipe at the edge. This cone is generally created by a variable thickness sand cushion between the tank bottom plate and its foundation. Furthermore, the tank bottom is generally made up of slightly overlapped fillet welded individual plates. Thus, the surface between the bottom plate and the sand cushion contains a series of rough steps. Under these conditions, it is reasonably conservative to estimate:

$$
(\operatorname{con}) \geq 0.7
$$

However, under other base plate details, a conservative (COF) might be less.

For the example tank, $W_{T_{e}}=57.6$ kips, $P_{a}=14.0 \mathrm{psi}$, and $\left(\Sigma T_{B}\right)=635.7$ kips have been previously computed. Thus, from Equations $(H-51)$ through $(H-53)$ :

$$
\begin{aligned}
W_{V_{e}} & =2600 \mathrm{kips} \\
V_{S C} & =2260 \mathrm{kips}
\end{aligned}
$$

For base shear sliding, it is recomended that the inelastic energy absorption reduction factor $k$ be taken as unity. Combfing the above $V_{S C}$ with the previously computed base shear response, $V_{S H}=1310 \mathrm{kips}$ for $S M E_{e}=0.27 \mathrm{~g}$, from Equation $(H-$ 1) one obtains:

$$
\text { SME }_{V}=\frac{2260 \mathrm{kips}}{1310 \mathrm{kips}}(0.27 \mathrm{~g})=0.47 \mathrm{~g}
$$

based on base shear. Since SME $V$ substantially exceeds SME S $_{M}$ base shear does not govern the seismic margin capacity of this tank. This situation is nearly always the case.

\section{H.3.6 Fluid Pressure Capacity}

It is recommended that the CDFM hoop membrane stress capacity of the tank she 11 , $\sigma_{a}$, be taken as the ASME Code (15) seismic design limit for primary stress of $2.0 S_{M}$ or $37.5 \mathrm{ksi}$ for SA240-Type 304 stainless steel. Using this stress limit, the pressure capacities, ${ }{ }_{C A}$, at capacity evaluation locations of the example tank are given in Table $\mathrm{H}-\mathrm{l}$. A hoop membrane stress failure mode of a steel tank with 
full penetration butt-welded joints is certainly ductile. For this case an inelastic energy absorption seismic response reduction factor of $k=0.8$ can be easily justified for HCLPF capacity evaluations. Using the capacity pressure, $P_{C A}$, the hydrostatic pressures, $P_{S T}$, and the maximum seismic induced hydrodynamic pressures, $P_{S M}$, listed in Table $H .1$ together with $k=0.8$, the SME associated with fluid pressure can be computed using Equation (H-1). Table $H-1$ presents these computed $S M E_{p}$ at several capacity evaluation locations for the example tank. Note that the lowest computed $S M E_{p}$ is $1.3 \mathrm{~g}$ which is many times greater than $S M E_{M}$ SO that it does not govern. Even if $k=1.0$ had been used, the lowest $S_{M E}$ would have been $1.0 \mathrm{~g}$ from which the same conclusion would be reached. Actually, the fluid pressure capacity never seems to govern the seismic capacity either by the CDFM capacity calculation procedure or from seismic experience for normal designed flat bottomed steel tanks with butt-welded side plates. Therefore, pressure computations are included more for completeness than for SME capacity evaluations.

\section{H.3.7 Other Capacity Checks}

For the example tank, the fluid slosh height, $h_{s}$, was estimated to be $1.41 \mathrm{ft}$. for $\mathrm{SME}_{e}=0.27 \mathrm{~g}$. Based on Figure $\mathrm{H}-1$, this slosh height would have to exceed about $3.4 \mathrm{ft}$ before any significant roof damage might be expected. Thus from Equation $(H-1)$, SME $S \geq 0.65 \mathrm{~g}$ and will not govern. Even if roof damage might be expected, such damage is unlikely to impair the ability of the tank to contain fluid for at least a few days after an earthquake.

For tanks on soil sites, one should also check the SME capacity of the tank foundation and this check sometimes governs.

Lastly, the possibility of piping failure or the failure of nozzles where such piping is attached to the tank should be checked. Such failures will likely lead to loss of tank contents. In fact, a significant fraction of the cases of seismic induced loss of tank contents have been due to such failures when the piping contained poor seismic details. A check of seismic details of piping and their attachment to such tanks should be made during the seismic walkdown. A SME evaluation of piping and nozzles is only necessary when potentially poor seismic details are observed. Otherwise, this failure mode can be screened out during the walkdown. The issues involved are:

1. Are heavy pipe valves or long piping runs being supported through the piping nozzles off of either the tank side walls or the bottom plate, 
or are they independently supported? If heavy valves or long piping runs are being supported off the tank, then the ability of the nozzles and the tank side wall or bottom plate to withstand the imposed seismic induced inertial forces should be checked. Methods outlined in Welding Research Bulletin 107 (21) may be used to compute local stresses in the tank shel1, whereas the strength acceptance criteria for vessels contained in Section 6 can be used for stress capacity. If heavy valves or long piping runs are independently supported as is the normal case, then for SME levels up to 0.5 these inertial checks at piping attachment points should generally be unnecessary based upon seismic experience and judgment.

2. Is there sufficient piping flexibility to accommodate relative seismic anchor movements (SAM) between where the piping is supported from the tank shell and where it is independently supported? Almost any type of flexibility loop in the pipe between the tank and independent piping supports should be sufficient for SME levels up to $0.5 \mathrm{~g}$ so no evaluation should generally be necessary. However, if a straight run of pipe exists between where the pipe is independently rigidly supported and the tank shell, the piping nozzle and tank shell should be evaluated for their ability to withstand the expected relative SAM.

\section{H.4 Discussion of Seismic Capacity}

For the example tank, the HCLPF SME PGA capacity of $0.29 \mathrm{~g}$ was governed by overturning moment which is nearly, always the case. Again, it should be noted that this HCLPF statement is conditional on the SME response spectrum anchored to this SME PGA of $0.29 \mathrm{~g}$ not being exceeded by a future ground motion at more than $16 \%$ of the natural frequencies within the frequency range and direction of interest. The overturning moment response and thus the SME capacity is primarily governed by the horizontal impulsive response mode with an estimated frequency of about $6 \mathrm{~Hz}$. Thus, the frequency range of interest is about 5 to $7 \mathrm{~Hz}$. For a circular tank, the direction of interest is the direction of largest horizontal ground motion. Thus, the HCLPF statement is conditional on the SME response spectrum anchored to a PGA of $0.29 \mathrm{~g}$ not being exceeded at more than $16 \%$ of the natural frequencies between 5.0 and $7.0 \mathrm{~Hz}$ in the direction of largest horizontal ground motion. 
Within the frequency range of 5.0 to $7.0 \mathrm{~Hz}$, the SME $5 \%$ damped response spectrum used for this example tank had a spectral amplification factor of 2.12 .

Therefore, the SME $5 \%$ damped spectral acceleration becomes $(2.12)(0.29 \mathrm{~g})=$ $0.61 \mathrm{~g}$. Therefore, rather than defining the HCLPF SME capacity of this tank in terms of its PGA of $0.29 \mathrm{~g}$, an improved HCLPF SME capacity statement for this tank would be as follows:

"The HCLPF SME capacity of this example tank is a $5 \%$ damped, $84 \%$ nonexceedance probability spectral acceleration between 5.0 and $7.0 \mathrm{~Hz}$ of $0.61 \mathrm{~g}$ in the direction of largest horizontal ground motion."

Again, it should be noted that this capacity is a HCLPF capacity and not the median capacity which is expected to be more than twice as great.

\section{H.5 References}

1. Lockheed Aircraft Corporation and holmes \& Narver, Inc., Nuclear Reactors and Earthquakes, TID-7024, prepared for the US Atomic Energy Commission, Washington, D.C., August 1963.

2. Kennedy, R.P., "Above Ground Vertical Tanks", Section 2.2, Appendix C, Recommended Revisions to Nuclear Regulatory Commission Seismic Design Criteria, NUREG/CR-1161, Lawrence Livermore National Laboratory, prepared for U.S. Nuclear Regulatory Comission, December, 1979.

3. Veletsos, A.S., and J.Y. Yang, "Dynamics of Fixed-Base Liquid Storage Tanks", Presented at U.S.-Japan Seminar for Earthquake Engineering Research with Emphasis on Lifeline Systems, Tokyo, Japan, November, 1976, PP 314-341.

4. Veletsos, A.S., and J.Y. Yang, "Earthquake Response of Liquid Storage Tanks", Advances in Civil Engineering Through Engineering Mechanics, Proceedings of the Engineering Mechanics Division Specialty Conference, ASCE, Raleigh, North Carolina, 1977, ppl-24.

5. Veletsos, A.S., "Seismic Response and Design of Liquid Storage Tanks", Chapter 7, Guidelines for the Seismic Design of Oil and Gas Pipeline Systems, ASCE, $198 \overline{4}$.

6. Veletsos, A.S., and Yu Tang, "Interaction Effects in Vertically Excited Stee? Tanks", Dynamic Response of Structures, ASCE, March, 1986, pp 636-643.

7. Veletsos, A.S., and Yu Tang, "Oynamic of Vertically Excited Liquid Storage Tanks", Journal of Structural Engineering, Vol. 112, No. 6, ASCE, June, 1986, pp 1228-1246.

8. Haroun, M.A. and G.W. Housner, "Seismic Design of Liquid Storage Tianks", Journal of the Technical Councils of ASCE, Vol. 107, No. TCl, 1981, pp 191207. 
9. Haroun, M.A. and G.W. Housner, "Complications in Free Vibration Analys is of Tanks", Journal of the Engineering Mechanics Division, Vol. 108, No. EM5, ASCE, 1982 , pp 801-818.

10. ASCE Standard and Commentary - Seismic Analysis of Safety-Related Nuclear Structures, ASCE 4-86, September, 1986.

11. Newmark, N.M. and W.J. Hall, Development of Criteria for Seismic Review of Selected Nuclear Power Plants, NUREG/CR-0098, Nuclear Regulatory Commission, May 1978.

12. Priestly, M.J.N., et. al., "Seismic Design of Storage Tanks", Bullet in of the New Zealand National Society for Earthquake Engineering, Voi. 19, No. 4, December 1986.

13. Priest1y, M.J.N., Seismic Design of Storage Tanks, Recommendations of a Study Group of the New Zealand National Society for Earthquake Eng ineering, December 1986.

14. Rotter, J.M., Local Inelastic Collapse of Pressurized Thin Cylindrical Steel Shells Under Axidl Compression, Research Report, School of Civil and Mining Engineering, University of Sydney, Australia, 1985.

15. Subsection NC3300, ASME Boiler \& Pressure Vessel Code, 1983.

16. Buckling of Thin-Walled Circular Cylinders, NASA SP-8007, National Aeronautics and Space Administration, August 1968.

17. Manual of Steel Construction, Eighth Edition, AISC, 1980.

18. Flugge, W., Stresses in Shel1s, Springer-Verlag, 1960.

19. Manos, G.C., "Earthquake Tank-Wall Stability of Unanchored Tanks", Journal of Structural Engineering, Vol. 112, No. 8, ASCE, August 1986, Pp. 1863-1880.

20. Haroun, M.A., and H.S. Badawi, "Nonlinear Axisymmetric Uplift of Circular Plates", Dynamics of Structures, ASCE, August 1987, pp 77-89.

21. K.R. Wickman, A.G. Hopper and J.L. Mershon, "Local Stresses in Spherical and Cylindrical Shells due to External Loadings", Welding Research Bulletin 107, August 1965. 
Table H-1.

Hydrostatic and Hydrodynamic Pressures and SME Capacity at Capacity Evaluation Locations

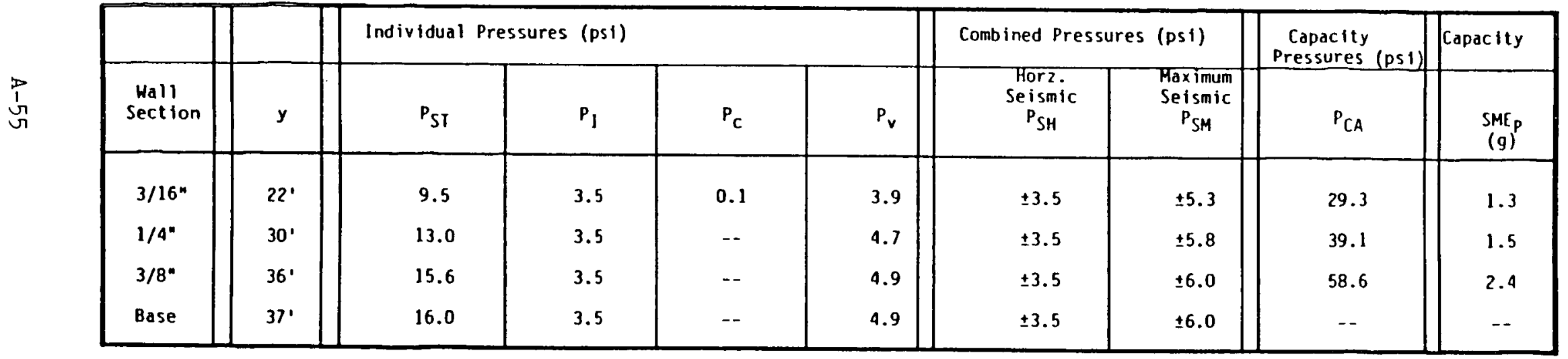


Table H-2.

Computation of Overturning Moment

Capacity, MSC

J

\begin{tabular}{|c|c|c|c|c|c|c|c|c|c|c|c|c|}
\hline Trtal & $(1+\cos A)$ & $c_{1}$ & $c_{2}$ & $c_{3}$ & $c_{4}$ & $\underset{k 1 p s}{T_{B 1}}$ & 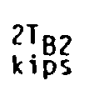 & $\begin{array}{l}2 T_{B 3} \\
\text { kips }\end{array}$ & $\begin{array}{l}T_{k i p s} B A \\
\text { ips }\end{array}$ & $\sum_{k i p s} I_{B}$ & $\underset{k i p / \text { in }}{C_{M}^{\prime}}$ & ${ }_{k i p-r t}^{14}$ \\
\hline 2.80 & 0.0578 & 4.40 & .449 & 286 & 53.9 & 107 & 214 & 214 & 199.4 & 734.4 & 9.09 & 23.100 \\
\hline 2.75 & 0.0757 & 3.84 & .513 & 186 & 41.0 & 107 & 214 & 214 & 140.7 & 675.7 & 7.21 & 21,900 \\
\hline 2.70 & 0.0959 & 3.41 & .575 & 102 & 32.2 & 107 & 214 & 214 & 100.7 & 635.7 & 5.87 & 20,800 \\
\hline
\end{tabular}


Materials: Shell \& Chairs: SA240 - Type 304 SST Bolts: A307 - 2" \&

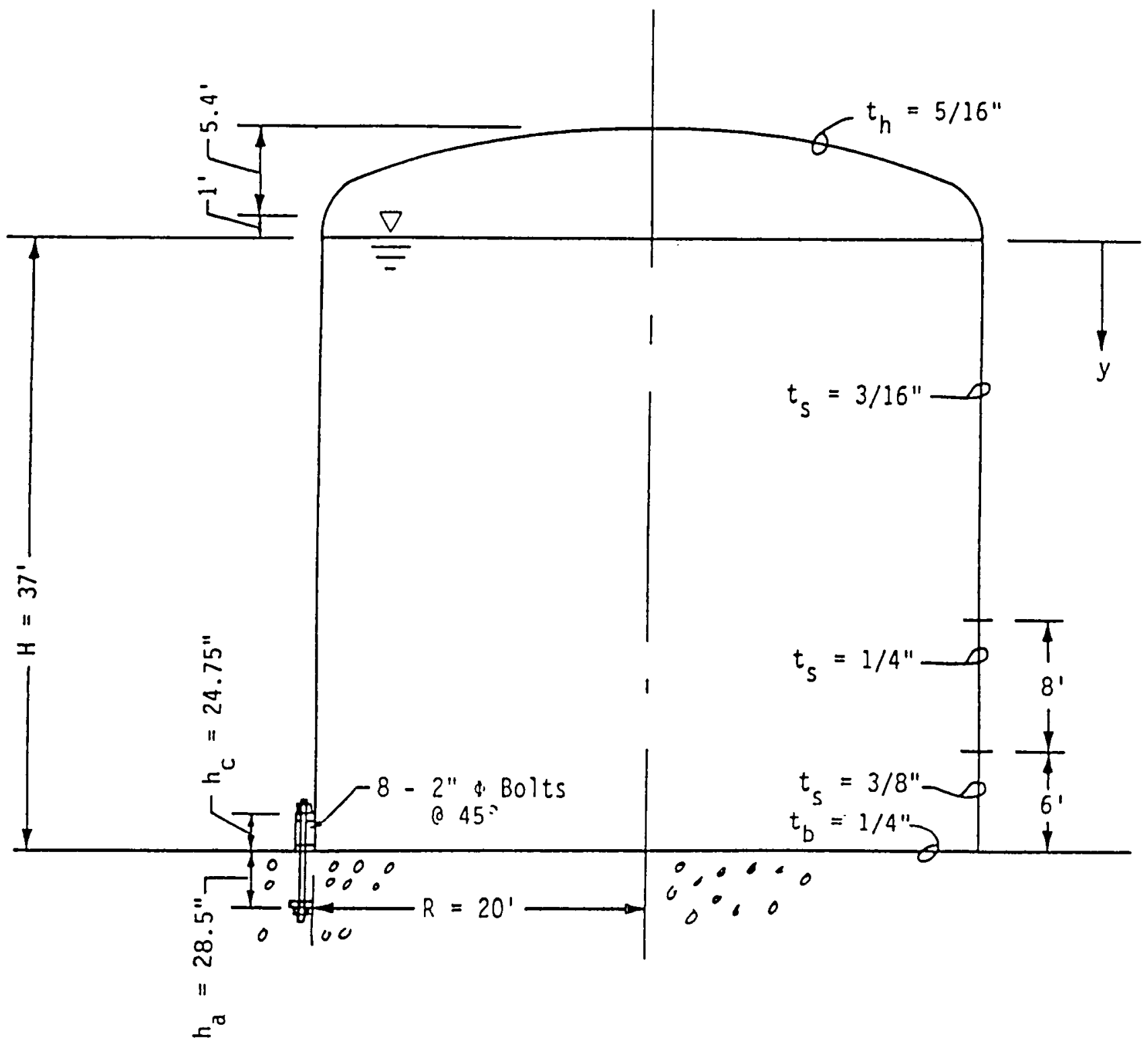

Figure H-1. Example Tank 
SHELL

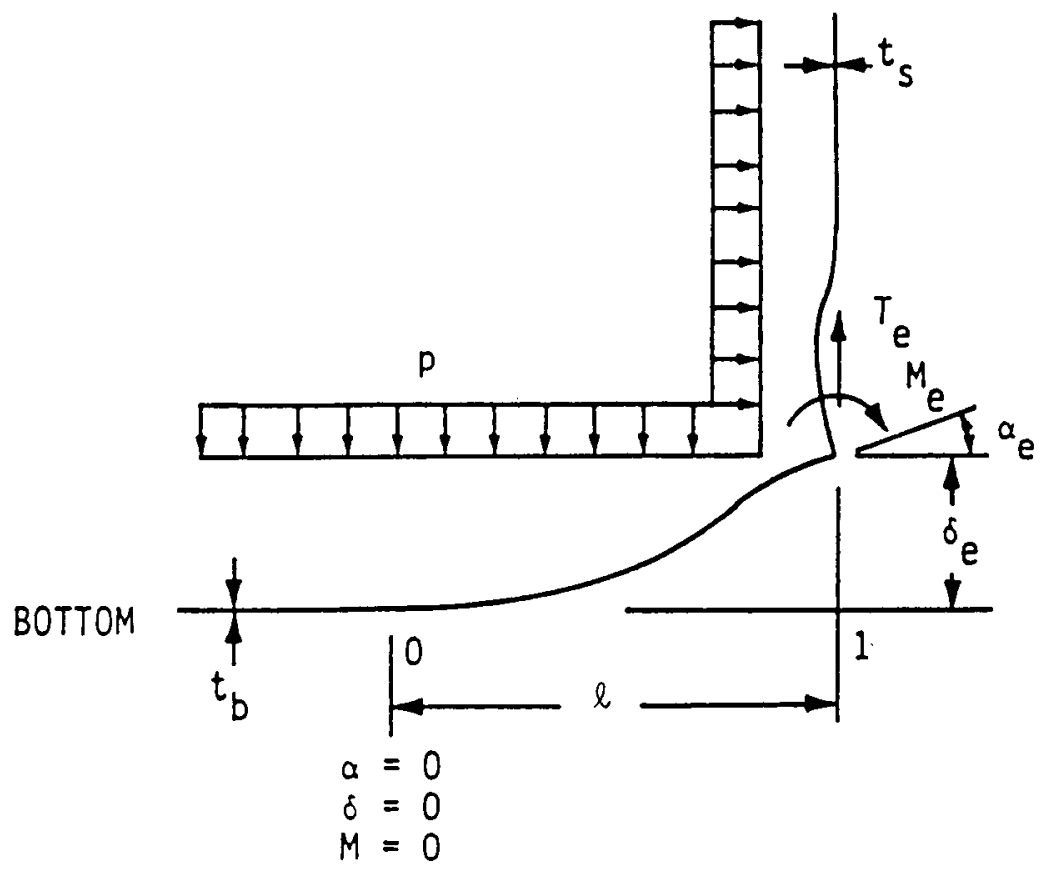

Figure H-2. Schematic Illustration of Tank Bottom Behavior Near Tensile Region of Tank Shell 

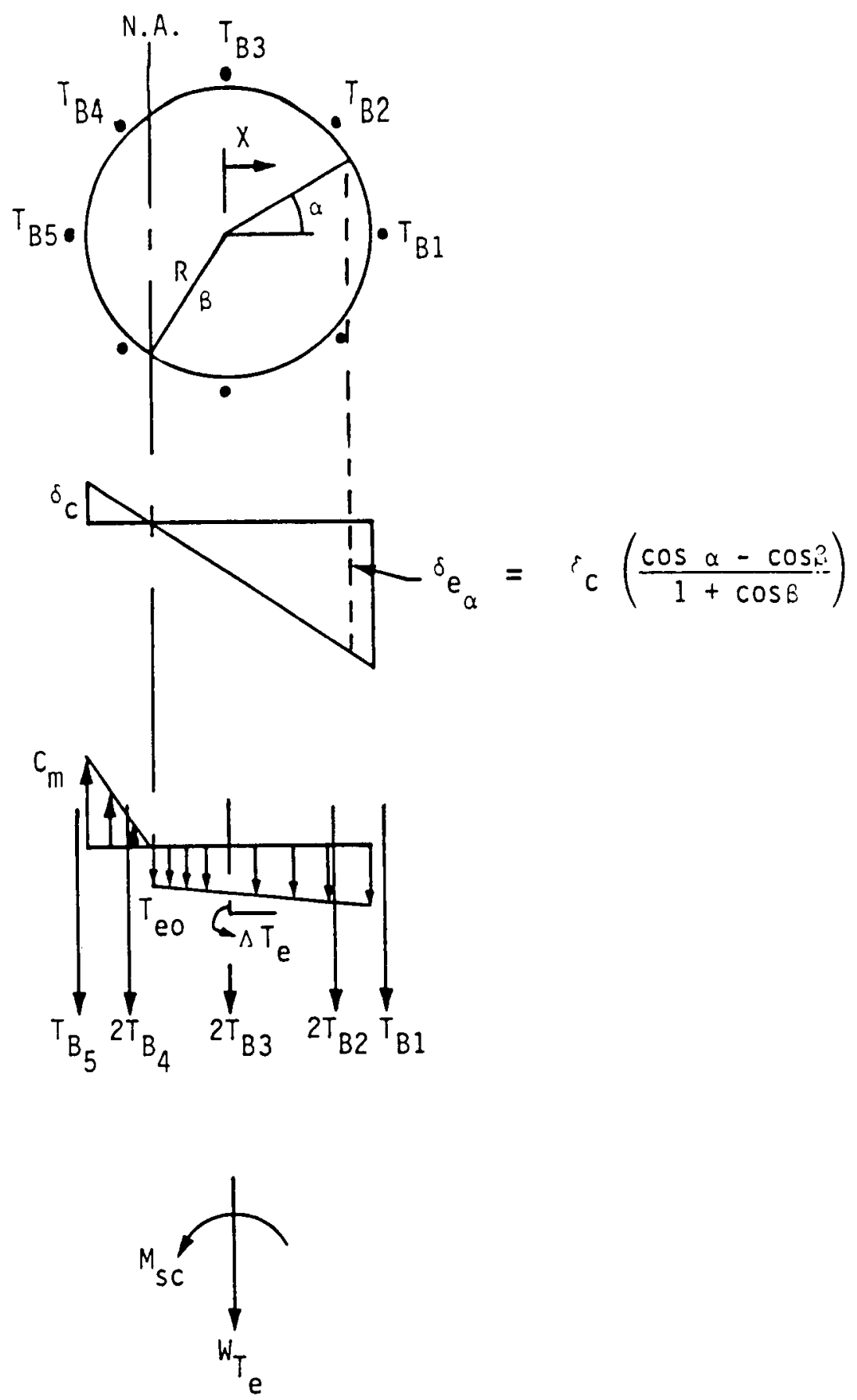

Figure H-5. Vertical Loading on Tank Shell at Base 
FLAT-BOTTOM VERTICAL WATER STORAGE TANK

FRAGILITY METHOD

By

R. P. Kennedy 


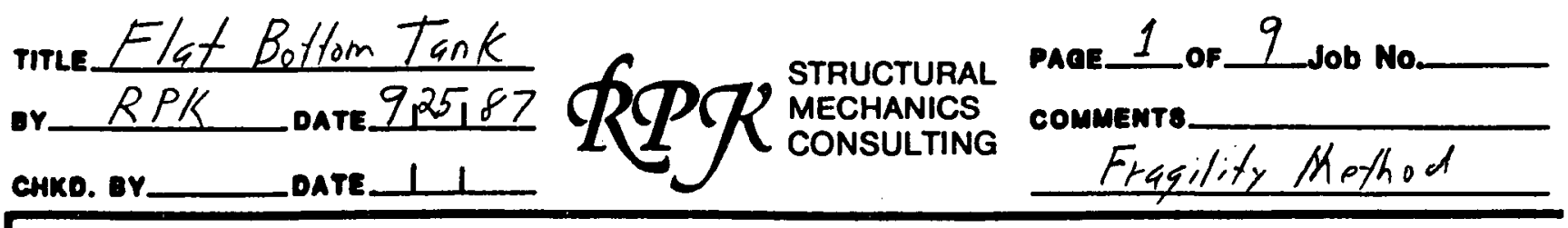

1) Tank Shown in Fig. 1

2) Capacity controlled by overturning moment (See Appendix $H$ CDFM (alcalations) due to latest horizontal component

3) Estimated Median Capacity SME

$$
\text { SHE } \left.=\frac{C_{\text {capacity }}}{\text { Response }_{e}} \text { (SHE }\right)
$$

Response for $S K E_{e}=0.6 \mathrm{~g}$

$$
\begin{aligned}
& M_{e}=M_{C P F M} \cdot\left(\frac{0.6 \mathrm{~g}}{S M E_{\text {CorM }}}\right) \cdot \frac{F_{D} F_{F}}{F_{G M H}}
\end{aligned}
$$

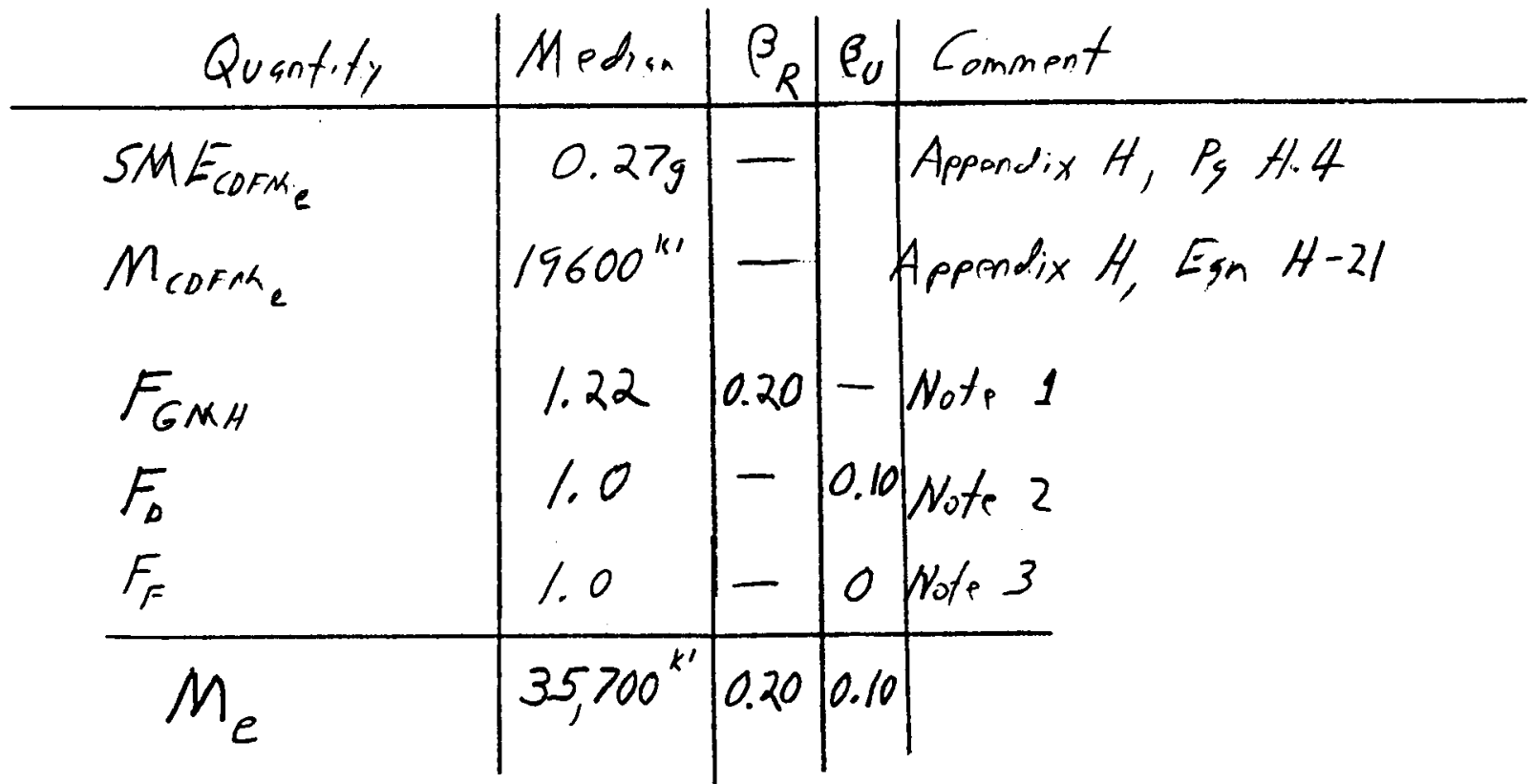

A-62 


$$
\begin{aligned}
& \text { ritue Flat Bottion Tank } \\
& \text { or RPK } \\
& \text { Date } 9,25187 \\
& \text { (1) }
\end{aligned}
$$

STRUCTURAL

MECHANICS

CONSULTING

PACE 2 of 9 Job No

COMMENT:

Fragitity Method

Note 1: $F_{G M A}=$ Horizonts/ Ground Motion Response Fortor

Median Tank Response is controlled by medial lanerest hotizontsl sportral respone at tank imputiop mode notural fregeoncy while SATE spection defined as 84\% NEP laresest horie. spertal tesponse

$$
\therefore \stackrel{V}{F}_{G M H}=F_{7}=1.22 \quad(\text { Table 1) }
$$

Mut include pesk \& valley varisbility

$$
\therefore \beta_{R_{\text {GM }}}=\beta_{R_{1}}=0.20 \quad \text { (Table 1) }
$$

Note 2: $F_{0}=$ Dsmping Uncertainty Fartor

Overfurning Momenty Controlled by Horiz. Impulive Mode at madian freguency, $\dot{f}_{ \pm}=6.0 \mathrm{~Hz}$ (Appendix H) using NUREG/(ex)0098 Madish Amplifiestion Factow.

$$
\begin{aligned}
& \text { CDFM: Dampin, } \left.=5 \% \quad A F_{6.0,5 \%}=2.12\right\} \quad F_{D}=1.0 \\
& \text { Median }: D_{\text {ampin, }}=5 \% \\
& -1.5 \beta_{0}: D_{\text {ampin, }}=3 \% \quad A F_{60,3 \%}=2.46 \\
& \beta_{D_{U}}=\frac{\ln (2.46 / 2.12)}{1.5}=0.10
\end{aligned}
$$

Note 3: $F_{F}=$ Fles. Shift Eftect

$\pm / \beta$ freg. tange $=5.5 \mathrm{~Hz}$ to $6.6 \mathrm{~Hz}$

Within this range AF does not change for CR0098 sperteom

$$
\therefore F_{F}^{v}=1.0 \quad \beta_{F_{u}}=0
$$

A-63 
TitLe $\frac{\text { Flat Bottom Tank }}{R P K}$ structural page 3 or 9 Job No. DATE 9125187 QR O

CHKD. Dr Date 11

CONSULTING. Fragility. Method

Other Response. Quantities Required for Capacity Evaluation

$$
\text { for SME }=0.6 \mathrm{~g} \text { (84\% NEP Largest Hotiz) }
$$

Pressures on Base

Static Pressure $: P_{S T}=16.0$ psi $\quad\left(P_{g} H .30\right.$, Appendix H)

Imputire Pressure $: P_{I}=P_{I_{\text {CORM }}} \cdot\left(\frac{0.6 \mathrm{~g}}{0.275}\right)\left(\frac{F_{D} F_{F}}{F_{G M H}}\right)$

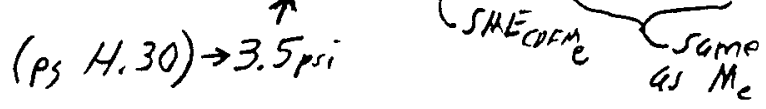

(1) $F_{0} \xi F_{v}$ same as

Vert. Pressure $: \quad P_{V}=P_{V_{\text {Cork }}}\left(\frac{0.6 \mathrm{~g}}{0.27 g}\right)\left(\frac{F_{b} F_{F}}{F_{\text {G MV }}}\right)$

for Me $_{e}$

$\left(P_{s} H .30\right) \rightarrow 4.9_{p s i}$

(2) F F MV is for vertical

$$
\left.\begin{array}{l}
F_{G M V}=\frac{F_{G}}{F_{g}}(0.67)=\frac{1.41}{0.67}(0.67)=1.41 \\
\beta_{R_{G M V}}=\beta_{R_{g}}=0.34
\end{array}\right\} \text { From Table } 1
$$

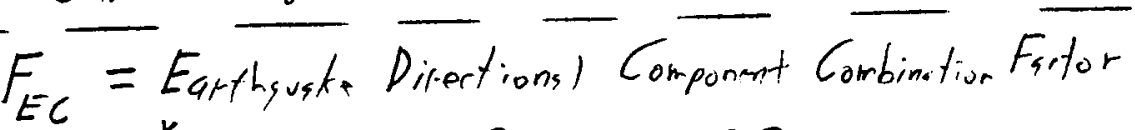

$$
\tilde{F}_{E C}=0.4 \quad \beta_{R_{E C}}=0.39
$$

Max Comp. $Z_{\text {ane }}$ Preserver $\left.: P_{C t}=P_{T T}+P_{I}+F_{E C} \cdot P_{V}\right\} E_{\text {sn }} H .22$

Min Comp Z on- Presenter: $\left.P_{C-}=P_{T}+P_{T}-F_{t c} P_{r}\right\} \xi \%$ H. 23

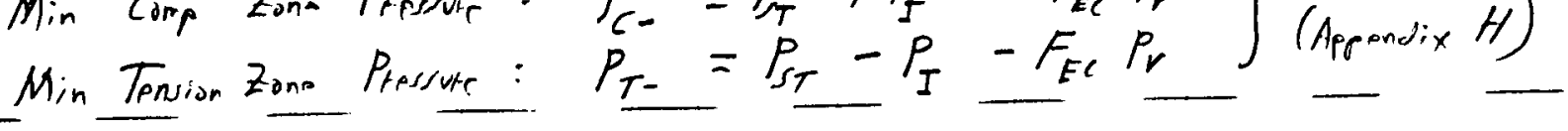

Effective Hold down Wt

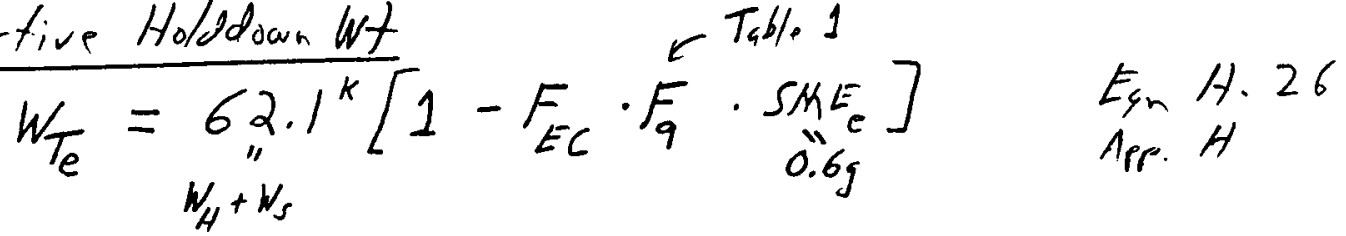

Apr. H

$$
\stackrel{v}{F}_{q}=0.48 \quad \beta_{R_{q}}=0.34
$$

A-64 
Tite Flat Botfom Tank or_RPK Date $\frac{9125187}{\text { chko. or }}$

aнko. DY. CONSULTING . COMMENTE

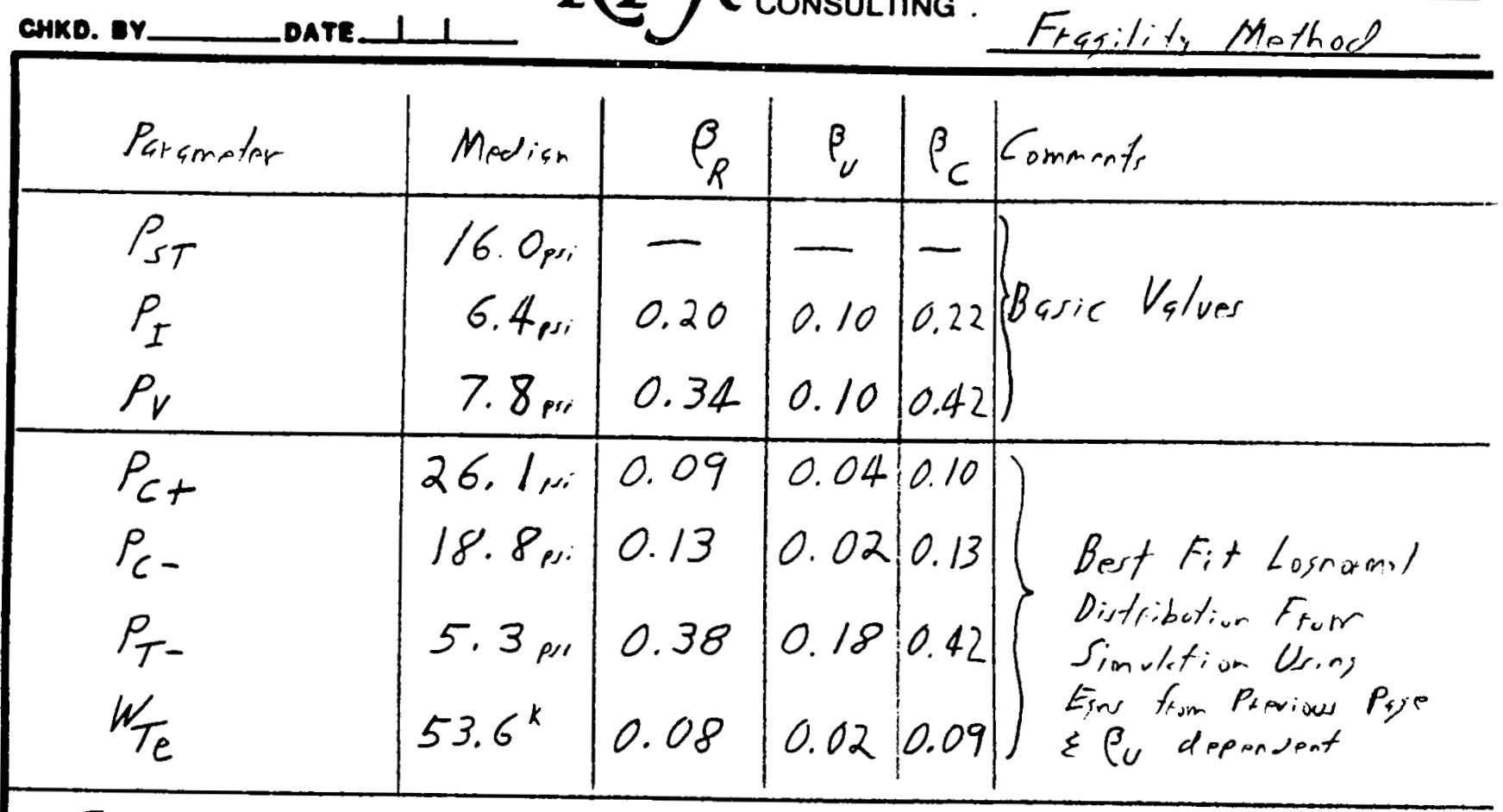

Caparity Computotions

Compressiver Caparily Tank Sholl

Controllood by Elophant Foot Buckling (Egn H.27, App. H)

$$
\begin{aligned}
& C_{m}=F_{E}\left(\frac{0.6 E_{s} t_{s}}{\left(R / t_{s}\right)}\right)\left[1-\left(\frac{P_{C+} R}{\sigma_{y e} t}\right)^{2}\right]\left[1-\frac{1}{1.12+S_{I}^{1.5}}\right]\left[\frac{S_{I}+\frac{\sigma_{r e}}{3 \sigma^{* i t}}}{S_{S}+1}\right] \\
& S_{I}=\left(\frac{R}{400 t}\right)=\left(\frac{240^{\prime \prime}}{400\left(3^{\prime \prime}\right)}\right)=1.6 \\
& E=27.7 \times 10^{3} \mathrm{ks} \quad t_{s}=3 / \mathrm{s}^{\prime \prime} \quad R=240^{\prime \prime} \\
& \therefore C_{m}=9.74 \% F_{E}\left[1-\left(\frac{640 P_{c t}}{\sigma_{y e}}\right)^{2}\right][0.682]\left[\frac{1.6+\frac{\sigma_{y p}}{36^{* i r}}}{2.6}\right] \\
& F_{E}=E_{\text {sn }} \text {. Elrof V V V ri,ble } \quad F_{E}=1.0 \quad \beta_{G_{E}}=0.11
\end{aligned}
$$
$\sigma_{0}$ but pxpattad to be elaser to $\sigma_{0}$

A- 65 


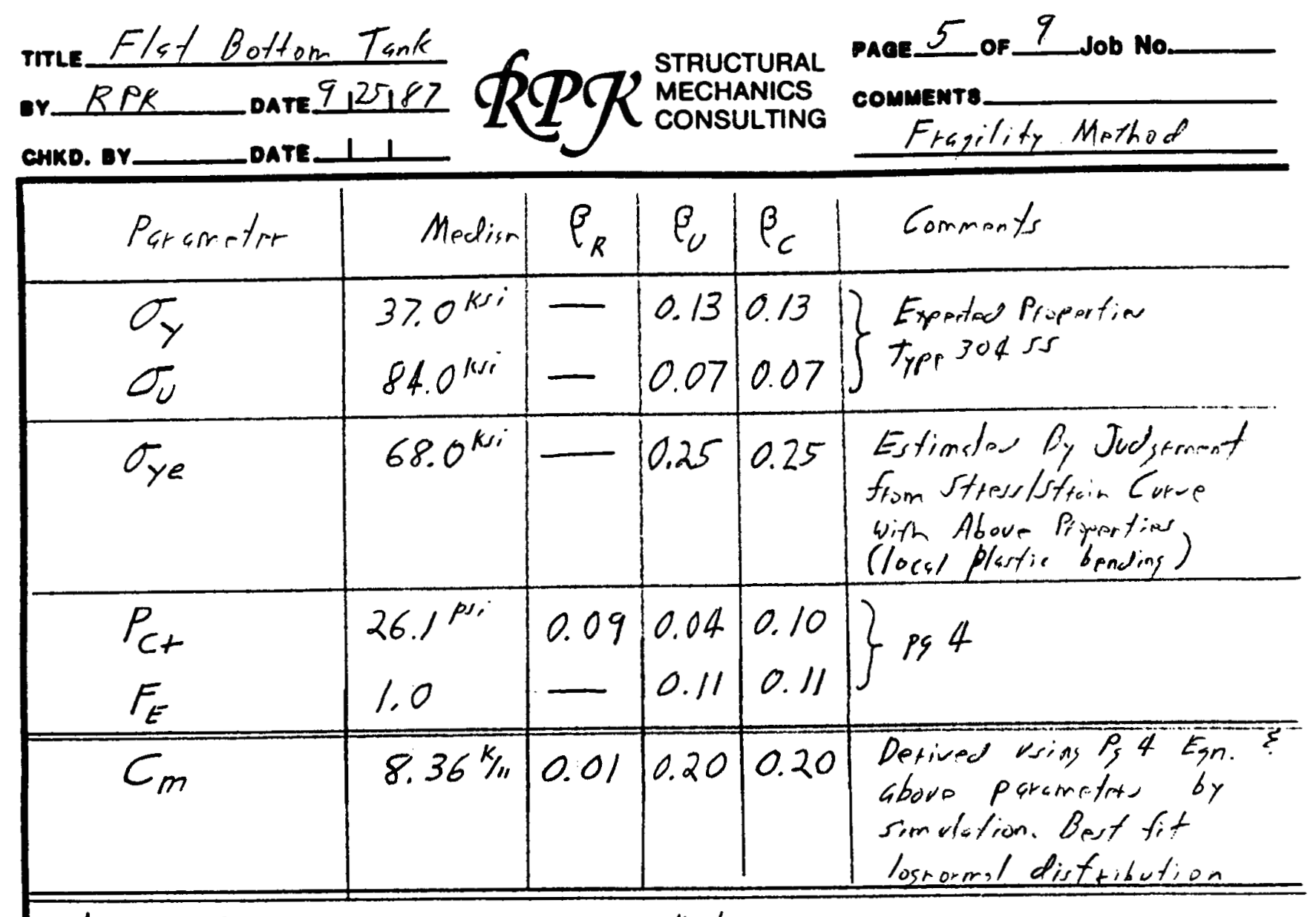

Anchor Bolt Tenion Caparity -2 " $\phi$ A307

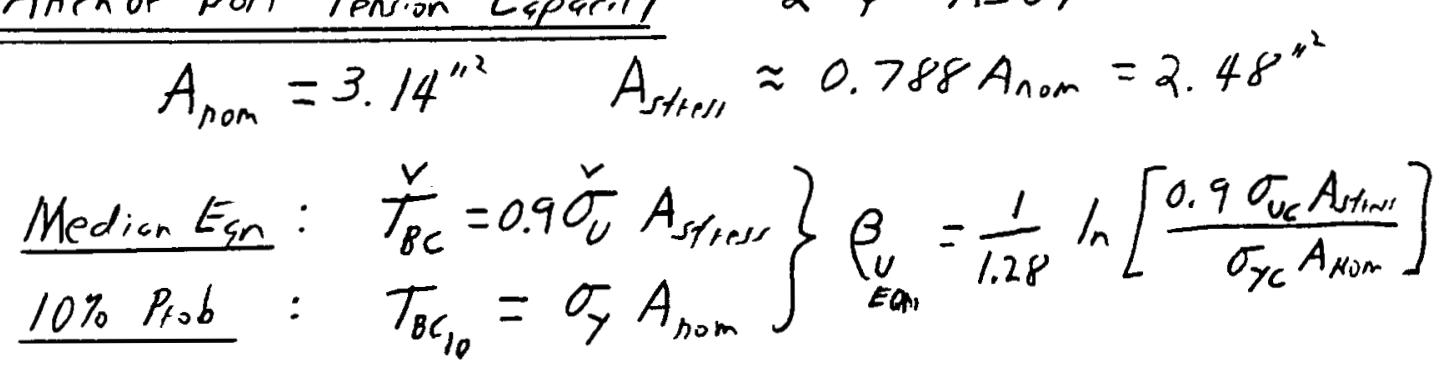

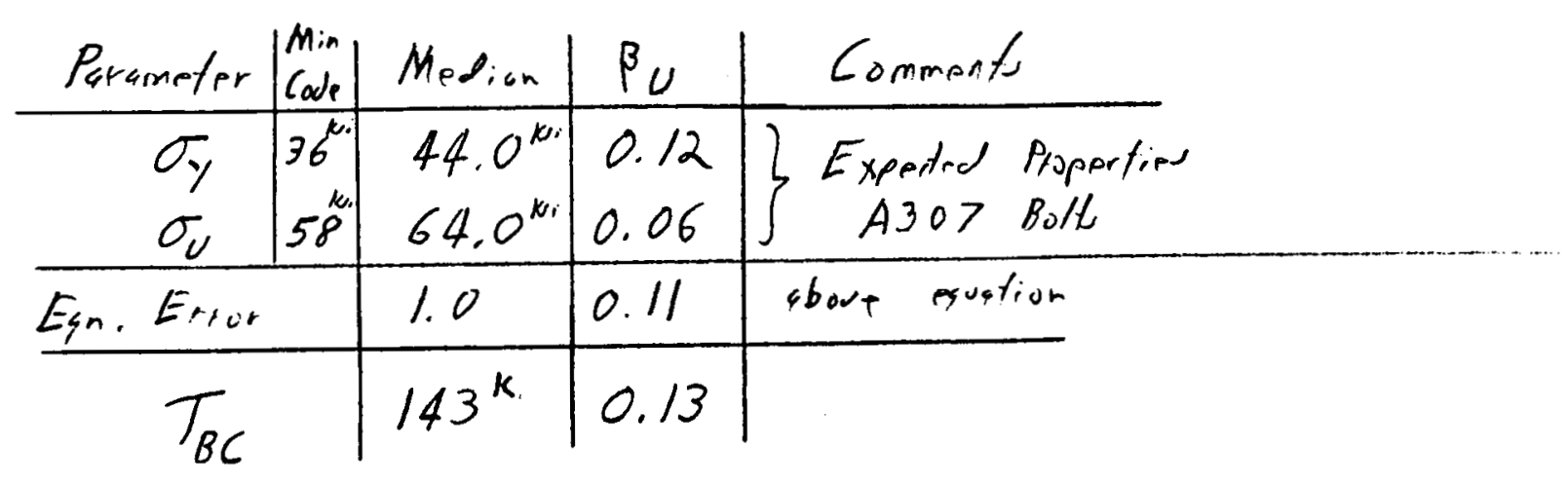

A-66 
TITLE $\frac{F / c t \text { rotting } / \text { ant }}{R P /}$

STRUCTURAL

MECHANICS

2HKO.

CONSULTING

PACE 6 of 9 Job No

COMMENT 8

Fragility Method

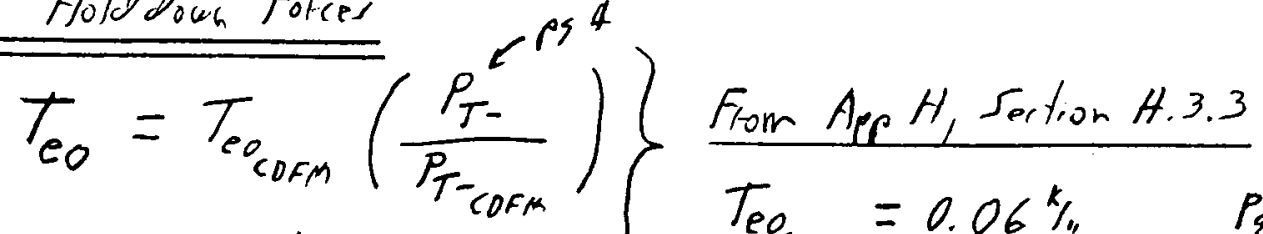

$$
\begin{aligned}
& T_{e_{1}}=0.27 \frac{k}{11^{2}} \\
& T_{\text {nO }{ }_{\text {CDFm }}}=0.06 \% \quad P_{9} \mathrm{H} .20 \\
& P_{T_{\text {corm }}}=10,5_{p r i} \quad E_{\text {qt }} \text { H. } 25
\end{aligned}
$$

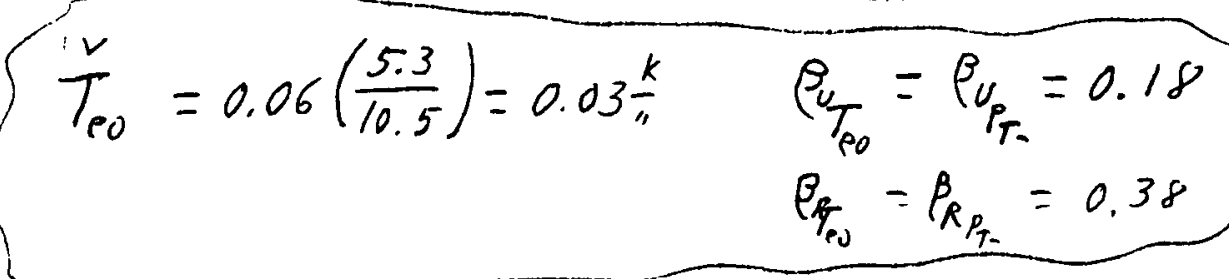

Overturning Moment $C_{\text {asperity }}: M_{S C}$

1) CDFM Formulation for M Sc is estimated to be at 95\% Confidence level ard is to u conservative for Median Greasily Estimate

2) Following Formulation is considered to be median centered.

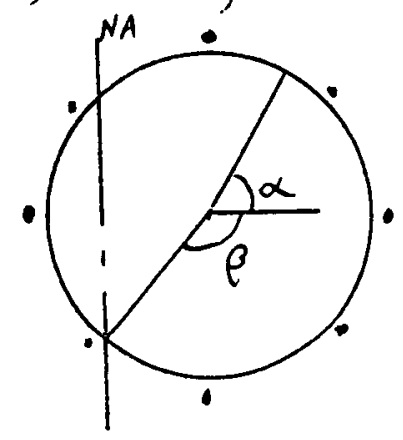

dips.

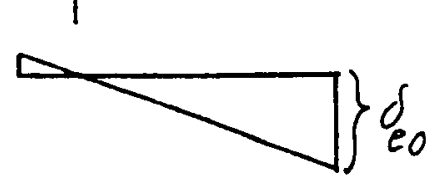

$$
\begin{aligned}
& \delta_{e_{\alpha}}=\delta_{e_{0}}\left(\frac{\cos \alpha-\cos \beta}{1-\cos \beta}\right) \\
& \Delta T_{e}=\delta_{e 0} T_{e 1} \\
& T_{B_{i}}=T_{B P}+K_{B}^{\prime}\left(\frac{\cos \alpha-\cos \beta}{1-\cos \rho}\right) \leq T_{B C} \quad K_{B}^{\prime}=\frac{\delta_{0} A_{B} E_{B}}{\left(h_{c}+h_{a}\right)} \\
& B_{1}=\left[\frac{1}{(\pi-\beta)}\right] \quad B_{3}=\left(\frac{\sin \beta-\beta \cos \beta}{1-\cos \beta}\right) \\
& B_{2}=2 \sin \beta \quad B_{4}=\left(\frac{\beta-\sin \beta \cos \beta}{1-\cos \beta}\right) \\
& C_{m}^{\prime}=B_{1}\left[\frac{W T_{e}+\sum T_{B_{i}}}{2 R}+T_{e 0} \beta+\Delta T_{e} B_{3}\right] \\
& \left.M_{S C}=C_{m}^{\prime} R^{2} B_{2}+\sum T_{B_{i}} R \cos \alpha i\right)+T_{e 0} R^{2} B_{2}+\Delta T_{e} R^{2} B_{4}
\end{aligned}
$$

$T_{D 5} 2 T_{C 4} \quad 2 T_{B 3} \quad 2 T_{B 2} \quad T_{B 1}$

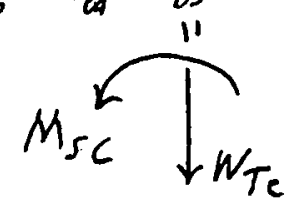

trialserres: assume $e$, find $C_{m}^{\prime}$

vary $\beta$ until $C_{m}^{\prime}=C_{m} \quad$ (es 5$)$

$-1$

A-67 


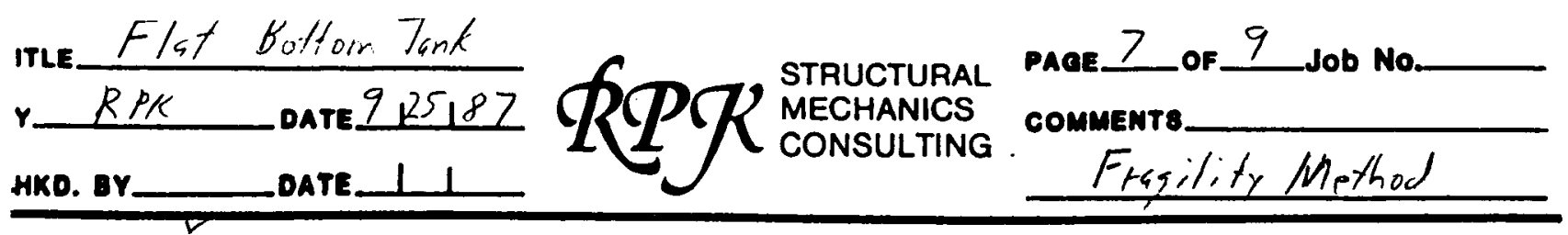

$$
\begin{aligned}
& \text { Find } M_{S C} \text { by wing all median ptopertiou in presiow formulation } \\
& \checkmark r^{5} \% \text { stmin Ouer Unanchored Lenjth } \\
& \delta_{e 0}=0.05\left(h_{c}+h_{a}\right)=0.05\left(24.75^{4}+28.5^{\prime \prime}\right)=2.66^{\prime \prime} \\
& \check{K}_{B}^{\prime}=0.05\left(A_{B}\right)\left(E_{B}\right)=0.05\left(3.14^{\prime \prime 2}\right)\left(29 \times 10^{3} \mathrm{ks}\right)=4550^{k}
\end{aligned}
$$

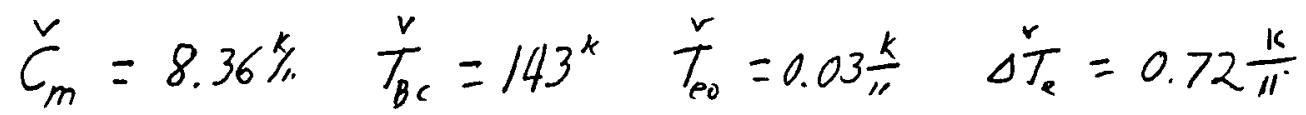

$$
\begin{aligned}
& \check{W}_{T_{e}}=53.6^{k} \quad R=20^{\prime}=240^{\prime \prime} \quad T_{B P}=0 \text { (Nopetenseror) }
\end{aligned}
$$

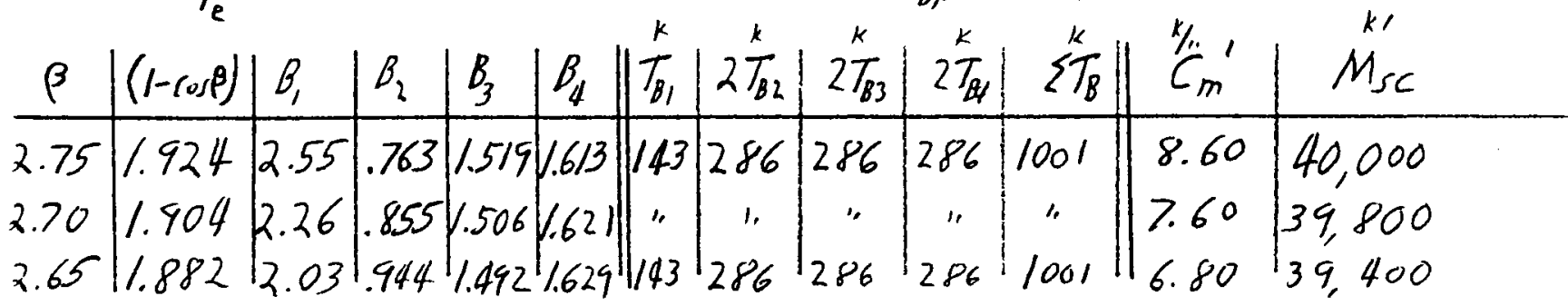

$$
\begin{aligned}
& \therefore \widetilde{M}_{S C}=40,000 \quad S \text { MNE }=\frac{40000}{35700}(0.60)=0.67 \mathrm{~g}
\end{aligned}
$$

Find MSC Variabilities

(1) Set $C_{m}$ to $-\beta_{u} V_{s} / u e: C_{m_{-\beta}}=8.36 \frac{k}{11} e^{-0.20}=6.84$

All else uncharjed

$$
M_{S C_{\mathbb{U}}}=39400^{\prime \prime} \quad \therefore \beta_{C_{C_{m}}}=\ln \left(\frac{40,000}{39400}\right)=0.02
$$

Insensitive to $C_{m}$ Varisbility

(2) Sot $\overline{T_{B C}}$ to $-\overline{P_{0}} \overline{V_{\text {alue }} \text {, all etre median }: \bar{T}_{B C}}=\overline{143 e^{-0.13}}=\overline{126^{\mathrm{K}}}$

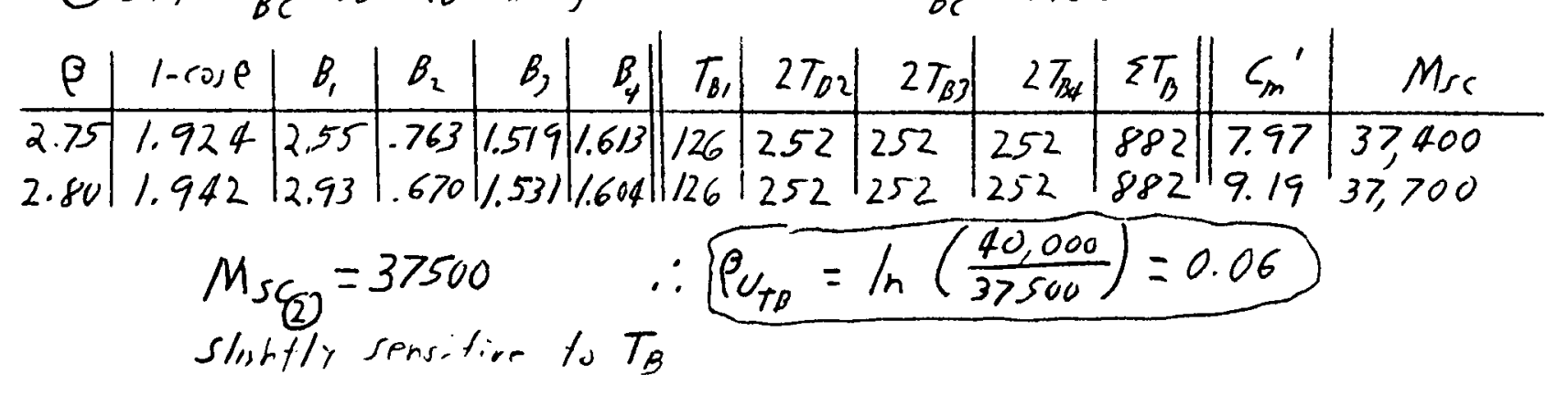

A -68 


$$
\begin{aligned}
& \text { TITLE Flat Bottom Tank } \\
& \text { or RPK DATE } \frac{9,25187}{1}
\end{aligned}
$$

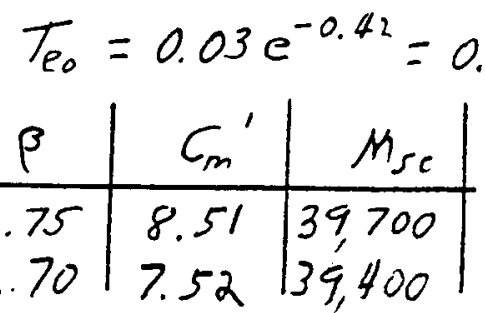

$$
\begin{aligned}
& M_{S C_{(3)}}=39700 \\
& \beta_{R_{e}}=\ln \left(\frac{40000}{39700}\right)=0.01 \text { Neslisible }
\end{aligned}
$$

\begin{tabular}{c|c|c|}
$\beta$ & $C_{m}{ }^{\prime}$ & $M_{5 c}$ \\
\hline 2.75 & 8.51 & 39,700 \\
2.70 & 7.52 & 39,400
\end{tabular}

(4) Invertigste Esostion Etror

CDFM Formulation (Section H.3.4, AppH) Assumed to be at 95\% Confidence Use medisn properties in CDFA Formulation

$$
\begin{aligned}
& \dot{C}_{m}=8.36 \frac{\mathrm{k}}{11} \quad T_{B C}=143^{\mathrm{k}} \quad T_{P 0}=0.03 \% \\
& \check{\delta}_{c}=\frac{C_{m} h_{c}}{E_{s} t_{s}}=\frac{8.36(24.75)}{27.7 \times 10^{3}(0.375)}=0.0199^{\prime \prime} \\
& \Delta \tilde{T}_{e}=\dot{T}_{e} \delta_{c}=0.27 \frac{k}{N_{1}}\left(0.0199^{\prime \prime}\right)=0.0054 \xi_{11} \\
& \check{K}_{B}=\frac{\delta_{C} A_{B} E_{B}}{\left(h_{C}+h_{a}\right)}=\frac{0.0199(3.14)\left(29 \times 10^{3}\right)}{(24.75+28.5)}=34.0^{\mathrm{K}} \\
& \check{W}_{T_{e}}=53.6^{\prime} \quad R=20^{\prime}=240^{\prime \prime} \quad T_{B P}=0 \\
& \begin{array}{|c|c|c|c|c|c|c|c|c|c|c||c|c}
\beta & (1+\cos \beta) & C_{1} & C_{2} & C_{3} & C_{4} & T_{B 1} & 2 T_{B 2} & 2 T_{B 3} & 2 T_{B 4} & \sum T_{B} & C_{m}^{\prime} & M_{C C} \\
\hline 2.70 & .0959 & 3.41 & .575 & 102 & 32.2 & 143 & 286 & 286 & 139.7 & 854.7 & 7.28 & 26,000 \\
2.75 & .0757 & 3.84 & .513 & 186 & 41.0 & 143 & 286 & 286 & 195.2 & 910.2 & 9.03 & 27,600
\end{array} \\
& M_{S C_{(B)}}=27000 \\
& \beta_{U_{\text {EWN }}}=\frac{1}{1.65} \ln \left(\frac{40000}{27000}\right)=0.24
\end{aligned}
$$

Equation is bigsest soutes of uncertainty. Much grestor

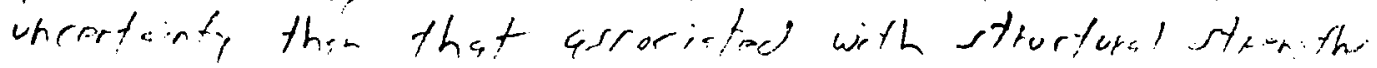

A-69 


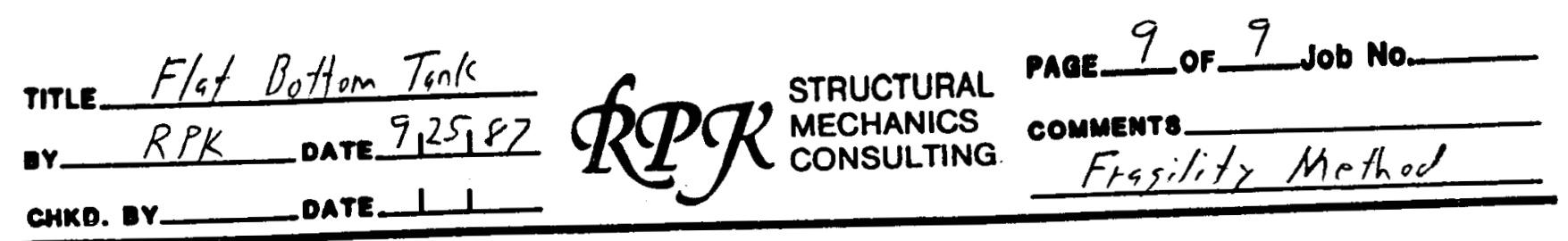

Variabilitips \& HCLPF Computation

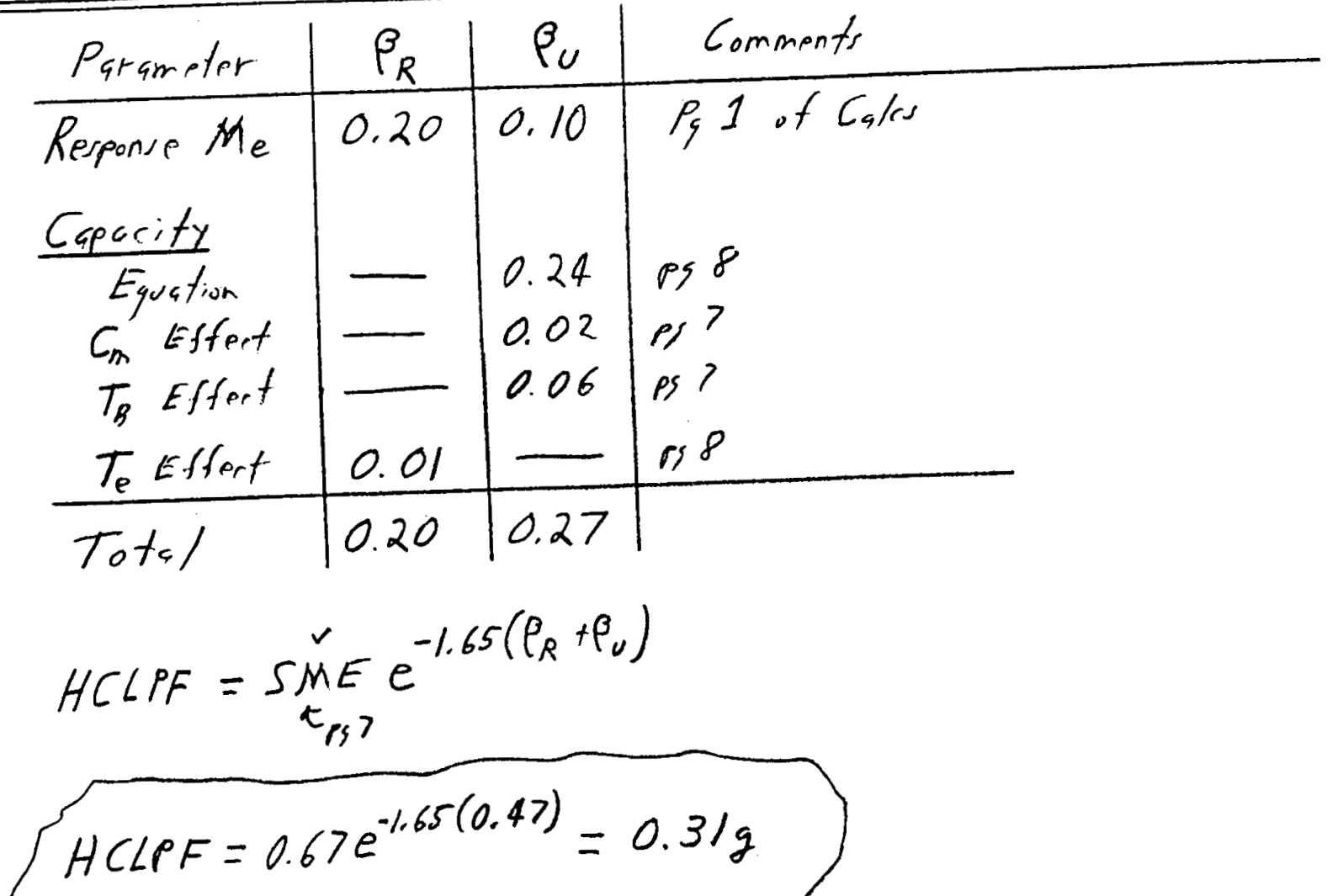

Excellent asteement with pfior CDFih value from Appendix $H$ of $0.29 \mathrm{~g}$. Therefore fresility method has prodveed reasonible results.

A-70 


\section{AUXILIARY CONTACTOR CHATTER IN MOTOR CONTROL CENTER \\ CDFM METHOD}

By

R. P. Kennedy

A-71 


$$
\text { mire MCC Aux Contartor Chythr }
$$

First d. for Gsbinot At Gride

From Ref (当)

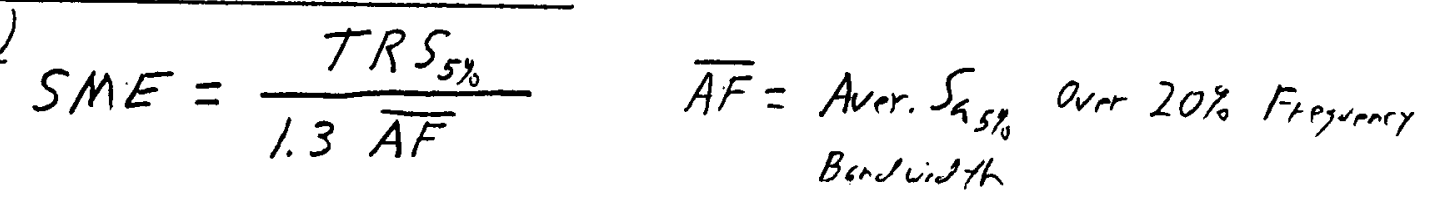$$
\text { TRS }=\text { GERS }=1.5 \mathrm{~g} \operatorname{Ref}(4) \xi F_{\text {ig }} 5
$$

$\overline{A F}_{5 y_{0}}=2.12 \quad \operatorname{RPf}(5)$ NUREG/RRoo48 around 6.5 He

$$
\therefore\left\{\begin{array}{c}
\text { CDFMM } \\
\text { at grad: }
\end{array} \text { SME }=\frac{1.5 \mathrm{~g}}{1.3(2.12)}=0.54 \mathrm{~g}\right.
$$

Next Cabinet Mounted High in Structure (Fig $6 \xi 7$ Flook Spertis)

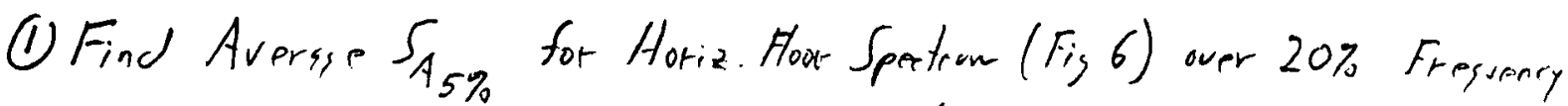
Bindwidth Centernd On Reronent Pesk $(6.7-8.2 \mathrm{H})$

$$
\bar{S}_{a_{4 \%}}=2.28 \mathrm{~g} \quad \bar{S}_{47 \%}=1.61 \mathrm{~g} \quad \therefore{\overline{S_{5 \%}}}_{5 \%}=1.99 \mathrm{~g}
$$

(2) These $\bar{S}_{a}$ are for $S M E_{e}=0.18 \mathrm{~g}$ since thet was lewel wed to senerote floor epertiom.

$$
\begin{gathered}
\therefore \overline{A F_{5 \%}}=\frac{S_{S_{5 \%}}}{0.18 \mathrm{~g}}=\frac{1.99 \mathrm{~g}}{0.18 \mathrm{~g}}=11.1 \\
\therefore \sqrt{\begin{array}{l}
\text { CDFAh } \\
\text { isis in } \\
\text { strot. }
\end{array}} \text { SME }=\frac{1.5 \mathrm{~g}}{1.3(11.1)}=0.10 \mathrm{~g}
\end{gathered}
$$

A-72 


\section{AUXILIARY CONTACTOR CHATTER IN MOTOR CONTROL CENTER}

FRAGILITY METHOD

By

R. P. Kennedy 


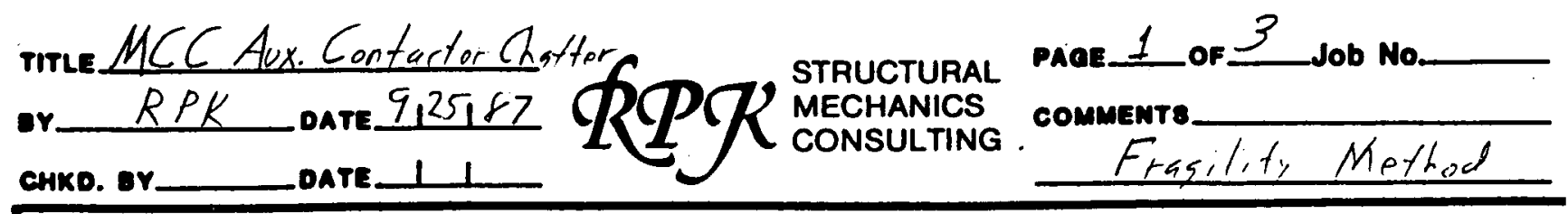

General Notes on Conservatism $\xi$ Variability of GERS Dots

(1) GERS are based upon broad spectrum inputs which are amplified through different cabinets in differing amounts and of differing frequencies so large variability in aux onfector chatter vs GERS

(2) GERS are not at HCLPF level. They represent a lower bound of a limited number of tests. Estimate aux contactor chatter GERS are 84\% Confidence of 90\% NEP for broad free. in pot

(3) GERS have specter conservation and greater variability when subjected to narrow frequency in pot such as Figurer 6 floor spertion then they have for blood fioguarey in pot.

(4) Cabinets amplify input at many frequencies in targe of $5 \% 15 \mathrm{~Hz}$ that are of prime interest for ax. contactor chatter.

(5) Based upon a review of limited fragility data for aux. contactor chatter, the following estimates are mode for the ratio of CAPACITY / GERS:

\begin{tabular}{l|c|c|c|c} 
CAPACITY / GERS & Median & $\beta_{R}$ & $\beta_{U}$ & HCLPF \\
\hline Broad Frequency Input Spartion & 1.45 & 0.11 & 0.23 & 0.83 \\
Narrow Fry. Input Spectrum & 1.74 & 0.11 & 0.25 & 0.96
\end{tabular}




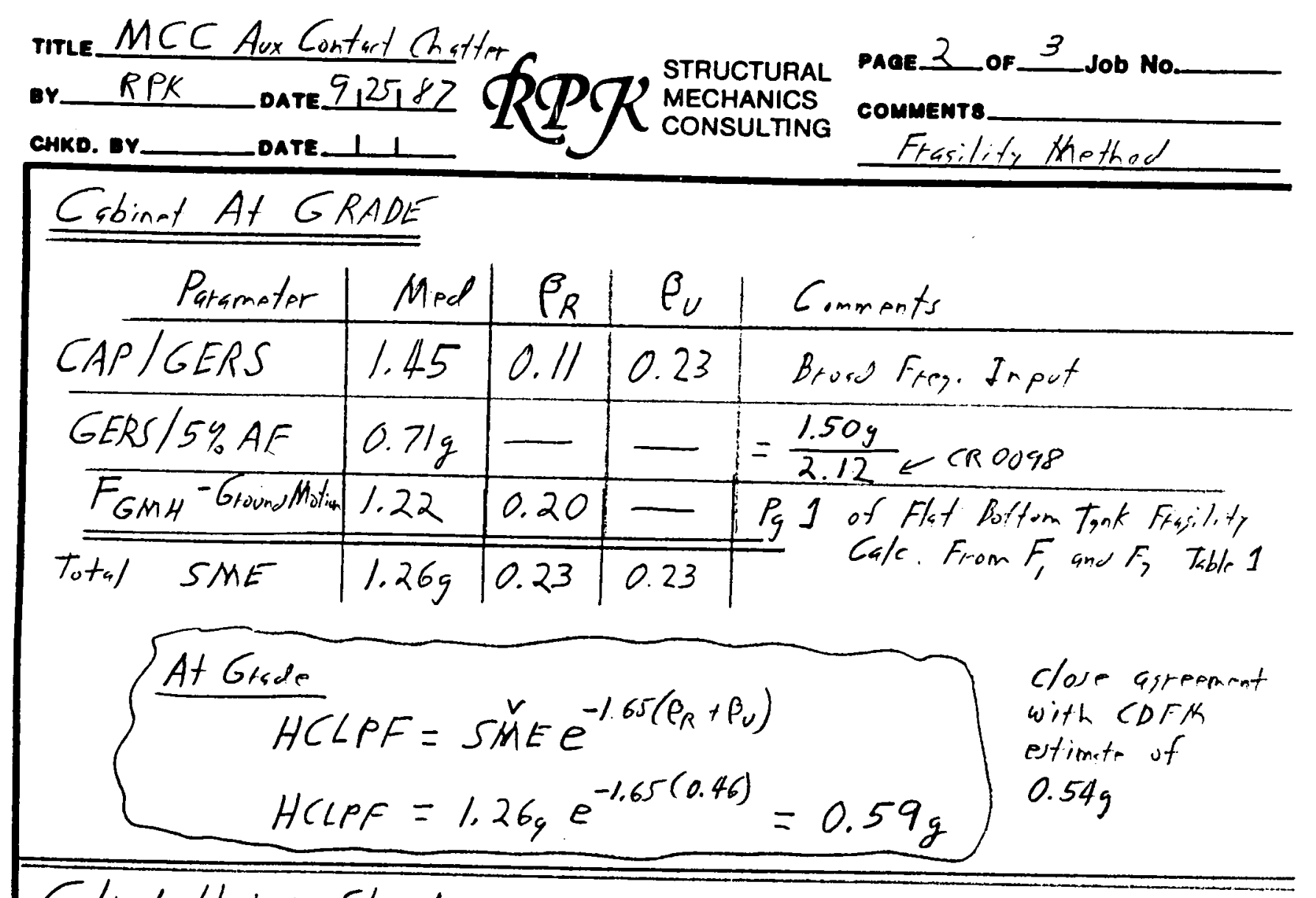

Cabinet High in Strurture

Must incorporste structure response varisbility factors due

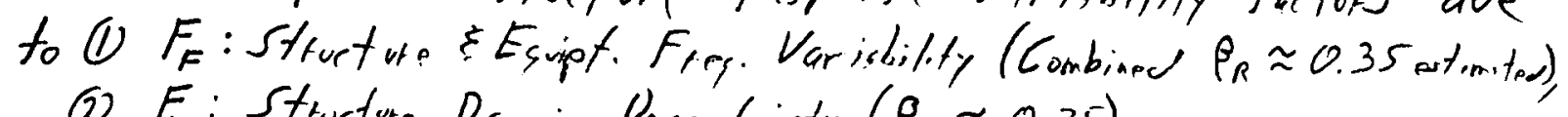

(2) $F_{0}:$ Stturture Damping Uncerfainty $\left(\rho_{U} \approx 0.35\right)$

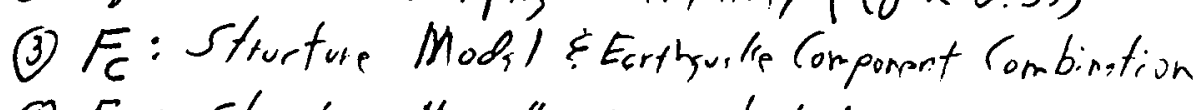

(4) $F_{\mu}$ : Sturture Moselling Variability

A) Generally these paremeless worts be estimsted based upon a detriled teriew of the strurfure response anslysis. How aver, this information is not cucilsble. There foure au ersise values will be used based on a teview of similer esficmstes from severel higher surlety PRA studies (heavily influorered by sperir/studips perfirmed on Disbo (corya)

B) Above estimstes will bo made for csip of esuipment very close \% teronant fresuency of strueture

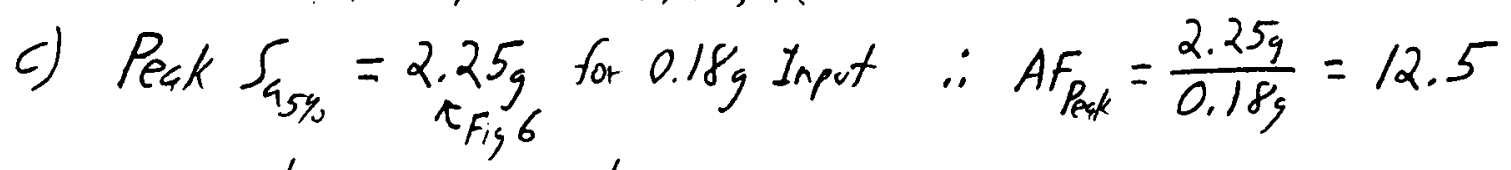

$$
\text { GERS } / A F_{B}=1.50, / 12.5=0.12 \mathrm{~g}
$$

$A-75$ 


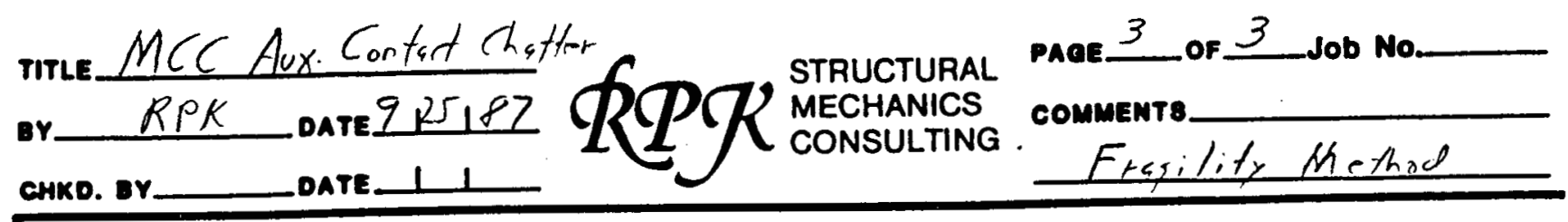

Hish In Struturr (Oont.)

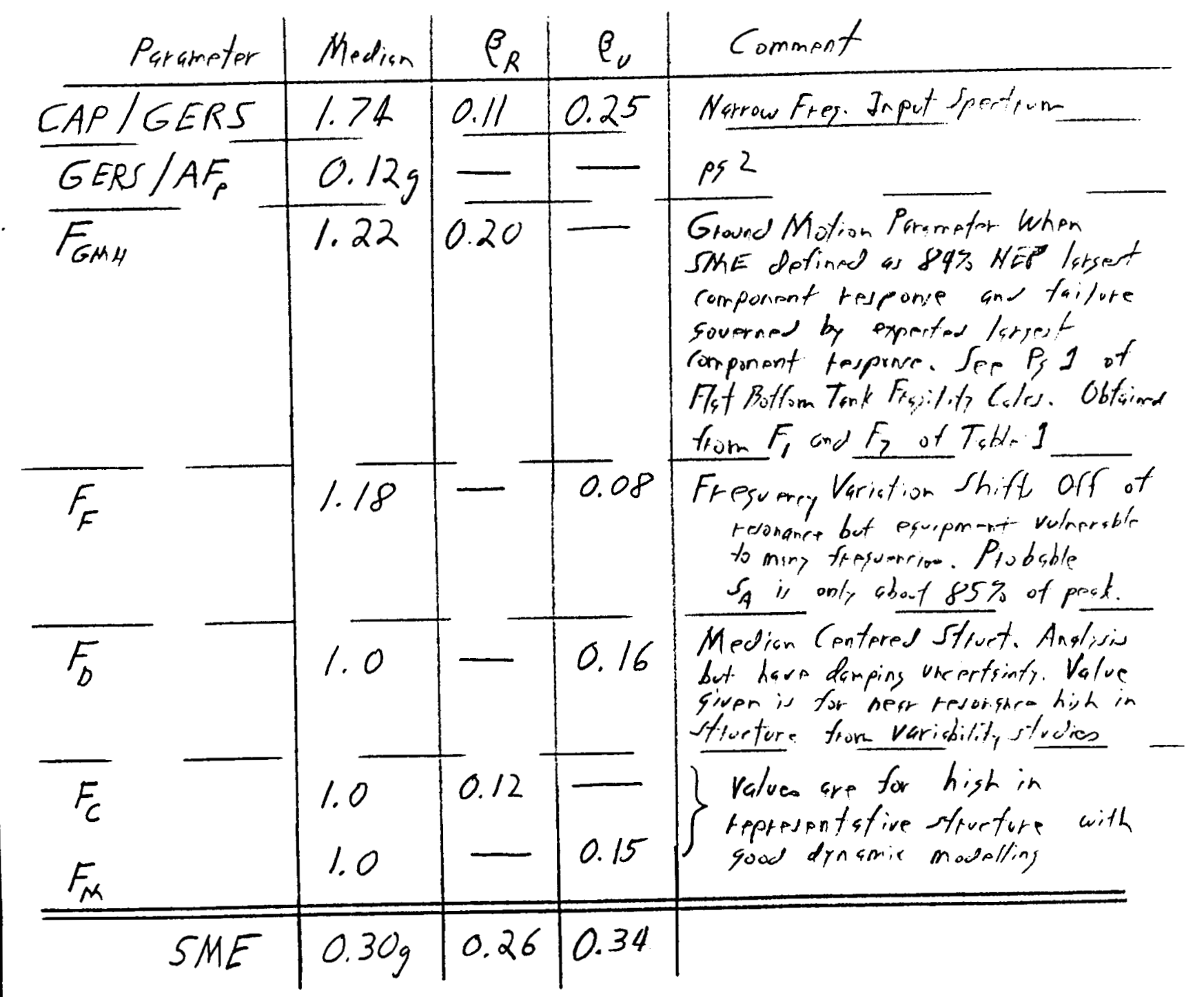

Hish In Strueture

$$
H C L P F=0.30 g e^{-1.65(0.60)}=0.11 \mathrm{~g}
$$

Very close to CDFM esfimste of $0.10 \mathrm{~g}$ so frasility estimote sppears to be reasoncble

A-76 
DIESEL GENERATOR ROOM STARTING AIR TANK SUPPORTS

CDFM METHOD

By

R. P. Kennedy

A -77 
Tires Stat in, Air Tank Support or RPK Dare 91/2187

cHко. oats
StRUCtURAL MECHANICS CONSULTING.

CDFM Method

Air Tank Shown in Figure 2 is Mounted High In Structure With Floor Sports Input Shown In Figs $6 \xi 7$ for $S M E_{e}=0.18 \mathrm{~g}$

(1) Seismic Capacity is Controlled by Combined Base Angle Bending and Anchor Bolt Failure Mode. Only this failure mode is computed in these example CDFM calculations.

(2) Anchor Bolts ate sufficiently embedded in concrete so that capacity is governed by steel and not by concrete

Horizontal Natural Frequency - Controlled by bending frexibility of base angles.

$$
\begin{aligned}
& l_{1,1.5^{\prime \prime} \rightarrow 1}^{T_{0} \uparrow} \\
& \alpha=\frac{\delta_{1}}{a} \\
& \frac{1}{d T_{v}-1} \\
& a=R_{B}\left(1-\cos 100^{\circ}\right) \\
& \delta_{1}=\frac{T_{L} l_{1}^{3}}{3 E_{2} I_{2}} \\
& M=2 T_{v} a \\
& T_{0}^{W} \cdot \underbrace{\delta_{x}} \quad \begin{array}{l}
M=W \cdot h_{C G}=K_{R} \alpha \\
\delta_{x}=\frac{W h_{C G}^{2}}{k_{R}}
\end{array} \quad \therefore K=\frac{k_{R}}{h_{C G}^{2}} \\
& k_{R}=\frac{M}{\alpha}=\frac{\left(2 a^{2}\right)\left(3 I_{L} E_{L}\right)}{l_{1}^{3}} \\
& \therefore K=\frac{\left(2 a^{2}\right)\left(3 \xi_{L} I_{L}\right)}{l_{1}^{3} h_{C \sigma}^{2}} \quad f_{H}=\frac{1}{2 \pi} \sqrt{\frac{K g}{W}} \text {. }
\end{aligned}
$$

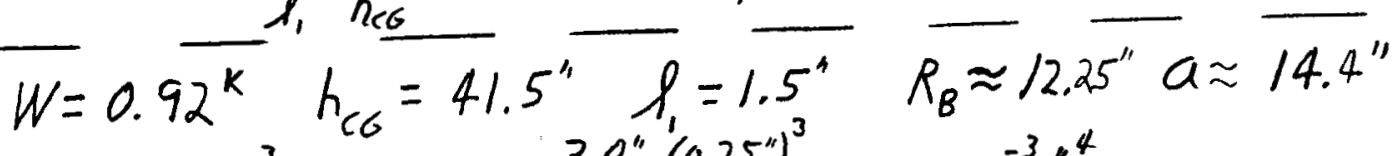

$$
\begin{aligned}
& E_{L}=29 \times 10^{3} \mathrm{ksi} \quad I_{L}=\frac{3.0^{\prime \prime}\left(0.25^{\prime \prime}\right)^{3}}{12}=3.91 \times 10^{-3.4}
\end{aligned}
$$

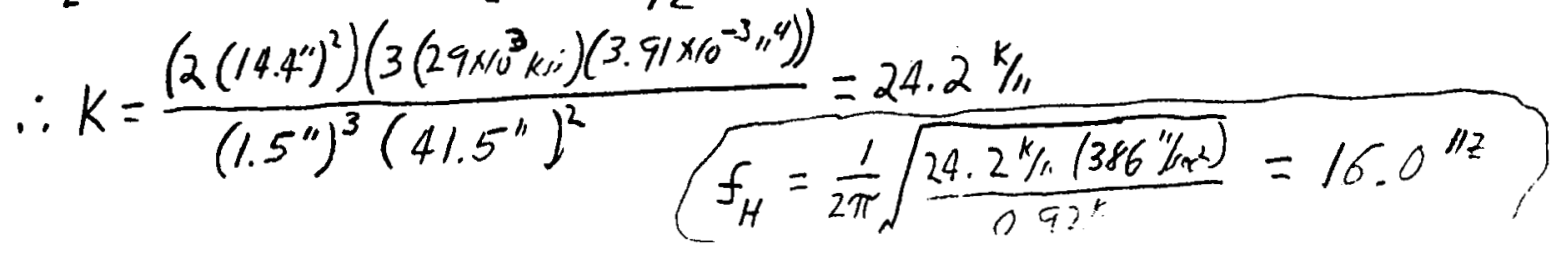

$A-78$ 


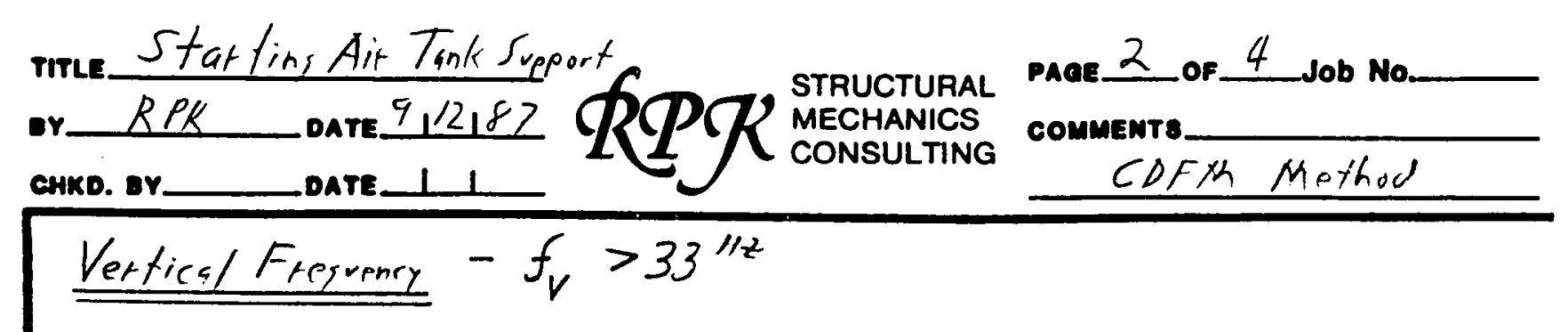

Determine Responre Amplificstion Factow oupr Ground SME

Hotiz: Damping $=5 \%$

Fig 6 Floor Spertian is Unhedocdened. To account for both structure and egoipment freguoney uncerteinty, enter Fig 6 with a fedored freguener, $f_{H}^{\prime}$

$$
\begin{aligned}
& f_{H}^{\prime}=0.80\left(f_{H}\right)=0.80\left(16.0^{H z}\right)=12.8 \mathrm{~Hz} \\
& S_{A_{12.8}}, 5 \%=0.60 \mathrm{~g} \\
& A F_{H}=\frac{0.60}{0.18-5 \text { SAE E }_{e}}=3.33
\end{aligned}
$$

Vert:

$$
A F_{V}=\frac{0.20}{0.18}=1.11
$$

Base Moment Response

$$
M_{R}=W \cdot h_{C G} \cdot A F_{H} \cdot S M E=0.92^{k}\left(41.5^{\prime \prime}\right)(3.33) \operatorname{SME}=127^{k *} \operatorname{SME}
$$

Effertive Wt Base

$$
\begin{aligned}
& W_{E}=W[1-0.4 \mathrm{AE} \text { SME }]=0.92^{\mathrm{k}}[1-0.4(1.11) \mathrm{SME}] \\
& W_{E}=0.92^{\mathrm{k}}[1-0.44 \mathrm{SME}]
\end{aligned}
$$

A-79 


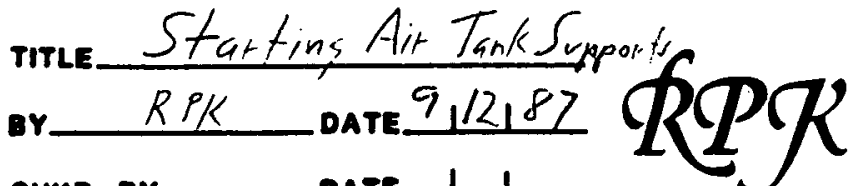

CHKO.
STRUCTURAL MECHANICS CONSULTING.
PAQE 3 or 4 Job No COMMENT8

CDFM Method

Caparity Cales

Bolts - 5/8" A307 Use 1.7* Part 1 AISC Cspacity (Eighthed)

$A_{\text {nom }}=0.3068^{\prime \prime} \quad V^{\prime}=$ Shes on bolt to rombine wirh tension

$$
\begin{aligned}
& T_{B}=A_{\text {Dom }}\left[1.7\left(26.0^{k i}\right)-\frac{1.8 V^{\prime}}{A_{\text {nom }}}\right] \leq T_{B C} \\
& \left.\begin{array}{l}
T_{B C}=1.7\left(6.1^{k}\right)=10.4^{k} \\
T_{B}=13.6^{k}-1.8 \mathrm{~V}^{\prime}
\end{array}\right\} \begin{array}{l}
\text { Use Whichever } \\
\text { Less }
\end{array}
\end{aligned}
$$

Base Ansle Will Yirld $\& G_{0}$ into membrain action with prying before bolt csparities are reached.
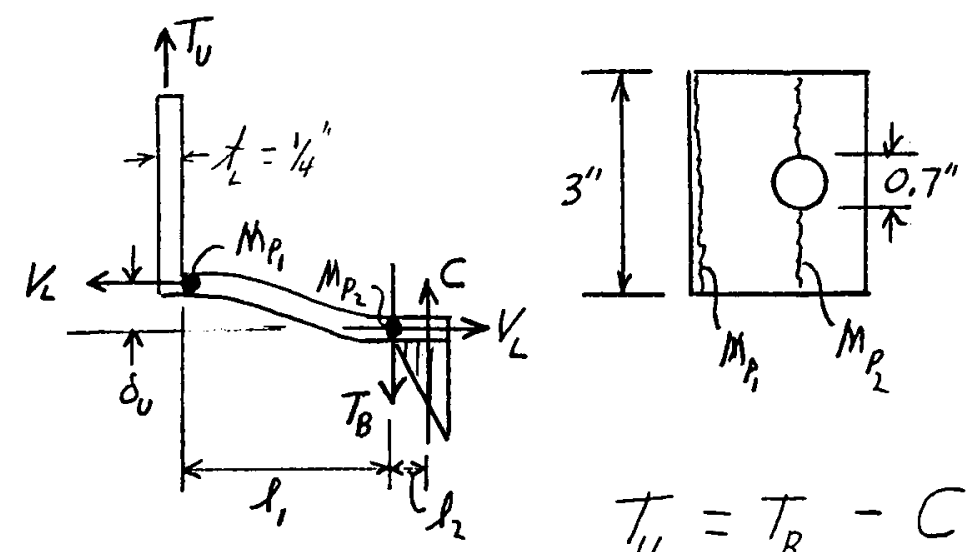

for CDFM

$M_{p}=90 \%$ Foll P\%stie Momart

$$
\begin{aligned}
& M_{p_{1}}=0.9 \frac{36^{\prime \prime \prime}\left(3^{\prime \prime}\right)\left(1_{4}^{\prime \prime}\right)^{2}}{4}=1.52^{k^{\prime \prime}} \\
& M_{P_{2}}=0.9 \frac{36^{k \prime i}\left(2.3^{\prime \prime}\right)\left(1_{4}^{\prime \prime}\right)^{2}}{4}=1.16^{k \prime \prime}
\end{aligned}
$$

$l_{1}=1.5^{\prime \prime} \quad l_{2} \approx 0.5^{\prime \prime}$

$T_{U}=T_{B}-C$

$C=M_{P_{2}} / h_{2}=1.16^{k \prime \prime} / 0.5^{\prime \prime}=2.3^{k}$

$$
T_{U}=11.3^{k}-3.06 \mathrm{~V} \leq 8.1^{k}
$$

(1) Combine (1) $\dot{1}(2)$

Also

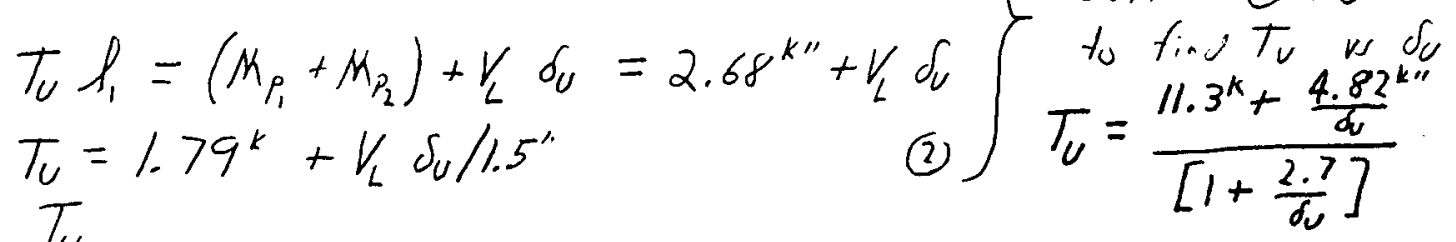

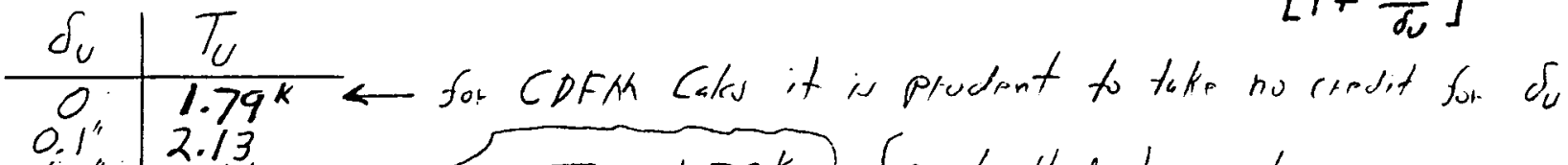

\begin{tabular}{l|l}
$0.1^{\prime \prime}$ & 2.79 \\
$0.2^{\prime \prime}$ & 2.44 \\
$0.3^{\prime \prime}$ & 2.74 \\
$0 .+16$ & 3.014
\end{tabular}

$$
\therefore T_{U}=1.79^{k}-\left\{\begin{array}{l}
\text { contiolled by ansle } \\
\text { plutir hinses }
\end{array}\right.
$$

$A-80$ 


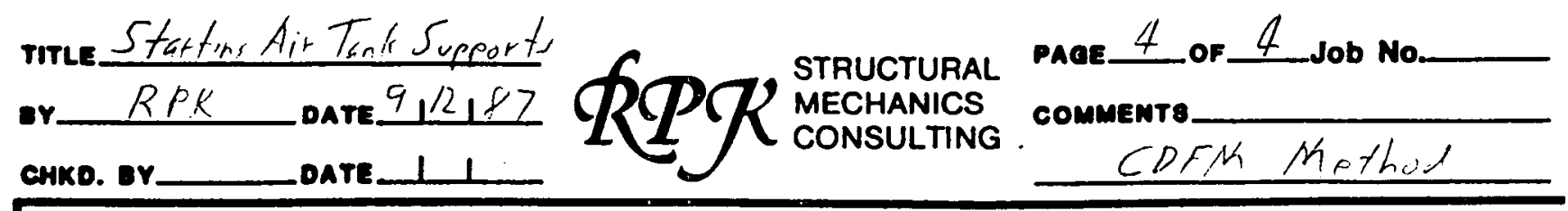

Moment Caparity

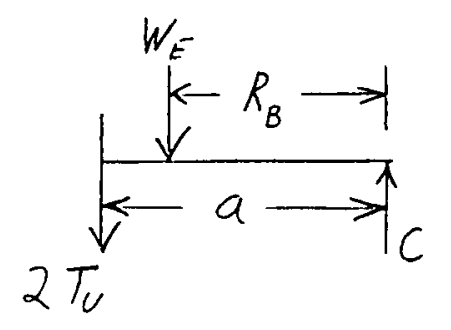

$$
M_{C}=2 T_{T} a+W_{F} R_{B}=M M_{R}
$$$$
\left.T_{U}=1.79^{k}\left(\mathrm{es}^{3}\right) \quad W_{E}=0.92^{k}[1-0.445 \mathrm{ME}] \mathrm{G}_{2} 2\right)
$$$$
M_{R}=127^{k \prime \prime} \text {.SME (P.2) }
$$$$
a=14.4^{\prime \prime} \quad R_{B}=12.25^{\circ} \text { (psi) }
$$$$
\text { SME }=\frac{2\left(1.79^{k}\right)\left(14.4^{\prime \prime}\right)+0.92^{k}\left(12.25^{\prime \prime}\right)}{127^{k \prime}+0.92^{\prime \prime}\left(12.25^{\prime \prime}\right)(0.44)}
$$$$
\text { SME }=0.48 \mathrm{~g} \text { CDFAM Mathod }
$$

$A-81$ 
DIESEL GENERATOR ROOM STARTING AIR TANK SUPPORTS

FRAGILITY METHOD

By

R. P. Kennedy

A-82 


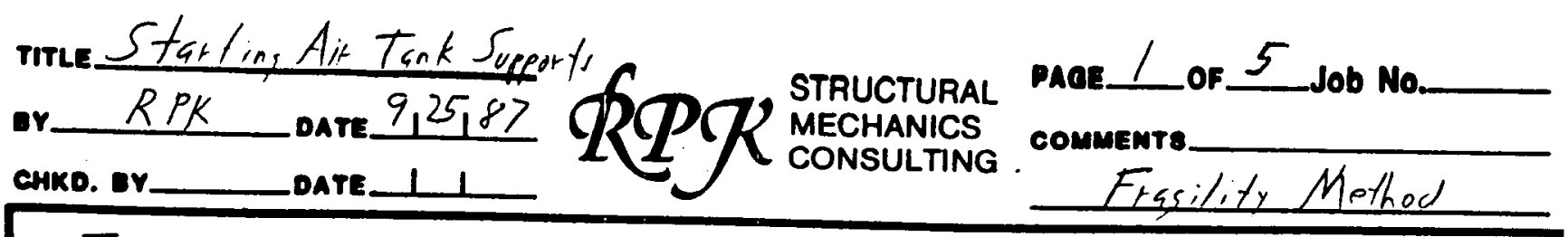

Fragility Cales follow Same Format A Previous CDFM Cales

Response Factors

Horiz:

Fresuency : CDFM Cales produred a low estinute of

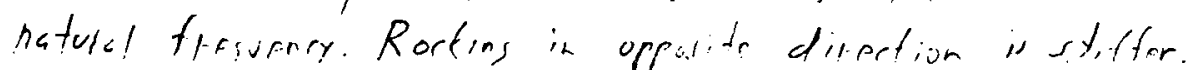

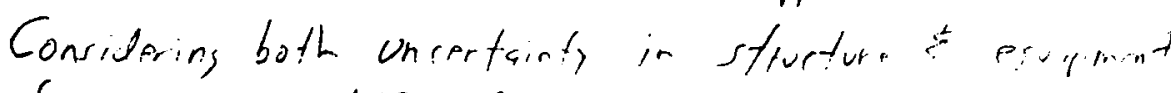

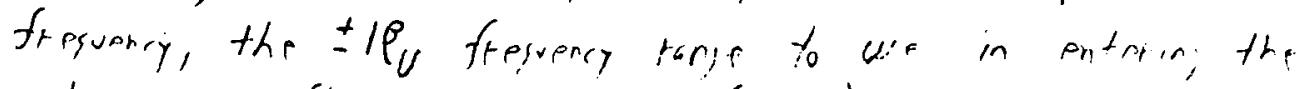
unbroclened flour spertuon plot (Fi, 6) "s elimitred to be:

$$
f_{H_{ \pm i \beta_{u}}}=12.5 \text { to } 24 \mathrm{~Hz}
$$

Equipt. Damping $: 5 \%=$ Medica; $7 \%=+1 \beta_{0} ; 4 \%=0.66 \beta_{0}$

From Fig $6: S_{A_{H}}$

$\left.\begin{array}{l|l|l} & 12.5 \mathrm{~Hz} & 24 \mathrm{~Hz} \\ \hline 4 \% & 0.63 \mathrm{~g} & 0.50 \mathrm{~g} \\ 7 \% & 0.60 \mathrm{~g} & 0.48 \mathrm{~g}\end{array}\right\} \beta_{E_{\mathrm{F}}}=\frac{\ln \left(\frac{0.63}{0.60}\right)}{1.66}=0.03$

$$
\begin{aligned}
& \beta_{v_{F}}=\frac{\ln (0.630 .50)}{2}=0.12 \\
& S_{A_{H}}^{V}=0.55 \mathrm{~g} \quad \beta_{V_{S_{N M}}}^{v_{v_{F}}}=\sqrt{0.12^{2}+0.03^{2}}=0.12 \\
& A^{2} F_{H}^{\prime}=\frac{S_{A_{H}}}{0.18 g g_{\text {SME }}}=\frac{0.55}{0.18}=3.06
\end{aligned}
$$

Vett: $\left(R_{\text {isid }}\right)$

Expres, $A F_{v}^{\prime}$ in torms of vert glound are (0.129)

$$
\text { (Fir } 7) \quad \therefore F_{v}^{\prime}=\frac{0.20}{0.12}=1.67 \quad \beta_{u}=0
$$

A-83 


$$
\text { or }
$$

Mut Add in Structural Response Varisbilly As to how it will effert thoor spertian away from resonant peck. Use estromita for hish in stiorture away from resonsere obtained trom Disblo Cingon Veriability Senitivity Study.

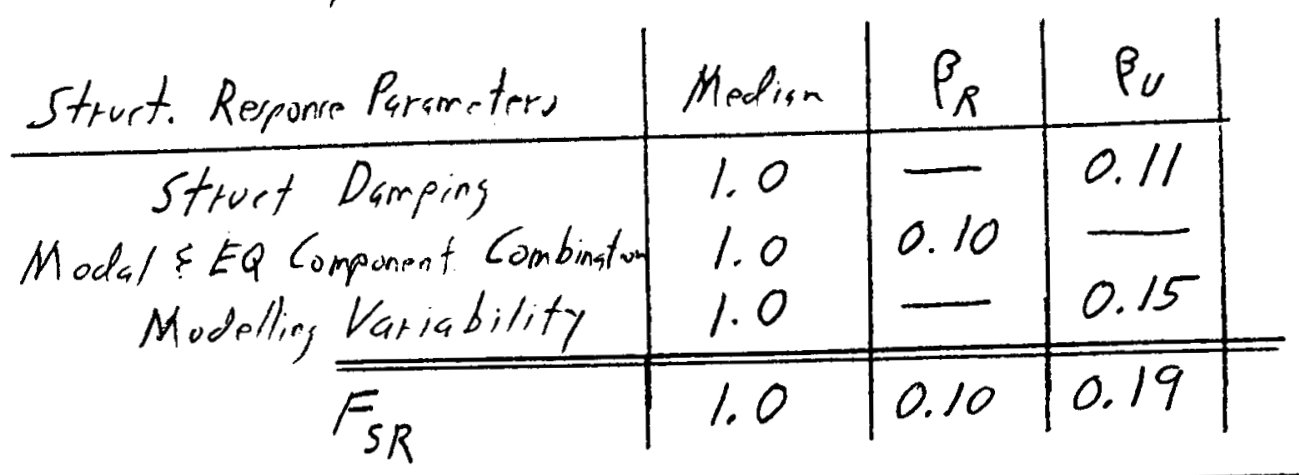

Combined Horiz Repponre $A F_{H}$

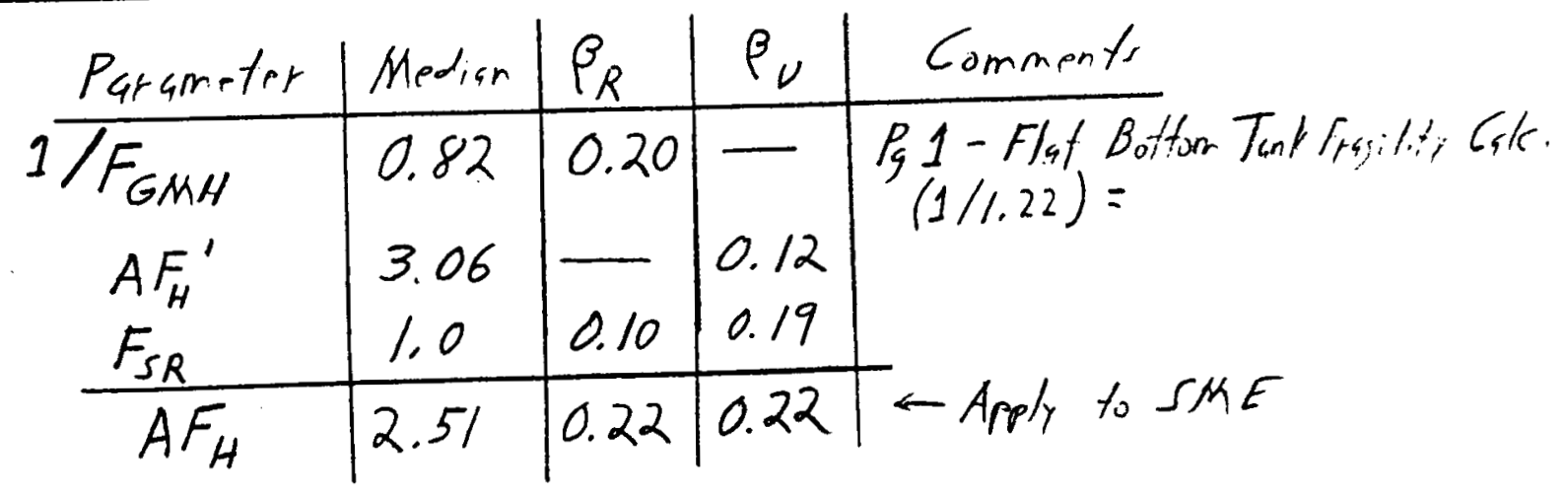

Combines Vert Repanre $A F_{r}$

\begin{tabular}{c|c|c|c|l} 
Parameter & Medim & $\rho_{R}$ & $\rho_{u}$ & Comments \\
\hline Vert / SME & 0.48 & 0.34 & - & $F_{q}$ from T.ble 1 of Text . \\
$A F_{V}^{\prime}$ & 1.67 & - & 0 & \\
$F_{S R}$ & 1.0 & 0.10 & 0.19 & \\
\hline$A F_{V}$ & 0.80 & 0.35 & 0.19 & $\leftarrow A_{\text {pply }}$ to SME
\end{tabular}

A-84 


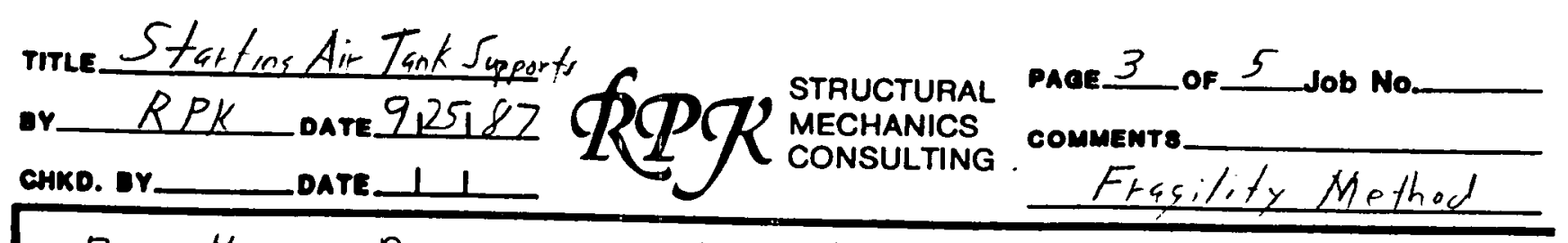

Base Moment Rerponse $\quad W=0.92^{k} \quad h_{c G}=41.5^{\prime \prime}$

$$
M_{R}=W \cdot h_{C G} \cdot A F_{H} \cdot S M E=38.2^{k \prime} \cdot A F_{H} \cdot S M E
$$

Eff. Busp Wt.

$$
W_{E}=W[1-\overbrace{F_{V C} \cdot A F_{V}}^{F_{V}} \cdot S M E]=0.92^{k}\left[1-F_{V} \cdot S M E\right]
$$

\begin{tabular}{l|l|l|l|l} 
Parameter & Median & $\beta_{\bar{R}}$ & $\beta_{V}$ & Cumments \\
\hline$F_{v c}$ & 0.40 & 0.39 & - & Vertical Component Combim.tiv Fartor \\
$A F_{V}$ & 0.80 & 0.35 & 0.19 & \\
\hline$F_{V}$ & 0.32 & 0.53 & 0.19 &
\end{tabular}

$\underline{\underline{B o l t} \text { Caparify }} 5 / 8^{\prime \prime} A 307 \quad A_{\text {dom }}=0.3068^{\prime 2} A_{\text {stis, }}=0.2260^{\prime \prime 2}$

$$
\begin{aligned}
& T_{B C}=F_{B} \cdot T_{B C_{D F A}}=0.9 \cdot \sigma_{u} \cdot A_{s t e r s}
\end{aligned}
$$

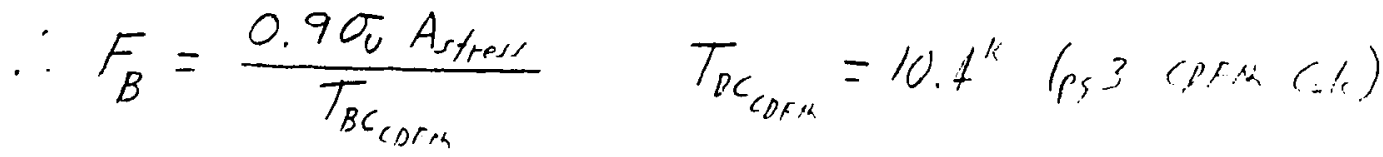

$$
\begin{aligned}
& \text { 10\% Prob: } T_{B C}=\sigma_{y} \cdot A_{\text {Aom }} \therefore \beta_{E Q N}=\frac{1}{1.28} \ln \left[\frac{0.9 \sigma_{u c} A_{\text {stres }}}{\sigma_{y C} A_{\text {rom }}}\right]
\end{aligned}
$$

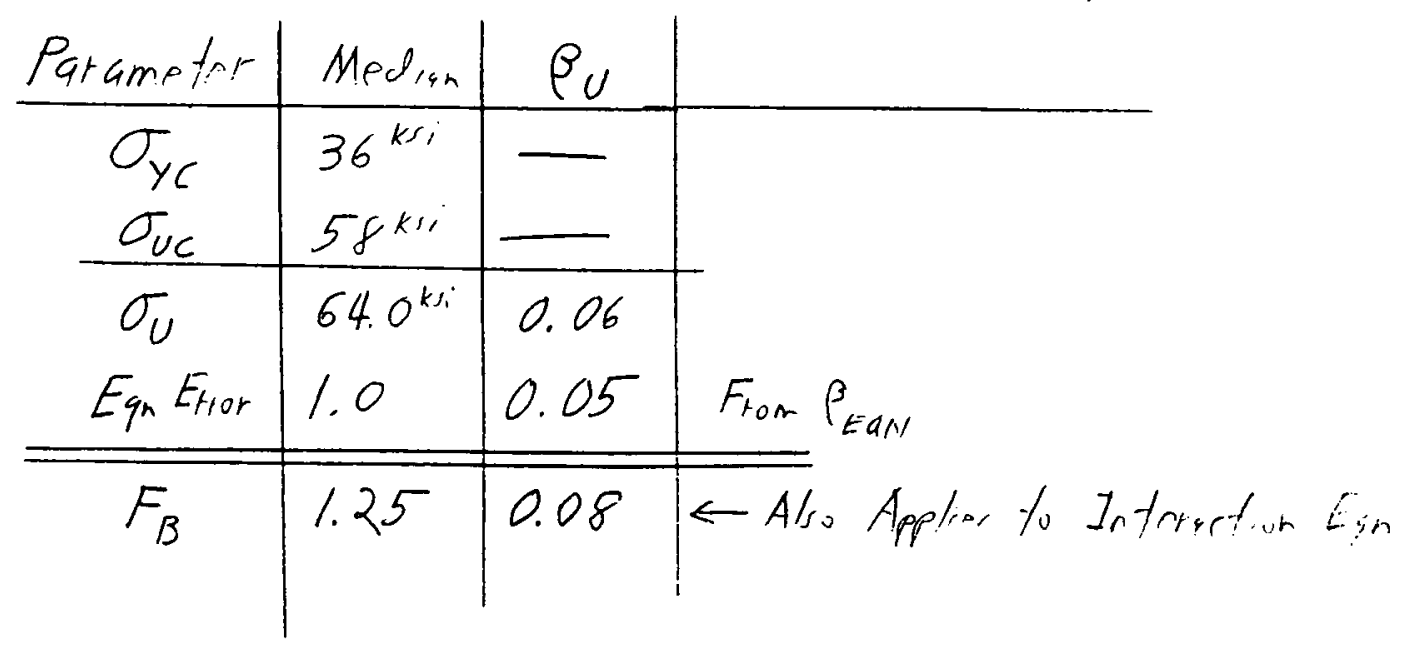

A -85 


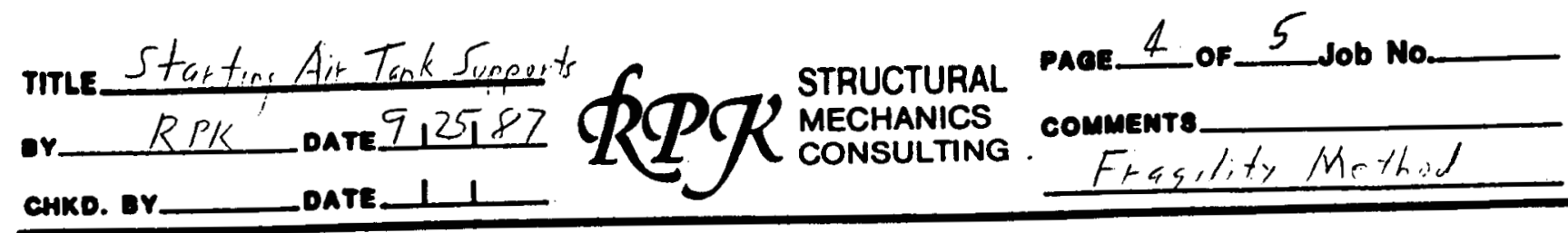

$$
\begin{aligned}
& \text { Angle Bending } \\
& M_{P}=F_{M_{P}} \cdot M_{P_{C O F M}}
\end{aligned}
$$

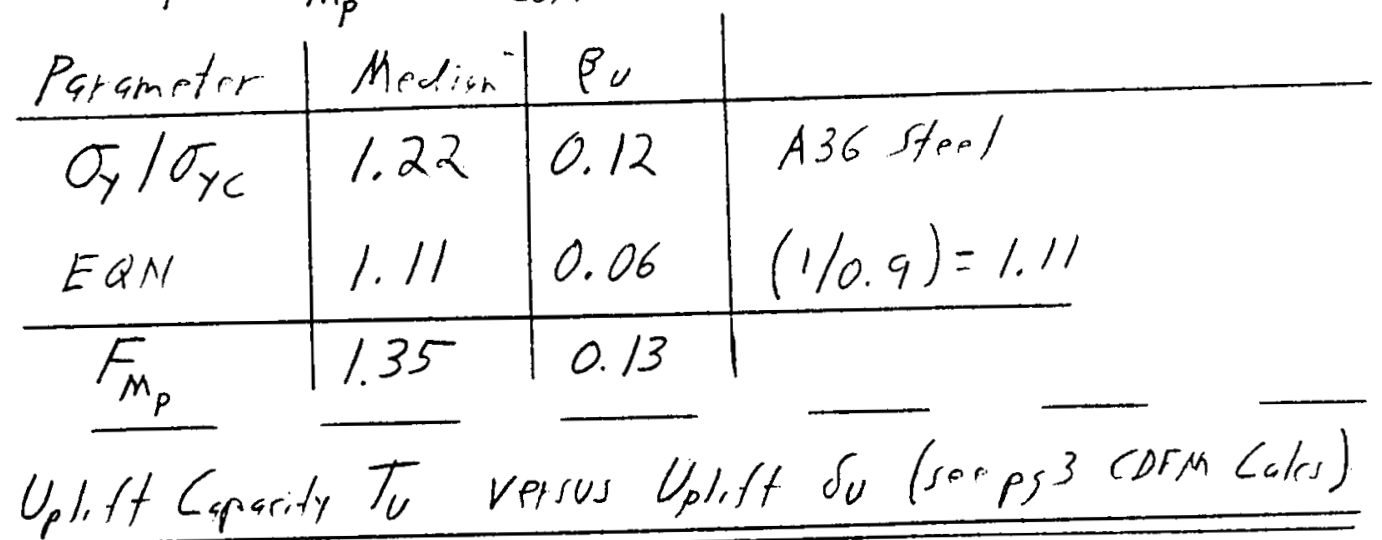

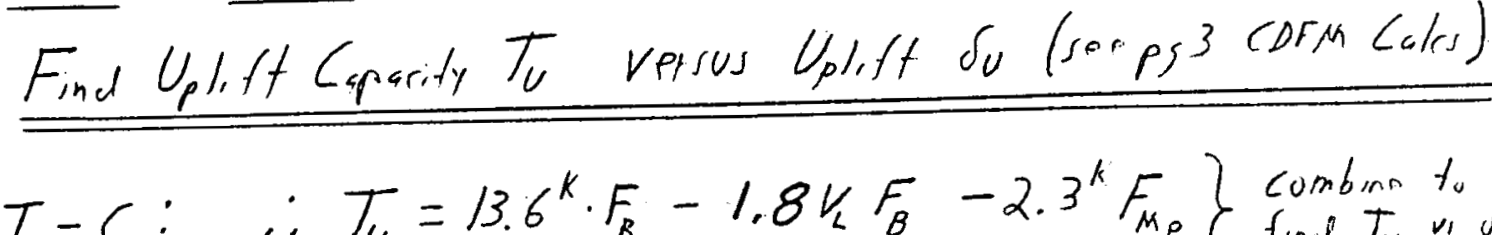

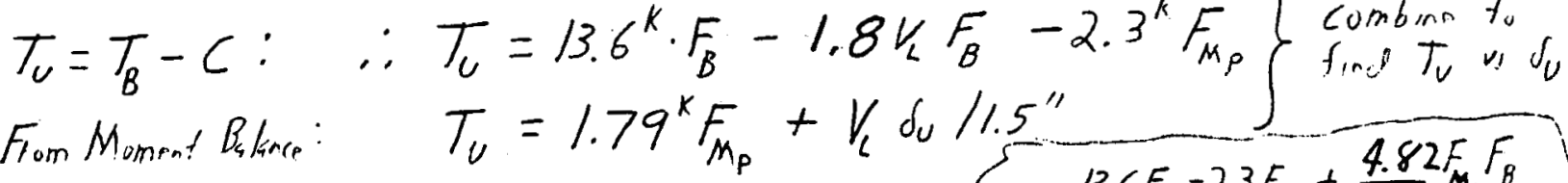

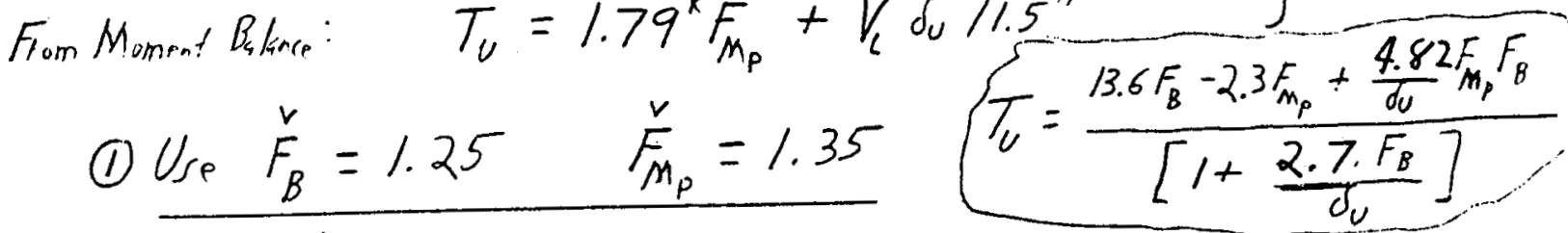

$$
\begin{aligned}
& \begin{array}{c|ccc}
\delta_{v} & T_{u} & \delta_{v}=0.3^{\prime \prime} \text { Estimate } \\
\hline 0 & 2.42^{k} & \delta_{u} \\
0.25^{\prime \prime} & 3.20^{k} \\
0.50^{\prime \prime} & 3.89^{k} \\
0.75^{\prime \prime} & 4.49^{k} \\
1.0^{\prime \prime} & 5.03^{k}
\end{array} \quad \begin{cases}T_{v}=3.34^{k} & \beta_{u}=0.14 \\
& T_{v_{95}}=2.65^{k}\end{cases}
\end{aligned}
$$

(2)

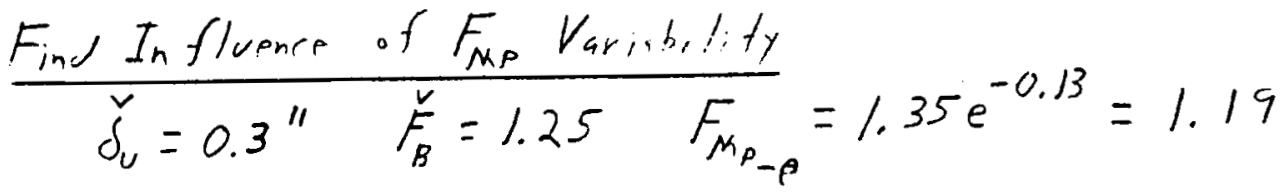

$$
\begin{aligned}
& \therefore T_{v_{(2)}}=3.12 \\
& \beta_{M_{p}}=\ln \left(\frac{3.34}{3.12}\right)=0.07
\end{aligned}
$$

A-86 


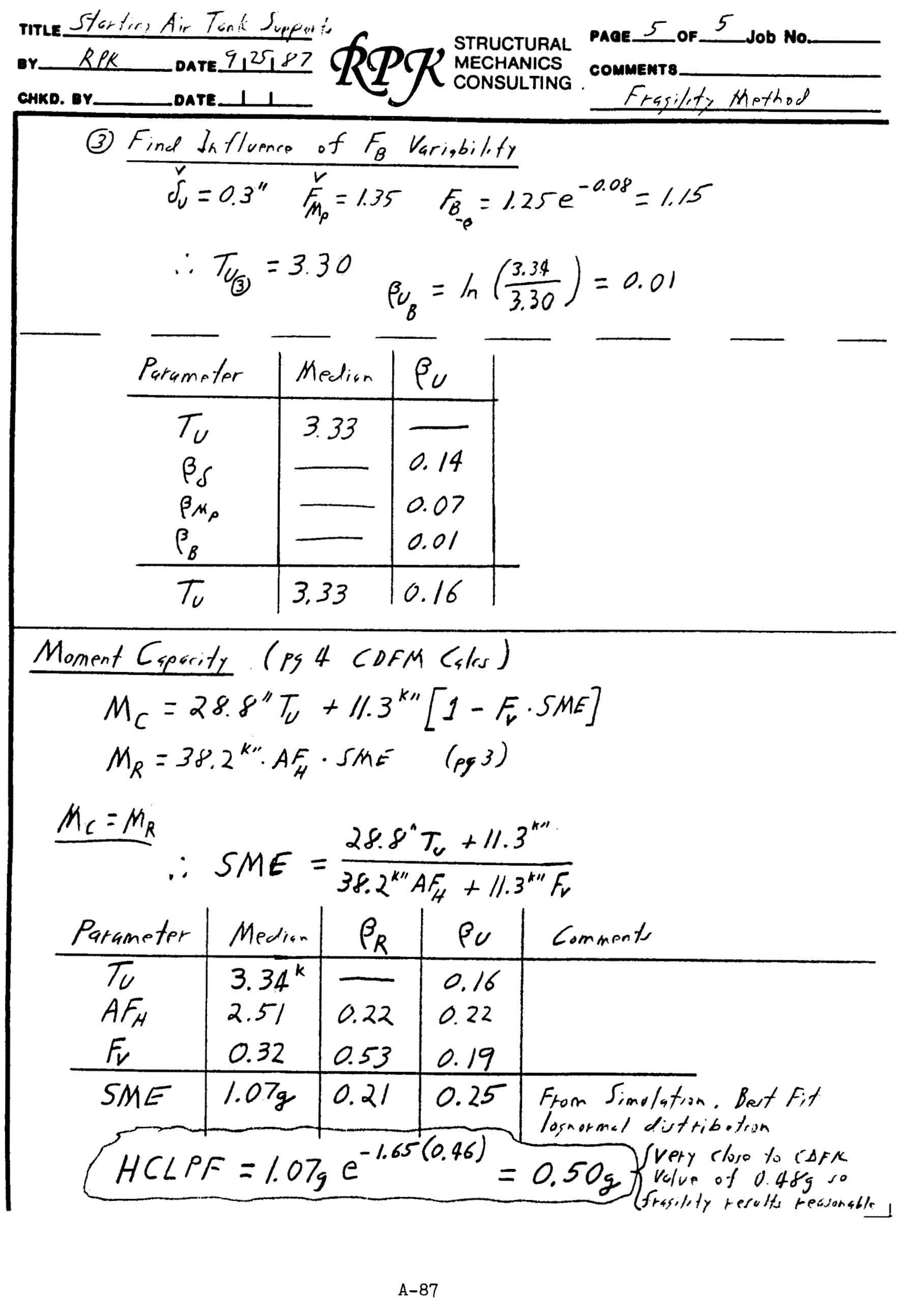


COMPONENT COOLING HEAT EXCHANGER SUPPORTS

CDFM METHOD

By

R. P. Kennedy 
rime Heat Exrhaniser Sup orts or BPK oar e 9, 1,87

STRUCTURAL

PACE / OF 2 Job No. MECHANICS COMMENTS

CHKO. B

Dare $\frac{1}{1}$

CONSULTING. CDFM Method

Heat Exchanger Shown in Fig 3 is Mounted High in Structure With

Floor Spectra Input Shown in Figures 6\% 7 for S/KE $=0.18 \mathrm{~s}$

(1) Seismic Capacity is Controlled by Longitudin, Shear on bolts and Only this failure mode capacity is shown in these example CDFMh celcolstion,

(2) Heat Exchanger is Bolted to Stead Support frame which is assumed to be rigid

Horizontal E Vertical Frequencies Exceed $33 \mathrm{~Hz}$

Determine Horiz $\&$ Vertical Response Amplification Factor

$$
\begin{aligned}
& S_{A_{H}}=A F_{H} \cdot S M E \quad S_{A_{V}}=A F_{V} \cdot S M E \\
& A F_{H}=\frac{0.38}{0.18}=2.11\left(F_{i g .6}\right) \quad A F_{V}=\frac{0.20}{0.18}=1.11 \quad\left(F_{i g} .7\right)
\end{aligned}
$$

Determine Base Response Per Sad dee

Longitudinal (one saddle has slotted bolt holes)

$$
V_{\text {tony }}=W \cdot A F_{H} \cdot \operatorname{SME}=23.5^{\mathrm{k}}(2 \cdot 11) \cdot \operatorname{SME}=49.6^{\mathrm{k}} \cdot 5 \mathrm{KE}
$$

Lateral

$$
\begin{aligned}
& V_{\text {lat }}=\frac{W \cdot A F_{H} \cdot \operatorname{SME}}{2}=24 \cdot 8^{\mathrm{k}} \cdot 5 \mathrm{SME} \\
& M_{\text {lat }}=V_{\text {lat }} \cdot h_{\text {cG }}=24.8^{\mathrm{k}}\left(25^{\prime \prime}\right) \cdot 5 \mathrm{ME}=620^{\mathrm{k}} \cdot 5 \mathrm{ME}
\end{aligned}
$$

Vector Sum Shear Per Bolt

Combine 100\% Long With 40\% Lat $540 \%$ Vest

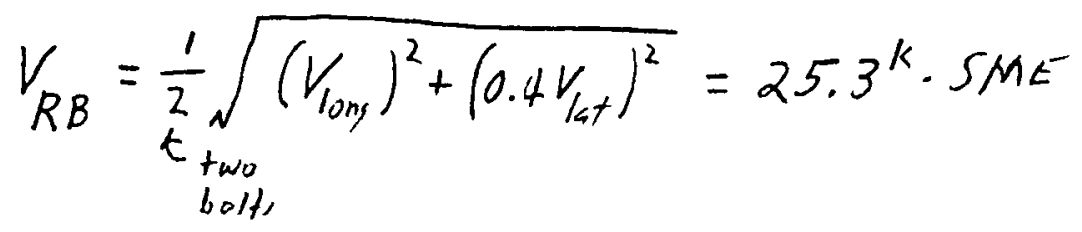

A-89 
TIrLE Heat Exchanspr Supeots

STRUCTURAL

PAOE 2 of 2 Job No or RPK OATE 9,13,87

MECHANICS COMMENT.

chko. Date 11

CONSULTING CDFM Method

Verticsl

$$
\begin{aligned}
& W_{e}=\frac{W}{2}\left[1-0.4 A F_{v} S M E\right]-V_{\text {long }}\left(\frac{h_{r c}}{s}\right) \\
& W_{e}=\frac{23.5^{k}}{2}[1-0.4(1.11) S M E]-49.6^{k}\left(\frac{25^{\prime \prime}}{216^{*}}\right) \text { SME } \\
& W_{e}=11.75^{k}-10.96^{k} S^{k} M E
\end{aligned}
$$

Capacity

First, assume bulf caparity controlled by sheer. Will subsequontly cherk combinad shear and tersion

$$
V_{C}=1.7 * \text { AJSC Part } 1 \text { Capacity (EighthEdition) }
$$

for $7 / 8 * A 307$ bolts

$$
\begin{gathered}
V_{c}=1.7\left(6.0^{k}\right)=10.2^{k} \\
\therefore V_{c}=V_{R B}: S \text { SME }=\frac{10.2^{k}}{25.3^{k}}=0.40 \text { CDFM Caparity }
\end{gathered}
$$

Check Tension Interation for $5 M E=0.40 \mathrm{~g}$

$$
\begin{aligned}
& T_{1}\left(24^{\prime \prime}\right)+T_{2}\left(4^{\prime \prime}\right)+W_{E}\left(14^{\prime \prime}\right)=M / 4 t \\
& T_{2}=\left(\frac{4}{24}\right) T_{1} \\
& \therefore T_{1}=\frac{M_{\text {lot }}-W_{E}\left(14^{\prime \prime}\right)}{24^{\prime \prime}+\frac{\left(4^{\prime \prime}\right)^{2}}{24^{\prime \prime}}}=\frac{M_{k t}-14^{\prime \prime} W_{E}}{24.67^{\prime \prime}} \\
& \text { L21" } \stackrel{k 4^{\prime \prime} \mid}{\longrightarrow} 40 \% \text { katemal at tim- of max shesr } \\
& M_{\text {lat }}=620^{k \prime \prime}(0.40 \mathrm{~g})(0.40)=99.2^{k \prime \prime} W_{E}=11.75^{k}-10.96^{k}(0.40)=7.37^{k}
\end{aligned}
$$

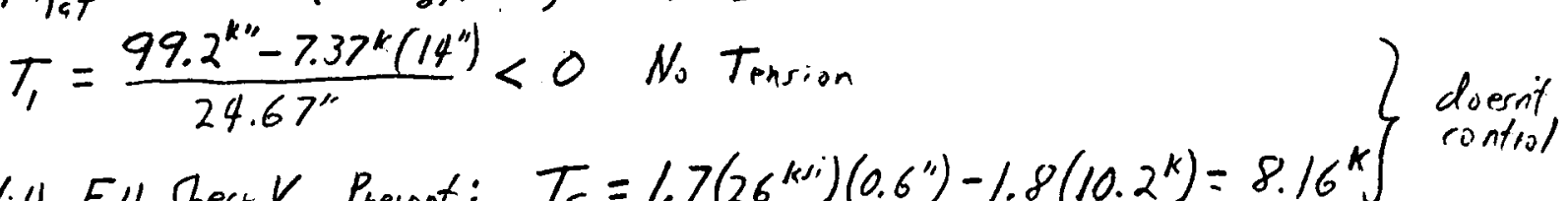

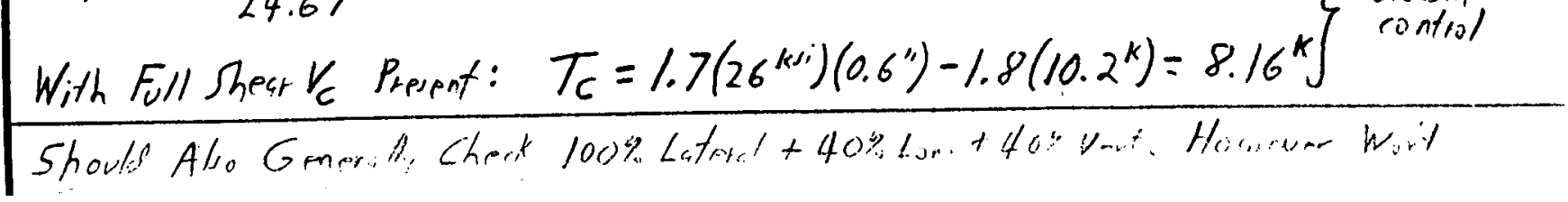

A-90 
COMPONENT COOLING HEAT EXCHANGER SUPPORTS

FRAGILITY METHOD

By

R. P. Kennedy 
rime. Heat Exchanser Support

STRUCTURAL

PAaE L OF 2 Job No.

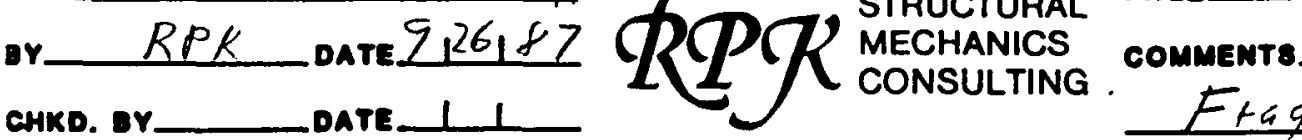

aHKo.

Etagilitr Method

(1) SME Capacity of Heat Exchanger Is Not Governed By

Largest Holiz Response Componant. It is Governed By Lonsitudinst

Response Component. SME is defined as 84\% NEP Latsert

Hotiz Responir.

$$
\begin{aligned}
& \frac{\text { Long Rerp }}{84 \% \text { Laspert Hotiz }}=\frac{F_{1} \cdot F_{2}}{F_{6}} \\
& \frac{\text { Vept Resp }}{84 \% \text { Latsest Hotid }}=F_{q}
\end{aligned}
$$$$
\frac{\text { Lat Respons }}{84 \% \text { Larpert Horiz }}=\frac{F_{1}}{F_{2} F_{6}}
$$

where $F_{1}, F_{2}, F_{6}$, and $F_{9}$ are all defined in Table 1 of int, odurtory trext

(2) Must Abro Inelude Sttuetural Rerponse Varisbility for High in. Stheline

Away From Resonater. Use Fre derived on pase 2 of freility method calcs for storting air tank supports.

(3) Since fres $>33^{1 / 2}, A F=2.11$ used in CDFM Cales without varisbility

(4) $\therefore$ Vertor Som Shear Per Bolt (see ps 1 of CPFM Gales)

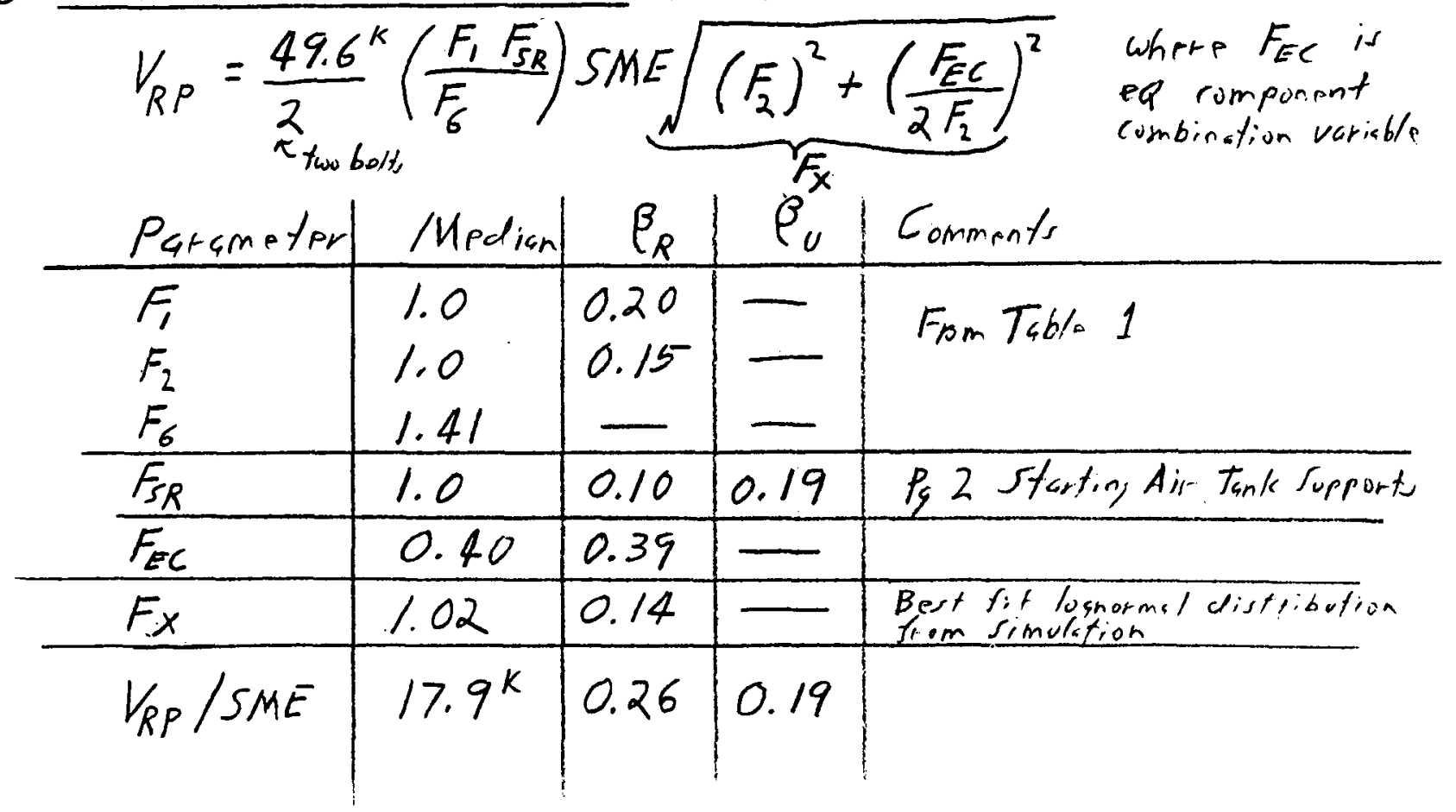

A-92 
Tirte. Heat Exchanser Support.

STRUCTURAL

pree 2 of 2 Job No MECHANICS cOMMENT8

CHKo. oare. CONSULTING

Fresility Method

Bolt Caparity (assume treads are in shear plane)

$$
\begin{aligned}
& V_{C}=0.55 A_{N_{0 m}} \sigma_{U} \\
& \sigma_{u c}=58^{k i} \\
& v_{y c}=36 \mathrm{ksi} \quad A_{\text {nom }}=0.6 \\
& E_{\text {gn }} E_{110 \mathrm{r}}: \beta_{E \otimes N}=\frac{1}{1.65} \ln \left(\frac{\sigma_{u r}}{\sigma_{y c}}\right)=0.29
\end{aligned}
$$

\begin{tabular}{c|c|c|c|} 
Parameter & Medicn & $\beta_{u}$ & \\
\hline$\sigma_{U}$ & $64^{k r}$ & 0.06 & \\
$E Q N$ & 1.0 & 0.29 & \\
\hline$V_{c}$ & $21.1^{k}$ & 0.30 &
\end{tabular}

$$
S M E=V_{C} /\left(V_{R P} / S M E\right)
$$

$$
\text { HCLPF }=1.18 e^{-1.65(0.62)}=0.42 \mathrm{~g}
$$

\begin{tabular}{c|c|c|c|} 
Parameter & Median & $\beta_{R}$ & $\beta_{U}$ \\
\hline $\begin{array}{c}\left.V_{R P} / \text { SME }\right) \\
V_{C}\end{array}$ & $17.9^{\mathrm{K}}$ & 0.26 & 0.19 \\
\hline SME & $1.18^{\mathrm{K}}$ & - & 0.30 \\
\hline
\end{tabular}

Veiy rlore to CDIM value of 0.40 g

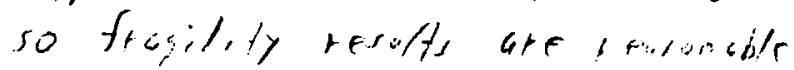

* In a fragilety study, one should cherk other fallore mudes to insure they do not tesulti in a leserer median copacity The failure modp whirh was cherked rontrols the HCLPI caparty but possibly not the medion.

A-93 


\section{CANTILEVER REINFORCED BLOCK WALL}

CDFM METHOD

By

R. P. Kennedy

A-94 
LE Bled Wall

RPK DATE $91 / 3,87$ OPOPUCTURAL

KD. BY

DATE

CONSULTING

pACE 1 or 4 Job No..

CDFK MiKthoO

Gantilever Blort Wall Showin in Fig 4 is Mounted Hist in Strueture $\xi$ Sobjerted to Flow Spertia Inpot Shown in Fig $6 \dot{\text { ? } 7}$

for $\operatorname{Sin} E_{e}=0.18 \mathrm{~g}$

(1) Seismic Laparity is Gouerned by Out-of. Plane Flexurel Bohavior about wall base and this is the onty fuilure madp cherkerd in these cales.

i) Wall it evaluated using sterath derisu mathod.

Conctete Block Properties

$$
\begin{aligned}
& f_{c}^{\prime}=3000 \text { pri Typr } S \text { Mortar }
\end{aligned}
$$

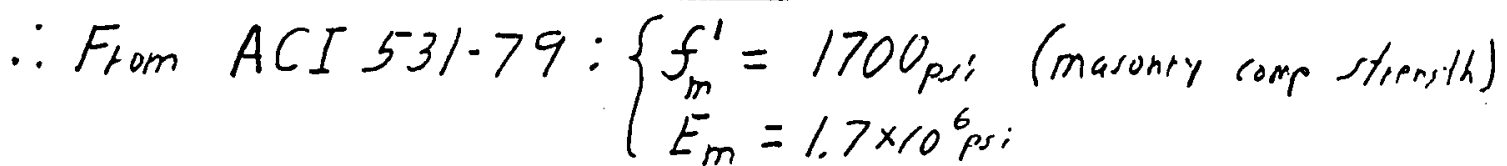

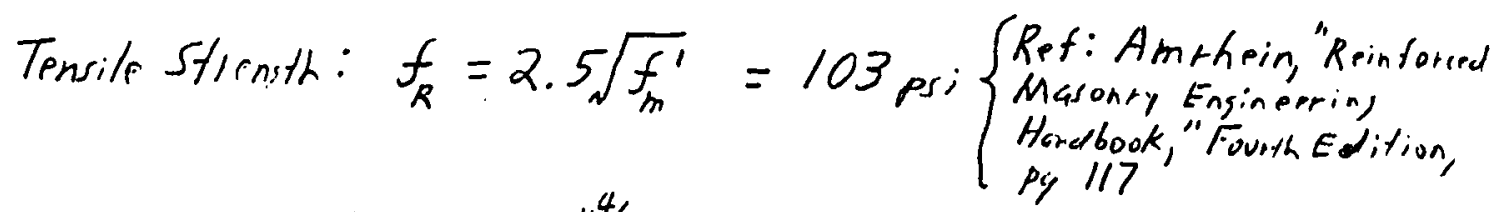

$$
\begin{aligned}
& \text { Gross Sertion: } I_{g}=1340^{114} / \mathrm{ft} \quad S_{g}=231^{113} / \mathrm{st} \\
& \text { Ctarkad Spetion: } I_{C R}=86^{14 / 8 t} \\
& \text { Weight: } \omega=111 \text { psf HA. } h=10^{\text {st }} \\
& \text { Natural Fieg Gross Section: } f_{\mathrm{g}}=12 \mathrm{~Hz}
\end{aligned}
$$

Ctarking Moment Caparity

$$
M_{C R}=f_{R} S_{g}=0.103 \mathrm{ks} ;\left(231^{\prime \prime 3} / \mathrm{H}\right)=23.8 \mathrm{~km} / \mathrm{s}
$$

A-95 
TITLE_ Block Wall

STRUCTURAL

PACE 2 OF 4 Job No.

MECHANICS COMMENT

chko.

Date 11

CONSULTING

CDFM Method

Ultimate Moment Amrhein, Ps 115

$$
\begin{aligned}
& M_{u}=\phi A_{s} f_{y}\left[d-\frac{9}{2}\right] \quad a=\frac{A_{s} f_{y}}{0.85 f_{m}^{\prime} 6} \quad \frac{\text { Specie Inspection }}{\phi=0.80} \\
& A_{s}=0.233^{\prime \prime 2} \% \quad f_{y}=60^{\mathrm{kr} i} \quad d=5.81^{\prime \prime} \\
& \therefore a=\frac{0.233(60)}{0.85(1.7)\left(12^{\prime \prime \prime}\right)}=0.81^{\prime \prime} \\
& M_{u}=0.8(0.233)(60)\left[5.81-\frac{0.81}{2}\right]=60.4^{\mathrm{k \prime \prime} / \mathrm{s}}
\end{aligned}
$$$$
\phi=0.80
$$

Determine lIef $\xi$ Elastic freq. $f$

$$
\begin{aligned}
& I_{\text {eff }}=\left(\frac{M_{C R}}{M_{U}}\right)^{3} I_{g}+\left[1-\left(\frac{M_{\text {rR }}}{M_{V}}\right)^{3}\right] I_{C R} \quad\left(\frac{M_{R R}}{M_{v}}\right)^{3}=\left(\frac{23.8}{60.4}\right)^{3}=0.0612 \\
& I_{\text {eff }}=(0.0612)\left(1340^{11 / / 4 t}\right)+(0.9388)\left(86^{114 / 4 t}\right)=163^{114} / 1 \\
& f=f_{g} \sqrt{I_{\text {ers }} / I_{g}}=12 \mathrm{~Hz} \sqrt{\frac{163}{1340}}=4.2 \mathrm{~Hz}
\end{aligned}
$$

However wall will go substantially in elastic bot ore it fils and response will be governed by an effective in elastic frequency $f_{e}^{\prime}$. NUREG/CR-3805, Vol. 1, May 1984 (Kennedy, et. al.) will be used to estimate $f_{e}^{\prime}$ and inelastic spertial response

(1) Estimate Inelastic Base Rotation Capacity During EQ

Non-Cyclic Base Rotation Capacity to given by ACI 349, App. C

$$
r_{u}=(0.0065)\left(\frac{0.85 d}{a}\right)=0.040 \text { radians }
$$

However, under 3 to 5 strung response cycles from EQ should limit to to lesser value.

$$
r_{v}^{\prime}=2 / 3 r_{v}=2 / 3(0.040)=0.026 \text { radians }
$$

A-96 


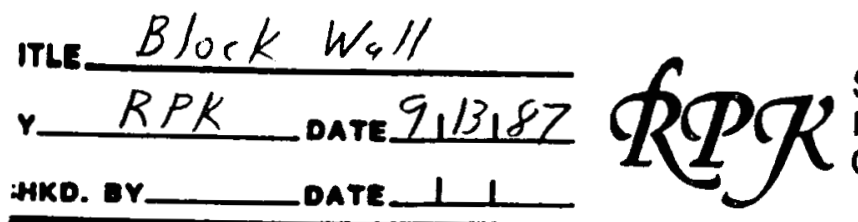

STRUCTURAL

MECHANICS

PACE 3 or 4

Job No.

CDFM shethod

(2) Determine Elastic s In clastic deformation of wall $C G$.

$$
\begin{aligned}
& \text { Elastic: } \quad \delta_{e_{c G}}=\frac{w h^{4}}{22.6 E I_{\text {eff }}}=\frac{0.111^{k} / 1^{2}\left(10^{1}\right)^{4}\left(144^{\left.11^{2} / 2\right)}\right.}{22.6\left(1.7 \times 10^{3} \mathrm{kr}\right)\left(163^{\left.\mathrm{m}^{4} / 1\right)}\right)}=0.026^{\prime} \\
& I_{n+1 \text { asti }} \delta_{C G}=\delta_{e_{C G}}+r^{\prime}\left(\frac{h}{2}\right)=0.026^{\prime}+0.026\left(5^{\prime}\right)=0.156^{\prime}
\end{aligned}
$$

(3) Determine Secant ? Eff. Fregreneies EE Eff. Damping

$$
\begin{aligned}
& f_{s}=f \sqrt{\delta e_{c G / \delta_{C G}}}=4.2 \mathrm{~Hz} \sqrt{\frac{0.026}{0.156}}=1.7 \mathrm{~Hz} \\
& f_{e}^{\prime}=0.15 f+0.85 f_{s}=0.15(4.2)+0.85(1.7)=2.1 \mathrm{~Hz} \\
& {f_{e}}^{\prime} \approx 10 \%
\end{aligned}
$$

(4)

$$
\frac{\text { Determine Eff } S_{A}}{S_{A_{e f f}}=K \cdot S_{A}\left(f_{e}^{\prime}, P_{e}^{\prime}\right)}
$$

Where $k$ is an inelastic response reduction factor.

$$
k=\left[f_{s} / f_{e}^{\prime}\right]^{2}=0.67
$$

However $\operatorname{Ref}(3)$ limits $k \geq 0.8$ for the CDFM Method unless a nonlinear response analysis is performed.

(5) Account for Freq Uncertainty

The Fig 6 Floor Spertic a unbrocdened and must be frequency shitted to account for structure frequency uncertain ty.

Furthermost; $f_{e}^{\prime}$ is unretain $\xi$ should be freq. shifted to account for uncertainty. To account for both, use highest sportily arcoloration within range 0.7 to 1.4 times $f_{e}^{\prime}$

$$
f_{e}=1.5 \mathrm{~Hz} \text { to } 2.9 \mathrm{~Hz}
$$

A -97 


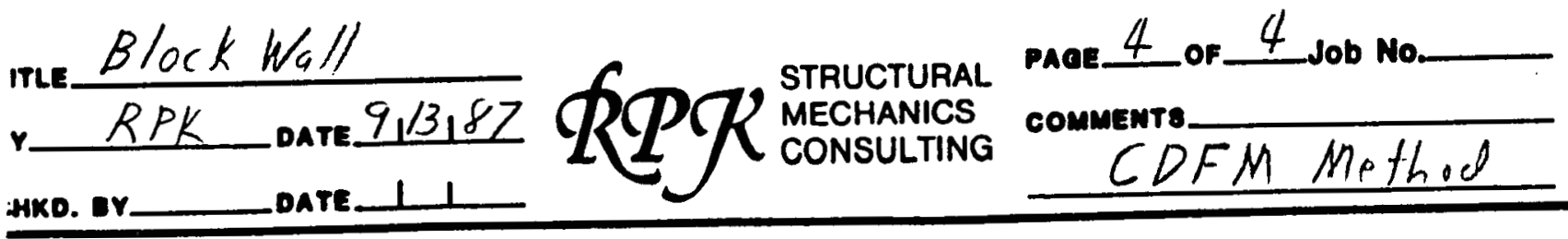

(6) Determine $A F_{H}$

$$
\begin{aligned}
& S_{A(2.9 \mathrm{~Hz}, 10 \%)}=0.33 \mathrm{~g} \quad \text { from } F \text { ig } 6 \text { for } S M E_{\mathrm{e}}=0.18 \mathrm{~g} \\
& S_{A_{\text {eff }}}=0.8(0.33 \mathrm{~g})=0.26 \mathrm{~g} \\
& A F_{H}=\frac{S_{A_{\text {Aff }}}}{0.18 \mathrm{~g}}=\frac{0.26 \mathrm{~g}}{0.18 \mathrm{~g}}=1.47
\end{aligned}
$$

(7) Determine Base Moment, $M_{R}$

$$
M_{R}=\frac{w h^{2}}{2} A F_{H} \cdot \operatorname{SME}=\frac{0.111^{\mathrm{kSF}}\left(10^{\prime}\right)^{2}(12 ! !)}{2}(1.47) \operatorname{SME}=97.9 \frac{\mathrm{kn}}{1} \cdot \operatorname{SME}
$$

(6) Determine SME

$$
\text { SME }=\frac{M U}{\left(M_{R} / S M E\right)}=\frac{60.4^{\mathrm{k} \prime \prime} / 1}{97.9^{\mathrm{k} / 1} / 1}=0.62 \mathrm{~g} \text { CDFM }
$$

A-98 
CANTILEVER REINFORCED BLOCK WALL

FRAGILITY METHOD

By

R. P. Kennedy

A-99 


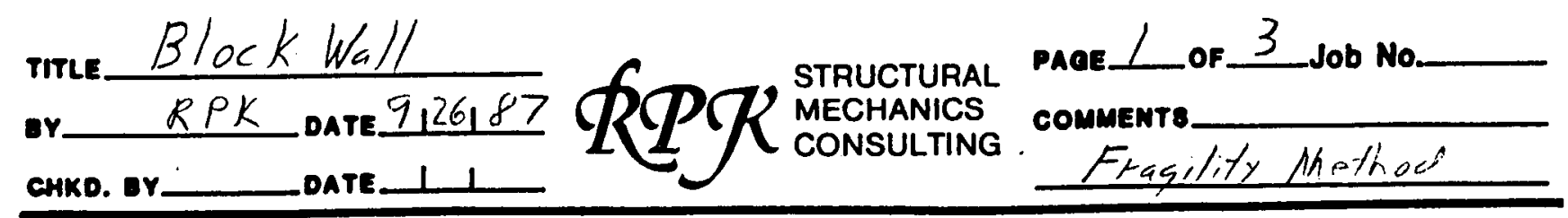

Moment Capacity Mu

$$
M_{u}=F_{\phi} \cdot A_{s} f_{y}\left[d-F_{a} \frac{a}{2}\right] \quad a=\frac{A_{s} f_{y}}{0.85 f_{m}^{\prime} b} \quad A_{s}=0.233 \frac{n^{2}}{1}
$$

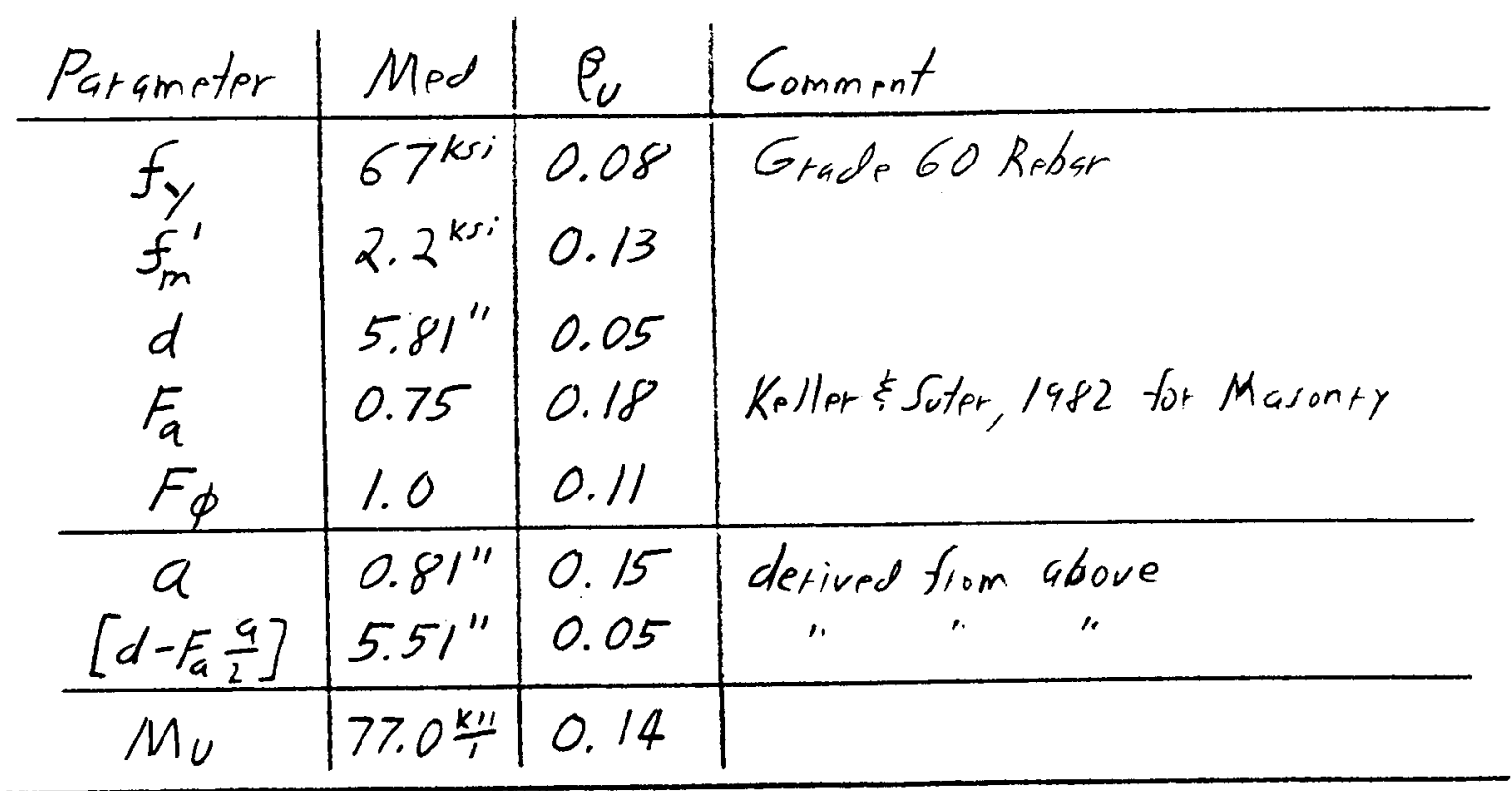

Rotational Capacity

$$
\begin{aligned}
& r_{c}{ }^{\prime}=F_{s} F_{v}(0.0065)\left(\frac{0.85 d}{9}\right) \\
& \begin{array}{c|c|c|l}
P_{\text {parameter }} & \text { Med } & \beta_{v} & \multicolumn{1}{|c}{\text { Comments }} \\
\hline F_{s} & 0.80 & 0.12 & \text { Seismic Cydie Reduction Factor } \\
F_{v} & 1.40 & 0.32 & \text { En Variability Factor } \\
d & 5.81^{\prime \prime} & 0.05 & \\
a & 0.81^{\prime \prime} & 0.15 & \\
\hline r_{v}^{\prime} & 0.044 & 0.38 &
\end{array}
\end{aligned}
$$

$A-100$ 


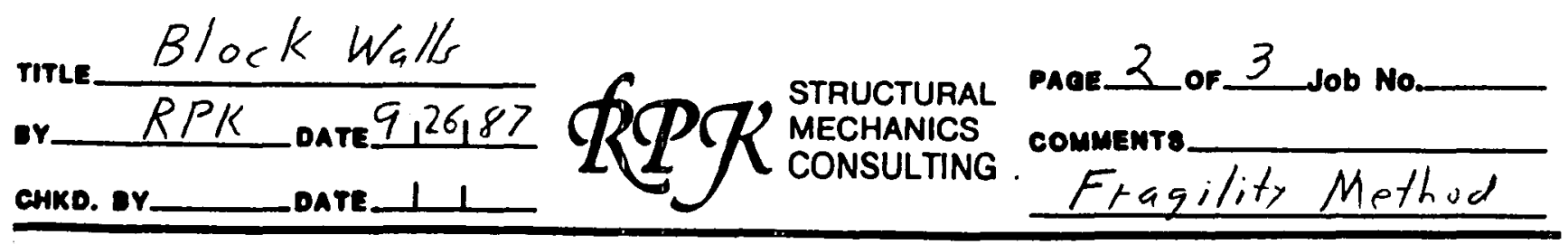

Elastic \&Inelastic Deformation Uncertainty $\xi$ Freq. One.

(based on values k equations used in (DFM evaluation)

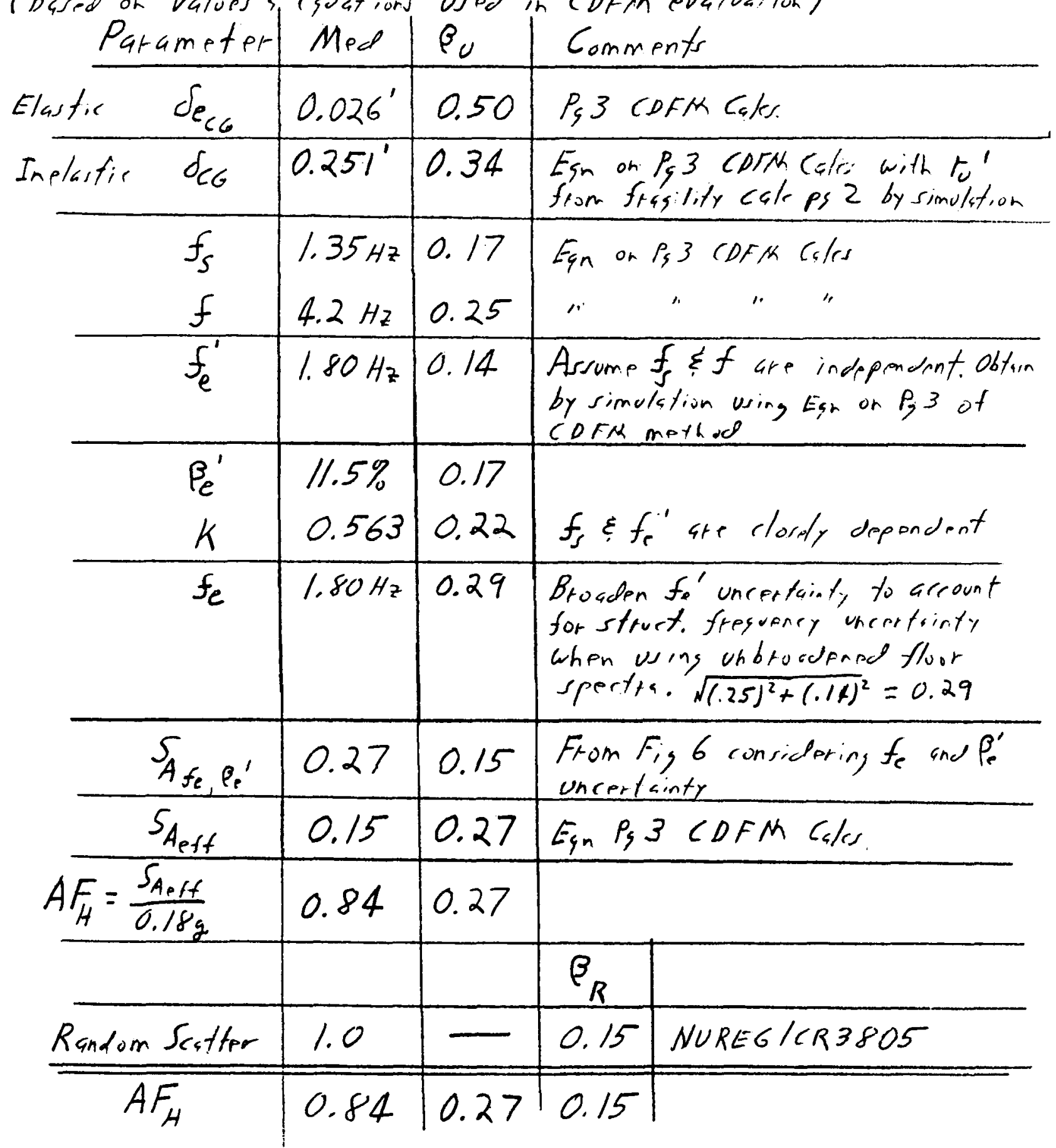

$A-101$ 


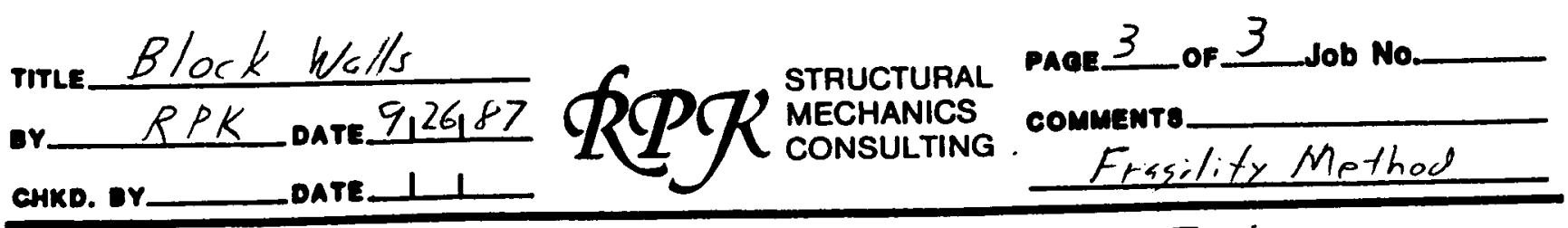

Incorporate Struetura/ Response \& Gtound Motion Fartots

(1) SME Caparity of Block $W_{a} l l$ is soverned by floot response out-of-planp of wall $c$ not by largest horiz responis comporent. SME is defined as 84\% NEP lariest horiz. Hesponse

$$
\frac{\text { Out-of-plane tesponie }}{84 \% \text { NEP largest horiz }}=\frac{F_{1} F_{2}}{F_{6}} \quad \begin{aligned}
& F_{1}, F_{2}, F_{6} \text { from } T_{a b} \text { of infredurtory text. }
\end{aligned}
$$

(2) Must also include strurtursl response variability for high in strueture away fiom resonance. Use $F_{S R}$ derived on pose 2 of frasility coles for starting sir tank supports.

(3) Moment Response SME

$$
\begin{aligned}
& \left(M_{R} / S M E\right)=\frac{M_{R C O F M}}{A F_{C D F M}} \cdot A F_{H} \cdot F_{S R} \cdot\left(\frac{F_{1} F_{2}}{F_{6}}\right) \\
& \text { SME }=M_{U} /\left(M_{R} / S M E\right)
\end{aligned}
$$

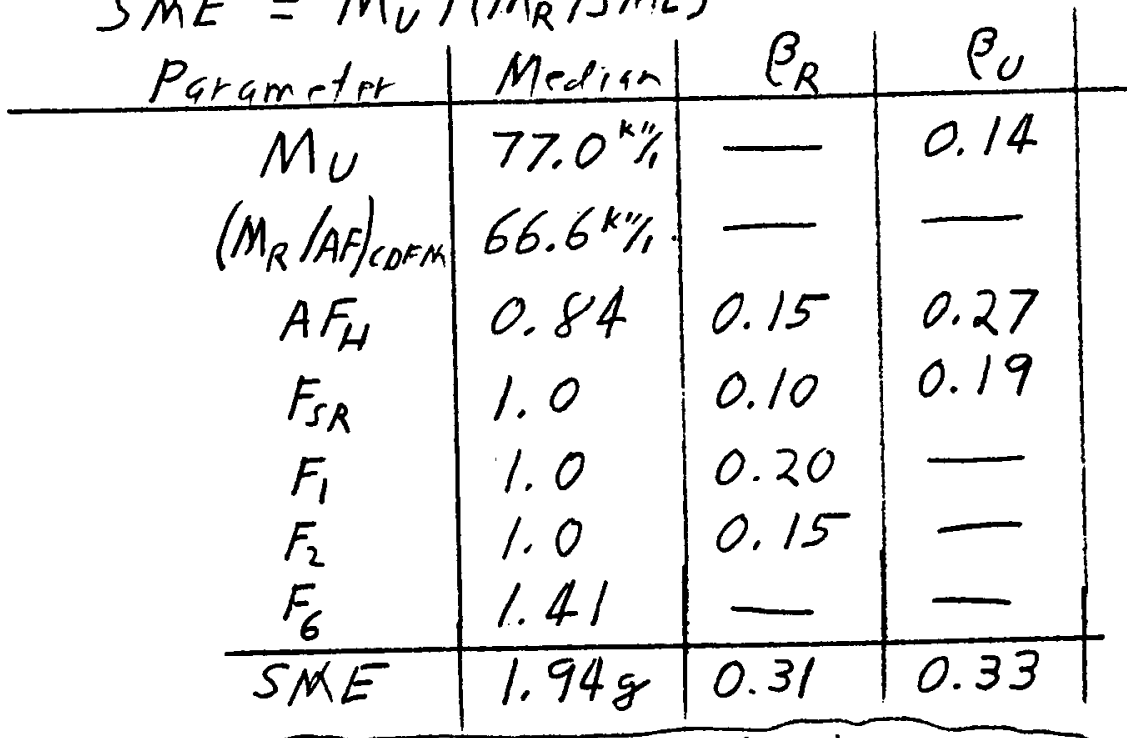

$$
\begin{aligned}
& H C L P F=1.94 \mathrm{~g} e^{-1.65(0.64)}=0.67 \mathrm{~g} \text { corm value of } 0.62 \mathrm{~g}
\end{aligned}
$$




\section{APPENDIX B \\ EQE ENGINEERING, INC.}

B-1 


\section{SUMMARY OF HCLPF CAPACITY CALCULATIONS FOR SELECTED COMPONENTS PERFORMED BY EQE INCORPORATED}

\section{Component 1: Flat Bottom Storage Tank}

Failure of the vertical storage tank is defined to be gross loss of fluid contents. This is assumed to occur when the tank shell buckles. Horizontal seismic load initiates uplift of the tank shell from its foundation. This uplift is resisted by the anchor bolts, the tank bottom plate, and the tank weight. The anchor bolts, with the modified chair detail, are permitted to yield, so long as their behavior is ductile, since yielding does not directly result in loss of fluid contents. Shell compressive stresses progressively increase until buckling occurs.

The following HCLPF capacities have been obtained:

$$
\begin{array}{ll}
\text { Fragility Analysis Method: } & 0.26 \mathrm{~g} \\
\text { CDFM Method } & : 0.29 \mathrm{~g} \\
\text { Median Capacity } & : 0.54 \mathrm{~g}
\end{array}
$$

These estimates were not revised in the Second Round Calculations following the Study Group discussion.

\section{Component 2: Motor Control Center}

The cabinet has a fundamental frequency of $6.5 \mathrm{~Hz}$ which is a typical electrical cabinet frequency that results in significant amplification of base motion input. The functional failure mode of the motor control center is governed by chatter of auxiliary contactors on the motor starters. This may result in spurious signals and may adversely affect the equipment controlled by the motor starter. The median fragility was derived using relay and contactor chatter data evaluated during the SSMRP program [Cover et al 1985]. However, the uncertainties for that 
fragility derivation were large due to the fact that some non-typical very sensitive devices were included in the data base. Therefore, Generic Equipment Ruggedness Spectra (GERS) [ANCO, 1987] were used to establish a lower bound capacity and the uncertainty on strength was derived from the acceleration capacity range between the median and GERS capacity. The HCLPF was then derived from the median capacity and the uncertainties in capacity, building response and equipment response. The deterministic HCLPF capacity was derived using the EPRI methodology and GERS. GERS were reduced by a factor of 1.3 in accordance with the EPRI Seismic Margin Criteria Methodology. In addition, operability during an earthquake is only a concern if there are interlock circuits. Therefore, the 0.87 reduction factor on GERS for interlock circuits, as stated in the GERS report [ANCO, 1987], was used to reduce the HCLPF capacity.

An additional failure mode investigated was operability after the earthquake. ANCO GERS and other generic test data have been used for this HCLPF capacity derivation. Two locations for the cabinet were studied: ground level and high-up in the building. The following HCLPF capacities have been calculated for the MCC:
Case
Median Capacity
(g)
HCLPF Capacity (g)
In-Structure
Fragility Method CDFM Method
Function During
0.36
0.07
0.09
Function After
1.16
0.21
0.26

Ground Mounted
Function During
1.58
0.39
0.47
Function After
5.06
1.18
1.45

These estimates were not revised in the Second Round Calculations. 


\section{Component 3: Starting Air Receiver Tank}

The starting air receiver tank is a vertical, skirt supported cylindrical tank which is anchored to the building floor by three angles. welded to the tank skirt and bolted to the floor. The leg of the angle is much weaker in bending than the anchor bolts. The angle leg is very ductile in bending and the failure mode is low cycle fatigue. When fracture of the angle occurs, the air tank is assumed to fail through failure of the attached piping.

A low cycle fatigue analysis was conducted to determine the ductility limit at failure, assuming 5 cycles of strong motion input. The resulting failure ductility was used in the fragility analysis to determine the median acceleration capacity. The HCLPF capacity was then computed from the median capacity and the derived uncertainties of the important variables that contribute to building response, equipment response, strength and ductility.

In the EPRI deterministic analysis method, bending of the angle leg was also the governing failure mode. In this case the computed bending stress was compared to the ASME Component Support Code allowable stress for plate bending. In addition, a load factor of 0.8 to account for ductility was used to reduce the seismic load before comparison to the code allowable. This load factor is specified in the EPRI deterministic criteria for ductile failure modes.

This tank is assumed to located high up in the building. Since the nozzle loads were not specified, the analysis has not included any effect of nozzle loads. The following HCLPF capacities have been calculated:

$$
\begin{array}{lr}
\text { Fragility Analysis Method: } & 0.44 \mathrm{~g} \\
\text { CDFM Method } & : 0.53 \mathrm{~g} \\
\text { Median Capacity } & : 1.55 \mathrm{~g}
\end{array}
$$

These estimates were not revised in the Second Round Calculations. 


\section{Component 4: Horizontal Heat Exchanger}

The failure mode governing the median capacity of the horizontal heat exchanger was combined tension and shear of the anchor bolts. Tension results from overturning of the heat exchanger in the lateral direction while shear results from inertial loads in both horizontal directions. When this anchorage failure occurs, the heat exchanger is assumed to fail through failure of the nozzles and attached piping. The HCLPF capacity was computed from this median capacity and the derived uncertainties in building response, equipment response and strength.

The governing failure mode for the EPRI deterministic HCLPF capacity calculation was pure shear of the anchor bolts. This change in governing failure mode results from the fact that dead weight resists the overturning and tensile stresses do not develop in the bolts at the HCLPF capacity level but do develop at the median capacity level. The difference in the calculated HCLPF is small, however, for the two failure modes of the anchor bolts.

Two locations for this heat exchanger have been studied: ground mounted and high-up in the building. Since nozzle loads were not specified, they have not been considered. The following HCLPF capacities have been calculated:

Case

Ground Mounted

In-Structure
Median Capacity

(g)

$$
1.87
$$

1.08
HCLPF Capacity (g)

Fragility Method CDFM Method

These estimates were not revised in the Second Round Calculations.

\section{Component 5: Reinforced Block Hall}

The block wall is represented as a vertical cantilever fixed at its base. Seismic capacity is controlled by out of plane bending moment at the base of the wall. The wall is capable of withstanding seismic 
excitation levels in excess of those causing initial yielding through ductile response. Failure occurs when the ductility demand reaches a maximum permissible value. This maximum allowable ductility corresponds to a deformation level at which the load carrying capacity begins to significantly degrade.

The sample block wall ultimate strength and load-deflection relationship is determined following the recommendations of the ACI-SEASC Task Committee on Slender walls. The capacities are estimated using two different stiffness assumptions: equivalent elastic-plastic loaddeflection curve and secant stiffness. The following HCLPF capacities have been calculated for block wall:

$$
\begin{array}{ll}
\text { Fragility Analysis Method: } & 0.48 \mathrm{~g} \\
\text { CDFM Method } & : 0.48 \mathrm{~g} \\
\text { Median Capacity } & : 1.55 \mathrm{~g}
\end{array}
$$

In the Second Round Calculations, median damping of $10 \%$ was used along with a median ductility of 3 . The revised capacity estimates are:

HCLPF Fragility Analysis Method: $0.63 \mathrm{~g}$

HCLPF CDFM Method $\quad: 0.63 \mathrm{~g}$

Median Capacity $\quad: 2.10 \mathrm{~g}$

\section{References}

1. Cover, L. E., M. P. Bohn, R. D. Campbe11, D. A. Wesley, "Handbook of Nuclear Power Plant Fragilities", Seismic Safety Margins Research Program, NUREG/CR-3558, June 1985

2. ANCO, "Generic Seismic Ruggedness of Power Plant Equipment", Prepared by ANCO Engineers for Electric Power Research Institute, EPRI NP-5223, May, 1987 


\section{COMPONENT 1}

FLAT BOTTOM STORAGE TANK 
EQE

ENGINEERING. PLANNING AND MANAGEMENT CONSULTANTS

SHEET NO

JOB NO $87218.0,10 B$ HCLPF Comparizen BY PSH DATE 9-30-8? CLIENT LLUL SUBJECT Trave CHKD MKR DATE $10-5-87$

TANK HCLPF CAPACTYY

B-8 
EQF

ENGINEERING. PLANNING AND MANAGEMENT CONSULTANTS

JOBNO 87218.01 JOB_HCLPF COmperison

CLENT LNV SUBJECT Tonk BY PSH DATE $9-30-87$ CHKD NKR DATE $10-5-87$

Summory

The tonk HCLF copecity is determined following the Maine Yonker RWST colculations CRef. ID, with revisions incorporated to account for the revised anchor bolt choirs. The tonk HCLFF cosclity is calcubted to be:

$$
\begin{aligned}
& \text { Fragility enalysis }-0.26 \mathrm{~g} \\
& \text { CDFM - } 0.29 \mathrm{~g}
\end{aligned}
$$

B-9 
ERE

ENGINEERING PLANNING AND MANAGEMENT CONSULTANTS

JOB NO $87218.01 \mathrm{JOB}$ HCLPF Comparing CLIENT LUNL SUBJECT Tank
SHEET NO. $T-2$

BY PAH DATE $9-30 \& 7$

CHK'D MK DATE $10-5-87$

Median Seismic Response

From Maine yankee Rust ales (Red. 1): Impulsive mode frequency $=5.6 \mathrm{~Hz}$ Sloshing made frequency $=0.273 \mathrm{~Hz}$

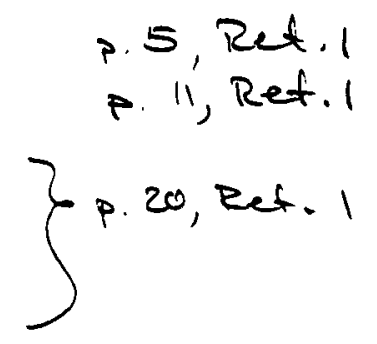

Median Ease Moment Capacity

The tank base monet capacity will be determined Using the same approach developed for the maine yankee RW ST. With the new bolt choirs (Ref. 2), the bolts control the tank capacity.

Anchor Bolt Capacity - 2" \$ ABOTA.B.

Belt yield force governs w/ new chairs installed.

yield On Nominal Area

Min. yield stress $=36 \mathrm{mi}$

Estimate $f_{g}^{2} \approx 44 \mathrm{kri}$ Typical for $A 36$ steel
$A_{b}=3.14 \mathrm{in}^{2}$

$$
\begin{aligned}
\check{P}_{y} & =44(3.14) \\
& =138^{\circ}
\end{aligned}
$$

$B-10$ 
ERE

ENGINEERING. PLANNING AND MANAGEMENT CONSULTANTS

SHEET NO. $T-3$

JOB NO $87218,01 \mathrm{JOB}$ HCLPE Comparison BY DATE $9-30-87$
CHKD MIR DATE $10-5-57$

CLIENT SiNK SUBJECT Tone

Tank Axial Load

Estimate $\dot{A}=0.5 \mathrm{~g}$

$$
\begin{aligned}
& P_{D L}=63.74 \\
& \text { P. } 3 \text {, Ret. } 1 \\
& P_{R Q}=5.08^{\circ} \\
& \text { p. } 20 \text {, Ret. } 1 \\
& \text { For } 0.3 \mathrm{~g} \text { horiz. PEA } \\
& P_{\text {NET }}=-63.7+5.08\left(\frac{0.5}{5.3}\right) \\
& =-55.2^{k}
\end{aligned}
$$

Bottom $R$ Holdown Force

Estimate $\tilde{A}=0.5 \mathrm{~g}$

see p.68, Ret. 1

$$
\begin{aligned}
& S_{\text {er }}=0.378 \text { For } 0.3 \text { ghoriz. PEA, p.16, Ret, } 1 \\
& S_{\text {er }}=0.378\left(\frac{0.5}{0.3}\right) \\
&=0.63 g \\
& \begin{aligned}
\omega & =62.4(37)[1-0.4(0.63)] \\
& =1727 \text { pet } \\
& =0.0120 \mathrm{ksi}
\end{aligned}
\end{aligned}
$$

Anchor Bolt Dimensions - See Pr BA, Ret. 1

$h_{1}=$ Dimension from top of chair to bottom of tank

$$
\begin{aligned}
& =0.75+24+0.75+1.5 \text { from tow sotrom to cotton of } \\
& =27^{4}
\end{aligned}
$$

$h_{2}=$ Dimension from top of chair to top of anchor Th $=39+(27-11.5)$ $39^{\prime \prime}=h_{2}$ for old chair $=54.5^{\prime \prime}$

B-11 
EDE

ENGINEERING. PLANNING AND MANAGEMENT CONSULTANTS

JOB NO 87218.01 JOB HCLPF Comparison CLIENT LEAN SUBJECT Tent BY PS

SHEET NO $T-4$

DATE $9-30-87$

CHKD MK DATE $10-5-87$

Program Input

Program TANKER calculates the tank capacity following the derivation in App. B, Ret. 1 . See p.70, Ret. Io r ot h input.

$$
\begin{aligned}
& R=\text { Tank radius }=240^{\prime \prime} \\
& t_{s}=\text { bul thickness }=0.375^{n} \\
& t_{b}=\text { cotton Pethickness }=0.25 \text { " } \\
& E_{3}=\text { shell wotton Flex mod of elasticity }=28,000 \mathrm{us} \\
& n=\text { of bolts }=8
\end{aligned}
$$$$
D=\text { Bolt diameter } 2^{\prime \prime}
$$$$
h_{1}=27^{\prime \prime}
$$$$
h_{2}=54.5^{\prime \prime}
$$$$
E_{0}=\text { Bolt modulus of elasticity }=29,000 \mathrm{mi}
$$$$
\omega=0.0120 \mathrm{ksi}
$$$$
f_{\text {se }}=\text { Shell buckling stress }=23 \mathrm{wi} \text { p.67, Ret. } 1
$$$$
P_{y}=138^{-}
$$$$
\text { FIT- } 2
$$

$$
\begin{aligned}
\text { Program Output } \\
\theta_{N_{A}}=0.4007 \mathrm{rod} \\
P_{S}=-1103.1 \mathrm{k} \\
P_{b}=890.0 \mathrm{Z} \\
P_{L}=157.9- \\
M_{S}=260,500 \mathrm{k-in} \\
M_{D}=46,020 \mathrm{ki} \\
M_{L}=10,780 \mathrm{~m} \\
\tilde{M}_{U}=317,300 \mathrm{k-in}
\end{aligned}
$$

B-12 
EQE

ENGINEERING. PLANNING AND MANAGEMENT CONSULTANTS

JOBNO 87218.01 JOB HCLEF COMparimea

CLIENT LUNC SUBJECT Tont
SHEETNO. T-5

BY PSH DATE $9-30-87$

CHKD MKK DATE $10 / 5 / 87$

Strength Futor

$$
\begin{aligned}
\bar{M}_{u} & =317,300 \mathrm{k}-\text { in } \\
M & =233,800 \cdots \cdots \\
F_{s} & =\frac{317,300}{233,800} \\
& =1.35
\end{aligned}
$$

Uncertainties

Shell Buckling Stress

$$
\begin{aligned}
& \text { Estimate } \sigma_{c r-1 \beta}=17.4 \mathrm{ksi} \\
& M_{-1 \beta}=302,200 \ldots i \\
& \beta=-\operatorname{Ln} \frac{302,200}{317,300} \\
& =0.05
\end{aligned}
$$

Botton R Holdown Force Estimat. no bottom R holdown is an extreme lower boud, 30 below the nean.

$$
\begin{aligned}
M-30 & =273,700 \text { k-in } \\
\beta= & -\frac{1}{3} \ln \frac{273,700}{317,300} \\
& =0.05
\end{aligned}
$$

A. B. Yield Stress

Estinate fymin = $36 \mathrm{ks}$ has how contidence of nonexceedence.

B-13 
EQ

ENGINEERING. PLANNING AND MANAGEMENT CONSULTANTS

JOBNO $87228.01 \mathrm{JOB}$ HCLPF COMpeniron

CLIENT LLNL SUBJECT Tank
SHEET NO $I-7$

BY PS Y DATE 9-30-87

CHKD MKS DATE $10: 5 i \bar{Z} 7$

Inelastic Energy Absorption Factor

A ductility Ref is was estimated for monotonic loading On PQ, $71 B$ to $7 D$. Howcuch, the hysteretic entry implied ty this ductility is available for on fo ne stroy motion cycle since the top lutes, wee deformed and in one discretion

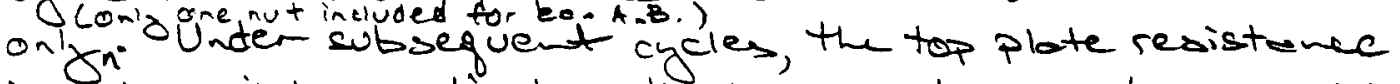
is not available until the displacements are those echicued in the previous cycle. Energy dissipation in the subsequent cycles is therefore small. To account for the reduced ene ry sorption capability, the following effective ductility is estimate b for three cycles strong motion.

$$
\begin{aligned}
\mu_{e} & =\frac{\mu-1}{n}+1 \quad n=\text { \# of cycles } \\
& =\frac{1-3-1}{3}+1 \\
& =1.1
\end{aligned}
$$

$\beta=7020$ Median dew ping for welded steel

$$
\begin{aligned}
q & =3.00 \beta^{-0.30} \\
p & =q+1 \\
r & =0.48 \beta^{-0.08} \\
\phi \mu & =(p \mu-q)^{-r} \\
q & =3.0(7)^{-0.30} \\
& =1.67 \\
p & =1.67+1 \\
& =2.67 \\
r & =0.48(7)^{-0.08} \\
& =0.41
\end{aligned}
$$

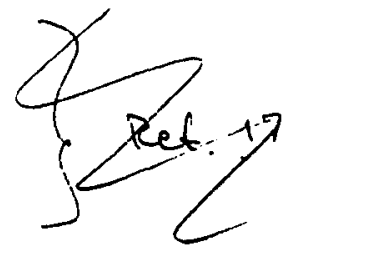


EDE

ENGINEERING, PLANNING AND MANAGEMENT CONSULTANTS

JOB NO 97218.01 JOB HCLFF Comparison

CLIENT LN
SHEETNO $1-8$

BY PSH DATE 9-30-89

CHKDMKR DATE $10 !=|\bar{E}\rangle$

$$
\begin{aligned}
\check{F}_{\mu} & =\frac{1}{\phi_{\mu}} \\
& =[2.67(1.1)-1.67]^{0.41} \\
& =1-1
\end{aligned}
$$

Variability

A lower bound $F_{\mu}$ of 1.0 is estimated to bc about 30 below the mean with randomness and uncertainty stout nagual.

$$
\begin{aligned}
\beta_{L} & =-\frac{1}{3} \ln \frac{1}{1.1} \\
& =0.03 \\
\beta_{R} & =\beta_{u}=\frac{1}{\sqrt{2}}(0.03) \\
& =0.02
\end{aligned}
$$

Spectral Shape Factor

Response is in the a amplified rome, with asinglemode dominus t

$$
\begin{aligned}
& F_{2 \alpha}=1.22 \\
& \beta_{R}=0.20 \\
& \beta_{1}=0
\end{aligned}
$$

B-15 
BERING, PLANNING AND MANAGEMENT CONSULTANTS

SHEET NO $T-9$

10. 87218.01 JOB HCLPF COnasurison BV PSH DATE 9-30-8) iT UNA SUBJECT Tank CHKDMIKR DATE $10 / 5 / E$ E 7

Dumping Factor

Median dumping of $7 \mathrm{Z}$. for welded steel structures of ai approaching yield was used to define the seismic loses.

$$
\frac{v}{F y}=1.0
$$

Variability

As noted on $P, 7,5^{\circ}$ lo damping is considered to be a lower bound. This value is estimated to be about or below the mean.

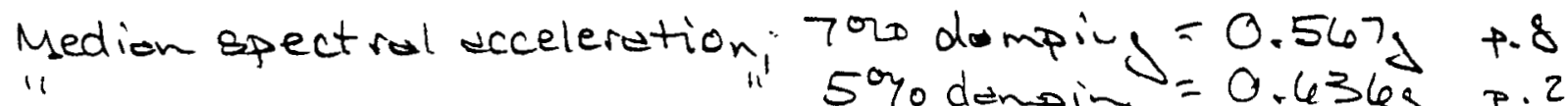

$$
\begin{aligned}
& 5 \% \text { damping }=0.636 \mathrm{~g} p .22 \\
& \beta_{u}=\ln \frac{0.6 \% 26}{0.507} \\
& =0.11 \\
& \beta_{R} \approx 0.2 \beta_{u} \\
& \text { estimated }
\end{aligned}
$$

Modeling Factor

The RUST is a relatively simple structure, with dynamic characteristics reasonably well-detinad.

Note, it all of the mas participating

$\beta_{U}=0.10$ estimated in the impulsive code is Bon above the

$$
\beta_{R}=\frac{1}{3} \ln \frac{1}{0.76}=0.04 \approx 0.10 \text { or }
$$

B-16 
EQ

ENGINEERING. PLANNING AND MANAGEMENT CONSULTANTS

SHEET NO $T-10$

JOB NO 87218.01 JOB HCLPF COmparison BY PEA DATE $9-30-87$
CHKD!KK DATE $10 / 5 / 8$ ?

CLIENT UN L

SUBJECT Tone

Modal Combination. Factor

The absolute sum of the modal responses is an upper bound estimated to be so above the mean.

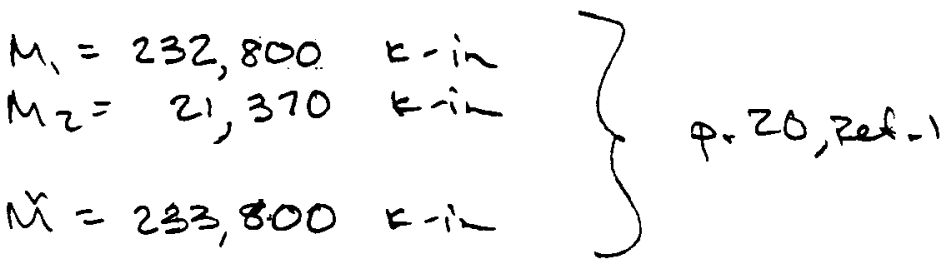

$$
\begin{aligned}
& M_{b s}=232,800+21,370 \\
& =254,200 k-\text { in } \\
& \beta_{R}=\frac{1}{3} \ln \frac{2000}{233,800} \\
& =0.03
\end{aligned}
$$

Combination of $E Q$ Components Factor

For a symmetrical cylindrical structure, the maximum horizontal response is the maximum due to \& single horizontal component. Vertical response has fit tie effect on the capacity.

$$
\begin{aligned}
& F_{R C}=1.0 \\
& \beta_{R}=0.01 \\
& \beta_{U}=0
\end{aligned}
$$

B-17 
ERE

ENGINEERING, PLANNING AND MANAGEMENT CONSULTANTS

JOB NO 87218-0 JOB HCLPF COMapurim Client LN SUBJECT Tank
SHEETNO. $T-11$ BY PST DATE $9-30-87$ CHKD NIKR DATE i j

SSI Soil Deumplificutio - Footer

The RWST is on rock.

$$
\begin{aligned}
& u \\
& F_{s 0}=1.0 \\
& \beta_{\pi}=\beta_{u}=0
\end{aligned}
$$

S8I Method of Analysis
$F_{M A}=1.0$

$\beta_{\text {R }}=0.01$

Estimated for rock founded structure

$\beta_{u}=0.05$

$c$

Grout Spectrum Direction Effect

Because asperity of the tank is governed by the higher of the two ground motion compose ct, the ground spectrum used (which is the higher the two conoponsto ) is medion-centered.

$$
\begin{aligned}
& \tilde{F}_{\Psi}=10 \\
& \beta_{\Psi}=0
\end{aligned}
$$

B-18 
EQE

ENGINEERING. PLANNING AND MANAGEMENT CONSULTANTS

JOBNO 87218.01 JOB HCLPE COMparimon CLENT LUNL SUBJECT Tank
SHEET NO. T-12 BY PSH DATE $9-30-87$ CHKD N.IKR DATE I'S/E?

Overall Frogility

$$
\begin{aligned}
& \ddot{F}=1.35(1.1)(1.22) \\
& =1.8 \\
& \tilde{A}=1.8(0.30 g) \\
& =0.54 \mathrm{~g} \\
& \begin{aligned}
\beta_{R} & =\sqrt{0.02^{2}+0.20^{2}+0.02^{2}+0.03^{2}+0.01^{2}+0.01^{2}} \\
& =0.20
\end{aligned} \\
& \begin{aligned}
\beta_{u} & =\sqrt{0.18^{2}+0.02^{2}+0.11^{2}+0.10^{2}+0.05^{2}} \\
& =0.24
\end{aligned} \\
& \text { HCLPF Capacity }=0.54 \mathrm{~g} \mathrm{e}^{-1.65(0.20+0.24)} \\
& =0.268
\end{aligned}
$$

B-19 
ERE

ENGINEERING. PLANNING AND MANAGEMENT CONSULTANTS

JOBNO. 87218.01 JOB HCUPF Comparison CLIENT LUNE
SHEET NO. T- 13 BY FSH DATE $9-30-87$ CHKD N K $:$ DATE $1 \mathrm{~J} / 5 / 87$

CDFM Copecit

Seismic Load

Some loos - as calculates in Ret. I which are based upon the median Nurze/CR-0048 spectrum scaled to $0.3 \mathrm{~g}$ ot 5 or damping.

From p. 25, Ret. 1

$$
\begin{aligned}
& V_{E Q}=14532 \\
& M_{E Q}=261,200 \mathrm{k}-\text { in } \\
& P_{E Q}=5.08 \mathrm{k}
\end{aligned}
$$

Ease Moment Capacity,

Allowable Bolt Capacity

lice nominal bolt are factored by 34 ks per URS anchorage guidelines (Ref. 3 ).

$$
\begin{aligned}
P_{\text {all }} & =34(3.14) \\
& =107 \mathrm{k}
\end{aligned}
$$

Rotten it Holdown Force

See p. 48, Ret. 1

Estimate HCLPF capacity $\approx 0.3 \mathrm{~g}$

$$
\begin{aligned}
\text { Suv } & =0.424 \mathrm{~g} \\
w & =42.4(32)[1-0.4(0.424)] \\
& =1917 \mathrm{pt} \\
& =13.3 \mathrm{Pzi} \\
& =0.0133 \mathrm{tsi}
\end{aligned}
$$

B-20 
EQE

ENGINEERING. PLANNING AND MANAGEMENT CONSULTANTS

JOBNO 87218.01 JOB HCYPF COmparivan CLIENT LLNL SUBJECT Tonk
SHEET NO. $T-14$ $B Y$ PSH DATE $9-30-87$ CHKDNKR DATE IS? ?

Tank Vertical Lood Estima $0.3 \mathrm{~g}$ MCLPF COPClity

$$
\begin{gathered}
P_{D L}=63.7^{4} \quad \text { P. 2, Ret.1 } \\
P_{\text {EQ }}=5.08^{\circ} \quad 0.3 J_{\text {POA }} \\
\begin{array}{c}
P_{\text {NET }}=-63.7+5.08 \\
=-58.6^{k}
\end{array}
\end{gathered}
$$

Program Input - sec p. T- -4

$$
\begin{aligned}
& t_{s}=0.315^{\prime \prime} \\
& t_{b}=0.25^{\prime \prime} \\
& E_{3}=28,000 \text { m: P. T-4 } \\
& n=8 \\
& D=2^{\prime \prime} \\
& h_{1}=27^{\circ} \\
& h_{2}=54.5^{\prime \prime} \\
& E_{1}=29,000 \mathrm{H}: \\
& \omega=0.0133 \mathrm{ks} \text {. } \\
& f_{50}=16.5 \mathrm{ks} \\
& P_{y}=107 \mathrm{k} \\
& \text { A. } T-13 \\
& P .47 \text {, Ret }-1 \\
& \text { P. } T \text { - B }
\end{aligned}
$$

Proqrom Qutput

$$
\begin{aligned}
& P_{S}=-857.4 \mathrm{~K} \\
& P_{-}=649.6 \mathrm{~K} \\
& P_{L}=149.2 \mathrm{C}
\end{aligned}
$$

$B-21$ 


\section{EQE}

ENGINEERING. PLANNING AND MANAGEMENT CONSULTANTS

SHEET NO. $T-15$

JOBNO 81218.01 JOB HCLPF COMparison

BY DATE $9-30-87$

CLIENT SUBJECT CHKDI:KK DATE $! 3 / 5 ! C ?$

$$
\begin{aligned}
& M_{s}=20,930 \mathrm{c} \text {-in } \\
& M_{D}=42,550 \mathrm{ki} \\
& M_{L}=10,540 \mathrm{c}-i \\
& M_{u}=255,000=-i
\end{aligned}
$$

$$
\begin{aligned}
\text { HCLPF Cepoeity } & =\frac{255,000}{261,200}(0.3 \mathrm{~g}) \\
& =0.298
\end{aligned}
$$


EDE

ENGINEERING. PLANNING AND MANAGEMENT CONSULTANTS

SHEET NO $T-16$

JOBNO $87218.01 \mathrm{JOB}$ HCLPF Comparison BY. DATE $9.30 \cdot 87$ CLIENT WUL SUBJECT Ton CHKD!K DATE MY 27

References

1. Heine yankee Seismic Margin Review, RWST Calculation, Jos No.

2. Memo, R.P. Kenned to HCLPF Panel, July 8, 1987.

3. URS Corporotion/Ooh-A. Blum a Associates, Engineers, "Seismic Anchorage Guidelines For Nuclear Plant Equipment", prepared for the Electric Power Research Institute, Draft, June, 1986.

$B-23$ 
Medion Copocity

TANK DATA

$\begin{array}{ll}\text { RADIUS } & =2.4000 E+02 \\ \text { SHELL THICKNESS } & =3.7500 E-01 \\ \text { BOTTOK PLATE THICKHESS } & =2.5000 E-01 \\ \text { SHELL YOUNG'S HODULUS } & =2.8000 E+07\end{array}$

ANCHORAGE DATA

NUKBER OE AHCHOF BOLTS =

ARCHE BOLT OIAMETER $=2.0000 \mathrm{0}+00$

GEO LENGTH OF BOLT $=12.7000$ E +01

. LENGTH OF BOLT $=5.4500$ E +01

TOUNE'S HODULUS $=2.9000 E+07$

FRECALCULATED DATA

EFFECTIVE FLUTD WEIBHT $=1.2000$ OOU

TAHK SHELL CRITICAL STRESS $=2.3000$ +04

LIMIT ON BULT CAFACITY $=1.3800$ E +05

HET VERTICAL BASE REACTION $=-5.5200 \mathrm{E}+04$

ITERATIOH PAFAMETERS

-

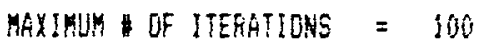

CONUEREENCE TOLERAHCE - a 1.00 


\begin{tabular}{|c|c|c|c|c|c|c|}
\hline & MEUTRAL AXIS & PS & $P B$ & $\mathrm{PL}$ & PSilh & XNORM \\
\hline & & & & & & \\
\hline 1 & $1.5708 E+00$ & $-4.1400 E+00$ & $8.9508 E+04$ & $4.3233 E+04$ & $-4.0073 E+06$ & $-3.9521 E+06$ \\
\hline 2 & $1.0472 E+00$ & $-2.835 X E+16$ & $3.6439 E+05$ & $7.4580 E+04$ & $-2.3963 E+06$ & $-2.3411 E+00$ \\
\hline 3 & $7.22735-0 !$ & $-1.9771 E+06$ & $6.4917 E+05$ & $1.0471 E+05$ & $-1.2232 E+06$ & $-1.1690 E+$ \\
\hline 4 & $5.0536 E-01$ & $-1.3888 E+06$ & $7.8960 E+05$ & $1.3596 E+05$ & $-4.6325 E+05$ & $-4.0805 E+$ \\
\hline 5 & $3.5542 E-01$ & $-9.7889 E+05$ & $9.6334 E+05$ & $1.6992 \mathrm{EE}+015$ & $1.543 E E+\hat{U} 5$ & $2.0958 E+$ \\
\hline$b$ & $4.3647 E-01$ & $-1.2008 E+06$ & $51 E+05$ & $964 E+05$ & $-2.0365 E+05$ & $-1.4645 E+$ \\
\hline 7 & $3.9790 \mathrm{E}-01$ & $-1.0955 E+06$ & $8.9384 E+05$ & $160 E+195$ & $-4.2854 E+04$ & $1.2346 \mathrm{E}+$ \\
\hline$g$ & $4.1761 E-0.01$ & $-1.1492 E+06$ & $8.6857 E+05$ & $899+05$ & $-1.2676 E+05$ & $-7.1581 E+$ \\
\hline 9 & 4.078 & $-1.1:$ & $E+05$ & $3 E+05$ & $-8.5787 E+04$ & $-3.0587 E+04$ \\
\hline 10 & 4.029 & EEtó & $3 E+05$ & $38 E+05$ & $-6.4580 E+04$ & $-9.3799 E+\hat{0} 3$ \\
\hline 11 & 4.00 & -1.1 & & $9 E+\hat{0} 5$ & $-5.5784 E+04$ & $1.4158 E+03$ \\
\hline 12 & 4.0 & -1.1 & $E+05$ & $2 E+115$ & $Q E+\hat{U} 4$ & $986 E+03$ \\
\hline 13 & & -1.1 & $E+05$ & $E+\dot{0} 5$ & $-5.6495 E+104$ & $-1.2951 E+03$ \\
\hline 14 & -01 & -1.10 & $E+i j 5$ & $1 E+\hat{U} 5$ & $-5.5142 E+04$ & $5.8291 E+01$ \\
\hline 15 & 4.00 & -1.1 & $E+05$ & $7 E+0.5$ & $-5.5819 E+0.4$ & $-6.192 E E+i j 2$ \\
\hline 16 & $E-\hat{0} 1$ & -1.10 & $E+105$ & $9 E+105$ & $-5.5480 \mathrm{E}+04$ & $-2.8045 E+02$ \\
\hline 17 & 4.0077 & -1.103 & $E+0 \hat{L} 5$ & $0 E+05$ & $-5.5311 E+04$ & $-1.1108 E+02$ \\
\hline 16 & $4.0075 E-01$ & -1.103 & $6 E+05$ & $1.5790 E+05$ & $-5.5226 E+04$ & $-2.6406 E+0 !$ \\
\hline 19 & $4.0074 E-01$ & -1.103 & $97 E+0.5$ & $1.5791 E+05$ & $-5.51 B 4 E+04$ & $1.5906 \equiv+01$ \\
\hline 20 & $4.0074 E-01$ & -1.103 & $8.8997 E+155$ & $1.5791 E+05$ & $-5.5205 E+04$ & $-5.2344 E+00$ \\
\hline 21 & $4.0074 E-01$ & $-1.1031 E+06$ & $8.8997 E+05$ & $1.5791 E+05$ & $-5.5194 E+04$ & $6.1719 E+C$ \\
\hline 22 & 4. $0074 E-01$ & $-1.1031 E+06$ & $8.8997 E+05$ & $1.5791 E+05$ & $-5.5200 E+04$ & $4.3750 E-$ \\
\hline
\end{tabular}


RESULTS OF ANALYSIS

(1) NeUtfal axis location:

$\begin{array}{ll}\text { DEGREES } & \text { RADIANS } \\ 22.9609 & 4.0074 E-01\end{array}$

$22.9609 \quad 4.0074 E-01$

(2) TENSILE FORCES IN AHCHOR BOLTS:

$\begin{array}{ccc}\text { BOLT } & \text { REF. ANGLE (DEGREES) } & \text { FORCE } \\ 1 & 190.0060 & 1.3800 \mathrm{E}+05 \\ 2 & 135.0000 & 1.3800 \mathrm{0}+05 \\ 3 & 90.0000 & 1.3800 \mathrm{E}+05 \\ 4 & 45.0000 & 9.9985 E+04\end{array}$

(3) DIFECT FORCES AT TANK EASE:

D.........

INGIVUDINAL FUFCE IN SHELL $=-1.1031 E+06$

U: UF ANCHOK BOLT FORCES $=8.8997 E+05$

DOTTOH PLATE HOLUOLN FORCE $=1.5791 \mathrm{E}+05$

TOTAL $=-5.5200 \mathrm{E}+04$

(4) RESTORING MOHENT:

-................

FFOM LONGITUDINALL FORCES IN SHELL $=2.6052 E+O B$ FRON ANCHOR BOLTS TENSILE FORCES - 4.602.3E+07 FROA BOTTOM PLATE HOLDOHN FORCE $=1.0784 E+07$

TOTAL $=3.173 \mathrm{JE}+0 \mathrm{~B}$ 


$$
9.4 / 4
$$

ADDITIONAL RESULTS:

MAXIMUM LENGTH OF UPLIFTED BOTTOH PLATE $=1.8519 E+U 1$

KAXIMUY UPLIFT DISPLACEMENT $\quad=5.3768 E-01$

HAXIKULM FIBEE STRESS IN BOTTOM PLATE $=6.5847 \mathrm{E}+04$ 


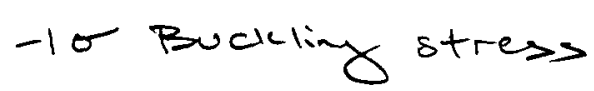

TANK DATA

$\begin{array}{ll}\text { RAOIUS } & =2.40000+02 \\ \text { SHELL THICKNESS } & =3.7500 E-01 \\ \text { BOTTOH PLATE THICKNESS } & =2.5000 E-01 \\ \text { SHELL YOUNG'S MODLLUS } & =2.8000 E+07\end{array}$

ANCHORAEE DATA

HUMBER OF ANCHOR BDLTS =

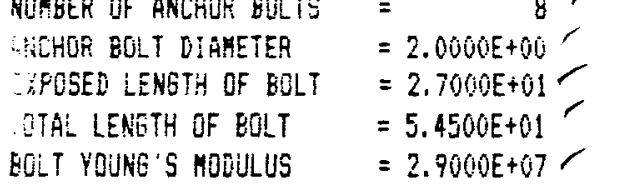

FRECALCULATED DATA

EFFECTIVE FLUIO WEIGHT $=1.2000 E+01$ TANK SHELL CAITICALL STFESS $=1.7400 E+04$ LIMIT QN BOLT CAFALITY $=1.3800 E+05$ NET VEFTICAL BASE REACTION $=-5.5200 E+04$

ITERATIOH PARAMETERS

MALIMUK OF ITERATIONS $=100$

CONUERGENCE TOLERANCE $=1.00$ 


\begin{tabular}{|c|c|c|c|c|c|c|}
\hline ER & NEUTRAL AXIS & PS & 98 & PL & PSUM & XNOERK \\
\hline & & -. & -- & -- & $\cdots$ & $\cdots$ \\
\hline 1 & $1.570 B E+00$ & $-3.1320 E+86$ & $6.7135 E+04$ & $4.0320 E+04$ & $-3.6240 E+06$ & $-2.96888 E+06$ \\
\hline 2 & $1.0472 E+00$ & $-2.1450 E+\hat{0} b$ & $2.7567 E+005$ & $6.9554 E+04$ & $-1.7997 E+06$ & $-1.7445 E+00$ \\
\hline 3 & $7.2273 E-01$ & $-1.4957 E+06$ & $5.9191 E+05$ & $9.7651 E+04$ & $-8.0612 E+05$ & $-7.5 \hat{0} 92 E+05$ \\
\hline 4 & $5.0536 E-01$ & $-1.0507 E+06$ & $7.6535 E+05$ & $1.2680 E+05$ & $-1.5852 E+05$ & $-1.0332 E+05$ \\
\hline 5 & 3. $5542 E-01$ & $-7.4055 E+05$ & $8.9679 E+\hat{W} 5$ & $1.5848 E+055$ & $3.1471 E+05$ & $3.6991 E+05$ \\
\hline 6 & $4.3647 E-(01$ & $-9.0843 E+05$ & $8.1916 E+005$ & $1.3956 E+05$ & $4.0285 E+04$ & $9.5485 E+04$ \\
\hline 7 & $4.7208 E-01$ & $-9.8201 E+015$ & $7.8412 E+05$ & $1.3266 E+005$ & $-6.5226 E+04$ & $-1.0 \hat{0} 26 E+0 \hat{0} 4$ \\
\hline$g$ & $4.5460 E-i 01$ & $-9.4591 E+05$ & $7.9569 \mathrm{E}+05$ & $1.3596 E+05$ & $-1.4270 E+04$ & $4.0936 E+04$ \\
\hline 9 & $4.6331 E-01$ & $-9.6412 E+105$ & $7.8969 E+05$ & $1.3427 E+05$ & $-4.0159 E+04$ & $1.5041 E+04$ \\
\hline 10 & $4.6777 \mathrm{E}-01$ & $-9.7310 E+015$ & 7. $86855+1.15$ & $1.3346 E+05$ & $-5.2791 E+6 j 4$ & $2.4090 E+03$ \\
\hline 11 & $4.69935-01$ & $-9.7757 E+005$ & $7.8548 E+05$ & $1.33065+05$ & $-5.9032 E+04$ & $-3.8324 E+03$ \\
\hline 12 & $4.69855-01$ & $-9.75354 E+05$ & $7.8616 E+0.5$ & $1.3326 E+0 \hat{5}$ & $-5.5918 E+04$ & $-7.1813 E+02$ \\
\hline 13 & 4.6831E-01 & $-7.7422 E+05$ & 7.865IE+0̂5 & $1.3336 E+05$ & $-5.4356 E+04$ & $8.4408 E+02$ \\
\hline 14 & 4. $6859 E-\pi 1$ & $-9.7478 \mathrm{E}+05$ & $7.8634 E+05$ & $1.3331 E+005$ & $-5.513 B E+04$ & $0.2406 \mathrm{E}+\hat{0} 1$ \\
\hline 15 & $4.6871[-0.1$ & $-9.750 t=05$ & $7.6625 E+05$ & $1.3328 E+105$ & $-5.55295+04$ & $-3.2791 E+02$ \\
\hline 16 & $4.6864 E-61$ & $-9.7493 E+05$ & $7.8629 E+0.5$ & $1.3330 E+0 \hat{0}$ & $-5.5332 E+044$ & $-1.3225 E+02$ \\
\hline 17 & $4.6861 E-01$ & $-9.74855+05$ & $7.8631 E+05$ & 1.3330E+05 & $-5.5235 E+04$ & $-3.4516 E+01$ \\
\hline 18 & $4.6859 E-01$ & $-9.7481 E+05$ & $7.8532 E+015$ & $1.3330 \mathrm{E}+0 \mathrm{5}$ & $-5.5186 E+04$ & $1.4313 E+01$ \\
\hline 17 & $4.6860 \mathrm{E}-01$ & $-9.7463 E+\dot{0} 5$ & $7.8632 E+05$ & $1.3330 E+05$ & $-5.5210 E+04$ & $-1.0453 E+0 !$ \\
\hline 20 & $4.68 t 0 E-01$ & $-9.7482 E+055$ & $7.8632 \mathrm{E}+0.5$ & $1.3330 E+05$ & $-5.5199 E+64$ & $2.0000 E+00$ \\
\hline 21 & $4.6860 \mathrm{E}-0 \mathrm{i}$ & $-9.7483 E+005$ & $7.8632 E+0.5$ & $1.3330 E+05$ & $-5.5205 E+\hat{v} 4$ & $-4.7031 \mathrm{E}+00$ \\
\hline 22 & $4.6860 \mathrm{E}-01$ & $-9.7483 E+05$ & $7.8632 E+05$ & $1.3330 E+05$ & $-5.5201 E+04$ & $-9.5313 E-01$ \\
\hline
\end{tabular}




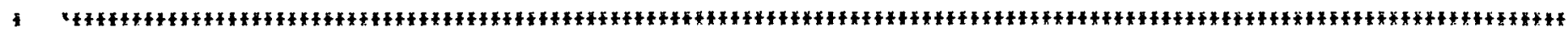

RESULTS OF ANALYSIS

$$
p 3 / 4
$$

(1) NEUTRAL AXIS LOCATION:

$\begin{array}{lr}\text { DEGREES } & \text { RADIANS } \\ 26.8487 & 4.6860 E-01\end{array}$

(2) TEMSILE FORCES IN ANCHOR BOLTS:

$\begin{array}{ccc}\text { BOLT } & \text { REF. ANGLE (OEGFEES) } & \text { FORCE } \\ 1 & 180.0000 & 1.3600 \mathrm{~B}+05 \\ 2 & 135.0000 & 1.3800 \mathrm{0}+05 \\ 3 & 90.0000 & 1.3800 \mathrm{0}+05 \\ 4 & 45.0000 & 4.8160 \mathrm{0}+044\end{array}$

(3) DIRECT FGRCES AT TANK EASSE:

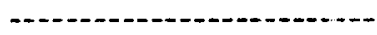

LONGITUDINAL FOFCE IN SHELL $=-9.748 .3 E+05$

SU: OF ANCHOR BOLT FORCES $=7.8632 \mathrm{E}+05$

BUTTOH FLATE HOLDOWN FORCE = $1.3330 \mathrm{OE}+05$

TOTAL $=-5.5201 E+04$

(4) RESTOAING KOAENT:

FROA LGNGITUDINAL FORCES IN SHELL $=2.2888 E+00$

FROM ANCHOR BOLTS TENSILE FORCES $=6.3613 E+007$

FROA BUTTOH PLATE HOLDOHM FORCE $=9.7444 E+66$

TOTAL $=3.0224 E+0 B$ 


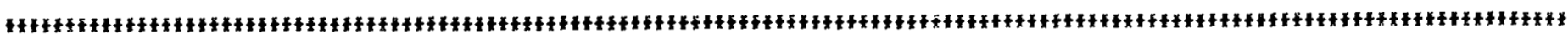
1) +BNORMAL. STY10-01-87 07:49:54

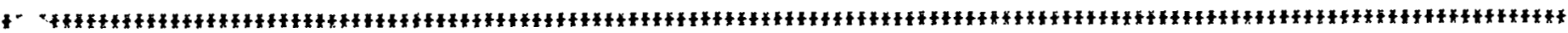

$$
p \cdot 4 / 4
$$

ADOITIONAL RESULTS:

MAXINUM LENGTH OF UPLIFIED BOTTOM PLATE $=1.59322 E+01$

KALIMUM UPLIFT DISPLACEHENT $\quad=3.9452 E-01$

SUM FIBRE STRESS IN BOTTOH PLATE $=4.8734 E+04$ 


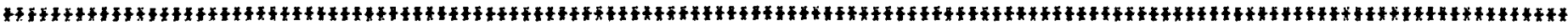 1)+6NORMAL. STY10-01-87 08:00:5!

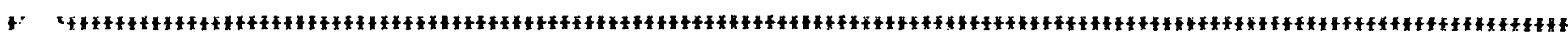

No Zotton R Hollown

P. $1 / 3$

IARK DATA

$\begin{array}{ll}\text { RADIUS } & =2.4000 E+02 \\ \text { SHELL THICKNESS } & =3.7500 E-01 \\ \text { BOTTOM PLATE THICKNESS } & =.0000 E+00 \\ \text { SHELL YOUNG'S MOOULUS } & =2.8000 E+07\end{array}$

SNCHORAGE DATA

HHSER OF ANCHOF EOLTS =

ANCHOF GOLT OIAMETEF $=2.0000 \mathrm{E}+00$

ER̈POSED LENGTH OF BOLT $=2.7000$ E +01

TOTAL LENGTH DF BOLT $=5.4500 \mathrm{E}+0 \mathrm{U}^{\prime}$

SOLT YDUNE'S MOOULUS $=2.9000 \mathrm{E}+\hat{+17} 7$

PFECALCULATED DATA

-

EFFECTIVE FLUIO WEIGHT $=1.2000$ E +01

TANK SHELL CRITICAL STRESS $=2.3000 E+04$

LIMIT ON BAT CAFACIIY $=1.3800 E+05$

NET VERTICAL GASE REACTIOK $=-5.52000+04$

I TEEATION PARAHETERS

MATIMULI OF ITERATIONS $=100$

CONVERGENCE TOLEFANCE $=1.00$ 


\begin{tabular}{|c|c|c|c|c|c|c|}
\hline ER I & MEUTFAL AXIS & PS & $\mathrm{PE}$ & PL & PSUH & XNORK \\
\hline & & -- & -- & -- & $\cdots$ & \\
\hline 1 & $1.5708 E+0 \hat{0}$ & $-4.14005+0 t$ & $8.950 \mathrm{BE}+0 \mathrm{0} 9$ & $.0000 E+00$ & $-4.0505 E+06$ & $-3.9953 E+106$ \\
\hline 2 & $1.0472 E+00$ & $-2.8353 E+06$ & $3.6439 E+0.5$ & $.0000 E+00$ & $-2.4709 E+06$ & $-2.4157 E+06$ \\
\hline 3 & $7.2273 \mathrm{E}-0 \mathrm{i}$ & $-1.9771 E+06$ & $6.4917 E+05$ & $.0000 E+00$ & $-1.3279 E+06$ & $-1.2727 E+0 \dot{0}$ \\
\hline 4 & $5.0536 E-01$ & $-1.3888 E+066$ & $7.8960 E+0.5$ & $.0000 E+00$ & $-5.992 i E+05$ & $-5.4401 E+$ \\
\hline 5 & $3.5542 E-01$ & $-9.7889 E+05$ & $9.6334 E+05$ & $.0000 E+00$ & $-1.5548 E+04$ & $3.9652 E+$ \\
\hline 6 & $4.3647 E-01$ & $-1.2008 E+015$ & $8.4751 E+05$ & $.0000 E+00$ & $-3.5329 E+0.5$ & $-2.9809 \mathrm{E}+$ \\
\hline 7 & $3.97905-01$ & $-1.0953 E+6 E$ & $8.5384 E+005$ & $.6000 E+60$ & $-2.0146 E+05$ & $-1.4626 E+$ \\
\hline 8 & $3.7723 E-01$ & $-1.0397 E+06$ & $9.2473 E+05$ & $.00000+000$ & $-1.1395 E+05$ & $-5.8750 \mathrm{E}+$ \\
\hline 9 & $3.6648 E-01$ & $-1.0092 \mathrm{E}+06$ & $9.4290 E+05$ & . OOUNOE + & $-6.6315 E+04$ & $-1.1115 E+$ \\
\hline 10 & $3.6099 E-01$ & $-9.9417 E+05$ & $1 E+05$ & $.0000 \mathrm{E}+00$ & $54 E+104$ & \\
\hline 11 & $3.6375 E-01$ & -1.00 & & $.0000 E+00$ & $B E+04$ & \\
\hline 12 & $3.6512 \mathrm{E}-01$ & -1.00 & & $E+00$ & $E+104$ & -4.9 \\
\hline 13 & $3.6443 E-01$ & $-1.00 .36 E+0.06$ & 9.4 & $.0000 E+100$ & $-5.7050 E+\hat{V} 4$ & $-1.8499 \mathrm{Q}+\mathrm{C}$ \\
\hline 14 & $3.6409 E-01$ & $-1.0027 E+06$ & $9.4716 E+05$ & $.0000 E+00$ & $-5.5494 E+04$ & $-2.9400 E+02$ \\
\hline 15 & $3.6392 E-01$ & $-1.0022 E+0 E$ & $9.4747 E+05$ & $.0000 E+00$ & $-5.4715 E+04$ & $4.8469 E+02$ \\
\hline 16 & 3. $6490 \mathrm{E}-01$ & $-1.0024 E+06$ & $9.4732 E+155$ & $.00000 E+000$ & $-5.5104 E+0.4$ & $9.6250 E+01$ \\
\hline 17 & $3.6405 E-01$ & $-1.6025 E+06$ & $9.4724 E+65$ & 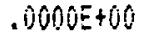 & $-5.5299 E+\hat{v} 4$ & $-9.8936 E+[1]$ \\
\hline 18 & $3.64035-0 !$ & $-1.0025 E+06$ & $9.4728 E+05$ & $.0000 \mathrm{E}+000$ & $-5.5201 E+04$ & $-1.3750 E+90$ \\
\hline 19 & $3.6401 \mathrm{E}-01$ & $-1.0025 E+06$ & $9.4730 E+05$ & $.00000+00$ & $-5.5154 E+04$ & $4.6375 E+01$ \\
\hline 20 & $3.6402 \mathrm{E}-01$ & $-1.00125 E+\hat{U} E$ & $9.4729 E+05$ & 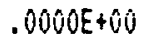 & $-5.5177 E+1.4$ & $2.2563 E+0 !$ \\
\hline 21 & $3.6402 E-01$ & $-1.0025 E+06$ & $9.4728 E+05$ & $.0000 E+00$ & $-5.5169 E+04$ & $1.0625 E+01$ \\
\hline 22 & $3.6402 \mathrm{E}-0.1$ & $-1.0025 E+06$ & $9.4728 E+05$ & $.00000+00$ & $-5.5195 E+\hat{v} 4$ & $4.6250 \mathrm{E} E+\hat{U} \hat{O}$ \\
\hline 23 & $3.6402 E-01$ & $-1.0025 E+06$ & $9.4728 E+05$ & $.0000 E+00$ & $-5.5199 E+04$ & 6.2500E-01 \\
\hline
\end{tabular}


(1) MEUTRAL ALIS LOCATION:

$\begin{array}{lr}\text { DEGREES } & \text { RADIANS } \\ 20.8571 & 3.6402 E-0 !\end{array}$

(2) TENSILE FORCES IH ANCHOR BOLTS:

$\begin{array}{ccc}\text { EOLT } & \text { REF. ANGLE IDEEREES } & \text { FORCE } \\ 1 & 180.000 \hat{0} & 1.3800 \mathrm{fE}+05 \\ 2 & 135.0000 & 1.3800 \mathrm{E}+05 \\ 3 & 90.0000 & 1.3800 \mathrm{E}+05 \\ 4 & 45.0000 & 1.2864 E+05\end{array}$

(3) DIRECT FUGCES AT TANIE BASE:

$$
\begin{aligned}
& \text { LONGITUDINAL FORCE IN SHELL }=-1.0025 E+006 \\
& \text { SUS GF ANCHOR BOLT FORCES }=9.4728 E+05 \\
& \text { EOTTOH PLATE HOLDOHN FORCE }=.0000 \mathrm{E}+00 \\
& \text { TOTAL }=-5.5199 \mathrm{E}+04
\end{aligned}
$$

(4) RESTOFINE HOMENT:

FROS LONGITUDINAL FORCES IN SHELL $=2.3743 E+08$ FROH ANCHOR BOLTS TENSILE FORCES $=3.6297 \mathrm{E}+07$ FROH BOTTOH PLATE HOLDOWN FORCE $=.0000 E+00$ TOTAL $=2.7373 E+0 B$ 


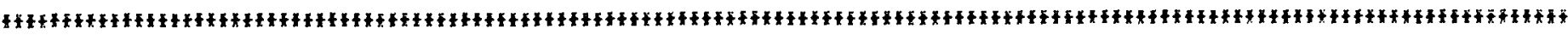
1)+QNOKMAL.STY10-01-B7 08:35: j4

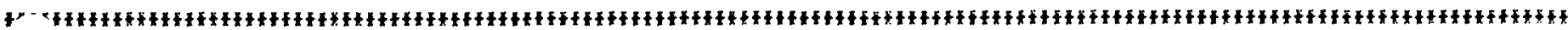

Lower Bound A.B. Yield Stress

TAHK DATA

$\begin{array}{ll}\text { RADIUS } & =2.4000 \mathrm{E}+02 \\ \text { SHELL THICKNESS } & =3.7500 \mathrm{E}-01 \\ \text { BOTTOM PLATE THICKNESS } & =2.5000 \mathrm{E}-01 \\ \text { SHELL YDUNG'S MODULUS } & =2.80000 \mathrm{E}+07\end{array}$

ANCHOFAGE DATA

\begin{tabular}{|c|c|}
\hline NUMBER OF ANCHOR BOLTS & $=$ \\
\hline ANCHOR BULT DIAHETER & $=2.0000 \mathrm{E}+00$ \\
\hline EXFOSED LENGTH OF BOLT & +01 \\
\hline TOTAL LENGTH DF EOLT & $=5.4500 E+01$ \\
\hline EOLT YOUNS'S MODULUS & \\
\hline
\end{tabular}

FEECALEULATED DATA

EFFECTIVE FLUID HEIOHT = $1.200 \hat{O}+0$ I TANK SHELL CRITICAL STRESS = $2.3000 \mathrm{OE}+04$ LIMIT ON EOLT CAPACITY $=1.1300 E+05$ NET VEFTICAL BASE REACTION= $-5.5200 E+0.04$

I TERATION PARAMETERS

MaxIMUM DF ITERATIORS $=100$

COHIVERENCE TOLERANCE $=1.00$ 


\begin{tabular}{|c|c|c|c|c|c|c|}
\hline$R$ & NEUTKAL AXIS & PS & $P B$ & $\mathrm{PL}$ & PSUH & XNORK \\
\hline & & & -- & -- & & \\
\hline 1 & $1.570 B E+\hat{0} 0$ & $-4.1400 E \div 06$ & $8.9508 E+04$ & $4.3233 E+04$ & $-4.0073 E+06$ & $-3.9521 E+06$ \\
\hline 2 & $1.0472 E+00$ & $-2.6353 E+O b$ & $3.6439 E+05$ & $7.4580 E+04$ & $-2.3963 E+06$ & $-2.3411 E+06$ \\
\hline 3 & $7.2273 E-01$ & $-1.9771 \mathrm{E}+06$ & $5.7417 E+05$ & $1.0471 E+05$ & $-1.2982 E+06$ & $-1.2430 E+06$ \\
\hline 4 & 5. $0536 \mathrm{E}-01$ & $-1.3888 E+06$ & $6,6460 E+05$ & $1.3596 E+05$ & $-5.8825 E+05$ & $-5.3305 E+05$ \\
\hline 5 & $3.5542 \mathrm{E}-01$ & $-9.7889 E+0 \hat{5}$ & $7.9100 \mathrm{E}+05$ & $1.6992 E+05$ & $-1.7964 E+04$ & $3.7236 E+04$ \\
\hline 6 & $4.3647 E-\hat{0} 1$ & $-1.20008 E+06$ & $7.2251 E+115$ & $1.4964 E+05$ & $-3.2865 E+185$ & $-2.7345 E+05$ \\
\hline 7 & $3.9790 \mathrm{E}-01$ & $-1.0053 \mathrm{E}+06$ & $7.6884 E+05$ & $1.5 \mathrm{~B} 60 \mathrm{E}+05$ & $-1.67 E 5 E+05$ & $-1.1265 E+$ \\
\hline 8 & $3.7723 \mathrm{E}-0 !$ & $-1.0387 E+06$ & $7.9100 \mathrm{E}+05$ & $1.6390 \mathrm{E}+05$ & $-8.3782 E+04$ & $-2.8582 E+$ \\
\hline 9 & $3.6648 \mathrm{E}-01$ & $-1.0092 E+06$ & $7.9100 E+05$ & 1. $6681 E+055$ & $-5.1406 E+04$ & $3.7940 E+03$ \\
\hline 10 & 3.7189E-0! & $-1.0241 E+06$ & $7.9100 \mathrm{E}+05$ & $1.6533 E+05$ & $-6.7721 E+04$ & $-1.2521 E+0.0$ \\
\hline in & $3.6920 \mathrm{E}-01$ & $-1.0167 \mathrm{E}+06$ & $7.9100 \mathrm{E}+05$ & $1.6606 E+05$ & $17 E+04$ & $-4.3969 E+03$ \\
\hline 12 & $3.6784 E-101$ & $-1.0129 E+06$ & $7.9100 E+05$ & $1.6643 E+05$ & $-5.5509 E+04$ & $-3.09927 E+$ \\
\hline 13 & $3.6716 \mathrm{E}-01$ & $-1.0111 E+06$ & $7.9100 \mathrm{E}+05$ & $1.6662 E+05$ & $-5.3461 E+04$ & $1.7392 E+03$ \\
\hline 14 & 3.675E-01 & $-1.0120 E+06$ & $7.9100 E+05$ & $1.6653 E+05$ & $-5.4486 E+04$ & $7.1423 E+02$ \\
\hline 15 & $3.6767 E-01$ & $-1.0125 E+0 t$ & $7.9100 E+05$ & $1.6648 E+05$ & $-5.4998 E+04$ & $2.0242 \mathrm{E}+02$ \\
\hline 16 & $3.677 \Delta E-01$ & $-1.0127 E+06$ & $7.9100 \mathrm{E}+05$ & $1.6646 E+i j 5$ & $-5.5254 E+04$ & $-5.4391 E+01$ \\
\hline 17 & $3.6771 \mathrm{E}-0 !$ & $-1.012 t E+j j 6$ & $7.9100 \mathrm{E}+005$ & $1.6647 E+05$ & $-5.5126 E+\hat{0} 4$ & $7.4094 E+01$ \\
\hline 19 & $3.6774 E-i j 1$ & $-1.0127 E+06$ & $7.9000 \mathrm{E}+0 \mathrm{5}$ & $1.6646 E+055$ & $-5.5189 E+04$ & $1.0875 E+01$ \\
\hline 19 & $3.6775 E-01$ & $-1.6127 E+06$ & $7.9100 \mathrm{E}+\hat{0} 5$ & $1.6645 E+015$ & $-5.5221 E+04$ & $-2.0891 E+01$ \\
\hline 20 & $3.6774 E-01$ & $-1.0127 E+00$ & $7.9100 \mathrm{E}+\hat{0} 5$ & $1.6646 E+05$ & $-5.5205 E+104$ & $-4.9375 E+00$ \\
\hline 21 & $3.6774 E-61$ & $-1.0127 E+106$ & $7.9100 E+05$ & $1.6646 E+05$ & $-5.5197 E+04$ & $2.9375 E+00$ \\
\hline 22 & $3.6774 E-01$ & $-1.0127 E+06$ & $7.9 ! 00 E+05$ & $1.664 t E+0 \hat{U} 5$ & $-5.5201 E+04$ & $-1.0469 E+00$ \\
\hline & & $-1.0127 E+06$ & $7.9100 \mathrm{E}+05$ & $1.6646 E+05$ & $-5.5200 E+04$ & $7.8125 E-1$ \\
\hline
\end{tabular}




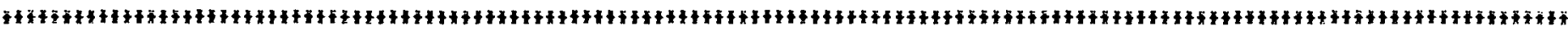
1)+QNOEFAL. STY10-0!-87 08:35:34

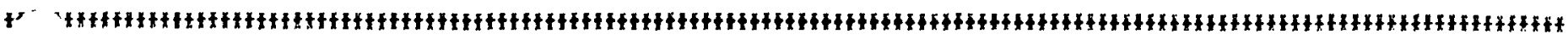

RESULTS OF ANALYSIS

(1) MEUTRAL AXIS LOCAIION:

$\begin{array}{lr}\text { DEGREES } & \text { RADIANS } \\ 21.0699 & 3.6774 E-01\end{array}$

(2) TENSILE FORCES IN ANCHOR BOLTS:

\begin{tabular}{ccc}
\hline BOLT & REF. ANGLE IDEEREES! & FOREE \\
1 & 150.0000 & $1.1300 E+05$ \\
2 & 135.0000 & $1.1300 E+05$ \\
3 & 90.0000 & $1.1300 E+05$ \\
4 & 45.0000 & $1.1300 E+05$
\end{tabular}

(3) DIRECT FORCES AT TANK ERSE:
LONGITUOINAL FCRCE IN SHELL
SUM OF ANCHOF BOLT FORCES
$=-1.0127 \mathrm{E}+0.6$
BOTTOUY FLATE HOLDOWN FOREE
$=7.9100 E+05$
$=1.6646 E+05$
TOTAL $=-5.5200 \mathrm{E}+\hat{0} 4$

(4) RESTORING HOHENT:

FROM LONGITUDINAL FORCES IN SHELL $=2.3970 E+08$

FROH ANCHOR BOLTS TENSILE FORCES $=2.7120 E+07$

FROM BOTTOM PLATE HOLOOHN FORCE $=1.0994 E+07$

TOTAL $=2.7789 E+08$ 


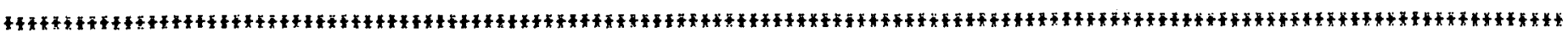
1) +QNOFMAL. STY10-01-87 08:35:34

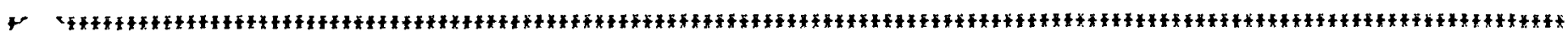

ADOITIONAL RESULTS:

$0.4 / 4$

A

MAXIMUN LEMGTH OF UPLIFTED BOTTOH PLATE $=1.9353 E+01$

RAXIKUA UPLIFT DISPLACEMENT $\quad=6.412 \mathrm{EE}-01$

MAXIMUH FIBRE STRESS IN BOTTOK PLATE $=7.1912 E+04$ 
TANK DATA

$\begin{array}{ll}\text { RADIUS } & =2.4000 E+02 \\ \text { SHELL THICKMESS } & =3.7500 E-01 \\ \text { BOTTOM PLATE THICKNESS } & =2.5000 \mathrm{E}-01 \\ \text { SHELL YOUNG'S MODULUS } & =2.8000 \mathrm{E}+07\end{array}$

ANCHORAGE DATA

$\begin{array}{ll}\text { WUMBER OF ANCHOF BOLTS } & = \\ \text { ANCHQR BOLT DIAHETER } & =2.00000+00 \\ \text { EXPOSED LENGTH OF BOLT } & =2.70000+01 \\ \text { TOTALL LENGTH OF BOLT } & =5.4500 E+01 \\ \text { BOLT YOUNG'S MODULUS } & =2.9000 \mathrm{E}+07\end{array}$

PRECALCULATED DATA

EFFECTIVE FLUID WEIGHT $=1.3300 E+01 \varnothing$ TANK SHELL CRITICAL STRESS $=1.6500 \mathrm{E}+04$ LIMIT ON EOLT CAFACITY = $1.0700 E+05$ NET VEFTICAL BASE REACTION= $=5.8600 E+04$

ITERATION PARAMETERS

MAXIKUM * OF ITERATIONS = 100

CONVERGENCE TOLERAMCE $=1.00 \mathrm{j}$ 


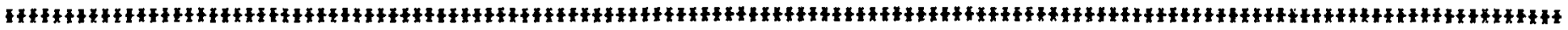
1 $>+4$ NORKAL. STY10-06-87 10:45:49

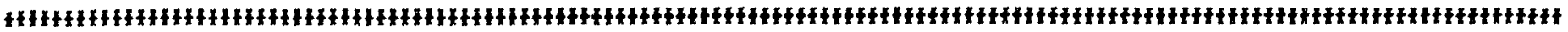

IHTERHEDIATE RESULTS

\begin{tabular}{|c|c|c|c|c|c|c|}
\hline ITER & MEUTRAL AXIS & PS & $P B$ & PL & PSUK & XNORY \\
\hline & - & & - & & & \\
\hline 1 & $1.5708 E+00$ & $-2.9700 \mathrm{E}+0 \mathrm{~b}$ & $6.4212 E+04$ & $4.2979 E+09$ & $-2.8628 E+06$ & $-2.8042 \mathrm{E}+06$ \\
\hline 2 & $1.0472 E+00$ & $-2.0340 E+06$ & $2.6141 E+65$ & $7.4141 E+04$ & $-1.6985 E+06$ & $-1.6399 E+06$ \\
\hline 3 & $7.2273 E-01$ & $-1.4183 E+06$ & $4.89711 E+05$ & $1.0409 E+05$ & $-8.2452 E+05$ & $-7.6592 E+05$ \\
\hline 4 & $5.0536 \mathrm{E}-01$ & $-9.9632 E+05$ & $6.0645 E+05$ & $1.3516 E+05$ & $-2.5471 E+05$ & $-1.9611 E+05$ \\
\hline 5 & $3.5542 E-01$ & $-7.0225 E+05$ & $7.3109 E+05$ & $1.6893 E+05$ & $1.9777 E+05$ & $2.56 .77 E+05$ \\
\hline 6 & 4. $3647 E-0 !$ & $-8.6145 E+0)^{5}$ & $6.4800 \mathrm{E}+05$ & $1.4876 E+05$ & $-6.4687 E+04$ & $-6.0865 E+03$ \\
\hline 7 & $3.9790 E-01$ & $-7.8576 E+05$ & $6.8123 E+05$ & 1.576.7E+05 & $5.3149 E+04$ & $1.1175 E+05$ \\
\hline 8 & $4.1761 E-01$ & $-8.2444 E+0.5$ & $6.6310 E+05$ & $1.5299 E+05$ & $-8.3509 E+03$ & $5.0249 E+04$ \\
\hline 9 & $4.2714 E-\hat{v} 1$ & $-8.4314 E+05$ & $6.5522 E+05$ & $1.5002 E+05$ & $-3.7090 E+04$ & $2.1504 E+034$ \\
\hline 10 & 4.3183E-01 & $-8.5234 E+0.5$ & $6.5153 E+05$ & $1.4978 E+05$ & $-5.1028 E+04$ & $7.5723 E+03$ \\
\hline 11 & $4.3415 E-01$ & $-8.5690 \mathrm{VE}+\mathrm{U} 5$ & $6.4975 E \div 05$ & $1.4927 E+05$ & $-5.78900 E+04$ & $7.0953 E+002$ \\
\hline 12 & 4. $3531 E-01$ & $-8.5918 E+05$ & $6.4887 E+\hat{0} 5$ & $1.4901 E+05$ & $-6.1296 E+04$ & $-2.6763 E+03$ \\
\hline 13 & $4.3473 E-01$ & $-8.5804 E+05$ & $6.4931 E+05$ & $1.4914 E+05$ & $-5.9596 E+04$ & $-9.9564 E+02$ \\
\hline 14 & $4.3444 E-01$ & $-8.5747 E+05$ & $6.4953 E+05$ & $1.4920 E+05$ & $-5.8743 E+104$ & $-1.4338 E+02$ \\
\hline 15 & $4.3470 E-01$ & $-6.5719 E+0.5$ & $6.4964 E+05$ & $1.4924 E+05$ & $-5.8317 E+04$ & $2.9303 E+02$ \\
\hline 16 & $4.3437 E-011$ & $-8.5733 E+05$ & $6.4958 E+05$ & $1.4922 E+05$ & $-5.8530 E+04$ & $6.9828 E+01$ \\
\hline 17 & $4.3441 E-01$ & $-8.5740 \mathrm{E}+05$ & $6.4955 E+05$ & $1.4921 \mathrm{E}+0 \mathrm{E}$ & $-5.8637 E+04$ & $-3.6688 E+01$ \\
\hline 18 & $4.34395-01$ & $-8.5737 E+105$ & $6.4957 E+05$ & $1.4922 \mathrm{E}+0.5$ & $-5.8584 E+i j 4$ & $1.6109 E+01$ \\
\hline 19 & $4.3440 E-01$ & $-8.5738 E+05$ & $6.4956 E+05$ & $1.4921 E+05$ & $-5.8610 E+04$ & $-9.8906 E+60$ \\
\hline 20 & $4.3439 E-01$ & $-8.5738 E+05$ & $6.495 i E+i j 5$ & $1.4922 E+05$ & $-5.8597 E+04$ & $2.5781 E+000$ \\
\hline 21 & $4.344 \hat{v} E-\hat{v} 1$ & $-8.573 \mathrm{BE}+055$ & $6.4956 E+05$ & $1.4922 E+05$ & $-5.8603 E+04$ & $-3.1094 E+00$ \\
\hline 22 & $4.3439 E-011$ & $-8.5738 E+05$ & $6.4956 E+05$ & $1.4922 E+05$ & $-5.86000+04$ & $-2.9688 E-01$ \\
\hline
\end{tabular}

$2 / 4$ 
(1) NEUTRAL AXIS LOCATION:

$\begin{array}{ll}\text { DEGREES } & \text { RADIAHS } \\ \text { 24.8890 } & 4.34375-01\end{array}$

(2) TENSILE FORCES IN ANCHOR BOLTS:

\begin{tabular}{ccc}
\hline EOLT & REF. ANGLE (ŨEGFEES) & FORCE \\
1 & 180.0000 & $1.0700 E+05$ \\
2 & 135.0000 & $1.0700 E+05$ \\
3 & 90.0000 & $1.0700 E+05$ \\
4 & 45.0000 & $5.7281 E+04$
\end{tabular}

(3) DIRECT FORCEE AT TANK. BASE:

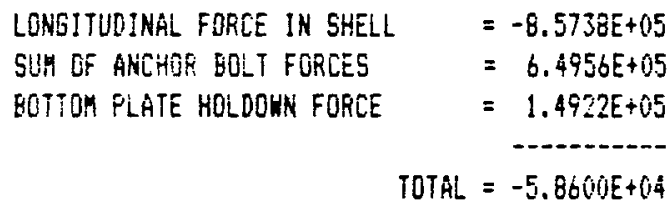

(4) RESTORING MOMENT:

FROM LONGITUDINAL FORCES IN SHELL $=2.019 .3 E+00$

FROM ANCHOR BOLTS TENSILE FORCES $=4.2555 E+07$

FROM BOTTOM PLATE HOLDOWN FORCE $=1.0541 E+07$

TOTAL $=2.5502 E+O B$ 
ADDITIONAL RESULTS:

MAXIMUH LENGTH OF UPLIFTED BOTTOM PLATE $=1.5935 E+01$

MAXIMUM UPLIFT DISFLACEMENT $\quad=3.2672 \mathrm{E}-01$

MAXIMUM FIGRE STRESS IN BOTTOM PLATE $=5.4038 E+04$ 


\section{COMPONENT 2}

\section{MOTOR CONTROL CENTER}


EDE

ENGINEERING. PLANNING AND MANAGEMENT CONSULTANTS

SHEET NO. 1

JOBNO\&Z-218. “' JOB HCLPF STUNYY BY ROC DATE $10-187$

CLINT ENL SUBSET MOTOR CONTROL CENTER

CHKD MK DATE $10 / 4 / 87$

DEVELOP FRAGILITY OF MOTOR CONTROL CENTER,

AVAILABLE INFORmation AND GROUND RULES ARE:

1) CABINET HAS $\mathrm{fn}_{n}=6.5 \mathrm{~Hz}$

2) No qualification test, thus USE ANCO GERS DR OTHER GENERIC SOURCE

3) CABINET IS MOUNTED IN STRUCTURE FOR WHICH UMFIIAN FLOOR SPECTRA ARE GIVEN FOR A $0.18 \mathrm{G}$ PEAK GROUND ACCEL. ALSO DO CASE FOR GROUND MOUNTED

4) $E Q$ is DFFINED AS $84 \%$ NED EARTHQUAKE

5) InVESTIGATE AUXILIARY cONTACTOR CHATTFR FRAGILITY "function During E.Q." ALSO DEUECOP FRaGiLITY For "Function AFter eQ"

References:

1. Generic Seismic Rugged ness of Rower Plant Equipment EPRI NP -5223, May 1987, Prepared by Arcs

2. Evaluation of Nuclear Power Plant Seismic Margin, Draft EPRI Report, Pro pared ky NTS Enqueerive, RPK consulting. Pickle Lon f6orrick, woodward Clyde Associates and Duke Power. Co. Aug, 1987

B- 44 
QR

NGINEERING. PLANNING AND MANAGEMENT CONSULTANTS

SHEET NO 2

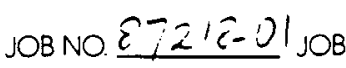

HCLPF SUDsY

BY ROC DATE $10-1-57$

CLIENT LLIVL SUBJECT

MC C

CHKD $M N_{R}^{2}$ DATE $101+\varepsilon 7$

Do two cases for mac's,

1) Function during earthquake assuming unenergized auxiliary contractors and relays which are in interlock circuits.

2) Only function after earthquake is important (ie), circuit diagram review indicates no can sequence of relay chatter.

The 6ERS report has TRS; for 13 tests, in 5 of which some form of functional failure occurred and 8 of which no anomalies were observed. The "functional during the $E Q$ " GERs are a lower bound of the failures white the "functional af ter" are an upper bound of success but not above any failure case that was not functional a filer the sersinic test. we cannot derive any real statistical median

B-45 
BF

:NGINEERING. PLANNING AND MANAGEMENT CONSULTANTS

JOB NO. $82218-01 \mathrm{JOB}$ HCLPF Sta: SHEET NO 3

CLIENT $\stackrel{i}{-} N !$ SUBJECT $M \subset C$ BY ROC DATE $10 / 2 / 87$ CHKD MFR DATE $10 / 4 / 87$

fragility. from tho GERS as presented since we do not know the cabinet frequencies us the frequency content of the in put motion. wo must than makes same approximate estimates of th broad banked fragility curve.

One other source of elate from the ssm RP program lists a median relay chatter fragility at $2.07 \mathrm{~g}$ for a broad banded spectrum from 5-500 年. The median was derived from 17 shock tort inmecs, switchgear and control cabinets during the SAFEGUARD ProGRAM. large $\left(\beta_{c^{2}} 1.96\right)$ due to the fact that some very sensitive relays were included in the original testing. This obviously biased tho median.

From the GERS we can deduce that there are MCC's that would function during an $E Q$ with greater than $4 \mathrm{~g} S_{a}$ at the base, Likewise, since than is not very much

$B-46$ 
NGINEERING, PLANNING AND MANAGEMENT CONSULTANTS

JOB NO $872 / \varepsilon-0)_{\text {JOB }}$ HCLPE Sid

SheEt No 4

CLINT LLNL SUBJECT $M C C$ BY PC DATE $10 / 1 / 87$ CHKD M KR DATE 10/4/87

date, there may be failures to function be low $105 \mathrm{~g}$. Let us use the $\sin R \mathrm{P}$ median and estimate a $95 \%$ confidence value: using the lower bound GERS, the suggested 0.87 factor for interlock circuits, Rel .1, and the EPRE seismic margin reduction factor of 1.3, Ref. 2 . (ute that 552 AP suggests a reduction of 1.5 for A-46, however, this is a licensing issue for SSE) since SImP median is biased by low capacity relays, Increase sSmRP by $20 \%$ as best median estimate Median $=1.2(2.07)=2.18 \mathrm{~g}$

HCLPF on MCC alone is $\frac{1.5(0.87)}{1.3} \approx 1.0 \mathrm{~g}$

$$
\beta=\frac{1}{1,65} \ln \frac{2.48}{1.0}=0.55
$$

Tho upper 5\% confidence bind is then

$$
5 \% \text { Cont }=2.48 e^{1.65(0.55)}=6.15 \mathrm{~g}
$$

This appears reasonable with the information available,

B-47 
QI

NGINEERING. PLANNING AND MANAGEMENT CONSULTANTS

JOB NO. $\mathrm{JOB}$ HCLPF STUdy

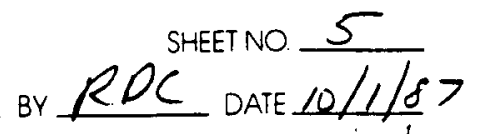

CLIENT SUBJECT MC CHE MK DATE $10 / 4$ i 7

Thus for function during the EO.

$$
\begin{aligned}
& S_{a}=2.48 \mathrm{~g} \\
& \beta_{u}=0.55
\end{aligned}
$$

this is contingent updo meeting tho 6 tEes ground rules that the individual relays and contractors have a $4.5 \mathrm{~g}$ minimum $Z P A$ input chatter capability.

Now, for function after the earthquake, the $400 \mathrm{~g}$ GERS is in the lower tail of the fragility curve. The only readily referencoabl source for "function after" would be the "trip" fragility fran the ssmRP program, This value was derived from some 16 shock torts of $M C C_{5}$, switchgear and control panels.

use $S_{a}^{v}=7.97 \mathrm{~g}$ from SSMRP

Estimate the HCCPF as GERS $/ 1,3=-\frac{4}{1.3}=3.08 \mathrm{~g}$

B-48 
GINGERING. PLANNING AND MANAGEMENT CONSULTANTS

OBNO $\mathrm{JOB}$ HC: PF Study SHEET NO 6

cLIENT

SUBJECT $\wedge 1 C C$ CHKD MR DATE $10 / 4 / 87$

$$
\beta=\frac{1}{1.65} \ln \frac{7.97}{3.08}=0.58
$$

This is lower than the B of 0.77 from sSMRP due to the fact that there man have

keen some inherently weak components in the SAFEGUAROS test data plus tho tests include t other types of cabinets as well as incl's.

Derive strength factors mounted In Structure

Ref. GIRS and instructure plots an pg s- $8+9$

For "Function during the EQ.", $S_{a}=1.65 \mathrm{~g}$ at $6.5 \mathrm{Hg}$,

$$
\begin{aligned}
& F_{S}=\frac{2.48}{1.65}=1.50 \\
& \beta_{u}=0.55
\end{aligned}
$$

For function after the $E Q$

$$
\begin{aligned}
& F_{s}=\frac{7.97}{1.65}=4.83 \\
& \beta_{L}=0.58
\end{aligned}
$$

B-49 
QE

:NGINEERING. PLANNING AND MANAGEMENT CONSULTANTS

SHEET NO 7

JOBNO.

$\mathrm{JOB}$

$$
\text { HCLPF } \sin \sigma y
$$

BY RDC DATE CL/1/87

CLIENT

SUBJECT

MCC

CHKD M KR Date $10 / 4 / \varepsilon 7$

Ground Mounted. - "Functim During Quake"

$5 \% \quad S_{a}=2.12(0.18)=0.38 \mathrm{~g}$

( 2.12 is $5 \%$ omplo

F.S. $=\frac{2.48}{0.38}=6.53$

from NURE 60098

Mefim soectral Shayes)

$\beta_{u}=0.55$

Ground Mounted-Function Aftor Gualer

$$
\begin{aligned}
& F_{S} S_{1}=\frac{7.97}{0.38}=20.97 \\
& \beta_{S}=0.58
\end{aligned}
$$

B-50 
GERS-MCC. 3

$12 / 1 / 86$

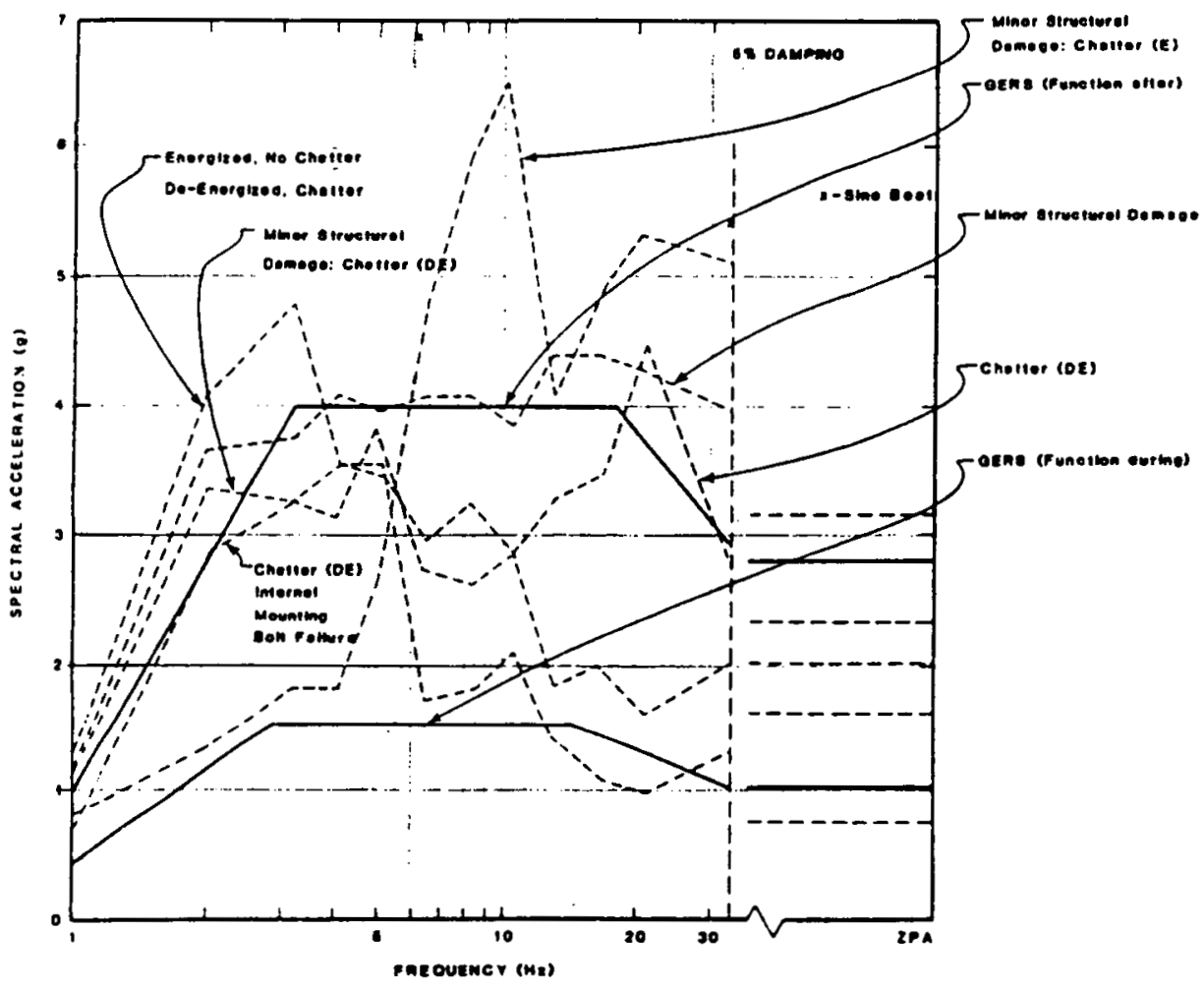

Figure 2. Comparison of GERS with failure data: function during and after for MCC. 


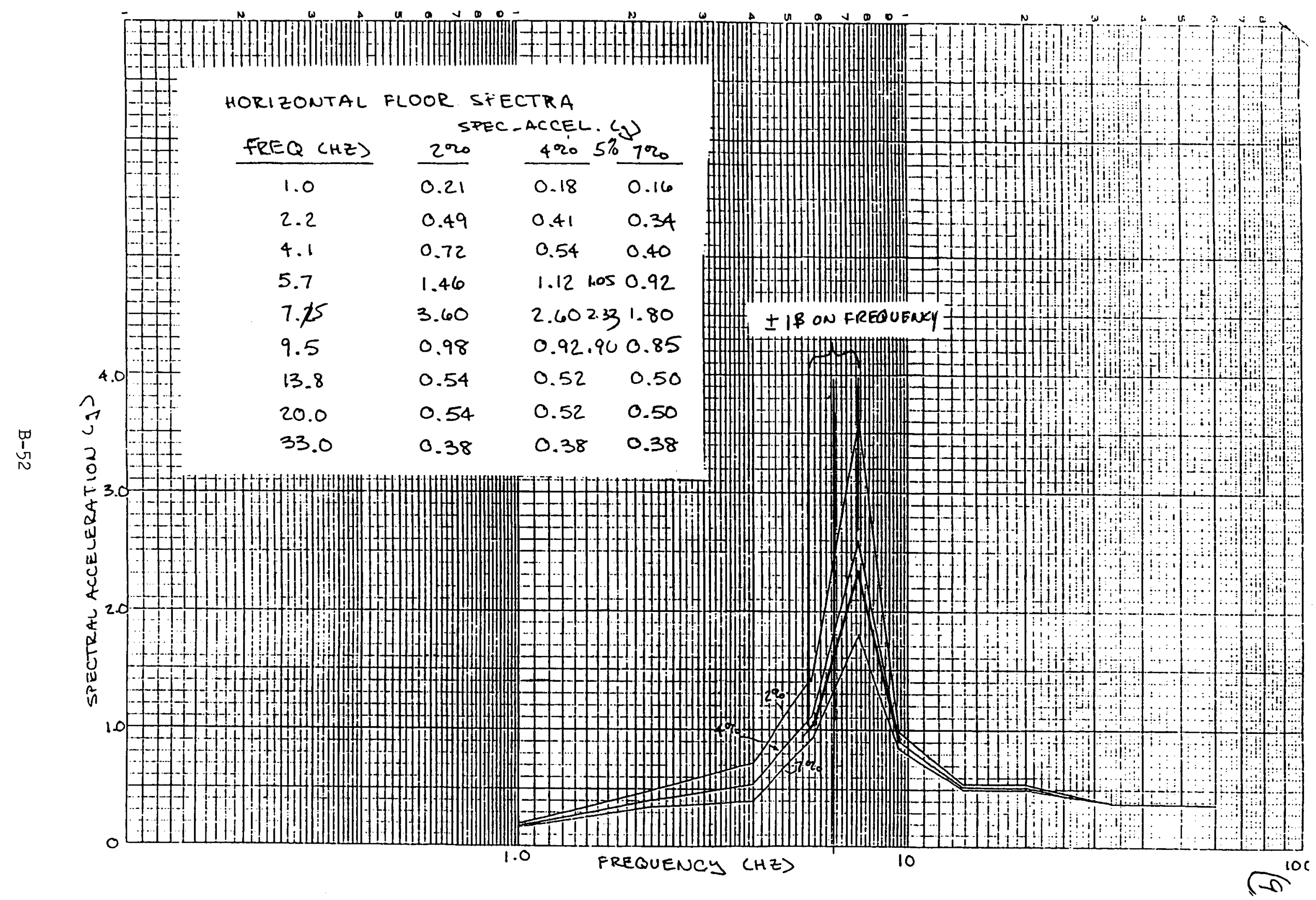


EDE

ENGINEERING. PLANNING AND MANAGEMENT CONSULTANTS

JOB NO

$J O B$

HCLPF Sinai

SHEET NO. $\%$

CLIENT

SUBJECT

$M C C$

DATE $10 / 1 / 87$

CHKD MIR DATE IS/4:87

Equipment Response factor

variables are:

method of analysis

Modeling

Dumping

Mode Combination

Earthquake Component Combination

inetherd of Analysis:

The frequency was given and assumed to be derived from analysis: or insitu modal testing. Since we are comparing a SDOF response to a broad burled spectiung to determine tho capacity factor, some error may result which will be addressed in other variables. There is no aeliborete bias in tho respond o calculation such as in a design situation whore 1.5 path $S_{4}$ is commonly

B-53 
ENGINEERING. PLANNING AND MANAGEMENT CONSULTANTS

JOB NO. JOB HCLPF Sim SHEET NO 11

CLIENT SUBJECT NCO BY $\frac{\mathbb{R} D C}{11 K R}$ DATE $\frac{10 / 1 / 87}{1.1 / 87}$

used, thus, this variable is $N A$,

Modeling

There are two sources of uncertainty here:

1) Frequency (stiffness) error

2) Inaccevary in predicting response

Frequency:

Assume the error in frequency prediction is represented by a cav ( $\sim B$ ) of 0.15

$$
\begin{aligned}
& \pm 1 \beta f_{n}=6.5 e^{ \pm 0.15}=\begin{array}{c}
7.55 \\
5.59
\end{array} \\
& S_{a_{f}=7.55}=2.33 \\
& \beta_{u}=\ln \frac{2.33}{1.65}=0.35
\end{aligned}
$$

Response Prediction:

A SDOF representation of response for corp orison to GERS is likely not biased but there are other modes which can contribute leather mono er loss)

This uncertainty is small compared to frequency error. Assume $R_{0}=0.1$

$$
B u_{M}=\left(0.35^{2}+0.1^{2}\right)^{1 / 2}=0.37
$$

B-54 
ED

ENGINEERING. PLANNING AND MANAGEMENT CONSULTANTS

SHEET NO -12

JOB NO $J O B$ HCLPF Study BY RDC DATE 10/2/27

CLIENT SUBJECT $M C C$

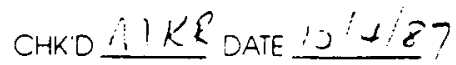

Damping:

$5 \%$ is indian

$$
F_{D}=1.0
$$

Assume $7 \%$ as $t / \not B$

$$
\begin{aligned}
& \beta_{D_{u}}=\ln \frac{S_{a_{5}}}{S_{a_{7}}}=\ln \frac{1.65}{1.35}=0.2 \\
& \beta_{R} \approx 0.2 B_{u}=0.04
\end{aligned}
$$

MULE COMBINATION:

Treated as souk system, This uncertainty is considered in mobletiry; therefore, MC is NA,

EARTHQUAKE COMPONENT COMBINATION:

The fragility is based on biaxial test input used in developing GERS and in the SAFEGUARDS program, thus,

$$
F_{E C c}=1.0
$$

Relays tend to be sensitise to unidirectional in pul any but, from summary of relay test data in EPRI Margin study, Appending, A, Refs,

$B-55$ 
EDE

ENGINEERING. PLANNING AND MANAGEMENT CONSULTANTS

SHEET NO 13

$J O B N O$ $J O B$ HCLPF Study BY ROC DATE /O/2/87

CLIENT SUBJECT $M C C$ CHKD MKR Date $10 / 4 / \varepsilon 7$

and GeRS study, Appendix A, Ref: 1, we could expect tho differences be tween broad bundled randan in put biaxial tests and single axis input be no more than about $1 \%, 7=1.93$.

Let 1.93 te $3 \beta_{R}$

$$
\beta_{R_{E C C}}=1 / 3 \ln 1.93=0.12
$$

EQUIPMENT Response:

$$
\begin{aligned}
F_{R E}= & F_{m} F_{D} F_{E C C}= \\
& (1)(1)(1)=1.0 \\
\beta_{R}= & \left(0^{2}+0.04^{2}+0.12^{2}\right)^{k}=0.13 \\
\beta_{u}= & \left(0.37^{2}+0.2^{2}+0\right)^{1 / 2}=0.42
\end{aligned}
$$

This apples to "in-structure" case.

For ground mounted case the modeling uncertainty duetutequency sheftuig is essentially zero since s we are an tho flat portion of tho spectrum.

$B-56$ 
NGINEERING. PLANNING AND MANAGEMENT CONSULTANTS

SHEET NO 14

JOBNO. $J O B$ HCLPE Sin n

CLIENT SUBJECT rice BY $R D C$ DATE $10 / 2 / 8>$

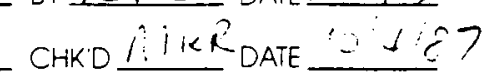

The damping uncertainty is:

$$
\begin{aligned}
& \beta U_{D}=\ln \frac{S_{a_{50}}}{S_{0_{71}}}=\ln \frac{2.12}{189}=0.11 \\
& \beta_{R_{D}}=0.2 B_{0}=0.02
\end{aligned}
$$

For Ground Case!

$$
\begin{aligned}
& F_{R E}=1.0 \\
& \beta_{R}=\left(0+0.2^{2}+0.12^{2}\right)^{1 / 2}=0.12 \\
& B_{U}=\left(0.1^{2}+0.11^{2}+0\right)^{1 / 2}=0.15
\end{aligned}
$$

B-57 
ERE

ENGINEERING. PLANNING AND MANAGEMENT CONSULTANTS

SHEET NO 15

JOB NO $\mathrm{JOB}$ HCLPF Sining DATED $0 / 2 / 87$

CLIENT SUBJECT NC BY $20 C$ CHKO -Date jilin?

structural response

Use PSH values as appropriate.

for In Structure Case:

spectral Shape:

$$
\begin{aligned}
& F_{\text {SS }}=1.22 \\
& \beta_{R}=0.20
\end{aligned}
$$

EQ Direction Content:

$$
\begin{aligned}
& F_{E Q D}=1.1 \\
& \beta_{R}=0.15
\end{aligned}
$$

Damping

$$
\begin{aligned}
& F_{D}=1.0 \\
& \beta_{U_{D}}=0.14 \\
& B_{R_{b}}=0.03
\end{aligned}
$$

modeling

$$
\begin{aligned}
F_{m} & =1.0 \\
\beta_{U} & =0.16 \\
F_{S S I} & =1.0 \\
\beta_{R} & =0.01 \\
B_{U} & =0.05
\end{aligned}
$$

$B-58$ 
ERE

ENGINEERING. PLANNING AND MANAGEMENT CONSULTANTS

JOB NO $\mathrm{JOB}$ HCLPF sind SHEET NO 16 CLIENT SUBJECT NC BY REC DATE $10 / 2 / 87$ CHKD MIR DATE $10 / 4 / 87$

Structure Inelastic Response.

for in-structure cos, structural inelastic: respire results in lower frequency which could increase mc response. bowers, for tho "function during EU" case, the relay chatter capacity is low and the structure is probably elastic, therefore, this is ignored.

For "function after the $E Q$ ", tho structure is likely to las in elastic, but, the mc is also probably gelding around the anchorage location so that both feywencios shift, feeding to negate the effect.

For both cases let

$$
\begin{gathered}
F_{S S R}=10 \\
\beta U_{S I R}=0.1 \\
F_{S R}=1.22(1.1)(1)(1)(1)(1)=1.34 \\
\beta R_{S R}=\left(0.2^{2}+0.15^{2}+0.03^{2}+0+0.01^{2}+0\right)^{1 / 2}=0.25 \\
\beta U_{S R}=\left(0+0+0.14^{2}+0.16^{2}+0.05^{2}+0.1^{2}\right)^{1 / 2}=0.24
\end{gathered}
$$

B-59 
ERE

ENGINEERING. PLANNING AND MANAGEMENT CONSULTANTS

JOB NO

CLIENT
$\mathrm{JOB}$

SUBJECT

$$
\text { LC! PE }
$$

SHEET NO 17 BY RPC Date 10/2/87 CHKD MR DATE $10 / 4 / 87$

For ground mounted case:

spectral Shape and EQ Direction Content are applicable,

$$
\begin{aligned}
& F_{5 R}=1.22(1.1)=1.34 \\
& F_{R}=\left(0.2^{2}+0.15^{2}\right)^{1 / 2}=0.25 \\
& \beta_{u}=0
\end{aligned}
$$

In Structure - "Function During"

$$
\begin{aligned}
& \not=(1.5)(1.0)(1.34)(0.18)=0.36 \mathrm{~g} \\
& B_{R}=\left(0+0.13^{2}+0.25^{2}\right)^{1 / 2}=0.28 \\
& B_{u}=\left(0.55^{2}+0.40^{2}+0.24^{2}=0.12=0.72\right. \\
& H C L P F=0.36 e^{-1.65(0.72+0.28)=}=0.07 \mathrm{~g}
\end{aligned}
$$

In Structure - Function After

$$
\begin{aligned}
& \hat{A}=(4.83)(1.0)(1.34)(0.18)=1.16 \mathrm{~g} \\
& \beta_{R}=\left(0+0.13^{2}+0.25^{2}\right)^{1 / 2}=0.28 \\
& B_{U}=\left(0.58^{2}+0.42^{2}+0.24^{2}\right)^{1 / 2}=0.76 \\
& H C L P F=0.21 \mathrm{~g}
\end{aligned}
$$

B-60 
EQ

ENGINEERING, PLANNING AND MANAGEMENT CONSULTANTS

SHEET NO 18

JOB NO $J O B$

HiLL PF STun BY ROC DATE $10 / 2 / 87$

CLIENT

SUBJECT

$M C C$

CHE MK E DATE $1014 / 8$

On Ground - "Function During EQ"

$$
\begin{aligned}
& A^{N}=(6.53)(1.0)(1.34)(0.18)=1.58 \mathrm{~g} \\
& \beta_{R}=\left(0+0.12^{2}+0.25^{2}\right)^{1 / 2}=0.28 \\
& \beta_{U}=\left(0.55^{2}+0.15^{2}+0\right)^{1 / 2}=0.57 \\
& H C L P F=1.58 e^{-1.65(.57+.28)}=0.39 \mathrm{~g}
\end{aligned}
$$

On Ground, "Function After"

$$
\begin{aligned}
& \tilde{A}=(20.97)(1.0)(1.34)(0.18)=5.06 \mathrm{~g} \\
& B_{R}=\left(0+0.12^{2}+0.25^{2}\right)^{1 / 2}=0.28 \\
& B U=\left(0.58^{2}+0.15^{2}+0\right)^{1 / 2}=0.60 \\
& H C L P F=e^{-1.65(0.28+0.60)}=1.18 \mathrm{~g}
\end{aligned}
$$

B-61 
IGINEERING. PLANNING AND MANAGEMENT CONSULTANTS

SHEET NO 19

OB NO $\mathrm{JOB}$ HCLPF St min BY $R D C$ DATE $10 / 2 / 87$

cLIENT SUBJECT a) $c$ CHKD DATE $: 0.4 / 8$ ?

EPRI DETERMINIST METHOD.

Eq + structural response are median.

compare dEMAND TO GERS/113

FOR ASSUMED INTERLOCK CIRCUITS, REDUCE Gers by 0.87 FACTOR,

IN STRUCTURE, "FUNCTION DURING"

$$
\text { * HCLPF }=\frac{0.87(1.5)(0.18)}{1.3(1.65)}=0.11 \mathrm{~g}
$$

IN STRUTURE - "FUnCTION AFTER"

$$
\text { * HeLP }=\frac{4.0(0.18)}{1.3(1165)}=0.34 \mathrm{~g}
$$

ON Ground - Function During

$$
H C C P F=\frac{0.87(1.5)(0.18)}{1.3(2.12)(0.18)}=0.97 \mathrm{~g} v 30.39 \mathrm{~g}
$$

ON Ground - Function After

$$
\text { HCCPF }=\frac{4.0(0.18)}{1.3(2.12)(0.18)}=1.95 \mathrm{~g} \text { vs } 1.19 \mathrm{~g}
$$

+ Sa at $6.5 \mathrm{Hg} \mathrm{fn}_{\mathrm{n}}$ used for HCLPF. Spectrum is an linear slope so average oven $20 \%$ bandwidth is $S_{0}$ atbistly worst case would be for - frequency shifted spectrum.

B-62 


\section{EQE}

ENGINEERING. PLANNING AND MANAGEMENT CONSULTANTS

SHEETNO 2

JOB NO

HCLPF Stwoy

$T a n k$

BY $20 C$ Date $9 / 29 / 87$ $\mathrm{JOB}$ SUBJECT Air Recrive CHKD MKR DATE $10 / 6 / \bar{t} 7$

CLIENT SUBJEC

VENDOR CACCS DID NOT DETERMINE NATURAL FREQUENCY. TANK AND SKIRT ARE RIGID BUT MUUNTING angLES ARE FLEXIBCE.

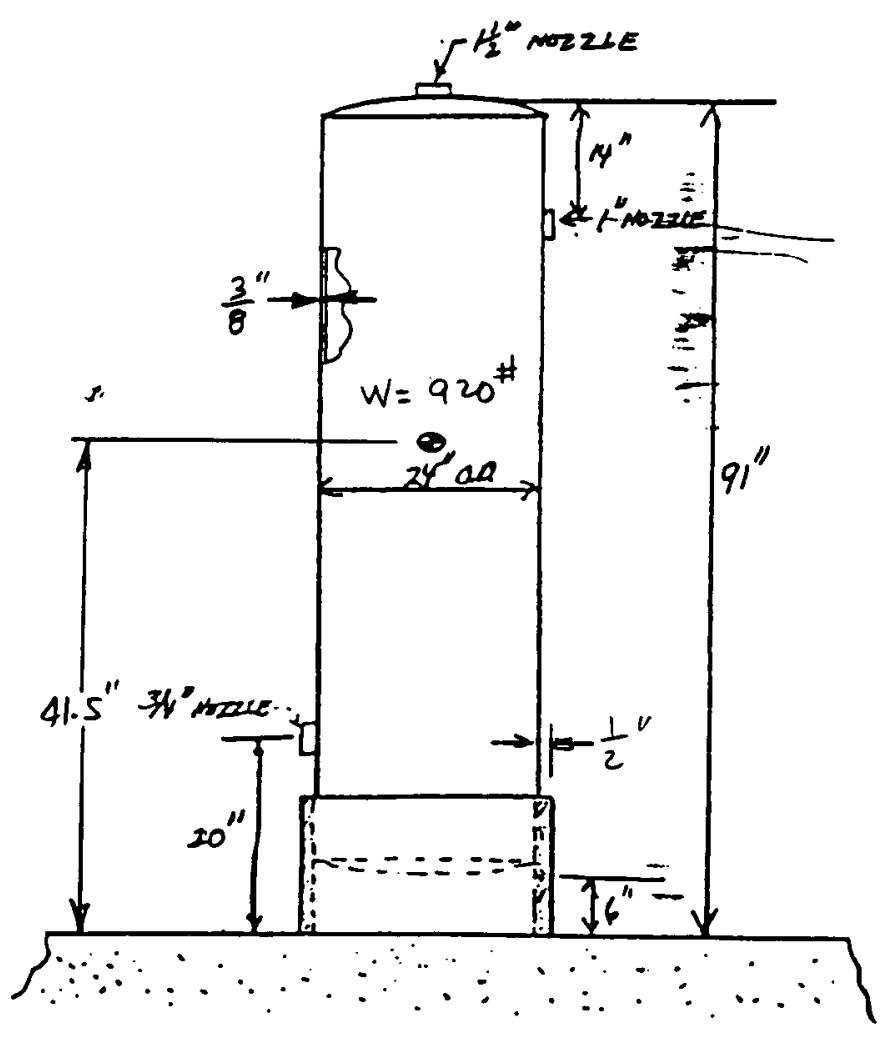


ENGINEERING. PLANNING AND MANAGEMENT CONSULTANTS

JOB NO $J O B$ HCLPE Sings SHEET NO $\frac{20}{20}$

CLIENT SUBJECT MACC BY ROC DATE $10 / 2 / 87$ CHKD MKS DATE $12 / 4 / 87$

Shit t RRS by $-15 \%$

perk is then at $6.37 \mathrm{Hg}$. Let peak bo at fo of component. The HCLPF would than be based upon maintaining a $5 F$ of 1.3 average oven a $20 \%$ band width a bout the peak.

For peak at $6.5 \mathrm{~kg}, \pm 10 \%=5.85-7.15 \mathrm{lg}$.
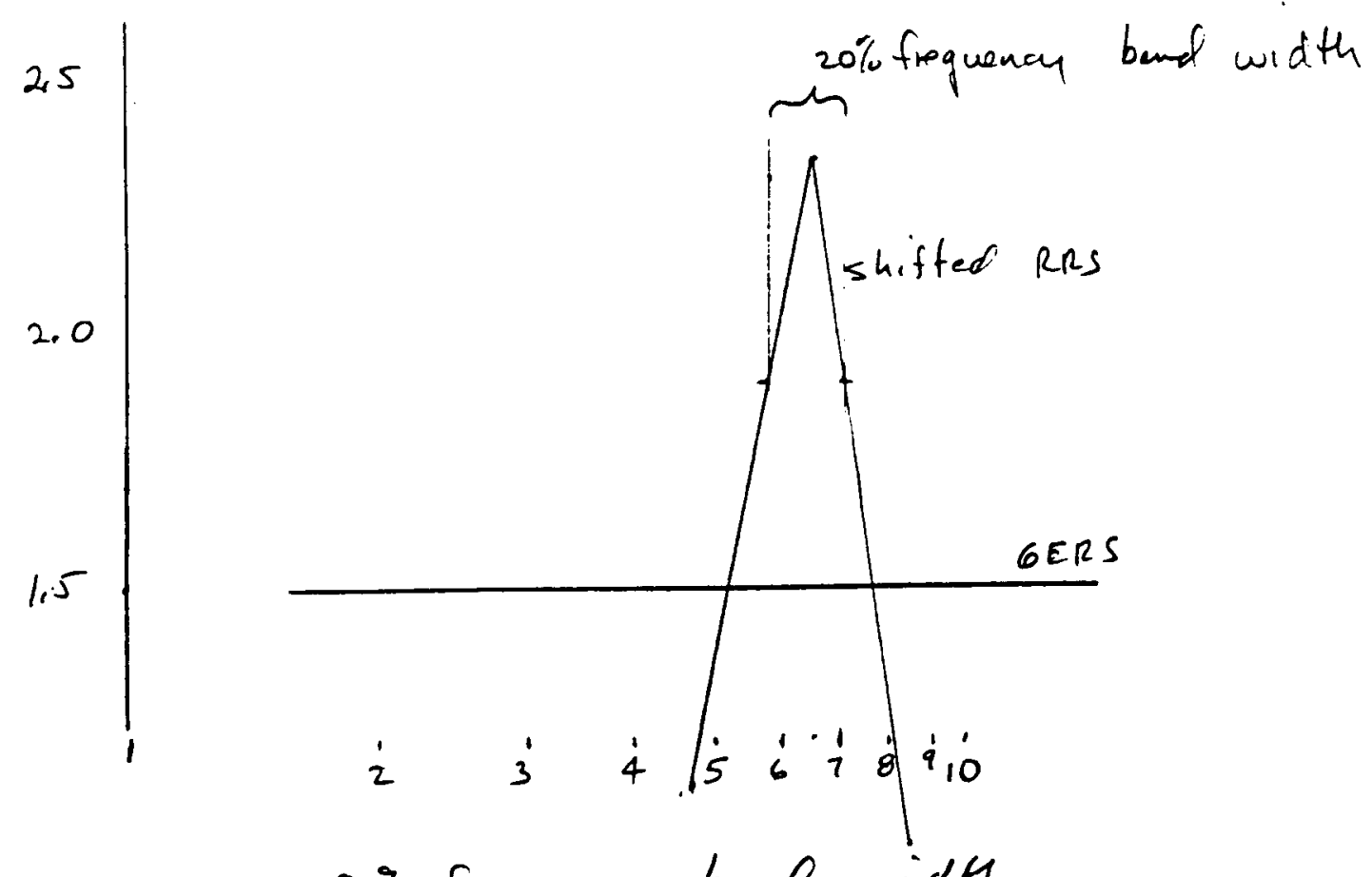

An $\mathrm{Sa}$ over $20 \%$ frequency band width

$$
\overline{s_{a}}=2.11 \mathrm{~g} \text {. }
$$

B-64 
EDE

ENGINEERING. PLANNING AND MANAGEMENT CONSULTANTS

JOB NO $J O B$ HELPF Simon

SHEET NO 21

CLIENT SUBJECT $M C C$ BY ROC DATE $10 / 2 / e 7$ CHKDMKE DATE $10 / 4 / 87$

In Structure - shitted frequency - 'Function During"

$$
H C L P F=\frac{0.87(1.5)(0.18)}{2.11(1.3)}=0.086 \mathrm{~g} \text { vo } 0.06 \mathrm{by}
$$

In Structure - shifted frequency - Function After"

$$
H C C P F=\frac{4.0(0.18)}{2.11(1.3)}=0.26 \mathrm{~g} \mathrm{vs}
$$

0.21 by fragility

Frequency shifting does not apply to ground mounted case as RRS is brodbanded flat spectrumiat $f_{n}$ of component, thus. shift ting results in same answer.

B-65 


\section{COMPONENT 3}

\section{STARTING AIR RECEIVER TANK}


EDE

ENGINEERING. PLANNING AND MANAGEMENT CONSULTANTS

SHEET NO $/$

Јов NO 87-218.0' JOB HCLPF STUDY BY ROC SHER NO $\frac{1}{C \text { DAR } 9 / 297}$ CLINT $\angle L N L$ SUBECT DG STARTING AIR RECEIVER CHKO MKR DATE 10;5/87?

DEVELOP FRAGILITY OF STARTING AIR REceiver tank,

REFERENCE:

GILBERT ASSOCIATES CALCULATICN, FILE /.362/d-1 $7 / 14 / 79$

Assumptions

1) TANK IS MOUNTED IN STRUCTURE

2) FLOUR SPECTRA ARE FROM BUILUING ANALYSIS USING 7\% DAMPING BUT ASSUME THAT THEY ARE MEDIAN CENTERED. $Z P A=0.18 \mathrm{~g}$

3) FARTHQUAKE IS DEFINTD AS $84 \%$ NED FOR THE LARGER OF TWO COMPONENTS OF $E Q$.

4) NUZzLE LUADS ARE NUT PRUUIDED, THEPEFURE IGNORE NOZZLE REACTIONS IN ANALYSIS

B-67 


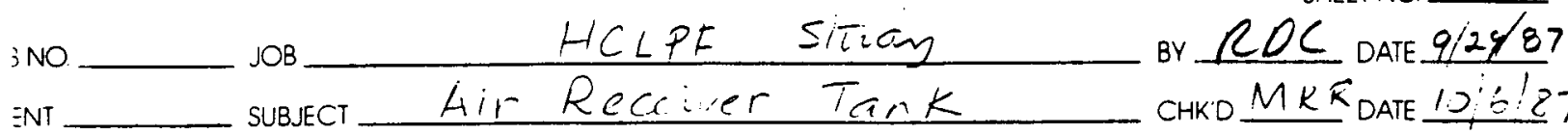
ENT CHKD MKK DATE 10:':?7

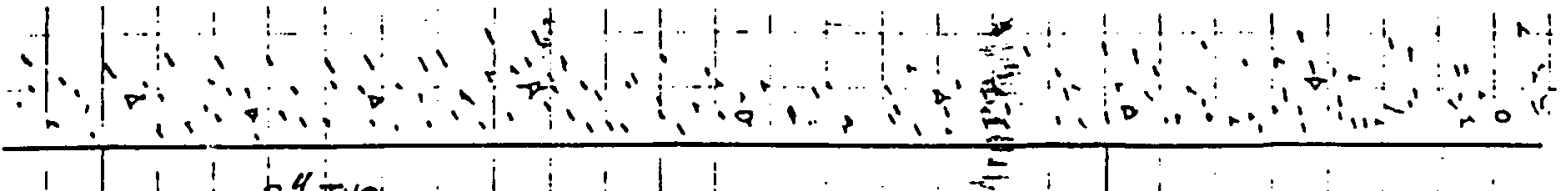

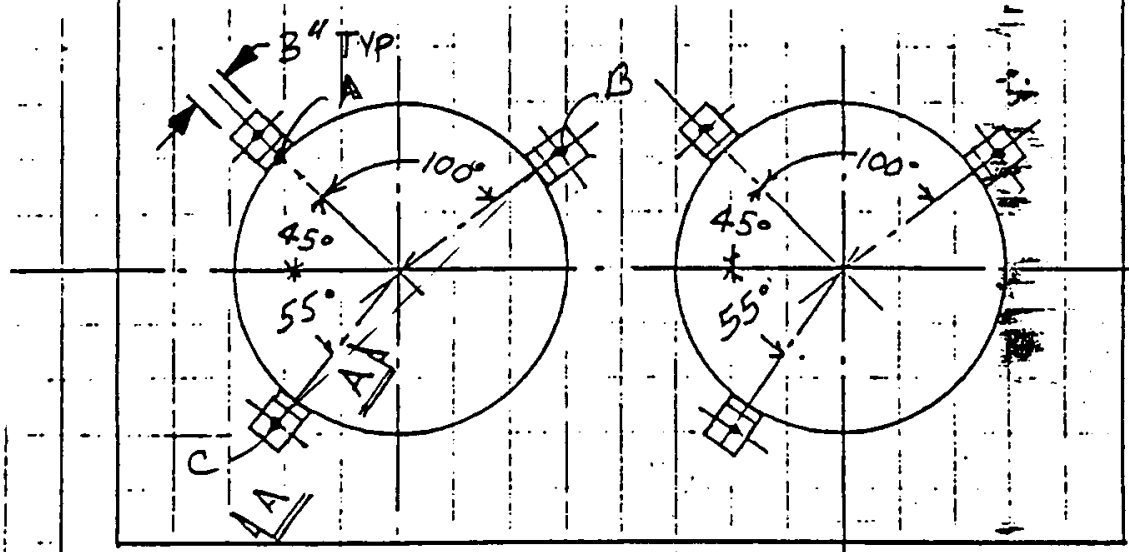

\section{1}

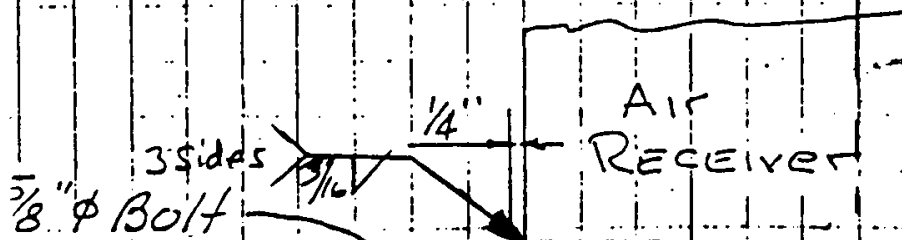
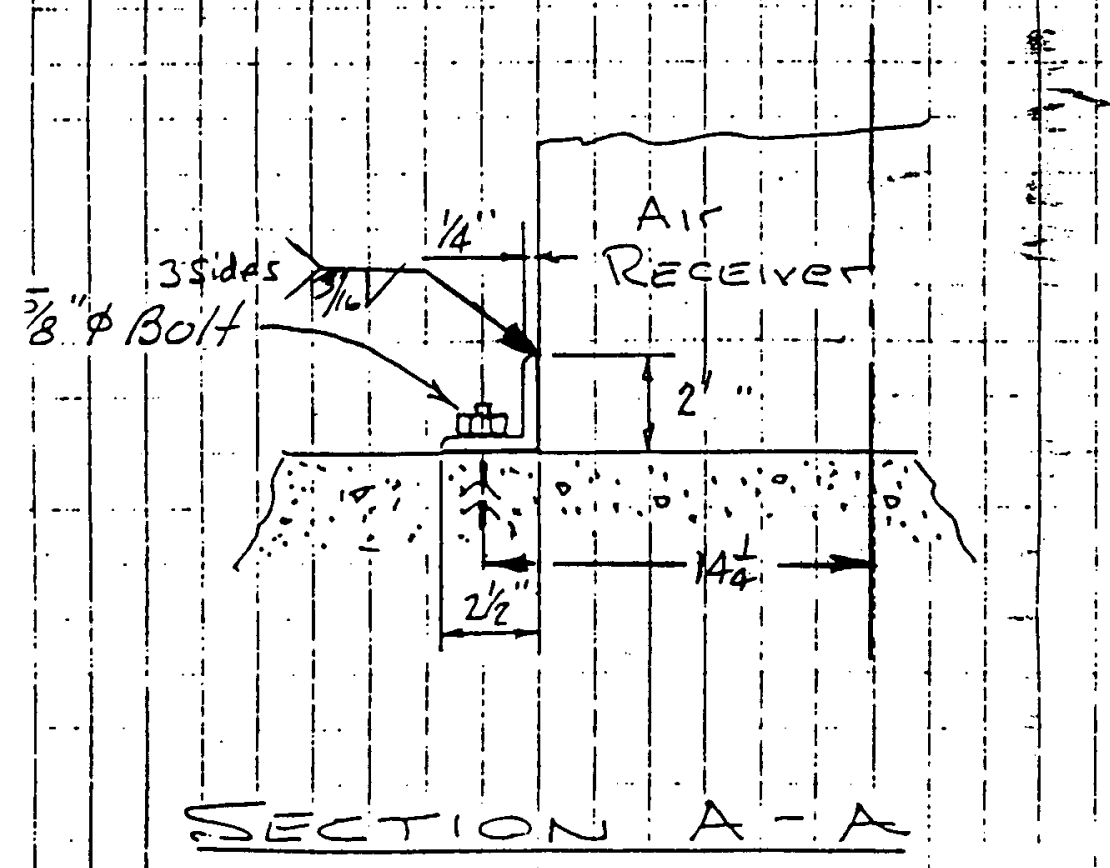
EDE

ENGINEERING. PLANNING AND MANAGEMENT CONSULTANTS

SHEET NO. $q$

JOB NO $\mathrm{JOB}$

HCLPF Sandy BY ROC DATE $\frac{4}{29 /(2)}$

CLIENT SUBJECT Air Receiver Tank

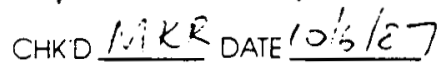

ESTIMATE NATURAL FREQUENCY

FOR MUST FLEXIBLE DIRECTION FOR ANGLES, TANK ROCKS ABOUT A RESULTING in TENSION ON BOLTS AT B

lg lateral at taIS CG produces

$$
\begin{aligned}
& M_{O T}=920^{*}\left(41.5^{\prime \prime}\right)=38,180, \mathrm{~N} \mathrm{H}^{\#} \\
& \text { ForCE } / \text { BLT }=\frac{M_{0 T}}{2(d)} \\
& d=12.5^{\prime \prime}+14.25 \sin 10^{\circ}=14.97^{\prime \prime} \\
& F_{b}=\frac{38,180}{2(10.97)}=1275^{\#}
\end{aligned}
$$

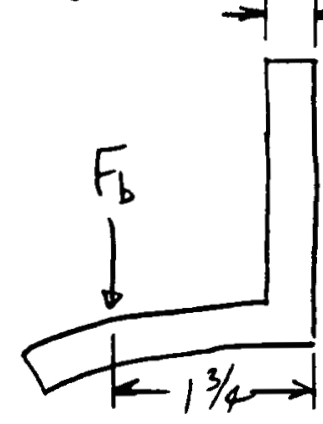

(Note: Bolt is not considered stiff enough in rotation to force guided cantilever bending shape. Likewise, prying action is not considered as this phoncmenen is negated a flor bolt stretch and rotation) Bracket Acts os cantilever Bon $\sim / \frac{1}{2}$ "long. $\delta=\frac{F b l^{3}}{3 E I}=\frac{1275(1.5)^{3}(12)}{3\left(29 \times 10^{60}\right)(3)(0.25)^{3}}=1.266 \times 10^{-2}$ in

B-69 
ENGINEERING, PLANNING AND MANAGEMENT CONSULTANTS SHEET NO 5

JOB NO $J O B$

HCDFF STAin BY ROC DATE $9 / 29 / 87$

CLIENT SUBJECT Air Tearier Ter CHKD NiKE DATE $0.6 / 87$

Deflection at $C G$ of Tank $=\frac{41.5}{19.97}(0.01266)=0.0357 \mathrm{in}$

$$
f_{n}=\frac{1}{2 \pi} \sqrt{\frac{g}{f_{s}}}=\frac{1}{2 \pi} \sqrt{\frac{3+6.4}{0.0351}}=1607 \mathrm{~Hz} \text {. }
$$

This is on a flat portion of the flow spectrum. so a more accurate calculation is not warranted. Use 16,7 os fin. for elastic system. If angle bracket yields, system is much softer,

Anglo bracket appears wank relater to bolt.

$5 / 8$ - 11 but has thread stress area of $0.2256 / \mathrm{m}^{2}$

yield of bolt occurs at $0.2256(3600)=8122$ Put effective load at edge of hole for case where angle bracket rotates plastically, to lo is $\sim 11 / 160,9$.

B-70 
ERE

ENGINEERING. PLANNING AND MANAGEMENT CONSULTANTS

SHEET NO. 6

JOB NO $J O B$ HCLPF Study BY PC DATE $9 / 30 / 8 ;$

CLIENT SUBJECT Air Receiver Tank CHE MIR DATE $1016 / 87$

Limit moment at full hinge is

$$
\begin{aligned}
& M_{L}=\frac{\sigma_{y} t^{2} l}{4} \\
& M_{L}=\frac{36,000(0.25)^{2}(3)}{4}=1687 \mathrm{in} \\
& F_{L}=\frac{1687}{1.156}=1960
\end{aligned}
$$

Much waler than bolt.

$$
\begin{aligned}
& \bar{\sigma}_{y}=1.25 \sigma_{y} \text { code } \\
& \overline{m_{L}}=1.25(1687)=2109 \mathrm{in} \#^{*} \\
& \overline{F_{L}}=1.25(1460)=1825 \#
\end{aligned}
$$

Anchor bolt detail is not given.

Assume shell anchor as worst case.

From NP 5228, mean pull out strength for $5 / 8$ expansion anchors with minimum embed mont is $9.51 k$. which is 5.2 times the load required to form a plastic hinge in the mounting bracket. Thus, the mounting bracket is clearly tho wack link.

$\mathrm{B}-71$ 
EDE

ENGINEERING. PLANNING AND MANAGEMENT CONSULTANTS

$J O B N O$ $J O B$ HCLPF Sting: SHEET NO 7

CLIENT

SUBJECT Lir derive- Tank BY ROC DATE $q / 30 / 87$ CHKD: $1=2$ DATE is $6 / 87$

check capability of bracket to develop hinge moment.

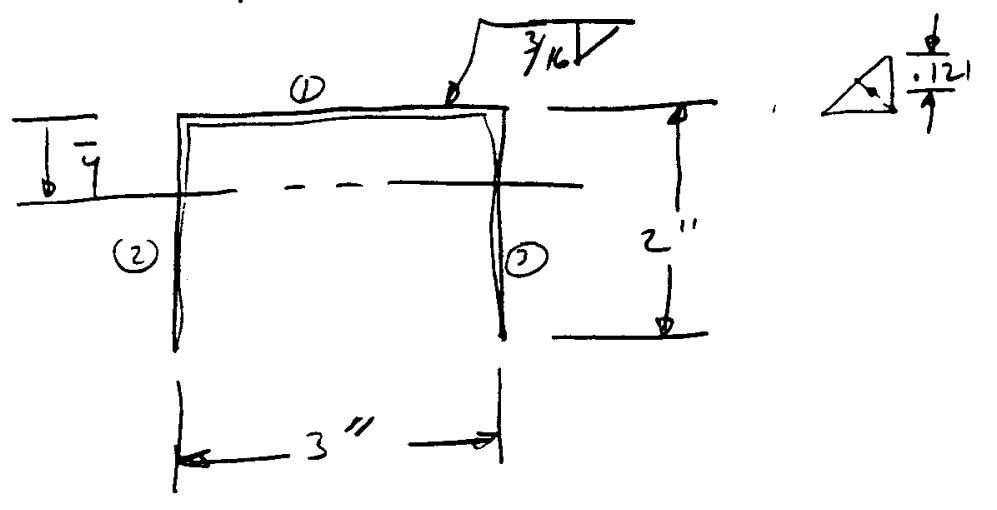

$$
\begin{aligned}
& \begin{array}{cccc}
10 & 4 & 4 & A 4 \\
1 & .398 & 0.121 & .0482 \\
2 & .263 & 1.0 & .263 \\
3 & .263 & 1.0 & .263 \\
& .924 & & .574
\end{array} \\
& \bar{y}=\frac{\sum A_{4}}{\sum A}=\frac{.574}{.924}=.621 \\
& \begin{array}{ccccc} 
& A & d & A_{d}^{2} & I_{44} \\
1 & .398 & .500 & .100 & - \\
2 & .263 & .379 & .038 & .088 \\
3 & .263 & .379 & \frac{.038}{.176} & \frac{.088}{.176}
\end{array} \\
& I=.176+.176=.352 \mathrm{in}^{4} \\
& \text { Af } \bar{M}_{2}=1687 \mathrm{in} \\
& f_{v}=\frac{1687(1.379)}{.352}=6609 \mathrm{psi}
\end{aligned}
$$

weld can carry limit moment

B-72 
ERE

ENGINEERING. PLANNING AND MANAGEMENT CONSULTANTS

JOB NO $\mathrm{JOB}$ HCLPF Sind,

CLIENT

SUBJECT Air Recover Tai< BY $2 D C^{\text {SHEET NO }} \frac{\mathrm{d}}{\mathrm{Late} / 32 / 87}$ CHR: $\because$ - DATE 06 '?

Failure mode is clearly plastic bounding of bracket, is, low cycle tatigio.

Assume 5 cycles

Adjust cols fatigue curse to remove factor of safety. In low cycle range, Fo. = 20 on cycles.

From fatigue curve with no F.S., Sa for 5 cycles is $\sim 4 \times 10^{6} \mathrm{ps} /$. Tho so was derived from strain canticlled fatigue tests. For a defined ductility limit, the angle bracket bending can be considered to bo Strain controlled.

$$
\varepsilon_{a}=\frac{4 \times 10^{6}}{29 \times 10^{6}}=.138=13.8 \% \text {. }
$$

There is plastic strain concentration so that the effective nominal strain is less. $k_{e} \approx 1 / n$ where $n=0.2$ for low alloy stael Ref: NB 3228.5 of section Arm Cate.

B-73 


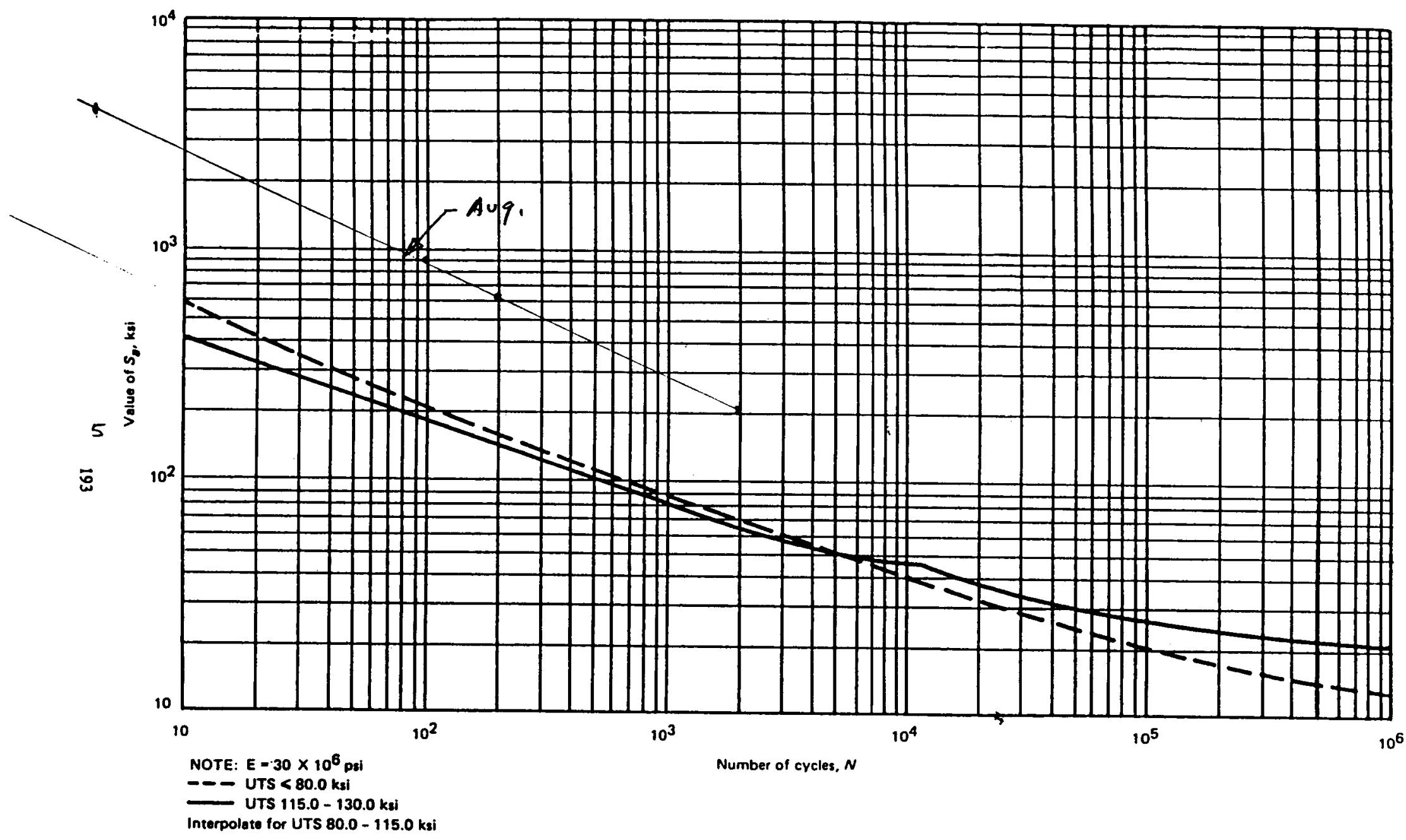

FTG. I-9.1 DESIGN FATIGUE CURVES FOR CARBON, LOW ALLOY, AND HIGH TENSILE STEELS FOR METAL TEMPERATURES NOT EXCEEDING 700॰

Table 1-9.1 Contains Tabulated Values and à Formula for Accurate 
EDE

ENGINEERING. PLANNING AND MANAGEMENT CONSULTANTS

JOB NO $J O B$

HCLPF Sind:

SHEET NO 9

CLIENT

SUBJECT

Air Recover Tank

BY ROC DATE $\mathscr{Q} / 30 / e 7$

CHKD MR DATE $10 ; 6 / \varepsilon 7$

$$
k_{e}=5
$$

nominal strain $=\frac{0.138}{5}=0.0276$

$0.2 \%$ offset yield strain is $\frac{1.2(36 \mathrm{~cm})}{29 \times 10^{6}}+0.02$

$$
\begin{gathered}
\varepsilon_{y}=3.49 \times 10^{-3} \\
\mu \sim \frac{0.0276}{3.99 \times 10^{-3}}=7.9 \quad \operatorname{say} 8
\end{gathered}
$$

Fy based on elastic frequency may be interpolated from $F_{\mu}$ in highly anglitied range and $F_{y}$ in reid range,

From Nowmarkf Fidel Pager.

$$
\begin{aligned}
F_{u}= & {[p \mu-q]^{r} } \\
p & =q+1 \\
q & =3.0 \gamma^{-0.3} \text { for amplified acceloution range } \\
r & =0.48 \gamma^{-0.08} \text { for a amplified acceleration range } \\
r & =\% \text { critical camping taken as } 5 \% \\
g & =3.0(5)^{-0.3}=1.851 \\
r & =0.48(5)^{-0.05}=0.422
\end{aligned}
$$

$B-75$ 
ERE

ENGINEERING. PLANNING AND MANAGEMENT CONSULTANTS

JOB NO $\mathrm{JOB}$ HCLPF Sind: PC DATE $\frac{10}{9 / 30187}$

CLIENT SUBJECT Air Receiver Tank CHKD UKR DATE IDS?

$$
F_{\mu}=[2.85(\mu)-1.857]^{0.422}
$$

Modify 4 for duration

$$
\begin{aligned}
& \mu^{*}=1.0+C_{D}(\mu-1.0) \\
& \text { For steel, } C_{D} \sim 1.0 \\
& \mu^{*}=1.0+1.0(\mu-1.6)=\mu_{1} \\
& F_{\mu}=[2.851(8)-1.851]^{0.92 L}=3.6
\end{aligned}
$$

Fur rigid range,

$$
\begin{aligned}
& F_{\mu} \sim \mu^{0.13} \\
& F_{\mu} \sim 8^{0.13}=1.31
\end{aligned}
$$

Let $F_{\mu}=3.6$ at spectral peak at $7 \frac{1}{2} \mathrm{H}$ $F_{u}=1.31$ at 33 ts .

At $16.7 \mathrm{HJ}$

$$
\begin{aligned}
\log F_{\mu} & =\log 1.31+\left[\frac{\log 33-\log 16.7}{\log 33-\log 7.5}\right][\log 3.6-\log 1.31] \\
\log F_{\mu} & =0.319 \\
F_{\mu} & =2.08
\end{aligned}
$$

$B-76$ 
ERE

ENGINEERING, PLANNING AND MANAGEMENT CONSULTANTS

SheET NO 11

JOB NO $\mathrm{JOB}$

HCLPF study BY $\frac{K D=}{2}$ DATE $\frac{9 ; 30,27}{10 / 6,67}$

CLIENT SUBJECT Air Receiver Tank

Effective peak study shows that Eu can approach 100 , thus assume that $F_{\mu}=1.0$ is $M_{0}(-2.33 \beta)$ case

$$
\begin{aligned}
& B_{c}=\frac{1}{2.33} \ln \frac{2.08}{1}=0.32 \\
& B_{C}=0.8 B_{C}=0.26 \\
& B_{C}=0.6 B_{c}=0.19
\end{aligned}
$$

Strength Factor

Bolt reaction at limit load $=1825^{\text {th }}$ (P as)

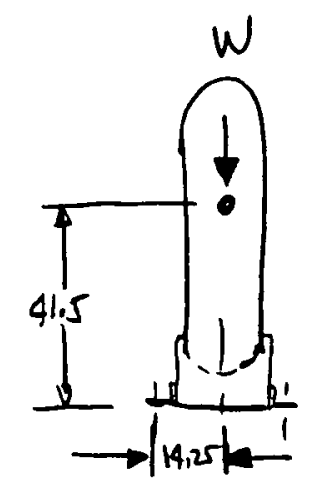

Bolt reaction per $g$ lateral $=1275^{\text {\# }}\left(P_{9}, 4\right)$ But reaction per $g$ vertical - $\operatorname{SMA}$ ( $A$ is on edp $e$ as shown on PG 3.

$$
f_{b}=-\frac{w\left(1-g_{v}\right)(14.25)}{2(19.47)}=-0.476(w)\left(1-g_{v}\right)
$$

B-77 
ERE

ENGINEERING. PLANNING AND MANAGEMENT CONSULTANTS

SHEET NO. 12

JOB NO. $\mathrm{JOB}$

HCLPF Study

BY ROC DATE $\frac{1 / 30 / 87}{1028}$

CLIENT

SUBJECT

Air Reaver Tank

CHKD MKR DATE :0,0:57

From median cantered fluor spectra,

$\mathrm{Sq}_{\mathrm{H}}$ at $16.7 \mathrm{~Hz}, 5 \%$ damping $=0.51$

Save (Rigid) $=0.2$

Using 10070, $907,40 \%$ phasing

$$
\begin{aligned}
& F_{b}=0.51(1275)-0.476(920)(1-0.2(0.4)) \\
& F_{b}=247
\end{aligned}
$$

Scale up $E Q$ until $F_{b}=F_{L}=1825^{\#}$

$$
\begin{aligned}
1825 & =F_{s}(0.51)(1275)-0.476(920)\left(1-F_{s}(0.2)(0.4)\right) \\
1825 & =650 F_{S}-438+35.03 F_{S} \\
F_{S} & =3.30
\end{aligned}
$$

There are a number of uncertainties associated with strength which include tho material yield strength, tho effective length of tho cantilever lea of tho bracket and the direction of rocking (is, the strength morel is for in axis t. Some quick studies of rocking abut other axes and including two horizontal components of $F Q$

B-78 
EQF

ENGINEERING. PLANNING AND MANAGEMENT CONSULIANTS SHEET NO. 13

JOBNO $J O B$ HCLPF stindy CLIENT SUBJECT Air Recirver Tark BY 2 OL DATE $9 / 30 / 87$ CHKD MKR DATE $10: 6 / 87$ indicate thed the musel used is medicun corteredo phasing will be consudeved in equipmant ripance factur. Material STRENGTH

Code valup is $95 \%$ Confidenis

$$
\beta_{U_{M}}=\frac{1}{1.65}(\ln 1.25)=0.14
$$

Othor unoto tie, add $B u=0.10$

$$
B u_{s}=\left(0.14^{2}+0.10^{2}\right)^{\frac{1}{2}}=0.17
$$

EquipmFNT RESPONSE FACTOR

IMETHOIS OF INACYYSIS -

THERE IS NO DECIBERATE HOMS in THE ANALYSIS METHOD, THUS, THIS FACTUR DUES NOT aPPLI, VARIABILITIES in analysis hre pICEED UP IN EUACUATION OF OTHER VARIABEES.

MUDELING

THE TWO CONSIDERATIONS ARE

1) FREQUENCY

2) MODEC A.CCURACY

B-79 
EDE

ENGINEERING. PLANNING AND MANAGEMENT CONSULTANTS

JOB NO $\mathrm{OB}$

HCLPF Study;

SHEET NO. 14

CLIENT

SUBJECT

Fir Receiver Tar

BY CDC DATE $9 / 30, S\rangle$

CHKD MR DATE $10 / 6 / 87$

FREQUENCY

Stiffness of angle. support man have been optimistic but seismic excitation in a different direction might have results in a stiffer model. Model was simple but crude ( rotary inertia was not considered but effect is small)

Estimate that $\beta_{F}$ is 0.2

$-1 B$ Frequency $=16.7 e^{-0.2}=13,71:=$

From horizontal spectrum

$\frac{S_{a_{13.7}}}{S_{a_{16,7}}}=1.0$ No change .

At $-2 \beta \quad f=1.6 .7 e^{-2(0,2)}=11.2113$

$$
\begin{aligned}
& \frac{S_{a_{11.2}}}{S_{a_{16.7}}}=\frac{0.70}{0.51}=1.373 \\
& \beta_{f}=\frac{1}{2} \ln 1.373=0.16=\beta_{u}
\end{aligned}
$$

MODEL

Use $\beta_{u}=0.1$ for error in actual model

for representing bolt reaction.

MODELING

$$
\beta_{M_{U}}=\left(0.16^{2}+0.1^{2}\right)^{1 / 2}=0.19
$$

B-80 
ERE

ENGINEERING. PLANNING AND MANAGEMENT CONSULTANTS

JOB NO. $\mathrm{JOB}$ HCLPF Sind SHEET NO. 15

CLIENT SUBJECT Air Reviver Tank BY.RPC Date q/30/87 CHKD MK DATE $10 / 6 / \overline{1}$

DAmPING

$5 \%$ Consubised Median.

Use $7 \%$ as $+1 \beta$

At $16.7 \mathrm{Hg}$

$$
\beta_{0}=\frac{S_{a_{5} \pi}}{S_{a_{7 \pi}}}=\ln \frac{0.51}{0.50}=0.02
$$

consider all Bu.

Modes combinateai

Most dynamic resporio is rigid body tacking, thus, response is all in a single mole,

Let $\beta_{m c}=\beta_{R} \sim 0.05$

Earthquake Component Combination

Analysis was done looking at one horizontal compare with $40 \%$ of vertical comment effective, consider both horizontal comports in phase as a $+3 B$ case and one horizontal and the vertical componat in phase as $a+3 B$ case,

B-81 
ENGINEERING. PLANNING AND MANAGEMENT CONSULTANTS

JOB NO. $J O B$ HCLPE Sind
ir Reviver BY $K D C^{\text {SHeEt No }} \frac{159}{9 / 3487}$

CLIENT SUBJECT Lir Reviver Fork CHKD $\because$ KR DATE i $/ 6 / 87$

iterizontal Phasing

Only a single direction horizontal component was used in the strength calculation. A median horizontal acceleration vector would be (using 1007,907, 40\% phasing)

$$
\begin{gathered}
\bar{S}_{0}=\left(1^{2}+0.42\right)^{1 / 2}=1.08 \\
F_{E C C}=\frac{1}{1.08}=0.93 \\
\beta_{E C C}=\frac{1}{3} \ln \frac{\sqrt{2}}{1.02}=0.09=\beta_{R}
\end{gathered}
$$

Vertical Phasing

consider $H+V$ in phase.

Use equations on $\mathrm{Pq}, 11 \mathrm{t} / 2$ for bolt reaction due to horizontal and vertical loading

B-82 
EDE

ENGINEERING. PLANNING AND MANAGEMENT CONSULTANTS

JOB NO $\mathrm{JOB}$ L - L PF Sind SHEET N 16

CLIENT SUBJECT Air Receiver Tank BY ROC DATE $\frac{16}{30 / 87}$ CHKD MIR DATE $10 / 6 / 87$

$$
\begin{aligned}
& F_{b}=1275 \mathrm{~g}-\frac{w\left(1-g_{v}\right)(14.25)}{2(11.97)}=1275 \mathrm{~g}-0.976 \mathrm{w}\left(1-g_{v}\right) \\
& S_{a}+=0.51 \mathrm{~g} \\
& S_{a v}=0.2 \mathrm{~g} \\
& F_{b}=0.51(1275)-0.476(92 \mathrm{w})(1-0.2)=563^{\prime}
\end{aligned}
$$

scale up to $F_{b}=F_{L}=1825 \#$

$$
\begin{aligned}
& 1825=F_{S}(0.51)(1275)-0.976(920)\left(1-0.2 F_{5}\right) \\
& 1825=650 \mathrm{Fs}-438+87.6 F_{5} \\
& F_{S}=\frac{2263}{737.6}=3.07 \text { compared to } 3.3 \\
& \beta=\frac{1}{3} \ln \frac{3.3}{3.07}=0.025 \text { say } 0.03 \text { all } P_{R} \\
& F_{R E}=F_{m} F_{D} F_{m C} F_{E C C}=(D)(1)(1)(0.93)(1.0)=0.93 \\
& B_{R E}=\left(0^{2}+0^{2}+0.05^{2}+0.09^{2}+0.03^{2}\right)=0.11 \\
& B U_{R E}=(0.192+0.02+0+0)^{1 / 2}=0.19
\end{aligned}
$$

$B-83$ 
EQ

ENGINEERING. PLANNING AND MANAGEMENT CONSULTANTS

JOB NO $\mathrm{OOB}$ HCLPF Study BY $R \mathcal{L}^{\text {SHEET NO } \frac{17}{q / 30 / 87}}$ CLIENT SUBJECT CHKD MKR DATE 10/6/87

STRUCTURAL RESPONSE FACTOR

USE PSF ANALYSIS IS APPROPRIATE.

Spectral Shape (Peak to Real Variation and EQ
Direction Content)

$$
\begin{aligned}
& \tilde{F}_{s s}=1.35 \\
& \beta_{R}=0.25
\end{aligned}
$$

$$
\text { Direction (enters) }
$$

Damping -

$$
\begin{aligned}
& \mathscr{F}_{D}=1.0 \\
& \beta_{R}=0.03 \\
& \beta_{U}=0.14
\end{aligned}
$$

Modeling

$$
\begin{aligned}
& \mathscr{F}_{M}=1.0 \\
& B_{R}=0 \\
& B_{U}=0.16
\end{aligned}
$$

$$
\begin{aligned}
& \frac{S S I}{F_{S S I}}=1.0 \\
& B_{R}=0.01 \\
& B_{U}=0.05 \\
& F_{S R}=1.35(1)(1)(1)=1.35 \\
& B_{R}=\left(0.25^{2}+0.03^{2}+0+0.01^{2}\right)^{1 / 2}=0.25 \\
& B_{U}=\left(0+0.11^{2}+0.16^{2}+0.05^{2}\right)^{1 / 2}=0.22
\end{aligned}
$$

B-84 
EQ E

ENGINEERING, PLANNING AND MANAGEMENT CONSULTANTS

JOB NO $\mathrm{JOB}$ HCLPF study CLIENT SUBJECT Air Record Tr ic

SHEET NO 18 BY $20 C$ Date $2 / 30 / 27$ CHKD MK DATE IsiS/\&?

FRAGiLITY.

$$
\begin{aligned}
& \tilde{A}=F_{s} F_{M} F_{R E} F_{R S}(0.18)= \\
& (3.3)(2.08)(0.93)(1.35)(0.18)=4.55 \mathrm{~g} \\
& \beta_{R}=\left(0+0.262+0.11^{2}+0.25^{2}\right)^{1 / 2}=0.38 \\
& \beta_{u}=\left(0.17^{2}+0.19^{2}+0.19^{2}+0.22^{2}\right)^{1 / 2}=0.39 \\
& H C L P F=1.55 e^{-1.65(0.38+0.39)}=0.440
\end{aligned}
$$

calculate hCLPF By fRI method.

MOUNTING BRACKET WOULD GOVERN USING APPENDIX F CRITERIA FOR MIME COM PINENT SUPPORT.

FOR Class 3 plate $t$ shell component support, primary bonding allowable is $1.5 \times$ membreap allowable of 1.25 or 0.75 $0.750=0.7(58,00)=40,600$ psi governs Allowable $=1.5(40,600)=60,900 \mathrm{ps} /$

B-85 
XE

NGINEERING. PLANNING AND MANAGEMENT CONSULTANTS

SHEET NO $18 a$

JOBNO. $J O B$

HCLPF STuck BY RPS DATE $9 / 30 / 87$

CLIENT

SUBJECT

Air Exciter Tank CHKDMKR DATE $10 \%$ / 187

Our Base Fragility considered one horizontal direction acting in the weakest axis. However, we have two components of horizontal earthquake at the fluor level. the $100 \%, 40 \%, 40 \%$ phasing rule is applicable to the margin criteria, thus we can scale up our horizontal model load by

$$
F=\left(1^{2}+0.4^{2}\right)^{1 / 2}=1.08
$$

B-86 
EDE

ENGINEERING. PLANNING AND MANAGEMENT CONSULTANTS

SHEET NO 19

JOB NO $J O B$ HCLPF Sivan BY ROC DATE $9 / 30 / 2)$

CLIENT

SUBJECT

Air Reviver $a_{1} K$

CHKD MKF DATE io /E/E7

$$
\sigma_{b}=\frac{m c}{I}=\frac{F_{b}(1.50)(0.125)(12)}{3\left(0.25^{3}\right)}=48 F_{b}
$$

(Note that $l$ taken as. $115^{\prime \prime}$ to counter of both holp for elastic deterministic calculation.)

$$
F_{b}=\frac{60,900}{48}=1269 \#
$$

From Equations on $P_{y}, 12$

$$
\begin{aligned}
& 1269=1.08(051) F_{S}(1275)-0.476(920)\left(1-F_{s}(0.2)(0.4)\right) \\
& 1269=702 F_{S}-438+35.03 F_{S} \\
& F_{S}=2.32 \\
& H C L P F=2.32(0.18)=0.42 \mathrm{~g}
\end{aligned}
$$

In addition., in tho EPRI method, we can use 0.8 as seismic load factor Thus HCLPF $=\frac{0.42}{0.8}=0.53 \mathrm{~g}$ compared to 0.449 by fragility method.

Alternatively, the code method allows use of limit a analysis which in this case would result in the same answer

B-87 
EDE

ENGINEERING. PLANNING AND MANAGEMENT CONSULTANTS

SHEET NO 20

$J O B N O$

$\mathrm{JOB}$

HCLPF stun

SUBJECT

Air Recliner Tank

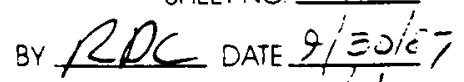

CLIENT

Note, that if the linear support rules

are used and the bending is considered as bending of a compact section, the allowable is

$F\left(0.755_{4}\right)$ whew $F$ is lesser of 2 or

$$
1.167 \frac{S_{U}}{S_{4}}-\text { governs }
$$

$$
F=1.88
$$

$\sigma_{\text {all }}=1.88(0.75) 5_{4}=50,764$ compared $t$

60,900 if angle log bending is considered a plate bending.

ACS criteria would allow $1.7\left(0.755_{7}\right)=45$, guess

Thus, HCCPF varies for EPRE mather depending upas classification of component and interpretation of code,

B-88 


\section{COMPONENT 4}

\section{HORIZONTAL HEAT EXCHANGER}


ENGINEERING, PLANNING AND MANAGEMENT CONSULTANTS

SHEET NO 1

ЈОВ NO 87-218.01 ОOB HCLPF STU OY BY ADC DATE $9-26-87$ CLINT LINK SUBSET COMPONENT HEAT EXCHANGER CHKDMKRDAE IO; $3 / 87$

DEVELOP FRAGILITY DESCRIPTION OF COMPONENT HEAT EXCHANGER.

REFERENCES: II ATLAS INDUSTRIAL MFR. CO. DRAW D-1260-4 3/-240 COMPONENI COULING HEAT EXCHANGER, FEB. 7,1967

2) WOODWARD CLYDE CALCULATIONS OF COMPONENT COOLING HEAT EICHANGFE For GINNA, 3-4-80

3, GILBERT ASSOCIATES CALCULITTUNS, COMPONENT COOLING HEAT EYCHPIUGER, FILE $1: 36.2 / C-1,6-21-79$.

Assumptions AND GRound RuLES:

1. TANK is mOUNTED AT GKADE (DO 1650 FOR CASE OF TANK MOUNTED IN STRUCTURE)

2. EAKTHQUAKE is DEFINED AS E4T IJE= FUP THE LARGER OE TWO com PONENTS of $E, Q$.

3 LUR SPECTRA ARE FROM BUILDING ANALYSIS USING $7 \%$ DAMPING BUT ASSUME THAT THEY ARE MEDIAN CENTERED. IPA $=0.18 \mathrm{~g}$,

4. NOZZLE LOADS ARE NOT PROVIDED, HFNEE 16 NORE NOZZLE LOAOS IN ANALYSIS.

B-90 
EQ E

ENGINEERING. PLANNING AND MANAGEMENT CONSULTANTS

SHEET NO 2

JOB NO $\mathrm{JOB}$

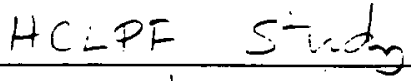
BY $R O C$ DATE $9-26-87$

CLIENT SUBJECT

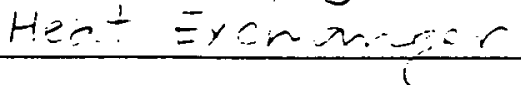
CHKD MKGDATE $2 \equiv i^{\circ}$

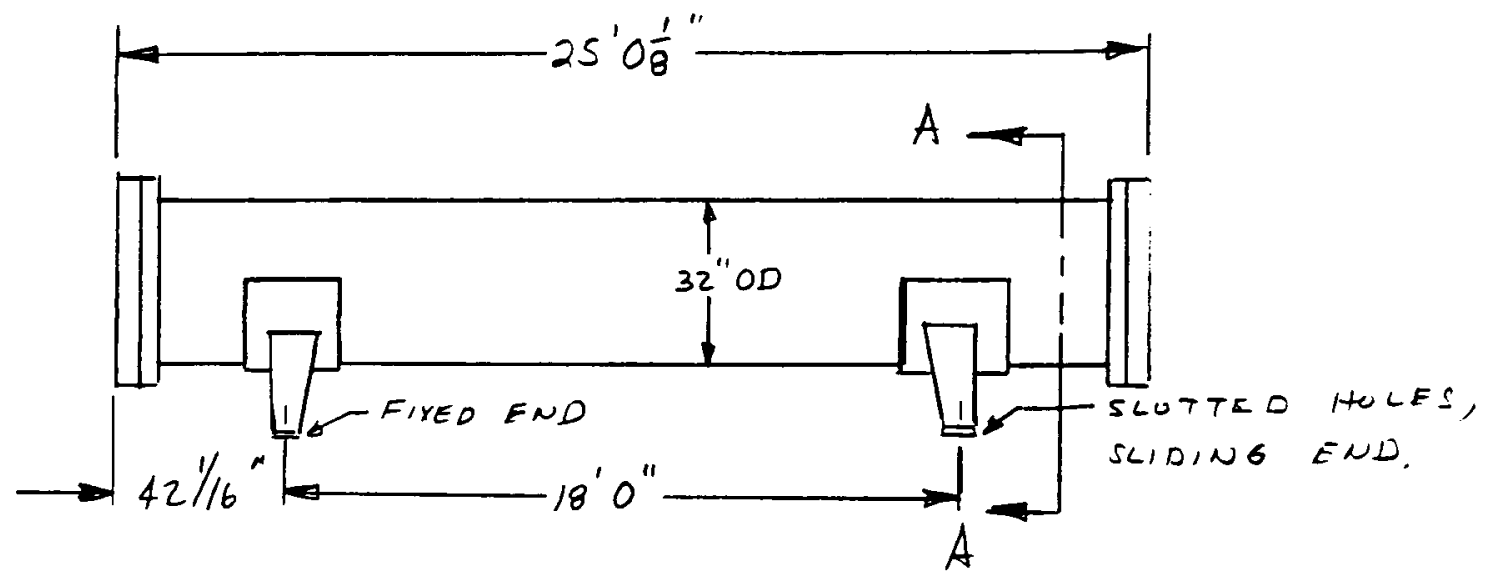

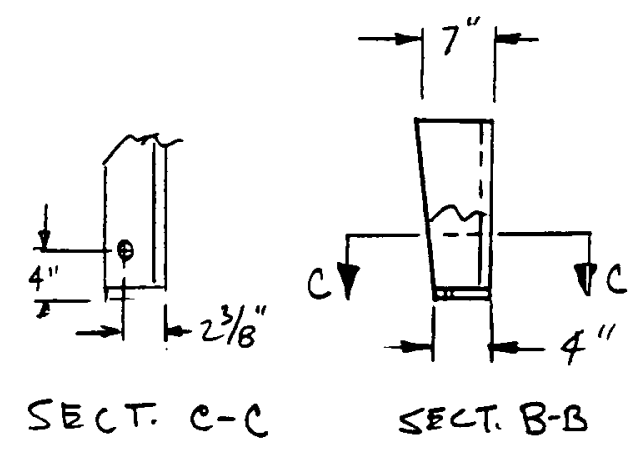

WT- EMPTY $=15,000 \angle B$

$$
\text { FULL }=23,500 \mathrm{LB}
$$

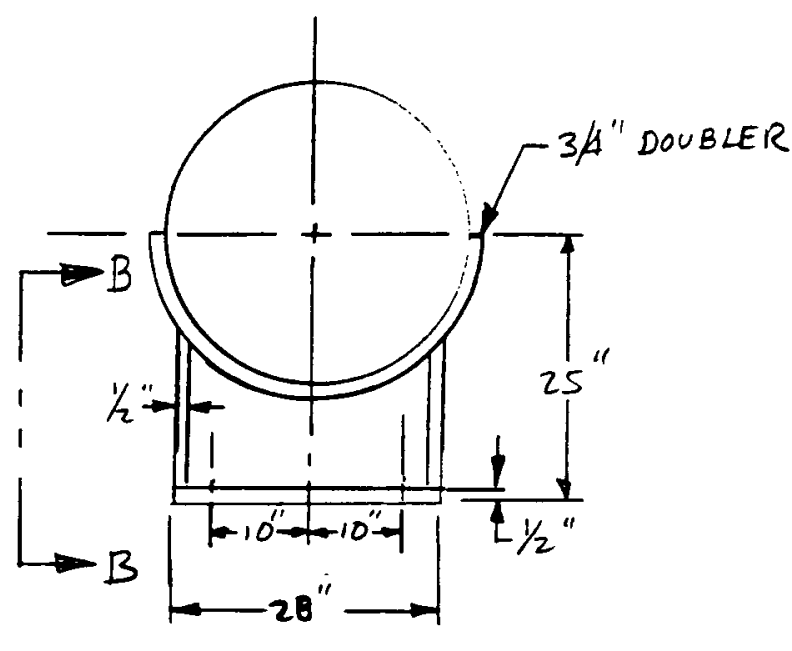

SECT AAA

HEAT EXCHANGER IS MOUNTED ON RIGID FRAME.

B-91 
ENGINEERING. PLANNING AND MANAGEMENT CONSULTANTS

SHEET NO. 3

JOB NO $J O B$ HCLPF Sion BY ROC DATE $9-26-87$

CLIENT SUBJECT Heat Excmaneen CHKDMK 2 DATE

DETERMINE GOVERNING FAILURE MODE

and calculate mEDIAN capacity.

THERE ARE THREE AREAS OF CONCERN:

1) LONGITUDINAL BENDING OF SADDLE AND

SADDLE/ DOUbLER WELD (nOte that COMPLETE CROSS SECTION IS NOT EFFECTIVE IN MENDING AS WAS ASSUMED IN REFS $2+3$,)

2. BOLT LUADING DOminated BY LATERTC $\angle O A D S$.

3. BASE PLATE BENDING FROM BOLT REACTION (NOTE, REFS 2 +3 USEI) INCORRECT DIMENSIONS FOR THU ANALYSIS).

CASE, LONGITUDINAL BENDING OF SADDLE, USE ONLY LONGITUDINAL EQ LOAD FOR PRELIMINARY CHECK ON RELATIVE STREISGTIS,

B-92 
ERE

ENGINEERING. PLANNING AND MANAGEMENT CONSULTANTS

SHEET NO 4

JOB NO

$\mathrm{JOB}$

HCLPE STing

CLIENT

SUBJECT

th et ixiniment BY. ROC DATE-26-27 CHR MIR DATE IO/ $/ 4 / \varepsilon 7$

estimate longitudinal frequency onLy oNe SADDLE CARRIES LONGITUDINAL LOKD. SADDLE aCTS AS A cANTILEVER beAm FIXED AT THE DOUBLER PLATE.

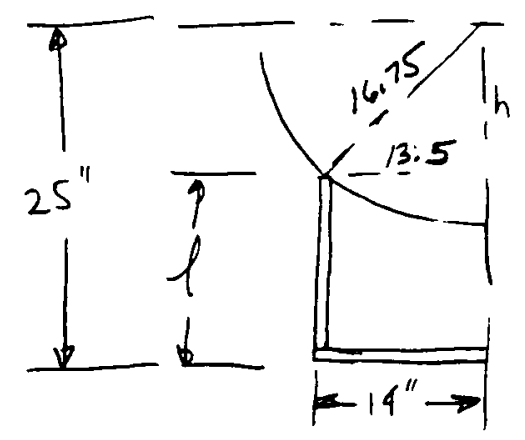

$$
l=25-\left(16.75^{2}-13.5^{2}\right)^{\frac{1}{2}}=15008^{\prime \prime}
$$

COMPLETE SADDLE CROSS SECTION IS NOT EFFECTIVE IN BENDING. ASSUME THAT THE SADDLE IS EFFECTIVELY 2 TX $\times \frac{1}{2}$ ANGLES, OF $l=15$, 08 "

$$
4 T 7 \sqrt{\sqrt{2}^{1}}
$$

$\begin{array}{cccc}\text { Element } & A & y & A y \\ 1 & 3.5 & 0.25 & 0.875 \\ 2 & \frac{3.25}{6.75} & 3.75 & \frac{12.187}{13.06}\end{array} \quad \bar{y}=\frac{13.06}{6.75}=1.935$

$\begin{array}{cccccc}\text { Ely, } & A & d & A d^{2} & I_{0} & I=32.158 \mathrm{IN}^{4} \\ 1 & 3.5 & 1.685 & 9.937 & 0.073 & I=3 E R \text { ANGLE } \\ 2 & 3.25 & 1.815 & \frac{10.706}{20.643} & \frac{11.443}{11.515} & I=64.321 \mathrm{~N}^{2}\end{array}$

B-93 
EDE

ENGINEERING. PLANNING AND MANAGEMENT CONSULTANTS

JOB NO $\mathrm{JOB}$ HCLPF study SHEET NO. 5

CLIENT

SUBJECT

Heat Exchanger BY ROC DATE $9-26-87$ CHE M MK DATE $\frac{10,4 / \varepsilon 7}{2}$

lg static deflection is:

$$
\begin{aligned}
f & =\frac{p l 3}{3 E I}=\frac{23,500(15.08)^{3}}{3\left(29 \times 10^{60}\right)(64.32}=0.0144^{11} \\
K & =\frac{23,500}{0.0144}=1.63 \times 10^{6} \\
f_{n}=\frac{1}{2 \pi} \sqrt{\frac{K}{m}} & =\frac{1}{2 \pi 1} \sqrt{\frac{1.63 \times 10^{6}(386,4)}{23,500}}=26 \mathrm{~Hz}_{3}
\end{aligned}
$$

SYSTEM is BL MOST RIGID.

LOOK AT PLASTIC CAPACITY OF SADDLE WELD TU DOUBLER, NO WELD DETAIL is GIVEN. ASSUME THERE is SUFFICIFUT WELD METAL TO DEVELOP FULL STRENGTH GE PLATE. THUS THE CAPACITY IS BASED CIS A $7 X>X \frac{1}{2}$ ANGLE BEING EFFECTIVE

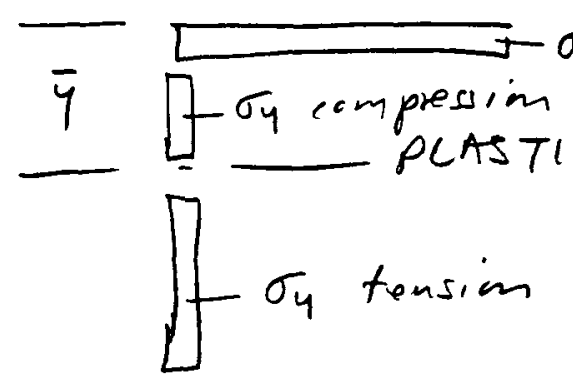

Use $\sigma_{4}$ median of A $516-60 \sim 44 \mathrm{ks}$,

B-94 
EDE

ENGINEERING. PLANNING AND MANAGEMENT CONSULTANTS

SHEET NO 6

JOB NO.

CLIENT
$\mathrm{JOB}$

HCLPF Singing

Heat ix e haves:-
BY RC DATE $9-x-87$ CHKD RIER DATE 10/4/ ET

PLASTIC NA BY TRIAL, ASSUME $\bar{y}=13 / 9$ "

$\sum M_{N A} \quad$ Try $\bar{y}=13 / 4$

$$
\begin{gathered}
\sigma_{y}(1 / 2)(7)(1 / 2)+\sigma_{y}(1 / 4)\left(\frac{1}{2}\right)(5 / 8)=\sigma_{y}\left(\frac{1}{2}\right)\left(5 \frac{1}{4}\right)\left(2 \frac{5}{8}\right) \\
5.64 \sigma_{y}=6.89 \sigma_{y}
\end{gathered}
$$

Try $\bar{y}=2^{\prime \prime}$

$$
\begin{gathered}
\sigma_{y}\left(\frac{1}{2}\right)(7)(13 / 4)+\sigma_{y}\left(1 \frac{1}{2}\right)\left(\frac{1}{2}\right)(3 / 4)=\sigma_{y}\left(\frac{1}{2}\right)(5)\left(2 \frac{1}{2}\right) \\
6.687 \sigma_{y}=6.25 \sigma_{y}
\end{gathered}
$$

Try $1.93^{\prime \prime}$

$$
\begin{aligned}
\zeta_{y}(1 / 2)(7)(1.68)+\sigma_{y}(1.93)(0.715)\left(\frac{1}{2}\right) & =\sigma_{y}\left(\frac{1}{2}\right)(5.07)(2.535) \\
6.39 & =6.926 \sigma_{y}
\end{aligned}
$$

$N A \sim 1.93^{\prime \prime}$

Moment capacity per log $=(6.39+693) F_{4}=12,82 \sigma_{4}$

Capacity of both logs $=2\left(12.82 ; \sigma_{y}\right.$

$$
\begin{aligned}
& m=2(12.82)(44000)=1.128 \times 10^{6}, \mathrm{~N} \\
M_{\text {applied }}= & 15.08 \mathrm{~W} \mathrm{Sa} \\
\mathrm{Sa} \operatorname{LimiT}= & \frac{1.128 \times 106}{15.08(23,50)}=3.18 \mathrm{gs}
\end{aligned}
$$

B-95 
ENGINEERING. PLANNING AND MANAGEMENT CONSULTANTS

SHEET NO $\longrightarrow$

JOB NO $\mathrm{JOB}$

HCLPF Since BY $R D C$ DAT $9 / 26 / 87$

CLIENT SUBJECT

Heat Exchanger CHE R I KR DAR

CAPACITY COULD BE LOWER IF WELD is NOT CAPABLE OF DEUELUPING PARENT METAL STRENGTH BUT SOME CREST MAY BE TAKEN FUR DUCTILITY.

CHECK CAPACITY INS BENDING IN OPPOSITE DIRECTION. BUCKLING OF GUSSET PLATE WOULD CONTROL. GUSSET PLATE DEF NUT QUALIFY AS $A$ C...P PACT SECTION PER NF 3322.1 (d)(1) (d) PER NF 3322./(dj(1) (b)(2)

$$
\begin{aligned}
& b_{f} / 2 t_{f}=7 / 2(0.5)=7<65 / \sqrt{5_{4}}=65 / \sqrt{44}=9.8 \\
& F_{b}=S_{y}\left[0.79-0.002\left(b_{f} / 2 t_{f}\right) \sqrt{5_{y}}\right]=0.70 F_{y} \\
& 0.66 F_{4} \text { for compact sections would govern }
\end{aligned}
$$
Faulted Allowable would be the smaller of $2\left(F_{b}\right)$ or $1.167\left(\frac{S u}{S y}\right) F_{b}$

Use specified values for $\frac{S_{v}}{S_{4}}=\frac{60}{35}$

$$
\begin{aligned}
& 1.167 \frac{S_{u}}{S_{4}} F_{b}=2.0 \mathrm{Fb}=2(0.66)=1.32 \mathrm{Fy} \\
& M_{\text {Faulted }}=\frac{1.32 F_{y} F}{c}=\frac{(1.32)(44)(64.32)}{5.065}=737 \mathrm{ink}
\end{aligned}
$$

B-96 
EQ

ENGINEERING. PLANNING AND MANAGEMENT CONSULTANTS

JOB NO $J O B$ HC -FF Sm=SHEET NO. 8

CLIENT SUBJECT

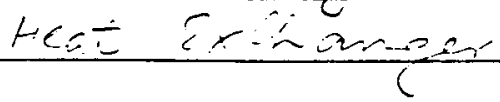
BY ROC DATE $\overline{\mathscr{2} / 26 / 8} 7$ CHKD $11 k \dot{k}$ DATE $2+? 7$

There is nominally a FS of 1.5 remairser for buckley in faulted allowarlos.

Estimated midian buckling capacity is -

$$
1.5(737 \text { ink })=1.106 \times 10^{6} \text { in \# composed }
$$

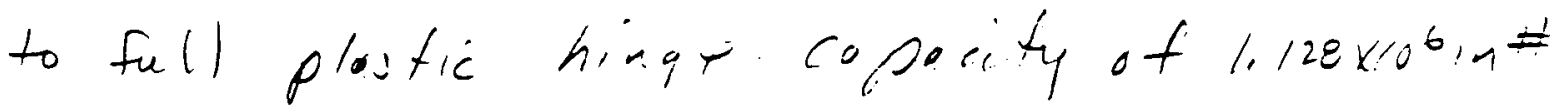

chick other follow ait is. If this

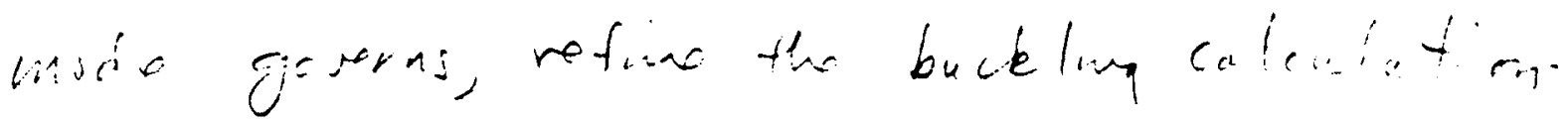

Carte that bolts wore found to nave significantly less capacity so concerns about buckling and weld area are relieved)

B-97 
EDE

ENGINEERING, PLANNING AND MANAGEMENT CONSULTANTS

JOB NO $\mathrm{JOB}$

HCLPF sind

SHEETNO 9

CLIENT

SUBJECT

Heat Exchanger

BY ROC DATE $9 / 26 / 8=$

CHKD MikzOATE's:-if?

CHECK BOLT STRENGTH

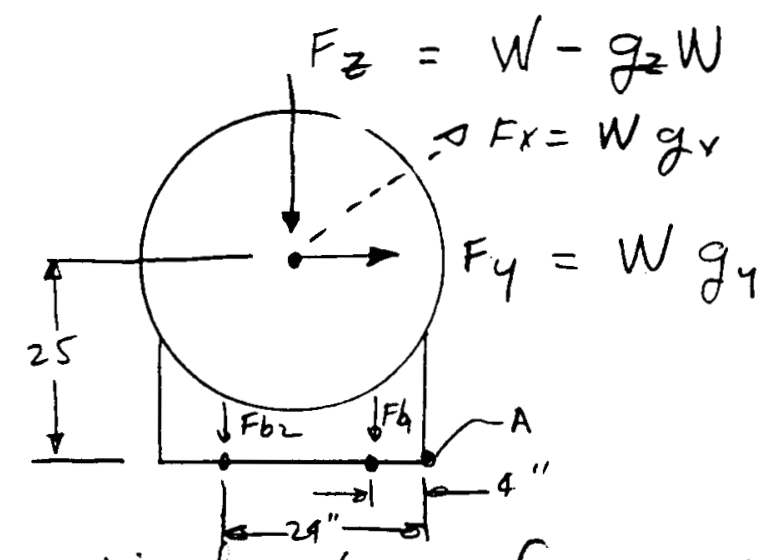

Bolts see direct shear from $x$ and $y$ loads and direct tension from Fy overturning Tank is rigid in the vertical direction and is about $26 \mathrm{~Hz}$ in $x$ direction. Assume $y$ direction is rigid. At ground, the vertical acceleration is $2 / 3$ of horizontal. In the structure, the $\mathrm{V} / \mathrm{h}$ ratio is lower. $(\tau / \mathrm{K})$. Use ground for this relative strength calculation At $26 \mathrm{H}_{3}$ the $\mathrm{Sa}_{4}$ is about $1.13 \times \mathrm{ZPA}$. For median calculations, assume $g_{z}=2 / 39 y$ and $g_{x} \sim 0.9 g_{y}$

B-98 
EQ

ENGINEERING. PLANNING AND MANAGEMENT CONSULTANTS

JOB NO $\mathrm{JOB}$

HCLPE Sing

SHEET NO 10

CLIENT

SUBJECT

$H e=5 x+5 x=2$ BY ROC DATE $9 / 26 / 87$ CHR MK DATE $10 / 4 / \varepsilon$ ?

$\sum M_{A}$.

$14 F_{z}-25 F_{y}+2\left[24 F b_{2}+4 F b_{1}\right]=0$

If Tank and Saddle rotate as a rigid

bo ty, $F b_{1}=4 / 24 F b_{2}$

$$
\begin{aligned}
& 14(w)\left(1-2 / 3 g_{4}\right)-25 w g_{4}+2\left[24 F b_{2}+\frac{4(4)}{24} F b_{2}\right]=0 \\
& 329000-219333 g_{4}-587,500 g_{4}+49.332 F b_{2}=0 \\
& F b_{2}=\frac{806833 g_{4}-329000}{49.332}=16355 g_{4}-6669
\end{aligned}
$$

with $100 \%, 40 \%, 40 \%$ phasing

$$
\begin{aligned}
& 14(23,500)\left(1-\frac{0.4(2) g_{y}}{3}\right)-25(23,50) g_{4}+2\left[24 F b_{1}+\frac{4(4)}{24} F b_{2}\right]=0 \\
& 329,0 w-87733 g y-587,500 g_{4}+49.332 F b_{1}=0 \\
& F b_{2}=\frac{675233 g y-329 \mathrm{wm}}{49.333}=13687 g y-6669
\end{aligned}
$$

Shear Stress, use 100\%, 40\%, co\% Phasing,

$$
g_{x}=0.4(0.9)(1.13) g_{y}=0.406 g_{4}
$$

4 bolts take $y$ load, 2 bolts take $x$ load,

$f_{v}=\frac{23,500 g_{4}}{4}+\frac{0,406 g_{4}(23,500)}{L}=10,645 g_{4}^{\#} /$ bolt

B-99 
EQ

ENGINEERING. PLANNING AND MANAGEMENT CONSULTANTS

JOB NO $\mathrm{JOB}$ HCLPF smoky SHEET NO 11 CLIENT SUBJECT Heat Frinererer BY ROC DATE $9 / 26 / 87$ CHKDMKR DATE Dis :Z?

It threads in bolt cont take direct shear then the failure plane is one. for either shear and tension on the full area or tension only on the thread area.

Tousim only on thread area.

Bolts are $7 / 8^{\prime \prime}-9$ INC $\left.A-367, S_{y}=36 k s,\right\}$ code

$$
\begin{aligned}
& \text { Stress area }=0.461 \mathrm{in}^{2} \\
& \text { Full area }=0.601 \mathrm{in}^{2}
\end{aligned}
$$

At code UTS,

$$
\begin{aligned}
F_{b}=0.461(58,000) & =13687 g_{4}-6669 \\
g_{4} & =2.44<5 a d d l e / \text { Doubler weld. }
\end{aligned}
$$

For Tensing shear in full area.

use cole Parabolic Equation and. $F_{v}=0.6 F_{t}$

$$
\begin{aligned}
& \left(\frac{f_{t}}{F_{t}}\right)^{2}+\left(\frac{f_{v}}{F_{v}}\right)^{2}=1.0 \\
& \left(\frac{13687 g y-666 g}{(0.601)(58,000)}\right)^{2}+\left(\frac{10,695 g y}{0.6(0.601)(5860 v)}\right)^{2} \leq 1.0 \\
& 0.154 g^{2}-0.150 g+0.0366+0.259 g^{2} \leqq 1.0
\end{aligned}
$$

B-100 
ERE

ENGINEERING. PLANNING AND MANAGEMENT CONSULTANTS

SHEET NO 12

JOB NO $\mathrm{JOB}$ HCLPE Sm dat BY ROC DATE $/ 26 / 27$

CLIENT SUBJECT $1-6=t+\cdots-0,0 e r$ CHKDNILKDATE' Jig/

$$
\begin{aligned}
& 0.413 g^{2}-0.150 g-0.9634=0 \\
& g=\frac{0.150 \pm \sqrt{0.150^{2}+4(0.413)(0.9636)}}{2(0.413)}
\end{aligned}
$$

$$
g_{y}=1.72
$$

governs bolt fichus.

Actual ultimate material strength may bo used, For low strength corbm steel such as $A 307$, the mean $4 T 5$ is abut 1.2 times code. (Rel. Nurr6/CR-2137, 5A-106B STL),

$$
g \sim 1.72(1.2)=2.06 \mathrm{~g} \text { capacity }
$$

Now, sep if plate can develop the ultimate strength of the bolt.

$$
\begin{aligned}
F_{U L T}= & 13687 g_{4}-6669= \\
& 13687(2.06)-6669=21,526
\end{aligned}
$$

B- 101 
EDE

ENGINEERING. PLANNING AND MANAGEMENT CONSULTANTS

SHEET NO 13

JOB NO.

CLIENT
$J \mathrm{OB}$

LCLFF Ind

SUBJECT

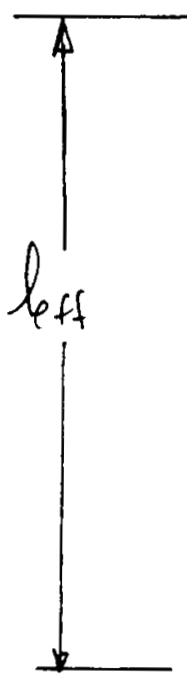

$-$

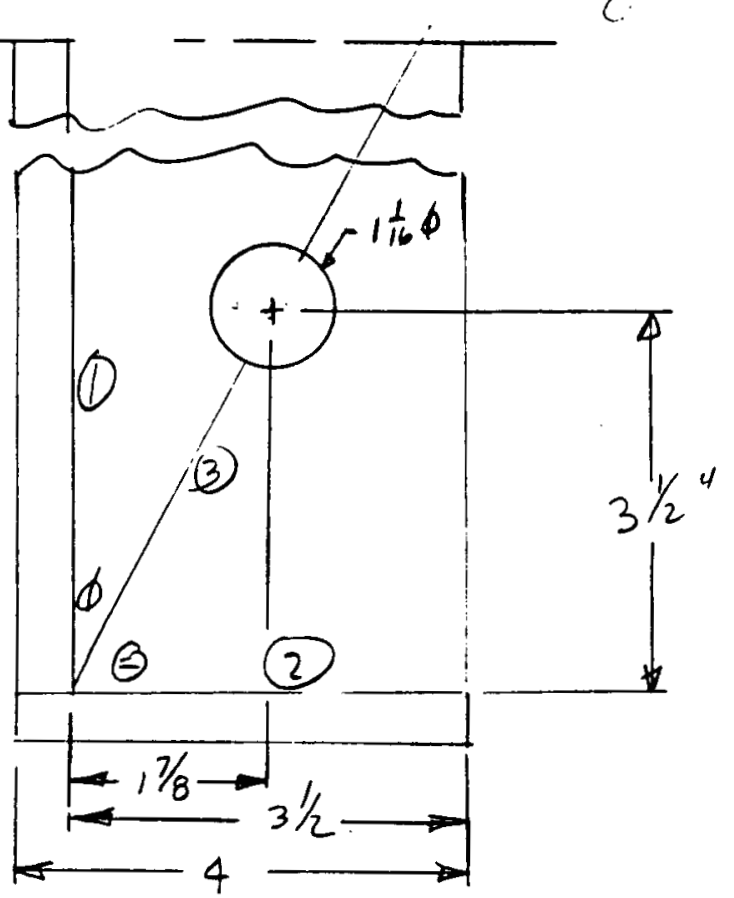
BY PC DATE $9-26-8$. CHKD MKR DATE 10/4/8\%

NO WELD DETAIL GIVEN. ASSUME FULL PENETRATION

ASSUME 3 YIELD LINES FURIM AT (S), (2) $f(3)$

left $=2(3 / 2 " \prime)=7^{\prime \prime}$

Limit Moment $=$
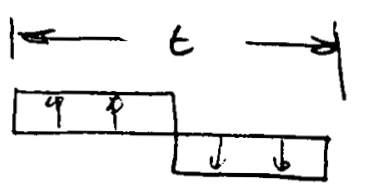

$$
M_{L}=2\left[\sigma_{y} \frac{t}{2} \cdot \frac{t}{4}\right]=\frac{\sigma_{y} t^{2}}{4} / \text { in }
$$

$\bar{\sigma}_{y} \approx 44 \mathrm{ks} /$

$$
\begin{aligned}
& M_{L_{1}}=\frac{7(44)\left(0.5^{2}\right)}{4}=19.25 \mathrm{in} \mathrm{k.} \\
& M_{L_{2}}=\frac{3.5(44)\left(0.5^{2}\right)}{4}=9.625 \mathrm{in} \mathrm{k} \\
& M_{L_{3}}=\frac{\left[\left(7^{2}+32^{2}\right)^{1 / 2}-1 \frac{1}{16}\right] 44\left(0.5^{2}\right)}{4}=18.60 \mathrm{~m} \mathrm{k}
\end{aligned}
$$

B-102 
ENGINEERING. PLANNING AND MANAGEMENT CONSULTANTS

SHEET NO 14

$J O B N O$ $\mathrm{OB}$ HCLPF $\quad 5-\ldots=r$ BYRDC DATE 9-26.87

CLIENT SUBJECT

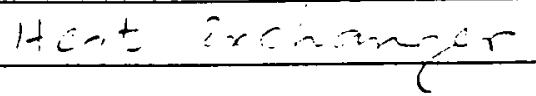
CHKDMKRDATE Di

$$
\begin{aligned}
& \text { work }=F_{b} \delta=M_{L_{1}} \alpha_{1}+M_{L_{2}} \alpha_{2}+M_{L_{3}} \alpha_{3}+M_{L_{3}} \alpha_{c} \\
& \alpha_{i}=\frac{f}{l_{i}} \\
& \alpha_{1}=\frac{\delta}{1.875} \\
& \alpha_{2}=\frac{f}{3.5} \\
& \alpha_{3}=\alpha_{1} \cos \theta \text { when } 0=90^{\circ}-\tan ^{-1} \frac{3,5}{1.875}=28.179^{\circ} \\
& \alpha_{4}=\alpha_{2} \sin \phi \\
& F f=\delta \sigma_{y} t^{2}\left[\frac{7}{(4) 1 \% 8}+\frac{3.5}{4(3.5)}+\frac{6.76 \cos \phi}{4(17 / 8)}+\frac{6.76 \sin \phi}{4(3.5}\right] \\
& F=44000(0.5)^{2}[0.933+0.25+0.79+0.228] \\
& F=24211 \#
\end{aligned}
$$

Bolt tension at ultimate failure in shear and tensing is 22,622 thus the bottom plate can develop sufficient resistance to fail the attachment bolts.

Develop fragility curve based on bo it failure.

B- 103 
EQ F

ENGINEERING, PLANNING AND MANAGEMENT CONSULTANTS

JOB NO $\mathrm{JOB}$ HCLPF Striving SHEeT NO $\frac{15}{9-29-87}$

CLIENT SUBJECT

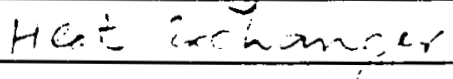

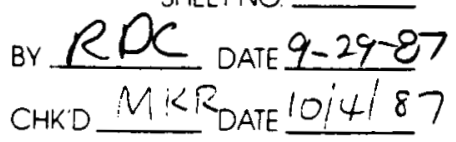

Recalculate capacity of bolts in terms of gl using in. Structure spectra, Ref. Pa, 10 for equations,

$$
\begin{aligned}
& g_{x} z P A=9070 g_{y} z P A \\
& g_{x} \text { at } 26 H_{y}=0.9\left(\frac{0.45}{0.38}\right) g_{y}=1.066 g_{y} \\
& g_{z}=\frac{0.2}{0.38} g_{y}=0.526 g_{4}
\end{aligned}
$$

Built tension Using $100 \%, 90 \%$, $90 \%$ Phasing

$$
\begin{aligned}
& 14 W\left(1-0.4(.526)_{g_{y}}\right)-25 W g_{y}+2\left[24 F_{b_{2}}+\frac{4(d)}{24} F_{b_{2}}\right]=0 \\
& 329,000-69222 g_{y}-587,500 g_{y}+49.332 F b_{2}=0 \\
& F_{b_{2}}=\frac{656722 g_{y}-329,000}{49.332}=13,312 g_{y}-6669
\end{aligned}
$$

Bull shear, Using 100\%, 40\%, $40 \%$ phasing

$$
\frac{23,500 g_{4}}{4}+\frac{0.4(11066) g_{4}(23,500)}{2}=10,805 g_{4} \# / 6.1 \%
$$

B-104 
EQ

ENGINEERING. PLANNING AND MANAGEMENT CONSULTANTS

JOB NO $J O B$

SCAPE SM ST

SHEET NO $\frac{16}{16}$

CLIENT

SUBJECT

Hest BY.RPC DATE $1 / 2 g / e Z$ CHKD MiKE DATE J is

$$
\begin{aligned}
& \left(\frac{13,312 z_{y}-6669}{0.601(58000)}\right)^{2}+\left(\frac{10,8859 y}{0.6(0.601)(58,000)}\right)^{2}=1.0 \\
& .1458 g_{y}^{2}-0.1461 g_{y}+0.0366+0.27099_{y}^{2}=110 \\
& 0.4167 g_{4}^{2}-.1461 g_{y}-0.4634=0 \\
& g_{4}=\frac{0.1461 \pm \sqrt{.1461^{2}+4(.4167)(.9634)}}{2(.4167)} \\
& g_{4}=1.7058
\end{aligned}
$$

For medium ult strength

$$
g_{4}=1.2(1.7058)=2.05 \mathrm{~g}
$$

B-105 
EDE

ENGINEERING, PLANNING AND MANAGEMENT CONSULTANTS

SHEET NO $\perp 7$

JOB NO $\mathrm{JOB}$ HCLPF Study By. $R D C$

CLIENT SUBJECT inert Erchowger CHKD DATE $28-8 ;$

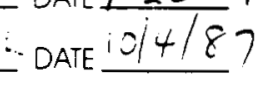

CAPACITY FACTOR

Median capacity for anchor bott falls was. $2.06 \mathrm{~g} 5 a \quad\left(P_{9}, 12\right)$

Code strength is $95 \%$ cenfidionce 50 if median $15 \sim 1,2 \times$ code.

$$
\beta_{0}=\frac{1}{165} \ln 1.2=0.11
$$

There is additional uncertainty in the failure strength and failure wa b of attachment bolts in combireep tension and ster. Estimate $\& \sim 0.15$. In addition, because of clearances in the bolt holes and local bending effects, the bolts will not be uniformly loaded as calculated. Assume $10 \%$ reduction in strength.

B-106 
ERE

ENGINEERING. PLANNING AND MANAGEMENT CONSULTANTS

SHEET NO 18

JOB NO $J O B$ ITCLPF Stu cig BY $\beta Q<$ Date $=\overline{i=\varepsilon / \bar{c}}$

CLIENT

SUBJECT

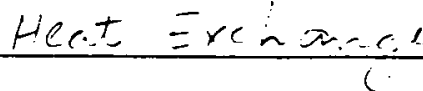

CHIC LIKE DATE $10 / 4 / 27$

Tho resulting strength is then

$$
\begin{aligned}
& S_{a}=\frac{2.06}{1.1}=1.87 \mathrm{~g} \text { in } y \text { dir. } \\
& \beta_{u}=\sqrt{0.11^{2}+0.15^{2}}=0.19 \\
& F_{s}=\frac{1.87}{0.3}=6.23 \text { for ground to be mar direction }
\end{aligned}
$$
(Note that $y$ dir is rigid $J Z P A$ is reference acceleration)

For In Structure case $\left(P_{9}, 16\right)$

$$
\begin{aligned}
& Z P A=2.05 / 1.1=1.86 \text { in } y \text { dir } \\
& F_{S}=\frac{1.86}{0.38}=4.90 \text { relating to in structure }
\end{aligned}
$$

DUCTILITY - Component is rigid, filum is brittle and UTS used for strength Ductility NA.

EQUIPMENT RESPONSE FACTOR

Tho sorites ore:

mother of analysis

Michel lie

Da in ping

note cribs

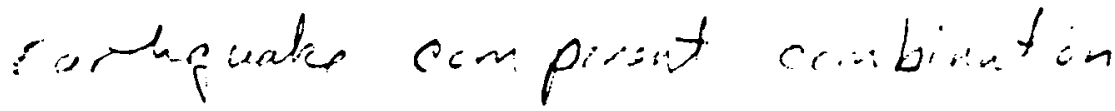

B-107 
ERE

ENGINEERING. PLANNING AND MANAGEMENT CONSULTANTS

JOB NO $\mathrm{JOB}$ HCIPF Stan BY SHEET NO. $\frac{19}{9-28-70}$ CLIENT SUBJECT Hin Exch aries CHR MR DATE $10 / 4 / 87$

Method of Analysis

NA for rigid body evaluation modeling error covered be low

Modeling

There are two considerations,

1) frequency error

2) riel accuracy

The lowest fundoinential frequency is

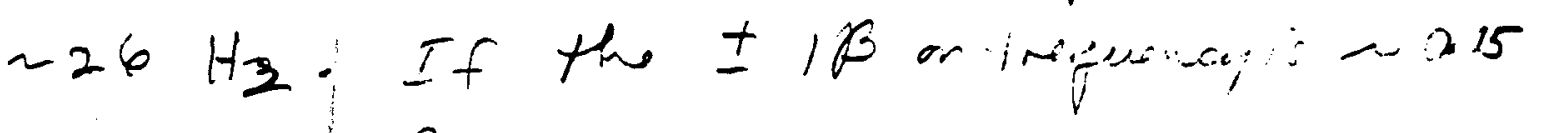
tim - if frequin. is

$$
f_{-1 / 5}=26 e^{-0.15}=22.3 \text { H. }
$$

From either the ground spectrum or the instructure spectrum the ratio of $S a_{27} \cdot s / S_{26}$ is o bunt $1.1 \%$.

$$
f_{5}=\ln 1.11=0.10
$$

Estimate the error in the hand calculation method to be represented by $\beta=0.10$

$$
\beta_{M}=B_{v}=\left(0.10^{2}+0.10^{2}\right)^{1 / 2}=0.14
$$

B-108 
EDE

ENGINEERING. PLANNING AND MANAGEMENT CONSULTANTS

JOB NO $\mathrm{JOB}$ HCLPF Strong

SHEET NO 20

CLIENT

SUBJECT

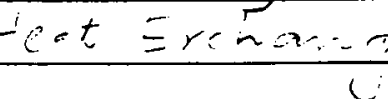
BY $20 C^{\text {SHEET NO DATE } 9 / 28 / 27}$ CHKD M KR DATE $10 / 4 i 27$

Damping

At $26 \mathrm{H}_{3}$, there is very little difference in Sa for different damping values.

Fain the ir structure sortie curves, There is only about $5 \%$. difference between: $4 \%$ and is. For Ex

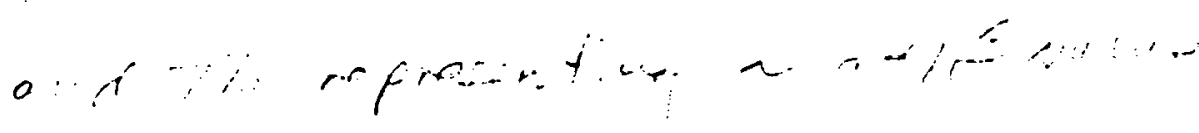

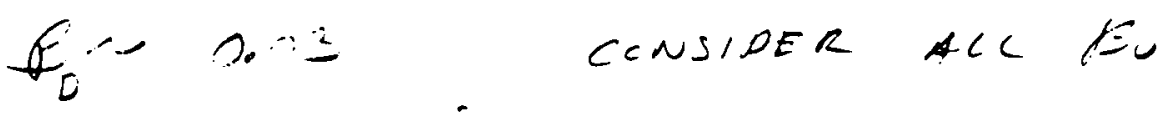

So would be similar for a ground inserted task.

Mode combinations

NA for a rigid body response,

Earthquake Component Combination;

The $100 \%$, 40\%, 40\% rule was used in tho capacity analysis as o medrair value.

B-109 
eNGINEERING. PLANNING AND MANAGEMENT CONSULTANTS

JOB NO $\mathrm{JOB}$ HCLPF Stud in SHEET NO 21 CLIENT SUBJECT Hest Exchanger BY. $R D C$ DATE $2 / 2 \times / e)$ CHKD MIR DATE $10 / 4 / 87$

Consider all components in phase to be a +3 B condition. Recalculate bolt strength assuming all components in phase, Ref pare 10 for capacity equations
for ground rose

$$
\begin{aligned}
& F_{b_{1}}=16,355 g_{y}-6669 \\
& f_{v}=\frac{23,500 g_{4}}{4}+\frac{0.9(1.13)(23,000) g_{y}}{2}=17,825 g_{4}^{\#} / b_{0} / t
\end{aligned}
$$

For tension and shoer combined on full body,

$$
\begin{aligned}
& \left(\frac{16,355 g_{4}-6669}{0.601(58,600)}\right)^{2}+\left(\frac{12825 g_{4}}{0.601(0.6)(58000)}\right)^{2} \leq 1.0 \\
& 0.220 \lg _{y}^{2}-0.1795 g_{4}+0.0366+0.726 g_{y}^{2} \leq 1 \\
& 0.94692-0.1795 g-0.9634=0 \\
& g=\frac{0.1795 \pm \sqrt{0.17952+4(0.946)(0.9634)}}{2(1946)}= \\
& g=1.108 \quad \text { based on cols properties. } \\
& g=1.2(1.108)=1.33 \text { band on medical strength }
\end{aligned}
$$

B-110 
ERE

ENGINEERING. PLANNING AND MANAGEMENT CONSULTANTS

JOB NO. $\mathrm{JOB}$

-C imPF SEnt SUBJECT
Heat Exchanger RP C DATE /28/87 CHE M KR DATE OO/ $/ 8$;

$$
\beta_{E C C}=\frac{1}{3} \ln \frac{2.06}{1.33}=0.15
$$

All $\beta_{R}$.

$$
\begin{aligned}
& F_{R E}=1.0 \\
& \beta_{R_{R E}}=0.15 \\
& \beta U_{R E}=\left(0.14^{2}+0.03^{2}\right)=0.14
\end{aligned}
$$

Assume same $\beta^{\prime}$ for in structure tank

STRUCTURAL REsPONSE

Use FRs for block walls (P 30 ) for in structure mounted tank except, effects of frequency shift for inelastic structure are not of concern but effect of inelasticity on in spectra ZPA may be considered to be ir bout the same, Relationship between so direction magnitude has been incised in fragility calculation but we need to include uncertainty.

B-111 
ENGINEERING. PLANNING AND MANAGEMENT CONSULTANTS

JOBNO. $\mathrm{JOB}$ HCLPF Sindh SHEET NO 23 CLIENT SUBJECT He=t Exch or BY RDC OATEq/20xe?

$$
\begin{aligned}
& F_{R S}=F_{S S} F_{E Q \text { oir }} F_{D} F_{M} F_{S S T} F_{\text {INE }} \\
& =1.22(1.0)(1.0)(1.0)(1.0)(1.0) \\
& \left.\begin{array}{c}
\text { Note, E.Q. Dir } \\
\text { Foctur inctedao } \\
\text { in copos: lycalc } \\
\text { thus, } F_{E D}=1.0
\end{array}\right) \\
& \text { Frs }=1.22 \\
& B_{R}=\left(0.20^{2}+.15^{2}+0.03^{2}+0+0.01^{2}+0.06\right)^{1 / 2}=0.26 \\
& B u=\left(0^{2}+0+0.19^{2}+0.16^{2}+0.05^{2}+0.06^{2}\right)^{1 / 2}=0.23
\end{aligned}
$$

For groune mounted Touk.

Peak to peate variation is not portinent to regia tank.

The E.Q direction cantant $B_{R}$ of $\sim 0.15$ is a ppropriate.

structume Damping, structural mode lmy and indestue structural respme are nat opplicatle.

Us SSI uncertainties

$$
\begin{aligned}
& F_{R S}=F_{E Q O V} \cdot F_{S S I}=1.0 \\
& \left.B_{R}=0.5^{2}+0.01\right)^{1 / 2}=0.15 \\
& B_{U}=\left(0.0+0.05^{2}\right)^{1 / 2}=0.05
\end{aligned}
$$

B-112 
EQF

ENGINEERING. PLANNING AND MANAGEMENT CONSULTANTS

JOBNO $\mathrm{JOB}$ $\frac{\text { HCLPF Stian }}{\text { HECi EvChanger }}$ SHEETNO 24

CLIENT SUBJECT BY RPC DATE $9 / 29 / 87$ CHKD MKK DATE

FRA GILITY

GROUND MUUNTED TANK.

$$
\begin{aligned}
\check{A} & =0.3\left(F_{C}\right)\left(F_{R E}\right)\left(F_{R S}\right) \\
& =0.3(6.23)(1.0)(1.0) \\
\check{A} & =1.87 \mathrm{j} \\
B R & =\left(0^{2}+0.15^{2}+0.15^{2}\right)^{1 / 2}=0.21 \\
B_{U} & =\left(0.19^{2}+0.14^{2}+0.05^{2}\right)^{1 / 2}=0.24 \\
H C C P F & =1.87 e^{-1.65(0.21+0.24 j}=0.89 q .
\end{aligned}
$$

IN STRUCTURT TANK

$$
\begin{aligned}
\check{A} & =0.18\left(F_{C}\right)\left(F_{12 E}\right)\left(F_{\text {KS }}\right) \\
& =0.18(4.90)(1.0)(1.22) \\
A & =1.08 \mathrm{~g} \\
B_{R} & =\left(0^{2}+0.15^{2}+0.26^{2}\right)^{\frac{1}{2}}=0.30 \\
B_{U} & \left.=0.19^{2}+0.14^{2}+0.23^{2}\right)^{1 / 2}=0.33 \\
\text { HCLPF } & =1.08 e^{-1.65 \cdot(0.30+.33)}=0.38 \mathrm{~g}
\end{aligned}
$$

B- 113 
ERE

ENGINEERING. PLANNING AND MANAGEMENT CONSULTANTS

JOB NO $J O B$ HCLPF Sim th BY $R C^{\text {SHeEt No }} \frac{25}{9 / 2 y / 0}$

CLIENT

SUBJECT

Heat Exchanger

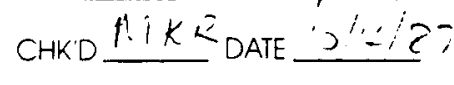

USING ERT MARGIN METHOD.

Q4\% Non Erreedance EN.

median Cantered Spectrum in structure.

Faulted Allowable fer A-307 Ferritic stol Bolts is:

$F_{t b}=$ lesser of $0,7 \mathrm{~S}_{4}$ or $S_{4}$

$S_{4}=36 \mathrm{ks}$, governs

$F_{u b}=$ lesser of 0.4250 or 0.654

$0.6 S_{4}=21.6 \mathrm{ks} 1$ governs,

GROUND MOUNTED TANK.

EPRI Margin Criteria Use 100\%, 40\%, 40\% rule with equal horizontal components.

Recompute $g$ capacity from equations on page 10

$$
\begin{aligned}
& \sum M_{A}=0 \\
& F_{b_{2}}=13,687 g_{4}-6669
\end{aligned}
$$

B-114 
ERE

ENGINEERING. PLANNING AND MANAGEMENT CONSULTANTS

JOB NO

CLIENT
$\mathrm{JOB}$

HCLPE Sin ring

Heat $=x-r a x-a$
SHEET NO 26 BYRD DATE $q / 2 q / 8)$ CHKO MR DATE $3 i+i z i$

For shear$$
g_{x}=0.9(1.13) g_{4} \quad \text { at } 26 \mathrm{~kg},
$$

$f_{v}=\frac{23,500 g_{y}}{4}+\frac{0.4(113) g_{y}(23,500)}{2}=11186 g_{y} / \mathrm{hol} t$

$$
\begin{aligned}
& \left(\frac{f_{t}}{F_{t_{b}}}\right)^{2}+\left(\frac{f_{v}}{f_{v b}}\right)^{2}=1.0 \\
& \left(\frac{13,6877_{y}-6669}{0.601(36,000)}\right)^{2}+\left(\frac{11,186 j 4}{21,600(0,601)}\right)^{2} \leq 1.0
\end{aligned}
$$

$$
\begin{aligned}
& 0.400 g_{y^{2}}-0.390 g_{4}+0.095+0.7425 g_{4}{ }^{2} \leq 1.0 \\
& 1.1425 g_{4}^{2}-0.39 g_{4}-0.905=0 \\
& g_{4}=\frac{0.39 \pm \sqrt{0.39^{2}+4(1.1425)(0.905)}}{2(1.1425)}
\end{aligned}
$$

$$
H C L P F=g_{y}=1.077 \mathrm{~g}
$$

compared to $0.89 \mathrm{~g}$ by

Fragility Method,

B-115 
ENGINEERING. PLANNING AND MANAGEMENT CONSULTANTS

JOB NO $J O B$

HCLPF SHan

SHEET NO 27

CLIENT SUBJECT $H C=\frac{1}{i} E x+i$ BY $2 M C$ Date $2 / 29 / 87$

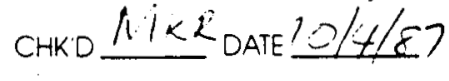

In structure lase - EPRI meth ed Ref $P_{4} .15$ for Equations.

$$
\begin{aligned}
& g_{x}=\frac{0.95}{0.38} g_{y}=1.184 g_{y} \text { at } 26 \mathrm{ltg} . \\
& g_{z}=0.526 g_{4}
\end{aligned}
$$

Tension

$$
f_{b_{2}}=13312 g_{4}-6669
$$

shoer.

$$
\begin{aligned}
& f_{v_{b}}=\frac{23,50094}{4}+\frac{0.4(1.184) 94(23,50)}{2}=11,490 \mathrm{~g}_{4}^{*} / \mathrm{rot}^{\mathrm{d}} \\
& \left(\frac{1331294-6669}{0.601(36006)}\right)^{2}+\left(\frac{1140094}{0.601(21600)}\right)^{2} \leq 1.0 \\
& 0.3786 g_{y}^{2}-0.3793 g_{y}+0.095+0.7766 g_{y}^{2}=1.0 \\
& 1,1552 g_{y} 2-0.3793 g_{y}-0.905=0 \\
& g_{y}=\frac{0.3793 \pm \sqrt{0.3793^{2}+4(1.1552)(0.905)}}{2(1.1552)} \\
& g_{y}=1.06 \mathrm{~g} \\
& H C L P F=\frac{1.06}{0.38}(0.18)=0.50 \mathrm{~g} c \mathrm{cmpared} \text { to } \\
& 0.38 \mathrm{~g} \text { by fragility method. }
\end{aligned}
$$

B-116 
Er

NGINEERING. PLANNING AND MANAGEMENT CONSULTANTS

SHEET NO. 28

JOB NO JOB HCLPF Study BY $R D$ DATE $10 / 2 \theta^{\prime}(U)$

CLIENT SUBJECT Heat Exchougey CHM DATE

Compare pure shear in anchor bolts for cow $H X$ to case analyzed for tension due to overturning and shear.

Bolt shear in uncleared end is

$$
\begin{aligned}
& F_{v}=\left[\left(\frac{W g_{x}}{2 A_{b}}\right)^{2}\left(\left(\frac{0.0)}{4 \mathrm{~W}_{y}}\right)^{2}\right]^{\frac{1}{2}}\right. \\
& A_{b}=.601 \mathrm{in}^{2}, \text { in full section } \\
& W=23,500
\end{aligned}
$$

In structure -

$$
\begin{aligned}
& 9 x=.45 \\
& g_{4}=.9(.38) \\
& \text { far } 0.18 \mathrm{~g} P 64 \\
& F_{v}-0.6 S_{4}=0.6(36)=21.6 \mathrm{ks} 1 \\
& f_{v}=\left[\left(\frac{23.5(.95)}{2(.601)}\right)^{2}+\left(\frac{0.4) 23.5(.9)(.38)}{4(.601)}\right)^{2}\right]^{1 / 2} \\
& f_{v}=8.9 \mathrm{ks} 1 \\
& \text { HCLPF }=\frac{21.6}{8.9}(.18)=0.44 \text { wm } 50 \text { for OT case. }
\end{aligned}
$$

Use $H C C P F=0.44 \mathrm{~g}$ for CDFm method for Tank mounted in structure. (Different failure mode than median calculation)

B-117 
ERE

ENGINEERING, PLANNING AND MANAGEMENT CONSULTANTS

JOB NO O OO HCLPF Study SHEET NO 29 SeE: Heat Exchanger. $\mathrm{CH} \times \mathrm{S}$

DATE $10 / 2 B / e T$ D. TE

Recompute CDFM HCLPF for ground mounted case considering pure shear in the anchor bolts.

from pg. 9, at $26 \mathrm{lt}$ in longitudinal $(x)$ direction, the $g_{x}$ is $\sim 1.13 \times Z P A$. Let lateral $Z P A\left(g_{y}\right)=0.9$ longitudinal $z P A(g x)$.

$$
\begin{gathered}
g_{x}-1.13(.3)=.34 \\
g_{4}=0.9(.3)=.27 \\
f_{v}=\left[\left(\frac{W g_{x}}{2 A_{b}}\right)^{2}+\left(\frac{0.4 W g_{y}}{4 A_{b}}\right)^{2}\right]^{\frac{1}{2}} \\
f_{v}=\left[\left(\frac{23.5(.34)}{2(.601)}\right)^{2}+\left(\frac{0.4(23.5)(.27)}{4(.601)}\right)^{2}\right]^{\frac{1}{2}}=6.73 \mathrm{kst} \\
\text { HCLPF }=\frac{21.6}{6.73}(.3)=0.96 \text { vs } 1.08 \text { for OT case }
\end{gathered}
$$

Use HCLPF $=0.96 \mathrm{~g}$ for ground mounted tank.

B-118 
ERE

ENGINEERING. PLANNING AND MANAGEMENT CONSULTANTS

JOB NO. 87218.01 JOB HCLPF COMPArison CLIENT LLNL SUBJECT BlOCK WO II
SHEET NO 30

BY SSH DATE $9-22-57$

CHR MIR DATE $12 \div ! \div ?$

$$
\begin{aligned}
\beta_{c} & =-\frac{1}{3} \ln \frac{1.0}{2 . y} \\
& =0.25 \\
\beta_{R}=\beta_{u} & =\frac{1}{\sqrt{2}}(0.25) \\
& =0.17
\end{aligned}
$$

Spectral Shape Factor (E Definition) - Structure Response The ewrthgucte is defined as the $84^{\circ} 20$ non-exceedence probability spectrum within the frequency range of interest
for the longer horiz. compton of of notion.

Peak to Peat Variation.

Estimate peck to peek variability, $\beta_{p p} \approx 0.22$

$$
\begin{aligned}
\check{F}_{p p} & =e^{0.20} \\
& =1.22
\end{aligned}
$$

EQ Directional Contemn

The calculated response is bared on the langer of the two hertz. components. Wall failure result e from inly one of the components. The following values are estimated -

$$
\begin{aligned}
& \breve{F}_{\psi}=1.1 \\
& \beta_{\psi}=0.15
\end{aligned}
$$

B-119 
ERE

ENGINEERING. PLANNING AND MANAGEMENT CONSULTANTS

JOB NO 87218.01 JOB HCLPF Comparison

CLIENT LLNL SUBJECT Bock WO.U
SHEET NO 31

BY PSH DATE 9-22-87

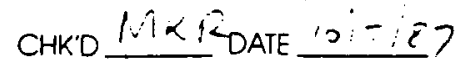

$$
\begin{aligned}
& \text { Total } \\
& F_{\text {st }}=1.22(1.1) \\
& =1.35 \\
& \beta_{\pi}=\sqrt{0.20^{2}+0.15^{2}} \\
& =0.25
\end{aligned}
$$

Damping - Structure Response

Floor spectra were generated for loo structure damping which is judged to be medium centered,

$$
F_{\delta}=1.0
$$

Variability

Estimate 7000 damping is th. 84 on s exceedarec value.

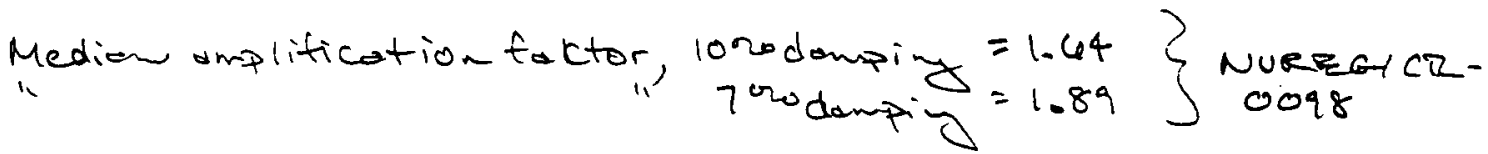

$$
\begin{aligned}
B_{u} & =\ln \frac{1.89}{1.64} \\
& =0.14
\end{aligned}
$$

Estimate $\beta_{R}=0.2 \beta_{U}$

$$
=0.03
$$

Hedelinf Factor- Structure Response

The building model is assumed to be median-cen-ered.

$$
\frac{r}{F_{M}}=1.0
$$

$B-120$ 
EQF

ENGINEERING. PLANNING AND MANAGEMENT CONSULTANTS

SHEET NO $\underline{22}$

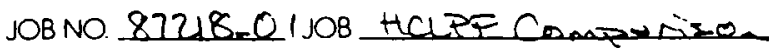

CLIENT LUNL SUBJECT Bock Woll
BV PSH DATE $9-22-87$

CHKD $14 \hat{K}$ DATE $10 / 5 / 87$

Estimate $\left.\begin{array}{rl}\beta_{m s} & \approx 0.15 \\ \beta_{x} & =0.30\end{array}\right\}$ Tyicalvalues

Fundamital freg. $27.2 \mathrm{~Hz}$

$$
\begin{aligned}
f_{+13} & =7.2 e^{0.30} \\
& =9.7 \mathrm{~Hz}
\end{aligned}
$$$$
\log A F=\frac{\log 1.64-\log 1}{\log 8-\log 33}(\log 9.7-\log 33)+\log 1
$$$$
A F=1.53
$$$$
\beta_{m+}=-\ln \frac{1.53}{1.64}
$$$$
=0.07
$$

$$
\begin{aligned}
f-\beta & =7.2 e^{-0.3} \\
& =5.3 \mathrm{~Hz}
\end{aligned}
$$$$
\beta_{m+}=0
$$

Estimote $\beta_{m}=0.04$

$$
\begin{aligned}
\beta_{v} & =\sqrt{0.15^{2}}+0.04^{2} \\
& =0.16
\end{aligned}
$$

B-121 


\section{EQE}

ENGINEERING. PLANNING AND MANAGEMENT CONSULTANTS

JOBNO $87218.01 \mathrm{JOB}$ HCLPF Comperison BY. PSH DATE $S-22-6]$

CLIENT SUBJECT Blockurall CHKDNKR DATE $10:=\mid \overline{2}$ ?

Soil-Structure Interaction

$F_{\text {SSE }}=1.0$

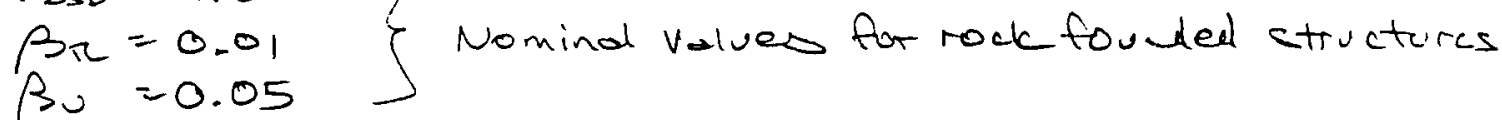

Structure Inclastic Respone

Structure inelestic response results in a reduction in structure frefuency, which would imply an increase in block wall responac. However, inelestic response of the block wall causes a reduction in fuall firequeniy un resuting shift awdy fro the floor spectrum peot. The block Soll response as calculated is judjed to be median-centered. Per Lin * Mahi-, the mean amplificution feltor for high period egut. response duc to nonlinearstructure resio. he is about lal for stiffuss degrading structures, Estinutc this is a upper boud for a nonlinear component.

$$
\begin{aligned}
\beta_{2} \approx \beta_{0} & =\frac{1}{1.45}<\frac{1.1}{1} \\
& =0.04 \ldots
\end{aligned}
$$




\section{COMPONENT 5}

\section{REINFORCED BLOCK WALL}




\section{EQE}

ENGINEERING. PLANNING AND MANAGEMENT CONSULTANTS

SHEET NO

JOBNO $.87218 .01 \mathrm{JOB}$ HCLPE Camponiron BY PSH DATE $9=30-87$

CLIENT LLNL_SUBJECT Flock USall CHK'DMKR DATE 10-5-87

BLOCK WALL HCLPF CAPACITY 


\section{EQE}

ENGINEERING, PLANNING AND MANAGEMENT CONSULTANTS

JOBNO. $87218.01 \mathrm{JOB}$ HCLPF COmparison BY. PSH DATEQ $9-28-87$

CLIENT LENL SUBJECT Block Woll

\section{Summary}

The somple tlock whll ultimate strength and lood-deflection relationship is determined following the recommendations of the ACI-SEASC Tark Committee On Slender Walls CRet. ID. Frogilities om developed for two cases using different stiftness assumptions:

1. Equivalent elastic-plastic load-deflection curve.

2. Secant stiffiness

The HCLPF cafacity for the first case is calculated to be $0.32 \mathrm{~g}$. This volue is likely conservative since it doesn t explicitly account for th wall response shifting down from the rising pa 1 of the floor spectrun, Wh HCLPF cepacity for tue second case is calculated to be 0.48 \&

The block wall HCLPF capacity thil't fine grester of thetwo calculated values:

HCLPF copacity $=0.48 \mathrm{~g}$.

By the CDFM mathod, the HCLPF Copecity is also culculated to te $0.48 \mathrm{~g}$. 


\section{EQE}

ENGINEERING, PLANNING AND MANAGEMENT CONSULTANTS

SHEETNO BW- 2

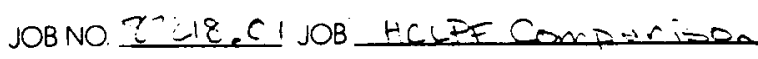
BY PSH

DATE $9-18-87$

CLIENT SUBJECT

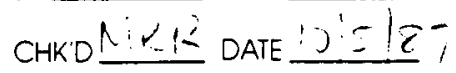

1.uteriol Properties

Rein Frcement

Estimated velues for Grade let reber:

$$
\begin{aligned}
& t_{\text {nam }}=69 \mathrm{kt} \\
& \text { EOV }=0.07
\end{aligned}
$$

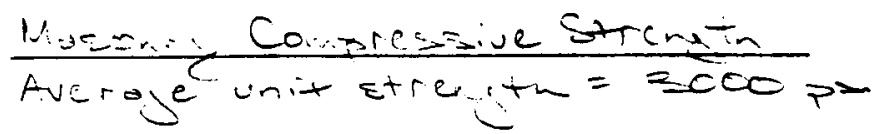

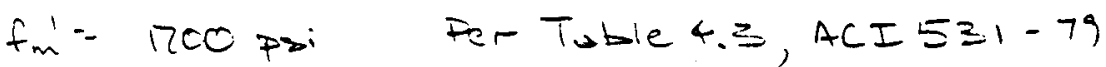

Fustimated values:

$$
\text { Medien fri } x \text { 2000 pi: }
$$

Merter Compressive Streath

Es-imic vilue for Type Smartor

$$
\begin{aligned}
\text { mon } & =1.4(1800) \\
& =2500 p>i
\end{aligned}
$$

$\operatorname{cov}=0.2$

\section{Other Necessory Det}

Other doto necessur to determine the w-ll HCLPF caparity is contained in the tronsmittal to the HeLPF Study Group (Ret. \&). 


\section{EQE}

ENGINEERING. PLANNING AND MANAGEMENT CONSULTANTS

SHEET NO Bw-3

JOBNO Q7IJZ, OI JOB HCLPF Conaporison

BY PSH DATE $9-21-87$

CLIENT LLNL_SUBJECT Bock Uall

CHKO I:KK DATE $13 / 5 / 87$

The force-deformation clive will be established followin: the recommedations of Ref.1. This paroch appears to be consistent with the results of other testing shown in Figures 3.7 and 3.8 af Ret. 2 .

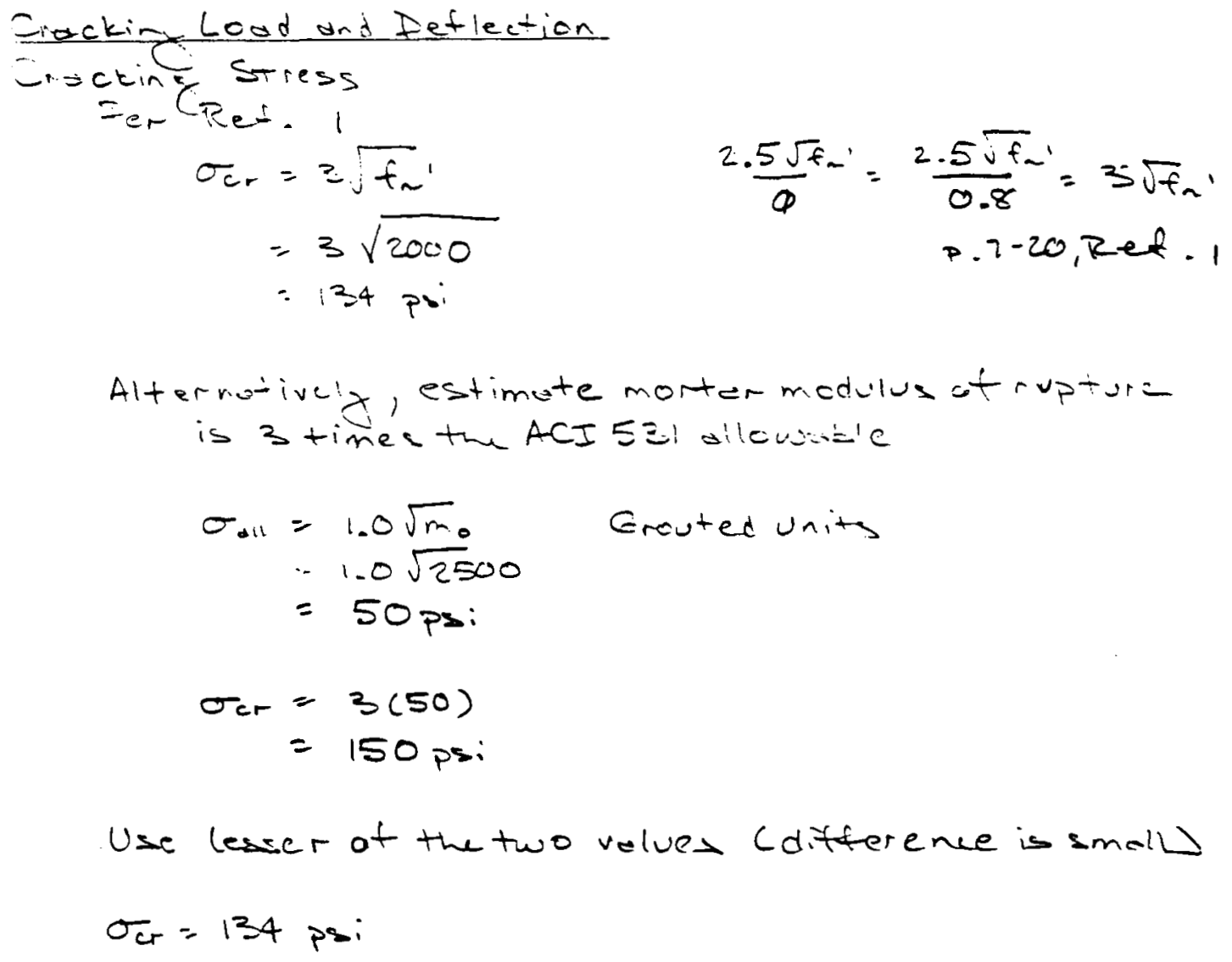

Use lesser of the two veluet (difference is small $\sigma_{c r}=134$ psi 
EDE

ENGINEERING, PLANNING AND MANAGEMENT CONSULTANTS

SHEET NO $: N-4$

JOB NO \&7218.01 JOB HCLPF Comparison BY PST DATE $9-21-87$ CLIENT LNL SUBJECT Block UN all CHKD $1: K R$ DATE! ): $-1<?$

Cracking Load

$$
\begin{aligned}
S & =\frac{4340}{11.63 / 2} \\
& =230 \mathrm{in} 3 / \mathrm{f}
\end{aligned}
$$

$$
M_{c r}=230(134)
$$$$
\text { - 30,800 in- } 1 \mathrm{~b} / \mathrm{ft}
$$$$
=2570 \mathrm{fr}-\mathrm{b} / \mathrm{G}
$$

$$
\begin{aligned}
\omega_{c r} & =\frac{2(2570)}{10^{2}} \\
& =\equiv 1 \text { psf }
\end{aligned}
$$

$$
\begin{aligned}
& \text { Cracking Defection } \\
& \text { =ar }=B 40 \mathrm{in}^{4} / \mathrm{f} \\
& E_{n}=1000 \mathrm{fm}^{\prime} \\
&=2.0 \times 10^{6} \mathrm{Ps} \\
& \Delta_{c r}=51(1)^{4}(1728) \\
& 8\left(2.0 \times 10^{6}\right)(1340) \\
&=0.041 \mathrm{in}
\end{aligned}
$$

B-128 
EQF

ENGINEERING. PLANNING AND MANAGEMENT CONSULTANTS

JOBNO 87218.01 JOB HCLPF Comparison CLENT LLNL SUBJECT Brock WUII
SHEET NO. 3W-5 BY PSH DATE $9-21-87$ CHKO MKK DATE j: $1 \bar{\varepsilon} 7$

$\frac{\text { Mield Load and Deflection }}{\text { Cracked Moment ot Inerti }}$

$$
\begin{aligned}
A_{s} & =0.31 \mathrm{in}^{2} / 16 " \text { \#5@16" } \\
& =0.233 \mathrm{in}^{2} / \mathrm{ft} \\
n & =\frac{29 \times 10^{6}}{2.0 \times 10^{\circ}} \\
& =15
\end{aligned}
$$$$
d=5.8^{\prime \prime}
$$

Ascume $(k d)>t_{f}$

$$
\begin{aligned}
& \frac{1}{2}(1 k)(k d)^{2}-\frac{1}{2}(5.9)[(k d)-1.5]^{2}-15(0.31)[5.8-(k d)] \\
& =0 \\
& 5.05(k d)^{2}+13.50(k d)-33.61=0 \\
& \text { Ckd }\rangle=1.57^{11}>t_{+} o k \\
& \pm=\equiv i r \\
& \text { Icr }_{\text {c }} \frac{1}{3}(16)(1.57)^{3}-\frac{1}{3}(5.9)(1.57-1.5)^{3} \\
& +15(0.31)(5.8-1.57)^{2} \\
& =104 \mathrm{in} / \text { block } \\
& =78 \mathrm{in}^{4} / \mathrm{fH}
\end{aligned}
$$

Yield Loed

$$
\begin{aligned}
f_{y m} & =69 \mathrm{ksi} \quad \quad \text { P. Bw-2 } \\
M_{y} & =\frac{69,000(78)}{15(5.8-1.57)} \quad \text { Nejlecting } 8 \text { aiel load } \\
& =84,800 \mathrm{in}-1 \mathrm{~b} / \mathrm{ft} \\
& =7070 \mathrm{ft}-1 \mathrm{~b} / \mathrm{t}
\end{aligned}
$$

B-129 


\section{EQE}

ENGINEERING, PLANNING AND MANAGEMENT CONSULTANTS

JOBNO 21218.CI JOB HCLPF CEmatrison BY PSH DATE $9-3.87$

CLIENT SUBJECT Black Woll CHKD $\because \because \because$ DATE

$$
\begin{aligned}
w_{y} & =\frac{2(7070)}{10^{2}} \\
& =141 \text { p }=t
\end{aligned}
$$

yield Deflection

Per Ref.1, the additionol deflection in excess of that ius ung cracking is based on the crocked memert
of inerfia.

$$
\begin{aligned}
\Delta_{y} & \left.=0.041+\frac{\left(141-\frac{51)(10}{4}\right)^{4}(1728)}{\left(2.0 \times 10^{4}\right.}\right)(78) \\
& =0.041+1.25 \\
& =1.29 \mathrm{in}
\end{aligned}
$$

Ultimete Strength

Fer Refs. 1" the witimate strength can be determined using the equivalent stress bleck uproach devaleped for reintorced concrete, txisl leed acting an estimet ed medien cepacity of about $1.5 \mathrm{~g}$ PEA will be included. Rebor Etrain-hüdeniz is neglected.

Eftective Axial Load

$$
\begin{aligned}
& P_{D L}=111(10) \\
& =1110 \mathrm{lb} / \mathrm{ft} \\
& \text { Wall weighs } 111 \text { p pt, p. 2, somple calc, } \\
& \text { Vert. } Z P A=0.20 \mathrm{x} \\
& =1.678 \\
& \begin{array}{l}
0.18 \mathrm{~g} \text { PEA } \\
1.5 \mathrm{~g} \text { PEA }
\end{array} \\
& P=1110[1-0.4(1.67)] \\
& 4000 \text { of mex vend respones } \\
& =370 \text { pif }
\end{aligned}
$$


EQF

ENGINEERING. PLANNING AND MANAGEMENT CONSULTANTS

SHEET NO BW-7

JOBNO. $87218.01 \mathrm{JOB}$ HCLPF Comporison BY PSH DATE $9-21-87$ CLIENT LLNL SUBJECT Block U2LU CHKDI'KK DATE $! 3 !<?$ ?

$$
\begin{aligned}
& f_{m}{ }^{\prime}=2000 \text { psi p. Bw-2 } \\
& a=\frac{0.233(69,000)+370}{0.85(6000)} \\
& 0.85(2000)(12) \\
& =0.81 \text { in }<t_{+} \text {ot } \\
& M_{u}=[0.233(69,000)+370]\left(5.8-\frac{0.81}{2}\right) \\
& \begin{aligned}
& 88,760 \mathrm{in}-16 / \mathrm{f} \\
= & 7400 \mathrm{fH}-16 / 4
\end{aligned} \\
& \omega_{0}=\frac{2(7400)}{10^{2}} \\
& =148 \text { p } t
\end{aligned}
$$

B-131 


\section{EQE}

ENGINEERING. PLANNING AND MANAGEMENT CONSULTANTS SHEET NO $3 w-8$

JOBNO 87218.01 JOB HCLPF COmperison BY PSH DATE $9-21-87$ CLIENT LLNL SUBJECT Bloch WhII

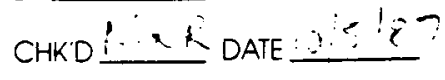

\section{Lood - Deflection Curve}

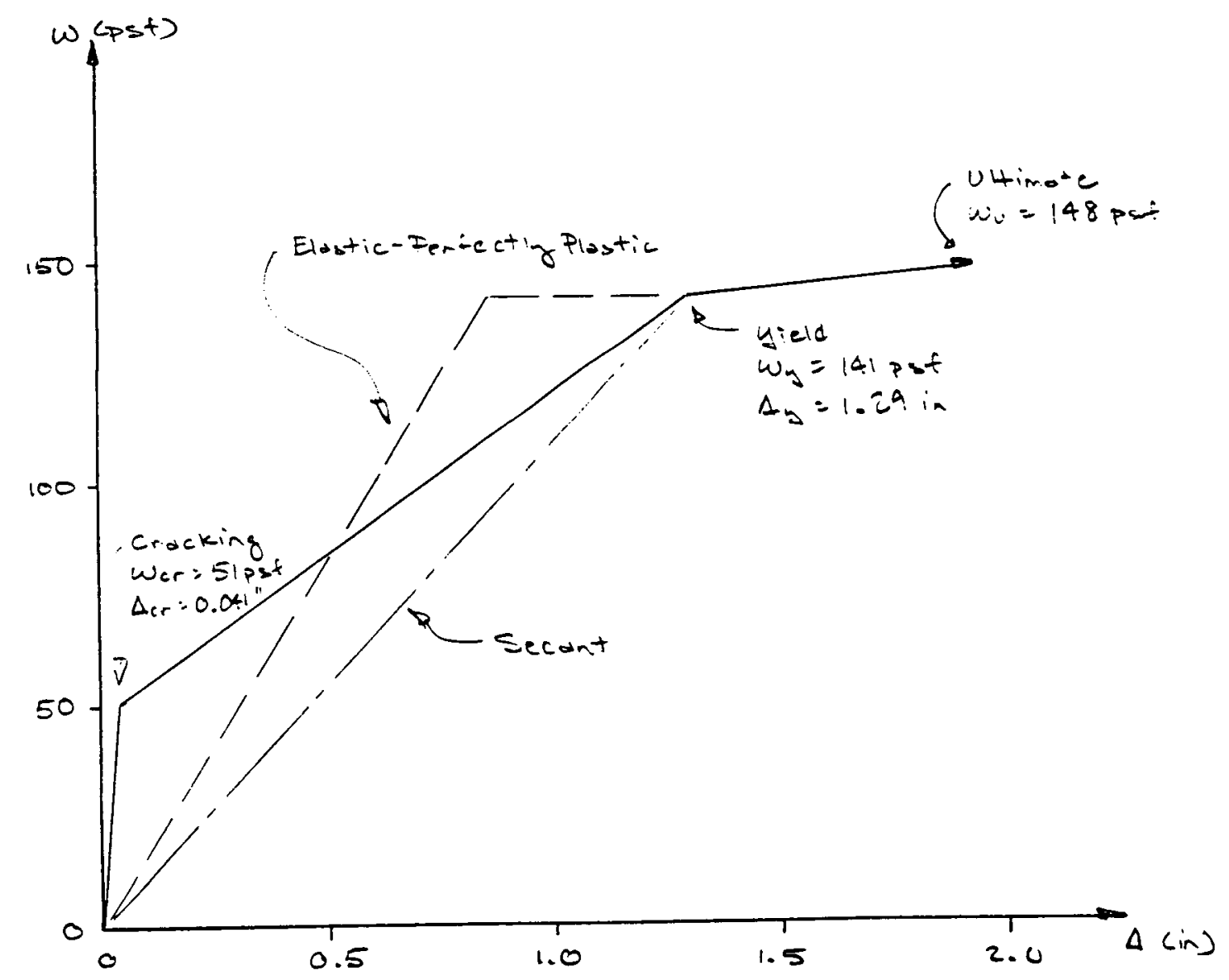


ERE

ENGINEERING. PLANNING AND MANAGEMENT CONSULTANTS

JOB NO 8728.01 JOB HCLPF COMparison CLENT LLNC SUBJECT Block Wall
SHEET NO BW-9

BY. PSHL DATE 9-20-87 CHM

DATE

i?

Equivalent Elastic Stiffness

Depending on the applied load, the wall stiffness may

van from the uncrocked ( $I=1340 \mathrm{in} 4 / \mathrm{ft}$ ) to the cracked CI 78 in $^{4} / f+$ ) values. Because the wall response and seismic cepecity will be sensitive to frequency and this sittings, two condiclate values will be investigated:

1. Elastic stiffness of the elastic-pertediyflestic load-deflection curve that preserves the tot 1 energy up to yield.

2. Secant stiffness to yield. (- viewer bound value).

Equivalent Elastic -Perfectly Plastic Curve

$$
\begin{aligned}
& E=\frac{1}{2}(51)(0.041)+\frac{1}{2}(141+51)(1.29-0.041) \\
&=121 \\
& \frac{1}{2}(141)\left(\Delta_{e}\right)+141\left(1.29-\Delta_{e}\right)=121 \\
& \Delta_{c}=0.8611 \\
& I_{e}=\frac{141(10)^{4}(1728)}{8\left(2.0 \times 10^{6}\right)(0.86)} \\
&=177 \mathrm{in} / \mathrm{f}
\end{aligned}
$$

Secant Stiffness

$$
\begin{aligned}
I_{e} & =141(10)^{4}(1728) \\
& =118 \mathrm{in}^{4} / \mathrm{ft}^{4}(1.29)
\end{aligned}
$$

B-133 
ERE

ENGINEERING PLANNING AND MANAGEMENT CONSULTANTS

JOB NO. 87218.O1 JOB HCLPFCOAparisen CLIENT LLNK SUBJECT Block W2.11
SHEET NO $3 w-10$ BY FSH DATE $9-21-87$

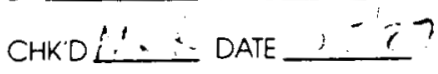

Fragility Based On Elastic - Perfect y Plastic Curve

Fundament 1 Frequency

$$
\begin{aligned}
& I_{e}=177 \mathrm{in} 4 / \\
& \bar{m}=0.0239\left(b-\sec ^{2} / \mathrm{in}^{2} / \mathrm{fd}\right. \\
& L=120^{11} \\
& E=2.0 \times 10^{\circ} \mathrm{psi} \\
& \omega=1.875^{2} \sqrt{\frac{2.0 \times 10^{6}(177)}{0.0239(120)^{4}}} \\
& =29.7 \mathrm{rps} \\
& f=4.7 \mathrm{~Hz}
\end{aligned}
$$

p. 3, sample call, Rets

Seismic Loads

Because block wall inelastic energy absorption will be explicitly accounted for, estimate median damping of
7020 .

$$
\begin{aligned}
S_{0} & =0.65 \mathrm{~g} \quad f=4.7 \mathrm{~Hz}, \xi=720 \\
\omega & =0.65(111) \\
& =72 \mathrm{ps} \\
V & =72(10) \\
& =720 \mathrm{plf} \\
M & =\frac{1}{2}(72)(10)^{2} \\
& =3600 \mathrm{ft}-1 \mathrm{~b} / \mathrm{tt}
\end{aligned}
$$

B-134 


\section{EQE}

ENGINEERING. PLANNING AND MANAGEMENT CONSULTANTS

SHEETNO BW-11

JOBNO 87218. C1 JOB HCLPF Comparisen BY PS DATE $0-21-87$ CLIENT SUBJECT CHKD! $!$ DATE

Strensth Foctor

$w=72$ pst

$\omega_{u}=148$ psf

$\tilde{F}_{s}=\frac{148}{72}$

$=2.05$

Uncertaint?

Nioterial Properties

strengthis primanily a function of twe reintoreme $=0$

$\begin{aligned} \beta & =\beta+y \\ & =0.07\end{aligned}$

Accurscy of Strentih Egution, Workmonshis

$A$ $\Phi$ fector of 0.8 is recommended fir utimote strentin design of masonry in Rets. Is.a 2.

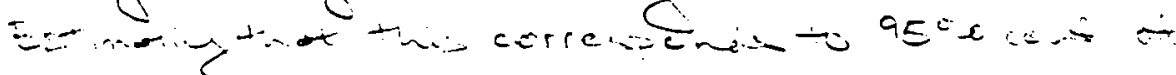
excederece

$$
\begin{aligned}
\beta= & -\frac{1}{1.4 \leq} \leq-\frac{0.8}{1.0} \\
& =0.14
\end{aligned}
$$

The following veriabilities con be determined from Fig. 4 of Ret. 3 :

For $\alpha=0.59$

$$
\begin{aligned}
\left(\frac{M_{y}}{M_{n}}\right)_{50 w_{0}} & =1.1 \\
\left(\frac{M_{0}}{M_{n}}\right)_{500} & =0.93 \\
E & =-\frac{1}{1.05} \ln \frac{0.93}{1.1} \\
& =0.10
\end{aligned}
$$




\section{EQE}

ENGINEERING. PLANNING AND MANAGEMENT CONSULTANTS

SHEETNO BW-12

JOBNO $97218.01 \mathrm{JOB}$ HCLPF COMPeriso. BY PSH DATE $9-21-87$

CLIENT LLNL SUBJECT Elock hisil CHKD MIKL DATE $1-?$

For $\alpha=0.33$

$$
\begin{gathered}
\left(\frac{\mu_{0}}{\mu_{n}}\right)_{5020}=1.0 \\
\left(\frac{\mu_{0}}{\mu_{m}}\right)_{500}=0.85 \\
B=-\frac{1}{1.65} \ln \frac{0.85}{1.0} \\
=0.10
\end{gathered}
$$

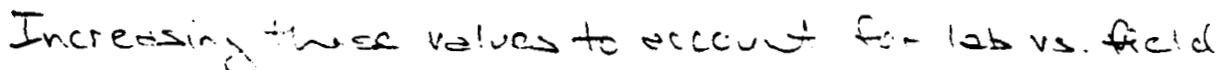

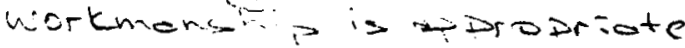

Estimate \& $\beta$ at $C .14$.

$B=\sqrt{0.07^{2}-0.14^{2}}$

$=0 . i \mathrm{~s}$

Increese to 0.18 to acceut for other souicey l block cite, relaur erea, ete.)

$\beta u=0.18$

\section{Inelastic Energ Absorption Factor}

loeding on the wolle tested in Ref. I was stopped when it wes judjed that feilure wasnear. Comsarison of meximum deflections to calculated yield deflections indicutes ratios varyin $\&$ from 1.1 to 3.0 with on overgeg of ubout 1.7 fur the concrete block walls, Comparable values ure exhibited bx Figs, 2.7 al 3.8 of Ref. 2. Estimate a medienductility tC octios! foilure of 2.0 .

The calculoted ultinote deflection is then:

$$
\begin{aligned}
\Delta_{u} & =2.0(1.3) \\
& =2.6
\end{aligned}
$$




\section{EQF}

ENGINEERING. PLANNING AND MANAGEMENT CONSULTANTS

SHEET NO BW-3

JOBNO 27218.01 JOB HCLPF Comperiser BY PSH DATEQ $-22-8]$

CLIENT LLNL SUBJECT Biock Wiell CHKD MKR DATE $10 / 5 ! 8 ;$

The effective ductility rotio for the equivalest elastic perfect $y$ plostic lood-deffection curve is then:

$$
\begin{aligned}
\mu_{e} & =\frac{\Delta u}{\Delta e} \\
& =\frac{2.6}{0.86} \\
& =3.0
\end{aligned}
$$

The inclest ic energy ubsorption factor will be determined usin. Riddell-Newriouklnay be conservetive since the estimated frequency is a the sot side of the peakl. Mecion domping of toro is judjed to be apprapriote when ductile res-ories is explicity acceutied for. Estimatc a duection coctficiant, $C_{n}$ of unity is pptopriste (EQ's centered on M6.3). $c_{1}=3.0(7)-0.30$

$-1.67$

$2=1.67-1$

$=2.67$

$r=0.48(7)^{-0.08}$

$=0.41$

$\ddot{F}_{\mu}=[2.67(3.0)-1.67]^{0.41}$

$=2.1$

Variability

Estimate $F_{\mu}=1.0$ is 30 below mean, rondemness and uncerteinty unc about egual. 
EQE

ENGINEERING. PLANNING AND MANAGEMENT CONSULTANTS

JOBNO 87218.0 JOB HCLPF COMPUirison CLENT LCNL SUBJECT Block WOII
SHEET NO BW-14 BY FSH DATE $9-22-57$ CHKD N/KR DATE $10 / 5 / 87$

$$
\begin{aligned}
\beta_{c} & =-\frac{1}{3} \ln \frac{1.0}{2.1} \\
& =0.25 \\
\beta_{R} & =\beta_{0}=\frac{1}{\sqrt{2}}(0.25) \\
& =0.18
\end{aligned}
$$

Szcctrol Siroce Factor (EQDefinition) - Structurc Tesi-snde The eurthqueke is defined as the 8406 non-exceedence probubility pectiun within the freguency ronce of interes for the lager heriz. compronct of notion.

Peak to Peak Variation

Estimate feek to plek variability, $\beta_{p p} \approx 0.20$

$$
\begin{aligned}
\check{F}_{p p} & =e^{0.20} \\
& =1.22
\end{aligned}
$$

EQ Directional Content

The colculated response is bered on the lenger of the two heriz. components. Woll foilure resulk from oniz one of the components. The following voluce afe estimated:

$$
\begin{aligned}
& \check{F}_{\psi}=1.1 \\
& \beta_{4}=0.15
\end{aligned}
$$

$B-138$ 
EQE

ENGINEERING. PLANNING AND MANAGEMENI CONSULTANTS

JOBNO 87218.0108 HCLPFCOmporiaen CLENT LLNL SUBJECT Bloek WD.U
SHEET NO. PW-15 $B Y . P S H$ DATE 9-22-87 CHKD NIKR DATE $0 / 5 / 87$

$$
\begin{aligned}
& \text { Totel } \frac{F_{s A}}{F_{s A}}=1.22(1.1) \\
& =1.35 \\
& \beta_{R}=\sqrt{0.20^{2}+0.15^{2}} \\
& =0.25
\end{aligned}
$$

Damping - Structure Resions

Foor spectro were generated for lCon strultura ciamping which is judged to fermedian centered at wall tailure. The structure fundamatal mode is at about $7.2 \mathrm{~Hz}$ (ampliticel ing-) $\dot{F}_{\delta}=1.0$

Verivbiity

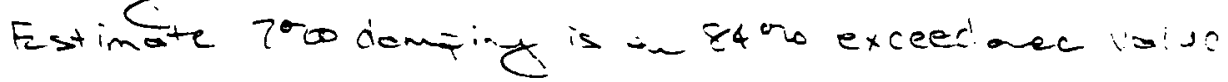

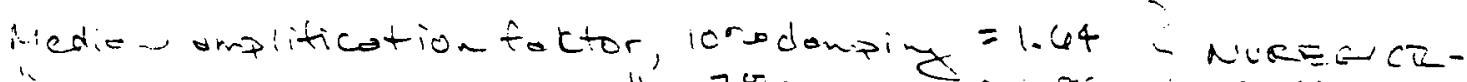

$$
\begin{aligned}
& 70 \text { oumping }=1.89-0098 \\
& E_{u}=\ln \frac{1.89}{1.64} \\
& =0.14 \\
& =0.03
\end{aligned}
$$

Hedeling Fuctor- Structure Response

The building nodel is assumed to be medion-centered.

$$
\vec{F}_{M}=1.0
$$

B-139 
EQE

ENGINEERING. PLANINING AND MANAGEMENT CONSULTANTS

SHEETNO. BW-16

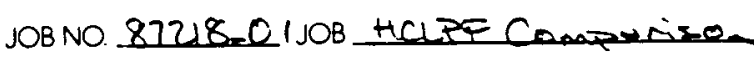

CLIENT LiNL SUBJECT Bock Woll
BY PSH DATE $9-22-87$

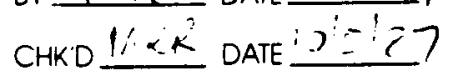

Estimate $\left.\begin{array}{rl}\beta_{m s} \approx 0.15 \\ \beta_{x}=0.30\end{array}\right\}$ Tyicalvolucs

Fundometol freg. $27.2 \mathrm{~Hz}$

$$
\begin{aligned}
f_{+1 / 3} & =7.2 e^{0.30} \\
& =9.7 \mathrm{~Hz}
\end{aligned}
$$$$
\log A F=\frac{\log 1.64-\log 1}{\log 8-\log 33}(\log 9.7-\log 33)-\log 1
$$$$
A F=1.53
$$$$
\beta_{m+}=-\ln \frac{1.53}{1.64}
$$$$
=0.07
$$

$$
\begin{gathered}
f-\beta=7.2 e^{-0.3} \\
=5.3+1 z \\
\beta_{m x}=0
\end{gathered}
$$

Estimote $\beta_{m t}=0.04$

$$
\begin{aligned}
\beta_{v} & =\sqrt{0.15^{2}}-0.04^{2} \\
& =0.16
\end{aligned}
$$

$B-140$ 


\section{EQE}

ENGINEERING, PLANNING AND MANAGEMENT CONSULTANTS

JOBNO 8?218.01 JOB HCLPF COMParison BY PSH DATE $9-22-82$

CLIENT SUBJECT Block woll

Soil-structure Interaction

$\left.\begin{array}{l}F_{S S E}=1.0 \\ \beta_{R}=0.01 \\ \beta_{0}=0.05\end{array}\right\}$ Nominal values for rock fou ded ctructurcs

Structure Inclustic Response

structure inelestic response results in a reduction in structure frequency, which would inply an incrcase in block wall response. However, inelastic response of the block wall causes a reduction in fus ll frequeniy un resulting shift away fre the floor spectrum peot. The black Sall repponis $\rightarrow$ celculated is judjed to be median-centerd Fer Lin * Mohine the mean amplitication finctor for hijh perid egmt. responte duc to nonlinearstructure resiente i: about 1.1 for stiffuss degradiz structures. Estimatc this is a upper boud for a nönlinear componen

$$
\begin{aligned}
\beta_{2} \approx \beta_{0} & =\frac{1}{1.05} \ln \frac{1.1}{1} \\
& =0.06
\end{aligned}
$$


EQF

ENGINEERING. PLANNING AND MANAGEMENT CONSULTANTS

JOBNO $87218.01 \mathrm{JOB}$ HCLPF Comperison CLIENT LLNL SUBJECT Bleck-WOall
SHEET NO. \$W-18 8Y PSH DATE $9-22-87$ CHKDMKR DATE :J:S!8?

Peok Broodeniy 4 Smoothing

The fleor fectro dre assumel to be row spectro.

$$
\begin{aligned}
& F_{s_{S_{1}}}=1-0 \\
& \beta_{n}=\beta_{u}=0 \\
& F_{s=2}^{v}=1.1 \\
& \beta_{0}=0.05
\end{aligned}
$$

Dompiny- Elock Woll

Estimerte Solo damping is a -lo voluc for block wall when inciestic response isexplicitiz accouted for.

$$
\begin{aligned}
s_{0} & =0.75 \\
\beta_{0} & =\ln \frac{0.75}{0.65} \\
& =0.14 \\
\beta_{2} & =0.2\left(B_{0}\right) \\
& =0.03
\end{aligned}
$$

B- 142 


\section{EQE}

ENGINEERING. PLANNING AND MANAGEMENT CONSULTANTS

SHEET NOQW 19

JOBNO $21218.0110 B$ HCLPF COmparison

BY PSH DATEq-22-d7

CLIENT SUBJECT Block Woll CHKO MKR DATE $10 / 5 / \varepsilon 7$

Modeling - Block W-ll

Estimote $\beta_{e}=0.10$

$$
\begin{aligned}
f=f_{+1 \beta} & =4.7 e^{0.10}=5.2 \mathrm{~Hz} \\
S_{0} & =0.8 \mathrm{~g} \\
\beta_{m f} & =\ln \frac{0.8}{0.65} \\
& -0.21 \\
f=f_{-1 \beta} & =4.7 e^{-0.10}=4.3 \mathrm{~Hz} \\
S_{0} & =0.5 g \\
\beta_{n f} & =-\ln \frac{0.5}{0.65} \\
& =0.26
\end{aligned}
$$

Fstimate $\beta_{u} \approx 0.25$ Cincreased to accunt tor use of effective elastic stiftress, derivation of fregility fram equiralet uniform lode, cte.). 
EQF

ENGINEERING. PLANNING AND MANAGEMENT CONSULTANTS

JOBNO $\$ 91218.01$ JOB HCLEF COMperison CLENT LWL SUBJECT Block WaU
SHEET NO $\$ 10-20$ BV PCH DATE $-22-87$ CHKD MIKR DATE $10 / 5 i \varepsilon 7$

Meda! Combinotion

Respore is predominomty intivenced by the findomental recil. Estin-ate $\beta_{R}=0.05$.

Combination of EQ Conporenty

Fuilule of the wall cecurs primorily due to response in esingle horizcriel direction, while verticol respo-se nes rinor effect on the resistarce. Estimate $E_{R}=0.05$

Overall Fregility Pormeters

$$
\begin{aligned}
\check{F} & =2.05(2.1)(1.35)(1.1) \\
& =6.4 \\
\check{A} & =6.4(0.18 g) \\
& =1.158 \\
\beta_{R} & =\left[0.18^{2}+0.25^{2}+0.03^{2}+0.01^{2}+0.06^{2}+0.03^{2}+0.05^{2}+0.05^{2}\right]^{1 / 2} \\
& =0.32 \\
\beta_{u} & =\left[0.18^{2}+0.18^{2}+0.14^{2}+0.16^{2}+0.05^{2}+0.06^{2}+0.05^{2}+0.14^{2}+0.25^{2}\right]^{1 / 2} \\
& =0.45
\end{aligned}
$$

B-144 


\section{EQE}

ENGINEERING. PLANNING AND MANAGEMENI CONSULTANTS

SHEETNO BW-24

JOB NO $87218.01 \mathrm{JOB}$ HCLPF COmp + rison BY. PSH DATE $9-25-67$

CLIENT UNL SUBJECT Blocle Wall CHKD MKK DATE $: 2 / 5 / \hat{c} ?$

HCLPF Casacity

$$
\begin{aligned}
\text { HCLPF Copacitg } & =1.15 \mathrm{~g} e^{-1.45(0.32+0.45)} \\
& =0.22 \mathrm{~g}
\end{aligned}
$$




\section{EQE}

ENGINEERING. PLANNING AND MANAGEMENT CONSULTANTS

SHEETNO 3w-22

JOBNO 97218.01 JOB HCLPE Comparison BY. PSH DATE $9-25-87$

CLIENT UNL SUBJECT Flock UDAll CHKD MKR DATE $13 / 5 / 27$

\section{Fropilit Boied On Secont Stiftness}

$$
\begin{aligned}
& \begin{array}{l}
\text { Fundamental Frequency } \\
I_{e}=118 \mathrm{in}^{4} 14
\end{array} \\
& f=4.7 \sqrt{\frac{118}{177}} \\
& =3.8 \mathrm{~Hz} \\
& \text { Seizmic Loods } \\
& S_{0}=0.40 \gamma \quad f=3.8+1 z, \xi=700 \\
& \begin{aligned}
\omega & =0.40(111) \\
& =44 \text { pot }
\end{aligned} \\
& V=44(10) \\
& =440 p^{4} \\
& M=\frac{1}{2}(4-4)(10)^{2} \\
& \text { - } 2200 \mathrm{ft}-\mathrm{lb} / \mathrm{fa}
\end{aligned}
$$

Strenth Foctor

$\omega=44$ pt

$\omega_{u}=148$ put

$F_{8}=\frac{148}{44}$

$=3.4$

$\beta_{0}=0.18$ 
ERE

ENGINEERING. PLANNING AND MANAGEMENT CONSULTANTS

JOB NO \$7218.0 JOB HCLPF COmparison CLIENT LLNL SUBJECT BlOCK Wo ll
SHEET NO. B 10.23 BY SSH DATE $9-25-87$ CHKD MK DATE $\{1-\mid \bar{\varepsilon}]$

Inelastic Energy sorption Factor

Rose effective ductility ratio and $F_{\mu}$ on ratio of ultimate to yield deflections. This is somewhat conservative since energy in the actual lood-deflection curve above the secant curve is neglected.

$$
\begin{aligned}
& \Delta_{y}=1.311 \\
& \Delta_{v}=2.6^{\prime \prime} \\
& \mu=2 \\
& \left.\breve{F}_{\mu}=[2.67(2)-1.6]\right]^{0.41} \\
& =1.7 \\
& \beta_{1}=-\frac{1}{3} \ln \frac{1}{1.7} \\
& =0.17 \\
& \beta_{R}=\beta_{U}=\frac{1}{\sqrt{2}}(0.17) \\
& =0.13 \\
& \frac{\text { Modeling }- \text { lect W.U }}{\text { Estimate } \beta_{+}=0.10} \\
& \begin{array}{c}
f=f_{+1 \beta}=3.8 e^{0.10}=4.2 \mathrm{HE} \\
S_{-}-0.48 \mathrm{~g}
\end{array} \\
& B_{\text {me }}-\ln \frac{0.48}{0.40} \\
& =0.18
\end{aligned}
$$

B-147 


\section{EQE}

ENGINEERING. PLANNING AND MANAGEMENT CONSULIANTS

SHEET NO BW 24

JOBNO 87218.01 JOB HCLPF COmporison BY PSH DATEQ $25-87$

CLIENT CWU SUBJECT Black Wr 11 CHKD MKR DATE $2:-\frac{1}{\mathrm{C}} \mathrm{C}$

$$
\begin{aligned}
f=f_{-1 \beta} & =3.8 e^{-0.10} \\
& =3.4 \mathrm{~Hz} \\
S_{-} & =0.38 \delta \\
\beta_{m s} & =-L-\frac{8.38}{0.40} \\
& =0.05
\end{aligned}
$$

Fstinate $F_{0}=0.20$ cheigted towends. The atitter side since this frejuemey should be more of -lawer bound, includes boost for use of unitirm pressure, ete.)

\section{Querall Fragility Porameters}

Other fectiors and variabilitics should be about the some as previously calculated for the eiestic-perfeaty plastic cace. Valves mox be somenuat difterest since wall response occurs in the freguency ronge where the floor epectra are not etroyly influened by structure response. However, the HCupf capaeity should nof be very sensitive to this proximstion. 
EDE

ENGINEERING. PLANNING AND MANAGEMENT CONSULTANTS

SHEET NO. BW-25

JOB NO 87218.01 JOB HCLPF Comparison

CLIENT LLNL SUBJECT Block Wd el) DATE $9-25-87$

$$
\begin{aligned}
& F=3.4(1.7)(1.35)(1.1) \\
& =8.4 \\
& \begin{aligned}
\tilde{A} & =8.6(0.18 \mathrm{~g}) \\
& =1.55 \mathrm{~g} v \mathrm{v} .1 .15 \mathrm{~g}
\end{aligned} \\
& \beta_{R}=\sqrt{0.32^{2}}-0.17^{2}+0.13^{2} \\
& -0.30 \\
& \text { vs. } 0.32 \\
& \beta_{u}=\sqrt{0.45^{2}-0.17^{2}}+0.13^{2}-0.25^{2}+0.20^{2} \\
& =0.41 \\
& \text { vs. } 0.45 \text {. }
\end{aligned}
$$

$$
\begin{array}{llllll}
\mu & F_{\mu} & N & \beta_{R} & \beta_{u} & \text { HCLPF } \\
3 & 2.1 & 1.9 & 0.32 & 0.45 & 0.5 \\
5 & 2.7 & 2.45 & 0.36 & 0.45 & 0.64
\end{array}
$$

B-149 
EE

ENGINEERING. PLANNING AND MANAGEMENT CONSULTANTS

JOBNO 97218.01 JOB HCLPF COmporison CLIENT LLNG SUBJECT Block WJII
SHEETNO. BW-26

$B Y$ PSH DATEQ-25-8?

CHKD MKR DATE $10 / 5187$

CDFM HCLPF Capacit

Materiol Properties

Use the following properties:

$$
\begin{aligned}
& f_{y}=60 \ldots . \\
& f_{m}^{\prime}=1700 \text { psi } \\
& m_{0}=1800 \text { psi }
\end{aligned}
$$

Secent Stiffucss

Crocking Loed al Defiection

$$
\begin{aligned}
\sigma_{c r} & =2.5 \sqrt{1700} \\
& =103 \text { psi }
\end{aligned}
$$

Toble 9-20, Ret. I

$$
\begin{aligned}
\text { Mer }= & 230(103) \\
= & 23,700 \mathrm{in}-16 / \mathrm{tt} \\
= & 1970 \mathrm{ft}-16 / \mathrm{tt} \\
\omega_{c r}= & \frac{2(1970)}{10^{2}} \\
= & 39 \text { pst } \\
E_{m}= & 1000(1700) \\
= & 1.7 \times 10^{6} \mathrm{ps} \\
\Delta_{c r}= & 39(10)^{4}(1728) \\
& 8\left(1.7 \times 10^{4}\right)(1340) \\
& 0.037 \mathrm{in}
\end{aligned}
$$

B-150 
EQ E

ENGINEERING. PLANNING AND MANAGEMENT CONSULTANTS

JOB NO 87218.01 JOB HCLPF Comparison

CLIENT LN N SUBJECT BlOck Wall
SHEET NO. BU. 27

BY PS DATE $0-25-87$

CHKD $\because \because k \hat{k}$ DATE $\supset ! 5 i \hat{c}]$

Yield Load and Deflection

$$
\begin{aligned}
n & =\frac{29 \times 10^{6}}{1.7 \times 10^{0}} \\
& =17
\end{aligned}
$$

$$
\begin{aligned}
& \frac{1}{2}(1 k)(k d)^{2}-\frac{1}{2}(5.9)[(k d)-1.5]^{2}-17(0.31)(5.8-6 k d)=0 \\
& 5.05(k d)^{2}+14.12(k d)-37.20=0
\end{aligned}
$$

(lad $) .1 .66^{\prime \prime}$

$$
\begin{aligned}
I_{c r}= & \frac{1}{3}(16)(1.66)^{3}-\frac{1}{3}(5.9)(1.66-1.5)^{3} \\
& +17(0.31)(5.8-1.44)^{2} \\
= & 115 \mathrm{in}^{4} / \mathrm{bleck} \\
= & 86 \mathrm{in}^{4} / \mathrm{f}
\end{aligned}
$$

$$
\begin{aligned}
M_{y} & =0.8 \frac{60,000(86)}{17(5.8-1.64)} \\
& =58,700 \mathrm{in}-16 / 6 t \\
& =4890 \mathrm{ft}-16 / \mathrm{ft} \\
\omega_{n} & =\frac{2(4890)}{10^{2}}
\end{aligned}
$$

- 98 pst

$$
\begin{aligned}
\Delta_{y} & =0.037+\frac{\left(98-\frac{20}{3}\right)(10)^{4}(1728)}{8\left(1.7 \times 10^{6}\right)(86)} \\
& =0.037+0.872 \\
& =0.91 \mathrm{in}
\end{aligned}
$$

B-151 
EQE

ENGINEERING. PLANNING AND MANAGEMENT CONSULTANTS

JOBNO 87218.01 JOB HCLPF COmpurison CLENT LUNL SUBJECT BlOCt UnWI
SHEET NO BW - 28

BY PSH DAIE $9-25-87$

CHKD MKR DATE $10 / 5 / 87$

Moment of Inertio

$$
\begin{aligned}
I_{e} & =\frac{98(10)^{4}(1728)}{8\left(1.7 \times 10^{6}\right)(0.91)} \\
& =137 \mathrm{in}^{4} / 4
\end{aligned}
$$

$$
\begin{aligned}
& \frac{\text { Ultimate Streyth }}{\phi=0.8} \\
& \therefore=\frac{0.233(60,000)+263}{0.85(1.700)(12)} \\
& =0.86 \mathrm{in}
\end{aligned}
$$

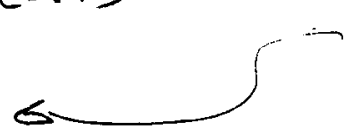

Effective Arial Loed

$$
\begin{aligned}
& \text { Estimate MCLPF } 0.58 \\
& \text { Vert. ZRA }=0.20 \mathrm{~g}\left(\frac{0.5}{0.18}\right) \\
& =0.56 \mathrm{~J} \\
& P=1110[1-0.4(0.56)] \\
& =86^{3} 3 / \mathrm{f4}
\end{aligned}
$$

Toble 7-4, Ret. 1

$$
\begin{aligned}
M_{u} & =0.8[0.233(60,000)+863]\left(5.8-\frac{0.86}{2}\right) \\
& =63,800 \mathrm{in}-16 / 4 \\
& =5310 \mathrm{ft}-16 / 4 \\
\omega_{0} & =\frac{2(5310)}{10^{2}} \\
& =106 p^{5 t}
\end{aligned}
$$

Scismic Response

$$
\begin{aligned}
\omega & =1.875^{2} \sqrt{\frac{1.7 \times 10^{4}(137)}{0.0239(120) 4}} \\
& =24 . \mathrm{rps} \\
f & =3.8 \mathrm{~Hz}
\end{aligned}
$$

Use roodoming

$$
s_{0}=0.408
$$

7020 denping.

B-152 
ERE

ENGINEERING. PLANNING AND MANAGEMENT CONSULTANTS

SHEET NO $W \omega-29$

JOB NO 8Ti18.01 JOB HCLPF COMPerivo DATE $9-25-87$ CLIENT WUL SUBJECT BlaCk $\omega=11$ BY CHKD MiR DATE $215: 87$

Since only slight in last ic energy absorption effect will the considered, estimate lon. damping ismedion.

$$
\begin{aligned}
& S_{d}=0.34 \mathrm{~g} \quad \text { ono dompity } \\
& w=0.34(111) \\
& =38 \text { pst }
\end{aligned}
$$

At the wall HCLPF capacity, estimate structure damping is about for median

$$
\begin{aligned}
F= & \frac{1.64}{1.89} \\
& =0.87
\end{aligned}
$$

Include 1.1 factor for mild ans ut of ductile behavior.

$$
\begin{aligned}
\text { HCLPF Capacity } & =\frac{106}{38}(0.81)(1.1)(0.18 \mathrm{~g}) \\
& =0.48 \mathrm{~g}
\end{aligned}
$$

$B-153$ 
EQF

ENGINEERING, PLANNING AND MANAGEMENT CONSULTANTS

JOBNO RI218.01 JOB HCIPF Cenparison

CLIENT LWN SUBJECT Eleck UL11
BY

CHKD MKR DATE $10 !=\mid E^{-}$,

References.

1. ACI-SEASCT Tasle Committee On Slender Walls, "Test Report On Slender Walls", 1982.

2. Omote, y.tal, "A Literature Survey-Transverse strength of Masonry Wialls ", Earthouluke Enginecrirg Research Center, UUCB/EERC-77/07, Mercin, 1977.

3. Suter, G.T. und G.A. Fenton, "Fiexural Copacity of Reintorced Mosonry tirmbers" ACIJarnal, JinuenFetruary, 986 .

4. Letter tronsmittol, M.K. Rovindro LEQE) to R.C. Murray (LLNL), JUly 16,1987 .

5. Lin, J. and S.A. Mohin, "Se ismic Response of Light Subsystems On Inelastic Structurcs," Juurnal the Structural Division, Asce, Februery,1985.

B-154 
बE

¿NGINEERING. PLANNING AND MANAGEMENT CONSULTANTS

STEET NO BW-31

JOBNO.87218.01 LOE HCLPF Comprison CLIEN LLNL SUEJECT Block Wall
BV. PSH DAIE 1-6-88

CHKZMMKR DAEE $1-7-88$

Round 2 Fraility Anolysis Colculation

The following modificotions will be made:

1. Use loro mediondomping.

2. Further inspection of testing in Ref. 1 indiceter that ratio of maximum to calculated gield deflections renge from 1.3 to 5.0 with an everage of ebout 2.3. Beceuse locding was stopped pric- to ectual failure, estimate - median ductility ygainst calculated ried of thort 3 .

On'y Eicont stiftues coles will be moditied.

$$
\begin{aligned}
& \text { For } f=3.8 \mathrm{~Hz} \text {, } 100 \text { demgin, } S_{0}=0.34 \mathrm{~g} \text {. } \\
& \omega=0.24(11) \\
& =38 \text { pst }^{\prime} \\
& \text { Etrenth Fector } \\
& F_{s}=\frac{148}{38} \\
& =3.9 \mathrm{~V}
\end{aligned}
$$

Inclestic Ener Absorption Fuctor

$$
\begin{aligned}
\Delta_{u} & =3(1.3) \\
& =3.9 . \\
A_{j} & =43 . \\
\mu & =\frac{3.9}{1.5} \\
& =3
\end{aligned}
$$

B-155 
EQE

ENGINEERING, PLANNING AND MANAGEMENT CONSULTANTS

JOBNO $\$ 7218.01$ JOE HCLPF COMperison CLEN LUN SUBJECT Block Wall
SHEET NO. $3 \omega-32$ BV. PSH DATE $1-6-88$

CHKD MIKR DATE $1-7-8 \hat{E}$

$$
\begin{aligned}
q & =3(10)^{-0.30} \\
& =1.50 \\
p & =1+1.50 \\
& =2.50 \\
r & =0.48(10)^{-0.08} \\
& =0.40, \\
\ddot{F_{\mu}} & =[2.50(3)-1.5]^{0.40} \\
& =2.01 \\
\beta_{c} & =-\frac{1}{3} \ln \frac{1}{2.0} \\
& =0.23 \\
\beta_{\pi} & =\beta_{0}=\frac{1}{\sqrt{2}}(0.23) \\
& =0.16 .
\end{aligned}
$$

Modeling - Block Woll

For $t=f+r_{0}=4.2 \mathrm{~Hz}$, loro donpiy, estimete $S_{c}=0.42 \mathrm{~g}$

$$
\begin{aligned}
\beta_{m f} & =\ln \frac{0.42}{0.34} \\
& =0.21
\end{aligned}
$$

For $f=f-1 /=3.4 \mathrm{~Hz}$, rou domping estimate $S_{-}=0.32 \mathrm{~g}$

$$
\begin{aligned}
\beta_{\text {nt }} & =-\ln _{\sim} \frac{0.32}{0.34}, \\
& =0.06
\end{aligned}
$$

Restimate $\beta_{m}=0.20$

B-156 
QF

:NGINEERING. PLANNING AND MANAGEMENT CONSULIANTS

SHEET NO $3 \omega-33$

JOB NO 87218.01 JOS HCLPF COmprrisoBV. PSH DATE $1-6.87$ ZLEN LUL SUEJECi Block Wall GTKE MKE DAE $1-7-88$

Damping - Block woll

Estinute 700 domping is -10

$$
\begin{aligned}
S_{2} & =0.40 . \gamma \\
\beta_{U} & =\ln \frac{0.40}{0.34} \\
& =0.14 \\
\beta_{R} & =0.2(0.16) \\
& =0.03
\end{aligned}
$$

Overall Frogilitz Parameters

Other values some as Setore

$$
\begin{aligned}
& \ddot{F}=3.9(2.0)(1.35)(1.1) \\
& =11.6 \mathrm{r} \\
& \begin{aligned}
A & =11.660 .189 \\
& =2.18
\end{aligned} \\
& \beta_{R}=\sqrt{0.16^{2}+0.25^{2}}+0.03^{2}+0.01^{2}+0.06^{2}+0.03^{2}+0.05^{2}+0.05^{2} \\
& =0.311
\end{aligned}
$$

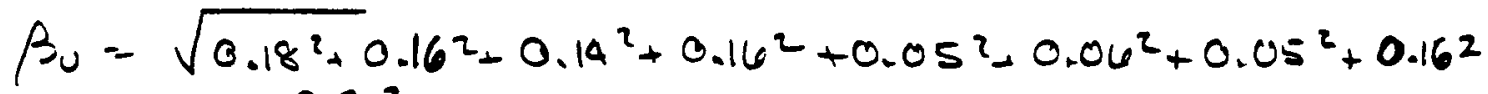

$$
\begin{aligned}
& +0.20^{2} \\
& =0.42 \mathrm{r} \\
& \text { HCLPF }=2.1 .9 e^{-1.65(0.31+0.42)} \\
& =0.63 \mathrm{~g} .
\end{aligned}
$$

B-157 
बह8

NGINEERING. PLANNING AND MANAGEMENT CONSULTANTS

O8NO 81218.01 jOB HCLPF COmperison ZIEN UNL SUEJE: Block WJall
SHEETNO BN-3Q

5V. PSH DATE $1-6 c-88$

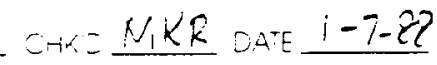

$2^{\text {nd }}$ Round CDFM Colculations

The following modifications will be made:

1. Use full $F_{\mu}=\frac{1}{0.8}=1.25$

2. Strocture is approeching yield, use lono structure damping.

$$
\begin{aligned}
\text { HCLPF } & =\frac{106}{3 z}(1.25)(0.18 \mathrm{~g}) \\
& =0.63 \mathrm{~g},
\end{aligned}
$$

B-158 


\section{EQF}

ENGINEERING. PLANNING AND MANAGEMENT CONSULIANTS

SHEET NO

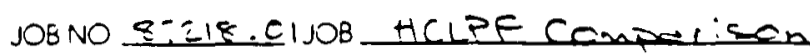

BY.

DATE i-1:-87

CLIENI LLUL

SJBJECT Sample Fork Wall

CHKD

DATE

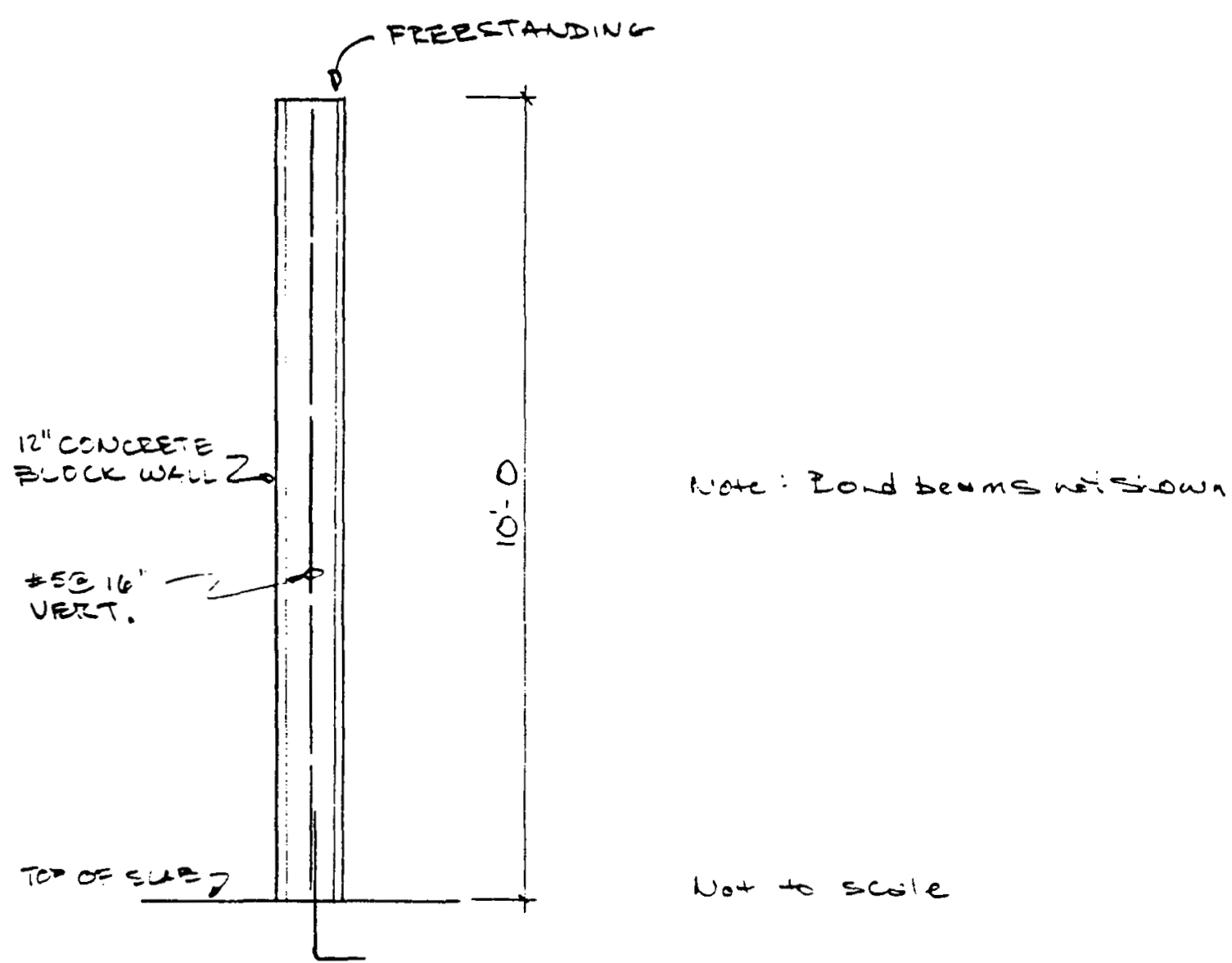

Woll is seratructed in running sond, cells w/ reinf. oroured suclid

Eleck: 12" hollow units, nermelweight, ASTM C90 Erorien

Mọrtar: ASTM C270 Type S

Vertical Reintorcement : \$5@16"0.C., Erade 60 steel

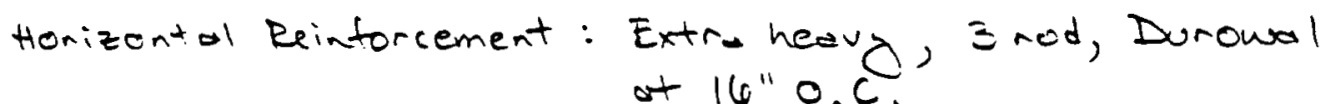




\section{EQE}

ENGINEERING. PLANNING AND MANAGEMENT CONSULTANTS

SHEET NO 2

NOBNO 91UT.CL JOB HCIPF COMParison

BY DATE $7-16-27$

CLIENT LUN SJBJECT Sample Bloct ULII

CHKO

DATE $=\ldots$

Assumptions:

1. Will is long compored to its height, iseloted fromtuc supperting structure st the top and cides. Can be medeled ds a one waz nember, tose supiorted, sponning verticall 2 .

2. Ne etreched ex:-izmert or ether masces.

2. Stwrelerd two cell =10 ck used:
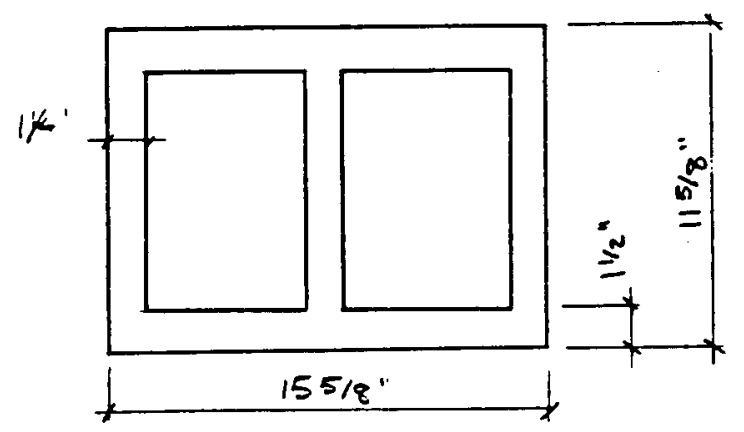

4. Wal weigh approximately 111 psf for cella groutei st 16 ".

5. Special inspection requiremetts ore met.

6. In-pie-c stresses are relatively Emall ad can be nejlected.

7. Average unit compressive streyth on net aree of 3000 psi. 
EQE

ENGINEERING. PLANNING AND MANAGEMENT CONSULTANTS

JOBNO RIZ1R.OI JOB HCLPF COmparison CLENT UNL SJBJECT Edraple Eock Wbl.
SHEET NO $\equiv$ BY DSA DATE $--\frac{-\varepsilon-2}{}$ CHKD DATE $=\ldots-\ldots$

Ve: $:$-not the well meets criterio in Appendix A to S.R.P. Estion $3-8.4$. Chact ageinst load Combination 2 (b) (4):

$$
D+L+T_{0}-E_{0}+E^{\prime}
$$

Jrerosed Etiffndy Geguerey (Upper Eoud)

$$
\begin{aligned}
& \left.I_{0} \approx \frac{1}{12}(16)(11.6)^{2}\right)^{3}-\frac{1}{12}(5.9)(8.63)^{3} \\
& =17 \text { \&1.na/biock } \\
& =1 \equiv 40 n^{4} / 6 x \\
& \bar{m}=\frac{1 i 1}{12\left(2-\varepsilon^{\prime}-t\right)}
\end{aligned}
$$

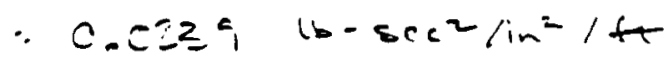

$$
\begin{aligned}
& \text { U. - cemi-essive strenth, nat areo }=\$ 000 \text { pai }
\end{aligned}
$$

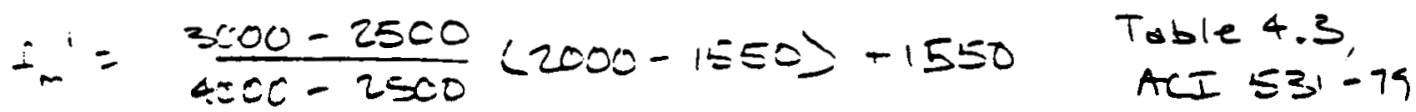

$$
\begin{aligned}
& =: 00 \text { poi }
\end{aligned}
$$

$$
\begin{aligned}
E_{m} & =1000 f_{m} \\
& =1.7 \times 10^{4} p=i
\end{aligned}
$$

B-161 
ER

ENGINEERING. PLANNING AND MANAGEMENT CONSULTANTS

SHEET NO 4

JOBNO 87TIS. C1 JOB HCLPF Comprrison BY DSU DATE $7-16-87$

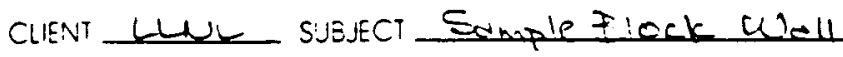
CHKD:DATE $-\div-\overline{7}$

$$
\begin{aligned}
& \omega=(1.875)^{2} \sqrt{\frac{1.7 \times 106(12-42)}{0.0239(120)^{4}}} \\
& =75 \mathrm{rps} \\
& t=12 H z \\
& \text { Spectrol acceleretion } 00.75 q \text { Too domped, troerenci, Et } \\
& \text { Maine un-kee sper-iun, ELb" } \\
& \text { turb./serv. bld } \\
& \text { Crocked Stiftecss Areiveny (Lower Foud') } \\
& \begin{aligned}
A_{s} & =\frac{0.31(12)}{16} \\
& =0.235 \mathrm{in}^{2} / 4
\end{aligned} \\
& n=\frac{29 \times 10^{4}}{1.7 \times 10^{6}} \\
& =17 \\
& a=\frac{11.63}{2} \\
& =5.8 \cdots \\
& \frac{1}{2}(12)(k d)^{2}-17(0.25=25[5-(k d)]=0 \\
& k d=1.65^{\prime \prime}>t_{t}=1.5^{\prime \prime} \\
& \frac{1}{2}(16)(k d)^{2}-\frac{1}{2}(5.9)[(k d)-1.5]^{2}-17(0.31)[5.8-(k d)]=0 \\
& 5.05(k d)^{2}+14.12(k d)-37.20=0 \\
& \text { (td) } 1.64 \text { " }
\end{aligned}
$$


EQF

ENGINEERING. PLANNING AND MANAGEMENT CONSULIANTS

JOBNO 87218.CJ JOB HCLPF COPwcit CLIENT LUL SUBJECT Somple Black $\omega_{0}$
SHEET NO 5 BY DATE ᄀᄀ-14-8า CHKD

DATE

$$
\begin{aligned}
I_{c r}= & \frac{1}{3}(16)(1.66)^{3}-\frac{1}{2}(6.9)(1.46-1.5)^{3} \\
& +17(0.3)(5.8-1.46)^{2} \\
= & 115 \mathrm{in}^{4} / \mathrm{block} \\
= & 86 \mathrm{in}^{4} / \mathrm{ft}
\end{aligned}
$$

$$
\begin{aligned}
f & =12 \sqrt{\frac{86}{1340}} \\
& =3.0 \mathrm{~Hz}
\end{aligned}
$$

Spectiol -cceleretion $\approx 0.450$

Out af Plane Sciernic Loeds

Encek fir spectrol acreleration of 0.75

$$
\begin{aligned}
\omega & =0.75(11) \\
& =837 \\
V_{\ldots .} & =83(10) \\
& =830 \mathrm{w} / \mathrm{ft} \\
M_{\text {mar }} & =\frac{1}{2}(23)(10)^{2} \\
& =4150 \mathrm{fr}-1 \mathrm{~b} / \mathrm{ft} \\
& =49,800 \mathrm{in}-1 \mathrm{~L} / \mathrm{t}
\end{aligned}
$$

B-163 
EQF

ENGINEERING PLANNING AND MANAGEMENT CONSULTANTS

JOBNE ETUL.01 JOB HCLPF Capaci-

CLIEN IURUL SUBJECT Sumple Bloct WhIII
SHEET NO L

$B Y$ PSH DAF $7-16-87$

CHKD

DA'E

Chece Reiffercemet Tensile Stress

Hlowable stress $=24,000$ ps. Sect.10.2.1. 1, ACI 5si-79,

$$
\begin{aligned}
& \text { Ectored allowaile }=2.0(26,000) \text { SRe Sectic } 3.8 .4 \text {, A.p. F } \\
& -48,000 \text { psi <c. iff } \\
& \therefore=49, \frac{800(5.8-1.66)(17)}{86} \\
& =40,800 \text { ps: }<48,00073 i \text { ex }
\end{aligned}
$$

Conservatively nylects axiol lood.

$$
\begin{aligned}
& \frac{\text { Ancic Hesonric Comprassive Stress }}{f_{m}{ }^{\prime}=1700 \text { psit }}
\end{aligned}
$$

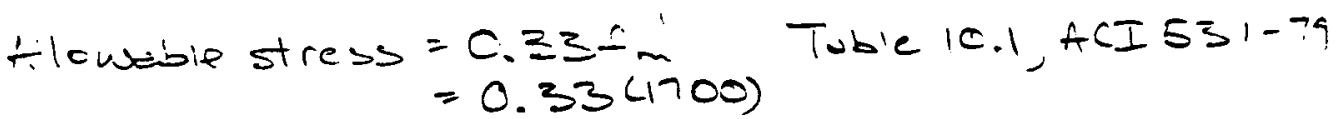

$$
\begin{aligned}
& \text { - } 560 \text { p }
\end{aligned}
$$

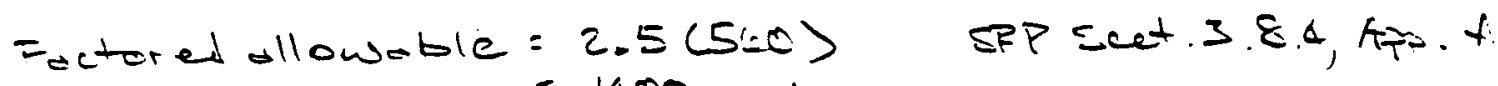
1400 ps:

$$
=\frac{49.800(1.64)}{86}
$$

-960 pri c 1400 psi or

fodequate maje if axial lood includad

B-164 


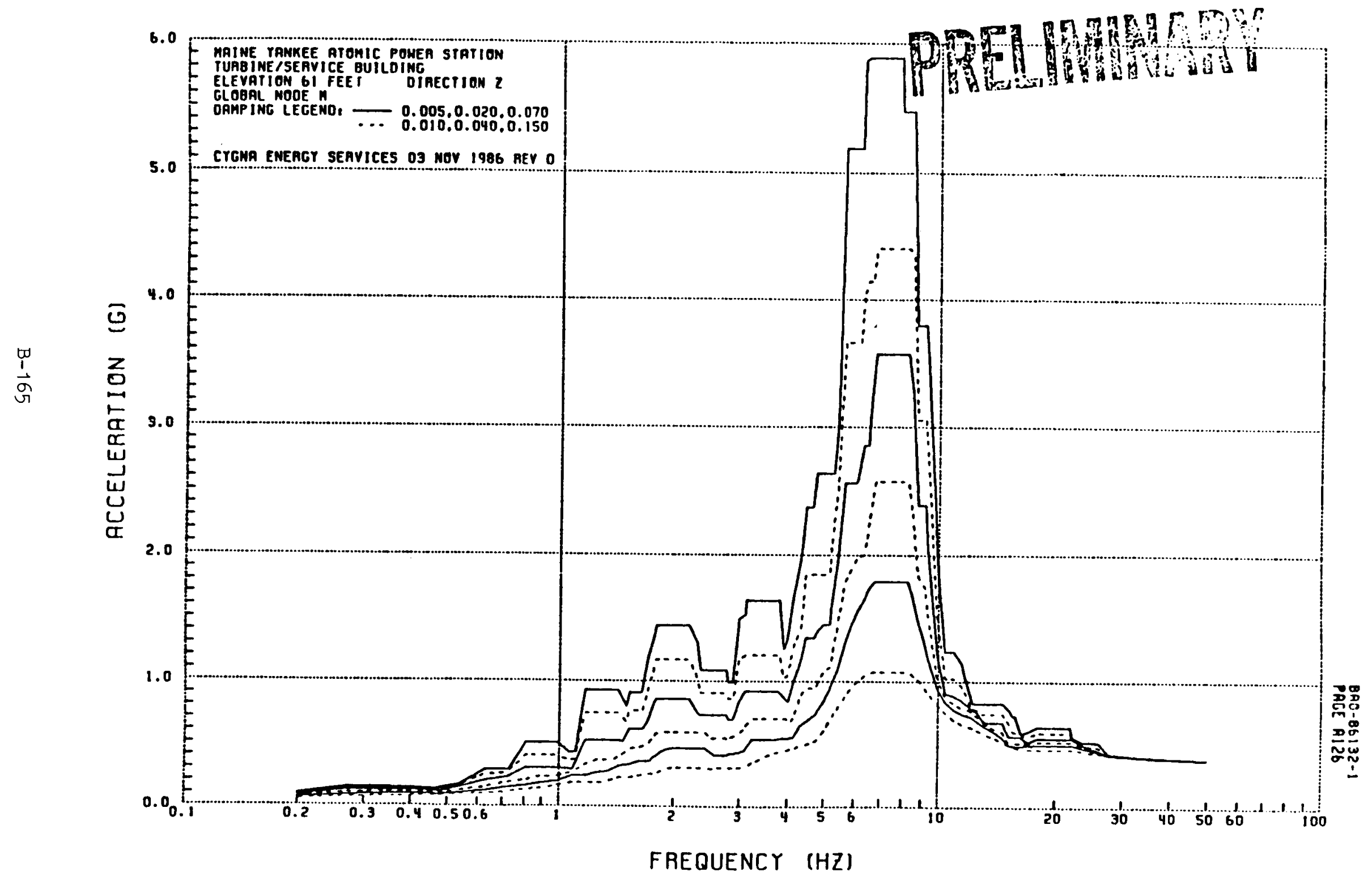


EQE

ENGINEERING. PLANNING AND MANAGEMENT CONSULTANTS

JOBNO \$7218.C1 JOB HCLPF Casit

CLIENT UdWL SUBJECT STimple ZlocE Wall
SHEET NO $\frac{7}{7-16-07}$

BY

DATE $7-16:-81$

CHKD DATE

Check Shear Stress

$$
\begin{aligned}
& \text { Allowab'c ctress }=1.1 \sqrt{f_{n}} \text { Table } 10.1 \text {, ACI 531-79 } \\
& =1-1 \sqrt{700} \\
& =45 \text { psi <50ps. }
\end{aligned}
$$

$$
\begin{aligned}
\text { Effectice sherered }= & {[5.9+2(1.25](5.8)} \\
& =48.7 \mathrm{in} / \text { slock } \\
& =37 \mathrm{in}^{2} / 4
\end{aligned}
$$

$$
\begin{aligned}
f_{v} & =\frac{830}{37} \\
& =22 \text { psi }<58 \text { psi ck }
\end{aligned}
$$

B-166 


\section{APPENDIX C JOHN W. REED}




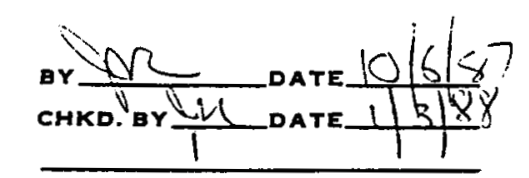

PROJECT
SUAJECT

PAGE 1 OF

JOE NO. $105-170$

SUMMRRY OF ANALYSES

$84 \%$ NEP MAX Norl3 Divection (9) ORIGINAL CALCS Revised Calcs

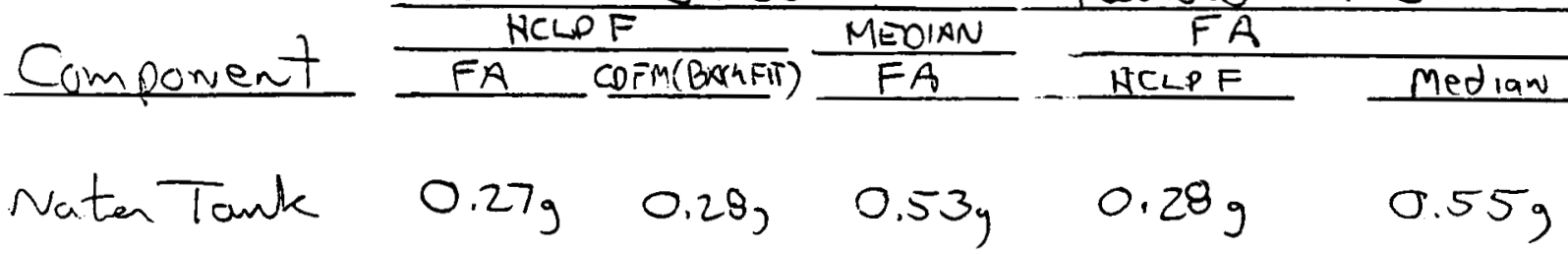
Aoxiliary Cont Chat:

Ground level $0.48 \quad 0.48 \quad 1.20 \quad$ Not Reused

Floor Leve! $0.11 \quad 0.10 \quad 0.43$

11

Stantion, Am Tant $0.43 \quad 0.41 \quad 1,40$

1)

teat Exchanger $0.39 \quad 0.36 \quad 1,0$

1)

$\begin{array}{llllll}\text { slock Wall } & 0.38 & 0.33 & 1.41 & 0.52 & 1.96\end{array}$

Rependix A - Gives $\left.\frac{v}{F}, a\right) \beta_{r}$ fr siffere of corracioni,

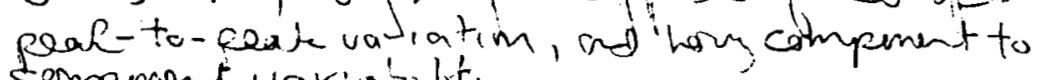
cempement variabilit?

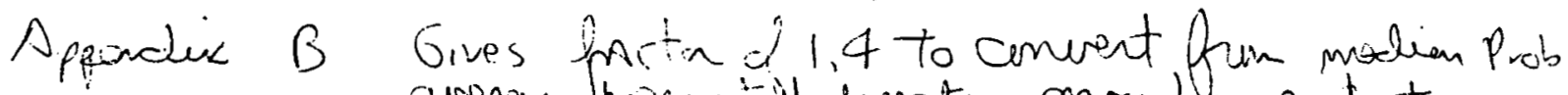

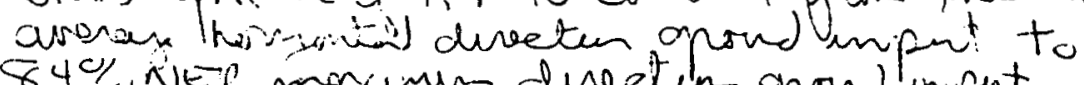
$84 \%$ J NEP monamen dereetin opond in put

Dppender C Desculer prosedure ard provide Mu bosis for bacle it ing a Rraghts andelypic HCLPF to a sinse determinstie anabyeir (1.e. CDFM)

Apporatix D Listings of onsel $\$$ Jack R. Benjamin \& Assoclates, Inc. Floor yosporins spectin usad

C-2 
BY

CHKD. BY

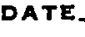

DATE

PROJECT

SUBJECT

PAGE 2

OF

General Assumptions \Comments (Special)

1. Vortical component $=3 / 3$ horugntal, No voualibly included in This nato

$27 \%$ damp n used as medium fo building This may be low-depende on sones state in building at median equipment level

3. Floor response speetron seen van high. This is why he HCPLF's seam low

4 Computer Programs are run on MATACAD Verlil by Matusoft

Jack R. Benjamin \& Associates, Inc.

Consulting Engineers

C-3 
origin

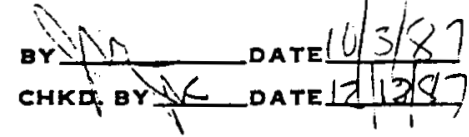

PROJECT

PAGE 1 OOF

FLAT-BOTIOM STORAGE TANK

Assumption (spp cion)

1. Capacitor is contiviles by archer bott yelling and buckling toul shell

2. To water hold dom force is o'tured

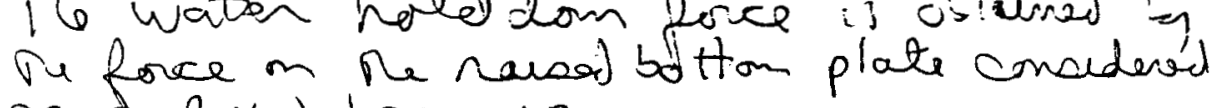
ar a fixed beam, lie

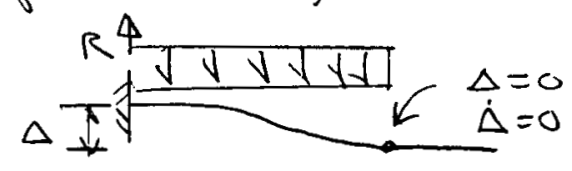

3 Seismic Torah Forces based on: Summary of TAal Calculations, by EQE.

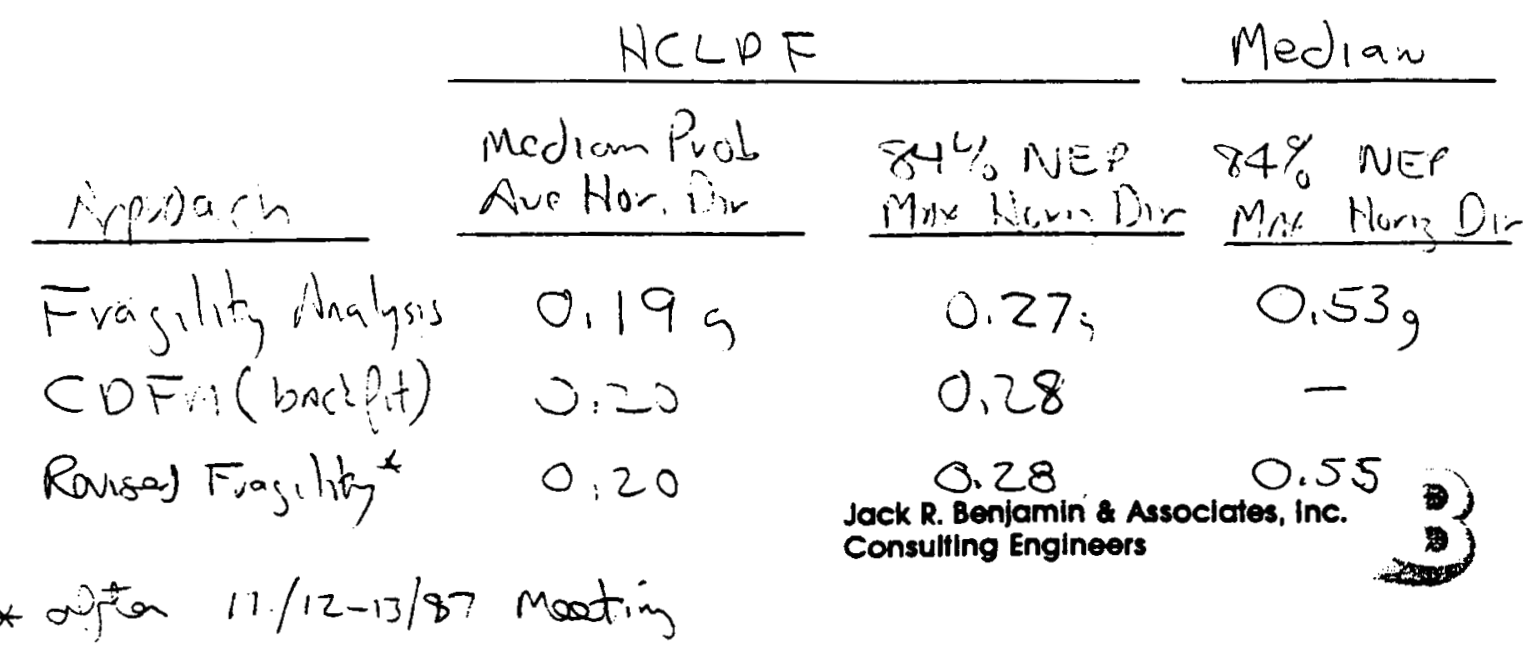

$\mathrm{C}-4$ 


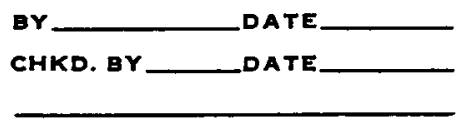

PROJECT

PAGE? $?$

SUBJECT

JOB NO.

Strengeh.

Progian TANK.HC2 goner in Calculation fr the motur aralupin (seofollowm pager)

$$
\text { pğa }=0.346 g
$$

Note. hat firie is baod on a modem level ground respmss spetrim (1.e. 50\% NEP) and ot moximum divectin motim (ie input scaled by 1.11 - see Kippendic B.)

pares 3-12 gue ar analyen

Jack R. Benjamin Associates, inc. Consulfing Engineers

C-5 
MCAD FILES TANK.HCE

CONSISTENT SET OF UNITS:

BASE UNITS:

in $\equiv 1 L$

$m \equiv 1 M$

$\sec \equiv 1 \mathrm{~T}$

DERIVED UNITS:

$$
\begin{aligned}
& f t \equiv 12 \cdot i n \\
& 1 b \equiv m \cdot \frac{i n}{\text { sec }} \\
& k \equiv 1000 \cdot 1 b \\
& g \equiv 32.2 \cdot \frac{f t}{2} \\
& k s i \equiv \frac{k}{2} \\
& M F a \equiv 0.144 \cdot k 5 i
\end{aligned}
$$

UEFINE MEDIAN FROFEFTIIES OF TANK:

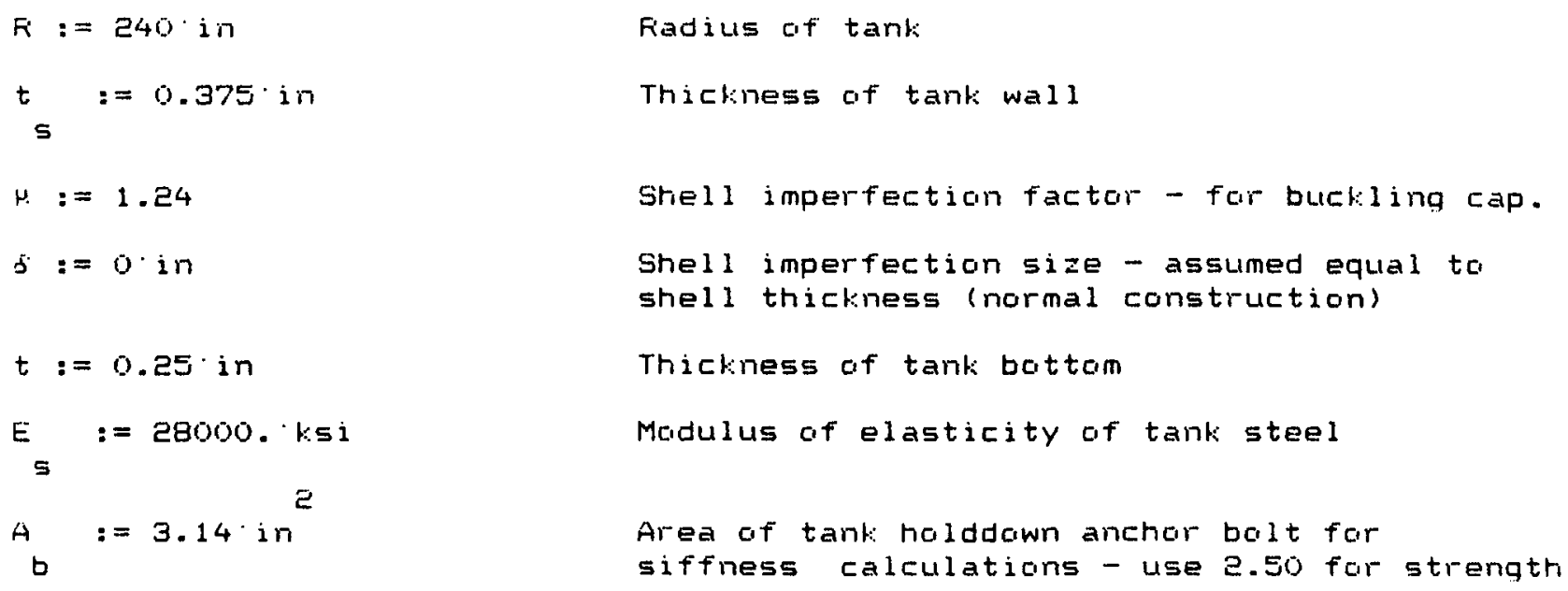




$$
\begin{aligned}
& \equiv \quad:=29000 \cdot k 5 i \\
& b \\
& \because \quad:=135 k \\
& \text { y } \\
& 7:=27 \text { in } \\
& 1 \\
& 7:=54.5 \cdot \mathrm{in} \\
& 2 \\
& f_{y}:=37 \cdot k 5 i \\
& =\quad:=63.7 \cdot \mathrm{k} \\
& ={ }_{E Q} \quad:=63.7 \cdot k
\end{aligned}
$$$$
\begin{aligned}
& s \quad:=62.4 \cdot \frac{1 b}{f t} \\
& n_{w}:=37 \cdot f t \\
& n:=8
\end{aligned}
$$$$
\text { i }:=1 \ldots n
$$$$
\theta_{i}:=(i-1) \cdot \frac{2 \cdot \pi}{n}
$$$$
\mathrm{VOH}:=\frac{3}{3}
$$$$
\operatorname{amp}:=1.89
$$

$\operatorname{inp} 1:=1.00$

inpe := 1.11

$\operatorname{lnp} 3:=1 \cdot 1$

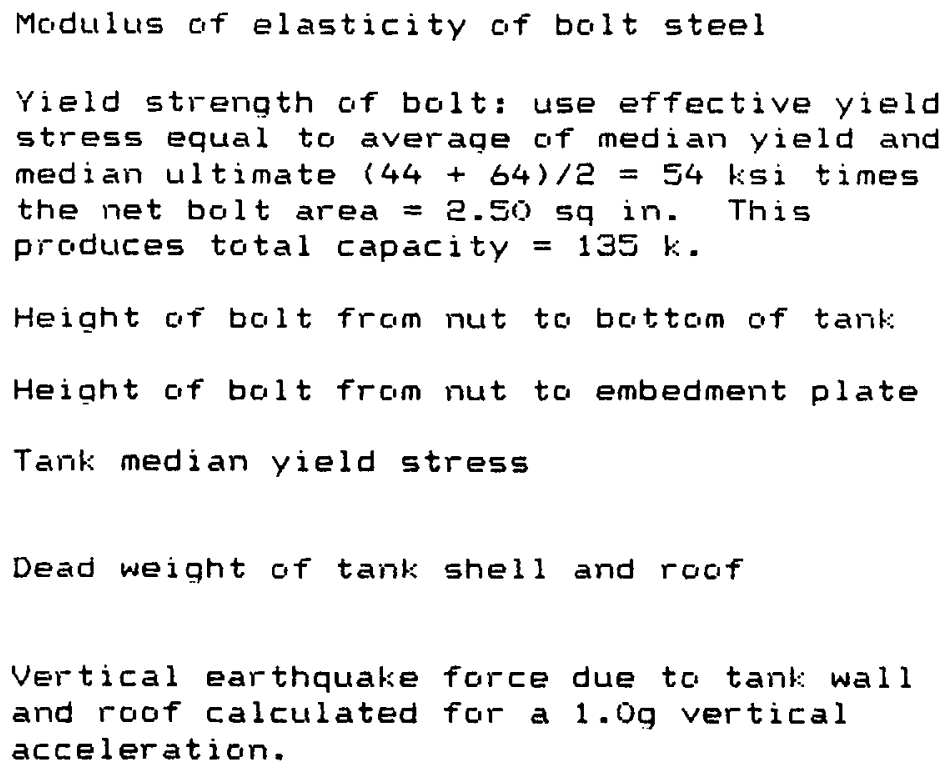

Density of water

Height of water in tank

Number af bolts

Loap over all bolts to define angle from symmetric axis to each bolt:

Angle from symmetric axis ta each bolt, i

Fatic of vertical to horizantal earthquate coimpanents

Vertical amplification far the fluid due ta tank radial expansion (median at $7 \%$ damping)

Input scale factor for higher spectral amplification relative to median input at $7 \%$ damping.

Scale factor for peak earthquake horizontal input cumpared to average of two horizontal components

Ductility increase factor which influences the vertical earthquake reduction in hold down forces 
$p:=\delta_{w} \cdot h h_{w} \cdot\left[\begin{array}{ll}1-.4 \cdot i n p 1 \cdot i n p 3 \cdot a m p v \cdot v 0 H \cdot \frac{a}{g} \\ g\end{array}\right]$ Water pressure at bottom of tank:

$w:=p \quad$ Effective pressure an tank battom

$I:=\left[\frac{1}{12}\right] \cdot\left[\begin{array}{l}3 \\ t\end{array}\right] \quad$ Moment of inertia of bottom plate per length

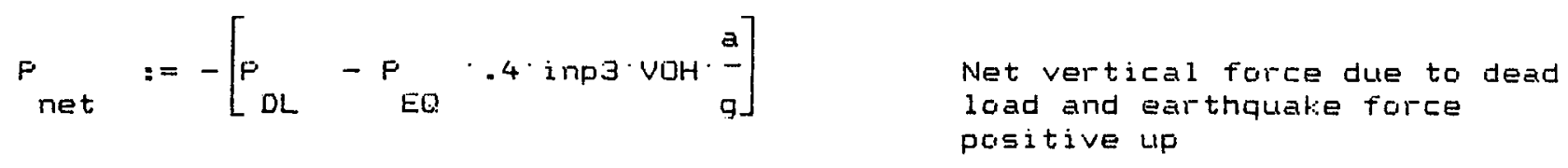

M : := 233800.inp1'inpe'k'in Net averturning mament due ta earthquake net $\quad$ (233800 k*in far $0.30 \mathrm{~g}$ for $7 \%$ damping average of two horizontal components)

DETERMINE SHELL BUCKLING CAFACITY:

Tank buckling capacity based on "Seismic Design of Storage Tanks" by M. J. N. Friestley, J. H. Wood and B. J. Davidson, Bulletin of the New Zealand National Society For Earthquake Engineering, Vol. 19, No. 4, December 1986.

$f_{C 1}:=0.6 \cdot E_{s}\left[\frac{t}{s} \frac{1}{R}\right] \quad$ Classical "perfect shell" buckling stress EUCKLING IN MEMERANE COMFFESSION (DIAMOND BUCKLING):

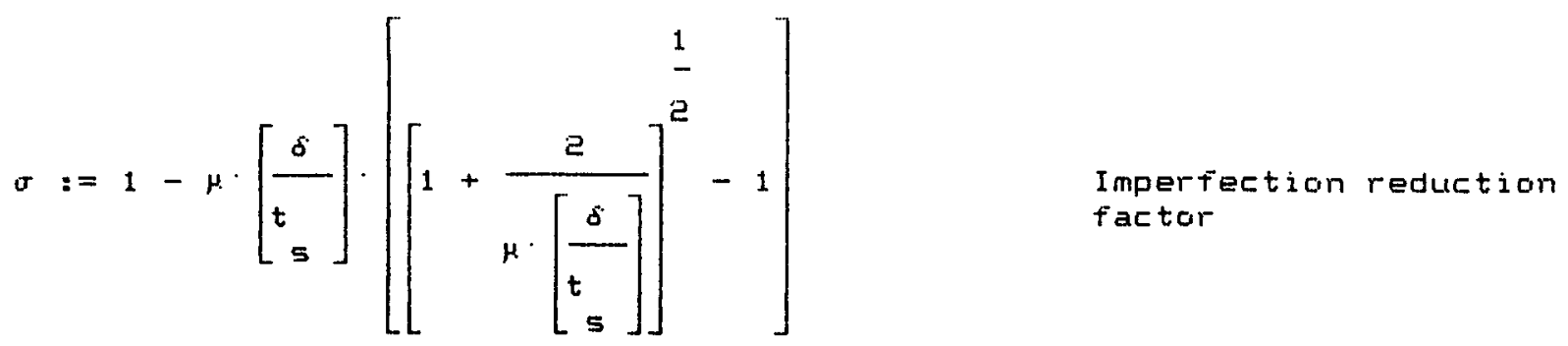

$\Gamma:=\frac{f^{y}}{\sigma \cdot f}$

Factar to determine THICK or THIN wall 


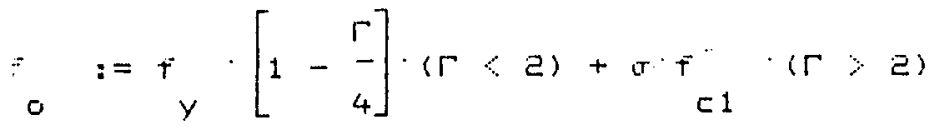

$$
\begin{aligned}
& \text { elastic THICH wall elastic THIN wall }
\end{aligned}
$$

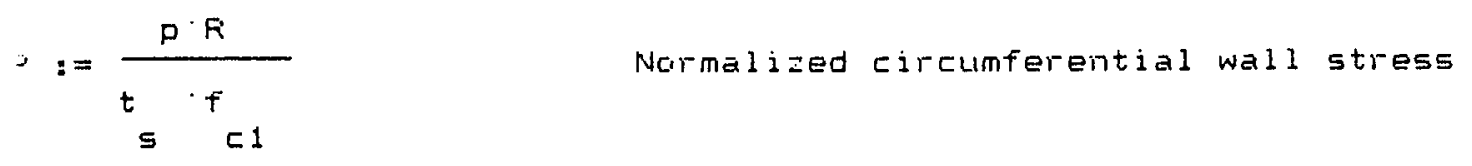

$y:=F \cdot(F<5)+5 \cdot(F>5)$ P is Limited to 5

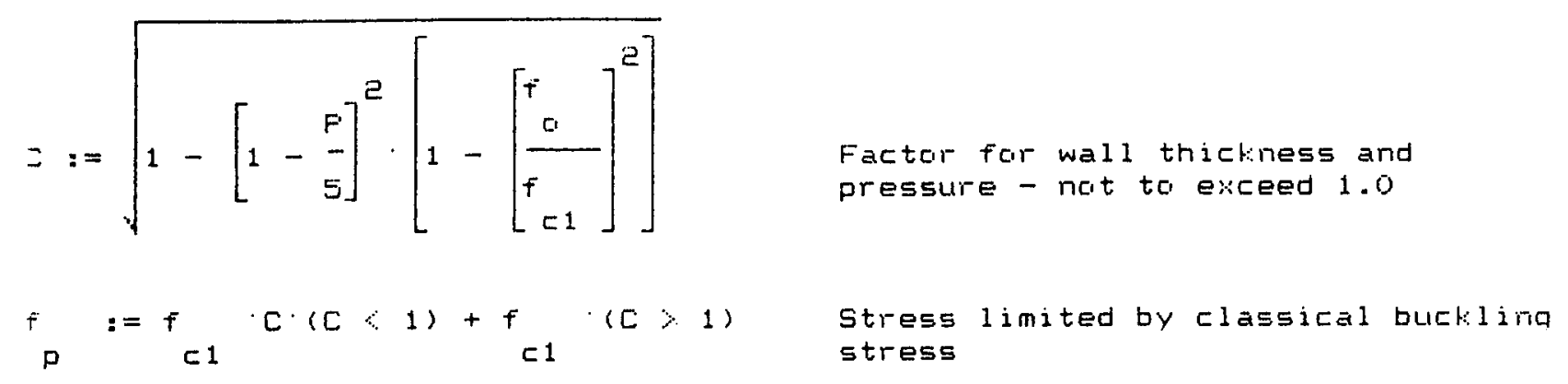

$f_{\text {md }}:=f_{\subset 1}\left[\begin{array}{r}t \\ \left.0.19+0.81 \cdot \frac{f}{t}\right] \subset 1\end{array}\right]$

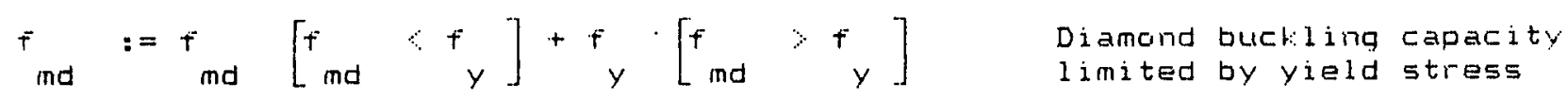

ELASTIC FLASTIC COLLAFSE (ELEFHANT FOOT BUCYLING):

$$
\begin{aligned}
& \frac{R}{t} \\
& \overrightarrow{3}=\frac{5}{400}
\end{aligned}
$$

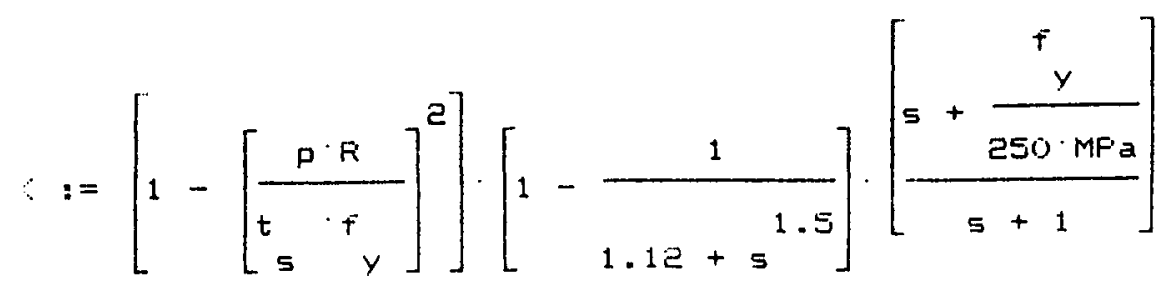




$$
\begin{aligned}
& f \quad:=f \cdot k(K<1)+f \cdot(K) 1) \text { Limit elephant fout buckling stress } \\
& \text { me c1 c1 to classical buctiling stress }
\end{aligned}
$$

MINIMUM BUCKLING STRESS:

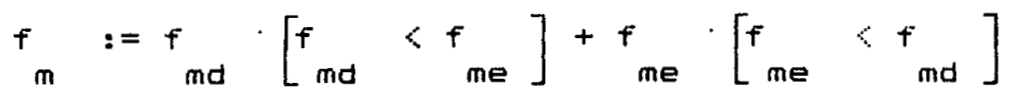
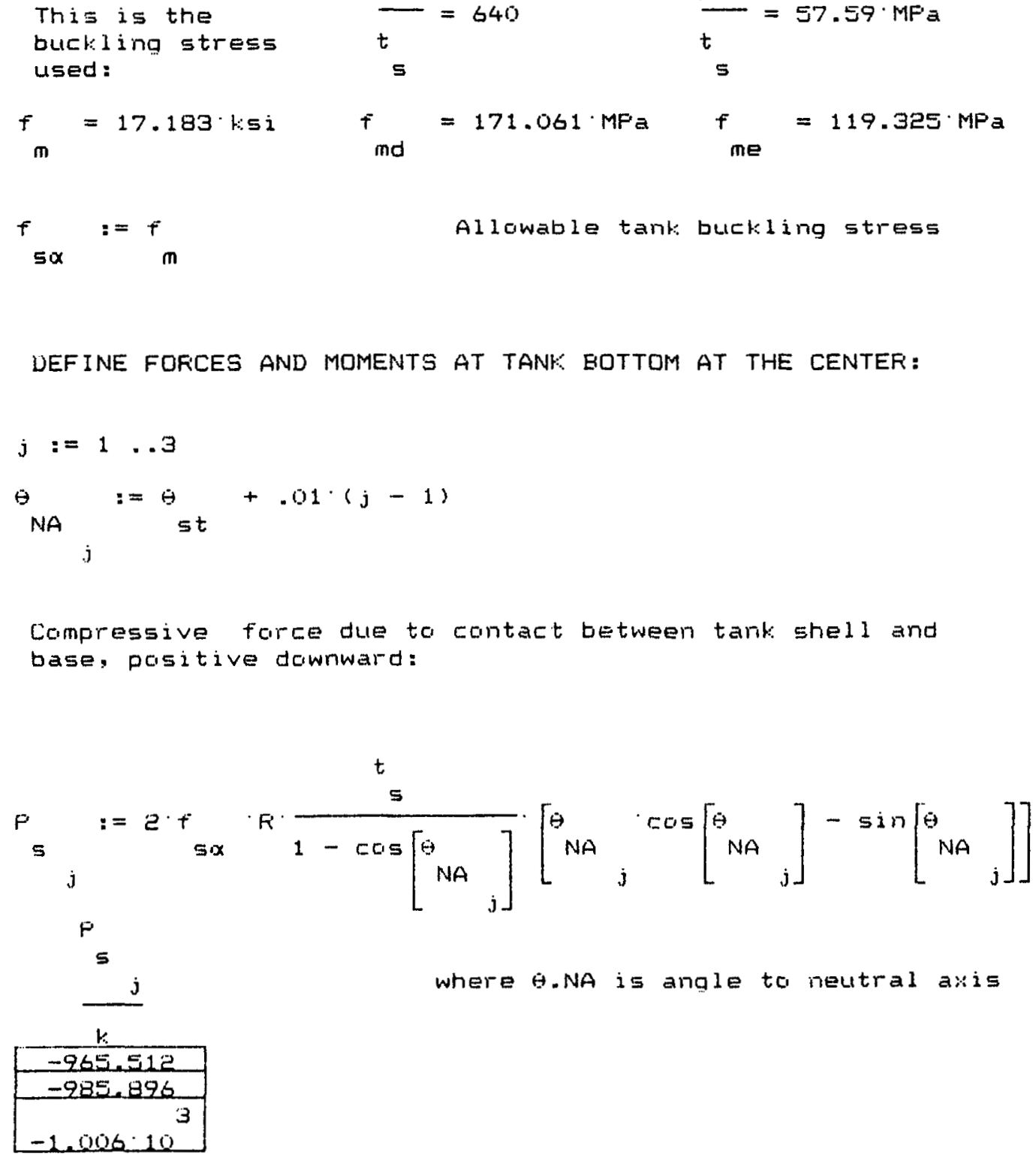

Allowable tank buckling stress

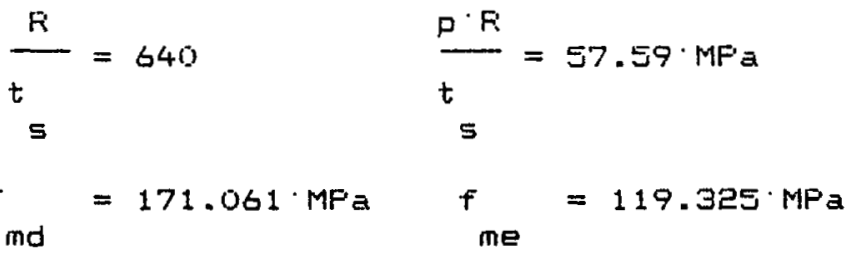

AT TANK: BOTTOM AT THE CENTER: 
Manent due to contact between tank shell aird base, when resists earthquake averturing:

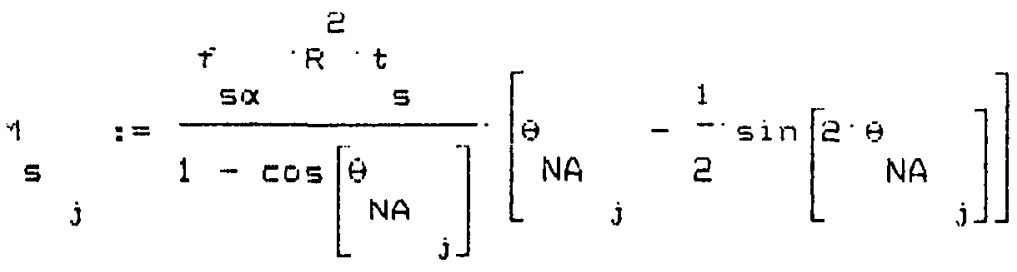

$M$

5

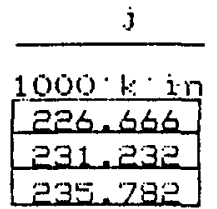

$\operatorname{const}:=\frac{A_{j} \cdot \operatorname{six}}{1-\cos \left[\begin{array}{ll}\theta_{N A} \\ \end{array}\right]} \cdot\left[\begin{array}{l}h \\ 1 \\ h \\ 2\end{array}\right] \cdot\left[\begin{array}{l}E \\ b \\ E \\ s\end{array}\right]$

$$
\begin{aligned}
& P e_{j}:=\operatorname{const} \cdot\left[\cos \left[\begin{array}{ll}
\dot{\theta} & \\
N A & \\
& j
\end{array}\right]-\cos \left[\begin{array}{l}
\theta \\
2
\end{array}\right]\right] \\
& P_{j}:=\operatorname{const} \cdot\left[\cos \left[\begin{array}{ll}
\theta & \\
N A & \\
& j
\end{array}\right]-\cos \left[\begin{array}{c}
\theta \\
3
\end{array}\right]\right] \\
& P_{j}=\operatorname{sonst} \cdot\left[\cos \left[\begin{array}{ll}
\theta & \\
N A & \\
& j
\end{array}\right]-\cos \left[\begin{array}{l}
\theta \\
4
\end{array}\right]\right] \\
& \sigma_{j}:=\operatorname{const}\left[\cos \left[\begin{array}{ll}
\theta & \\
N A &
\end{array}\right]-\cos \left[\begin{array}{ll}
\theta \\
5
\end{array}\right]\right]
\end{aligned}
$$

Modify bolt force to te greater than o but less than Fy:

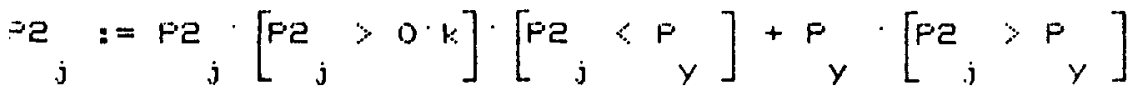

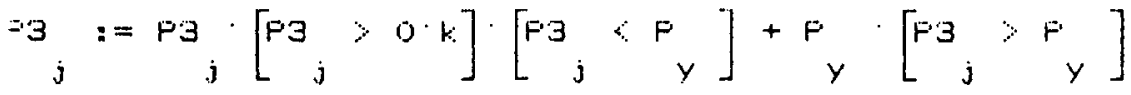




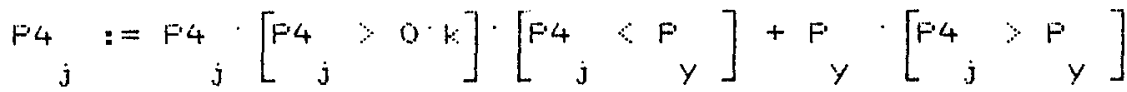

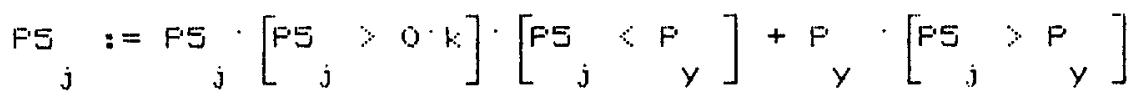

Force due to sum of all bolts, positive downward:

$$
\begin{aligned}
& \left.\mathrm{F}_{j}:=2 \cdot\left[\mathrm{F}_{j}+\mathrm{F}_{j}+\mathrm{F}_{4}\right]_{j}\right]+\mathrm{FS}_{j} \\
& \mathrm{~F}_{\mathrm{b}}
\end{aligned}
$$

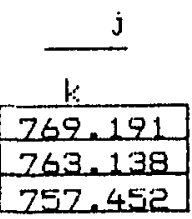

Mament due to all bolts, positive when resists earthquake overturning:

$M_{b}:=-F^{\prime} \cdot\left[2 \cdot F_{j} \cos \left[\begin{array}{l}\theta \\ e\end{array}\right]+2 \cdot F_{j}^{\prime} \cdot \cos \left[\begin{array}{l}\theta \\ 3\end{array}\right]+2 \cdot F^{4} \cdot \cos \left[\begin{array}{l}\theta \\ 4\end{array}\right]+F_{j} \cdot \cos \left[\begin{array}{l}\theta \\ 5\end{array}\right]\right]$

M

b j

$1000 \mathrm{k}$ in

62.236

63.263

664.228 
Total hold down force from water from bottom plate, positive downward: (This equation assumes that the there is no rotation at the tank: shell/battom plate interface as the plate picks up due to rocking.)

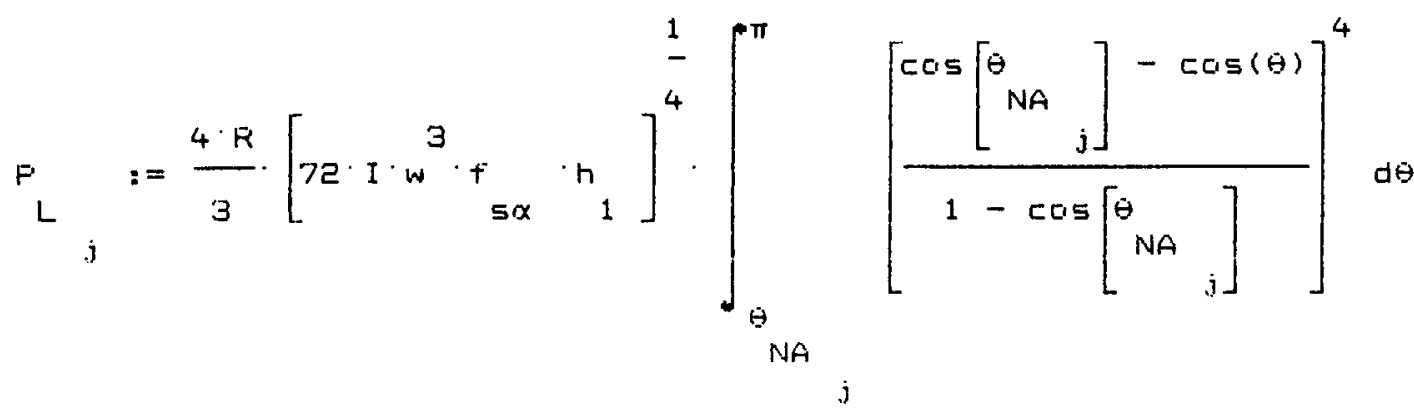

Total resisting moment from water un bottum plate, positive when resists ear thquake overturning:

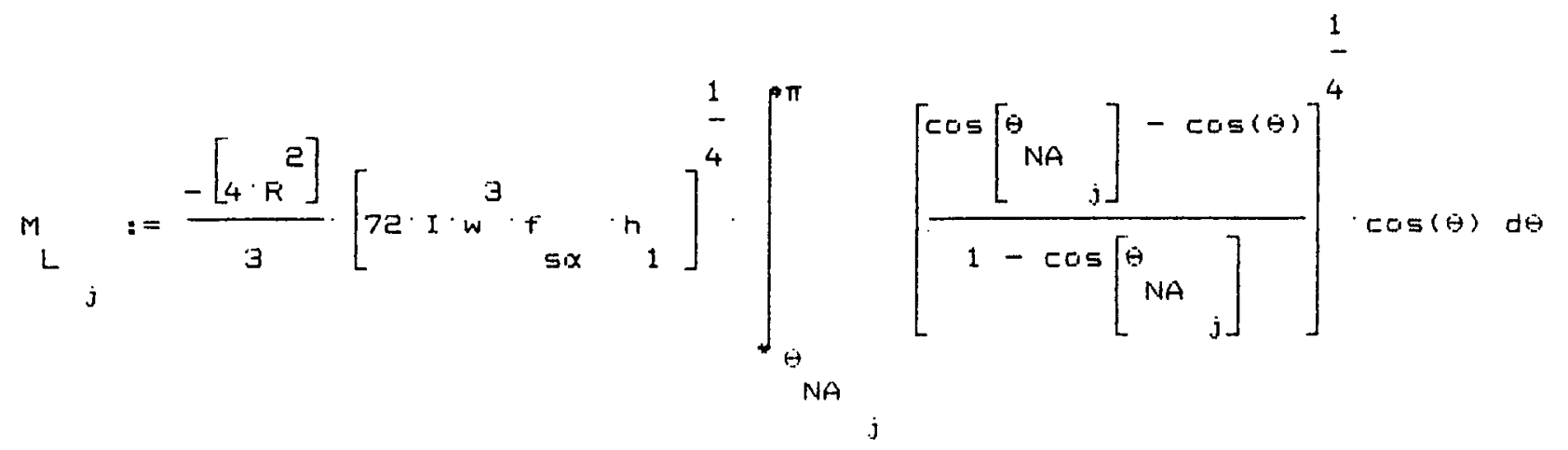<smiles></smiles>

$\frac{M_{j}}{\frac{j}{1000 \cdot 1 \cdot i n}} \frac{10.27}{\frac{10.23}{10.192}}$

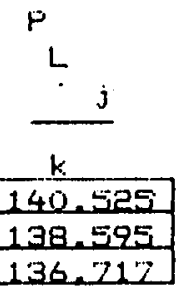

$F_{\text {NET }} \quad:=F+F_{b}+F_{i}-F$ net

Vertical equilibrium equation 

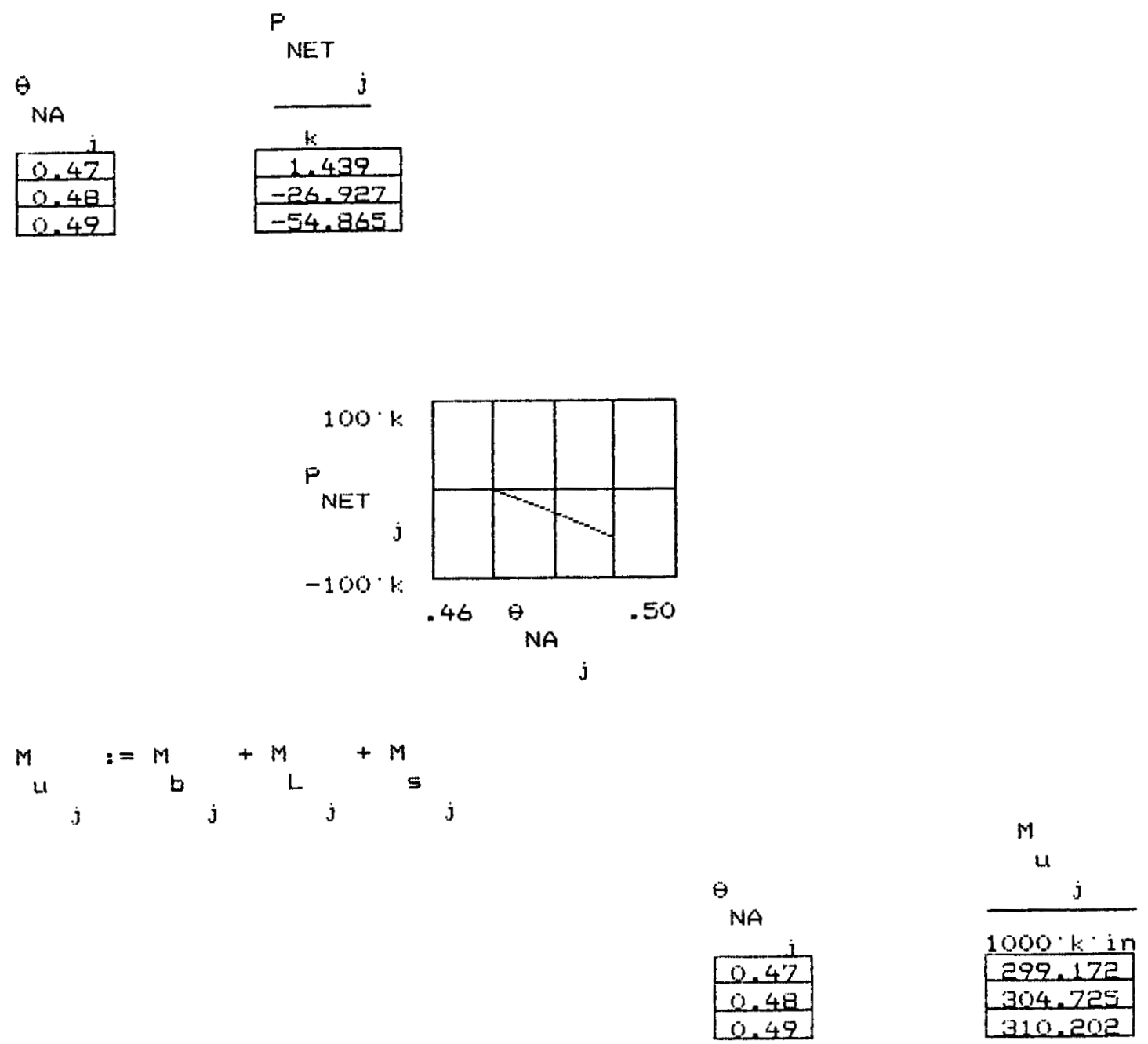

$a_{\text {CAF }} \quad:=\frac{M_{\text {net }}^{L}}{M} \cdot(.30 \cdot g)$

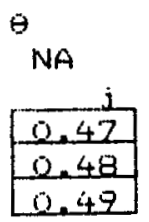

a

CAP

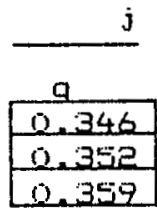




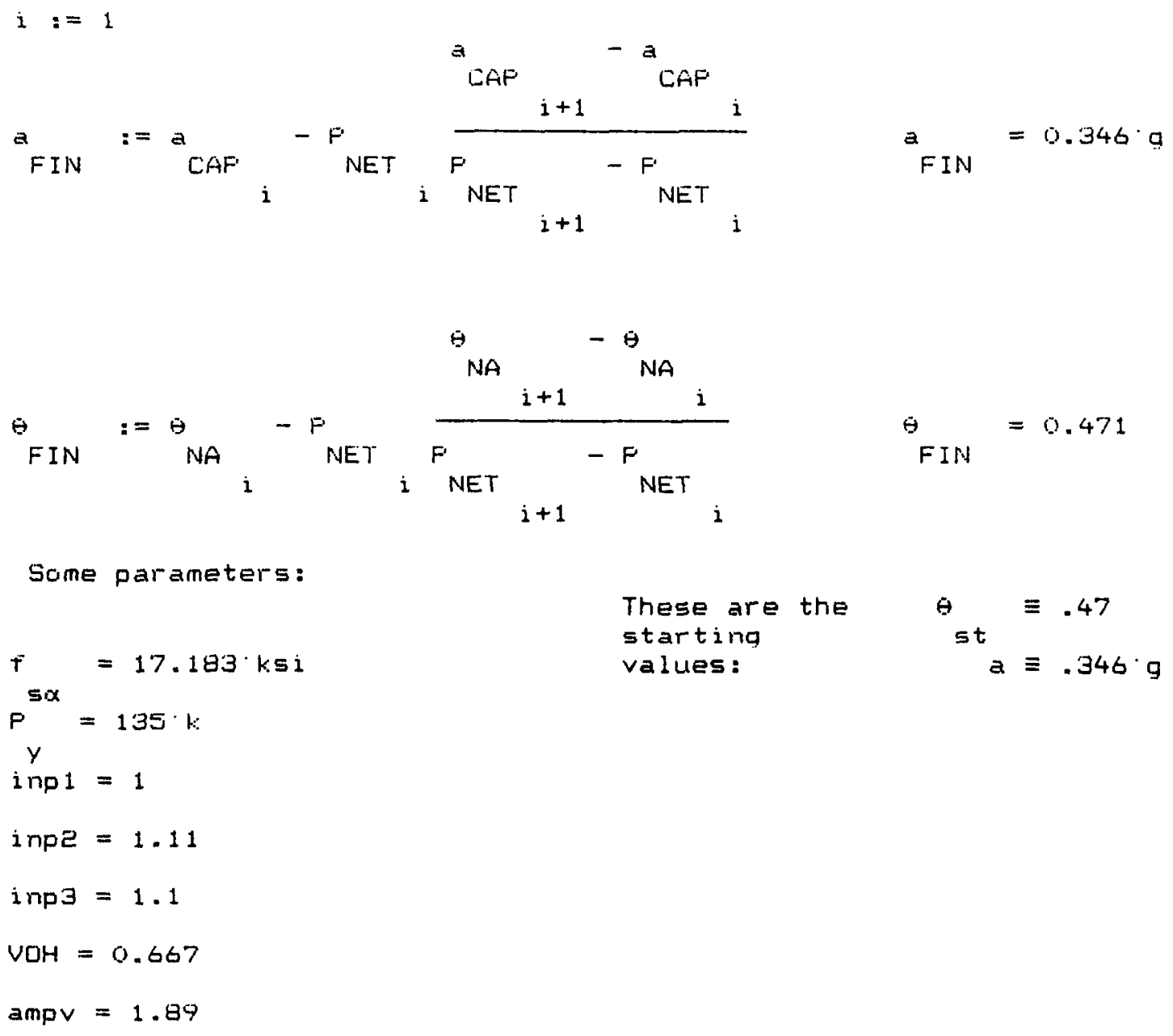




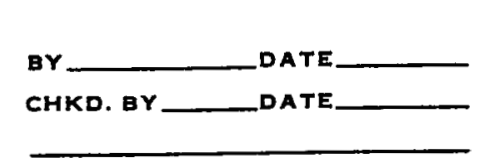

PROJECT

PAGE 13 OF

Develo, Fragritio Paramoters, F, 3, Bu \& NCLPF

Capncton

Strengeh

$$
\begin{aligned}
& \stackrel{v}{F}=0.346, / 0.346,=1.0 \quad \text { (seepage 12) } \\
& \rho_{r}=0
\end{aligned}
$$

uncertermis

1. Bult ceppity $\left(-1 \sigma=(44 \times 2,15)=110^{4}\right)$

TANk. HC2 agree Pga $=0,302$,

$$
P_{u}=\ln \frac{.346}{1302}=0.14
$$

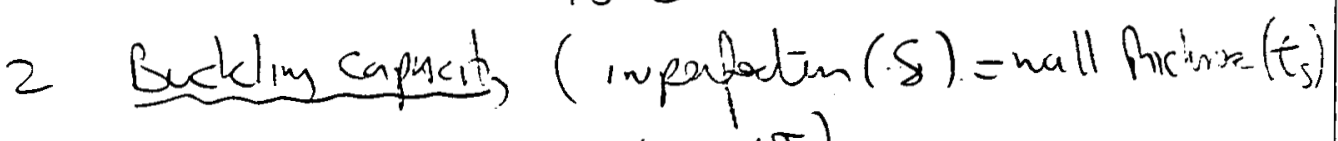
is $-1 \sigma)$

ThNK. Kez gur pga $=1334$,

$$
\rho_{v}=\operatorname{Dn} \cdot \frac{346}{1334}=\underline{0.04}
$$

3. Dolddouns water free $(-1 \sigma$ is $1 / 2$ modin forces) TANk. HC2 gives pga $=1324$ s

tutal

$$
\begin{aligned}
& p_{u}=\ln \frac{.346}{1324}=0.07
\end{aligned}
$$

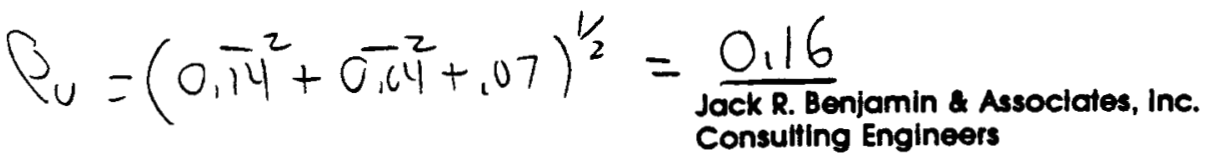

C-16 
Inolastic Respence

Bolts only yold 1 way (in tansion). Assume 3 maximun cuj cles of motion to stinato

$$
\begin{aligned}
& F_{N}=1.1 \\
& \beta_{c}=\frac{1}{2.33} \ln \left(\frac{1.1}{1}\right)=0.04 \\
& \beta_{r}=\beta_{2}=0.03
\end{aligned}
$$

Structure Reoperee

Ground Motion This parameter include:

1) Peal-to-peal spectum vovalitity

2) Noryontal Oreetim Vorralubts

3) Ejuipment Norzental Compmat Cous:

Toul capacity is contholled by maximen eanifurain heingental compomant. Factor of 1.11 to account for bias beiturean areray compones ( of 2 hory dir) ad madian of maximen duelu is rroluded in andupic (see inp $2=1.11$ on pg 4)

Randoumes for varealility of moximun compunat Cabout its madien is 0.1 .2 (see Appordix A)

Jack R. Benjamin \& Associates, Inc. Consulting Engineers

C-17 


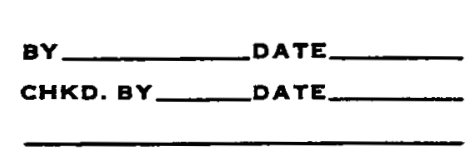

PROJECT

PAGE 15 OF

SUJECT

JOE NO.

Siwee I divedim contrals flere is no nowgratas dretem coucling

Fn peal-to-pal responce speetim varealibls analygiel touk fa $+1 \sigma$ in put, ie

$$
|n p|=e^{+.20}=1,22
$$

TANK.HC2 sure Pga $=.286$,

$$
\beta_{r}=\frac{1}{1} \ln \frac{346}{.286}=0.19
$$

Combined

$$
\begin{aligned}
& \stackrel{v}{F}=1.0 \\
& P_{u}=0 \\
& \rho_{r}=0.22\left(0,12^{2}+0.19^{2}\right)^{1 / 2}
\end{aligned}
$$

Oxmpins

Medim 2ampin is $7 \%$ (Analys perforna) pati -15 dempry is $5 \%$

from gound resporse spectra (frey om plateovof

$$
\begin{aligned}
& S_{a}(7 \%)=1.89 \\
& \text { spetra) } \\
& S_{a}(5 \%=2.12 \\
& \beta_{u}=\ln \frac{2,12}{1.89}=0.11 \\
& \rho_{r}=0 \\
& \stackrel{F}{F}=1,0
\end{aligned}
$$

C-18 
aY

CHKD. BY
PROJECT

SUBJECT

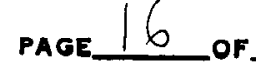

JOB NO.

Modelin

frepueng-since towk Arewerey $\left(\approx 6 h_{3}\right)$ is in vegion of ground vospme spetrer platew no variabilibj

$$
\begin{aligned}
& Y=1.0 \\
& \beta r=0 \\
& \rho_{u}=0
\end{aligned}
$$

Mode Shape - Assme contuvlled by Pirst mode \& analysis is rigorous

$$
\begin{aligned}
& \vec{F}=1.0 \\
& \rho_{r}=0 \\
& \beta_{u}=0 \quad \text { (estinate) }
\end{aligned}
$$

Mode Cemulutim

Assune no varealicts for mode effect

$$
\begin{aligned}
& \underline{v}=1.0 \\
& \rho_{r}=0 \\
& \rho_{r}=0
\end{aligned}
$$

Voriguntal Compowent Fhasin

Response contuollad in I direction (ie. max dir)

$$
\begin{aligned}
& \underline{v}=1,0 \\
& \beta_{v}=0 \\
& \beta_{v}=0
\end{aligned}
$$

SSI (Ruch site)

$$
\begin{aligned}
& \underline{F}=1,0 \quad \rho_{v}=0 \quad \beta_{v}=0.05 \text { Jack R. Benjamin \& Associates, Inc. } \\
& \text { (estivate) }
\end{aligned}
$$

C-19 


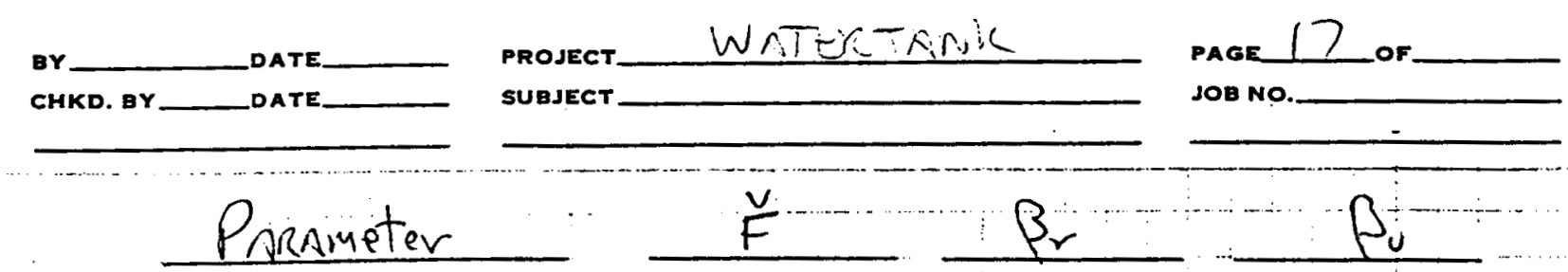

Expact,

$\begin{array}{llll}\text { Strenget } & 1,0 & 0 & 0.16 \\ \text { Inelastic Response } & 11 & 0.03 & 0.03\end{array}$

Equipment Response

Spectral Shape

Dampin)

Medeling-Frequency

- Modes hapi

Mode Comsinatim

Horiz. Component Phasing

Sivucture Response

Grand Motion

Dampin)

Modeliw ... Fuaguency

Mode SLape 110

Mode Combinatrow

Horiz Component Phasing)

SSI

Imelastic Response

Combined:

I.O

1,0

0

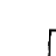

$$
\begin{aligned}
& \check{a}_{\text {pga }}=(1.1)(0.346) \mathrm{g}=0.38 \mathrm{~g} \quad \check{a}_{\text {pga }}(\text { para } 84 \%)=1.4(.38)=0.53 \mathrm{~g} \\
& \text { NCCP } \bar{F}_{m}(0.38) e^{-1.65(.20+.20)}=J .19 ;
\end{aligned}
$$

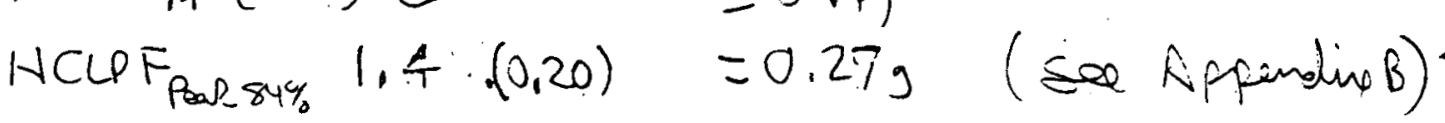

C-20 


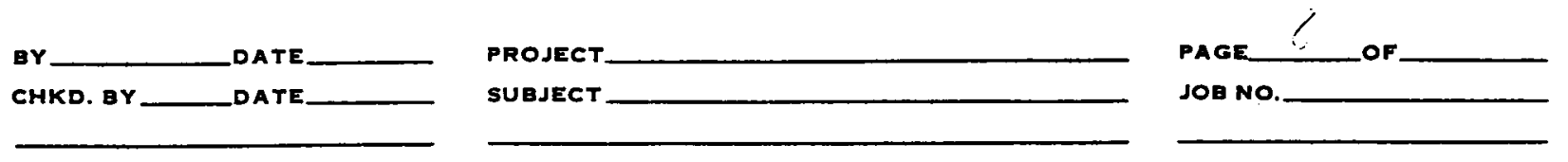

$\frac{\text { Compute NOLPF Divectly }}{\text { (See Appendex C) }} \sum x \cdot \beta=1,65(122+, 20)=0,0,69$

Parameter Consorvations x, B Determenistic Iniput

Strangeh $95 \%$ NEP $\quad(1,65)(0.16)=.26 \quad$ Doit Cap $=110^{2}-1 \sigma=0.14$

Buchly $8=t_{s}-1 \sigma=0,04$

$=1 / 2$ Hold water $-1 T=\frac{0.07}{0.25}$

Inelastie Rospase $\ln \frac{1,1}{1}$

0.10

$$
\text { (ie } \cong-2,33 \sigma)
$$

Ground Motion $+1 \sigma$

Damping $+1 \sigma$

$$
\Sigma=0.69
$$

$$
F \mu=1,0(\operatorname{inp} 3=1,0)
$$

inpr $=1.11$

Tnpl $=e^{.20}=1.22$

increare $\operatorname{inp} \mid$ to $(1,22)(2,12)$

ates ampu $=2,12$

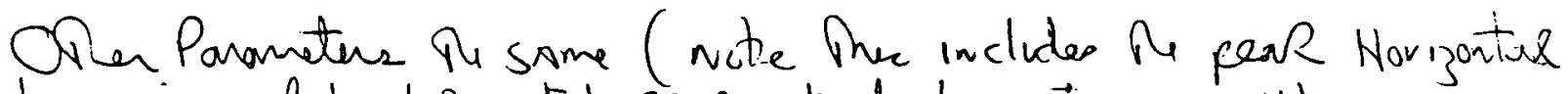

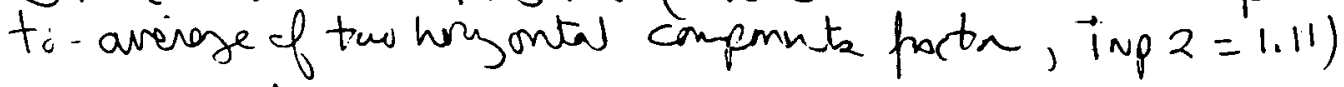

Sa Analupio on pays 19-28

Medem LCLN NCLP $F_{M}=0.20 \mathrm{~g}$

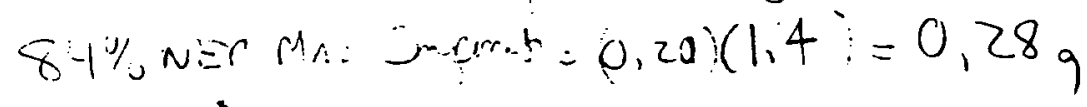

¿ see Apporshex B

Jack R. Benjamin \& Associates, Inc.

C-21 
CONSISTENT SET OF UNITS:

BASE UNITS:

$$
\begin{aligned}
& \text { in } \equiv 1 L \\
& m \equiv 1 M \\
& \text { sec } \equiv 1 T \\
& f t \equiv 12 \cdot i n \\
& 1 b \equiv m \cdot \frac{\text { in }}{\text { set }}
\end{aligned}
$$

DERIVED UNITS:$$
k \equiv 1000 \cdot 10
$$$$
g \equiv 3 e \cdot 2 \cdot \frac{f t}{\sec }
$$

$$
k s i \equiv \frac{k}{\text { in }^{2}}
$$

MFa $=0.144 \cdot 1.5 i$

DEFINE MEDIAN FFIOFEFTIES OF TANF:

$$
\begin{aligned}
& F:=240 \cdot \mathrm{in} \\
& \text { t : }=0.375 \cdot \mathrm{in} \\
& \mu:=1.24 \\
& \delta:=t \\
& t:=0.25 \cdot \mathrm{in} \\
& \text { E : }=28000.1 .5 i \\
& 5 \\
& A_{b}:=3.14 \cdot \text { in }^{2}
\end{aligned}
$$

Fiadius of tank:

Thickness of tant: wall

Shell imperfection factor - far buckling cap.

Shell imperfection size - assumed equal to shell thiclness (normal constructican)

Thickness of tank bottom

Madulus of elasticity of tant steel

Area of tank holddown anchar bolt far stiffness calculations - use 2.50 for strength 


$$
\begin{aligned}
& \begin{array}{l}
\mathrm{E}_{\mathrm{b}}:=29000 \cdot \mathrm{ksi} \\
\mathrm{F}_{\mathrm{V}}:=110 \mathrm{k}
\end{array} \\
& h:=27 \cdot \text { in } \\
& 1:=54.5 \cdot i n \\
& \text { e } \\
& f:=37 \cdot 1: 5 i \\
& P \quad:=63.7 \cdot k \\
& F_{E Q}:=63.7 \cdot k
\end{aligned}
$$

$$
\begin{aligned}
& \delta_{w}:=62 \cdot 4 \cdot \frac{1 b}{3} \frac{f^{3}}{h}:=37 \cdot f t \\
& w \\
& n:=8
\end{aligned}
$$$$
i:=1 \ldots n
$$$$
\theta_{i}:=(i-1) \cdot \frac{2 \cdot \pi}{n}
$$$$
\text { VOH }:=\frac{3}{3}
$$$$
\text { amp }:=2.12
$$$$
\operatorname{inp} 1:=1.22 \cdot 1 \cdot 12
$$$$
\text { inpe:=1.11 }
$$$$
\operatorname{inp} 3:=1.0
$$

Madulus of elasticity of bolt steel

Yield strength of bolt: use effective yield stress equalta average $44 \mathrm{ksi}$ times the net bolt area $=2.50 \mathrm{sq}$ in. This produces total capacity $=110 \mathrm{k}$.

Height of bolt from nut to bottom of tank Height of bolt from nut to embedment plate Tank median yield stress

Dead weight of tank shell and roof

Vertical earthquake force due to tank wall and roof calculated for a 1.0 gertical acceleration.

Density of water

Height of water in tank:

Number of balts

Loop over ali bolts to define angle from symmetric axis to each bolt:

Angle from symmetric axis to each bolt, i

Fiatio of vertical to horizontal earthquake components

Vertical amplification for the fluid due to tank radial expansion (median at $7 \%$ damping)

Input scale factor for higher spectral amplification relative to median input at $7 \%$ damping.

Scale factor for peak earthquake horizontal input compared to average of two horizontal components

Ductility increase factar which influences the vertical earthquake reduction in hold down farces 


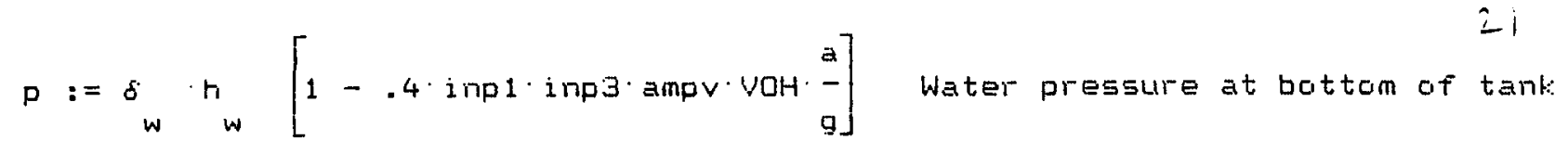

$w:=P$

Effective pressure on tank: bottoin

$I:=\left[\frac{1}{12}\right] \cdot\left[\begin{array}{c}3 \\ t^{3}\end{array}\right]$

Moment of inertia of bottom plate per length

$P_{\text {net }}:=-\left[\begin{array}{lll}F_{D L} & -F_{E Q} & .4 \cdot \operatorname{inp} 3 \cdot V O H \cdot- \\ g\end{array}\right]$

Net vertical force due to dead load and earthquake force pasitive up

$M_{\text {net }}:=233800 \cdot i n p 1 \cdot i n p 2 \cdot k \cdot$ in Net overturning moment due to earthquake
(233800 k*in for 0.309 for $7 \%$ damping
average of two horizantal compoments)

DETEFMINE SHELL BUCKLING CAFACITY:

Tank buckling capacity based on "Seismic Design of Storage Tanks" by M. J. N. Priestley, J. H. Wood and B. J. Davidson. Bulletin of the New Zealand National Society For Earthquake Engineering, Vol. 17, No. 4, December 1986.

$\left.f_{C 1}:=0.6 \cdot E_{s} \cdot\left[\frac{t}{s}\right]_{R}\right] \quad$ Classical "perfect shell" buckling stress BUCKLING IN MEMBFANE COMFFESSIDN (DIAMOND BUCKLING):

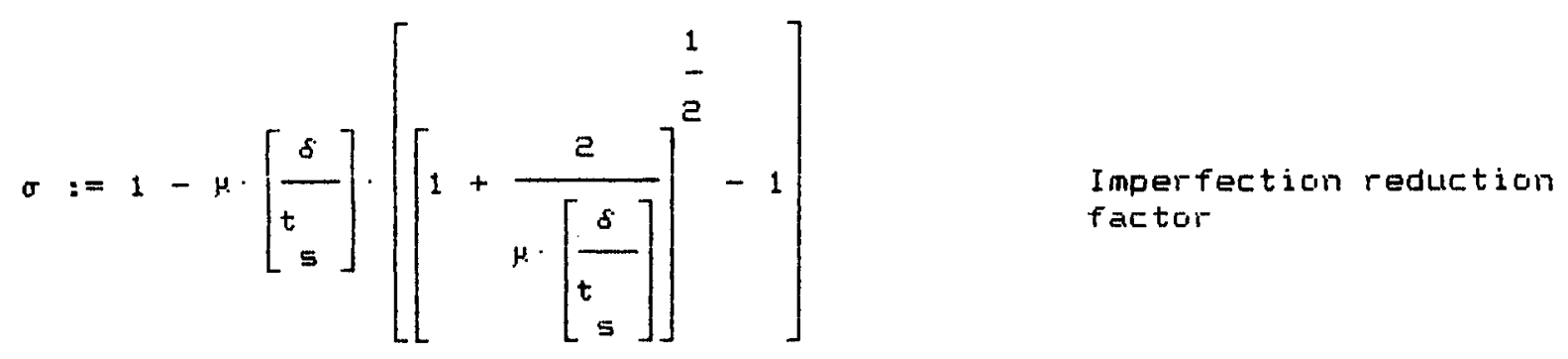<smiles>[Y]C(C)(C)C([3H])=[V]</smiles>

Factor to determine THICK or THIN wall 


$$
\begin{aligned}
& \tau_{0}:=\tau_{\gamma}\left[\begin{array}{r}
\Gamma \\
1- \\
4
\end{array}\right] \cdot(\Gamma<2)+i \tau_{\tau 1} \cdot(\Gamma>2) \\
& \text { Elastic THICF wall Elastic THIN wall } \\
& F:=\frac{P \cdot F}{t \cdot f} \quad \text { Normalized circumferential wall stress } \\
& F:=F \cdot(F<5)+5 \cdot(F\rangle 5) F \text { is Limited to } 5
\end{aligned}
$$

$$
\begin{aligned}
& L:=\sqrt{1-\left[1-\left[\begin{array}{r}
F \\
5
\end{array}\right]^{2} \cdot\left[1-\left[\begin{array}{c}
f \\
0 \\
f \\
c 1
\end{array}\right]\right.\right.} \\
& \text { Factor for wall thickness and } \\
& \text { pressure - nat to exceed } 1.0 \\
& \left.f_{p}:=f_{C 1} \cdot C \cdot(C<1)+f \quad(C) 1\right) \text { Stress limited by classical buckling }
\end{aligned}
$$$$
f_{\text {ma }}:=f\left(0.19+0.81 \cdot \frac{f}{f}\left[\begin{array}{c}
p \\
c 1
\end{array}\right]\right.
$$$$
\left.f \quad:=f \text { md }\left[\begin{array}{lll}
f & f \\
\text { md } & & y
\end{array}\right]+f \begin{array}{lll}
f & y \\
\text { md } & y
\end{array}\right] \quad \begin{aligned}
& \text { Diamond buckling capacity } \\
& \text { limited by yield stress }
\end{aligned}
$$

ELASTIC FLASTIC COLLAFSE (ELEFHANT FOOT BUCKLING):

$$
\begin{aligned}
& \frac{F}{t} \\
& s:=\frac{5}{400}
\end{aligned}
$$

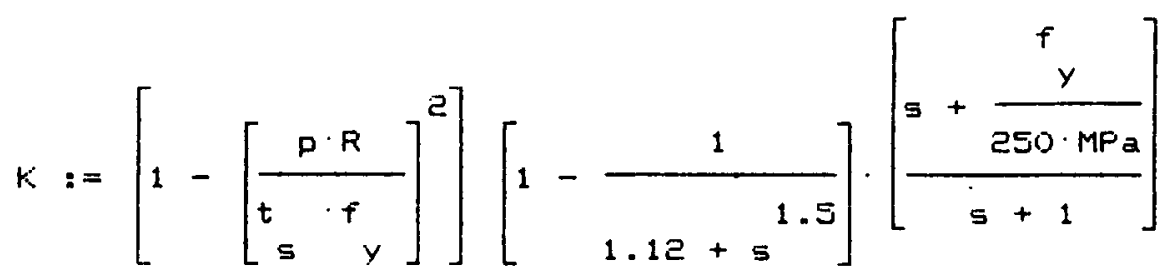




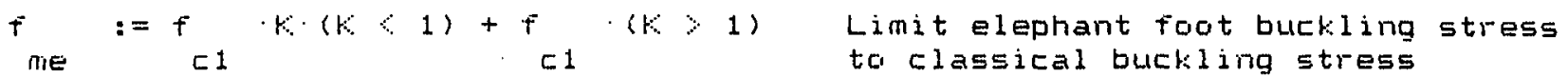

MINIMUM BUCKLING STRESS:

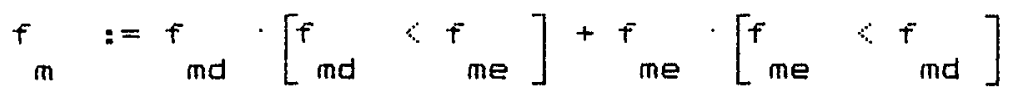

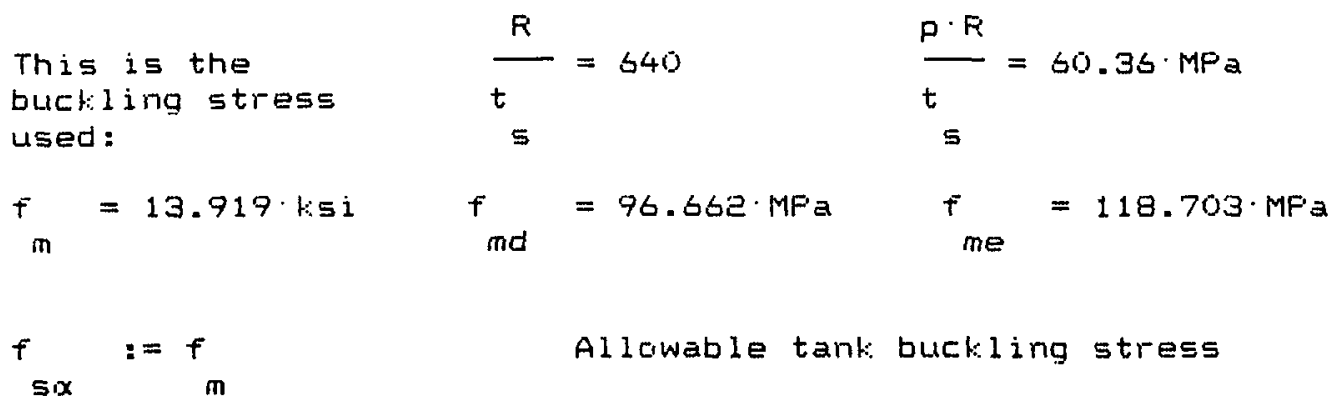

DEFINE FOFCES AND MOMENTS AT TANK BOTTOM AT THE CENTEF:

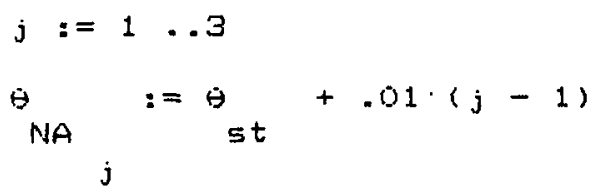

Compressive furce due to cantact between tank shell and base, positive downward:

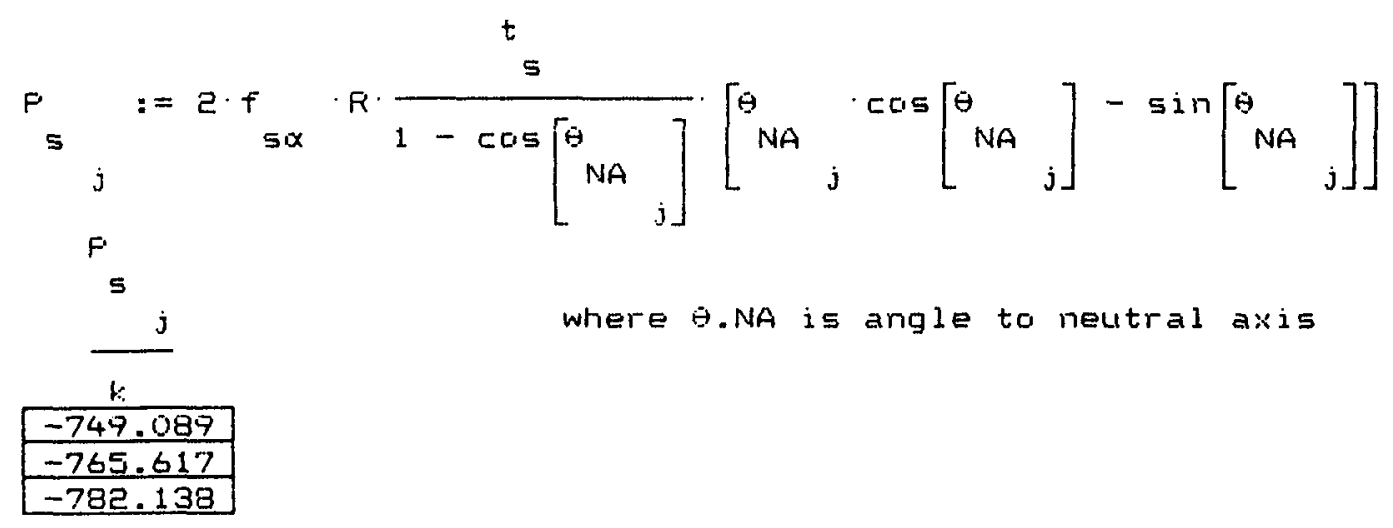


Moment due to contact between tank: shell and base, when resists earthquale overturning:

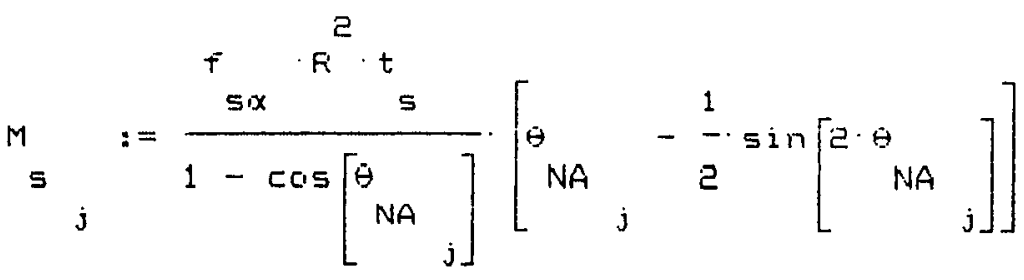

Force due to a single bolt:

$$
\begin{aligned}
& \mathrm{Fe}{ }_{j}:=\operatorname{const} \cdot\left[\cos \left[\begin{array}{ll}
\dot{\theta} & \\
N A & \\
& j
\end{array}\right]-\cos \left[\begin{array}{l}
\dot{\theta} \\
2
\end{array}\right]\right] \\
& F_{j}:=\operatorname{const} \cdot\left[\cos \left[\begin{array}{ll}
\theta & \\
N A & \\
& j
\end{array}\right]-\cos \left[\begin{array}{c}
\theta \\
3
\end{array}\right]\right] \\
& F_{j}:=\text { const } j\left[\cos \left[\begin{array}{ll}
\theta & \\
N A &
\end{array}\right]-\cos \left[\begin{array}{l}
\theta \\
4
\end{array}\right]\right] \\
& \mathrm{PS}_{j}:=\operatorname{const}{ }_{j}\left[\cos \left[\begin{array}{ll}
\dot{\theta} & \\
N A & \\
& j
\end{array}\right]-\cos \left[\begin{array}{l}
\theta \\
5
\end{array}\right]\right]
\end{aligned}
$$

Modify bolt force to be greater than o but less than Fy:

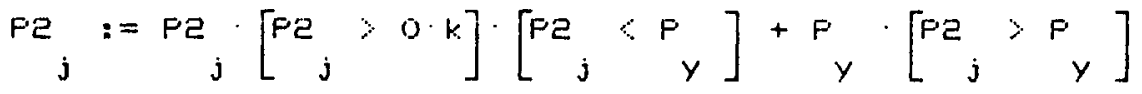

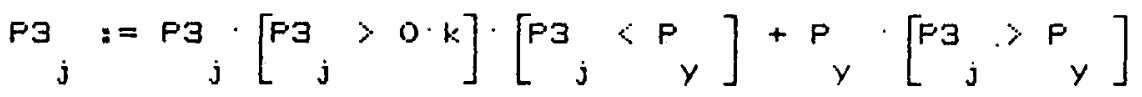




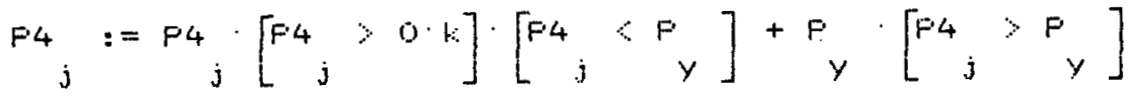

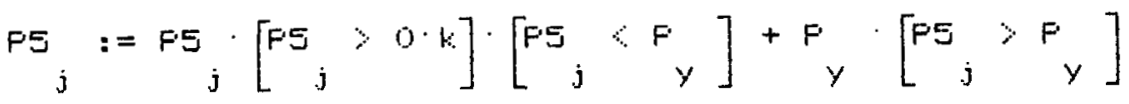

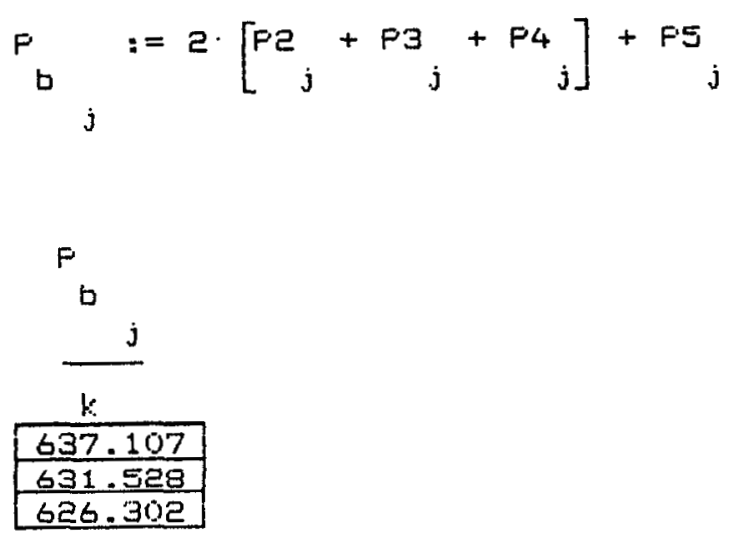

Moment due to all bolts, pasitive when resists earthquake overturning:

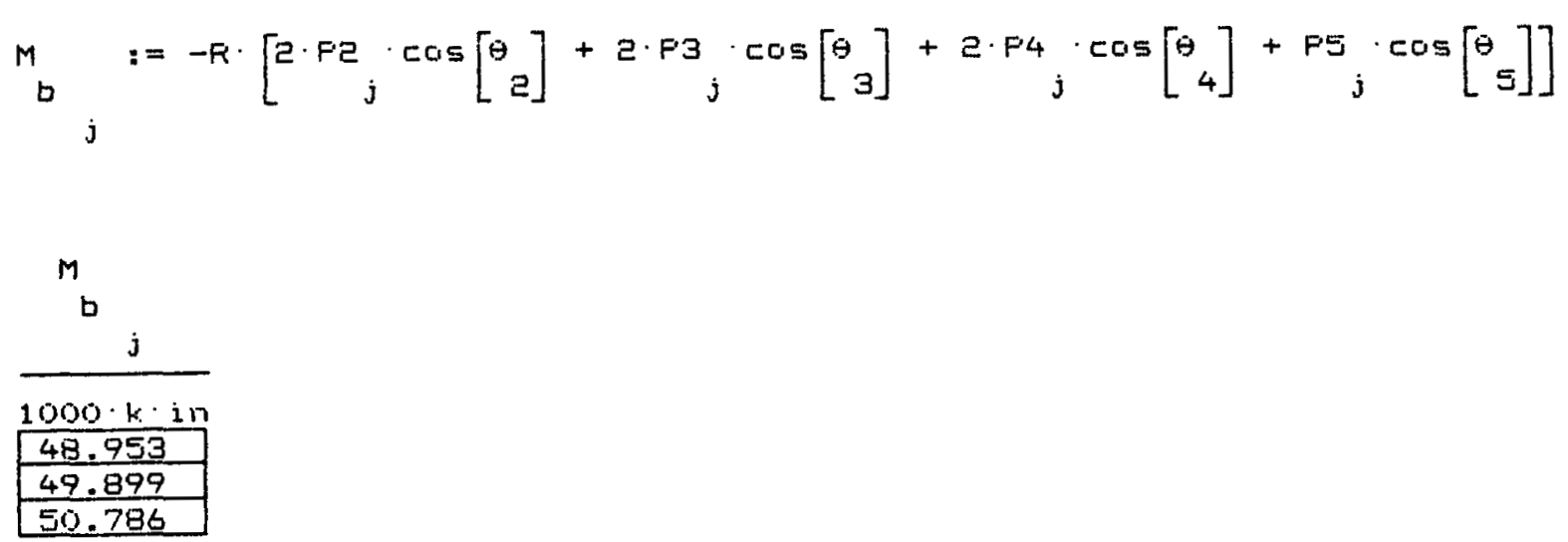


Tatal hold down force fram water from bottom plate, positive dowinward: This equation assumes that the there is no rotation at the tank: shell/bottam plate interface as the plate picks up due ta racking.)

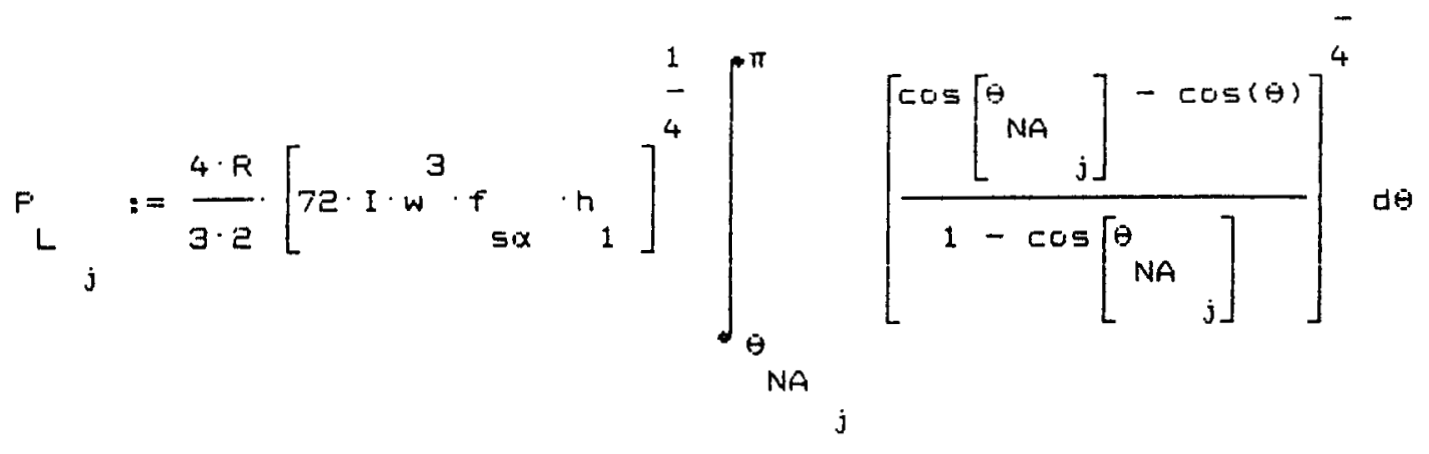

Total resisting moment from water on bottom plate, positive when resists earthquake averturning:

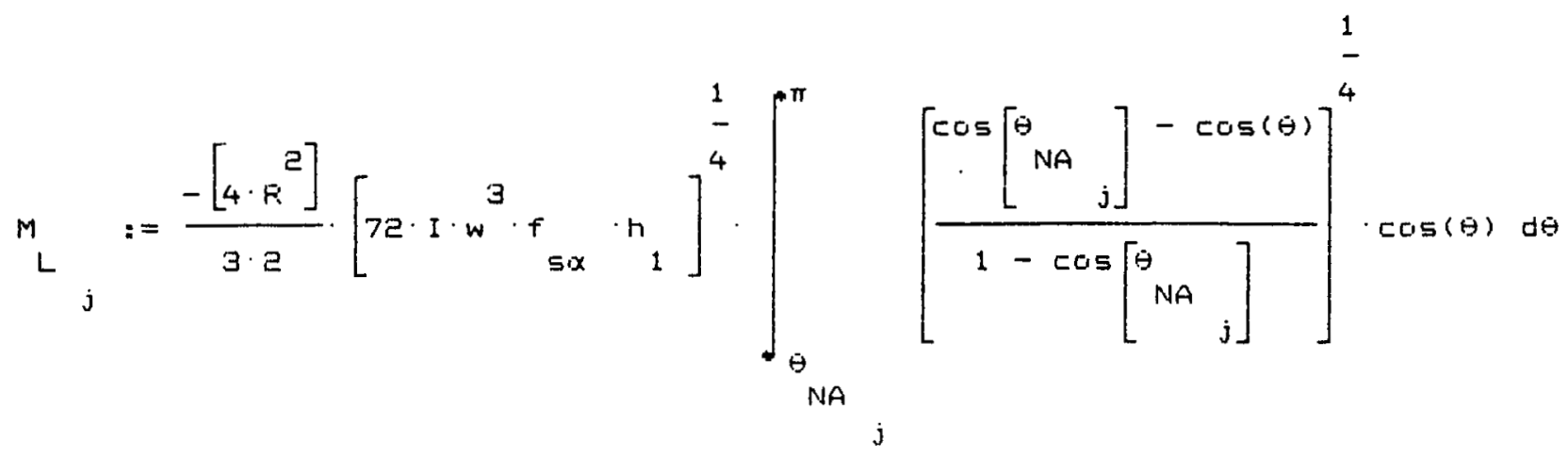

$$
\begin{aligned}
& w=0.014 \cdot \frac{t}{\text { in }^{2}} \\
& M \\
& \text { L } \\
& \text { j. } \\
& \text { P } \\
& \text { L } \\
& \text { j } \\
& j
\end{aligned}
$$$$
71.019
$$$$
70.017
$$$$
69.042
$$$$
F_{\text {NET }}:=F_{j}+F_{j}+F_{j}-F{ }_{j}-F_{\text {net }}
$$$$
\text { Vertical equilibrium equation }
$$ 

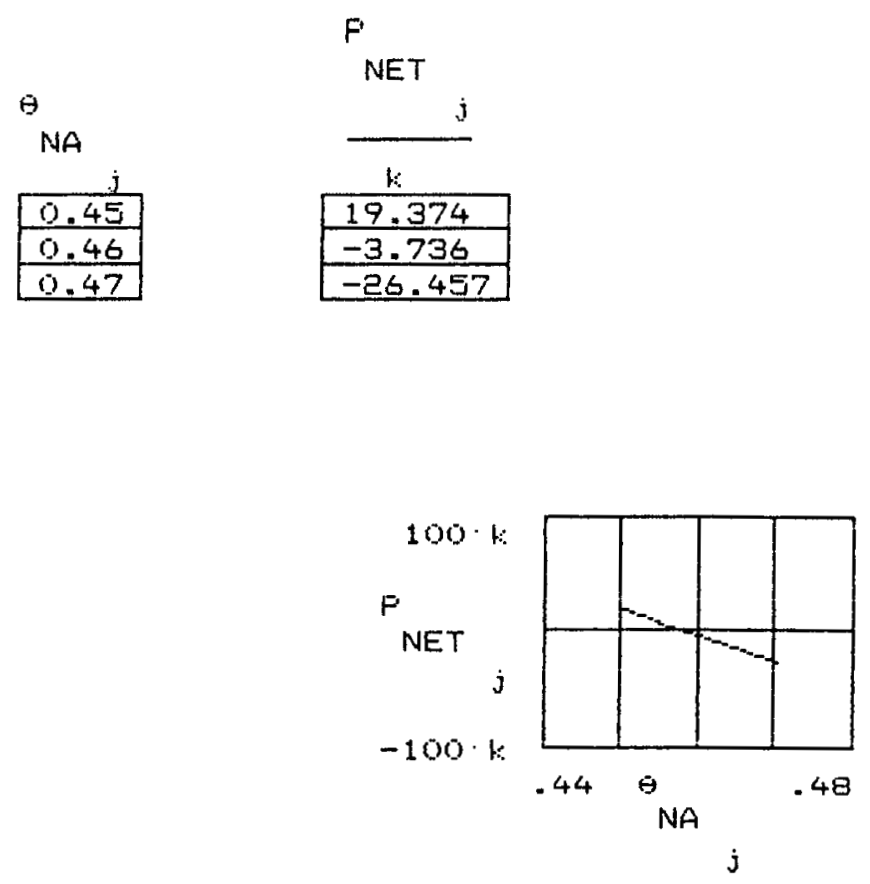

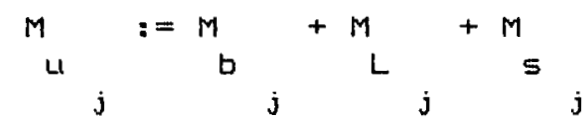

$\dot{\theta}$

\begin{tabular}{|c|}
\hline 0.45 \\
\hline 0.46 \\
\hline 0.47 \\
\hline
\end{tabular}

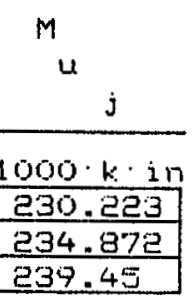

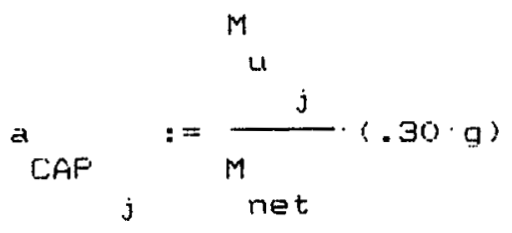

$\dot{\theta}$

NA
\begin{tabular}{|l|}
\hline 0.45 \\
\hline 0.46 \\
\hline 0.47 \\
\hline
\end{tabular}

a

CAF

\begin{tabular}{c|}
$\frac{j}{0}$ \\
\hline 0.195 \\
\hline 0.199 \\
\hline 0.203 \\
\hline
\end{tabular}




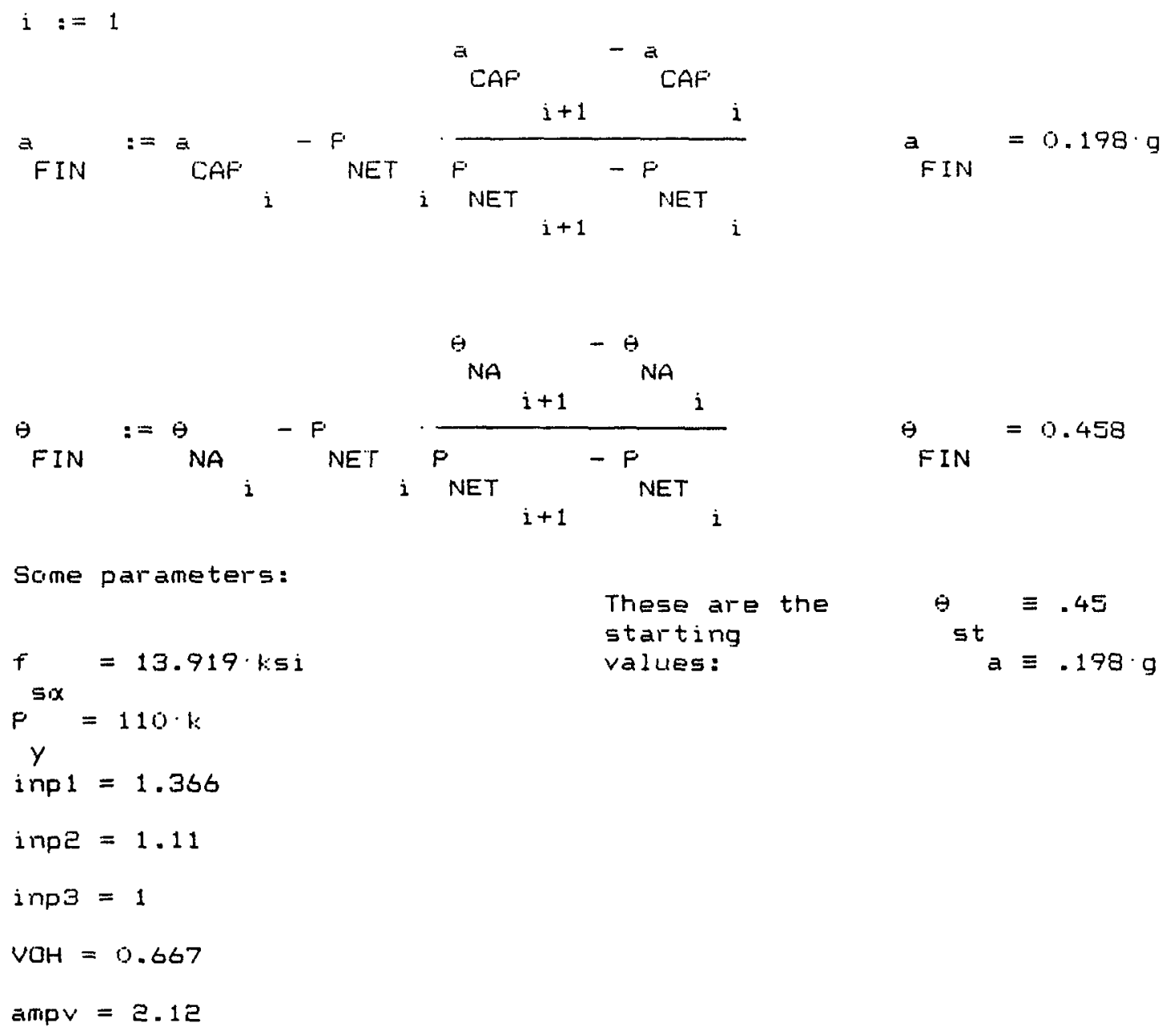




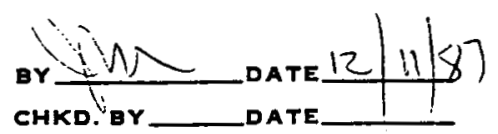

PROJECT

SUBJECT

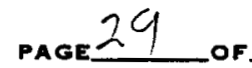

JOB NO.

Reysion to calculabions Subsorvent to Nov 12-13,1987 Metin)

Include inertial water pressue at tank bettom

Allowable bucklin, stress decreases

Wuld down furce on buttom plate increases

Revan TANK, HC2 (1.e. VTANK, NC2)

$$
\begin{aligned}
& C_{\text {Apacity }}=0.354 \mathrm{~g} \\
& a_{\text {pgas }}(\text { poar } 84 \%)=(1.4)(1.1)(.354)=0.55 \mathrm{~g} \\
& \text { NCLDE } F_{\text {peal } 84 \%}=0.55 \mathrm{e}^{-1.65(.20+.24)}=0.28 \mathrm{~g}
\end{aligned}
$$

Jack R. Benjamin \& Associates, lnc. Consulting Engineers

$c-32$ 


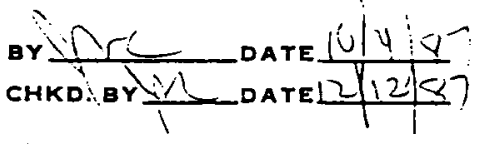

PROJECT
SUBJECT

PAGE 1

JOB No. $105-17 !$

AUXILARY CONTACTOR CATER NT GROUND

: Spain, EPRI Gers * (see pays D-3q)

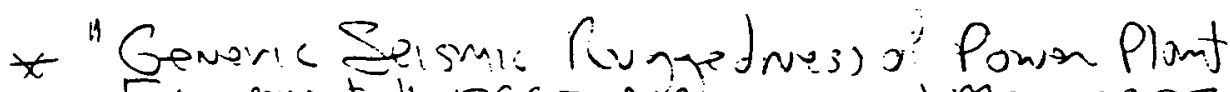

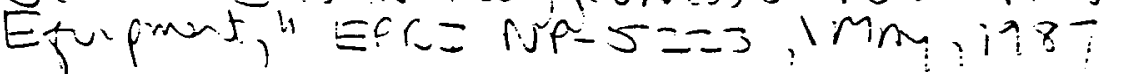

2. Failure (see page 2)

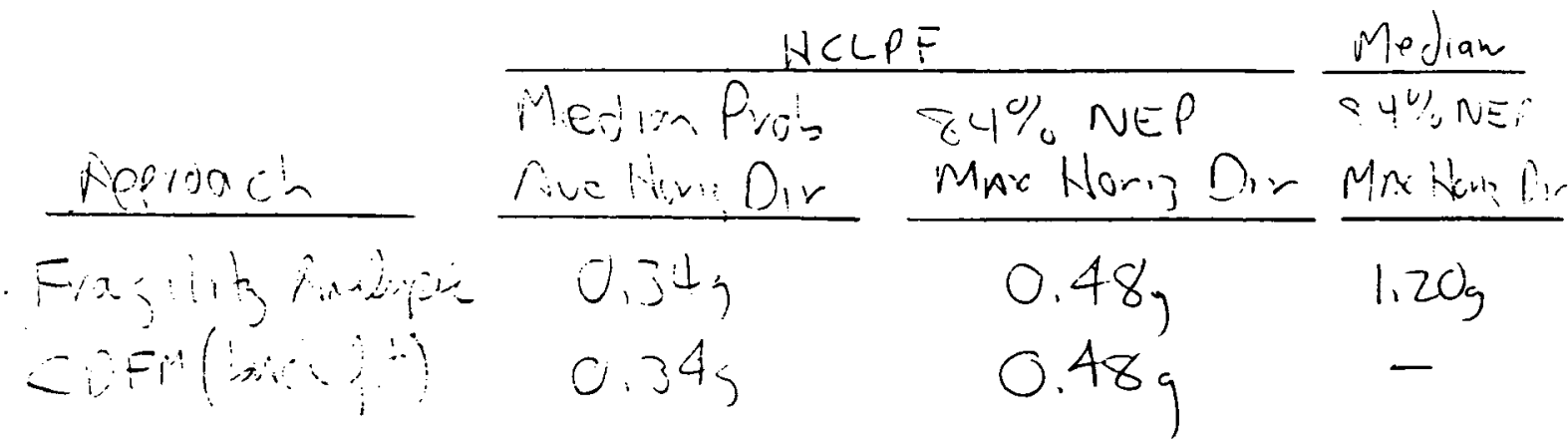

Jack R. Benjamin \& Associates, Inc. Consulting Engineers

$\mathrm{C}-33$ 


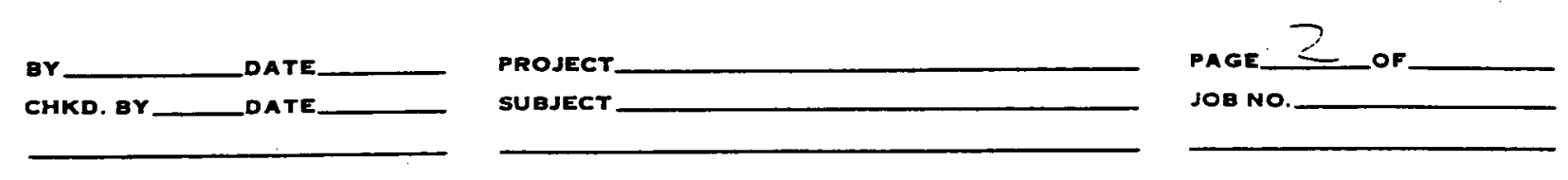

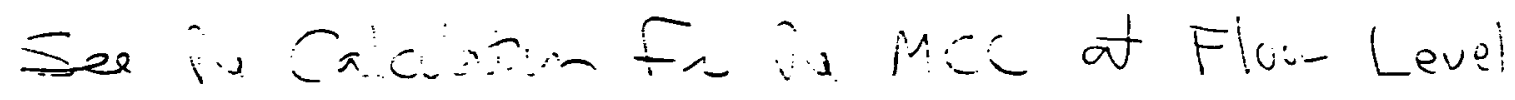
pr discessin o jonesi Assumptirm

To divenuree here is ow the oposid rospence spederin ( $5 \%$ dampes melered to $1 \mathrm{~g}$ ) is in inpt

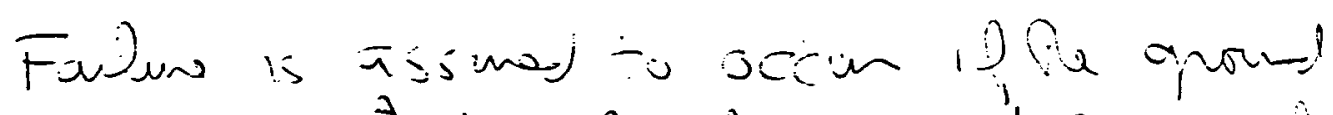

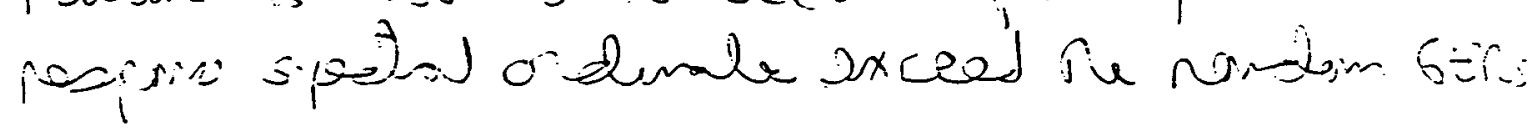

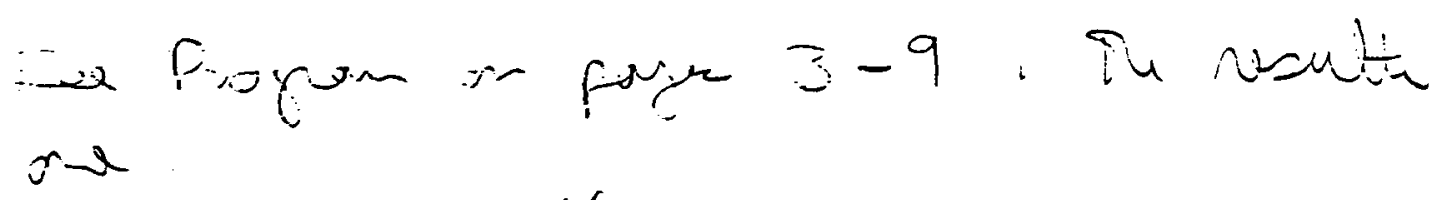

$$
\begin{aligned}
p_{3}^{2} & =0.8579 \\
p & =0.27
\end{aligned}
$$

Note jat an could here bex obtanal dereth,

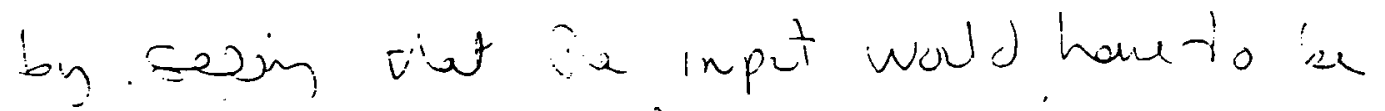

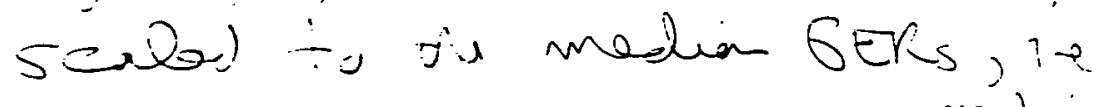

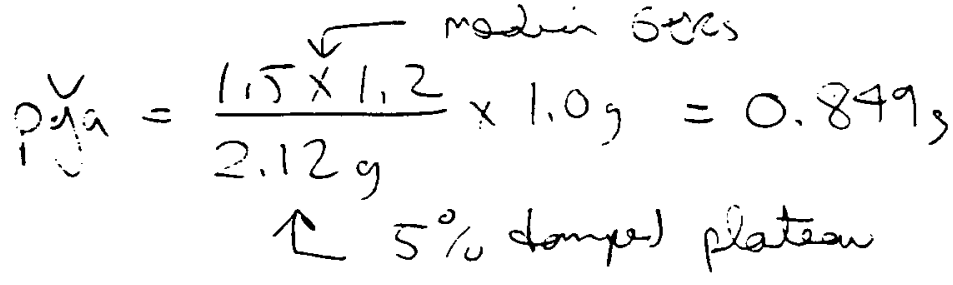

$$
\begin{aligned}
& p=0.27 \text { (2um in Gtrs) } \\
& \begin{array}{l}
\text { Jack R. Benjamin \& Associates, Inc. } \\
\text { Consulting Engineers }
\end{array}
\end{aligned}
$$

C-34 


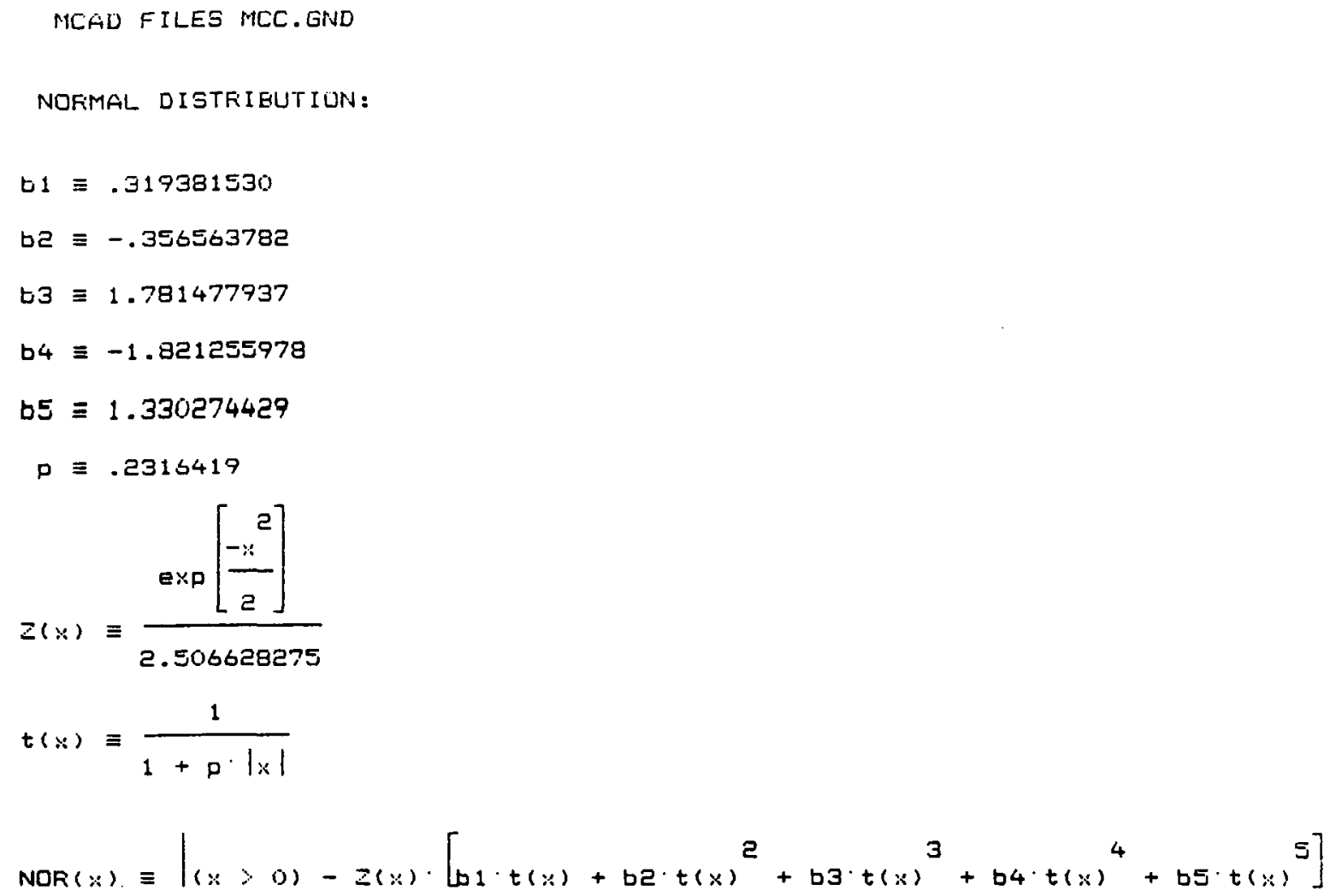


FEAD IM AND FLOT THE EASIC FLOOR FESFONSE SFECTFUM GEFS:

is

$i:=0 . .40$

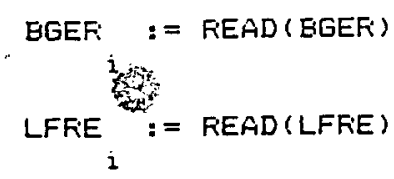

3

$s 1:=$ cSpline(LFFE, BGEF)

$1:=1 \quad .600$
Median GEFis values

Lagarithms of the corresponding frequencies

This fits a cubic spline through the GEFS values

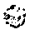

$$
i \operatorname{inerp}\left[51, \text { LFRE, BGER, } \ln \left[\frac{k}{10}\right]\right]
$$

4

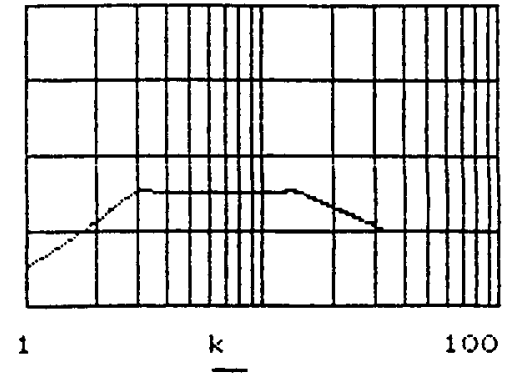

10

READ IN THE MEDIAN GROUND RESPONSE SFECTRUM NORMALIZED TO 1.OG:

$1:=0 \quad .40$

GRSF : = READ (GFSP)

Median grcund respanse spectrum values 1

LFFG $:=$ FEAD (LFRG)

Logarithms of the corresponding frequencies

s3 := cspline (LFFG, GRSP) 
This flincticin computes 1 if either the Sa value is greater than the GEFis, otherwise 0 and sums the values civer all $n$ trials:

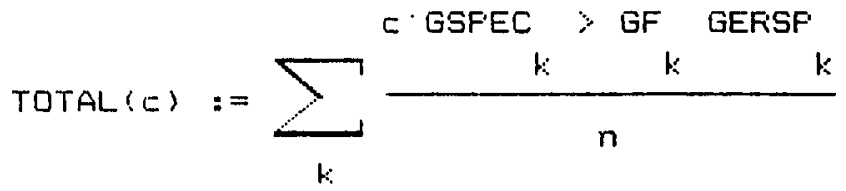

$N:=10$

$j:=0 \ldots N$

$\operatorname{pga}_{j}:=j \cdot 10+.5$

$\% \operatorname{fail}:=\operatorname{TOTAL}\left[\begin{array}{ll}\text { Pqa } \\ & j\end{array}\right]$

pga \begin{tabular}{l} 
\% fil \\
\hline 0.5 \\
\hline 0.6 \\
\hline 0.7 \\
\hline 0.8 \\
\hline 0.7 \\
\hline 1 \\
\hline 1.1 \\
\hline 1.2 \\
\hline 1.3 \\
\hline 1.4 \\
\hline 1.5 \\
\hline 0.025 \\
\hline 0.235 \\
\hline 0.395 \\
\hline 0.56 \\
\hline 0.715 \\
\hline 0.82 \\
\hline 0.895 \\
\hline 0.94 \\
\hline 0.965 \\
\hline 0.98 \\
\hline
\end{tabular}

This calculaticin take the probabilities of failure and the associated peak graund acceleration values and performs a least squares fit in the log-prabability domain to obtain the "best $f$ it" median and $\beta$ value.

$$
\begin{aligned}
& y I_{j}:=\ln \left[p q a_{j}\right]
\end{aligned}
$$

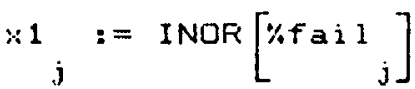




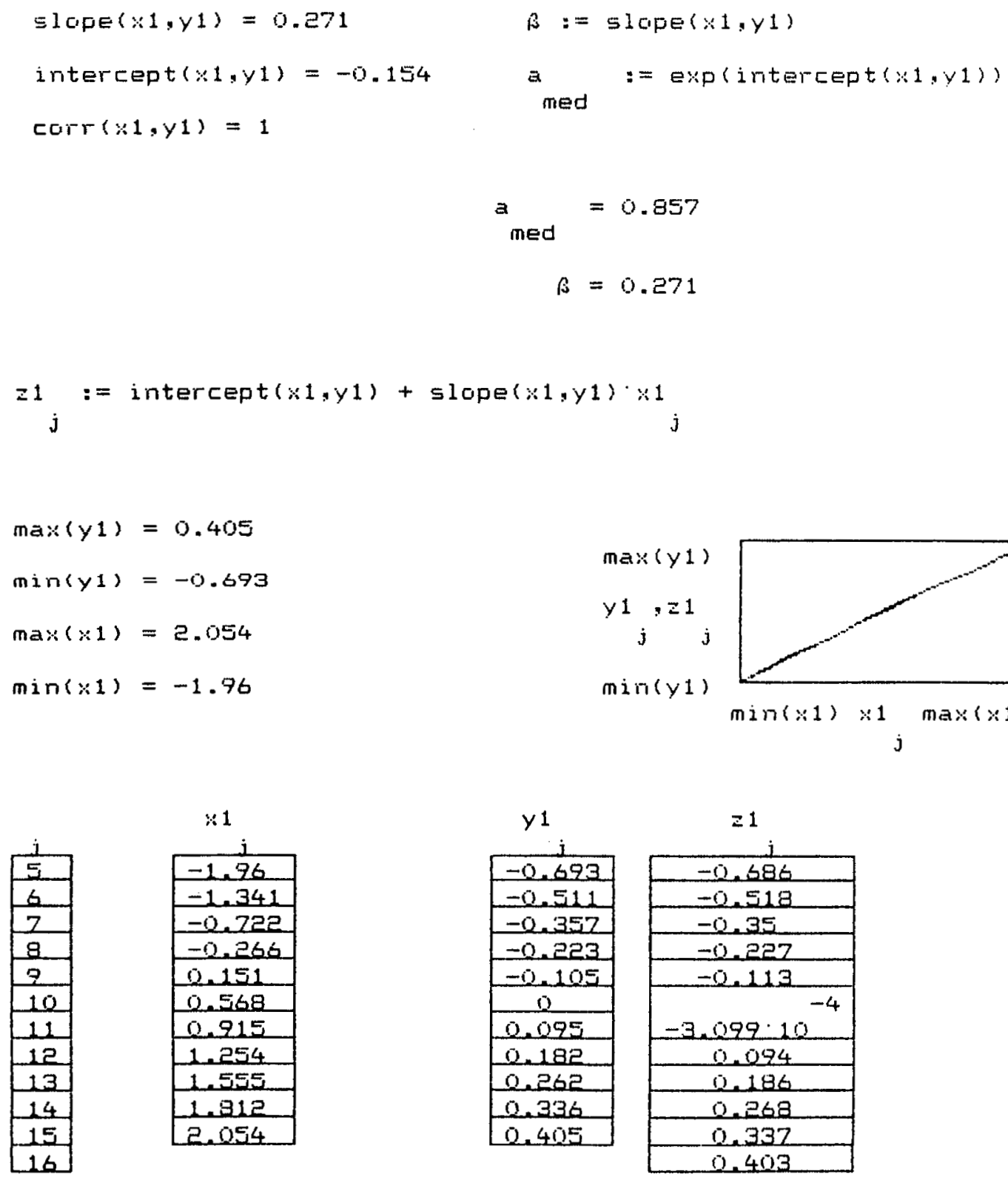

\begin{tabular}{|l|}
\hline$j$ \\
\hline 5 \\
\hline 6 \\
\hline 7 \\
\hline 8 \\
\hline 9 \\
\hline 10 \\
\hline 11 \\
\hline 12 \\
\hline 13 \\
\hline 14 \\
\hline 15 \\
\hline 16 \\
\hline-1.96 \\
\hline-0.722 \\
\hline 0.266 \\
\hline 0.151 \\
\hline 0.568 \\
\hline 0.915 \\
\hline 1.254 \\
\hline 1.555 \\
\hline 1.912 \\
\hline 2.054 \\
\hline
\end{tabular}

$y^{1}$
\begin{tabular}{|c|}
\hline-0.693 \\
\hline-0.511 \\
\hline-0.357 \\
\hline-0.223 \\
\hline-0.105 \\
\hline 0 \\
\hline 0.095 \\
\hline 0.182 \\
\hline 0.262 \\
\hline 0.336 \\
\hline 0.405 \\
\hline
\end{tabular}

\begin{tabular}{c}
$=1$ \\
\hline$j$ \\
\hline-0.386 \\
\hline-0.518 \\
\hline-0.35 \\
\hline-0.227 \\
\hline-0.113 \\
\hline-0.099 .10 \\
\hline 0.094 \\
\hline 0.186 \\
\hline 0.268 \\
\hline 0.337 \\
\hline 0.403 \\
\hline
\end{tabular}


This is the $5 \%$ damped spectrum

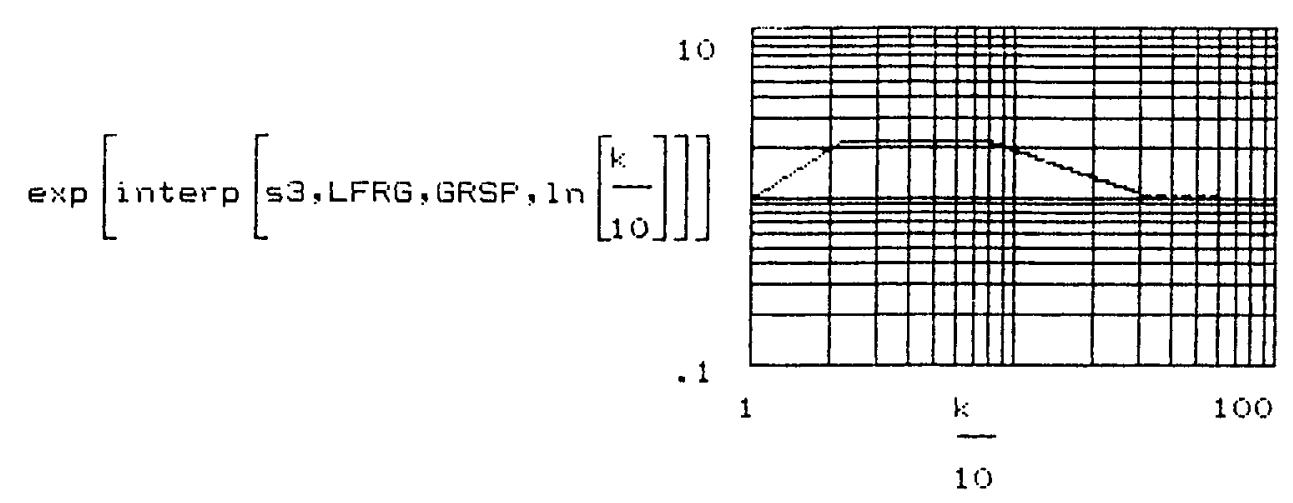

DETEFMINE FOINTS ON FRAGILITY CUFVE BASED ON LATIN HYFEFCUBE SIMULATIDN CONSIDEFING THE FOLLOWING VAFIABLES BEING UNCERTAIN:

$\begin{array}{ll}\text { Equipment frequency: } & \beta=0.20 \quad \text { median }=6.5 \mathrm{hz} \\ \text { GEFS: } & \quad \Leftrightarrow=0.27 \quad \text { median }=1.2 \times\end{array}$

Total of $n$ simulations are performed
$n:=200$
$k:=1 \ldots n$

$E F_{k}:=0.5 \cdot \exp \left[0.20 \cdot \operatorname{INOR}\left[\frac{k-1+r \operatorname{lnd}(1)}{n}\right]\right]$

This creates $n$ equipment fre values - nate it is based oi: a combined variability

$G F \quad:=1.2 \cdot \exp \left[0.27 \cdot \operatorname{INOK}\left[\frac{k-1+\operatorname{rnd}(1)}{n}\right]\right]$

This creates in factors to scale the GEFS capacity 
$p:=1 \quad \ldots 10$
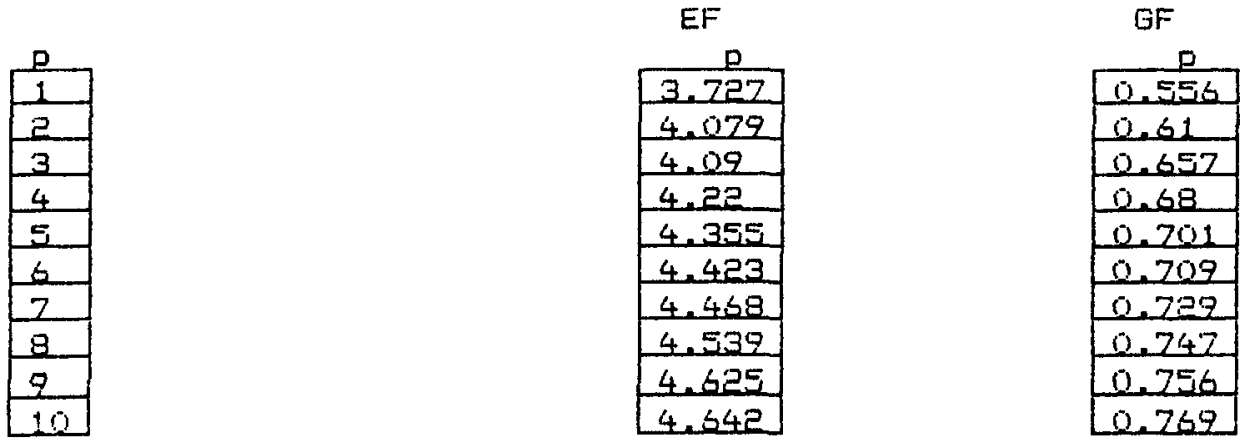

WFITE (EF) $:=E_{k}$
WRITE (GF) $:=G F$

The program is transferred to Dos where the files are randomly mixed using program CADMIX

$\mathrm{EF}_{k}:=\mathrm{FEAD}(\mathrm{EF})$
$\mathrm{GF}:=\mathrm{FEAD}(\mathrm{GF})$

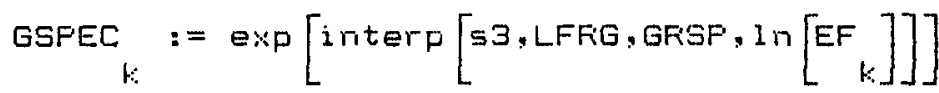

Ground spectrum values at

randoin firequencies

GEFSF $_{t:}:=\operatorname{interp}\left[s 1\right.$, LFFE, BGEF, In $\left.\left[\begin{array}{ll} \\ t\end{array}\right]\right]$

GEFs capacity values at

random frequencies
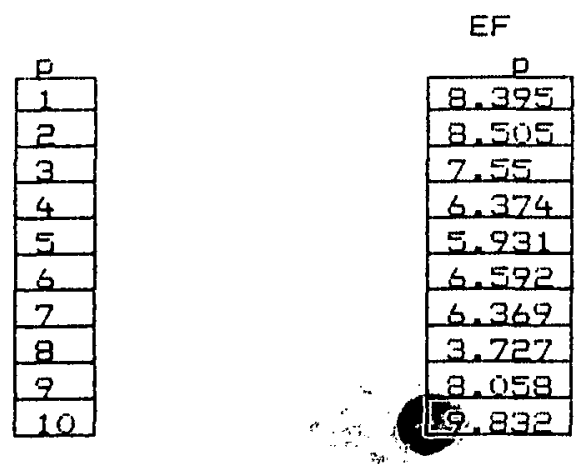

GF
\begin{tabular}{c} 
D \\
\hline 1.248 \\
\hline 1.979 \\
\hline 0.945 \\
\hline 1.067 \\
\hline 1.066 \\
\hline 1.109 \\
\hline 1.261 \\
\hline 1.045 \\
\hline 0.9 \\
\hline 1.093 \\
\hline
\end{tabular}

GSFEC
\begin{tabular}{|l|}
\hline 2.026 \\
\hline 2.062 \\
\hline 2.127 \\
\hline 2.114 \\
\hline 2.116 \\
\hline 2.113 \\
\hline 2.114 \\
\hline 2.116 \\
\hline 2.111 \\
\hline 1.894 \\
\hline
\end{tabular}

GF - GEFSF

\begin{tabular}{|l|}
\hline$p$ \\
\hline 1.872 \\
\hline 2.969 \\
\hline 1.418 \\
\hline 1.6 \\
\hline 1.599 \\
\hline 1.664 \\
\hline 1.391 \\
\hline 1.564 \\
\hline 1.35 \\
\hline 1.64 \\
\hline
\end{tabular}




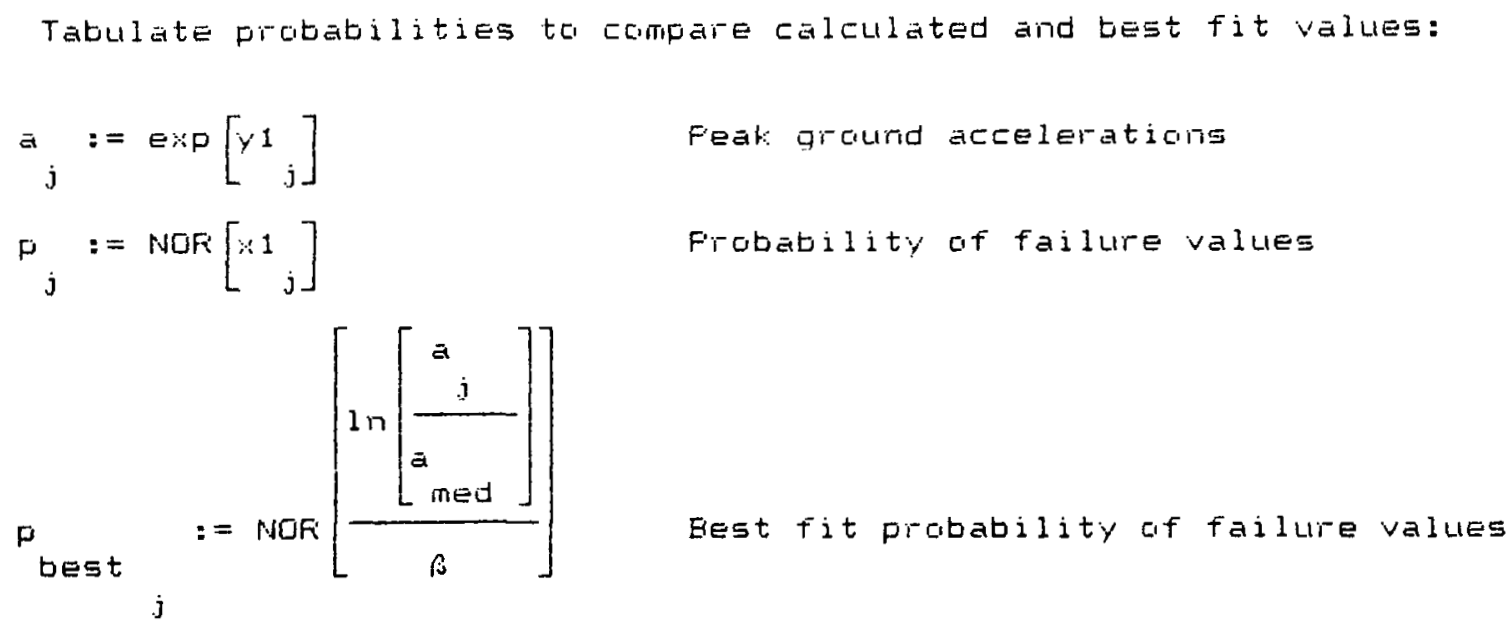

$a_{j}:=\operatorname{Exp}\left[\begin{array}{ll}y^{1} & \\ & j\end{array}\right]$

$P_{j}:=\operatorname{NDF}\left[\because \begin{array}{ll}1 \\ & j\end{array}\right]$

$P_{\text {best }}:=N O F\left[\frac{\ln \left[\begin{array}{l}a \\ a \\ m e d\end{array}\right]}{f}\right]$

Feat: grcund acceleratigin

Frabability of failure values

Eest fit probability uf failure values

\begin{tabular}{|l|}
\hline$j$ \\
\hline 5 \\
\hline 6 \\
\hline 7 \\
\hline 8 \\
\hline 9 \\
\hline 10 \\
\hline 11 \\
\hline 12 \\
\hline 13 \\
\hline 14 \\
\hline 15 \\
\hline 16 \\
\hline 0.5 \\
\hline 0.2 \\
\hline 0.8 \\
\hline 0.7 \\
\hline 1 \\
\hline 1.1 \\
\hline 1.2 \\
\hline 1.3 \\
\hline 1.4 \\
\hline 1.5 \\
\hline 1.6 \\
\hline
\end{tabular}

\begin{tabular}{|c|c|}
\hline$p$ & best \\
\hline$\frac{j}{0.025}$ & 0.012 \\
\hline 0.09 & 0.059 \\
\hline 0.235 & 0.160 \\
\hline 0.385 & 0,323 \\
\hline 0.56 & 0.497 \\
\hline 0.715 & 0.654 \\
\hline 0.82 & 0.776 \\
\hline 0.895 & 0.863 \\
\hline 0.94 & 0.918 \\
\hline 0.965 & 0.954 \\
\hline 0.98 & 0.974 \\
\hline 0.785 & 0.986 \\
\hline
\end{tabular}




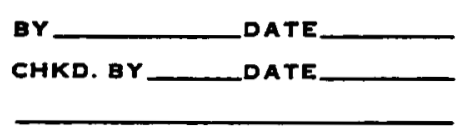

PROJECT

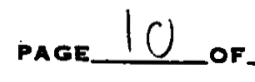

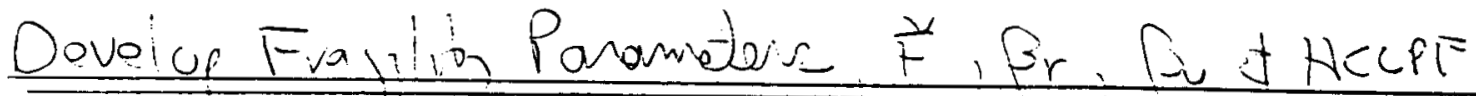

Supretin

Strengeh (see pore 2)

$$
\begin{aligned}
& \tilde{F}=0.86 / 0.80)=60 \\
& \rho_{u}=0.2- \\
& \rho_{r}=0
\end{aligned}
$$

Inolast,c rospores

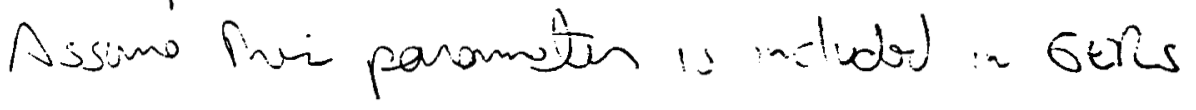

Response

Ground Motion This paramelie includs

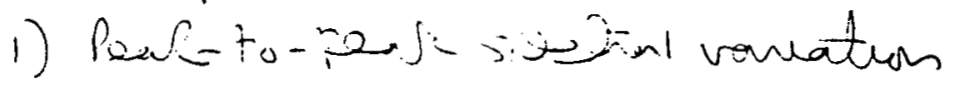

2) Nownin's Luster vonablich

3) Efurpment horantall componit cupk

Cissure cutieal elenortesurding is small Fran Appordix $A$.

$$
\begin{aligned}
& \tilde{F}=1,0 \\
& \rho_{r}=0.25 \\
& \rho_{u}=0
\end{aligned}
$$

Jack R. Benjamin \& Associates, Inc. Consulting Engineers

$C-42$ 
BY

CHKD. BY

DATE

PROJECT

SUBJECT

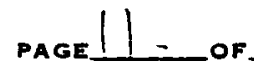

JOB NO.

Oampin:

Modion $5 \%$

$-103 \%$

$$
\frac{\text { Sai Gond, t } \left.6,5 h_{3}\right)}{2,12}
$$

2.46

$$
\beta_{2}=\frac{1}{1} \ln \frac{2.46}{2.12}=0.15
$$

Modelin

frefineng - included in simulitin (a small wizj)

Mode srape-(assume include) in GERs)

Mude Cmbinatent

Assime included in Ge'rs

Novinata) Compmimt Phacing

Assume includes in SERs

SSI (ROL Site)

$$
\stackrel{v}{F}=1,0 \quad \rho_{2}=q_{c}=0
$$

$$
\text { * } \begin{aligned}
\tilde{F} & =1,0 \\
\rho_{r} & =0 \\
\rho_{0} & =0
\end{aligned}
$$

Jack R. Benjamin \& Associates, Inc. Consulting Engineers

$C-43$ 


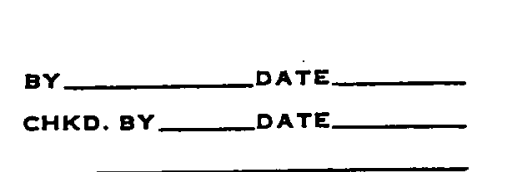

$\frac{P}{2}$

Parameter

Capacity
PROJECT
SUBJECT
PAGE 12 OF

$\stackrel{\mathrm{F}}{ }$

$\beta_{r}$

$\beta_{u}$

Inelastic Response 1,0 o 0.27

Equipment Response

Spectral Shape

Damping,

Medelin-Frequency

- Modeshaps

Mode Comsinatim

Horiz. Component Phasing

Structure Response

Grand Motion 1.0

1,0

0

0

Damping)

Modeling-Frapuency LO

Mode Shape 100

Mode Combination $\perp_{2} 0$

$N / A$

Noria Component Phasing 1.0

SSI ? 1,0

Inelastic Response
Combined: $\frac{n / x}{1,0} \frac{\pi / x}{0,25} \frac{n, \pi}{0,31}$

$\breve{a}_{p g a}=(1.0)(0.86)-\quad=0.86, \quad \check{a}_{p g a}\left(p_{e n} 84 \%\right)=0.86 \times 1,4=1,20 \mathrm{~g}$

$\operatorname{NCLP} F_{m}(.86) e^{-1.65(.25+.31)}=0.343$

NCLPF Pars 84\% $1.4(.34)=0.799$ (see Appardin B)

0

$\mathrm{C}-44$ 


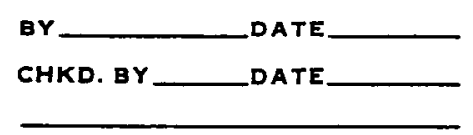

PROJECT.

SUBJECT

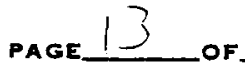

JOB NO

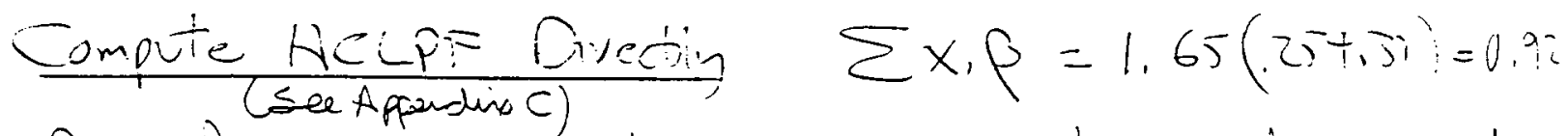

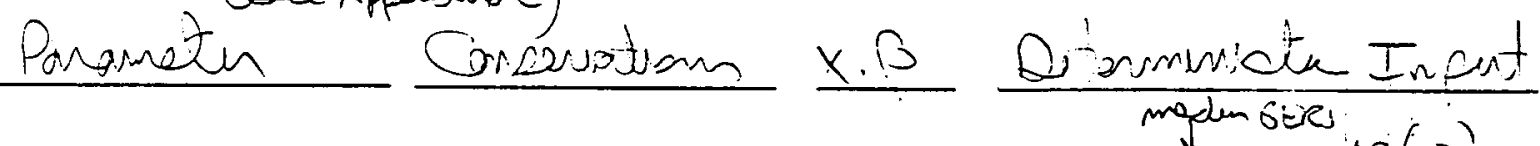

Stronguh $97,5 \%$ NEP $(1,96)(.27)=0.53 \quad(1,5)(1,2) e^{-0.96(127)}=1,06$

Ground Moten $+1 \sigma$

Oamping

$-1 \sigma$

0.25

$e^{.25}=1,28$

2.46 NCLPF

$L_{3} \%$ dimpis 5 A

Dil odier paromites of mederom value

Molim lewel NCLPF $=\frac{1,06}{(2,46)(1,28} \mathrm{g}=0,34 \mathrm{~g}$

$84 \%$ max componas $=(0,34,(1.4)=0,48 \mathrm{~g}$

¿se Appondix B

Jack R. Benjamin \& Associates, Inc.

Consulting Engineers

$\mathrm{C}-45$ 


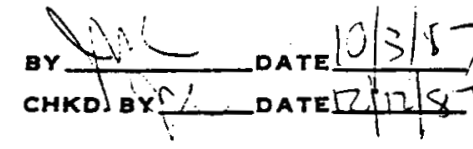

PROJECT

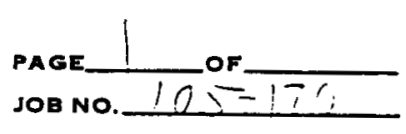

AUXILIARY CONTACTOR CHATTER RT FLOOR LESS-

1. Capacity: EPRI Ger's' (see pi D-39)

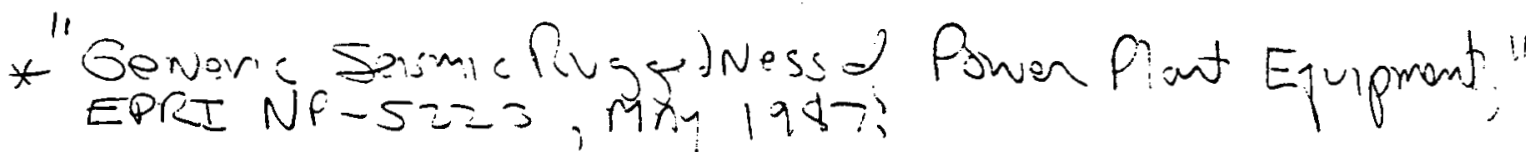

2. Failure (see page 2)

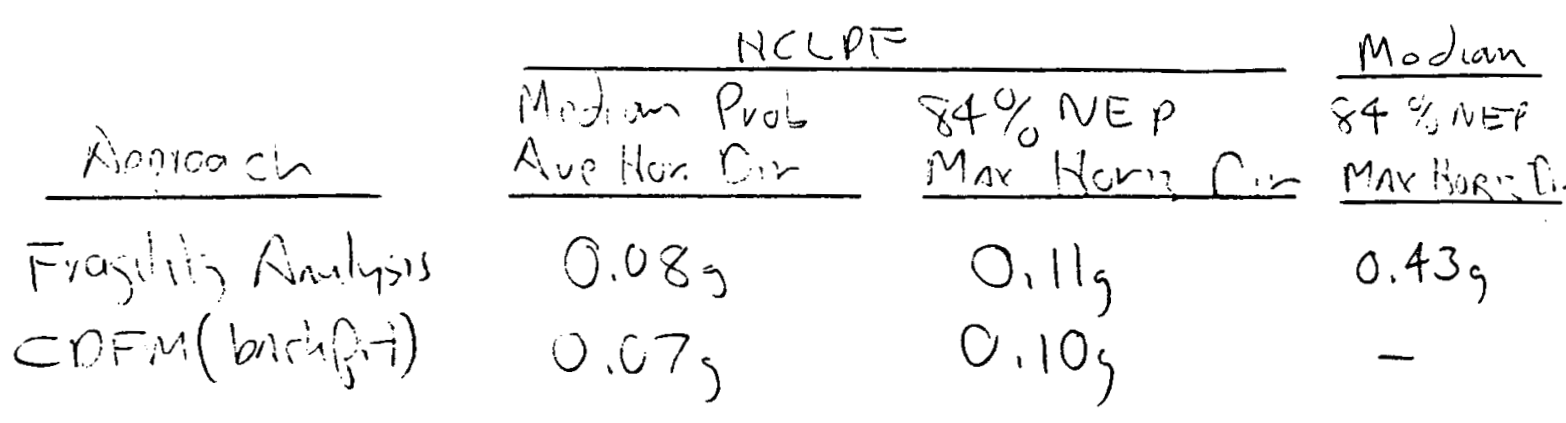

Jack R. Benjamin \& Associates, Inc. Consulting Engineers

$\mathrm{C}-46$ 
Ele medion Fracion Curse ure ostarnal

by simulition

Qhe GERs capreión Function was depinos in Pollows?

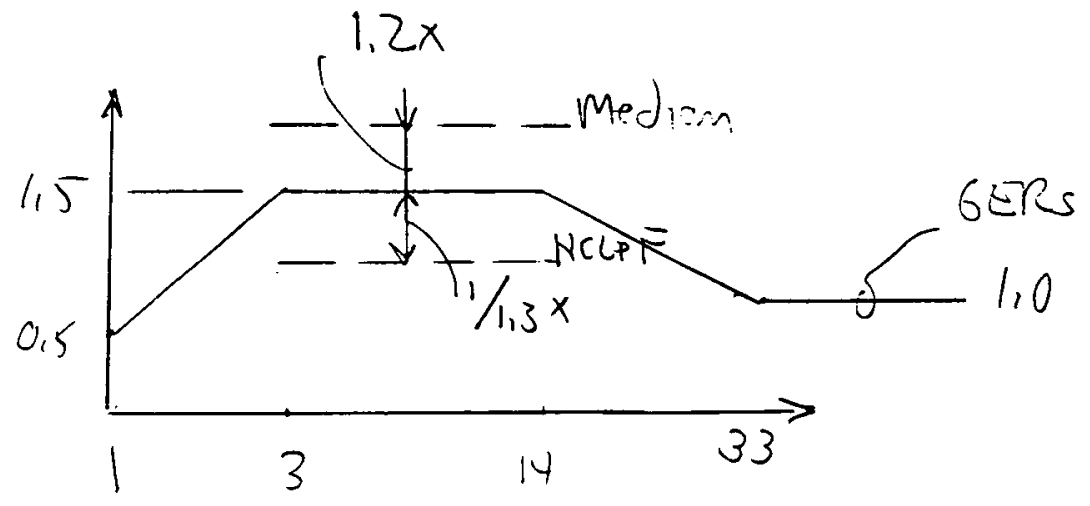

$$
\beta_{v}=\frac{\ln 1,2 \times 1,3}{1,65}=0.27
$$

Failue use concrieres to occun if eirlen of ve pollswing occur:

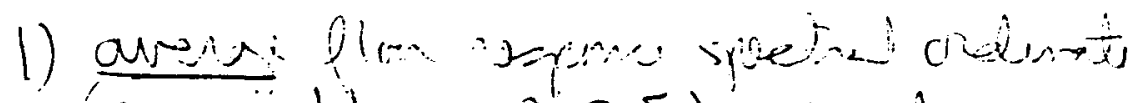
(averaing) Joven-0.2f) ixceads the randim Geres

or 2) Th Plon nxpenes spothal cidenat excoeds 1,5 x The randim Gers

Note: Rve speeta were prelt, broad herce this definitur conced iave used 1) will in average aperal to in spectum Jack R. Benjamin \& Associates, Inc. Consulfing Engineers

C-47 


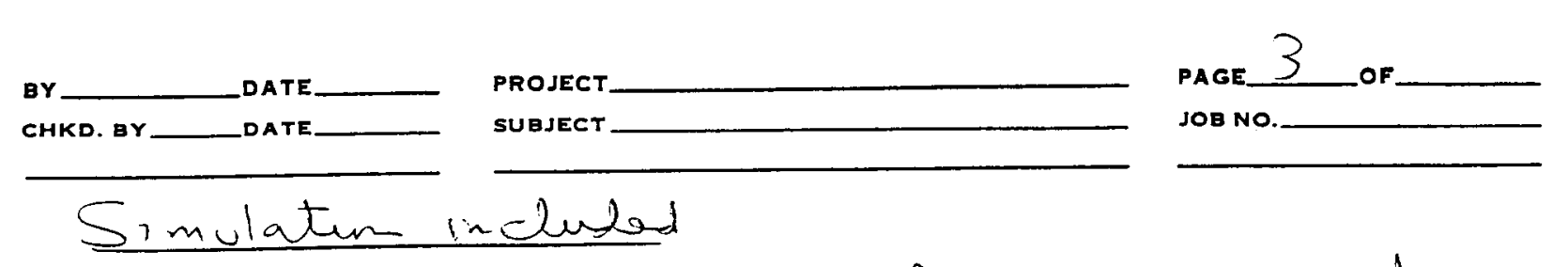

1. Sire pound varponse spectrum war valued

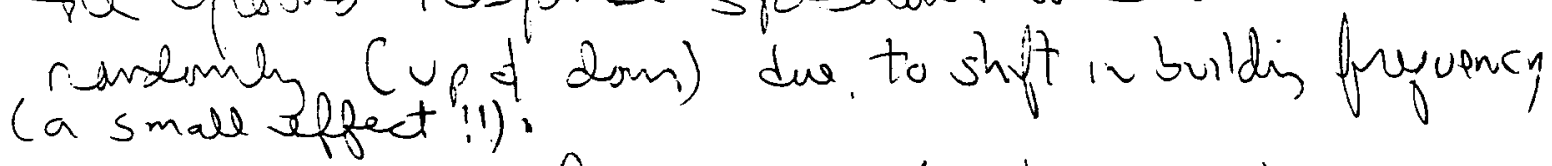

2. The augment frequency (at a combine) (P) fr vaviakhts m bo in Me erumpent and building Regueraj) was varied

3 De GERs wa varied randoms (up down)

The floor vosponee spectrum we held constant at $5 \%$ damping

page 4-13 que th aralupie ar vent ts

$$
\begin{aligned}
& \operatorname{\rho g}_{a}=0.31 \mathrm{~g} \\
& \beta_{u}=0.53
\end{aligned}
$$

Jack R. Benjamin \& Associates, Inc. Consulting Engineers

$C-48$ 
MCAD FILES MCC. FFA

NOFMAL DISTFIEUTION:

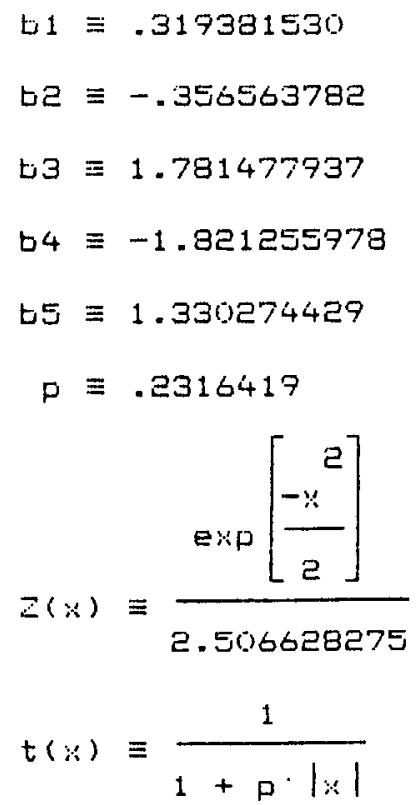



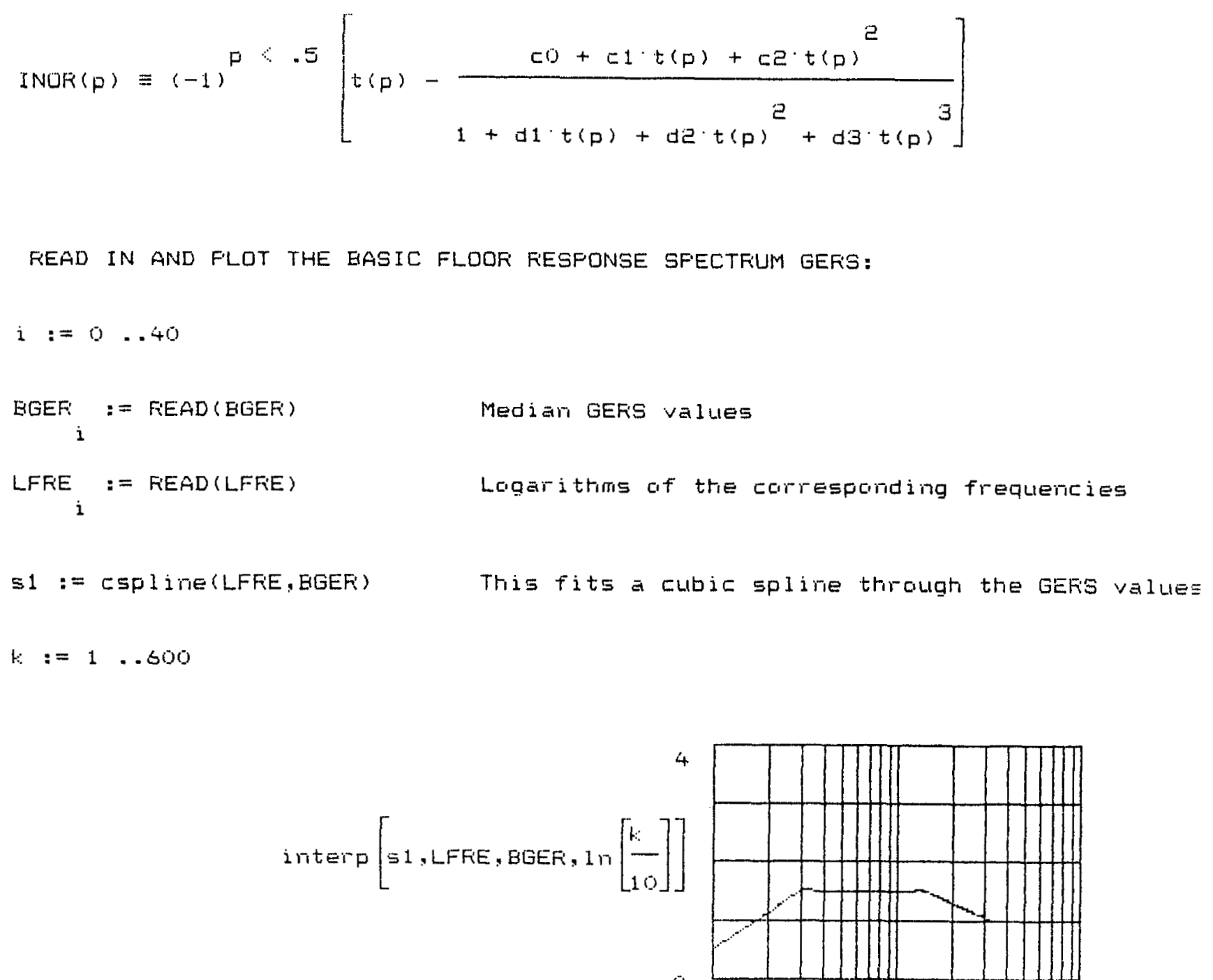

1

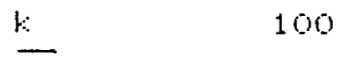

10

READ IN AND FLOT THE HORIZONTAL FLOOF RESFONSE SFECTRUM:

$j:=0 \quad .45$

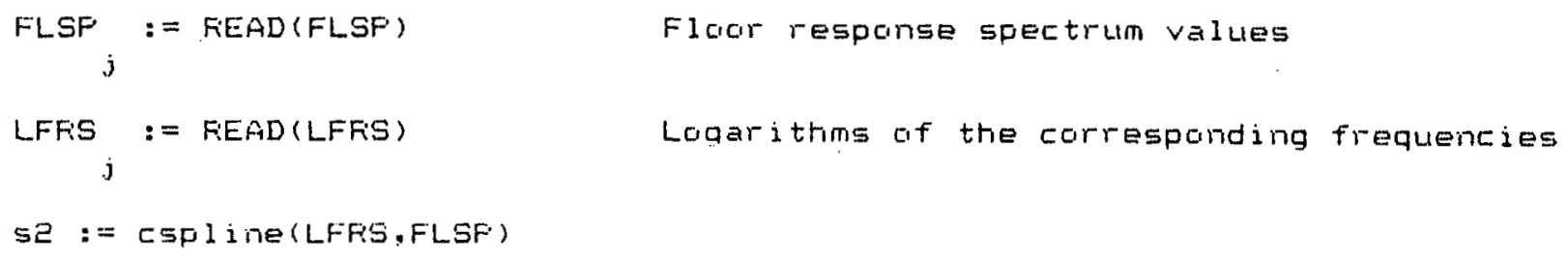


FEAD IN THE HOFIZONTAL FLOUF FESFONSE SFECTFUM A'VEFAGED DVEF O.2f FFEQUENCY BAND
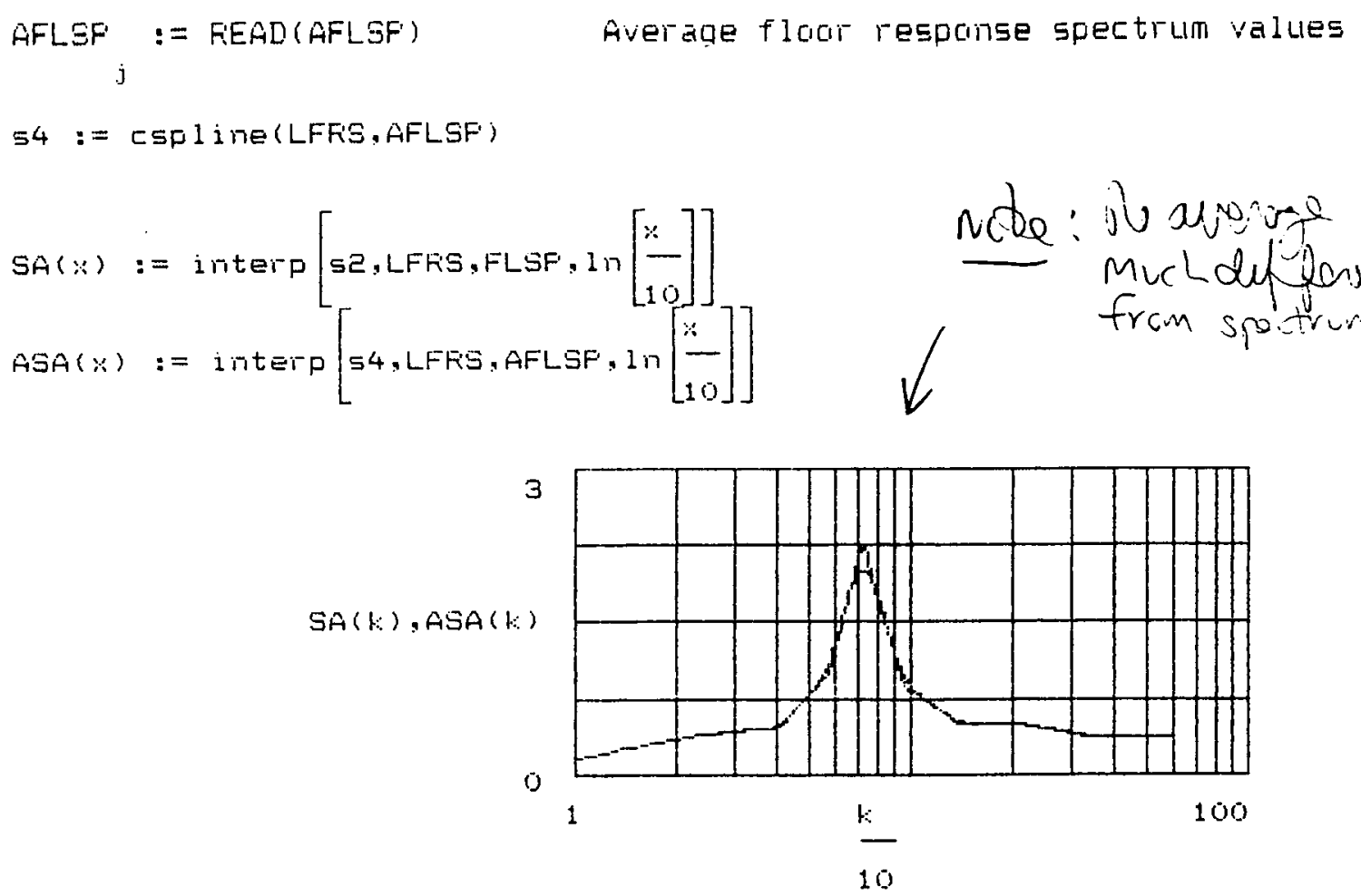

FEAD IN THE MEDIAN GFOUND FESFONSE SFECTFUM NORMALIZED TO 1.00 :

$1:=0 \quad \ldots 40$
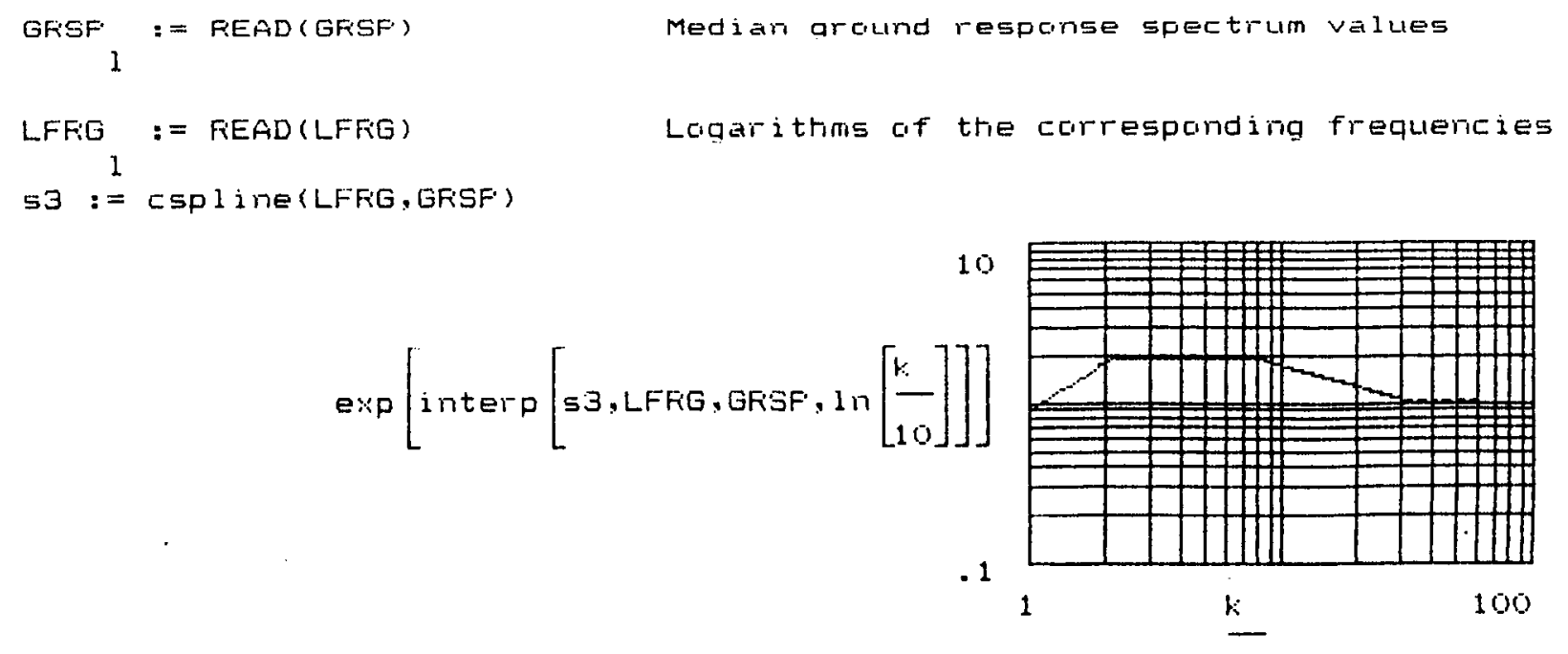

10 
DETEFMINE FOINTS ON FRAGILITY CUR'VE BASED ON LATIN HYFEFCUEE SIMULATION CONGIDEFING THE FOLLOWING VAFIAELES BEING UNCEFTAIN:

\begin{tabular}{|c|c|c|c|}
\hline Building & frequency: & $s=0.25$ & median $=7.2 \mathrm{hz}$ \\
\hline $\begin{array}{l}\text { Equipment } \\
\text { Camb ined }\end{array}$ & $\begin{array}{l}\text { frequency: } \\
\text { uncertainity: }\end{array}$ & $\begin{array}{l}0=0.20 \\
B=0.32\end{array}$ & median $=0.5 \mathrm{hz}$ \\
\hline GERS: & & $B=0.27$ & median $=1.2 \%$ \\
\hline
\end{tabular}

Total of $n$ simulations are performed

$n:=200$

k: $:=1 \ldots n$

$\mathrm{EF}:=7.2 \cdot \exp \left[0.25 \cdot \operatorname{INOR}\left[\frac{k-1+\operatorname{rnd}(1)}{n}\right]\right]$

This creates $n$ building fred values

COHST := exp (interp (53,LFRG,GFSF, 1n(7.2))

This canstant is the graund CONST $=1.89$

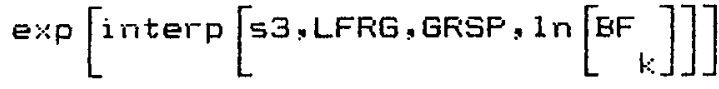

$$
\begin{aligned}
& \text { FF } k:=7 \text { CONST } \\
& E_{1 .}:=6.5 \cdot \operatorname{Exp}\left[0.32 \cdot \operatorname{INOF}\left[\frac{1-1+r n d(1)}{n}\right]\right] \\
& \operatorname{GF}:=1.2 \cdot \operatorname{Exp}\left[0.27 \cdot \operatorname{INOF}\left[\frac{k-1+\operatorname{rnd}(1)}{n}\right]\right]
\end{aligned}
$$

spectrum amplification at the building frequency, 7. ah 2

This creates in factors ta scale the fluar spectra for different building frequencies

This creates $n$ equipment fr values - note it is based a a combined variability for building and equipment frequencies

This creates $n$ factors to scale the GEFis capacity fui the variability in the GERS 
$P:=1 \ldots 10$ The first ten sets of samples before randomly mixing

\begin{tabular}{|c|c|}
\hline D & $F F_{D}$ \\
\hline 1 & 0.999 \\
\hline 2 & 0.998 \\
\hline 3 & 0.998 \\
\hline 4 & 0.998 \\
\hline 5 & 0.998 \\
\hline 6 & 0.998 \\
\hline 7 & 0.998 \\
\hline 8 & 0.398 \\
\hline 9 & 0.998 \\
\hline 10 & 0.998 \\
\hline WRI TE (FF) & $:=F F$ \\
\hline WR I TE (EF) & $:=E F$ \\
\hline WRITE (GF) & $:=\mathrm{GF}$ \\
\hline
\end{tabular}

\begin{tabular}{|c|c|}
\hline$E F$ & GF \\
\hline$p$ & $p$ \\
\hline 2.611 & 0.566 \\
\hline 2.918 & 0.64 \\
\hline 3.184 & 0.642 \\
\hline 3,314 & 0.67 \\
\hline 3,435 & 0.699 \\
\hline 3.486 & 0.714 \\
\hline 3.501 & 0.723 \\
\hline 3.709 & 0.739 \\
\hline 3.758 & 0.758 \\
\hline 3.835 & 0.762 \\
\hline
\end{tabular}

The program is transferred to Dos where the files are randomly mixed

$$
\begin{aligned}
& F F_{l:}:=\operatorname{FEAD}(F F) \\
& \mathrm{EF}_{l:}:=\operatorname{FEAD}(E F) \\
& G F_{k}:=\operatorname{FEAD}(G F)
\end{aligned}
$$$$
\text { FSPEC }:=\text { interp }\left[52, \text { LFFS, FLSF, In }\left[\begin{array}{l}
\text { EF } \\
k
\end{array}\right]\right] \begin{aligned}
& \text { Floor spectral values at random } \\
& \text { frequencies }
\end{aligned}
$$

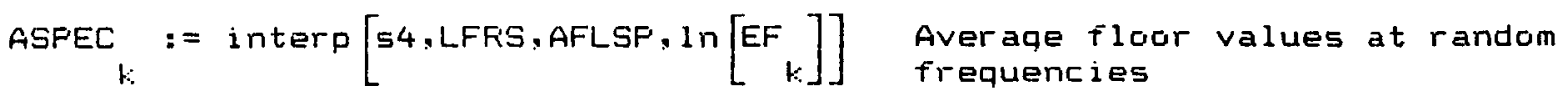$$
\text { GEFSF : = interp }[51, \text { LFFE, BGER, In }[\text { EF } k] \text { GERs capacity values at random freq. }
$$ 

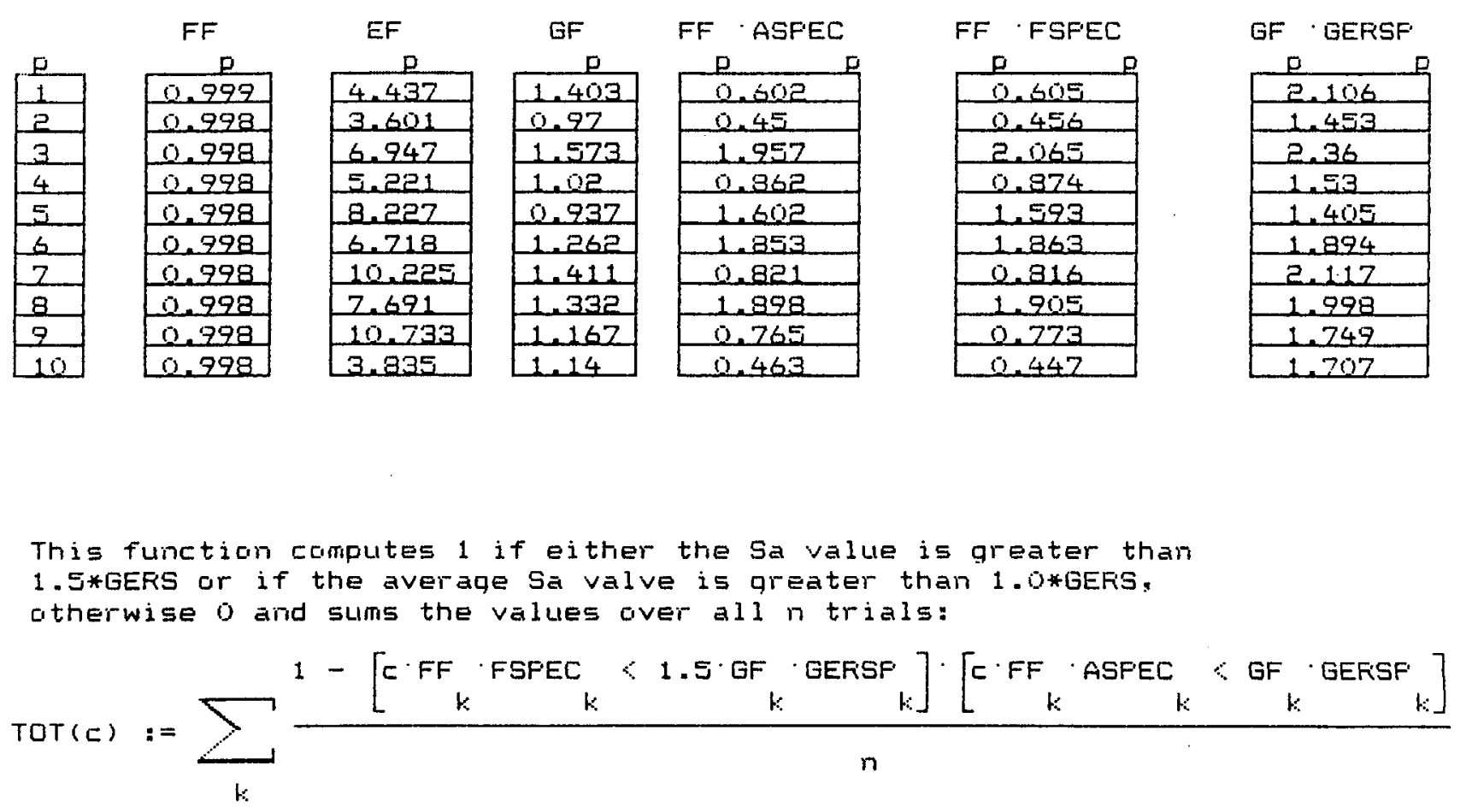

$$
\begin{aligned}
& a:=0.11 \\
& N:=10 \\
& j:=0 \ldots N \\
& \text { pga }:=\exp \left[\ln (a)+j \frac{\ln (N)}{N}\right] \\
& \% f_{j a i l}:=\operatorname{POa}\left[\frac{j}{0.18}\right]
\end{aligned}
$$

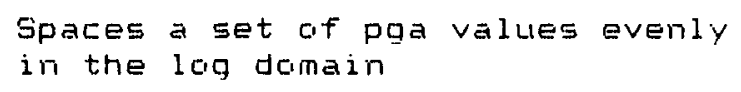

pqa
\begin{tabular}{|l|}
\hline 0.11 \\
\hline 0.138 \\
\hline 0.174 \\
\hline 0.219 \\
\hline 0.276 \\
\hline 0.348 \\
\hline 0.438 \\
\hline 0.551 \\
\hline 0.674 \\
\hline 0.374 \\
\hline 1.1 \\
\hline
\end{tabular} \mid \begin{tabular}{l|}
\hline 0.03 \\
\hline 0.05 \\
\hline 0.15 \\
\hline 0.315 \\
\hline 0.46 \\
\hline 0.585 \\
\hline 0.74 \\
\hline 0.84 \\
\hline 0.725 \\
\hline 0.975 \\
\hline 0.995 \\
\hline
\end{tabular}

WFITE(FGA) $:=p_{j}{ }_{j}$
WRITE(FAIL) $:=\%$ fail ${ }_{j}$ 
MLAD FILES LOGNOFM. FIL

This progiam take the calculated probabilities of failure and the asscieiated peat: around acceleraticin values and performs a least squares fit in the lop-protatility domain to obtain the "test fit" median and $\beta$ value.

NOFMAL DISTFIIBUTION:

$$
\begin{aligned}
& b 1 \equiv .317381530 \\
& \text { ьอ } \equiv-.356563782 \\
& b 3 \equiv 1.781477937 \\
& \text { b4 } \equiv-1.821255978 \\
& \text { 口5 } \equiv 1.330274429 \\
& p \equiv .2315417 \\
& Z(x) \equiv \frac{\exp \left[\frac{2}{2}\right]}{2.506628275} \\
& t(x)=\frac{1}{1+p \cdot|x|}
\end{aligned}
$$$$
\operatorname{NOF}(x) \equiv \mid(x) 0)-z(x) \cdot\left[b 1 \cdot t(x)+b 2 \cdot t(x)^{2}+b 3 \cdot t(x)^{3}+b 4 \cdot t(x)^{4}+b 5 \cdot t(x)^{5}\right] \mid
$$

\section{INVEFISE NOFMAL DISTFIEUTION:}

$$
\begin{aligned}
& c 0 \equiv 2.515517 \\
& c 1 \equiv .802853 \\
& c 2 \equiv .010328 \\
& d 1 \equiv 1.432788 \\
& d 2 \equiv .189269 \\
& d 3 \equiv .001308
\end{aligned}
$$$$
t(p) \equiv \sqrt{\ln \left[\frac{1}{((p) .5)-p)^{2}}\right]}
$$ 
$\operatorname{INOF}(p) \equiv(-1)^{p<.5} \cdot\left[t(p)-\frac{c 0+c 1 \cdot t(p)+c t^{2}(p)^{2}}{1+d 1 \cdot t(p)+d z \cdot t(p)^{2}+d 3 \cdot t(p)^{3}}\right]$

$$
\begin{aligned}
N:= & 10 \\
j: & =0 \ldots N \\
y 1 & :=\text { READ (FGA) } \\
x 1 & :=\text { FEAD (FAIL) }
\end{aligned}
$$
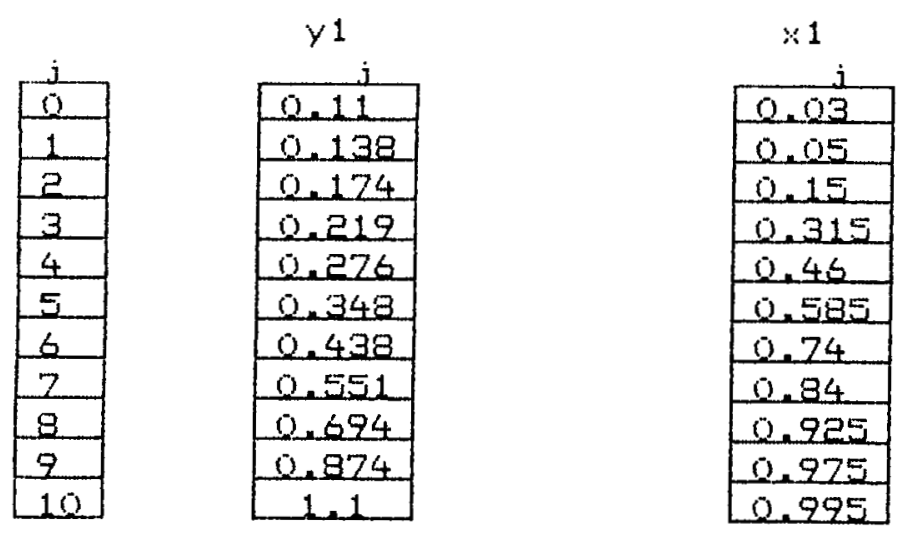

$$
\begin{aligned}
y_{j} & :=\ln \left[\begin{array}{ll}
y & 1 \\
& j
\end{array}\right] \\
x 1_{j} & :=\operatorname{INOF}\left[\begin{array}{ll}
x 1 \\
\end{array}\right]
\end{aligned}
$$

$$
\begin{aligned}
& \text { slcpe }\left(\times 1, y_{1}\right)=0.527 \\
& \text { intercept }(x 1, y 1)=-1.185 \\
& \operatorname{corr}(\times 1, y 1)=0.997
\end{aligned}
$$

$$
\begin{aligned}
& B:=5 \operatorname{lope}(x 1, y 1) \\
& a_{\text {med }}:=\exp (\text { intercept }(x 1, y 1))
\end{aligned}
$$

$$
\begin{gathered}
a_{\text {med }}=0.306 \\
\theta=0.527 \\
C-56
\end{gathered}
$$




$$
z_{j}:=\text { intercept }(x 1, y 1)+\operatorname{slope}(x 1, y 1) \cdot x 1 j_{j}
$$

$\max \left(y_{1}\right)=0.095$

$$
\begin{aligned}
& \min \left(y_{1}\right)=-2.207 \\
& \max (\times 1)=2.576 \\
& \min (\times 1)=-1.881
\end{aligned}
$$
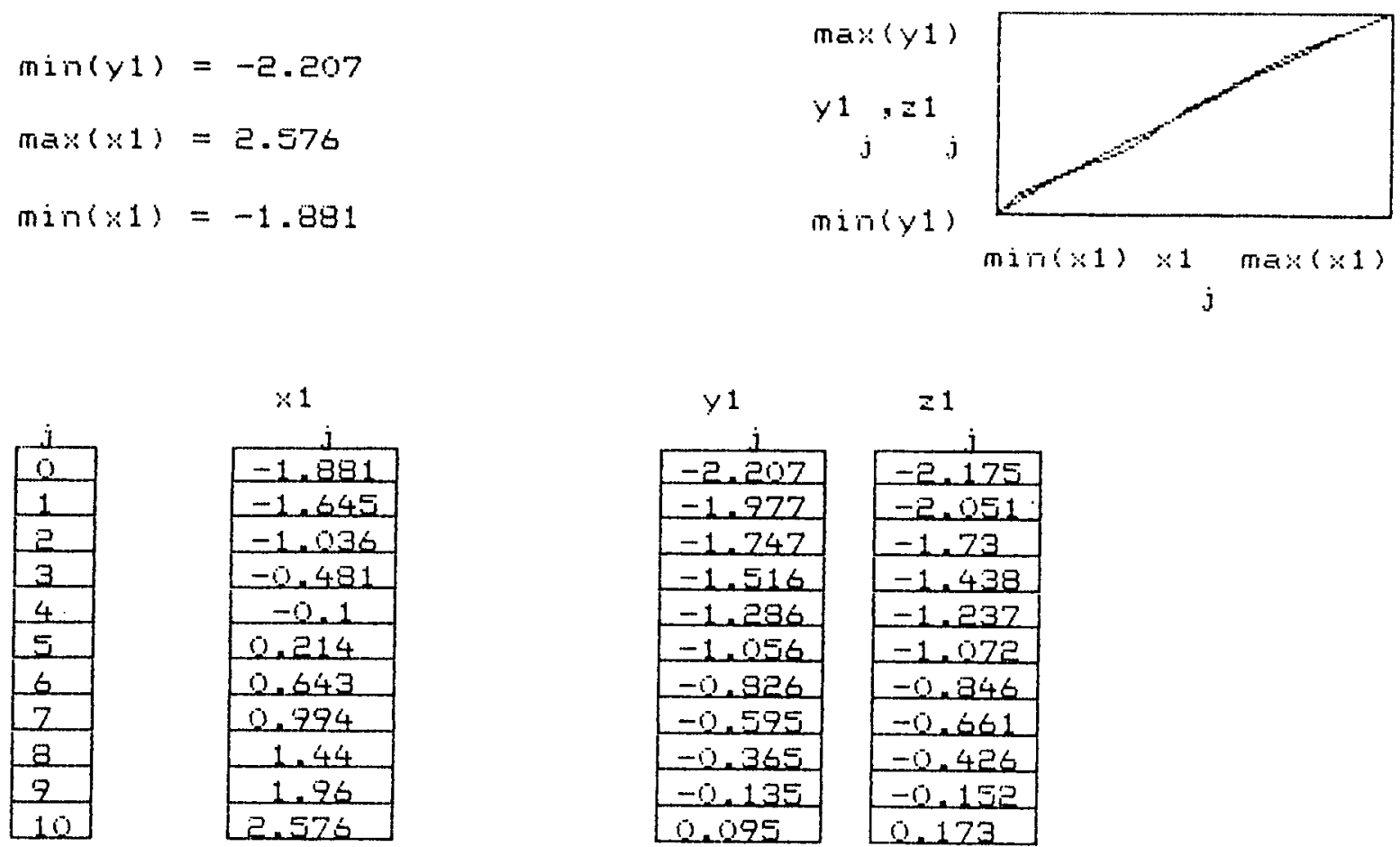

\begin{tabular}{c}
$\times 1$ \\
\hline-1.891 \\
\hline-1.645 \\
\hline-1.036 \\
\hline-0.481 \\
\hline-0.1 \\
\hline 0.214 \\
\hline 0.643 \\
\hline 0.794 \\
\hline 1.44 \\
\hline 1.96 \\
\hline 2.576 \\
\hline
\end{tabular}

$y 1$
\begin{tabular}{|c|}
\hline-2.207 \\
\hline-1.777 \\
\hline-1.247 \\
\hline-1.516 \\
\hline-1.236 \\
\hline-1.056 \\
\hline-0.326 \\
\hline-0.585 \\
\hline-0.365 \\
\hline-0.135 \\
\hline 0.095 \\
\hline
\end{tabular}

\begin{tabular}{|c|}
21 \\
\hline-2.175 \\
\hline-2.051 \\
\hline-1.73 \\
\hline-1.438 \\
-1.237 \\
\hline-1.072 \\
\hline-0.846 \\
\hline-0.661 \\
\hline-0.426 \\
\hline-0.152 \\
\hline 0.173 \\
\hline
\end{tabular}

Tabulate probabilities to compare calculated and test fit values:
$\therefore \quad:=\exp \left[\begin{array}{ll}y_{1} \\ \\ & j\end{array}\right]$
Feat: ground acceleratians
$P_{j}:=\operatorname{NOF}\left[\begin{array}{ll}x 1 \\ \\ & j\end{array}\right]$
Frobatility of failure values

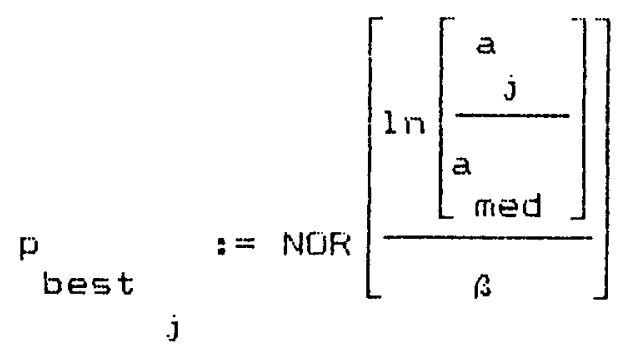

Eest fit probability of failure values 


\begin{tabular}{|c|}
\hline$j$ \\
\hline 0 \\
\hline 1 \\
\hline 2 \\
\hline 3 \\
\hline 4 \\
\hline 5 \\
\hline 6 \\
\hline 7 \\
\hline 8 \\
\hline 9 \\
\hline 10 \\
\hline 0.11 \\
\hline 0.124 \\
\hline 0.219 \\
\hline 0.276 \\
\hline 0.348 \\
\hline 0.438 \\
\hline 0.551 \\
\hline 0.694 \\
\hline 0.874 \\
\hline 1.1 \\
\hline
\end{tabular}

\begin{tabular}{|c|c|}
\hline 曰 & P best \\
\hline$\frac{j}{0.03}$ & 0.026 \\
\hline 0.05 & 0.066 \\
\hline 0.15 & 0.143 \\
\hline 0.315 & 0.264 \\
\hline 0.46 & 0.423 \\
\hline 0.585 & 0.596 \\
\hline 0.74 & 0.752 \\
\hline 0.84 & 0.868 \\
\hline 0.925 & 0.94 \\
\hline 0.975 & 0.977 \\
\hline 0.975 & 0.972 \\
\hline
\end{tabular}




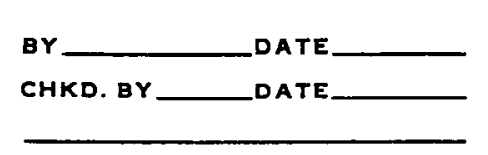

PROJECT

PAGEM OF

Develop Frasilí, Poraindeder.

Capreity

Strongth ( sap paop 1i)

$$
\begin{aligned}
& \tilde{F}=0.319 / 0.31 g=1.0 \\
& \rho_{u}=0.53 \\
& \rho_{r}=0
\end{aligned}
$$

Italastic lesponse

Assume Ris parameter is included in GerR

$$
\tilde{F}=1.0 \quad \rho_{r}=\beta_{u}=0
$$

Ejuipment Response

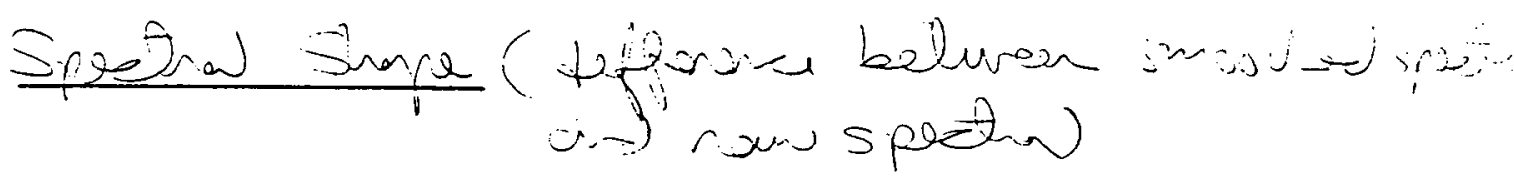

$$
\begin{aligned}
& F=1,0 \\
& p=0 \\
& \text { bu }=005 \text { (astinatin) }
\end{aligned}
$$

Drompiny

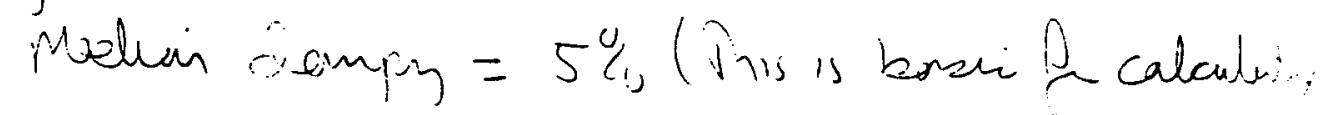

$$
\begin{aligned}
& \breve{F}=1,0 \\
& -\sigma=3.5 \% \text { Gur. }
\end{aligned}
$$

C-59 

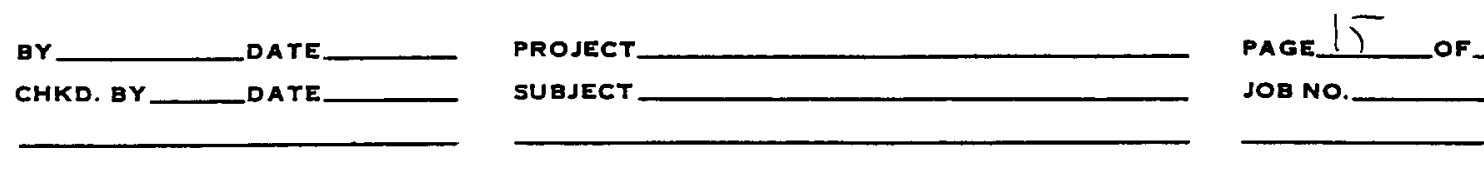

Ki.an MCC.FCA L $3.5 \%$ dimping Speta

$$
\begin{aligned}
& \check{S}_{a}=0.27 g \\
& \hat{P}_{1}=\frac{1}{1} \ln \frac{0.31}{0.27}=0.14 \\
& p_{r}=0
\end{aligned}
$$

Modolin

$$
\text { Freguary \& in ode Shapa- Assure ine }
$$

is noluded in in Sitrs

Mode Somjonstus

Assime includes in Gers;

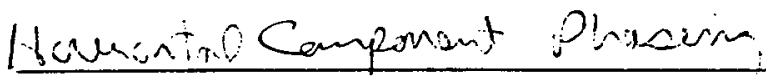

Assune includes in GerRs

Structure Rearmee

Sround Motion This Panamaten includes:

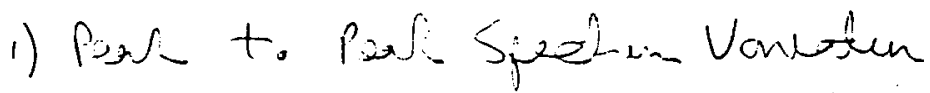

2) Norysutal Divitim Vavalies

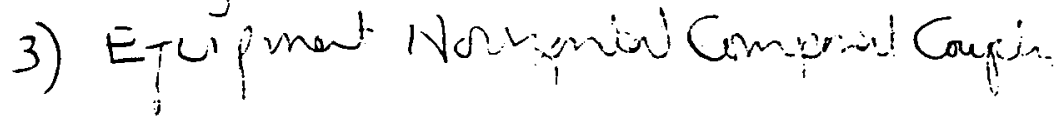

Assuie outen's denct Couptin. Sinall

Jack R. Benjamin \& Associates, Inc. Consulting Engineers

C-60 


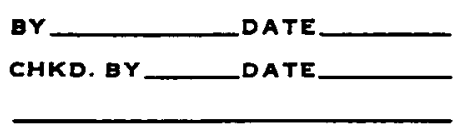

PROJECT

SUBJECT

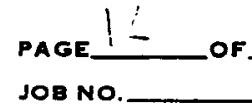

From Brizudio A:

$$
\begin{aligned}
& \check{F}=1,0 \\
& \rho_{V}=0,25 \\
& \rho_{u}=0
\end{aligned}
$$

Damping

$$
\begin{aligned}
& \text { Median: } 7 \% \\
& -15: 5 \% \\
& \beta_{0}=\frac{1}{1} \ln \frac{2.12}{1.89}=0.11 \\
& \text { Sra }=0 \\
& E=1.0 \text { (used } 7 \% \text { in strempleale) }
\end{aligned}
$$

Modeling

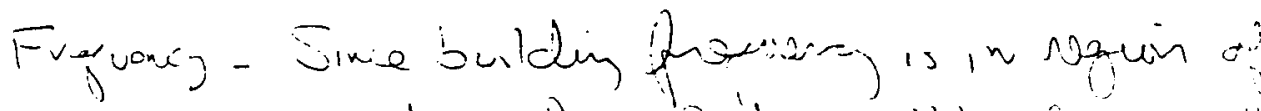

$$
\begin{aligned}
& F r=F=0 \quad F=1,0
\end{aligned}
$$

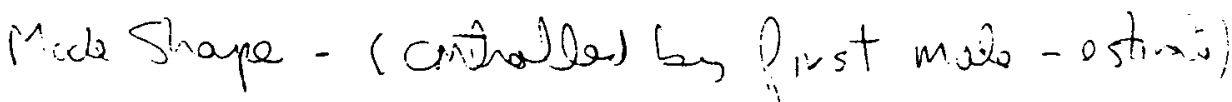

$$
\tilde{F}=1.0 \quad \rho_{r}=0 . \quad \rho_{u}=0.10(\varphi, t)
$$

Jack R. Benjamin \& Associates, Inc. Consulting Engineers

C-61 


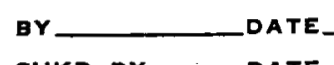

CHKD. BY
PROJECT

SUBJECT

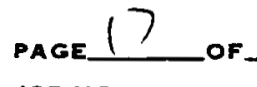

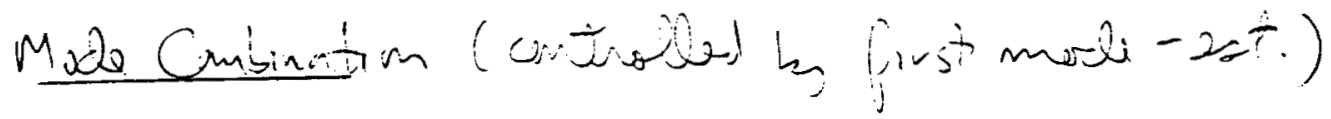

$$
\underline{v}=1.0 \quad \rho 0 y=f u=0
$$

Nornoutad Counferint lhacim

$$
F^{\nu}=1.0 \quad p_{r}=0.10 \quad \tilde{r}_{v}=0 \quad\left(2 x^{2}\right)
$$

SSI (roch Site)

$$
\forall=1,0 \rho_{v}=0 \rho_{v}=0.05\left(s_{t}\right)
$$

Inolastic Reparea (Strueturs ascuned lineon)

$$
\ddot{F}=1.0 \quad \rho_{r}=\rho_{v}=0
$$

Jack R. Benjamin \& Associates, Inc. Consulting Engineers

C-62 


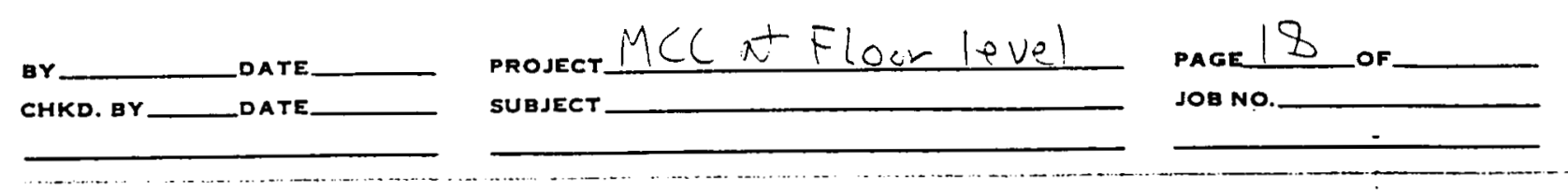

Parameter $-\stackrel{\check{E}}{\beta_{r}}-\beta_{u} \ldots$

Expact,

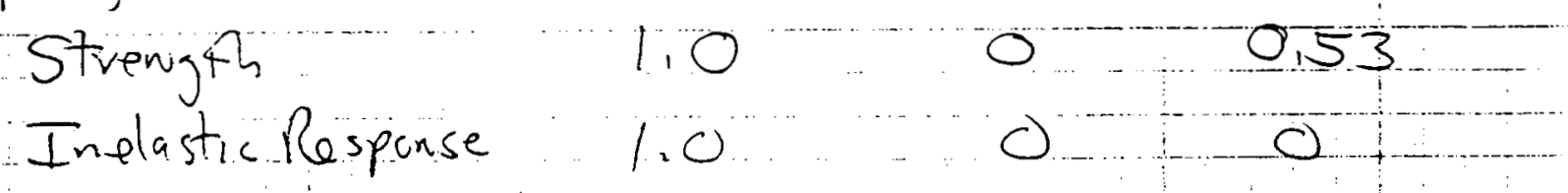

Equipment Response

Spectral Shape

Damping)

Modeling-Frguency $T 10$

- Modeshare ro

Mode Comsination

0.05
0.14

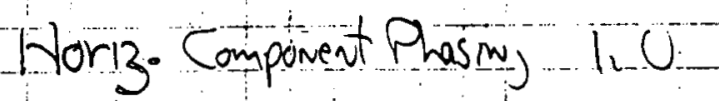

Sitructure Response

Grand Motion

1.0

0,25

Dampin)

Modeling - Fvofuency : 1,0

Modeshape lio

$0 \quad 0,11$

Mode Combinatiow 1.0

Horiz. Coupovent Phasing 1,0

0 o 0

0. 0.10

SSI

Imelastic Response

u $\quad 0$

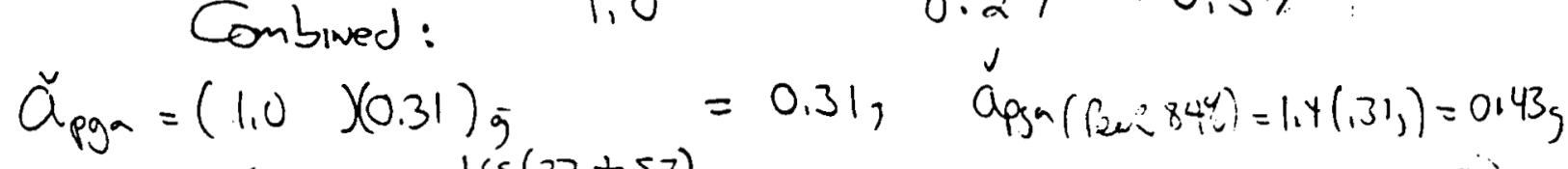

$$
\begin{aligned}
& \operatorname{NCCPF_{m}}(0.31) e^{-1.65(.27+.57)}=0.08 \mathrm{~g} \\
& \text { NCLPF Par 84\% } 1.4 \cdot(.08)=0,1 \mathrm{Lg} \text { (see Appendix B) }
\end{aligned}
$$

$c-63$ 


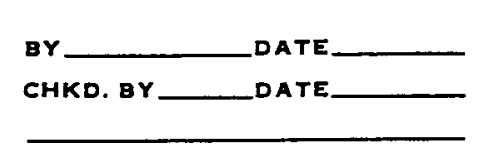

$\frac{\text { Compute NCLP }=\text { Divectly }}{\text { (see Apponsinc C) }} \sum X \cdot \beta=1,65(, 27+, 57)=1,3$

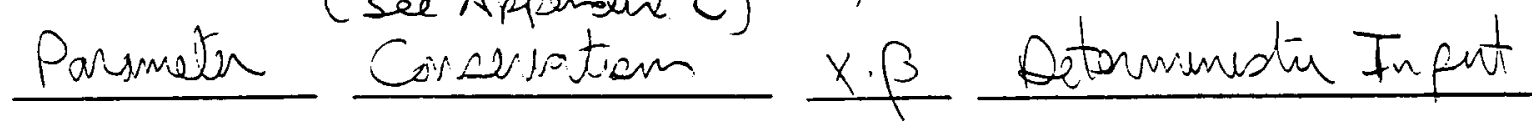

Strongch $95 \%$ NEP $\quad(1.65)(.53)=0.87$ Se discussin below

Ejup Dampin $-1 \sigma$

Grund Motim $+1 \sigma$

Buldy Drmpe $-1 \sigma$
0.14

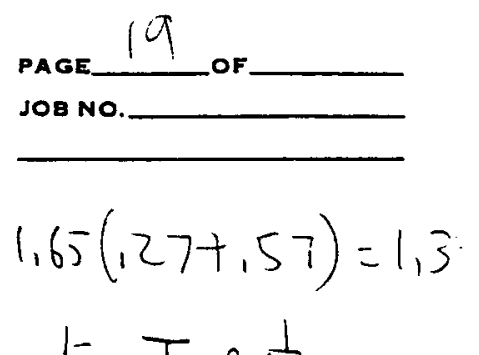

-

$$
\begin{aligned}
0.25 & e^{.25}=1.28 \\
\Sigma=\frac{0.11}{1.37} & \frac{2.12}{1.89}=1,12
\end{aligned}
$$

kep othan paranotore at medin level

Since the sinangi- we deternine by dros simulationLie couniting feuluere \& nun foulure in a Lation Hyprabe

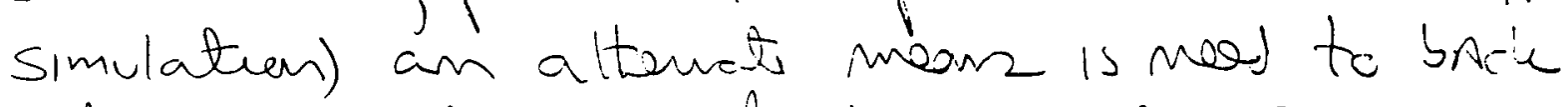

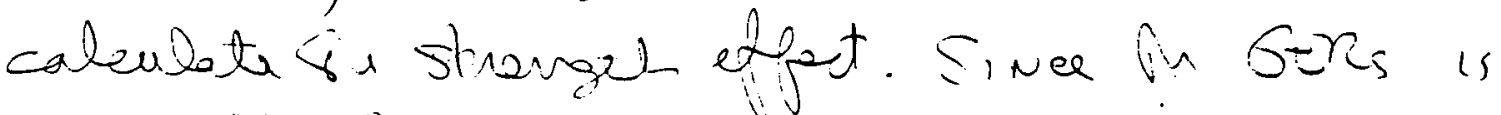
ossentide, flat in on $6.5 \mathrm{~h}_{3} \pm$ Noxim, one coubl

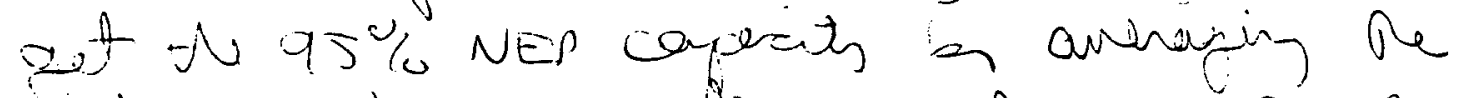

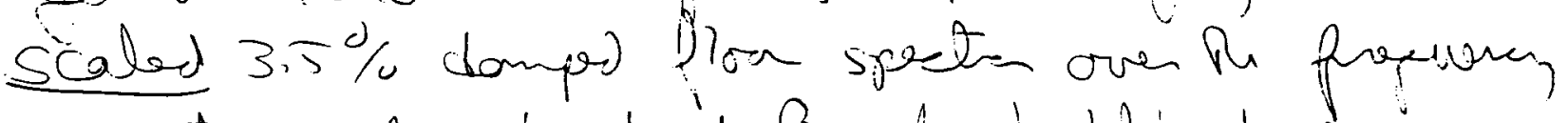
vareaten due to boil $\beta_{u}$ fr bulding \& eping

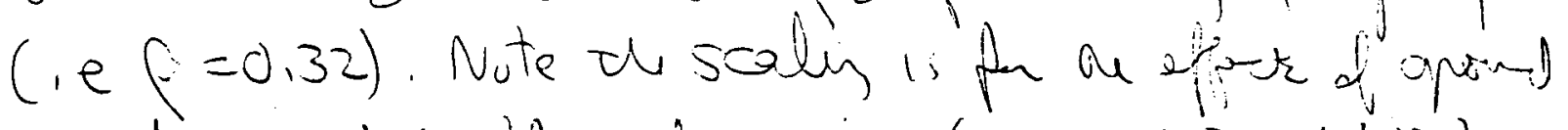
crortim ad builden damplive (1.e., $1.28 \times(12)$.

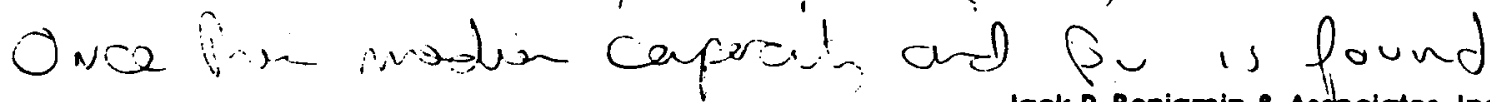
Jack R. Benjamin \& Assbciates, Inc.
Consulting Engineers

C-64 


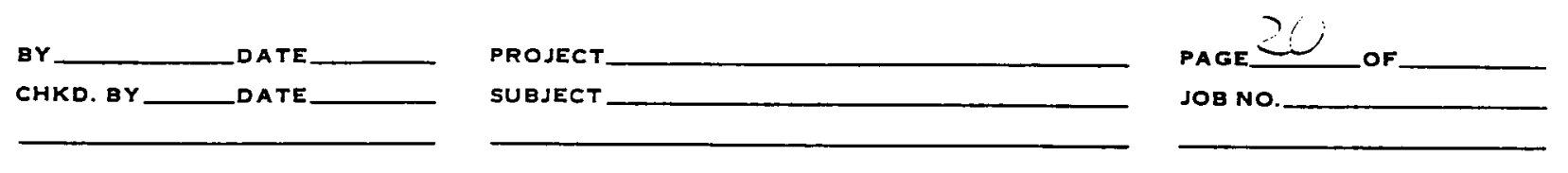

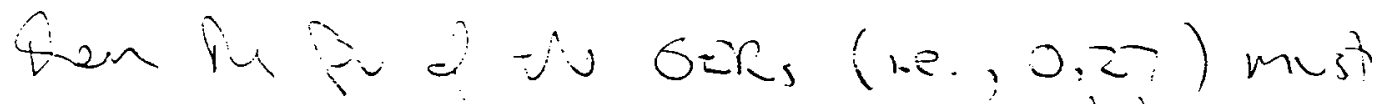

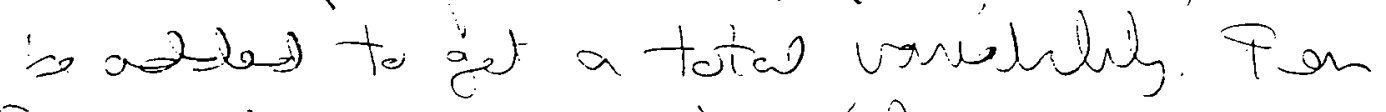
ia infut must in sealad (Rrm 0.19, pga) so vat pars is only a sic proin of exceding Te median GERs

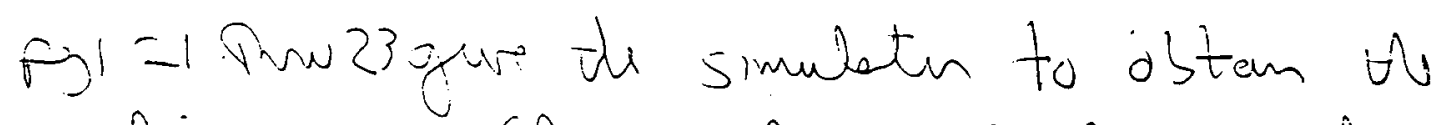
modien inpet (Mis is for 3.5\% fion speta. conacioding to 7\% buildy dompin ad meden gound motin in put scaled to O,lij pial, ie

$$
\begin{aligned}
& \check{S}_{a}=1,29 \mathrm{~J} \\
& \rho_{0}=0.50
\end{aligned}
$$

Scale pa ofu whet

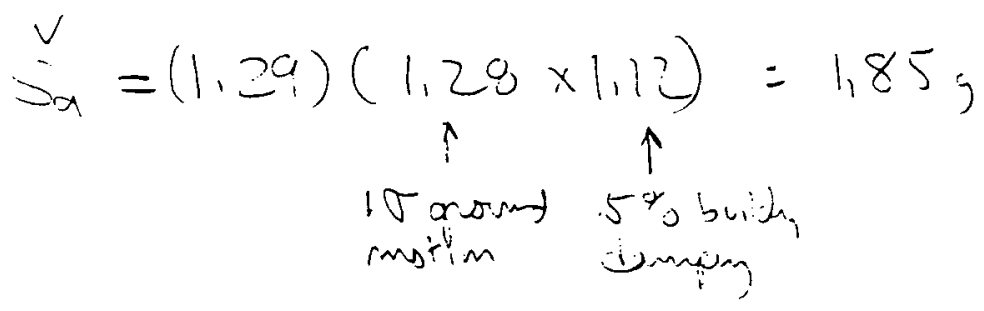

$$
\begin{aligned}
& \left.P_{u}\left(5.50^{2}+0 . \overline{27}\right)^{2}\right)^{\prime \prime}=0.57
\end{aligned}
$$

Now compre to Gets distubutin will

$$
\breve{S}_{n}=1.5 \times 1.2=1.8 \text {, }
$$$$
8=0.57
$$

Jack R. Benjamin \& Associates, Inc. Consulting Engineers

C-65 
MLAD FILES SFECT.SIM

NOFMAL DISTRIEUTION

$$
\begin{aligned}
& \text { t1 } \equiv .317381530 \\
& b 2 \equiv-.356563792 \\
& b 3 \equiv 1.781477937 \\
& b 4 \equiv-1.321255978 \\
& b 5 \equiv 1.330274429 \\
& p \equiv .2316419 \\
& Z(x) \equiv \frac{e \times p\left[\frac{-x}{2.506628275}\right.}{2.0} \\
& t(x) \equiv \frac{1}{1+p \cdot|x|}
\end{aligned}
$$

$\left.\left.\operatorname{NOR}(x) \equiv\right|_{(x)}>0\right)-z(x) \cdot\left[b 1 \cdot t(x)+b 2 \cdot t(x)^{2}+b 3 \cdot t(x)^{3}+b 4 \cdot t(x)^{4}+b 5 \cdot t(x)^{5}\right]$

INVERSE NOFMAL DISTFIIBUTION

$$
\begin{aligned}
& c 0 \equiv 2.515517 \\
& c 1 \equiv .802853 \\
& c 2 \equiv .010328 \\
& d 1 \equiv 1.432788 \\
& d 2 \equiv .189259 \\
& d 3 \equiv .001308
\end{aligned}
$$$$
t(p) \equiv \sqrt{\ln \left[\frac{1}{(p) .5)-p)^{2}}\right]}
$$

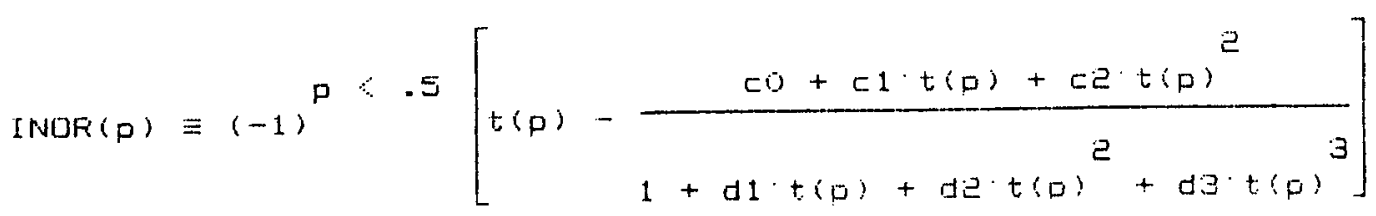




$$
\begin{aligned}
& \text { Freq }_{\text {hat }}:=0.5 \\
& \text { f }:=0.32 \\
& n:=199 \\
& i:=0 \ldots \\
& \text { Freq }_{i}:=\text { Freq }_{\text {hat }} \exp \left[\beta \cdot \operatorname{INOF}\left[\frac{i+r n d(1)}{n+1}\right]\right] \\
& \text { LFreq }_{i}:=\ln \left[\text { Freq }_{i}\right]
\end{aligned}
$$

m : $=45$

$j:=0 \ldots m$

$X L F F:=F E A D(X L F F)$

$X S A:=\operatorname{FEAD}(X S A)$ j

Read in the averaged $3.5 \%$ damped spectrum

$5:=\operatorname{cspline}(X L F R, X S A)$

$k:=1 . .600$

$$
\text { interp }\left[5, X L F F, \times S A, \ln \left[\frac{k}{10}\right]\right]
$$

4

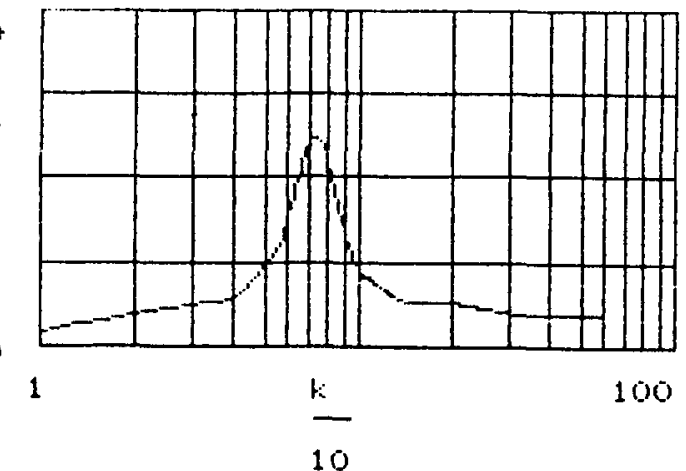

$$
\begin{aligned}
& S A_{i}:=\text { interp }\left[s, \times L_{F R} \times \text { XSA, LFreq }_{i}\right] \\
& \operatorname{LSA}_{i}:=\ln \left[3 A_{i}\right]
\end{aligned}
$$


Median := Exp(mean(LSA))

BEta : = stdev(LSA)

$$
\begin{aligned}
\text { Median } & =1.287 \\
\text { Beta } & =0.478
\end{aligned}
$$

\section{Finedian $:=\exp (\operatorname{mean}(L F r e q))$ \\ Fbeta := stdev(LFreq)}

$$
\begin{aligned}
\text { Fmedian } & =5.492 \\
\text { Fbeta } & =0.327
\end{aligned}
$$




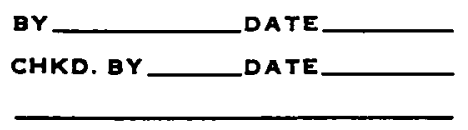

PROJECT

SUBJECT

$\hat{p}=0.57$

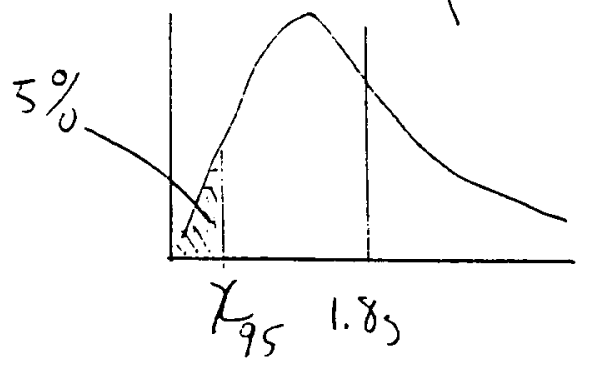

$$
x_{9.5}=1.0 e^{(-, 5)(1,5)}=0.705
$$

Fins de NCLEF is just:

Plow Lave: HCLFF $=\frac{0,704}{1.05} \times 0.18=0,07$

$$
\begin{aligned}
& \text { g to is cumpont }=(0.07)(1,4)=0,10 \\
& \tau_{\text {see Appudue B }}
\end{aligned}
$$

Jack R. Benjamin \& Associates, Inc. Consulting Engineers

C-69 


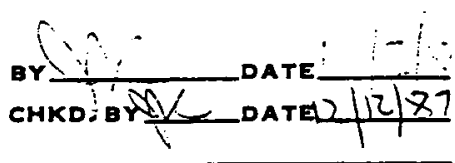

PROJECT

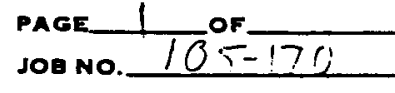

STARTINGAIR TANK

Assumption (creerial)

1. Foulue is 0.4in displiscement at C.G. of Tanr ( $\approx 1 \%$ duft). This assumer convectiy pifin, will fail at inis displacement

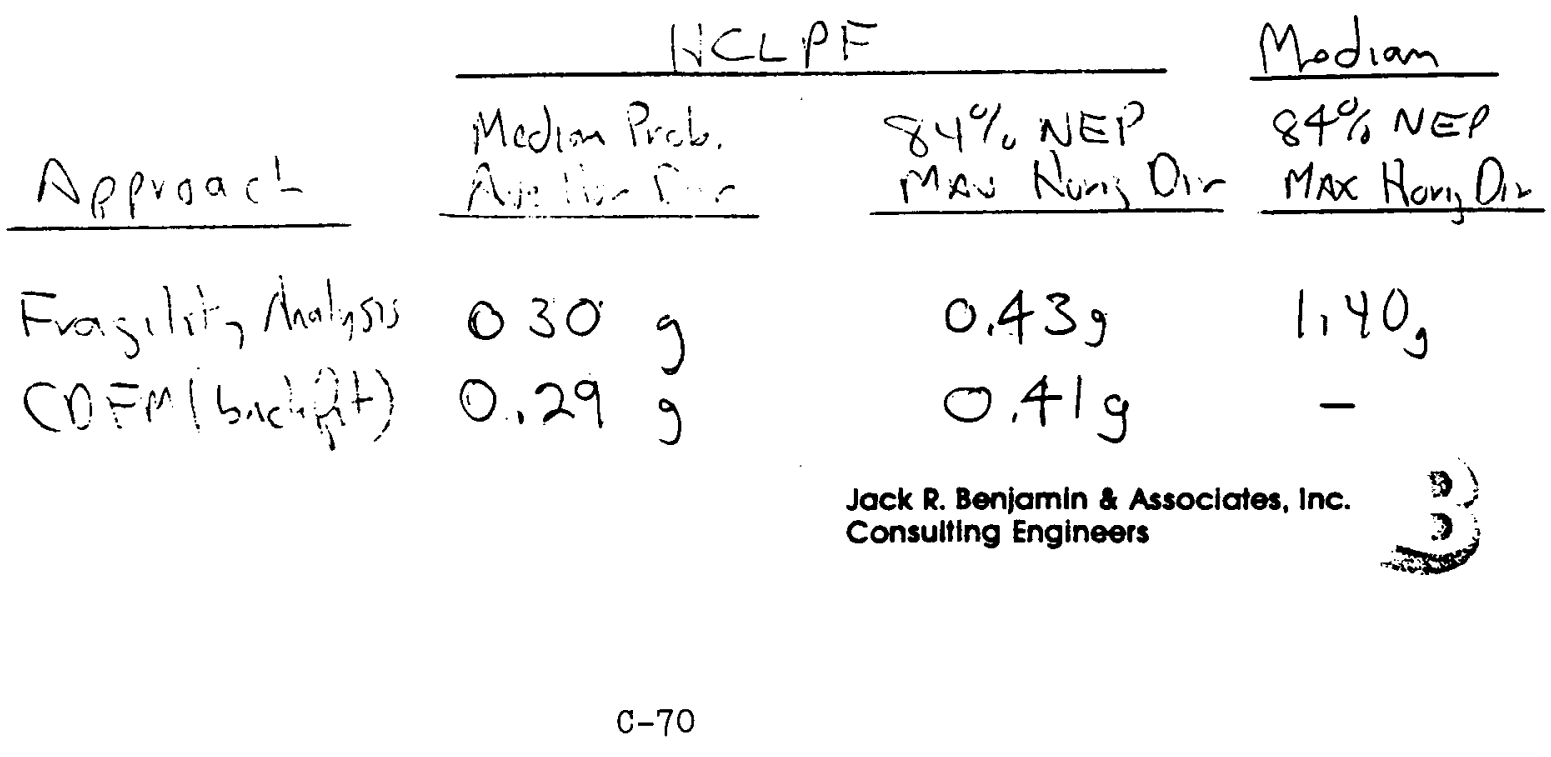


Calculate Carnation 2 Connection

bott: $5 / 8 \phi \sigma=1.2 \times 36=43.2$ les

PAGE_ OF

PROJECT

JOE No.

$$
\begin{aligned}
& \sigma=1.2 \times 36=43.2 \text { less } \\
& \text { Area }=.226 \mathrm{im}^{2} \quad \text { (across shend) } \\
& F_{\text {ten }}=(43.2)(.226)=9.8^{\mathrm{k}}
\end{aligned}
$$

Angle $2 \times 2 \frac{1}{2} \times 3^{n}$ long $1 / 4^{4}$ finch

$$
\begin{aligned}
M_{\text {CAp }}=S \sigma \quad S & =\frac{(3.0)(.25)^{2}}{6}=0,03125 \mathrm{in}^{2} \\
\sigma & =1.25 \times 36=45 \mathrm{ksi}
\end{aligned}
$$

Full plasticity acvesssection $\uparrow$ cope to mansion yeld

at Tank: $M_{\text {cap }}=(1.5)(103125)(45)=2.11 k-11$

at bolt $M_{\text {cap }}=2.11 \times \frac{3-3 / 4}{3}=1.58^{k-11}$

Assume fixed at tank \& bott: $T=\frac{M_{1}+M_{2}}{l}=\frac{2.11+1.58}{1.75}=2.11^{k}$

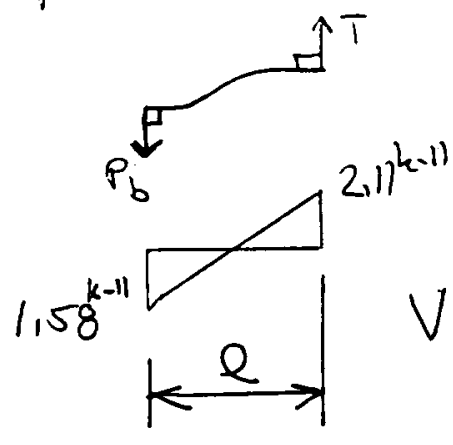

$$
\beta_{u}=\left(. \overline{1}^{2}+.04^{2}\right)^{1 / 2}=0.15
$$

Hence bending of o controls:

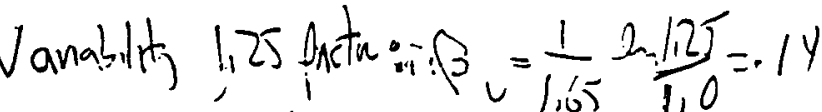
1,5 finch $B_{0} \frac{1}{1,65} \ln \frac{1,5}{1,4}=104$

Jack R. Benjamin \& Associates, Inc. Consulting Engineers

C-71 
BY

CHKD. BY
PROJECT

SUBJECT
OF

JOE NO.

Check Prying Actin:

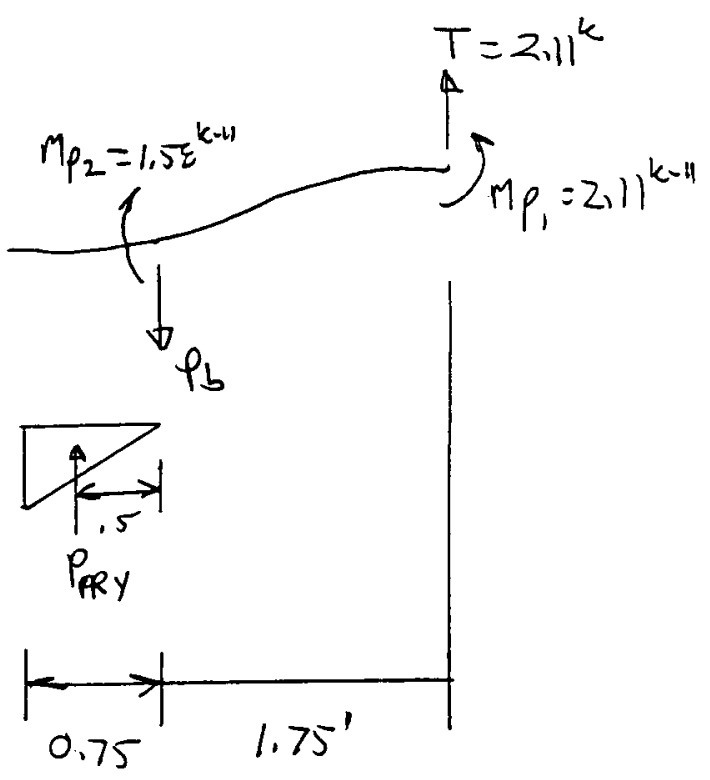

$$
\begin{aligned}
& P_{P R y}=\frac{1.58}{15}=3.16^{k} \\
& P_{b}=2.11+3.16=5.25^{k}<9.8^{k} \text { tenser capacity }
\end{aligned}
$$

This indicates tat angle bottom plate will become a mechanism before butt pails

Jack R. Benjamin \& Associates, Inc. Consulting Engineers

C-72 


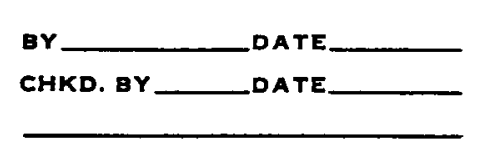

PROJECT

SUBJECT

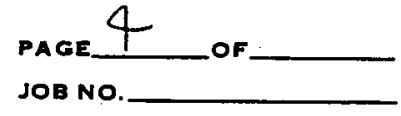

Calculate Frequent of Accumulator (hour)

$$
I_{\tan }=\frac{\pi}{4}\left[12^{4}-(12-\%)^{4}\right]=1942 \mathrm{kn}^{4}
$$

For $1^{k}$ load at C.G. displacement is: $\left(\Delta=\frac{P D}{3 E I}\right)$

$$
\Delta=\frac{(1)(41.5)^{3}}{(3)\left(29 \times 10^{5}\right)(1942} \cong 4 \times 10^{-4} \mathrm{in}
$$

(Joy small)

Most displacement is in support system

Support suction Stippross

$$
\frac{L-1.75^{\prime \prime}}{1 .}
$$

Angl: Moment, ranger between
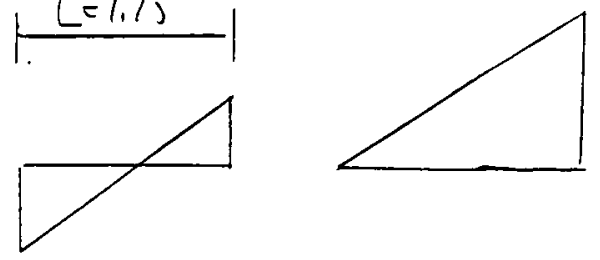

$$
\begin{aligned}
& I_{Y}=(3)(.25)^{3} / 12=003906 \mathrm{in} \\
& K_{\text {max: }}=\frac{(12)\left(29 \times 10^{3}\right)(1003906)}{(1.75)^{3}}=253.6 \mathrm{k} / 10 K_{\text {max }}=\frac{12 E I}{L^{3}} \quad K_{\text {man }}=\frac{3 E I}{L^{3}} \\
& k_{\text {min }}=1 / 4(253.6) \quad=63.4 \mathrm{k} / \mathrm{wn} \\
& =\frac{(307)\left(29 \times 10^{3}\right)}{6} \\
& =1484 \mathrm{k} / \mathrm{in}
\end{aligned}
$$$$
\text { Fixed-Fined } \bar{p} \text { ind- Prod }
$$

$$
\begin{aligned}
& A=(5 / 8) \frac{\pi}{4}=.367 \mathrm{in}^{2} \\
& l=6^{\prime \prime}
\end{aligned}
$$

Jack R. Benjamin \& Associates, Inc. Consulting Engineers

C-73 


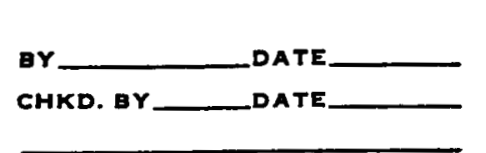

PROJECT

PAGE 5 OF

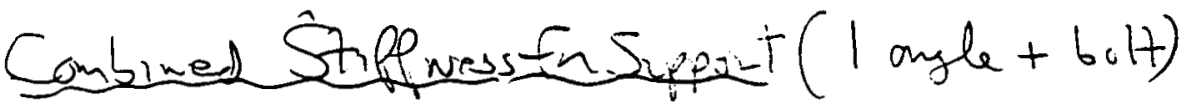

$$
\begin{aligned}
& \text { Miw: } \frac{1}{k}=\frac{1}{63.4}+\frac{1}{1484} \quad k_{\text {Min }}=60.8 \mathrm{k} / \mathrm{m} \\
& \text { Max: } \frac{1}{K}=\frac{1}{253.6}+\frac{1}{1484} \quad k=216.6 \mathrm{k} / \mathrm{in}
\end{aligned}
$$

Arcumulater Stidevess

From Force Analupie (brse retutin stuffunom, Tho)

$$
Z_{\theta}=\frac{M}{\theta}=\frac{10,77^{(N-1)}}{\psi} K \quad(\text { see paye 20) }
$$

where $k$ is singh ongl stiffiren

$$
\begin{aligned}
& \psi=\text { Normalyed displacement } \\
& \text { computed in foce aralugir } \\
& x_{\text {Lr }}: \psi=P / x_{x 1} \sin \theta \\
& y_{d i n} \psi=p 1 / x x^{2} \cos \theta
\end{aligned}
$$

Conimed $x \notin y$ valusrang Pfen alout 012 to. $025 \mathrm{k} / \mathrm{m}$ (See ph 25)

Ko rangle from $\frac{10,77}{.025}(60.8)=26,193 \mathrm{k}-11 / \mathrm{rad}$ to $\frac{10.77}{1012}(2166)=194,400 k-11 / \operatorname{vod}$ Jack R. Benjamin \& Associotes, Inc.
Consulting Engineers

C-74 


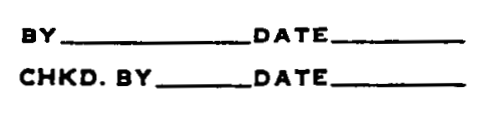

PROJECT

PAGE $b$ _OF

Using Table $8-10$ po iss From

Esmules 2 a national frepures at mosel Shape,

by Blevins

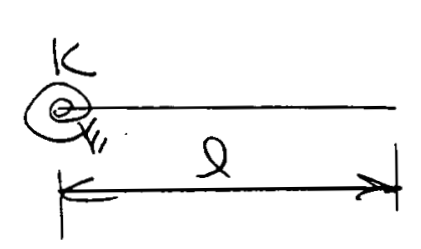

$$
\begin{aligned}
& L=91^{11} \quad m=920 /(386.4 * 91) \\
& I=1942 \mathrm{iw}^{\mathrm{y}} \quad=2,62 \times 10^{-5} \frac{\mathrm{k- \textrm {sec } ^ { 2 }}}{\mathrm{in}^{2}} \\
& E=29 \times 10^{3} \mathrm{ksi} \\
& K=194,400,26,193 \mathrm{k}-1 / \mathrm{rad}
\end{aligned}
$$

$$
\begin{aligned}
& \frac{K 2}{E I}=\frac{(194,400)(91)}{\left(29 \times 10^{3}\right)(1942)} \sim \frac{(26,193)(91)}{\left(29 \times 10^{3}\right)(1942)} \\
& =.314 \\
& =.042 \\
& \lambda_{1}(\text { Table } 8-10)=.979 \\
& .581 \\
& F_{1}=\frac{\lambda_{1}^{2}}{2 \pi L^{2}}\left(\frac{E I}{m}\right)^{1 / 2} \\
& =\frac{(.979)^{2}}{2 \pi\left(91^{2}\right)}\left(\frac{29 \times 16^{3} \times 1942}{2.62 \times 10^{-5}}\right)^{1 / 2}=\frac{(.581)^{2}}{2 \pi(91)^{2}}()^{1 / 2} \\
& =(.958)(28.2) h_{3} \quad(.338)(20.2) h_{3} \\
& =27 \mathrm{~h}_{3} \quad=9,5 \mathrm{~h}_{3}
\end{aligned}
$$

Assure modem, $\underset{\mathcal{F}}{\mathcal{L}}=\sqrt{27 \times 9,5}=16 \mathrm{~h}$

$$
\left.\beta=\frac{1}{4} \ln \frac{27}{9,5}=0,26 \text { (use } 0.25\right)
$$

C-75 
Ihis interpolates first mode values for Table 8-10 in Blevins Bual:

\begin{tabular}{|c|c|c|c|c|c|}
\hline $\begin{array}{l}t: \\
0\end{array}$ & $:=$ & .01 & ${ }^{\mu}$ & $:=$ & 41 419 \\
\hline $1:$ & $:=$ & .1 & $H_{1}$ & $:=$ & .7357 \\
\hline to & $:=$ & 1 & & $:=$ & $1.24 d$ \\
\hline k & $:=$ & 10 & ${ }_{3}$ & $:=$ & 1.723 \\
\hline$t_{4}$ & $:=$ & 100 & ${ }_{4}$ & $:=$ & 1.857 \\
\hline
\end{tabular}

$i:=0 . .4$

$\operatorname{Lagk}_{i}:=\log \left[\begin{array}{l}t: \\ i\end{array}\right]$

$s:=\operatorname{cspline}(\log l:, \mu)$

interp (s.LGgti,, $\log (.314))=0.979$

interp $(5 . \operatorname{Logk}, \mu .10 g(.042))=0.381$ 


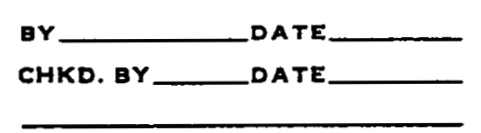

PROJECT

SUBJECT

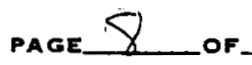

JOB NO.

cher vertical freeing (consider only 1. angle effective)$$
F=1 \sqrt{k}=1 \sqrt{608} \text { Min stigisio }
$$$$
=25.4 \mathrm{~h}_{3}
$$

$$
\begin{aligned}
S_{\text {max }} & =\sqrt{\frac{216.6}{60.8}}(25.4) \\
& =47,9 \\
\tilde{J} & =\sqrt{(25.4)(47.9}=35 \mathrm{~h}_{3}
\end{aligned}
$$

This frogueney is lithely to be consenonture (ie. low) since tee capris will concopins's to a vertical crecelerotion which is len than lg; hare, Te accumulator will not rise vertical

Nance Use $S_{A_{v}}=0.20 \mathrm{~g} / 0.10 \mathrm{~g}$ pga homing

$$
\begin{gathered}
P==\frac{1}{1.25} \ln \left(\frac{47.9}{35}\right)=0.25 \\
\text { P.0\% cmpldere }
\end{gathered}
$$

Jack R. Benjamin \& Associates, Inc. Consulting Engineers

C-77 


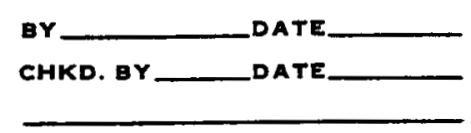

PROJECT.

PAGE 9 _ OF

JOB NO.

Analysis To Determine Fence in Bolts

Assumption: The accumulator rocks on a rigid body win ins gruficomi deformation relative to displacement in Anchor (bolt/angle). Note D te is $\approx 64 \mathrm{Fu}$ shell.

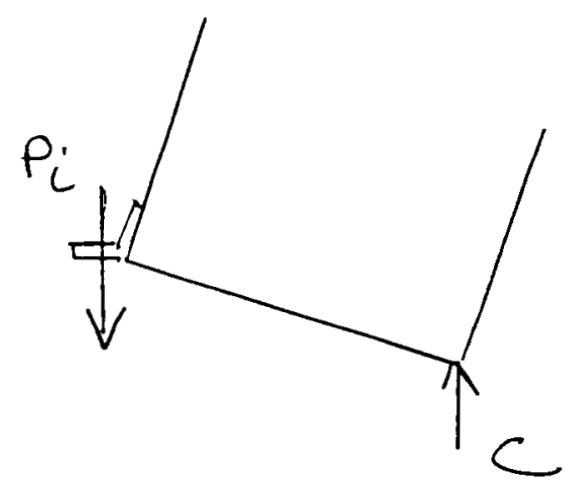

Following page que deviated for calculating bolt forces

The derivotim is followed by MATNCAY program fa finder forces by trial deer (ie $\Theta$, angle to compressive Revel, is unknom)

Jack R. Benjamin \& Associates, Inc. Consulting Engineers

C-78 
10

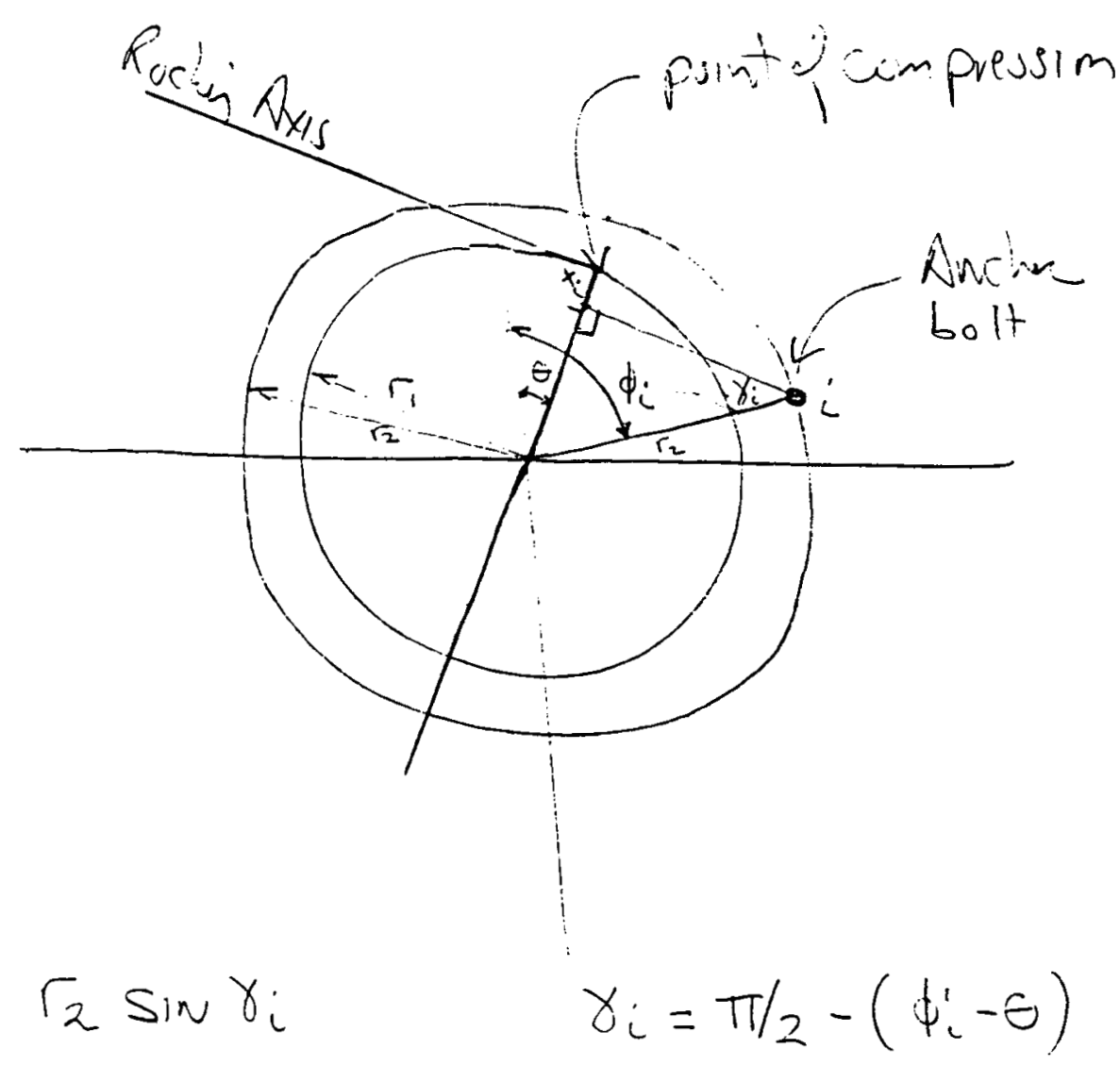

$$
\begin{aligned}
& x_{i}=r_{1}-r_{2} \sin \gamma_{i} \quad \gamma_{i}=\pi / 2-\left(\phi_{i}-\theta\right) \\
& 0 \leq x_{i} \leq r_{1}+r_{2} \\
& \left.x_{i}=r_{1}-r_{2} \cos i_{i}-\epsilon\right]
\end{aligned}
$$

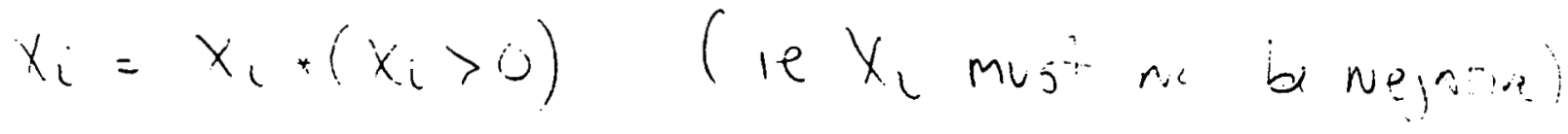

C-79 


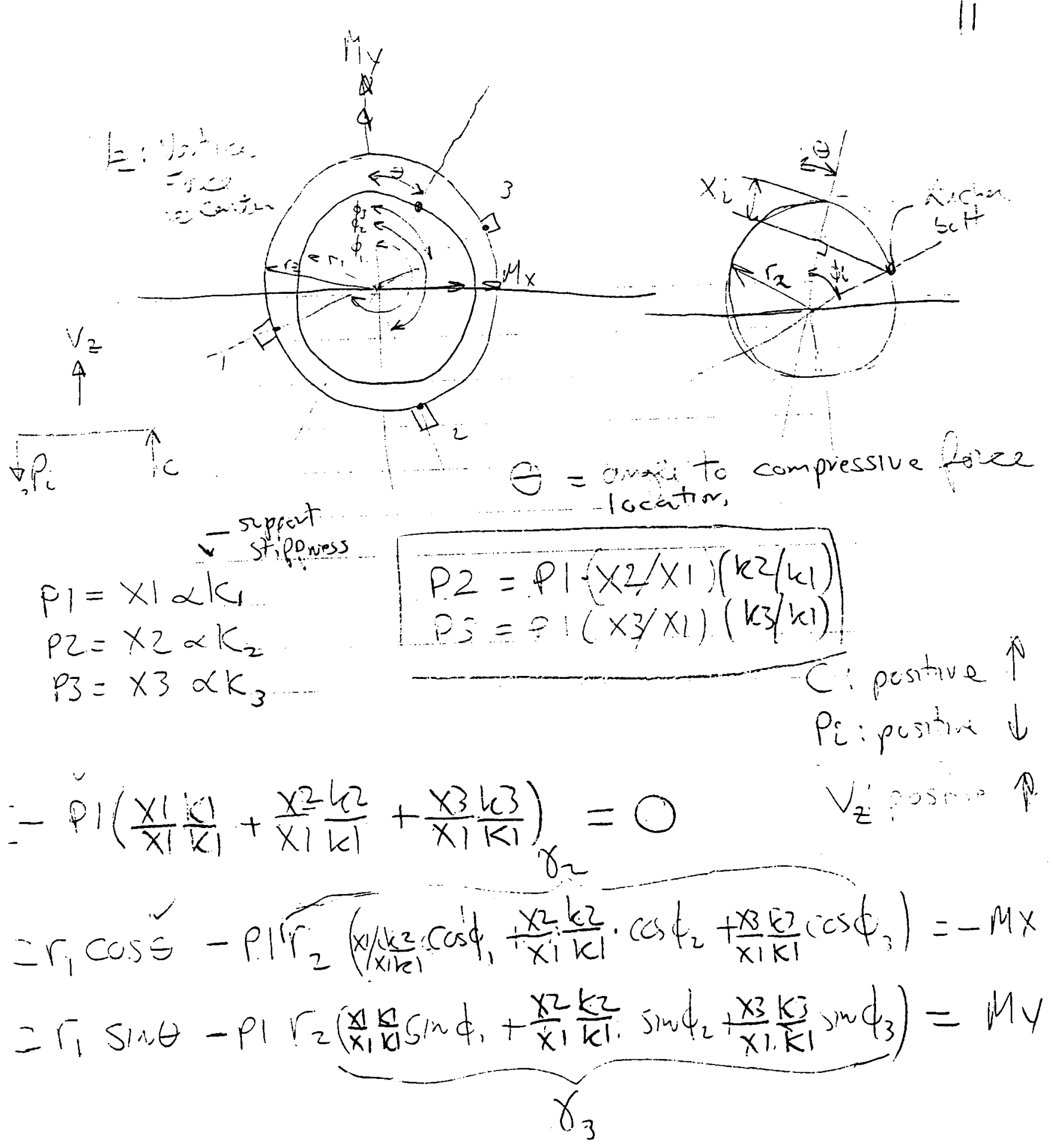

C-80 
12

$$
\begin{array}{r}
-x_{1}, p=-U \equiv \\
==x_{1},-V_{I}
\end{array}
$$$$
\begin{aligned}
& x_{1}=\leq x_{i} / x_{1} \\
& y_{2}=\leq \frac{x_{i} \cos t_{i}}{x !} \\
& y_{2}=\leq \frac{x_{i}}{x !}
\end{aligned}
$$

$$
\begin{aligned}
& \left(\gamma_{1} p 1-v_{7}\right) r_{1} \cos -r_{2} \gamma_{2} p 1=-M_{x} \\
& \left(\gamma_{1} p 1-v_{z}\right) r_{1} \sin \theta-r_{2} \gamma_{3} p l=m_{y}
\end{aligned}
$$

)

$$
\begin{aligned}
& \cos \theta=\frac{-M_{1}+r_{2} \gamma_{2} p_{1}}{r_{1}\left(\gamma_{1} p_{1}-V_{z}\right)} \\
& \cos \theta=\left[\frac{r_{2} \gamma=\rho 1-\mu_{x}}{r_{(}\left(\gamma_{1} p_{1}-v_{\bar{t}}\right)}\right] \\
& \sin \theta=\left[\frac{t r_{2} \gamma_{3} \cdot 1+r_{1}}{r_{1}\left(x_{1} p 1-v_{z}\right)}\right] \\
& \sin ^{2} \theta+\cos ^{2} \theta=1
\end{aligned}
$$

C-81 


$$
\begin{aligned}
& \left(r_{2} \gamma_{2} p 1-\mu_{x}\right)^{2}+\left(+r_{2} \gamma_{3} p !+r_{1}\right)^{2}=r_{1}^{2}\left(\gamma_{1} p 1-v_{z}\right)^{2} \\
& r_{2}^{2} \gamma_{2}^{2} p_{1}^{2}-2 M_{x} r_{-2} \gamma_{2} p_{1}+m_{x}^{2} \\
& r_{2}^{2} \gamma_{3}^{2} p_{i}^{\prime \prime}+2 M_{y} r_{2} \gamma_{3} p l+M_{y}^{2} \\
& -r_{1}^{2} \gamma_{1}^{2} p_{1}^{2}+2 v_{z} r_{1}^{2} \gamma_{1} p_{1}-r_{1}^{2} V_{z}^{2}=0 \\
& a p^{2}+b p 1+c=0 \\
& a=r_{2}^{2} \gamma_{2}^{2}+r_{2}^{2} r_{3}^{2}-r_{1}^{2} \gamma_{1}^{2} \\
& b=-2 M \times r_{2} \gamma_{2}+2 M_{1} r_{2} \gamma_{3}+2 v_{z} r_{1}^{2} \gamma_{1} \\
& c=n_{x^{2}}{ }^{2}+n_{y^{2}}{ }^{2}-r_{1}^{2} V_{z}^{2} \\
& P 1=\frac{-b \pm \sqrt{b^{2}-4 a c}}{2 a} \quad(p \text { inustint) } \\
& \theta=\cos ^{-1}\left[-\frac{r_{2} x_{2} p 1-\mu_{x}}{r_{1}\left(x_{1} p 1-v z\right)}\right]
\end{aligned}
$$

C-82 
14

digpderand at Cab

$$
\begin{aligned}
& \alpha=\frac{P 1}{k \cdot x} \\
& x \text { disp }=\alpha H \sin \theta \\
& y \text { disp }=\alpha N \cos \theta
\end{aligned}
$$

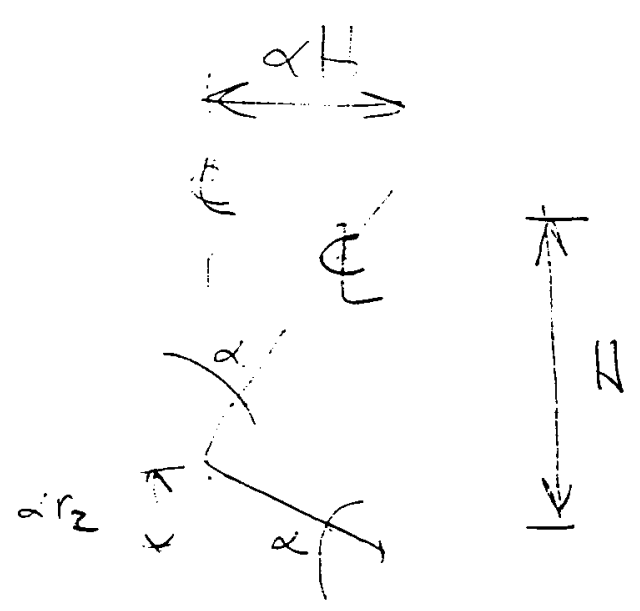


This program calculates the reacticins of a three support circular vertical tank where the tank rotates as a riqid body and the location of the compressive reaction must be abtained by trial and errar. The laading consists of a moment loading with a vector of 10 at an angle of $A$ with the reference axis and a second mament of 4 at 90 degrees to the vector af 10 .

$$
\begin{aligned}
& \text { deg } \equiv \frac{\pi}{180} \\
& i:=0 \ldots 15 \\
& A_{i}:=22.5 \cdot i \cdot d e g
\end{aligned}
$$$$
\text { This establishes the angle } A \text { at which the }
$$$$
\text { bolt forces will be calculated }
$$

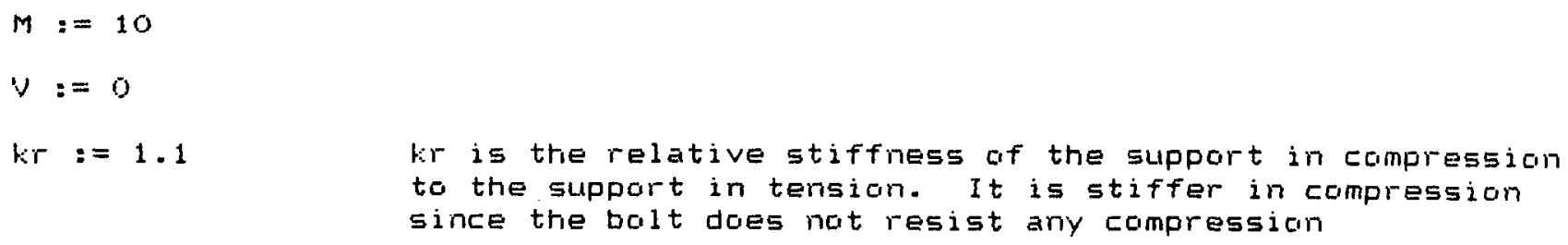




$$
\begin{aligned}
& 1_{i}:=r_{1}-r_{2} \cos [\phi 1-\dot{\theta}[i] \\
& \therefore e_{i}:=r_{1}-r_{2} \cos \left[\phi 2-\theta_{i}\right] \\
& \therefore 3:=r,-r_{i} \cos [\sin -\theta] \\
& k_{i}:=1 \cdot\left[\begin{array}{lll}
x 1_{1} & y & 0
\end{array}\right]+k r \cdot\left[\begin{array}{lll}
x 1 & < & 0
\end{array}\right] \\
& k e_{i}:=1 \cdot\left[\begin{array}{lll}
x 2 & y & 0
\end{array}\right]+k r^{\prime}\left[\begin{array}{lll}
x & < & 0
\end{array}\right] \\
& k 3_{i}:=1 \cdot\left[\begin{array}{lll}
\times 3 & y & 0 \\
i &
\end{array}\right]+1: r^{\cdot}\left[\begin{array}{lll}
\times 3 & 6 & 0
\end{array}\right]
\end{aligned}
$$$$
x z_{i}:=\left[\begin{array}{r}
k: 2 \\
i \\
k 1 \\
i
\end{array}\right] \cdot x e_{i}
$$$$
x_{i}:=\left[\begin{array}{l}
k 3 \\
i \\
k 1 \\
i
\end{array}\right] \times 3
$$
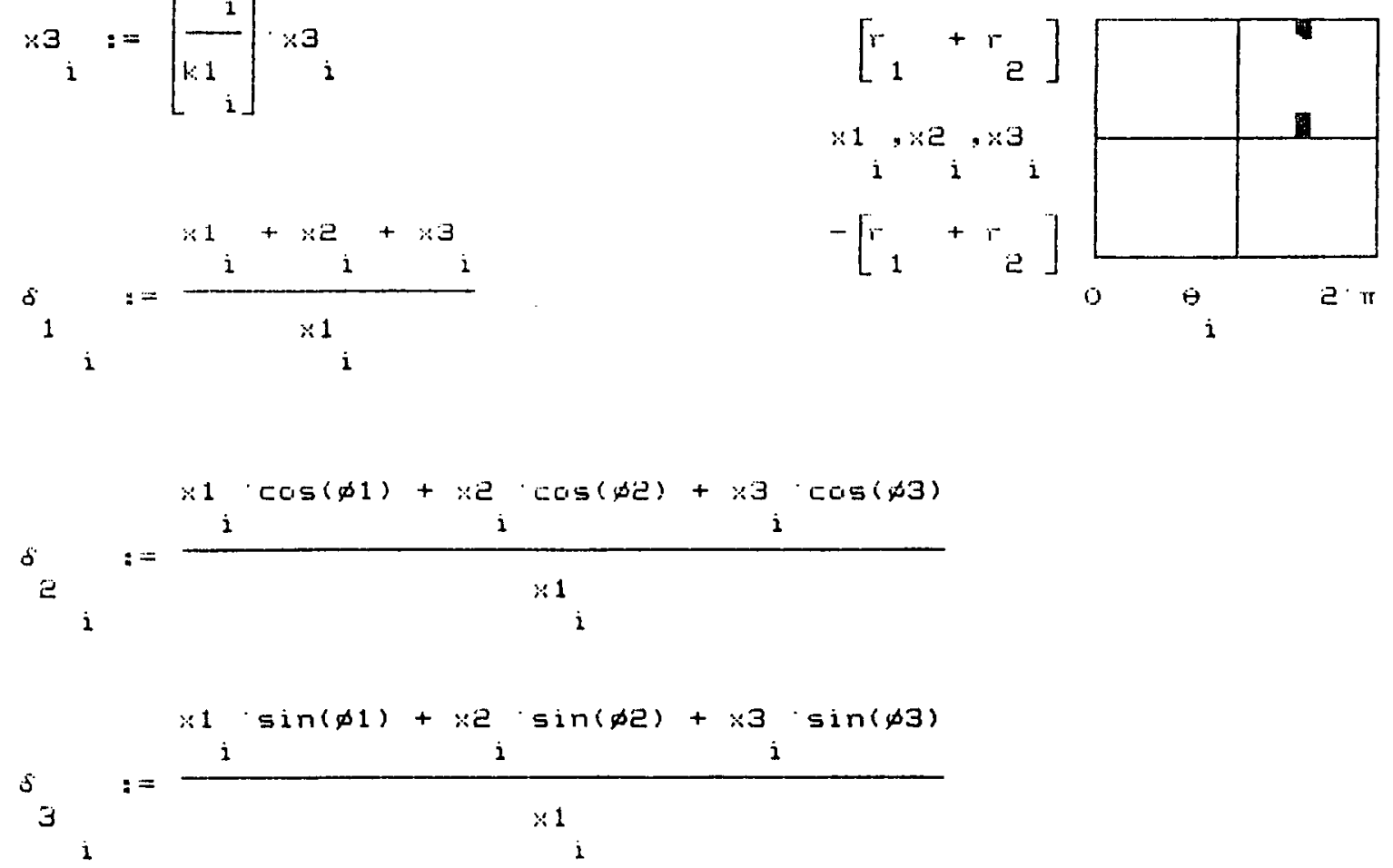


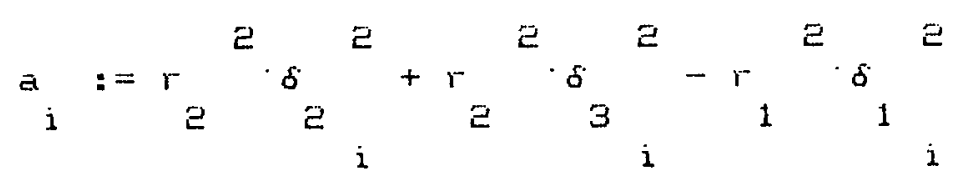

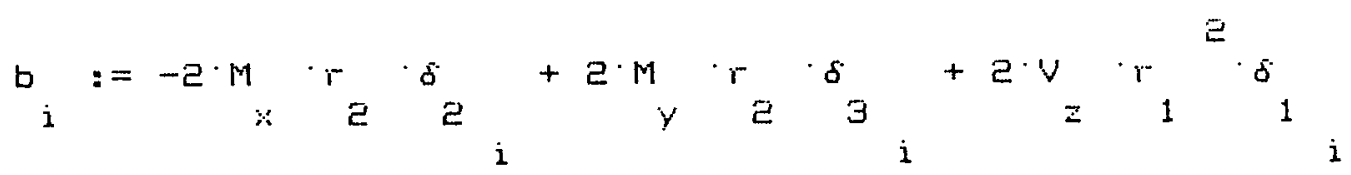

$$
\begin{aligned}
& c:=m^{2}+m^{2}-r_{1}^{2} v^{2} \\
& F_{i}^{1}:=\left[\frac{1}{e^{\cdot a}}\right] \cdot\left[-b_{i}+\left[\left[\begin{array}{lll}
b & 0 & 0 \\
i &
\end{array}\right]-\left[\begin{array}{lll}
b_{i} & 0
\end{array}\right]\right] \sqrt{\left.b_{i}^{2}-4 \cdot a \cdot c\right]}\right.
\end{aligned}
$$

$$
\begin{aligned}
& F e_{i}=F_{i} \cdot\left[\begin{array}{l}
\times 2 \\
i \\
\times 1 \\
i
\end{array}\right] \\
& F 3_{i}==F^{1} \cdot\left[\begin{array}{l}
\therefore 3 \\
i \\
\therefore 1 \\
i
\end{array}\right] \\
& c_{i}:=F 1 s_{i} \quad-v
\end{aligned}
$$

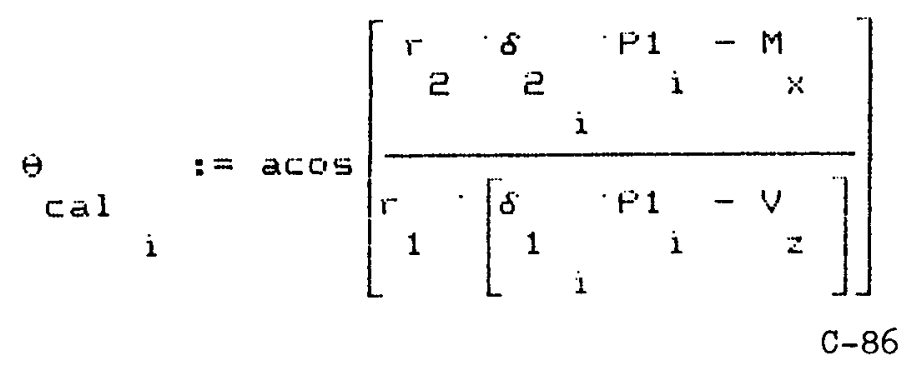




$$
\operatorname{cal}_{i} \quad:=\theta_{\operatorname{cal}}\left[\begin{array}{lll}
\theta & < & \pi \\
i &
\end{array}\right]+\left[\begin{array}{lll}
2 \pi & -\theta & \\
& c a l & \\
& & i
\end{array}\right] \cdot\left[\begin{array}{lll}
\theta & \gamma & \pi \\
i &
\end{array}\right]
$$

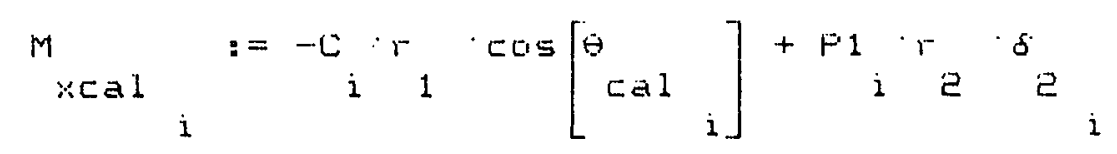

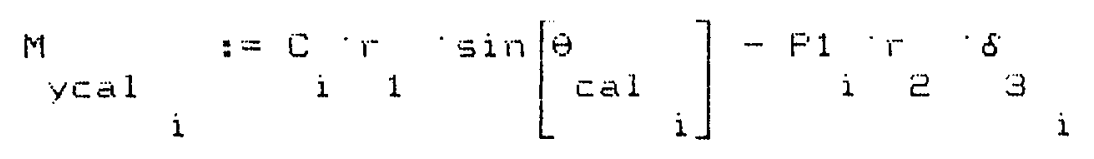

$$
v_{2 \operatorname{cal}} \quad:=P_{i}+F_{i}+F_{i}-C_{i}
$$

$\Theta_{d \in]_{i}}:=\theta_{\text {[a] }}-\theta_{i}$

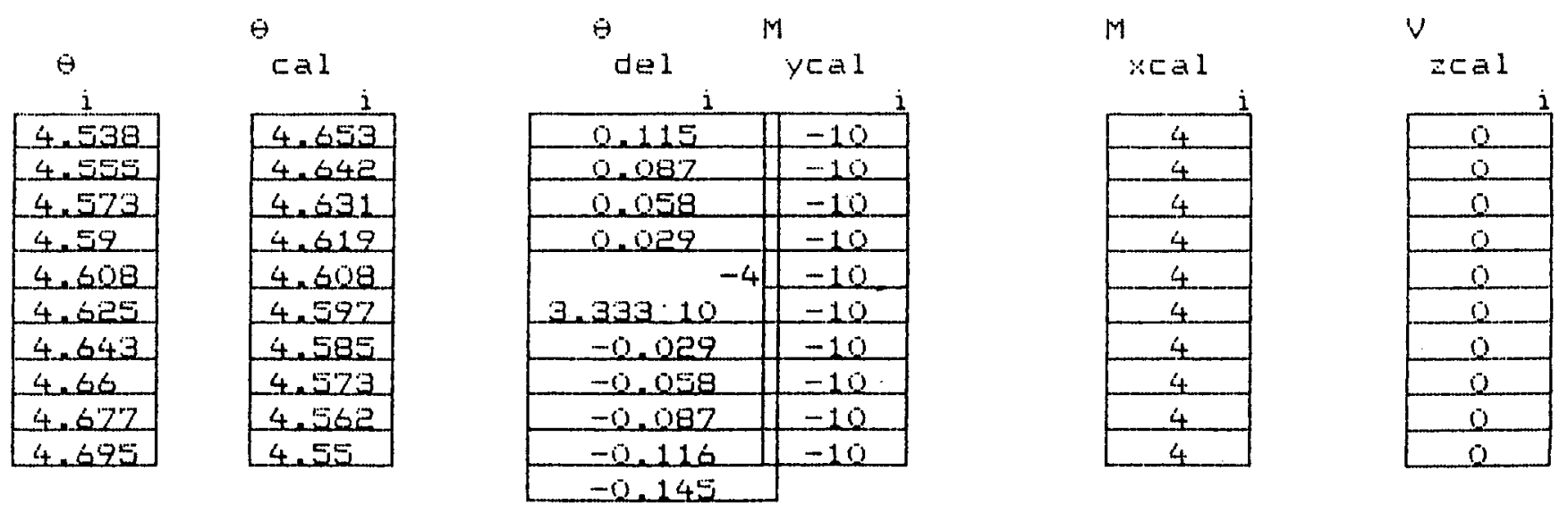

$$
\begin{aligned}
& t_{i}:=i \\
& s_{\text {cal }}:=\operatorname{cspline}\left[\begin{array}{ll}
t, \theta & \\
\text { cal }
\end{array}\right]
\end{aligned}
$$

$s:=c s p l i n e(t, \theta)$ 
$x:=5$

$\operatorname{rout}\left[\right.$ interp $\left.\left[\begin{array}{lll}s & \text { t, }, e_{\text {cal }} & x\end{array}\right]-\operatorname{interp}(s, t, \theta, x), x\right]=4.013$

$\theta_{\text {opt }} \quad:=\operatorname{interp}\left[s, t, \theta, r 00 t\left[\operatorname{interp}\left[s_{c a l}, t, \theta_{\text {cal }}, x\right]-\operatorname{interp}(s, t, \theta, x), x\right]\right]$ $k$

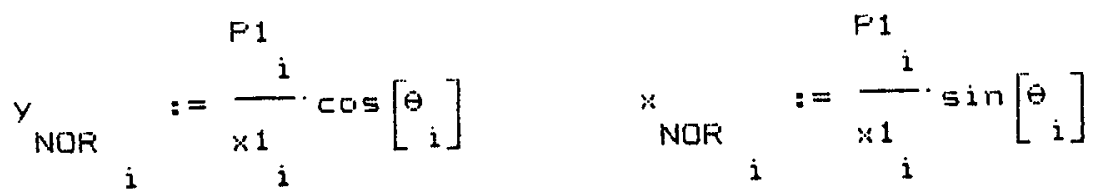

$j:=1$

$A_{k}=180 \cdot$ deg

F1

$\underline{i}$

\begin{tabular}{|c|}
\hline 0.413 \\
\hline 0.413 \\
\hline 0.412 \\
\hline 0.412 \\
\hline 0.412 \\
\hline 0.412 \\
\hline 0.412 \\
\hline 0.413 \\
\hline 0.413 \\
\hline 0.413 \\
\hline
\end{tabular}

$t \equiv B$

rance $_{1} \equiv 260 \cdot$ deg $\theta$

apt

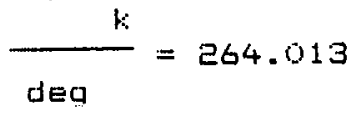

F'3

$c$

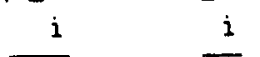

$\frac{j}{0.063}$

0.06

0.057

0.054

0.051

0.048

0.045

0.042

0.039

0.036

i.508

0.509

0.508

0.508

0.508

0.500

0.509

0.51

0.511

0.512

$\frac{0.059}{0.063}$

0.036

o.s1e

$\theta$
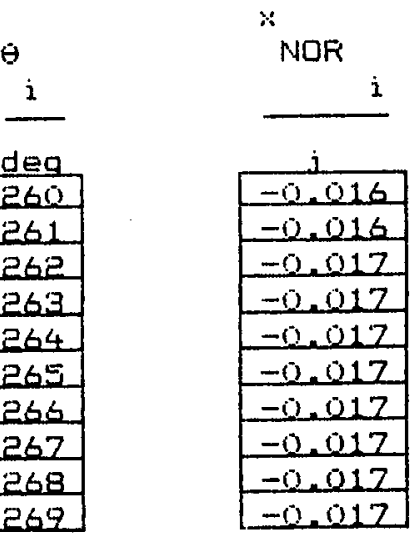

\begin{tabular}{|l|}
\hline 269 \\
\hline 260 \\
\hline 261 \\
\hline 262 \\
\hline 263 \\
\hline 264 \\
\hline 265 \\
\hline 266 \\
\hline 267 \\
\hline 268 \\
\hline 269 \\
\hline
\end{tabular}

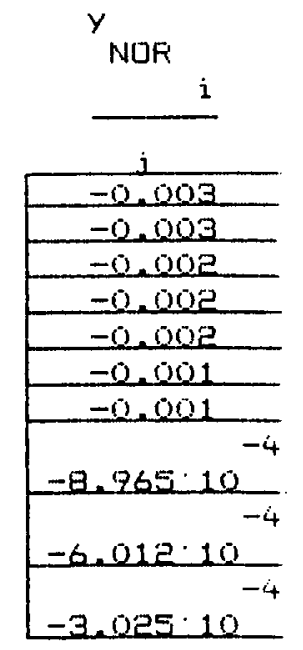


20

itis Five<smiles></smiles>

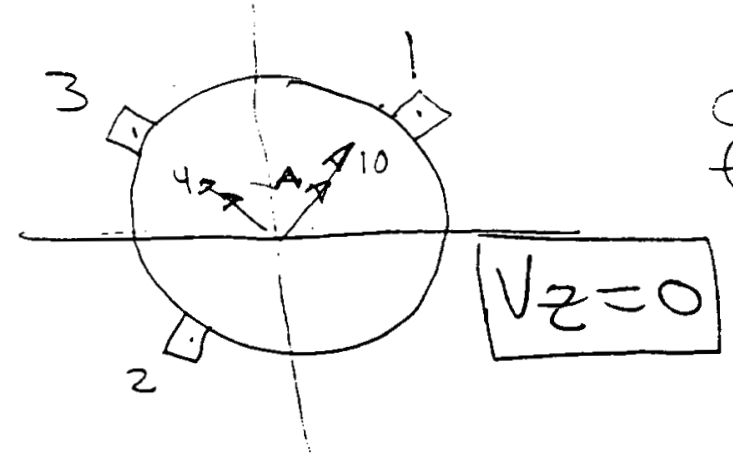

Comperese $\frac{10.77}{\text { fore }}=.410$

fore $(14,25+12)$

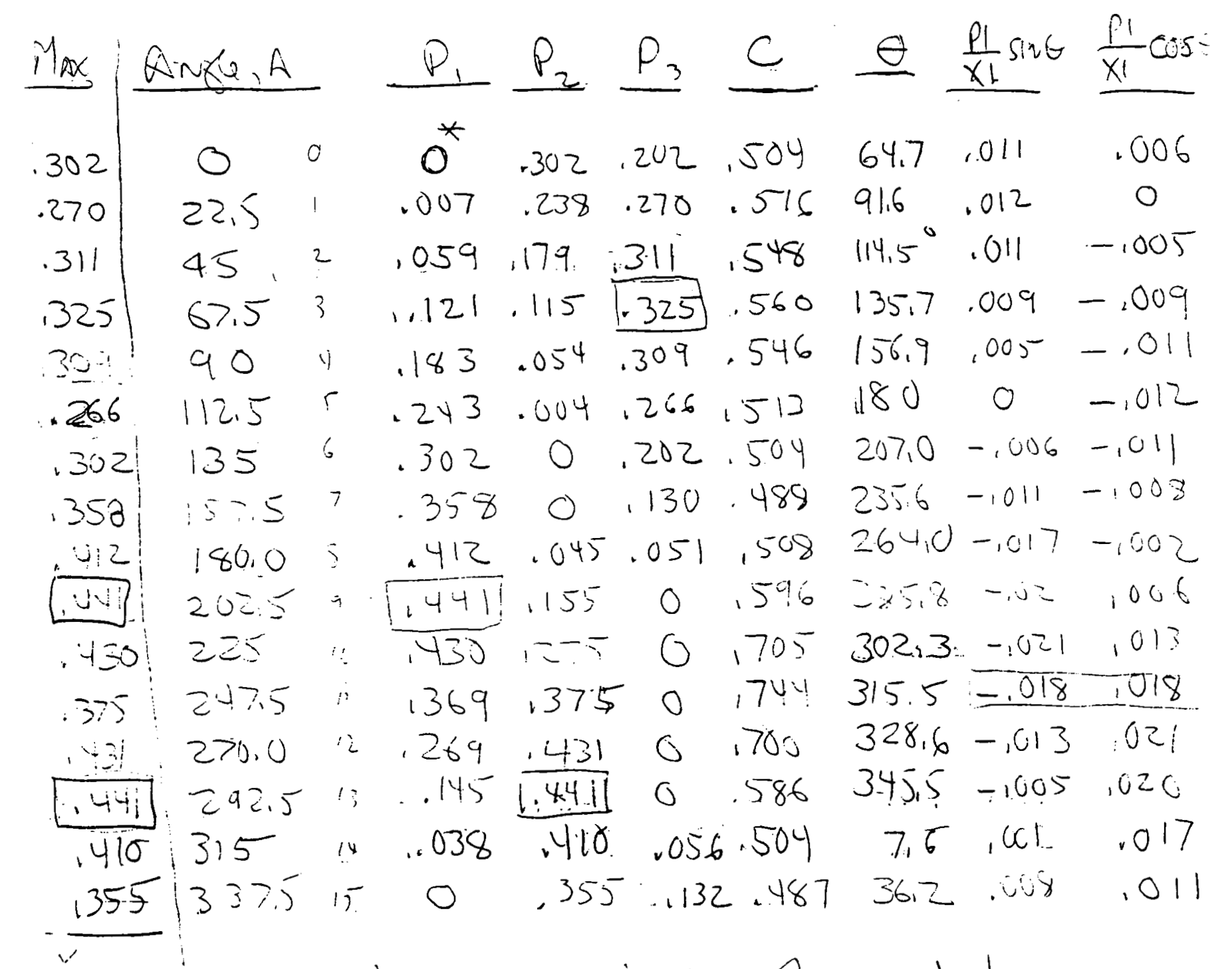

* Lisis run did not cilow 8le suppente to go in compossina-deffiorares is insirnifeosant

C-89 


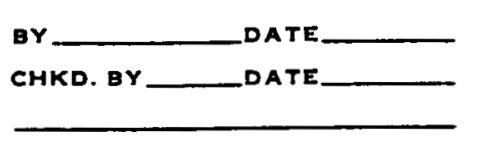

PROJECT

SUEJECT
PAGE 21 OF

JOE NO.

Pveliminan Capaction Nualias:

Fvom Free am hapis:

Medin firee $/ 10^{k-11}$ input $\cong 0.741^{k}=(\text { In bo t })^{\prime}$

Modian alloware angle cap $=2.11^{\mathrm{K}}$

$\Rightarrow$ Conesanding Momet at bx:2 $=(2.11(.441) 10=47.84 \mathrm{k}$

Free at C.g. $=\frac{47.84}{41.5}=1.15^{\mathrm{k}}$

$S_{a}=\frac{1.15}{920} g=1.25 \mathrm{~g}$

increre 2 iwelostic vapense

Nllow 0,4 in e Co $(\pi 1 \%$ buft $)$

$$
\text { Yiele) Frae in support } \approx \text { Fince in boit) } 2.11 \times \frac{1}{1,5}=1.41^{k}
$$

Proment at brese tome $=\frac{1.41}{.441} \times 10^{k-11}=31.9^{k-11}$

seepere 6

$$
\stackrel{4^{k-11}}{\longrightarrow} 0^{k-11}
$$


ductuldo:

$$
\begin{aligned}
& F=9.5 h_{3}: N=0.4 /\left(1.2 \times 10^{-3} \times 41\right)=8.1 \\
& F=27 h_{3}: N=0.4 /\left(1.6 \times 10^{-4} \times 41\right)=61.0
\end{aligned}
$$

convert to $7.5 \mathrm{~h}_{3}+33 \mathrm{~h}_{3} \quad$ Rddell/Newmant

e 7.5 h3: $\quad r=8.1 \times\left(\frac{7.5}{9.5}\right)^{2}=5.0 \quad F_{N}=[2.851(5.0)-1.851]^{1422}=2.89$

(8) 33h : $N=61 \times\left(\frac{33}{27}\right)^{2}=91.1 \quad F_{N}=(91.1)^{13}=1.80$

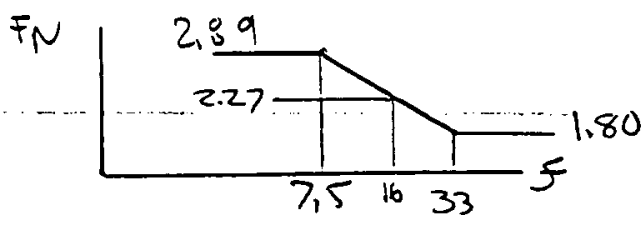

(e) Median: $f=16 h_{3}$ :

$$
\begin{gathered}
\ln F_{N}=\ln 2.59-\frac{\ln 16-\ln 7.5}{\ln 33-\ln 7.5}(\ln 2.89-\ln 1.80) \\
F_{N}=2.27 \\
\beta_{r}=0.11\left[F_{N-1 / 2}\right]=0.14 \quad F_{i t s} R_{1} d_{d o u}\left(N_{\text {tumn }}\right. \\
\beta_{U}=0.25 \quad \quad \text { (estinati) }
\end{gathered}
$$

Note: since $f=16 h_{3}$ is on stiff sicle of speetal plak , $F_{N}=2.27$ is too optimistic. see effect on $P \mathrm{Pg}_{2} 22 \mathrm{~A}-\mathrm{C}$

$$
\text { gre } 11 / 13 / 88
$$

Jack R. Benjamin \& A

C-91 


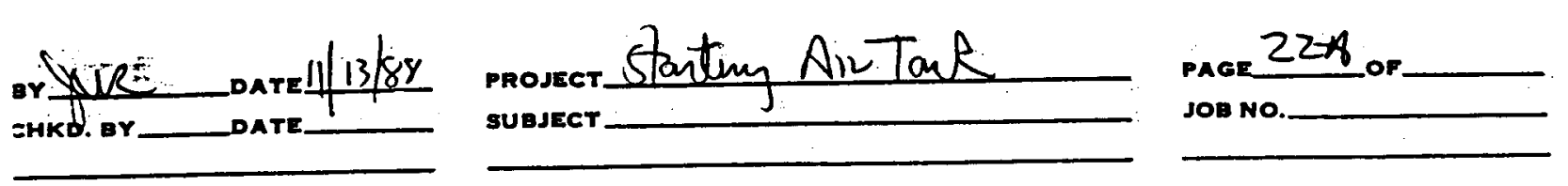
Reanalysis Perpormod Aftor Completion of Calculatime
(see pgs $23^{+}$for restof original calculation)

The better approol is to combre $S_{A} \& F_{N}$ into sinfy term Sa/FN. A time history was developed by fittin, real earihguake record to $5 \%$ domper flor spetrum and a nonlenear avalpin wos performas to obtain $F_{N}$ value , lie.

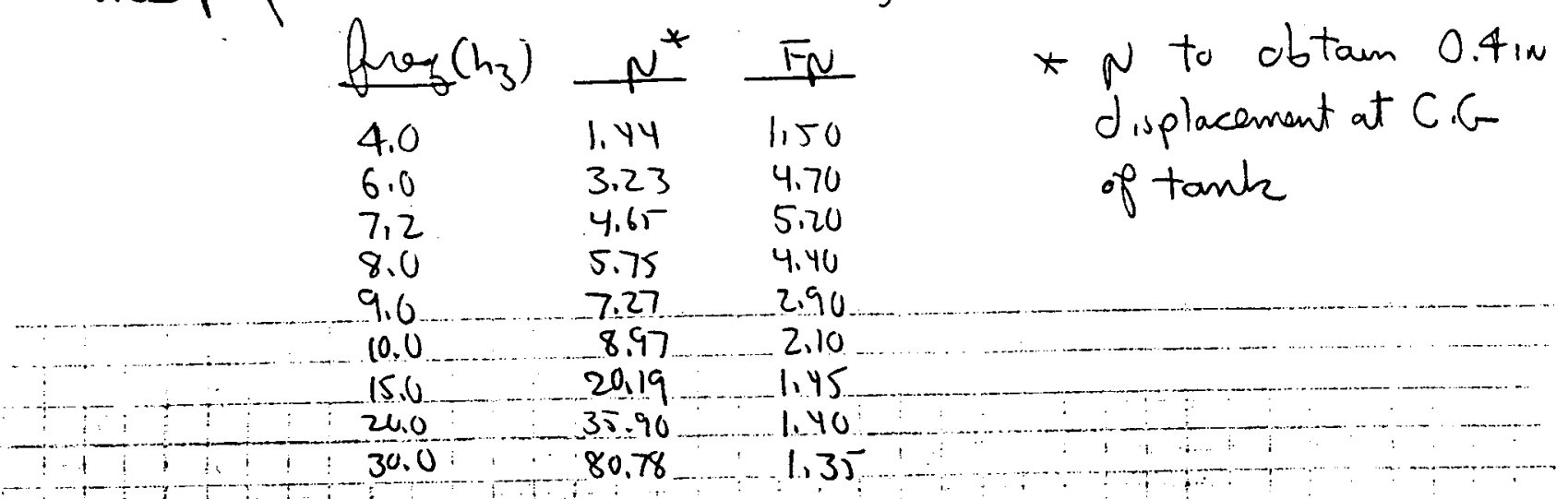

Page $22 B$ id $22 C$ compute $S_{n}$ (FN values and statistics are obtamod for uncertanty, in frequancy [i.e. $\operatorname{LN}\left(16 h_{3}, 0.35\right)$ ]. SalFr-medion-15-0.35g and $\beta=0.075 \operatorname{secps} 25$

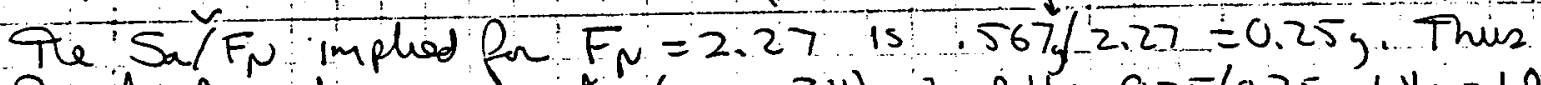

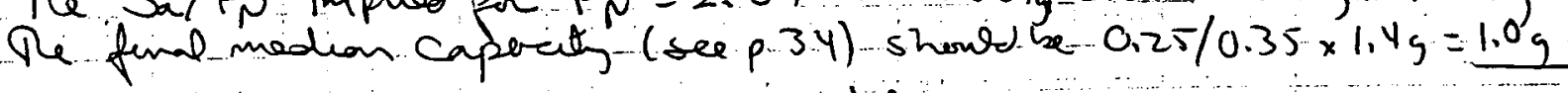
Nowever, in $\beta$ 's neod to be modified:

1) Bu due to firef is 0.07 not 0.30

2) Bu due to inslastic nepomis is estinated to be 0.15 not 0.25 3) \& - due to inelastic vespona is stinded to ke:

$$
\begin{aligned}
& F_{N}=.25 / .35 \times 2.27=1.62 \\
& \& \quad \rho_{r}=-111[1.62-1 / 2]=12
\end{aligned}
$$

Nowern, sxss to $\beta_{r}$ total. Tutal $\beta_{r} 11$ ond slintly increased: Bosed on those changer:

$$
\begin{aligned}
& \breve{a}=1.0, \beta_{r}=124 \beta_{\nu}=0,28
\end{aligned}
$$

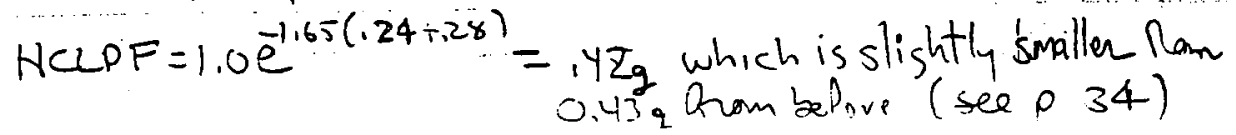

$$
\begin{aligned}
& \text { C-92 }
\end{aligned}
$$


MCAD FILES SAFU.SIM

OFIGIN $\equiv 0$

$y \equiv 0$

$\operatorname{inarm}(x) \equiv \operatorname{ragt}(\operatorname{cogarm}(y)-x y)$

Inverse normal distribution

Freq hat $_{\text {hat }}:=16$

$\beta:=0.35$

$n:=197$

$i:=0 \ldots n$

Freq $_{i}:=\operatorname{Freq}_{n \rightarrow t} \exp \left[\hat{s} \operatorname{inarm}\left[\frac{i+\operatorname{rnd}(1)}{n+1}\right]\right]$

LFreq $_{i}:=\ln \left[\begin{array}{rr}\text { Freq }_{i} \\ & \end{array}\right]$

$m:=45$

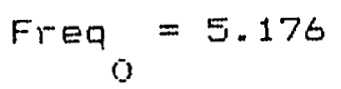

m: $=45$ $\operatorname{Freq}_{199}=40.227$

$j:=0 \ldots m$

$X L F F:=F E A D(H L F F)$

$x_{j}:=$ FIEAD $(H S A S)$

$\equiv 5:=c \equiv p 1$ ine (XLFFi,XSAS)

$1:=1 . .500$

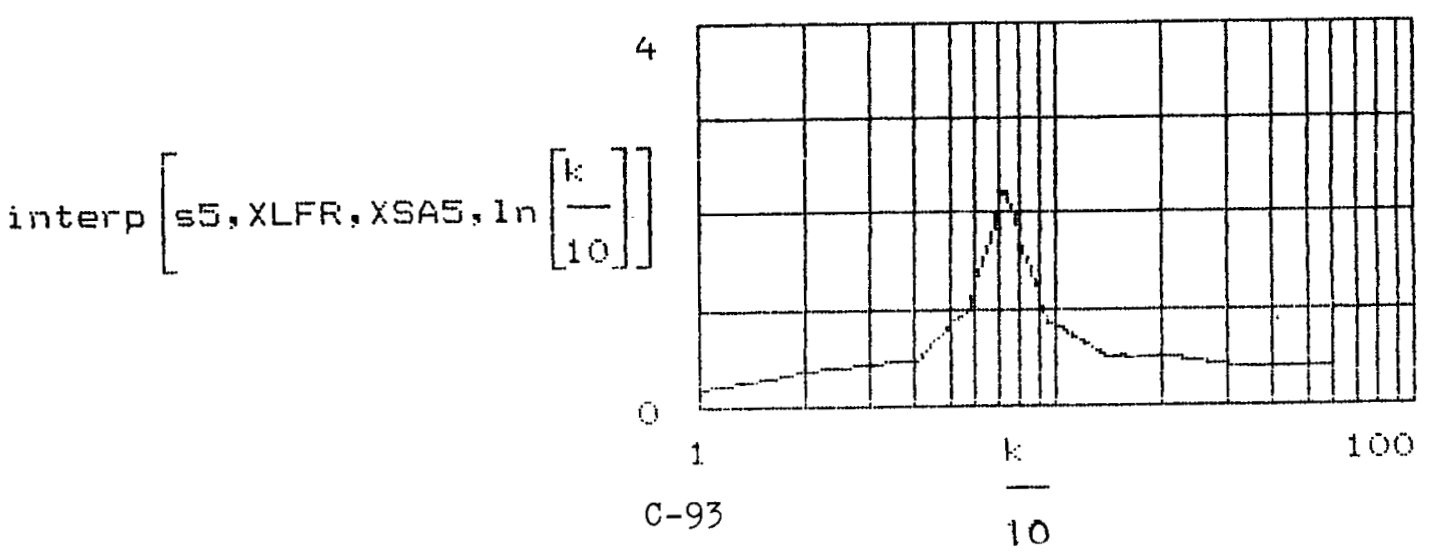


$S A:=\overline{(\text { interp }(S 5, X L F F, \times S A 5, L F r e q)}$

$$
\text { H.eq }:=\left[\begin{array}{r}
4.0 \\
6.0 \\
7.2 \\
3.0 \\
9.0 \\
10.0 \\
15.0 \\
20.0 \\
30.0
\end{array}\right]
$$

SaeFH. $:=\frac{\text { SA }}{\left[\frac{1 \text { interp }(\mu, F r e q, F \mu, F r e q)}{\text { F }}\right]}$

$1:=0 \ldots$

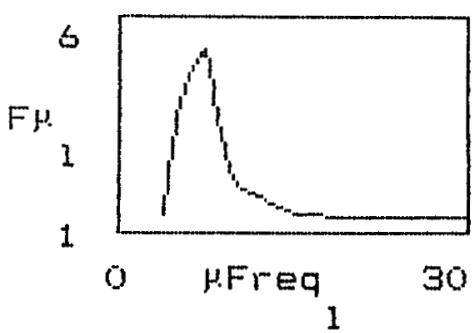

The peatied curve is Sa

The flat curve is $\mathrm{Sa} / \mathrm{FH}$

\section{LSA $:=\ln ($ SaeF $\mu)$}

Median $:=\exp ($ mean $(L S A))$

Eeta : = stdev(LSA)

Median $=0.35$

Eeta $=0.073$ $\operatorname{sp}(k), s \mu(1:)$

e

0

1

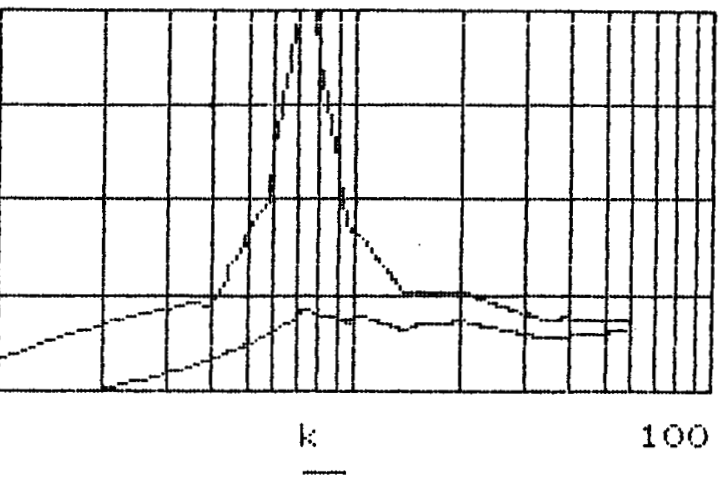

10

Fbeta : = stdev(LFreq)

$$
\begin{aligned}
\text { Fmedian } & =15.978 \\
\text { Fbeta } & =0.35
\end{aligned}
$$


Br

PROJECT

PAGE 23 . OF

JOB NO.

Sa coprect) $=(2.27)(1.25 \mathrm{~g})=2.84 \mathrm{~g}$

$$
\text { Floor caprent }=\left(\frac{2.84}{, 567}\right), 3 \overline{8}, \quad=1.90 \mathrm{~g}
$$

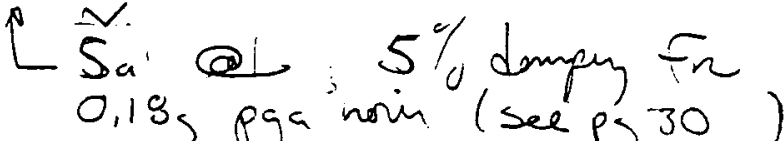

Ground capacit, $\left(\frac{2.84}{.567}\right)(118 g)=0.90 \mathrm{~g}$

Conospudy vertizer accelention a floor

$$
S_{a}=\frac{0,90 \mathrm{~g}}{0.18} \times 0.20=1,00 \mathrm{~g}
$$

Net downuged frice using $100=40-40 \mathrm{vde}$ is $\quad 920[1-(0,4)(1,00)] \cong 552 \mathrm{ks}$

The corvesponding Miriant e base is $47.84^{k-11}$

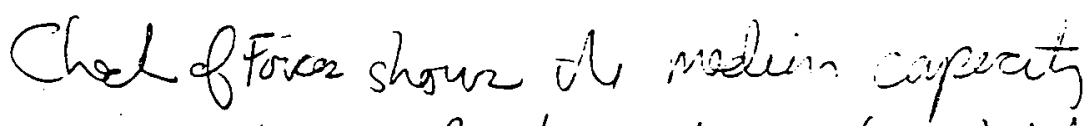
gove up by a fnctag $2 \pi 1,1$ ( due to helpiom)

Einal Capacit, Analinsis

Revised Net Downuad frace

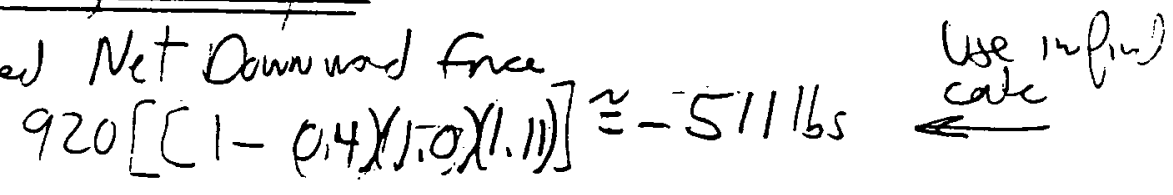

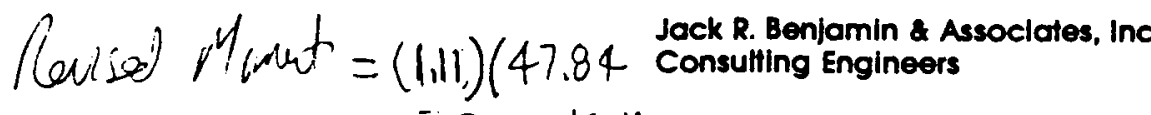
$=53.10 \mathrm{k}-4$

C-95 
Br

CHKD. BY DATE
PAGE 24

JOB NO

Fiesingute Maximum Farce in Aachen Fr Revisal Free fat

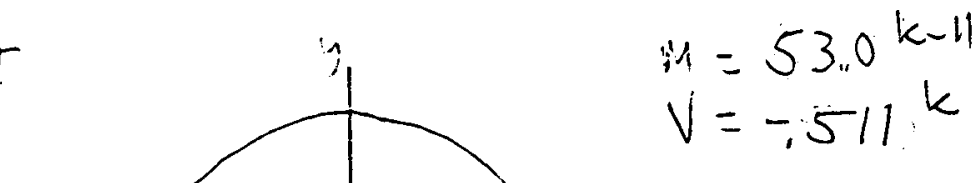

From pase 20 Angle $202.5^{\circ}$ and $292.5^{\circ}$ have Maximum farer in angle:

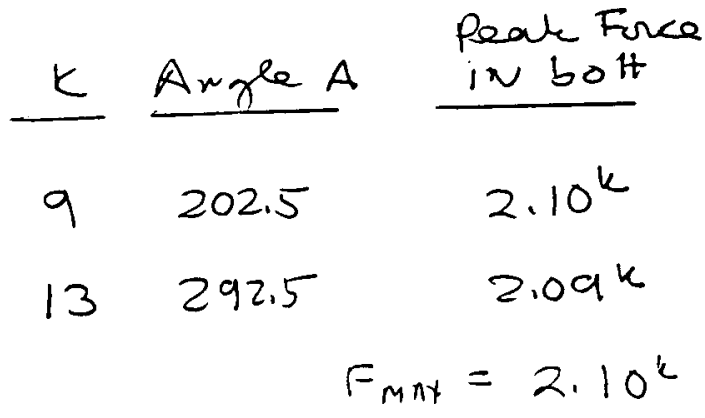

Fore e t $C \cdot G=\frac{53.0}{41.0^{4}} \times \frac{2.11}{2.10}=1.30^{k}$

$$
S_{a}=\frac{1,30}{1.92}=1.41, \mathrm{c}
$$

Jack R. Benjamin \& Associates, inc. Consulting Engineers

C-96 
BY CHKD. BY DATE

PROJECT

SUBJECT
PAGE $2 \dot{5}$ OF

JOB NO.

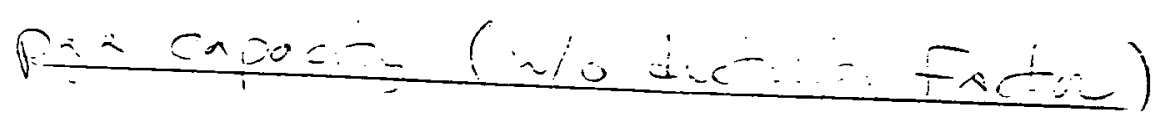

$P S==\frac{1.419}{.567} \times .18 \mathrm{~g}=0.45 \mathrm{~g}$

c $5 a 20,19 \mathrm{~g}$ pga hor: ( $5 \%$ damping) (see Sa call on pare 30 ).

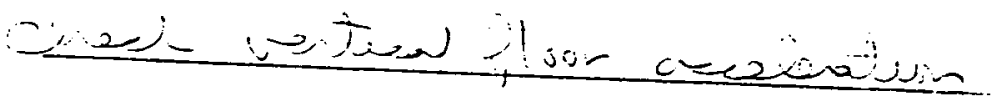

$$
\begin{aligned}
& \text { para w/dutis }=(.45)(2.27)=1.0 \mathrm{lg} \\
& S_{a v}=\frac{1.01}{0.18} \times .20=1.12 g\left(\operatorname{assman} 2(1.11)(1,0)=1.111_{g}\right) \\
& L \text { Epa }(0.18 \text {, by a ins }
\end{aligned}
$$

Here assured vertical load is ok

Jack R. Benjamin \& Associates, Inc. Consulting Engineers

C-97 


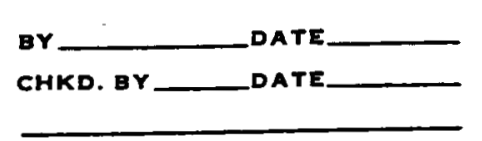

PROJECT

PACE 26 or

Develuor Fronitio Prisutr: = Or. A J NCLPE

Crepctin

Strougeh: $\quad \vec{F}=0.45 ; 10.45-y=1,0 \quad($ see paye 25$)$

$$
\begin{aligned}
& \beta_{r}=0 \quad 1 \\
& \beta_{u}=0.15 \text { (angle copacty-500 peye 2) }
\end{aligned}
$$

Inelassic Respase:

$$
\begin{aligned}
& \breve{F}=2.27 \\
& \beta_{r}=0.14 \\
& \beta_{u}=0.25 .
\end{aligned}
$$

(sel prez 22)

Equpowiti: Response

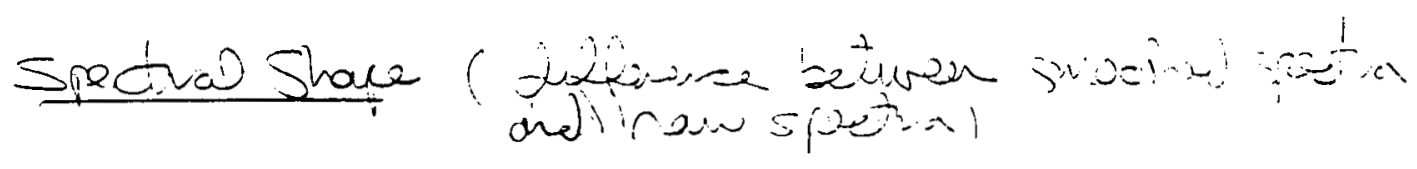

$$
\begin{aligned}
& \breve{F}=1.0 \\
& \beta_{r}=0 \\
& \beta_{u}=0.05 \quad \text { (estimate) }
\end{aligned}
$$

Jack R. Benjamin \& Associates, inc. Consulting Engineers

C -98 
Dampin)

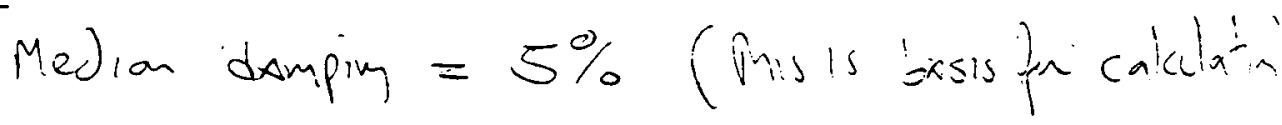

$$
\begin{aligned}
& \stackrel{\nu}{F}=1,0 \\
& -1 \sigma \cdot \text { dampen }=3.5 \% \\
& \frac{\text { her }}{16 h_{3}} \frac{S a(5 \%)}{.512 g} \frac{S_{a}(3.5 \%)}{.525 g} \quad(0.18 \text { ) hary paga } \\
& P_{u}=\frac{1}{1} \operatorname{2n} \cdot \frac{525}{1512}=0.03 \\
& p_{r}=0
\end{aligned}
$$

Modeling Freaars: Combine bein epuppunt of stuctue

$$
\begin{aligned}
& \beta_{u}=0.25 \text { bulding (est.) } \\
& \beta_{-}=0.25 \text { zquipiat }
\end{aligned}
$$

combined $\beta_{u}=0.35$

$$
\begin{aligned}
& \text { Qher } \quad \operatorname{Sa}(5 \%) \quad(0,10 \text {, hory } p \text { ga) } \\
& 16 \mathrm{~h}_{3} \quad 0.512 \mathrm{~g} \\
& 16 e^{-35}=11.2 h_{3} \quad 0.724 \\
& P_{u}=\frac{1}{1} \ln \frac{.724}{1512}=.35 \text { (too higli) }
\end{aligned}
$$

lockin at ploa reperse spoten shape Yhe valus is hif- Jack R. Benjomin \& Assoclates, inc. De simulation (see SPECT, sIMffallisen proye

C-99 
MCAD FILES SFECT.SIM

NOFMAL DISTFIEUTION

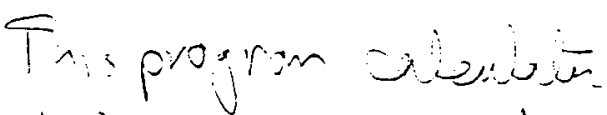

desinbutin $r$ - So den

七1 $\equiv .319381530$

be $\equiv-.356563792$

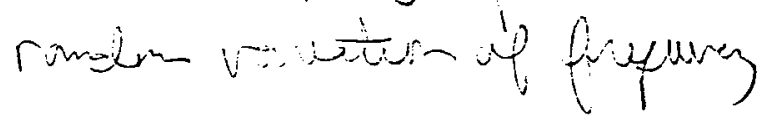

b3 $\equiv 1.781477937$

b4 $\equiv-1.821255979$

b5 $\equiv 1.330274429$

$p \equiv .2316419$

$I(x) \equiv \frac{\exp \left[\frac{2}{2}\right]}{2.506628275}$

$t(x) \equiv \frac{1}{1+p \cdot|x|}$

$\operatorname{NOR}(x) \equiv\left|(x y 0)-z(x) \cdot\left[b_{1} \cdot t(x)+b 2 \cdot t(x)^{2}+b 3 \cdot t(x)^{3}+b 4 \cdot t(x)^{4}+b 5 \cdot t(x)^{5}\right]\right|$

INUERGE NORMAL DISTFIEUTION

$$
\begin{aligned}
& c 0 \equiv 2.515517 \\
& c 1 \equiv .802853 \\
& c 2 \equiv .010328 \\
& d 1 \equiv 1.432788 \\
& d 2 \equiv .189269 \\
& d 3 \equiv .001308
\end{aligned}
$$$$
t(p) \equiv \sqrt{\ln \left[\frac{1}{((p)>.5)-p)^{2}}\right]}
$$

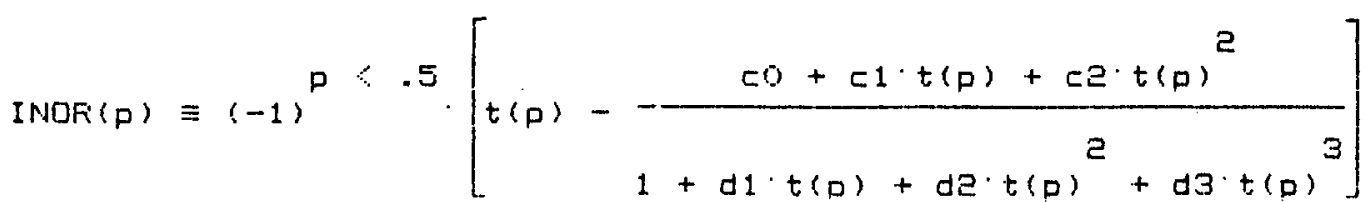




$$
\begin{aligned}
& \underset{\text { hat }}{\text { Freq }}:=15 \\
& 6:=0.35 \\
& \pi:=199 \\
& \text { i }:=0 \ldots n \\
& \text { Freq } i=\text { Freq }_{i} \exp \left[s \cdot \operatorname{INOF}\left[\frac{i+r \text { nd }(1)}{n+1}\right]\right] \\
& \text { LFieq }_{i}:=\ln \left[\begin{array}{cr}
\text { rreq }_{i} \\
\end{array}\right]
\end{aligned}
$$

$$
\begin{aligned}
& m:=45 \\
& j:=0 \ldots m \\
& \times L F F \quad:=\text { FEEAD (XLFF) } \\
& \text { XSAE }_{j}:=\text { FEAD (XSAE) }
\end{aligned}
$$$$
\text { SS:= CSPlinE(XLFF, KSAS) }
$$

$1::=1 \quad .600$

$$
\text { interp }\left[55, \times L F F, \times S A 5, \ln \left[\frac{1:}{10}\right]\right]
$$

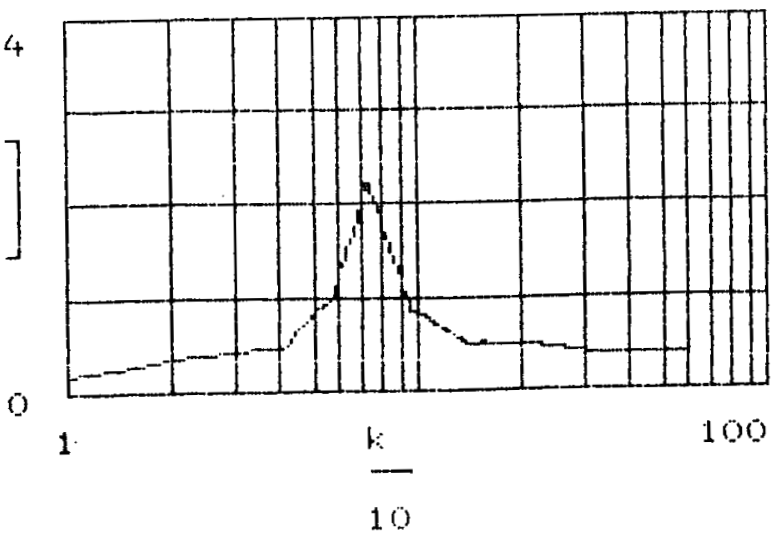

$$
\begin{aligned}
& S A_{i}:=\text { interp }\left[55, \times L F F, \times S A 5, \text { LFFEQ }_{i}\right] \\
& \operatorname{LSA}_{i}:=\ln \left[\begin{array}{c}
S A_{1} \\
i
\end{array}\right] \\
& \text { C-101 }
\end{aligned}
$$


Median := Exp(mean(LSA))

Beta: = stdev(LSA)

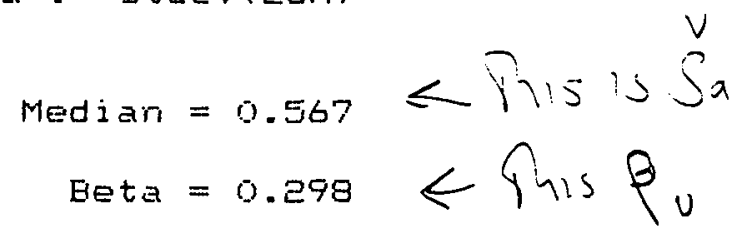

Finedian : = exp(mean(LFreq))

Fbeta : = stdev(LFreq)

$$
\begin{aligned}
\text { Fmedian } & =15.951 \\
\text { Fbeta } & =0.358
\end{aligned}
$$




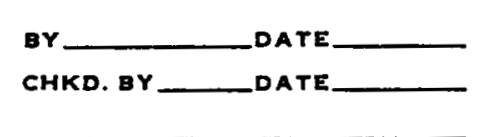

PROJECT

PAGE 31 OF

Modeliw, (Dveroonen) Continued

$$
\begin{aligned}
& P_{u}=0.30 \\
& \rho_{r}=0
\end{aligned}
$$

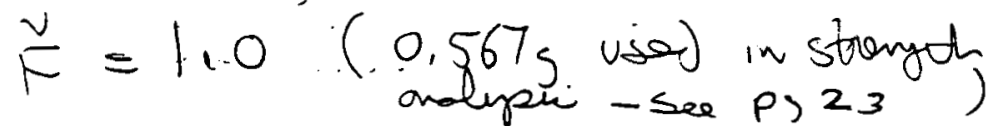

Mode shape: simple equipment in higi

$$
\text { figury som }
$$

$$
\stackrel{\sim}{F}=1.0 \quad \rho_{1}=\beta_{\nu}=0
$$

Mode Combination simple sisurture in hy frevan, dman (abe liven first mode shape $v$ wir OTMatber farlur mode)

$$
\stackrel{v}{F}=1,0 \quad \rho_{r}=\beta_{-}=0
$$

Horgentad Compunan. Phasinj

$$
\begin{aligned}
& \text { Bult Fice as } 100-40=2.10 * \text { (midin) } \\
& \text { But Force } n-1 / 100-100=2.617^{*}(3 \beta) \\
& P_{r}=\frac{1}{3}\left|\ln \frac{2.677}{2,10}\right|=0,07 \\
& e_{v}=0 \\
& \stackrel{v}{F}=1.0
\end{aligned}
$$

* Reron accumifin ( Ps 20)

Jack R. Benjamin a Assoclates, Inc. Consulting Engineers

$c-103$ 
BY

PROJECT

PACE 32 OF

HKD. BY DATE

SUBJECT

JOE NO.

Stvucture Response

Ground Motion Fin faramatin indudes:

1) peak-to fouk Spezium. Varioturn

2) Noriguntal Onestion Vaviabilitg

See Afpurdix A

3) Esurpment Honzontal Comiansat Couplim

$C=$ ratio of imina horizontal divectim rospense to combinad responce (ie ratio is
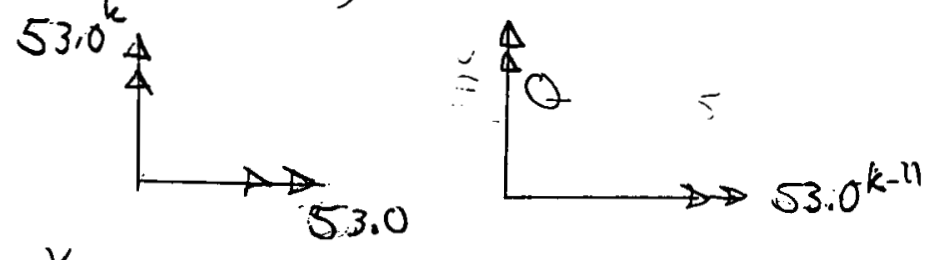

$$
\begin{aligned}
& \breve{P}_{b}=2,67^{k} \quad \ddot{P}_{3}=1.875^{k} \\
& C=\frac{2.617-1.875}{1.875}=0.40
\end{aligned}
$$

From DIR.FFT Rum (see Appendix A)

$$
\begin{aligned}
& \tilde{F}=1 / 1,02 \cong 0,98 \\
& \beta_{r}=0,29 \\
& \beta_{v}=0
\end{aligned}
$$

Dampin: Melem: $7 \%$

$\frac{\left.S_{A}(\text { Gon }) e 7.243\right)}{1.895}$

$-1 \mathrm{~T}: 5 \%$

2.12

C-104

Jack R. Benjamin Assoclates, inc. Consulting Engineers 


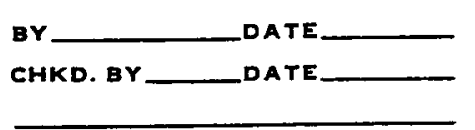

PROJECT

PAGE 33 OF

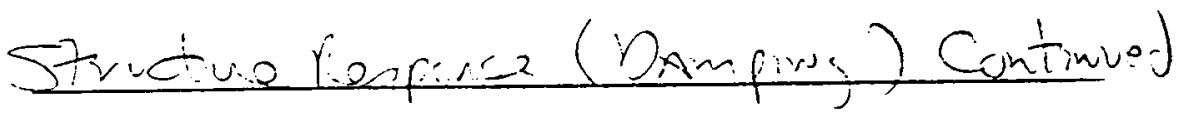

$$
\begin{aligned}
& \beta_{u}=\frac{1}{1} \ln \frac{2,12}{1.89}=0.11 \\
& \beta_{y}=0 \\
& \check{F}=1.0 \quad \text { (Used } 7 \% \text { m strengul cale) }
\end{aligned}
$$

Modeling

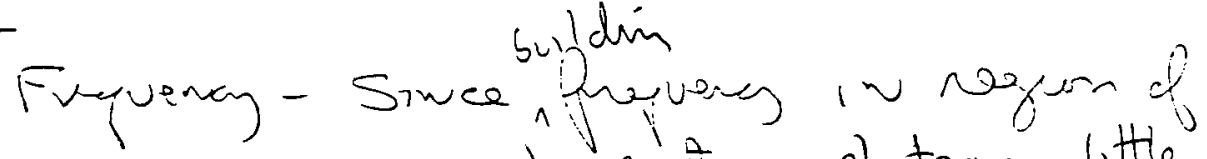
ground sigoin plateas litte

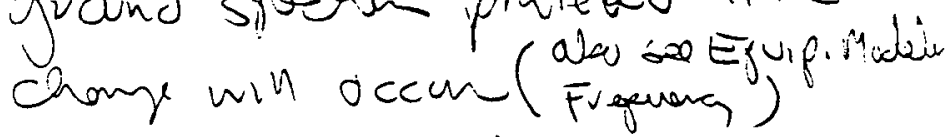

$$
\rho_{r}=\beta_{v}=0 \quad \check{F}=1.0
$$

Mode Siape - Assume conturlas by givest maste

$$
\tilde{F}=1.0 \quad \beta_{1}=0 \quad \beta_{2}=0.10 \text { (0.37 mast) }
$$

Mocde Combination, (Contudla) 3 first mode-estinatu)

$$
\breve{F}=1.0 P_{r}=\beta_{0}=0
$$

downented Cumpowsin Phasing

$$
\check{F}=1,0 \quad \rho_{2}=0,10 \quad \beta_{1}=0 \quad \text { (estimat) }
$$

SSI (Roch stie)

$$
\ddot{F}=1,0 \quad \beta_{r}=0
$$

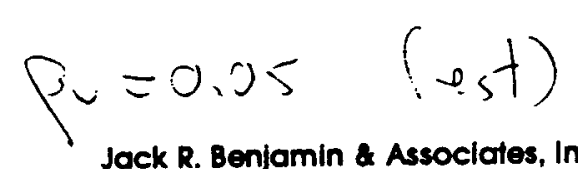

Jack R. Benjamin \& Asso

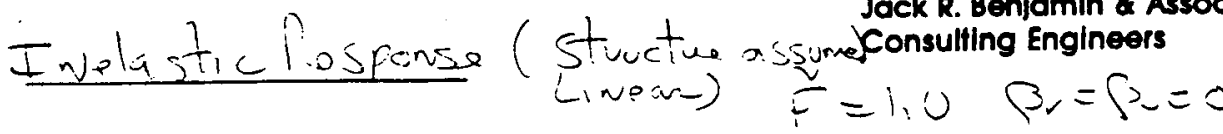

$$
\text { C-105 }
$$




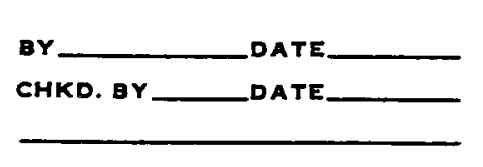

Pavameter

Capacty

Strongsin

Equipment Resconse

$\stackrel{v}{=}$

Pr

PAGE 34 OF

SUBJECT

JOB NO.

Invelastic Response

Spectival Shape

Dximpins

Modeliw - Fvequency

- Mode Shape

1.0

2.27

1,0

1,0

1,0

1.0

1.0

Mo de Combination

Horiz Compenent finsing 1,0

Structive Resporee

Ground Motion

Camping

Modeling-Freguenc:

- rodosinap

ibode Combiation.

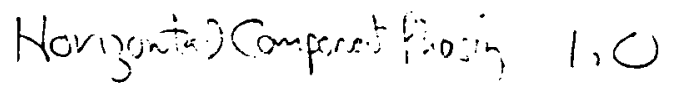

SSI

Inelasic Repase 1.0
0.98

1,0

1.0

1,0

1,0

1,0 fu

0.15

0,25

005

0,03

0.30

0

0

0

0

0.11

0

0,10

0

0

0.05

0.05

Combined

2.22

$v_{p g a}=(2.22)(0.45)$

$=1.00 \mathrm{~g}$

$\tilde{a}_{P g a}\left(P_{e a r} 84 \%\right)=1.0 \times 1.4=1.4 \mathrm{~g}$

HCLPF $=(1.00) e^{-1.65(.27+.45)}=0.30 .9$

Dondin

Jack R. Benjamin \& Associates, inc. Consulting Engineers

$H C L \bar{P}_{\text {Pan }} 8 \%=1.4(0.30)=0.43$ g

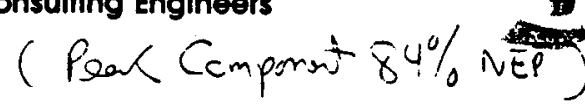

C-106 
BY

CHKD. BY

DATE
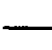

PROJECT

SUEJECT
PAGE 35 OF

JOE NO.

$\frac{\text { Simple NCl D Diveti1 }}{(\text { See Afperdex C) }} \sum x . p=1.55(.27+.45)=1.19$

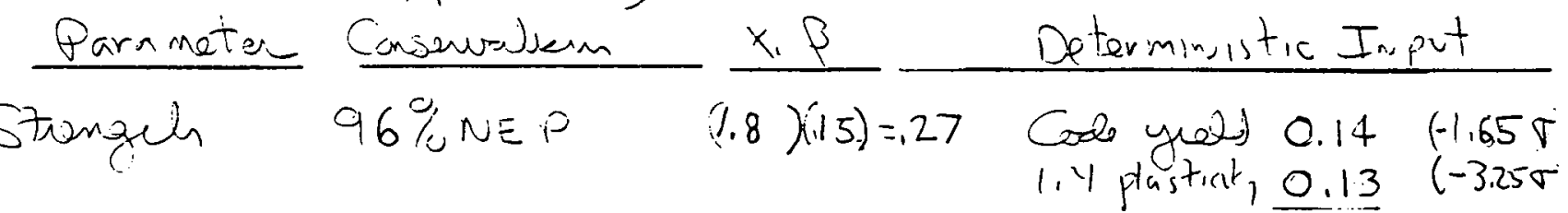

1.4 ptustiat, $\frac{0.13}{0.27}(-3.25 \sigma$

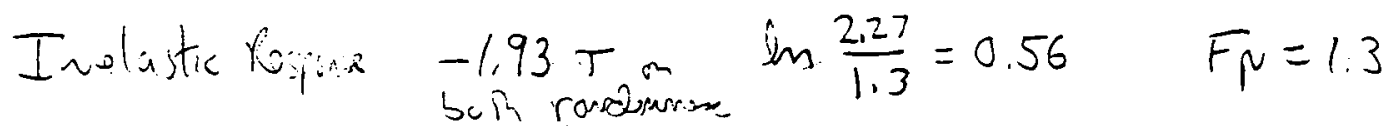
\& unceldarnsts

$$
(\gamma=156 / .29=1.93)
$$

Efup Frafurey $-0.5 \mathrm{~J}$

016

$(\gamma=.16 / .30=.5)$

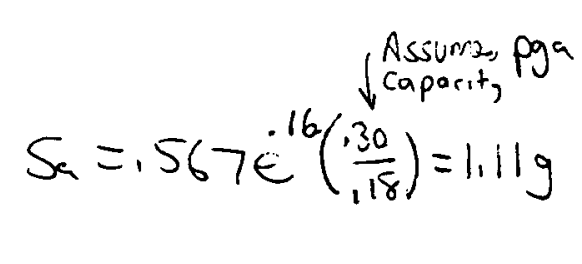

Groun Mstim +1.0 $0.20 \quad e^{20}=1.22$

$$
\Sigma=1,19
$$

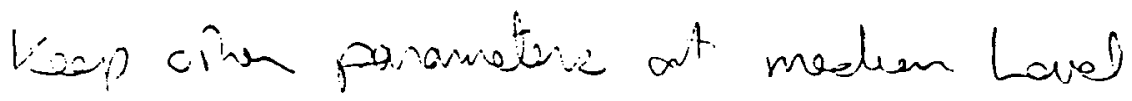

Jack R. Benjamin a Associates, Ine. Consulting Engineers

C-107 


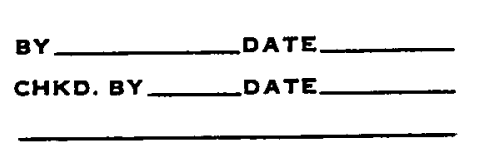

PROJECT

SUBJECT
PAGE 36 OF

JOB NO.

Develor in put to tons modol assumim 0.30 HCL =

at $0.5 T$ fingumy $S_{a}=0.567 e^{+.16}\left(\frac{.30}{.18}\right)=1.11 \mathrm{~g}$

increse fer t1.0Tapoud spetisn

$$
S_{a}=(1.22)(1.11)=1.35 \mathrm{~g}
$$

phonent at base of tink, $M$

$$
M=\frac{1.35}{1.41} \times \frac{53^{k-11}}{1.3}=\frac{39.1^{k-11}}{L_{\text {ductility fnicter }}^{k}}
$$

Vaticel jarce in towk, $v$

Epa. pr vont $: \frac{.30}{.18} \times .20 \times 1.22=0.41 \mathrm{~g}$

$$
V=.926[1-0.40(.41)]=.770^{k}
$$

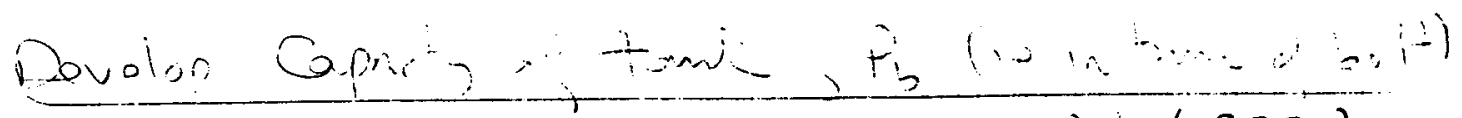

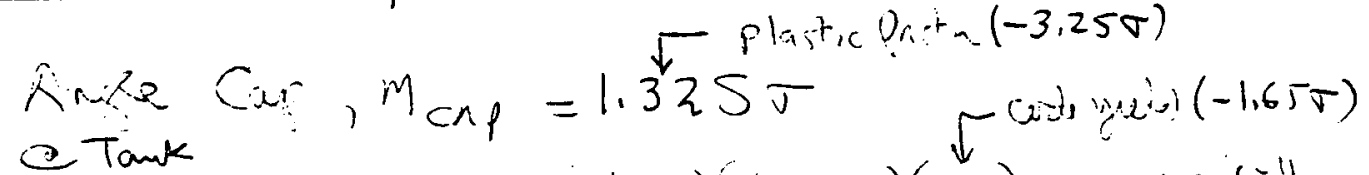

$$
=(1,32)(1,3125)\left(\frac{\pi}{36}\right)=1.49(11
$$

Ang cen at boit Mcnp $=1.49 \times \frac{3-3 / 4}{3}=1.12^{k-11}$

$$
T=(1.49+1.12) / 1.75=1.31^{k}
$$

Jack R. Benjamin \& Associotes, Inc. Consulfing Engineers

C-108 


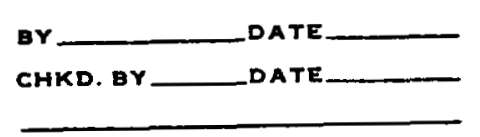

Analyeir fon Tour

Madian level HCLPF pgen $\cong \frac{1.31}{1.35} \times .30=0.29$ s

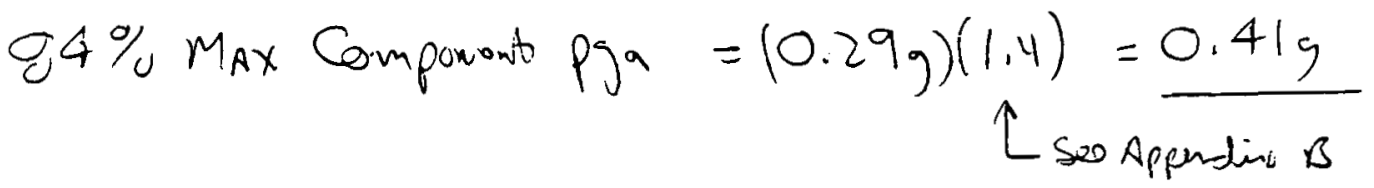

$$
\begin{aligned}
& M=39.1^{k-11} \\
& V=-.770^{k}
\end{aligned} \Rightarrow P_{b_{m A x}}=1.35^{k}>1,31
$$




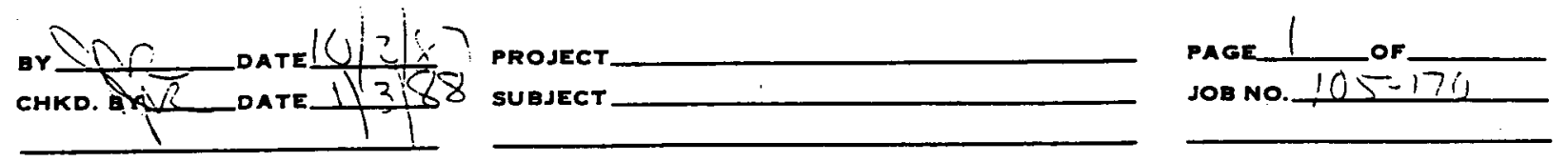

BDBSE WALL

Assimptian (spoeial)

1. No-zuiprat attiaced to wall. Mnx displocencit at top allowed $=6$ "

2 Bending at bare is failue moreb

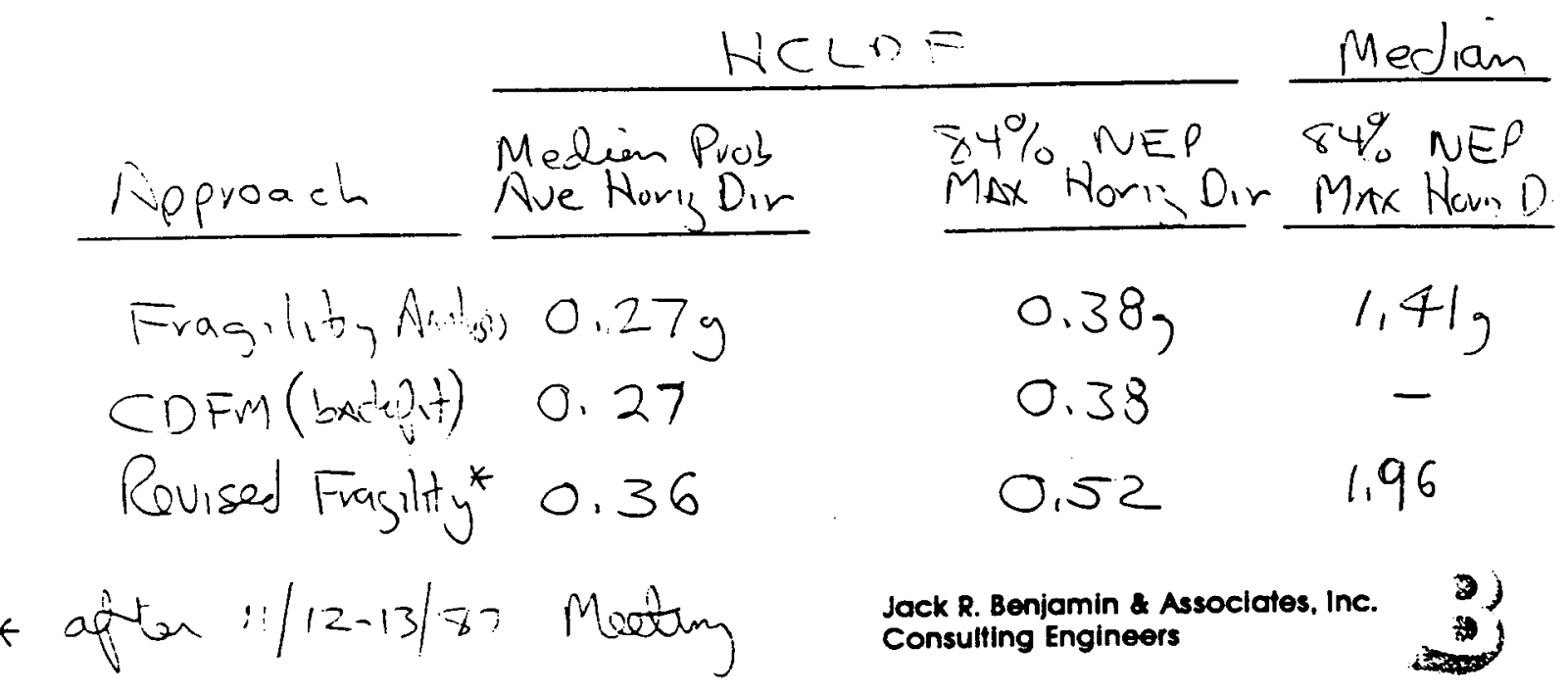

C-110 
BY.

PROJECT

PAGE ? OF

Calculate Stronsis it wail i

Median Capet? ('Vending at base is failure mode)

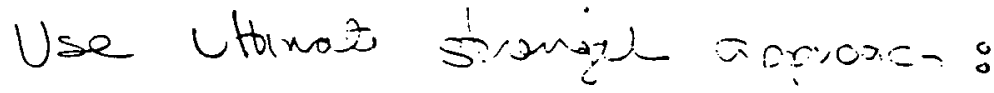

$$
\begin{aligned}
& f_{s}=(1.1)(60,000)=66,000,5 s: \\
& F_{c}^{\prime}=.85 S_{m}^{\prime}=.85(1700 p s i)=1450 \text { si Mypios }
\end{aligned}
$$

axing
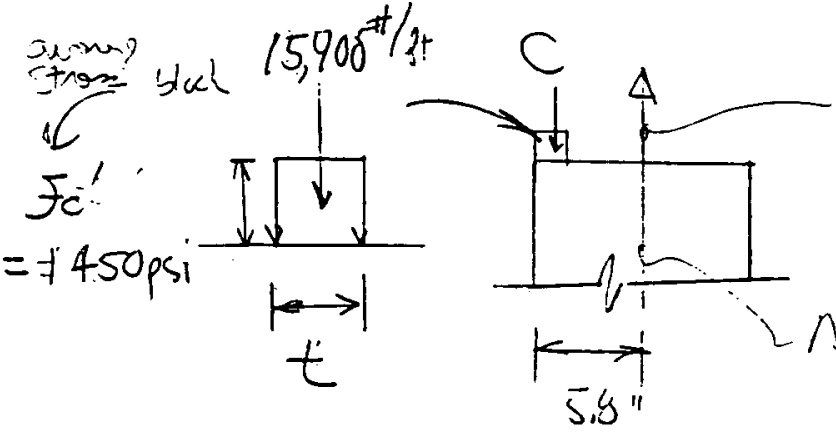

$$
\begin{aligned}
& T / 12^{\prime \prime}=\cdot 233 \times 66,000=15,400^{\circ} \\
& \text { C As/g; (\#ie16") }
\end{aligned}
$$

$$
\begin{aligned}
& t=\frac{15,400}{(12)(1430)}=0.90^{\prime \prime} \mathrm{C} 11^{\prime \prime 2} \text { heres wishing actsich shell of block } \\
& M_{C A}=15.4^{k}(5.8-0.90 / 2)=82.4^{k-11} / \rho_{t}
\end{aligned}
$$

Find Sa allowable

$$
\begin{aligned}
& \text { allowable } \\
& \rightarrow F_{a b l}=\frac{82.4}{60}=1,37^{2} \quad \breve{S}_{a}=\frac{1.37}{1,11}=1.24 \mathrm{~g}
\end{aligned}
$$

Sisplecent at COg is $\approx \frac{1,5}{2} \frac{S a}{j^{2}}$

Jack R. Benjamin \& Associates, Inc. Consulting Engineers

$\mathrm{C}-111$ 


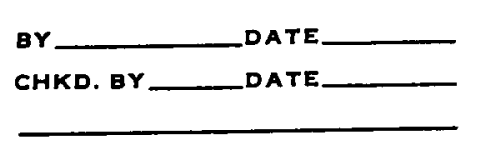

Vaviability

1. Placement of veinforcement dowels at botting of wall (assume $\pm 1^{\prime \prime}$ is $\pm 2 \sigma$ - speeal inspection)

En I" olliset!

$$
\begin{aligned}
& S_{a}=\frac{5.8-1-0.9012}{5.8-0.9012} \times 1.24=1.019 \\
& \text { ratiog } \quad \rho_{v}=\frac{1}{2} \ln \frac{1.24}{1.01}=0.10
\end{aligned}
$$

2. Fur vitimate compressive strengh (assme factor of $Y_{2}$ is $-2 \sigma$, speeial inspection)

$$
\begin{aligned}
& t=(0.90) \times 2=1.80 \text { (into groute) area revise } \\
& \text { t) } \\
& t=\frac{(1.80) \cdot(16)-(1.5)(16)}{8}+1.5=2.10^{4} \\
& S_{a}=\frac{5.8-2.10 / 2}{5.8-0.90 / 2} \times 1.24=1.109 \\
& \rho_{u}=\frac{1}{2} \ln \frac{1.24}{1.10}=0,06
\end{aligned}
$$

三Fr veinpurcament yeeb) stress (assue frotor of 1.0

$$
\text { is }-1 \sigma)
$$

$$
\beta u=\ln \frac{1,1}{1,0}=\underline{0,10}
$$

Combined Pu

$$
\begin{aligned}
P_{v} & =\left(\overline{0.10}^{2}+0 . \overline{06}^{2}+0 . \overline{10}^{2}\right)^{1 / 2} \begin{array}{c}
\text { Jack R. Benjamin \& Associates, inc. } \\
\text { Consuling Engineers }
\end{array} \\
& =0,15
\end{aligned}
$$

C-112 
BY

CHKD. EY
PROJECT

SUBJECT

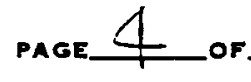

JOB NO.

Ductilit

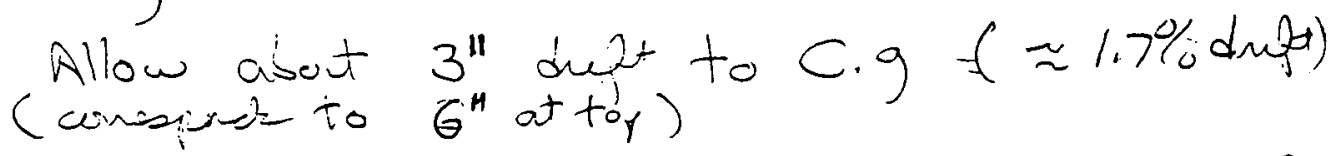

yold:

$$
\begin{aligned}
& \Delta_{c \cdot g}=1.5 / 2 \frac{S_{a}}{\omega^{2}}=\frac{0.75}{(2 \pi)^{2}} \frac{S_{a}}{f^{2}}=\frac{0.75,(386,4)}{(2 \pi)^{2}} \frac{S_{a / 9}^{9}}{g^{2}} \\
& =\frac{7.34}{F^{2}}(S y / 5)=\frac{2.34}{s^{2}}(1.24)=\frac{9.10}{F^{2}} \\
& \text { aqpura yida) } \mathrm{Se} \\
& \text { madien fry } \cong \sqrt{(3)(12)}=6 h_{3} \text { (use } \beta_{f}=0.25 \text { seps } 11 \text { ) } \\
& S_{a}(\text { yeds) }) \approx 1.24 \text {, }
\end{aligned}
$$

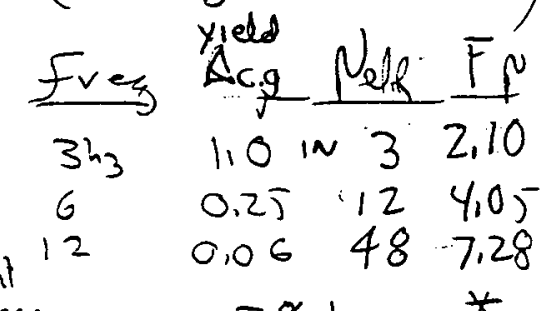

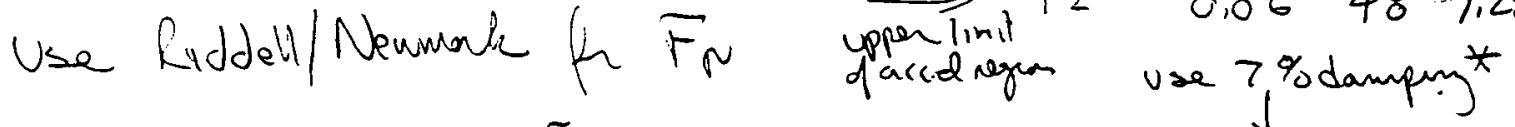

$$
\begin{aligned}
& F_{N}=\left[(q+1) N_{d_{f i}}-\delta\right]^{r} \text { (accelestm regen) } \quad q=3.0(7)^{-.3}=1.67
\end{aligned}
$$

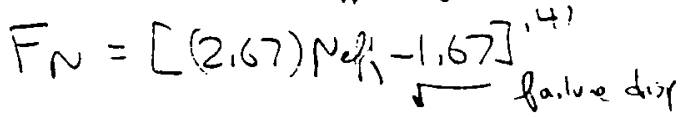

$$
\begin{aligned}
& r=0,48(7)^{-0.08}=.41 \\
& N_{2+3}=3 /\left(\frac{9.10}{f^{2}}\right)=0.330 f^{2}
\end{aligned}
$$

by simulatin. Fr (see pages 5-10 $\%$ DUCTR.SIM)

$$
\stackrel{v}{F}=3.92 \beta_{v}=0.24
$$

Vavasiling

$$
\begin{aligned}
& \rho_{v}=0.24 \\
& \rho_{r}=0.11\left[F_{p}-\frac{1}{2}\right]=0.37 \text { (Fits R.ddoll/Nenmonk dn! }
\end{aligned}
$$

* 10\% is better for pois farlure mode but $7 \%$ is max spéet rum Jack R. Bonjomin \& Assoclates, inc. avalaber.

C-113 
MLADF TLESSULL'IR.SIM

This program calculates the distribution on ductility factor for variability in firequency ot equipment

NOFMAL DISTFIIEUTION

$$
\begin{aligned}
& \mathrm{bI}_{1} \equiv .319381530 \\
& \text { เอ } \equiv-.356563782 \\
& \mathrm{~b} 3 \equiv 1.731477937 \\
& \text { b4 } \equiv-1.821255978 \\
& \text { b5 } \equiv 1.330274429 \\
& p \equiv .2316419 \\
& Z(x)=\frac{\exp \left[\begin{array}{c}
2 \\
-x \\
2
\end{array}\right]}{2.506628275} \\
& t(x)=\frac{1}{1+p \cdot|x|}
\end{aligned}
$$

$\operatorname{Nof}(x) \equiv \mid(x y 0)-2(x) \cdot\left[b 1 \cdot t(x)+b 2 \cdot t(x)^{2}+b 3 \cdot t(x)^{3}+b 4 \cdot t(x)^{4}+b\left(5 \cdot t(x)^{5}\right] \mid\right.$

$$
\begin{aligned}
& c 0 \equiv 2.515517 \\
& =1 \equiv .002453 \\
& c 2 \equiv .010328 \\
& d 1 \equiv 1.43278 d \\
& d 2 \equiv .189269 \\
& d 3 \equiv .001304
\end{aligned}
$$

$$
t(p) \equiv \sqrt{\ln \left[\frac{1}{((p) .5)-p)^{2}}\right]}
$$




\section{6}

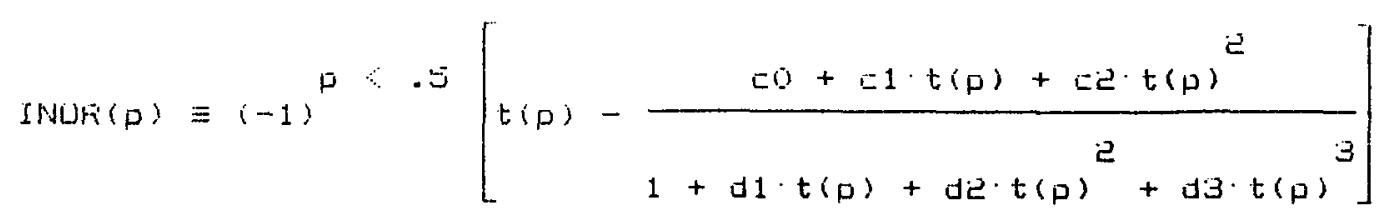

$n:=199$

$i:=0 \ldots n$

$\operatorname{dir}:=6 \cdot \exp \left[0.25 \cdot\right.$ INUR $\left.\left[\frac{i+r n d(1)}{n+1}\right]\right]$

Latin hypercube simulation of equipment Trequency

$\min (d i r)=2.014$

$\max (d i r)=11.58 E$

$n:=.330 \cdot d i r_{i}^{2}$

$n_{i}:=n_{i}\left[\begin{array}{lll}n_{i} & > & 1\end{array}\right]+1 \cdot\left[\begin{array}{lll}n & < & 1 \\ i & \end{array}\right]$

Calculate ductility from frequency

$\operatorname{dir}:=[2.67 \cdot n-1.67]^{.41}$

CaIculate ductility factar

$\min n(n)=1.338$

$\operatorname{mia} x(n)=45.033$

$\operatorname{nin}(d i r)=1.302$

$\max ($ dir $)=7.085$ 


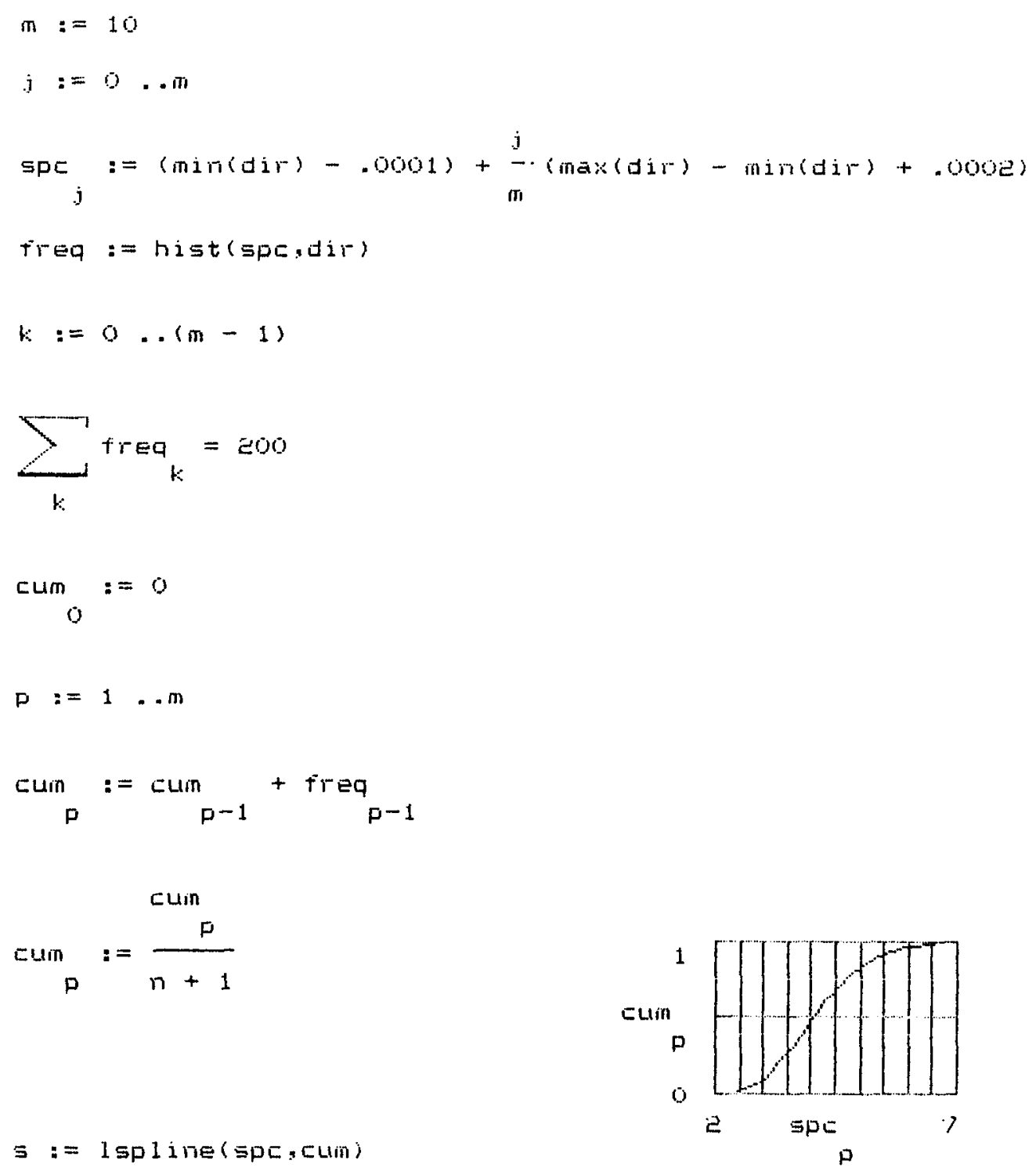

Find the median value:

$x:=4.0$

root(interp $(5$, spc, cum, $x)-.5, x)=4.034$

Find the + 10 value:

root(interp $(5, \operatorname{spc}$, cuin, $x)-.24134, x)=5$

\begin{tabular}{|c|c|c|}
\hline & CLIm & $s p c$ \\
\hline$j$ & $j$ & \\
\hline 0 & 9 & 1.302 \\
\hline 1 & 0.005 & 1.88 \\
\hline 2 & 0.015 & 2.458 \\
\hline 3 & 0.1 & 3.037 \\
\hline 4 & 0.31 & 3.615 \\
\hline 5 & 0.57 & 4.193 \\
\hline 6 & 0.78 & 4.772 \\
\hline 7 & 0.91 & 5.35 \\
\hline 8 & 0.965 & 5.928 \\
\hline$y$ & 0.77 & 6.507 \\
\hline 10 & 1 & 7.065 \\
\hline
\end{tabular}




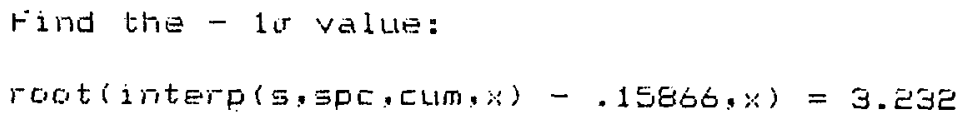

\begin{tabular}{|c|c|c|}
\hline & cum & SPC \\
\hline$j$ & $j$ & \\
\hline 0 & 0.005 & 1.38 \\
\hline 1 & 0.015 & 2.458 \\
\hline 로 & 0.1 & 3.037 \\
\hline 3 & 0.31 & 3.615 \\
\hline 4 & $0.5 ?$ & 4.173 \\
\hline 5 & 0.78 & 4.772 \\
\hline 6 & 0.91 & 5.35 \\
\hline 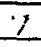 & 0.965 & 3.720 \\
\hline 8 & 0.79 & 6.507 \\
\hline
\end{tabular}

This program take the calculated probabilities ot tailure and the associated peak qround acceleration values and perforins a least squares fit in the Ica-probability domain to obtain the "best tit" median and fo value.

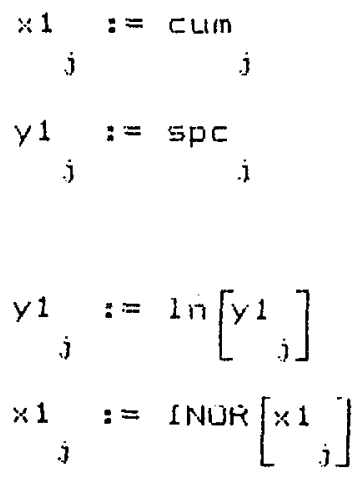




$$
\begin{aligned}
& \exists \text { lope }(x 1, y 1)=0.237 \\
& \beta:=\operatorname{sicpe}(x 1, y 1) \\
& \text { intercept }(x 1, y 1)=1.364 \\
& \operatorname{catr}\left(x 1, y_{1}\right)=0.791 \\
& a_{\text {med }}:=\exp (\text { intercept }(x 1, y 1))
\end{aligned}
$$

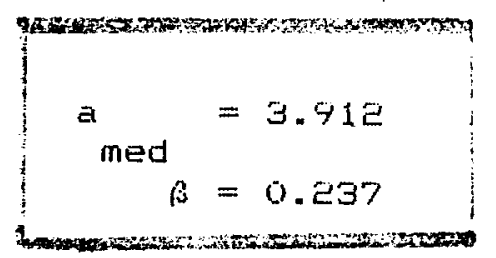

$21_{j}:=$ intercept $(x 1, y 1)+\operatorname{slope}(x 1, y 1) \cdot \times 1$

$\max \left(y_{1}\right)=1.873$

$$
\begin{aligned}
& \min (y 1)=0.631 \\
& \min (z 1)=0.752 \\
& \max (\times 1)=2.327 \\
& \min (\times 1)=-2.576
\end{aligned}
$$

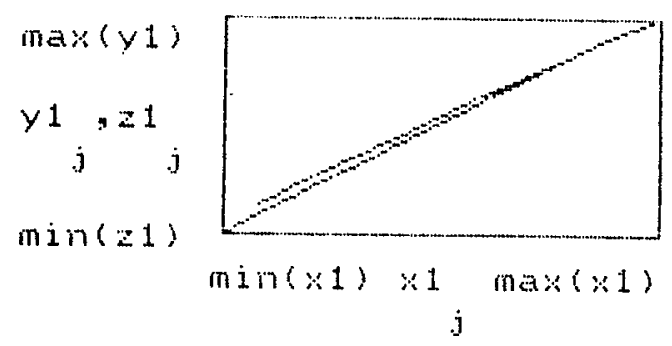
\begin{tabular}{|l|}
\hline 0 \\
\hline 1 \\
\hline 2 \\
\hline 3 \\
\hline 4 \\
\hline 5 \\
\hline 6 \\
\hline 7 \\
\hline 0 \\
\hline
\end{tabular}
$\because 1$

$\mid$\begin{tabular}{c|}
\hline-2.576 \\
\hline-2.171 \\
\hline-1.292 \\
\hline-0.475 \\
\hline 0.176 \\
\hline 0.772 \\
\hline 1.341 \\
\hline 1.812 \\
\hline 2.327 \\
\hline
\end{tabular}
$y 1$
21

\begin{tabular}{|c|}
\hline 0.631 \\
\hline 0.4 \\
\hline 1.111 \\
\hline 1.285 \\
\hline 1.434 \\
\hline 1.563 \\
\hline 1.677 \\
\hline 1.79 \\
\hline 1.873 \\
\hline
\end{tabular}

\begin{tabular}{|c|}
\hline 0.790 \\
\hline 0.649 \\
\hline 1.06 \\
\hline 1.946 \\
\hline 1.496 \\
\hline 1.547 \\
\hline 1.696 \\
\hline 1.794 \\
\hline 1.916 \\
\hline
\end{tabular}

Tabulate probabilities to compare calculated and best fit values:
a $\quad:=\exp \left[\begin{array}{ll}y & 1 \\ & j\end{array}\right]$

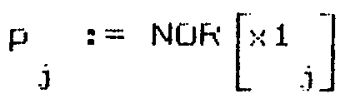

Feat: ground accelerations

Frobability of failure values 


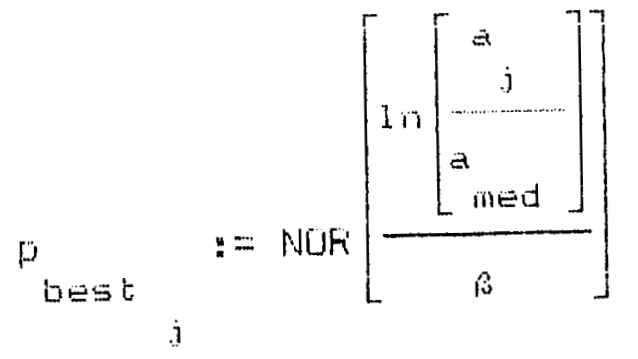

Best fit probability of failure values

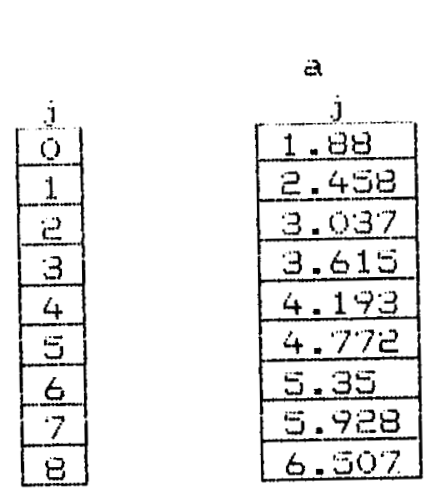

\begin{tabular}{c}
$P$ \\
$j$ \\
\hline 0.009 \\
\hline 0.015 \\
\hline 0.1 \\
\hline 0.31 \\
\hline 0.57 \\
\hline 0.78 \\
\hline 0.91 \\
\hline 0.965 \\
\hline 0.99 \\
\hline
\end{tabular}

$P$

best

0.001

0.025

0.143

0.37

0.615

0.799

0.706

0.96

0.984 
BY

CHKD. BY_DATE
PROJECT

SUBJECT
PAGE\I_OF

JOB NO.

Find PeakGround Acceleratim Capactin?

freguency of wall assumed to ronge 3 to $12 h_{3}$ Cassume This is $\pm 3 \sigma$ since wide range

$$
\beta_{v}=\frac{1}{6} \ln \frac{12}{3}=0,23 \quad \text { Use } 0,25
$$

frepof buldis is $7.2 h_{3}$-assume $P_{v}=0.25$ also combined frey varutum is $\sqrt{2}(.25)=0.35$ Simulate randonly M'frepuency vaunter to obtan medem $S a$ fr $7 \%$ damping (See SPECT.SIm on ment 3 pagen)

$$
\begin{aligned}
& \breve{S}_{a}=.855 \mathrm{~g} \leftarrow \text { consespud to } 0.18 \text {, } \\
& \rho_{u}=0,30
\end{aligned}
$$

(Nute if har) uses) Sa walue at medion frey of 6 h? Sa woub be $11 l_{5}$-anor foetr of 1,29 )

$$
p g a=\frac{\sqrt{2} \sec _{p g^{2}}}{0.855 \mathrm{~g}} \times 0.18=\underline{0.26 \mathrm{~g}}
$$

$c-120$

Jack R. Benjamin \& Associates, Inc. Consulting Engineers 
MLAD FILES SFECT.SIM

NOFIMAL DISTFIIEUTION

b1 $\equiv .317381530$

ט2 $\equiv-.356563782$

b3 $\equiv 1.781477937$

b4 $\equiv-1.821255978$

$b 5 \equiv 1.330274427$

$p \equiv .2316419$

$z(x)=\frac{\exp \left[\frac{5}{5}\right]}{5.506623275}$

$t(x) \equiv \frac{1}{1+p \cdot|x|}$

$\operatorname{NOF}(x) \equiv\left|(x y 0)-Z(x) \cdot\left[b 1 \cdot t(x)+b 2 \cdot t(x)^{2}+b 3 \cdot t(x)^{3}+b 4 \cdot t(x)^{4}+b 5 \cdot t(x)^{5}\right]\right|$

INVEFSE NOFMAL DISTFIIBUTION

$c 0 \equiv 2.515517$

$c 1 \equiv .802853$

$c \mathrm{ce} \equiv .010328$

$d 1 \equiv 1.432788$

$d 2 \equiv .189269$

$d 3 \equiv .001308$

$t(p) \equiv \sqrt{\ln \left[\frac{1}{((p) .5)-p)^{2}}\right]}$

$\operatorname{INOR}(p) \equiv(-1)^{p} .5\left[t(p)-\frac{c 0+c 1 \cdot t(p)+c 5 \cdot t(p)^{2}}{1+d 1 \cdot t(p)+d a+t(p)^{2}+d 3 \cdot t(p)^{3}}\right]$ 


$$
\begin{aligned}
& \underset{\text { Fret }}{\text { hiet }}:=6 \\
& \beta:=0.35 \\
& \text { i }:=199 \\
& i:=0 \ldots n \\
& \text { Freq }_{i}:=\text { Freq }_{\text {hat }} \exp \left[\beta \cdot \operatorname{INOR}\left[\frac{i+r n d(1)}{n+1}\right]\right] \\
& \operatorname{LFreq}_{i}:=\ln \left[\begin{array}{r}
\text { Freq }_{i} \\
\end{array}\right]
\end{aligned}
$$

$$
\begin{aligned}
& m:=45 \\
& j:=0 \ldots m \\
& x_{j}:=F E A D(X L F F) \\
& X 5 A 7:=F E A D(X 5 A 7)
\end{aligned}
$$$$
57:=\text { CEpline (XLFF, XSA7) }
$$$$
1:=1 . .600
$$

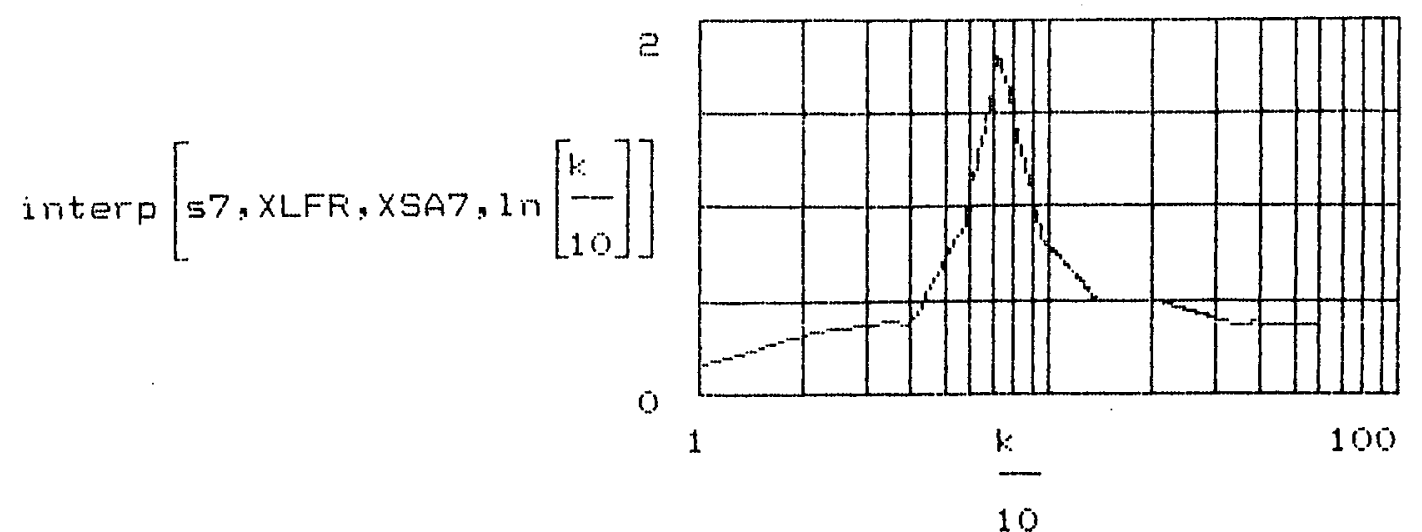

$$
\begin{aligned}
& S_{i}:=\operatorname{interp}[57, \times L F F, \times S A 7, \text { LFreq } \\
& L_{i}:=\ln \left[S A_{i}\right]
\end{aligned}
$$


Median : = exp (mean(LSA))

Eeta:= stdev(LSA)

$$
\begin{aligned}
\text { Median } & =0.855 \\
\text { Beta } & =0.298
\end{aligned}
$$

Fmedian : = Exp(mean(LFreq))

Fbeta: = stdev(LFreq)

$$
\begin{aligned}
\text { Fmedian } & =5.799 \\
\text { Fbeta } & =0.358
\end{aligned}
$$




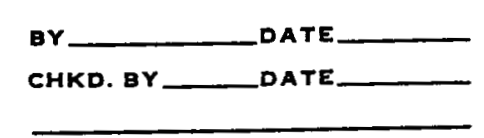

PROJECT

PAGE 15 OF

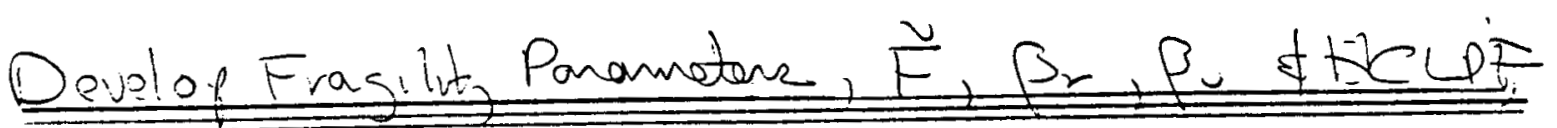

Capacity,

Strongish

$$
\begin{aligned}
& \tilde{F}=0.26 / 0.26=1.0 \quad \text { (see pagell) } \\
& \beta_{r}=0 \\
& \beta_{u}=0.15 \quad \text { (See pase 3) }
\end{aligned}
$$

Inelastic Response: (see pang 4)

$$
\begin{aligned}
& F^{2}=3.9 \\
& \beta_{r}=0.22 \\
& \beta_{u}=0.24
\end{aligned}
$$

Equipment Response

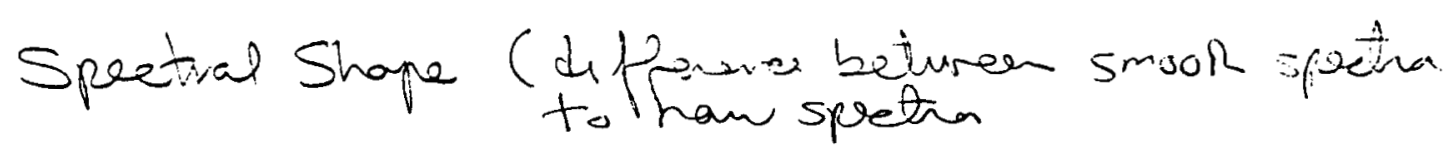

$$
\begin{aligned}
& \breve{F}=1,0 \\
& \beta_{r}=0 \\
& \rho_{u}=0.05 \quad \text { (estimate) }
\end{aligned}
$$

Jack R. Benjamin \& Associates, Inc. Consulting Engineers

$C-124$ 
Dampins

$$
\begin{aligned}
& \text { Medion dompin }=7 \% \text { (usa) ins calculation- } \\
& \text { Note: } 10 \% \text { woild have } \\
& \tilde{F}=1,0 \\
& \text { ben betton) } \\
& -1 \sigma \text { dempy }=5 \% \\
& 7 \% \breve{S}_{a}=0.855 \mathrm{~g} \quad(\text { See page } 11) \\
& 5 \% S_{a}^{2}=0,984 \text {, (ruwon SPECT, SIM) } \\
& \beta_{u}=\frac{1}{1} \ln \frac{.984}{.855}=0.14 \\
& \beta_{r}=0
\end{aligned}
$$

Note: If used spoded oneter at medem

$$
\begin{aligned}
& \text { fres }=6 h_{3}: \beta=\ln \frac{1,42}{1,1}=0,26 \\
& \text { (Bs defference!) }
\end{aligned}
$$

Modelin

frefung: combined boin exup $B=\&$ structurs BF in strangt cake. ( see poupll)

$$
\begin{aligned}
& \dot{F}=1,0 \\
& \rho_{r}=0 \\
& \rho_{v}=0.30 \quad \text { (see pagell) } \\
& \text { Jack R. Benjiamin \& Asso }
\end{aligned}
$$

Jack R. Benjamin \& Associotes, Inc. Consulting Engineers

$C-125$ 


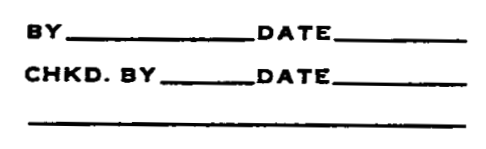

Mode shorpe: Simple eguipmat wir liweer mode shape - ist mode controls sine fallure mode is OTM at base (all higin moder contribute nolhing)

$$
\tilde{F}=1.0 \quad \beta_{r}=\beta_{u}=0
$$

Mode Combination (see mode shape discissim a'oue)

$$
F=1.0 \quad \beta_{v}=\beta_{v}=0
$$

Norizontal Compowent Phasiw)

Controlled by I divection mly. No contuistion from ofine divection

$$
\stackrel{v}{F}=1,0 \quad \beta_{v}=\beta=0
$$

Structure Response

Ground Motim this parameton includes:

1) Peal-to-Poak Spetim Uoreation

2) Nurizontal Divectim Variabilits

3) Egurpmat Norizuntal Compones Coupling Jack R. Benjamin \& Assoclates, Inc.
Consulting Engineers

$C-126$ 


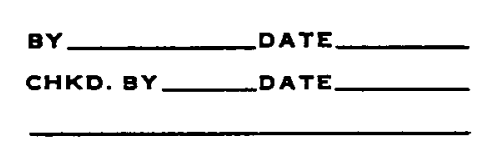

PROJECT

PAGE $\mid \&$ OF

SUBJECT

JOB NO

See Appadix A

$C=\operatorname{ratio} \&$ miwor horigutal divectim responce to combruse ivepine (ie ratio of smediona)

$c=0$ (only one divectin conilutly)

From DIR.FAT: $\tilde{F}=1.0$ pr $=0.24$

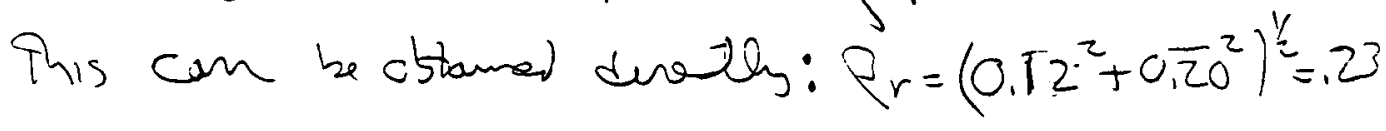

use $p_{r}=0.24$

$\tau$ Apperdiy $B$

Dampin : Medion: $7 \%$

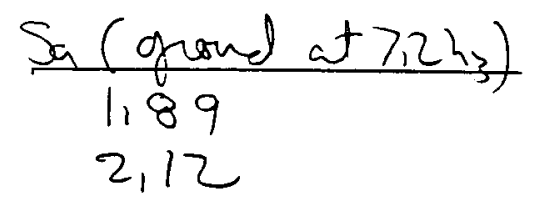

$$
\begin{aligned}
& -1 \sigma \quad 5 \% \quad 2,12 \\
& \beta_{u}=\frac{1}{1} \ln \frac{2,12}{1.89}=0,11 \\
& \beta_{v}=0 \\
& \check{F}=1,0 \quad \text { (used } 7 \% \text { m stangul (ake) }
\end{aligned}
$$

incleling:

bresung - sive buldiffrequery is in regin of ground spoctum plateav 1. Hie chong will occu (alw see Epupp.Moddan)

$$
\begin{aligned}
& \tilde{F}=1.0 \\
& \beta_{v}=\rho_{0}=0
\end{aligned}
$$

C-127 


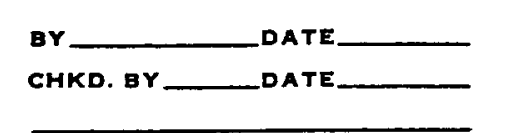

PROJECT

PAGE $1 \overline{9}$ OF

Mode shape - Assune cintrollej= Pirs mode

$$
\tilde{F}=1,0 \beta_{v}=0 \quad \beta_{v}=0,10 \text { (estimats }
$$

Mode Combination (Controllos by givst mosh-estinato)

$$
F=1,0 \quad \beta_{v}=\beta_{v}=0
$$

Dovizontal Compowent thasing

$$
\ddot{F}=1.0 \quad \beta_{v}=0.10 \quad \beta_{v}=0 \text { (estinat) }
$$

SST (Roch site)

$$
\tilde{F}=1.0 \quad \beta_{r}=0 \quad \beta_{2}=0.05 \text { (estinet) }
$$

Inelastic Reoponse (struetur assumas) to be linen)

$$
F^{\nu}=1.0 \quad \beta_{\nu}=\beta=0
$$

Jack R. Benjamin \& Associates, Inc. Consulting Engineers

C-128 


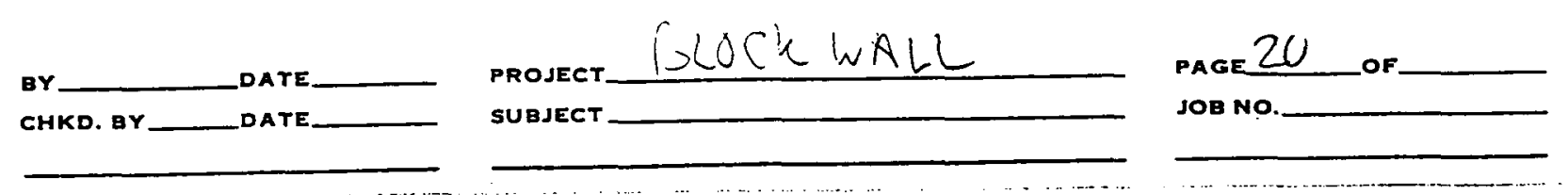

Parameter $\stackrel{\breve{E}}{\beta_{r}} \beta_{u}$

Capacity

Strength

Inelastic Response 3.9

$\begin{array}{ll}0 & 0.15 \\ 0.22 & 0.24\end{array}$

Equipment Response

Spectral Shape

0

Damping

0

Medeling-Fuguency -... It 0

- Mode Shape T io

0 .

O.

Mode Combination

o

Horns. Component Phasing T, $U$

0

0,05

0.14

0.30

0

$-0$

Structure Response

Grand Motion

Damping).

1,0

Modeling - Frequency

Mode Stare

Mode Combination

0.24

1,0

Mo do Contrition 1,0

C

0

Noria Component lasing

$\begin{array}{llll}\text { SST } & 1,0 & 0 & 0.05 \\ \text { Inelastic Response } & \frac{10}{3.9} & \frac{0}{0.34} & \frac{0}{0.47}\end{array}$

Combined: $\quad 3.9 \quad 0.34 \quad 0.47$

$$
\begin{aligned}
& \breve{a}_{\text {pga }}=(3.9,)(0.26) \mathrm{g} \quad=1,0 \mathrm{gg} \quad \text { agr }(\text { para } 84 \%)=1,4(1,01)=1,41 \mathrm{~s}, \\
& \text { NCCPF } F_{m}(1.01) e^{-1.65(34+.47)}=0.27
\end{aligned}
$$

C-129 
BY

PROJECT

PAGE 21 OF

JOB NO.

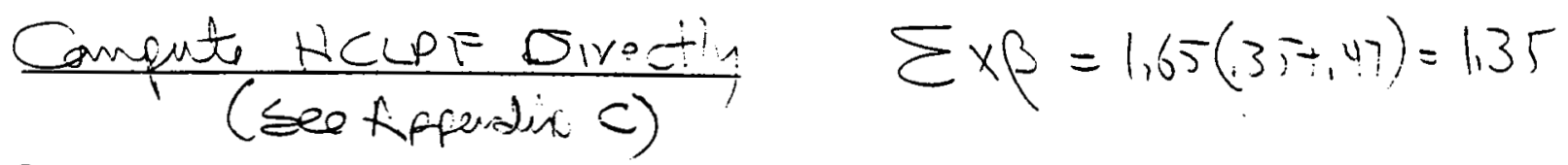

Pavameter Caservatism X.P Deterministic Input.

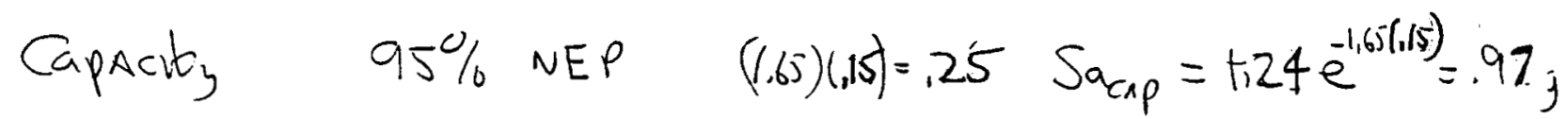

Inelastic leppose $-1.8 \sigma$

$1.8\left[\overline{22^{2}}+, \overline{24} \overline{2}^{2}\right]^{12}$

$0.59 \quad F_{N}=3.9-e^{-159}=2.76$

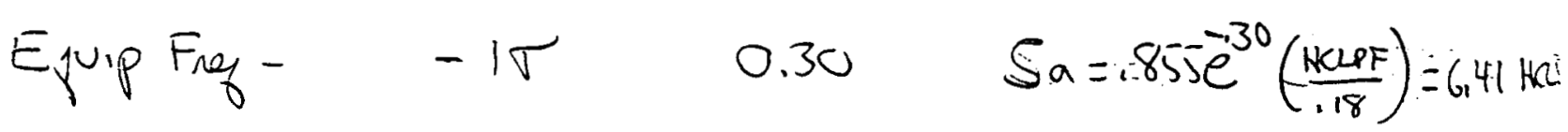

Ground Motin $=+1 \sigma \frac{0.20}{\sum=1.34} e^{120}=1.22$

Keap olien parametere of medion level

input: $(1.22)(641 \mathrm{NCCPF})=7,82 \mathrm{NCLPF}$

capriog $(0.97)(2.16)=2.10 \mathrm{~g}$

at modumlent: $H C L P F=2,10 / 7,82=0279$

at $84 \%$ Max Cimpinat NCLPF $=0.27 \times 1.4=0.39$

$\uparrow$ see Appondix B

Jack R. Benjamin \& Assoclates, Inc. Consulting Engineers

$c-130$ 


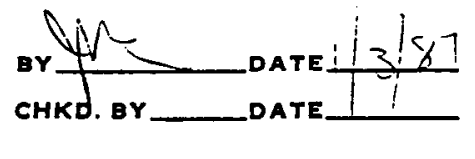

PROJECT

Reursed Anailysis - D20 wi... frolluve (wion

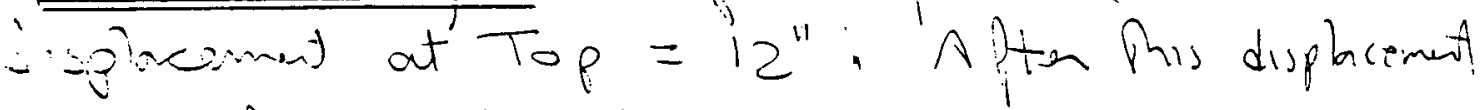
wall linely to collapse)

Revise page 4 , gele

$$
N_{\text {eff }}=\sigma^{n} /\left(\frac{9.10}{f^{2}}\right)
$$

$C$ yeld disploemat it CiG of wall as functin of aquep frey

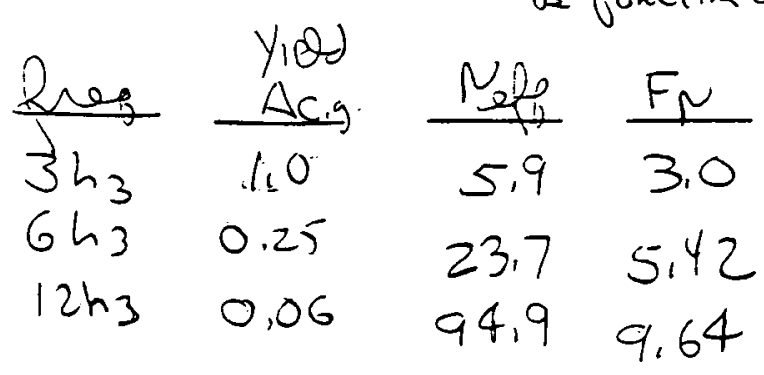

Bu simulatim (usin, DUCTK. sim-similon to page 5.10)

$$
\underline{F}=5.4 \quad \beta_{u=}=0.21 \quad \text { (use } 0.21 \text { is before) }
$$

Devisad Fragition Parametere (see ps 20)

$$
\begin{aligned}
& \breve{a}_{p j a n}=(5.4)(.26)=1.40, \quad \rho_{r}=0.34 \quad p_{l}=0.47 \\
& \text { HCLPF }=(1.40) e^{-1.65(.34+.47)}=0.37 \mathrm{~g} \\
& \text { NCLPFPad } 84 \%=1.4(137)=0.52 \mathrm{~g} \\
& \operatorname{apse}\left(p_{2}(84 \%)=(1.4)(1,40)=1.96 \mathrm{~g}\right.
\end{aligned}
$$

Jack R. Benjamin \& Associates, Inc. Consuliting Englneers

C -131 


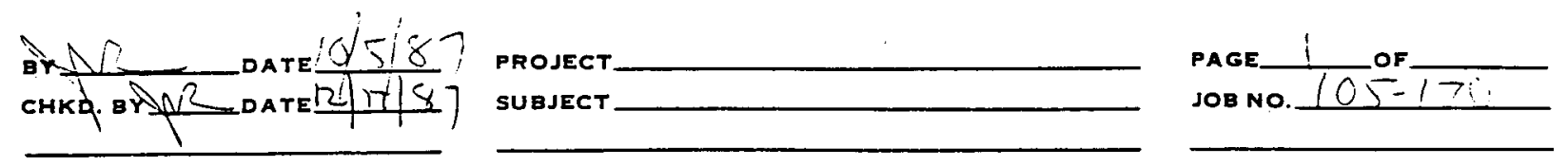

ComponemT coolinG NEAT EXCNANGER

Assumpions (spein)

1. No nuzjke loode considens in anajupic (nat

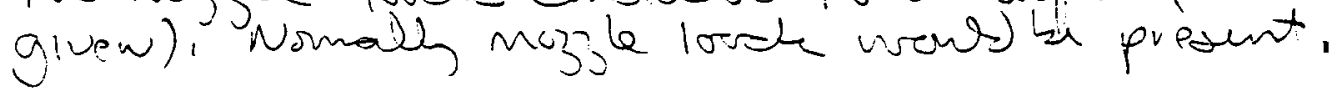

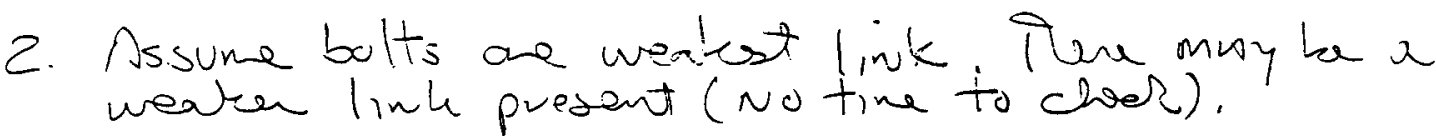
fonding of base plate at bottom al suppint

3. Ignar lexislibe \& suppent hame ( normally Pus eftes wowas be includes)

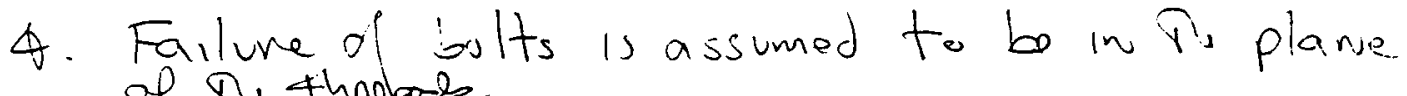
of The thabere

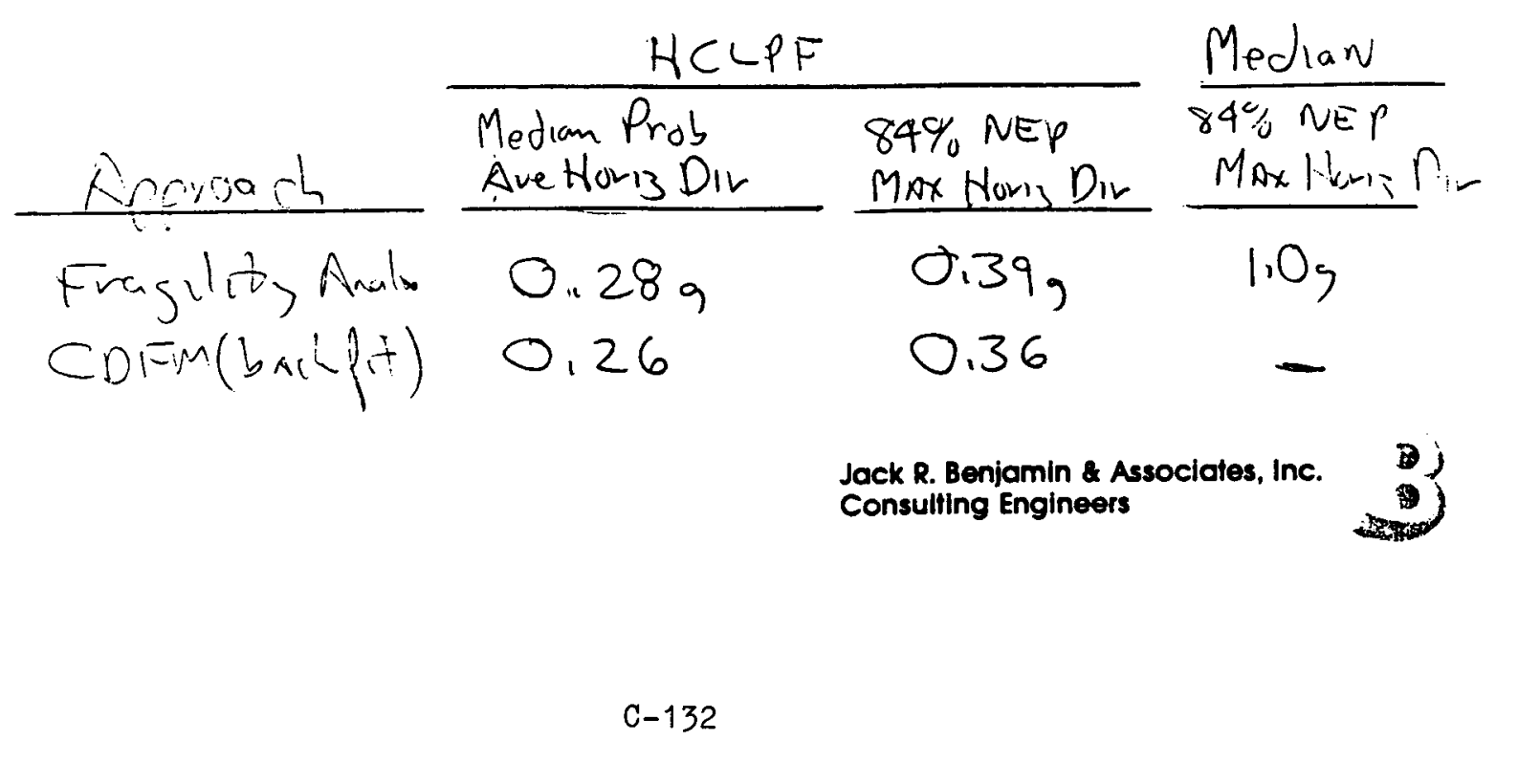




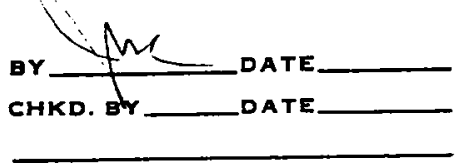

Sinengeh

Fuep in long $d x=33 \mathrm{~h}$ ( It is likeh Much less beeare of steel frame supportNormalin, This would be investiguted).

Frey in travopans direstion - assumed also zual to $33 \mathrm{~h} 3$

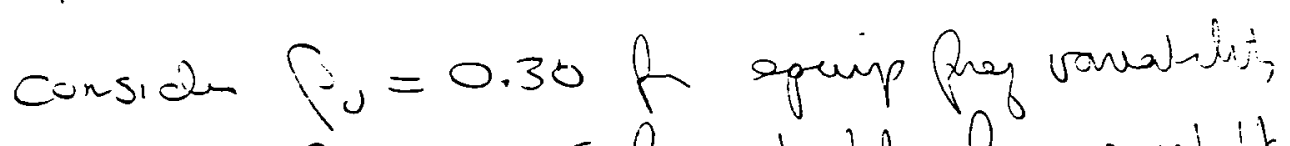

$\rho_{u}=0.25$ for buidenfry uninbilits

cumbined $\beta_{v}=0.39$

Fir 5\% dompin, the melien: $S_{a}=0.42$, (see poup $3-5$ Fon SPET. in $\quad \beta=0.14$ undurpiz)

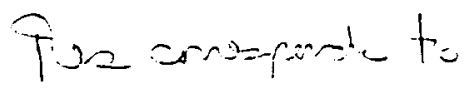

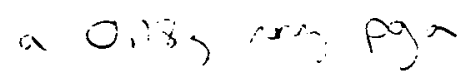

Initialin bstimat bolt caprety wir no tensem in bolts ( Bun cinot assimption)

$$
\begin{aligned}
& 7 g^{4} \text { \& } A-307 \\
& \text { bo } 17 s
\end{aligned}
$$

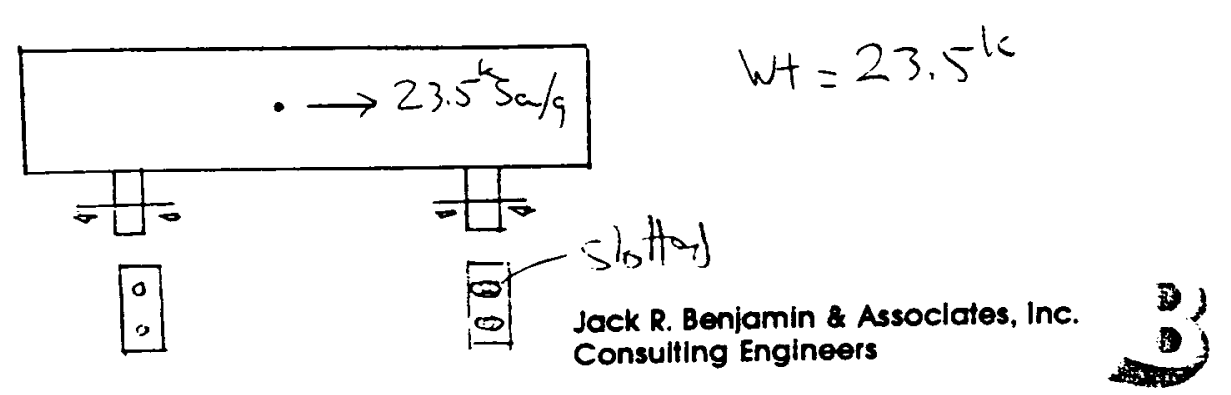

C-133 
MCAD FILES SFECT.SIM

NOFMAL DISTFIBUTION

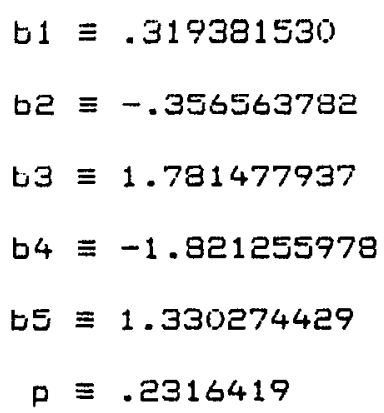




$$
\begin{aligned}
& \text { Freq } \\
& B:=0.39 \\
& i:=199
\end{aligned}
$$

$i:=0 \ldots n$

Freq $i=$ Freq $_{\text {hat }} \exp \left[6 \cdot \operatorname{INOR}\left[\frac{i+\operatorname{rnd}(1)}{n+1}\right]\right]$

LFreq $_{i}:=\ln \left[\begin{array}{rr}F_{r e q} \\ & i\end{array}\right]$

$$
\begin{aligned}
& m:=45 \\
& j:=0 \ldots i n \\
& X L F F i:=\text { FEAD (XLFF') } \\
& \text { XSAS }_{j}:=\text { READ (XSAS) }
\end{aligned}
$$

$55:=$ :spline (XLFF, XSA5)

$k:=1 . .600$

$$
\text { interp }\left[55, \text { XLFF, XSAS, } \ln \left[\frac{1:}{10}\right]\right]
$$

4

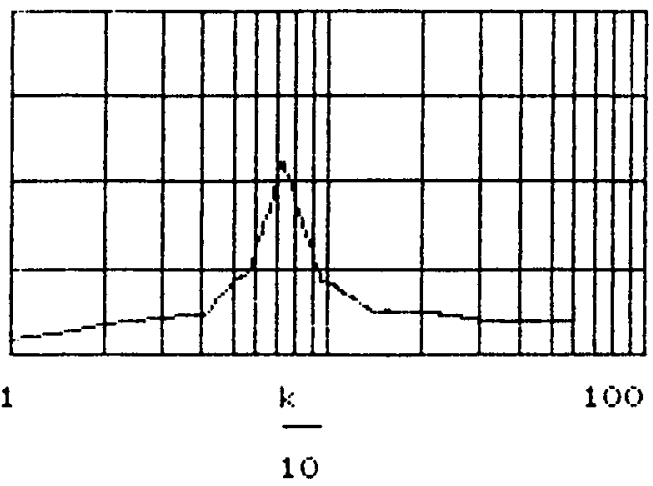

$$
\begin{aligned}
& S A_{i}:=\text { interp }\left[55, X L F R, X S A S, L_{F r E q}\right]
\end{aligned}
$$

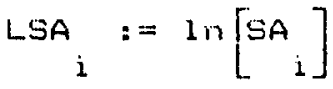


Mediair $:=\operatorname{Exp}(\operatorname{me} a n(L S A))$

Beta : = stdeV(LSA)

Median $=0.416$

Beta $=0.137$
Fmedian $:=$ Exp (mean(LFrEq)

Fbeta := stdev(LFreq)

Fmedian $=32.889$

Fbeta $=0.399$ 
BY

CHKD. BY

PROJECT

PAGE 6 OF

SUBJECT

JOB NO.

Conbicilin direction is longitudinal (cher assumption later)

Shear free on back bott:

Longitudinal component $\frac{23,5^{k} 5 / g}{2} \quad=11,75^{k} \mathrm{~s} / \mathrm{s}$

Transverse component $\quad \frac{23.5^{k}}{4} \times 0.45 \mathrm{a} / \mathrm{g}=2.35^{\mathrm{k}} \mathrm{5} / \mathrm{s}$

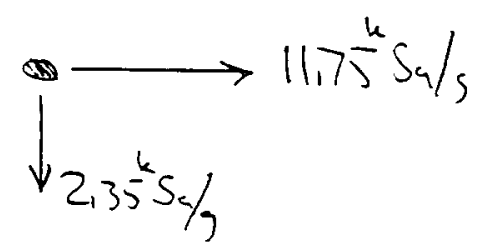

$$
V_{\text {eta }}=\sqrt{11.75^{2}+2.35^{2}}=11.98^{k} \mathrm{Sa} / \mathrm{g}
$$
$7 / 8$ \& $A-307$ bolt ultimate capacity note: include lain in elastic response

$$
\begin{aligned}
& \dot{v}_{v}=(60)(1.2)(0.60)=43.2 \text { less pyrometer } \\
& \sigma_{u} \frac{\text { modiam }}{\cos } \tau_{v} / \sigma_{0}
\end{aligned}
$$$$
\ddot{F}_{V}=A \sigma_{v}
$$

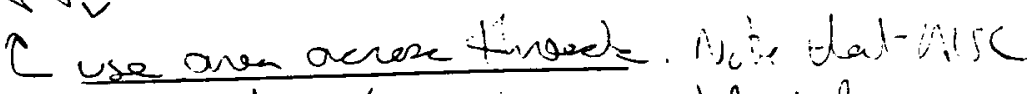

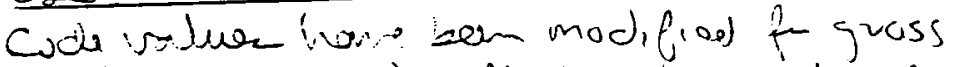
section one e, but really based on mit or ea

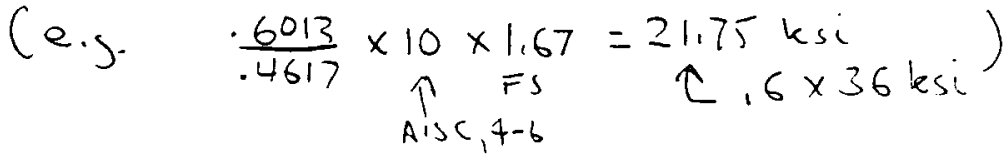

$$
F_{v}=(-4617)(43.2)=19.95 \mathrm{~K}
$$

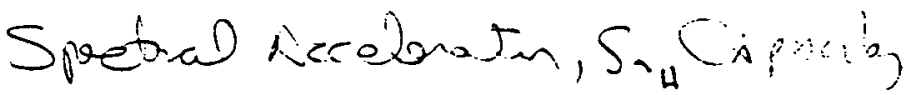

$$
S a_{H}=\frac{-1.9 .95}{11,00}=1.67 \mathrm{~g}
$$

Jack R. Benjamin \& Associates, Inc. Consulting Engineers

C -137 


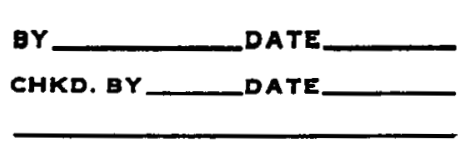

PROJECT

SUBJECT

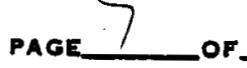

JOB NO.

Suace tonsia in bolts

$$
\begin{aligned}
& \text { pga capecty }=\frac{1.67}{0.42} \times .18 g=0.72 \mathrm{~g} \\
& \dot{S}_{a_{v}}=\frac{0.72 g}{0.18 g} \times \underset{S_{v}}{v}\left(a_{v g}=0.18\right) \\
& \uparrow
\end{aligned}
$$

Run SPECTISIM founticel dirom so dampin

$$
\begin{aligned}
f_{v}^{v} & =33 h_{3} \\
\rho_{u} & =0.39 \\
\Sigma_{r}(a=0,18) & =0.26 ; \quad \rho=0.40
\end{aligned}
$$

$$
S_{a v}=\frac{0.72}{0.18} \times 0.26,=7.04 \mathrm{~g}
$$

Moment in transuerse dircetin

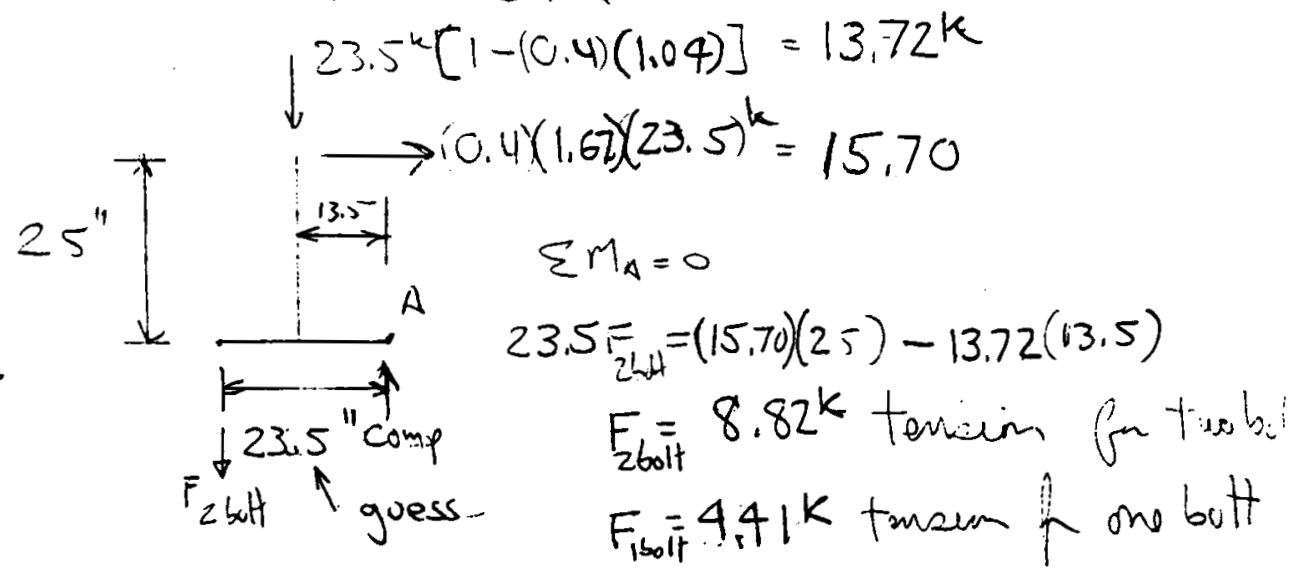

Noed to chech for shoar/tensim interaction

Jack R. Benjamin \& Assoclates, Inc. Consulting Engineers

$C-138$ 


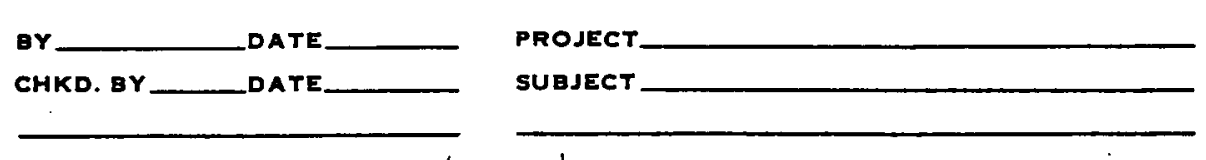

Bult Stress due to

Moment iv Longibudinal divectim (compression by inspectim)

Reeomput capacit, conselevin tensen in bo H

$$
\begin{aligned}
& \left(\frac{F_{+}}{F_{T}}\right)^{2}+\left(\frac{F_{v}}{F_{V}}\right)^{2}=1 \\
& F_{T}=(1.2)(60)(.4617)=33.24 \mathrm{k} \\
& \Sigma_{T}=\frac{0.4 \operatorname{SaH}(23.5)(25)-23.5\left[1-0.4\left(\frac{1.07}{1172}\right) \operatorname{Sa}_{H}\right] 13.5}{23.5 \times 2} \\
& \Sigma_{T}=5,00-6,75(1-.249 \text { Sa4 }) \\
& S_{T}=6.68 S_{a_{H}}-6.75 \\
& \left(\frac{6.68 S_{a_{k}}-6.75}{33.24}\right)^{2}+\left(\frac{11.98 \mathrm{Sa}}{1.9 .95}\right)^{2}=1 \\
& 0.04045 a_{M}^{2}-.08165 a_{N}+.0412+.40105_{a}^{2}-1=0 \\
& 379 S_{a_{N}^{2}}^{2}-.0816 \operatorname{San}_{-}-.9588 \\
& S_{a_{N}}=\frac{.0816 \pm \sqrt{(.0816)^{2}+(4)(\cdot 4010)(.9588)}}{(2)(.4010)} \\
& S a_{H}=1.65 g(\simeq 1.67 \text { slifht intrectur }) \\
& S_{A_{V}}=\frac{165}{1.67} \times 1.04 \mathrm{~g}=1.03 \mathrm{~g} \\
& \text { Pga }=\frac{1.65}{1.67} \times .72 g=0.7 \mathrm{lg}
\end{aligned}
$$




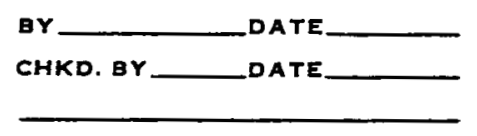

PROJECT

PAGE 9

SUBJECT

JOB NO.

Chech possiblin 8 at 100\% Horiz in transuerse divection contruols

$$
\left[\begin{array}{l}
\left(23.5 \mathrm{sa}_{\mathrm{H}} / \mathrm{s}\right) \\
\longrightarrow\left(23.5 \mathrm{Sa}_{M} / g\right)(0.4)
\end{array}\right.
$$

o

$\infty$

Sear on left bolt:

$$
\begin{aligned}
& \longrightarrow \frac{23.5}{2}(.4)=4.7^{4} \mathrm{sa} / \mathrm{g} \\
& \frac{23.5}{4}=5.88^{k} 5 \sqrt{g} \\
& \text { Vocta }=\sqrt{\left.(4.7)^{2}+5.88\right)^{2}}=7.53^{4} \mathrm{sa} / \mathrm{s}
\end{aligned}
$$

Momant in transuerse divection \& tensim on 1 bolt:

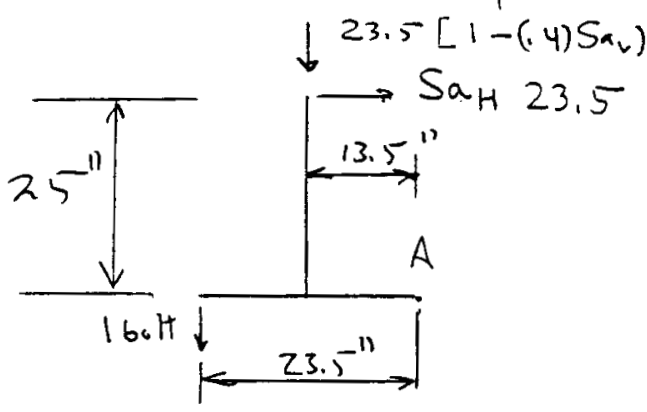

$$
\begin{aligned}
I_{T}= & \frac{S_{a_{H}}(23.5)(25)-23.5\left[1-0.4\left(\frac{1.07}{1.72} S_{a_{H}}\right] 13.5\right.}{23.5 \times 2} \\
S_{T}= & 12.5 S_{a_{H}}-6.75\left(1-.249 S_{a_{H}}\right) \\
S_{T}= & 14.18-6.75 \\
& \left(\frac{14.185 a_{14}-6.75}{33.24}\right)^{2}+\left(\frac{7.53 S_{a_{H}}}{1.9 .95}\right)^{2}=1.0
\end{aligned}
$$

$t_{y y} S_{a}=1165 g \quad \sum=.64<1.0 \quad$ Jack R. Benjamin \& Assoclates, Inc. here capecit, for in's loadin exceed 1,20-ohen direction controls

C-140 


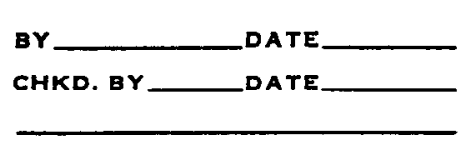

PROJECT

PAGE $\mid \bigcirc$ OF

JOB NO.

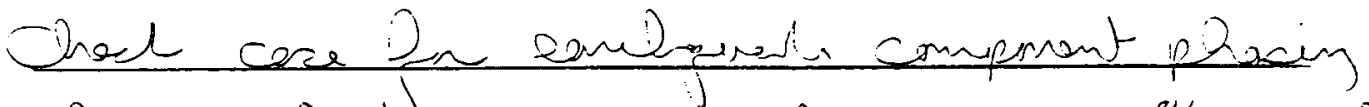

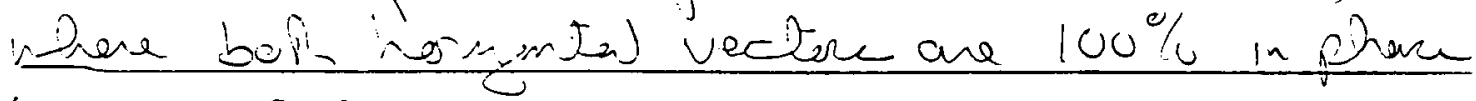
(to develop $\beta_{r}$ )

$$
\begin{aligned}
& 0 \longrightarrow \frac{23,5}{2}=11.75^{n} \mathrm{Sa} / \mathrm{g} \\
& \frac{23.5}{T}=5.875^{k} \mathrm{sa} / \mathrm{g} \quad \text { Vector }=13.14^{k} \mathrm{Sa} / \mathrm{s}
\end{aligned}
$$

$$
\begin{aligned}
& F_{T}=\frac{S_{a_{H}}(23.5)(25)-23.5\left[1-(0.4)\left(\frac{1.07}{1.72} S_{a_{H}}\right)\right] 13.5}{23.5 \cdot 2 \tau_{2} 2.115} \\
& F_{T}=12.5 S_{a_{H}}=6.75\left(1-.2495_{a_{H}}\right) \\
& \Sigma_{T}=14.18 \mathrm{Sa}_{N}-6.75 \\
& \left(\frac{14.18 \mathrm{Sa}_{\mathrm{H}}-6.75}{33.24}\right)^{2}+\left(\frac{13.14 .5 \mathrm{SaH}^{2}}{19.95}\right)^{2}=1 \\
& 0.1820 \mathrm{Sa}_{a^{2}}{ }^{2}-.1733 \mathrm{Sa}_{H}+.0412+.43385_{a}^{2}-1=0 \\
& 6158 . S_{a_{H}}^{2}-1733 S_{a_{H}}-.9588 \\
& S_{a_{N}}=\frac{0.1733 \pm \sqrt{(.1733)^{2}+(4)(.6158)(.9588)}}{(2)(.6158)} \\
& S_{a_{H}}=1.40 \text { g }
\end{aligned}
$$

consider this to $k$ : a 3.5 range

$$
\beta_{r}=\frac{1}{3} \ln \frac{1.67}{1.40}=0.06
$$

Jack R. Benjamin \& Associates, Inc. Consulting Engineers

C-141 


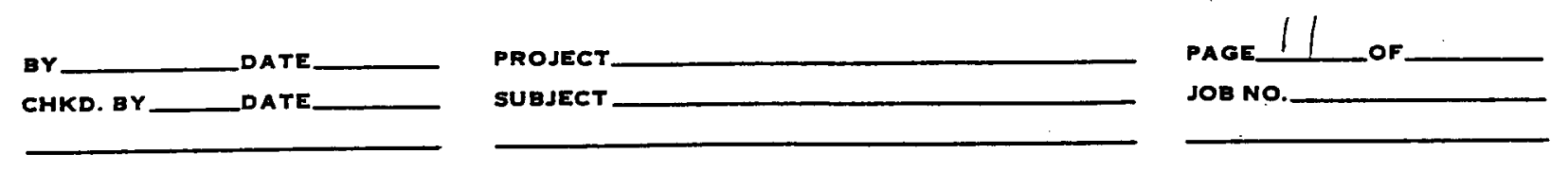

Develop Fragilit, Parameters, F, B, Pu, et HCLPF

Capacito

Strengin $\tilde{F}=0.71 \mathrm{~g}(0.7 \mathrm{~g}=1.0 \quad$ (see page 8 )

$$
\begin{aligned}
& \beta_{r}=0 \\
& \beta_{v}=\frac{1}{1.65} \ln \frac{1.2}{1.0}=0.11 \text { code to meden yuld } \\
& \oplus \frac{1}{1.65} \ln \frac{0,60}{0.5}=0.17 \quad \text { Shen } F_{y} \text { Hensin }_{F} F_{7} \\
& \beta_{u}=0.16
\end{aligned}
$$

Inelastre Response:

Capacty based on ultimat strangech of bult whed is predominately controlled by steer farlune thes no ductiliti assumed

$$
\ddot{F}=1,0 \quad \beta_{r}=\beta_{v}=0
$$

Efupment 16 siense

Spetral Shape (differve between smooked spectua

$$
\begin{aligned}
& \check{F}=1,0
\end{aligned}
$$

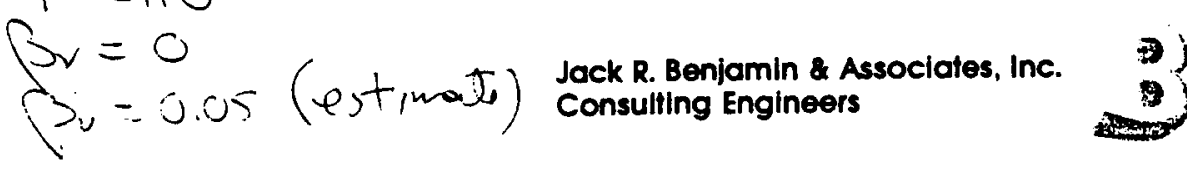

C-142 


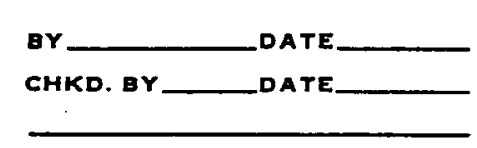

CHK

PROJECT

SUEJECT

Domping (hiy fegung resperses) Thus dompin effect neglible)

$$
\begin{aligned}
& \tilde{F}=1,0 \\
& \beta_{r}=0 \\
& p_{u}=0
\end{aligned}
$$

Modeling

$$
\begin{aligned}
& \text { Frequerg: From simulatim on fregueniz (horiz) } \\
& \beta_{v}=0.14 \\
& \widetilde{F}=1,0 \\
& \beta_{r}=0 \\
& \beta_{u}=0.14 \quad(\text { sel page } 5)
\end{aligned}
$$

Mole Shape: Primarly single mode no vorealutios

$$
\begin{aligned}
& \breve{F}=1,0 \\
& Q y=0 \\
& P u=0
\end{aligned}
$$

Mode Combination Simple structure in iff-fuefuenc)

$$
\tilde{F}=1,0 \quad \beta_{v}=\beta_{v}=0
$$

Jack R. Benjomin \& Associates, Inc. Consulting Engineers

C-143 


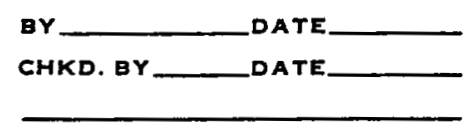

PROJECT

SUBJECT

JOGE 13 OF

Horyontal Compowent Phasin)

$$
\begin{aligned}
& \tilde{F}=1,0 \\
& P_{r}=0.06 \quad(\text { see page 10) } \\
& \beta_{u}=0
\end{aligned}
$$

Structore Respense

GroundMotion: = This parameter inclucles:

1) Pear-to- Pear Spotum vaviturn

2) Norizontal Divectim VAriability

3) Egurpmant Horrzontal Componant Curp.

See Apperdix A - Coupling is simall $(C<0.1$ )

$$
\begin{aligned}
& \vec{F}=1,0 \\
& \rho_{r}=0,25 \\
& \rho_{u}=0
\end{aligned}
$$

Dxmoing Medun: $7 \%$

$$
\begin{aligned}
& -105 \% \\
& \frac{\text { Sa (6round at 7,243) }}{1,894} \\
& \beta_{v}=\frac{1}{1} \ln \frac{2.22}{1.89}=0.11 \\
& \rho_{r}=0 \\
& \ddot{F}=1.0 \text { (used } 7 \% \text { in strempl cale) }
\end{aligned}
$$
Jack R. Benjamin \& Associates, Inc.
Consulfing Engineers

$C-144$ 


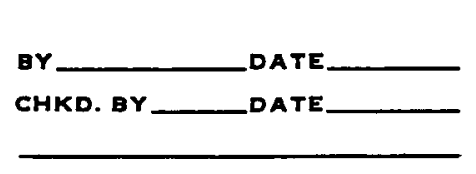

Modeling

Freguency - since buildin Rrepuery in regain of ground spectim platew litthe change will occur. See equpment modeting frequariy, whid includel ie plifet o bulding freweres vaviability on shefting floc resarn. specha

$$
\stackrel{v}{F}=100 \quad \beta_{v}=\beta_{v}=0
$$

Mode shape - Assune contvolled by first mode

$$
F=1.0 \beta_{r}=0 \quad \beta_{u}=0.10 \quad\left(0, t_{i n g}\right)
$$

Mode Conizininitin (controlled by first mode-estinats)

$$
\because=1,0 \quad \beta_{r}=\beta_{u}=0
$$

Horigntal Compmant Phasing

$$
\begin{aligned}
& F^{v}=1,0 \rho_{2}=0.10 \quad \beta_{2}=0 \text { (estimat) } \\
& \text { SST (Rech site) } \\
& \ddot{F}=1.0 \quad \beta_{2}=0 \quad \beta_{2}=0.05
\end{aligned}
$$

Imelastic Rezeonce (strueture assuma) to be (inean)

$$
\tilde{F}=\operatorname{iiv} C_{v}=C_{0}=0
$$

Jack R. Benjamin \& Associates, Inc. Consulting Engineers

C-145 


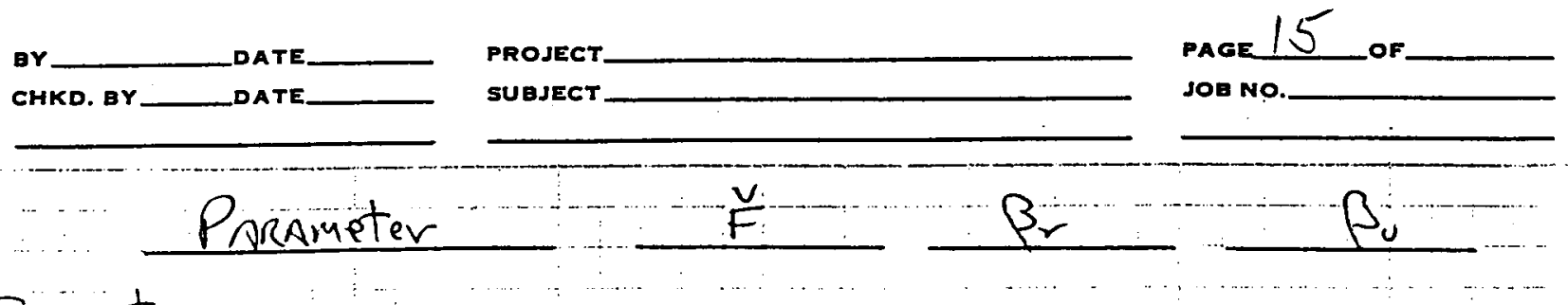

Expact,
Strength
1.2
$0 \quad 0.16$
Inelastic Response
c
0

Equipment Response

Spectral shape

110

$0 \quad 0.05$

Dampin - $\mathrm{I}_{1} \mathrm{O}+0,-\mathrm{O}_{-}$

Medeling Evequency

Mode Comsinatim i

$\operatorname{lic}$

Horiz- Component Phasw - T1O $\quad 0.06$

Structure Response

Grand Motion

Dampin.)

Modeling Fvervency $\quad T_{1} \mathrm{O}$

$$
\text { Mode Shape } 1,0
$$

Mode Combinatiow 1.0

Horiz. Component Phasing $I_{1}$

SSI

1.

Imelastic Response

Combined:

$\frac{1,0}{1,0}$

$\breve{a}_{\text {pga }}=(1.0)(0.71) \mathrm{g}$

NCLP $F_{m}(0.71) e^{-1.65(27+.28)}$

NCLF $1.4(0.28),=0.39$

$=0.71 \mathrm{~g}$
$=0.28 \mathrm{~g}$
$=0.39 \mathrm{~g}$

NCLPF Pas $84 \%$

$1.4 \quad(0.28) \mathrm{g}$ c)

c)

0.14
0
0

0.25

c

o

J

0 1 


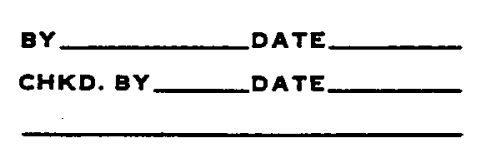

Compute NCLPE Directly (See Appendix C)

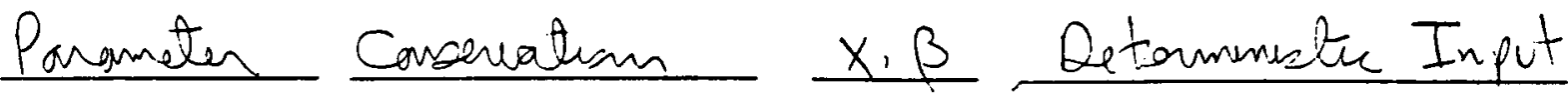

Strongch $998 \%$ HEP $\quad .46 \quad$ Code to median $; 1.65 \sigma=1.65(1,1)=1.18$

$$
\sum x \cdot \beta=1,65(.28+, 27)=0.91
$$

PAGE 16 or

PROJECT

$2.88 x$

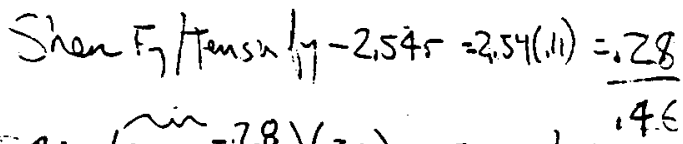

$\tau_{v}=\left(\lim _{0.6 e^{-28}}\right)(60)=27.2 k s^{4.46}$

$$
F_{V}=(27.2)(.4617)=10.92 \mathrm{k}
$$

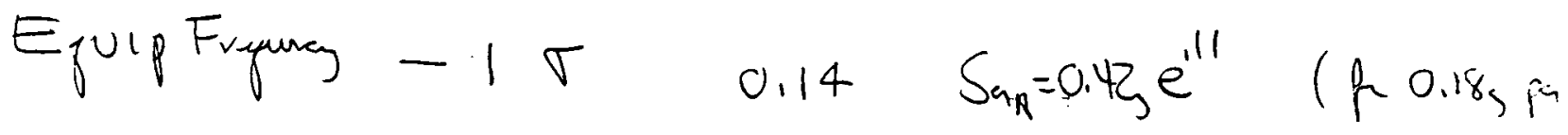

$$
\begin{aligned}
& =0.47 \mathrm{~g}
\end{aligned}
$$

Build Dumpy $+1 \sigma$

$\frac{0.11}{91 .}$

use $7 \% \quad F=\frac{2.12}{1.89}=1.12$

$S_{a_{H}}=(0,47)(1,22)(1,12)=0,64_{G}$

Use oiler farometwa $\left(p-0.18 ; p_{92}\right)$ medium value

Jack R. Benjamin \& Associates, Inc. Consulting Engineers

C- 147 
sr

CHKD. BY DATE

PROJECT

SUEJECT

Capxzing

$$
\begin{aligned}
& F_{V}=10.9 z^{k} \\
& F_{T}=(60)\left(.4(17)=27.70^{k}\right.
\end{aligned}
$$

Inpt: : $\operatorname{San}_{a_{N}}=0.64, \quad(R 0.18$, paga $)$

$$
\begin{aligned}
& S_{v}{ }^{\prime}=0.40 \mathrm{~g} \\
& (0.64 / 1.67 \times 1.04) \quad(\text { seep } 7)
\end{aligned}
$$

Solutim:

$$
\begin{aligned}
& \left(\frac{\xi_{+}}{F_{T}}\right)^{2}+\left(\frac{\xi_{v}}{F_{v}}\right)^{2}=1 \quad\left(\operatorname{sed} \operatorname{par}_{g} 8\right) \\
& \left(\frac{6.68 S_{\sin }-6.75}{27.70}\right)^{2}+\left(\frac{11.98 S_{\text {ant. }}}{10.92}\right)^{2}=1 \\
& 0.0582 S_{a}^{2}-11775 S a+.0594+1.204 S^{2}-1=0 \\
& 1.262 \mathrm{Sa}^{2}-11175 \mathrm{Sa}-.9406=0 \\
& S a_{A}=\frac{: 1175 \pm \sqrt{(.1175)^{2}+(4)(1.262)(9.9406)}}{(2)(1.262)} \\
& S a_{h}=0.91 \text {, (butt iv comp) } \\
& S_{A_{N}}=\frac{10.92}{11.98}=0.91 \mathrm{~g}
\end{aligned}
$$

$$
\begin{aligned}
& \text { Mod.an HCLPF }=\frac{0.91}{0.64} \times .18=\underline{\underline{0.269}} \\
& 84 \% \text { NEP M M NCLP }=0.26 \times 1.4=\underline{\underline{0.36}}
\end{aligned}
$$




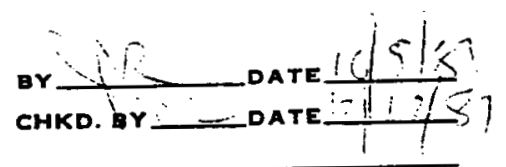

PROJEC

SUBJECT

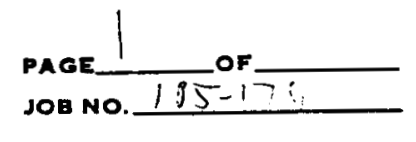

NPP=DO1X A

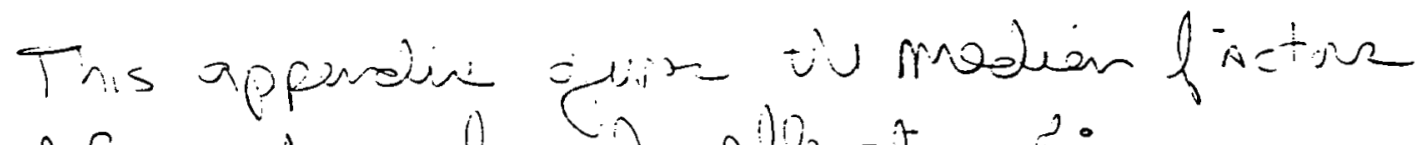
and $\beta_{F}$ value for he ploct of:

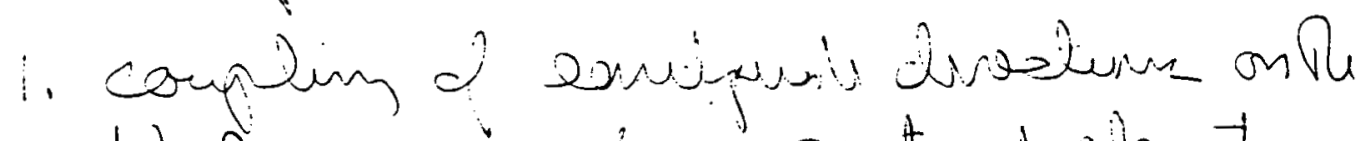
total necprise of a cruteal ilament in a crimparant (e.g. a bott holim Dim a tank)

2. peat to-peal opouth nespurse

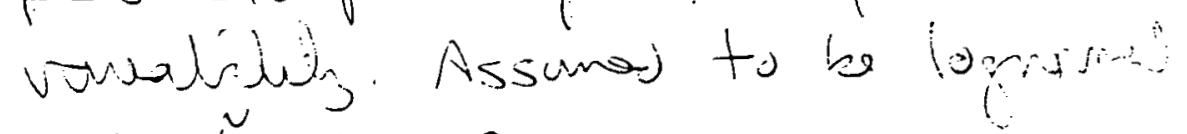
wi $\therefore \tilde{F}=1.0 \quad f=0.20$

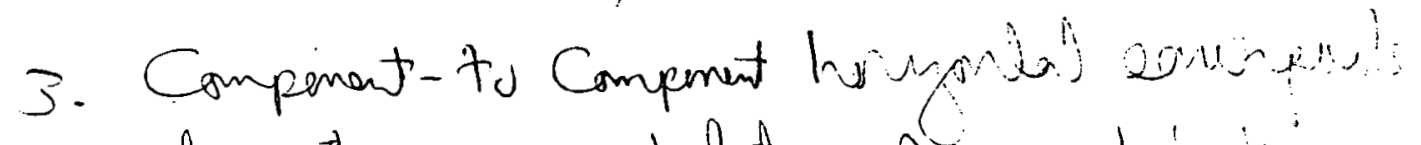

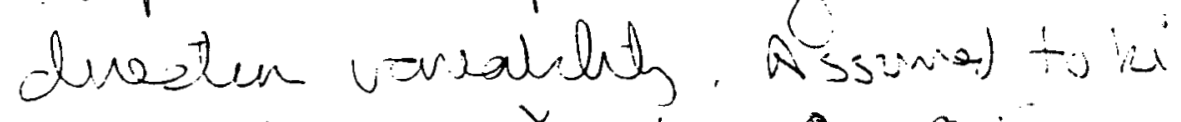
lopraine wid $\tilde{F}=1.0 \quad \beta=0 . i$ is

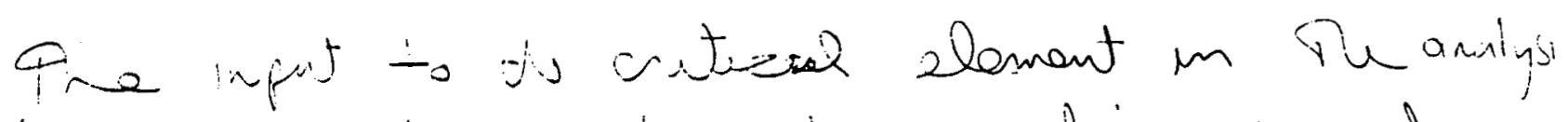

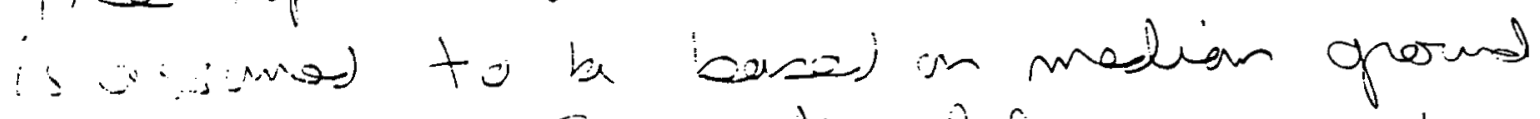
specta infut. Fe ratus 2 ing papmes due

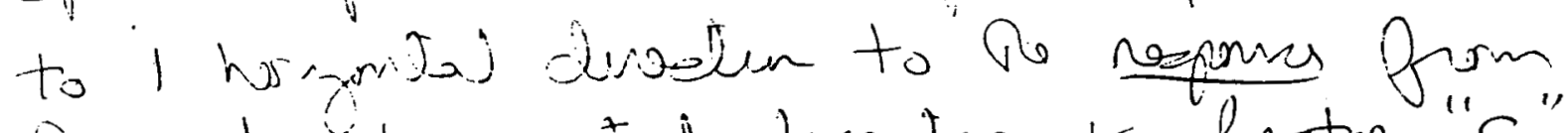

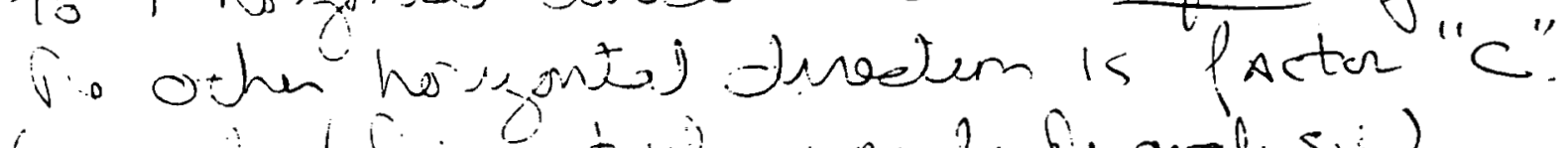

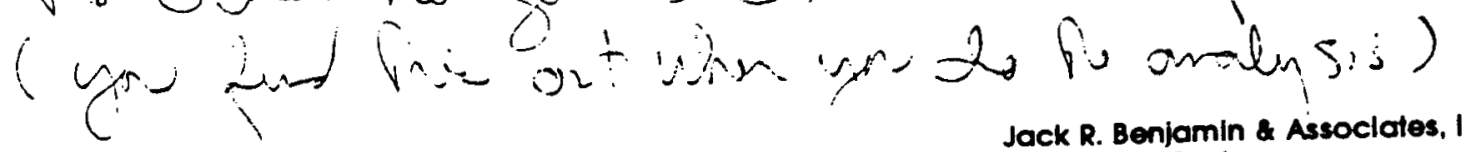

Jack R. Benjamin a Asso
Consulting Engineers

C-149 


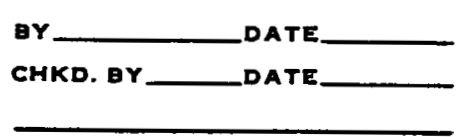

PROJECT.

PAGE $?$

Th peak to-pral distubuture for. cre two diveteme one assurmal to ba undependust (i.e. jus' beause one spetral of durate is high doesit mean ole corresperding one at $90^{\circ}$ is also hig)
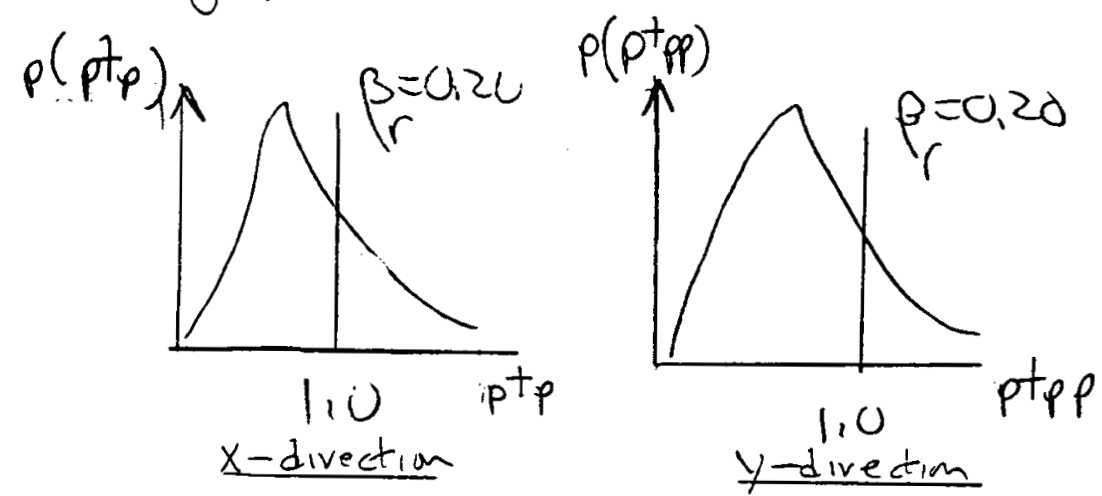

Each duetum compront relalue to u modion is also independent of ptp ad ptpp:

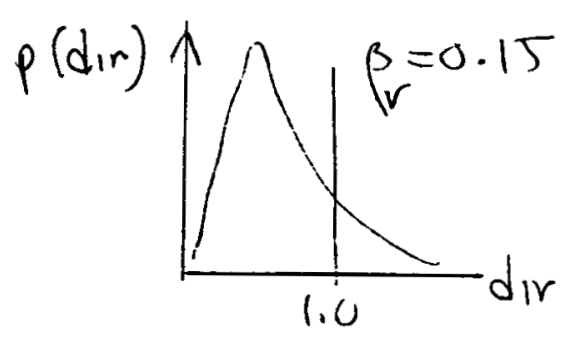

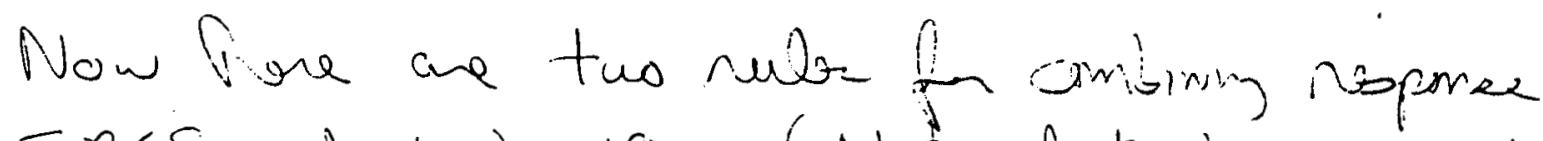
SRSS and 100-40. (Note Vat tis assunad that do vertical earidorata component is

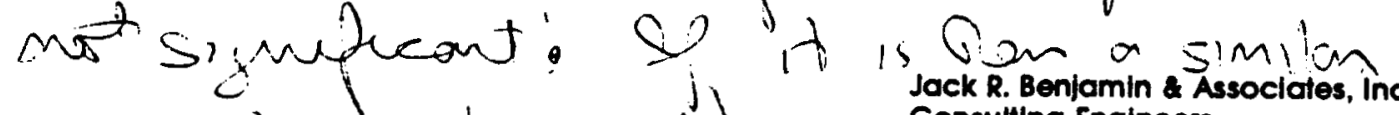
apinger Jon vaci) Jack R. Benjomin \& Asso

C-150 


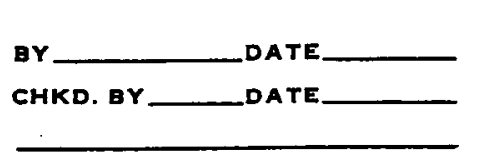

PROJECT

PAGE_S OF

SUBSECT

JOB NO

Th actol combras resparse relatur to $d$ caleulates comburad respense (ie Th aralupic use perform using to modien groond input fir de tuo horyontal drectins is sean dreetls:

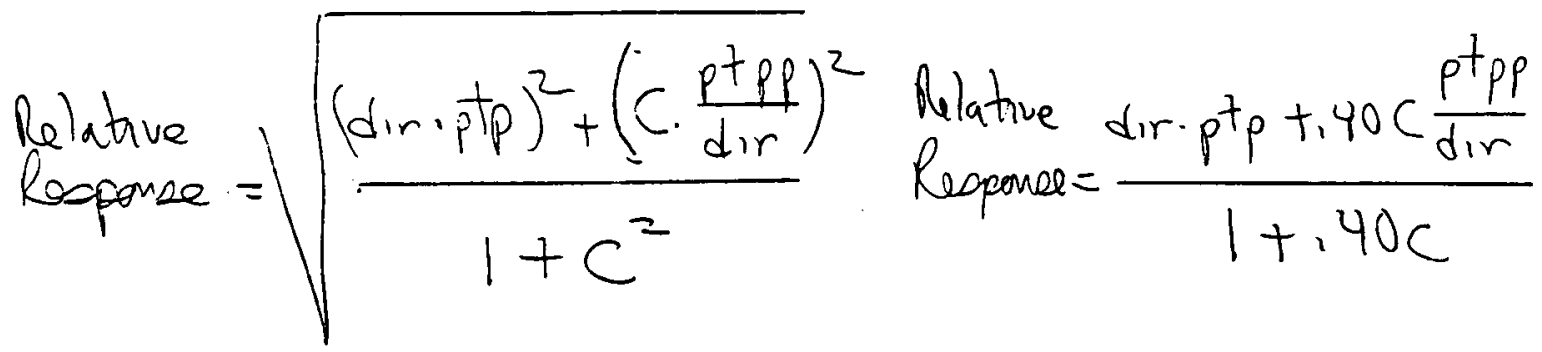

$$
\begin{aligned}
& \text { SRSS rule } \\
& 100-40 \text { vule }
\end{aligned}
$$

Following one ole two MATNCAD properam -

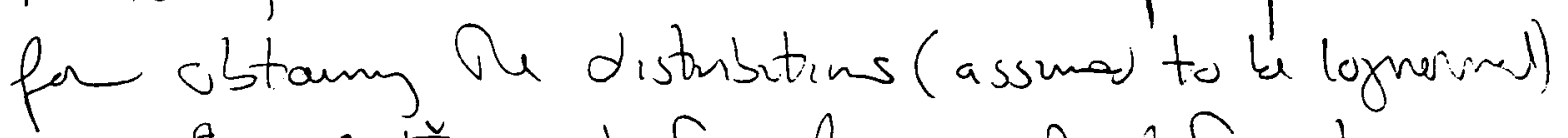
paramtere: $F$ ard for f a ece of fu two rules, l.e.

DIR. FAT: 100-40 mu

DIR. FAC: SRSS vule

On the last page is in medeen factors, $\breve{F}$ ad a ssocialas Br values of diffeient valus of $C$. There, knowing $C U$ conset $F$ and $\beta_{p}$ cen

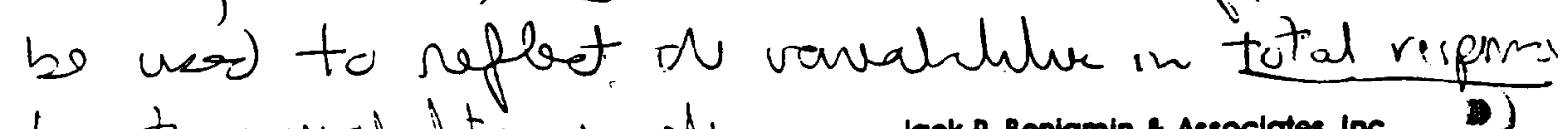
dere to vavainditur in d gorund motion.

C-151 
MCAU FILES DIFE.FAT

This program calculates the distributian on resporse for peak-to-peat: and direction variability when the combination method is the 10040 rule

NGFMAL DIETFIEUTION

$$
\begin{aligned}
& \text { b1 } \equiv .319381530 \\
& \text { เ2 } \equiv-.356563782 \\
& b 3 \equiv 1.781477937 \\
& \text { 七4 } \equiv-1.821255979 \\
& \text { 口5 } \equiv 1.330274427 \\
& p \equiv .2316419 \\
& z(x)=\frac{\exp \left[\frac{-x}{2}\right]}{2.506628275} \\
& t(x) \equiv \frac{1}{1+p \cdot|x|}
\end{aligned}
$$

$\operatorname{NOF}(x) \equiv\left|(x>0)-z(x) \cdot\left[b 1 \cdot t(x)+b 2 \cdot t(x)^{2}+b 3 \cdot t(x)^{3}+b 4 \cdot t(x)^{4}+b 5 \cdot t(x)^{3}\right]\right|$

\section{INUEFSE NOFMAL DISTRIBUTION}

$$
\begin{aligned}
& c 0 \equiv 2.515517 \\
& \tau 1 \equiv .002953 \\
& c 2 \equiv .010328 \\
& d 1 \equiv 1.432788 \\
& d 2 \equiv .189269 \\
& d 3 \equiv .001308
\end{aligned}
$$$$
t(p) \equiv \sqrt{\ln \left[\frac{1}{((p) .5)-p)^{2}}\right]}
$$ 
$\operatorname{INOR}(p) \equiv(-1)^{p<.5}\left[t(p)-\frac{c 0+c 1 \cdot t(p)+c 5 \cdot t(p)^{2}}{1+d 1 \cdot t(p)+d 5 \cdot t(p)^{2}+d 3 \cdot t(p)^{3}}\right]$

$n:=199$
$i:=0 \ldots n$

$\operatorname{ptp}{ }_{i}:=1.0 \cdot \exp \left[0.20 \cdot \operatorname{INOR}\left[\frac{i+r n d(1)}{n+1}\right]\right] a$

$\operatorname{dir}_{i}:=1.0 \cdot \exp \left[0.15 \cdot \operatorname{INOR}\left[\frac{i+r n d(1)}{n+1}\right]\right]$ a

$\min (d i r)=0.659 \quad a$

$\max (d i r)=1.555 \quad 0$

WRITE(DIF) $:=d i r \quad 0$

WRITE (PTF) $:=p t p \quad$ ${ }^{i}$

WRITE $(P T F F):=p t^{i} \quad \square$

i

Fandomiy mix both the direction and the two peak-to-peak arrays EXIT TO DOS and run CADMIX

dir $:=$ FEAD (DIF )

$i=\operatorname{READ}($ FTF $)$

$\dot{i}=$ READ (FTFF)

$c:=0 . .4$

1

\begin{tabular}{|c|c|c|}
\hline dit & $p t p$ & $p t p p$ \\
\hline & $c$ & c \\
\hline 1.087 & 1.033 & 0.796 \\
\hline 0.736 & 0.922 & 0.907 \\
\hline 1.189 & 1.013 & 1.643 \\
\hline 1.236 & 0.975 & 1,108 \\
\hline 1.194 & 1.026 & 1.346 \\
\hline
\end{tabular}




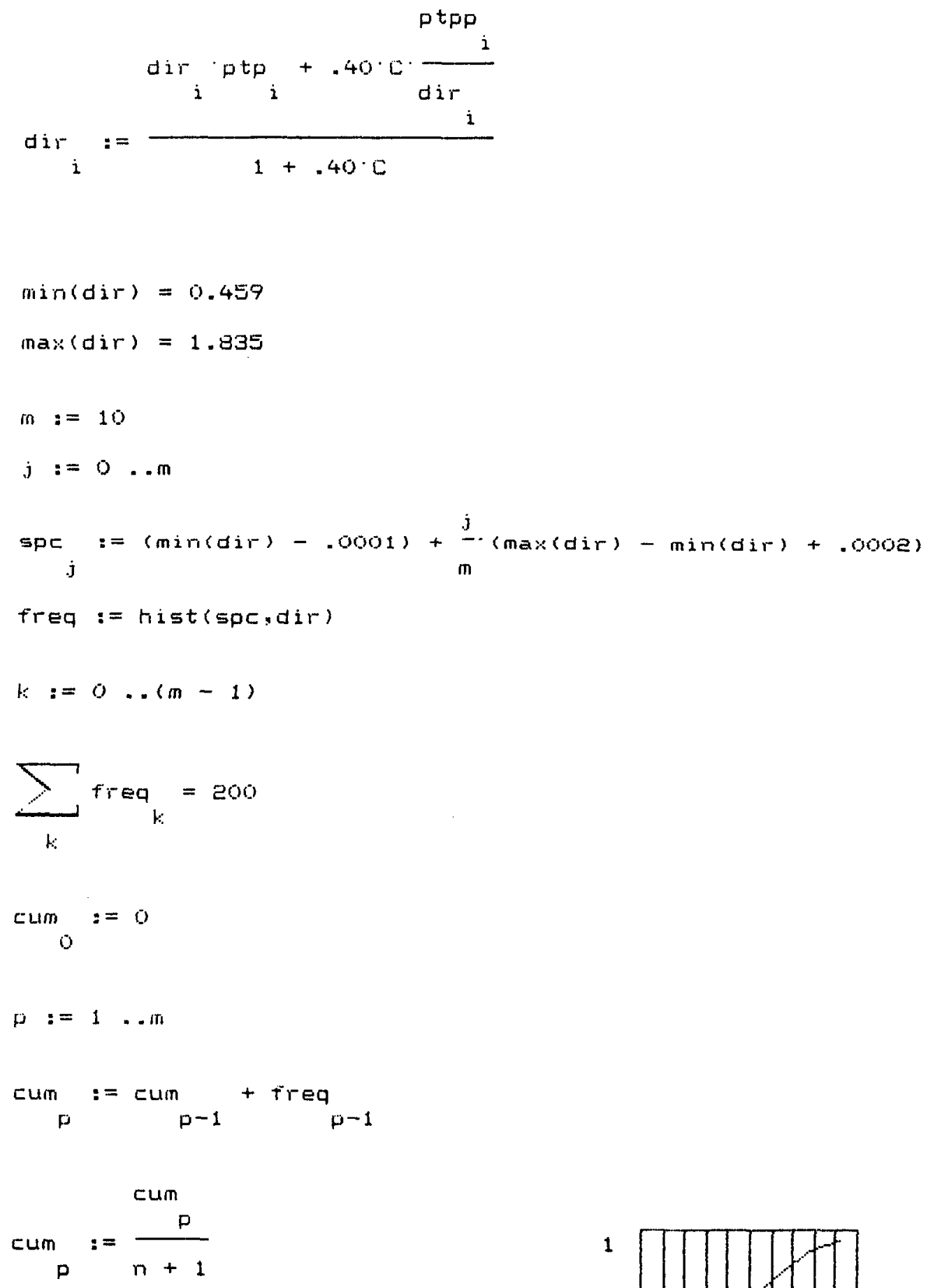


s:= lspline(spc, :um)

$$
\begin{aligned}
& \text { Find the median value: } \\
& x:=1.0
\end{aligned}
$$

rout(interp $(5, \operatorname{spc}$, cum $x)-.5, x)=0.999$

Find the + Ior value:

root (interp $(5.5 p c .($ cum,$x)-.84134 . x)=1.286$

Find the - la value:

rucit(interp $($ s.spc, cum, $x)-.15866, x)=0.779$

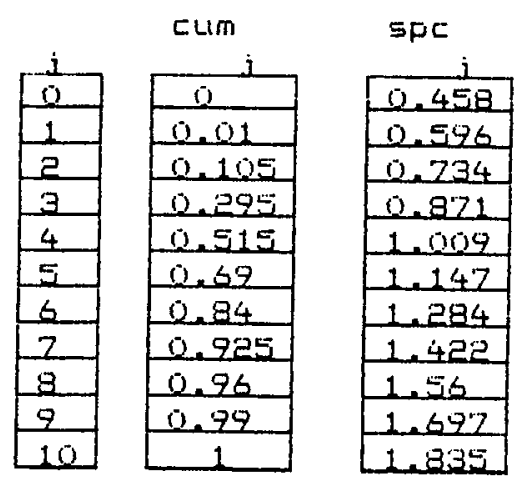

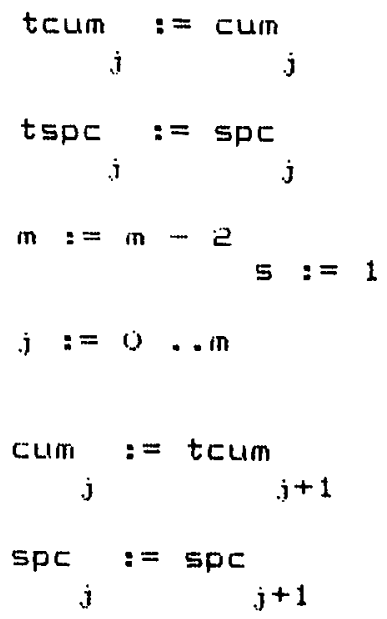

spc 
This program take the calculated probabilities of failure and the associated peak ground acceleration values and performs a least squares fit in the log-probability domain to abtain the "best fit" median and $\beta$ value.

$$
\begin{aligned}
& x 1:=c u m \\
& \text { j j } \\
& Y_{j}^{1}:=\operatorname{spc} \\
& y_{j}:=\ln \left[\begin{array}{ll}
y_{1} \\
& j
\end{array}\right] \\
& \because 1_{j}:=\operatorname{INOR}\left[\begin{array}{ll}
x 1 \\
& . j
\end{array}\right]
\end{aligned}
$$

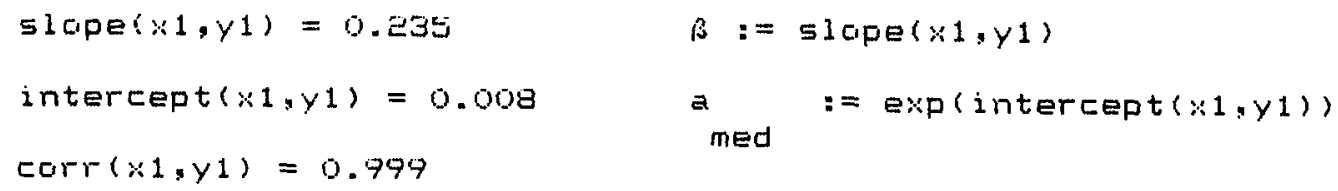
$c \equiv 0$
$\therefore \quad=1.000$
$\beta=0.235$

$z_{j}:=$ intercept $(\times 1, y 1)+\operatorname{slope}(\times 1, \times 1) \cdot \times 1$

$$
\begin{aligned}
& \max \left(y_{1}\right)=0.529 \\
& \min \left(y_{1}\right)=-0.517 \\
& \min (z 1)=-0.539 \\
& \max (x 1)=2.327 \\
& \min (x 1)=-2.327
\end{aligned}
$$

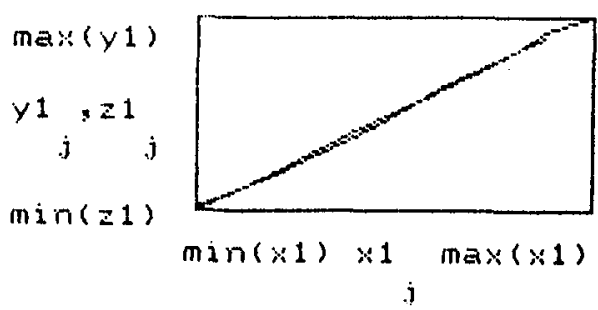




\begin{tabular}{|c|c|c|c|}
\hline & $\because 1$ & $y 1$ & \pm 1 \\
\hline$i$ & & i & i \\
\hline 0 & -2.327 & $-0,517$ & $-0,539$ \\
\hline 1 & $-1,254$ & -0.31 & -0.287 \\
\hline 2 & $-0,539$ & -0.138 & -0.119 \\
\hline 3 & 0.038 & 0.009 & 0.017 \\
\hline 4 & 0.495 & 0,137 & 0,124 \\
\hline 5 & 0.994 & 0.25 & 0.241 \\
\hline 6 & 1.44 & 0.352 & 0.346 \\
\hline 2 & 1.751 & (). 444 & 0.419 \\
\hline 8 & 2. 327 & 0.529 & 0,554 \\
\hline
\end{tabular}

Tatulate prababilities ta campare calculated and best fit values:
$\bar{a}:=\exp \left[\begin{array}{ll}r 1 \\ & j\end{array}\right]$
Feak: orcund accelerations
$\mathrm{P}_{j}:=\operatorname{NOF}\left[\begin{array}{ll}\therefore 1 \\ & j\end{array}\right]$
Frobatility cit failure values

$\operatorname{Pbest} i j_{\text {bes }}:=\operatorname{NOF}\left[\frac{\left[\begin{array}{l}a_{j} \\ a \\ \text { med }\end{array}\right]}{\beta}\right]$

Best fit probability of failure values
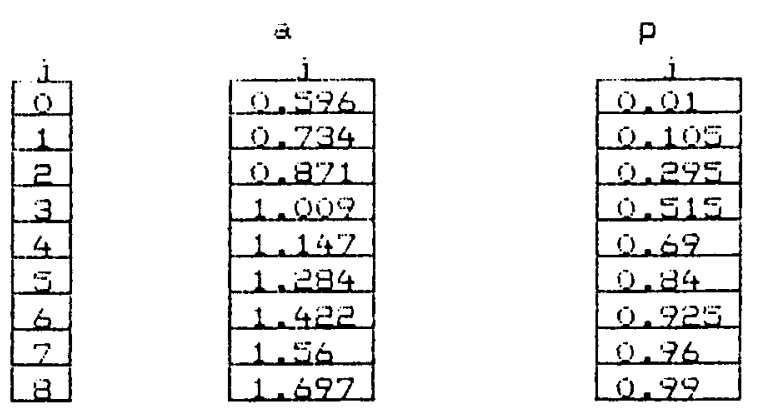

p

best

\begin{tabular}{|l|}
\hline 0.013 \\
\hline 0.038 \\
\hline 0.268 \\
\hline 0.502 \\
\hline 0.209 \\
\hline 0.349 \\
\hline 0.729 \\
\hline 0.762 \\
\hline 0.932 \\
\hline
\end{tabular}


MCAD FILES DIFI.FAC

This propram calculates the distribution on respanse for peak-to-peak and direction variability when the combination method is the sfiss rule

NOFMAL DISTRIEUTIUN

$$
\begin{aligned}
& \text { 七1 } \equiv .317381530 \\
& \mathrm{bD} \equiv-.356563782 \\
& \text { b3 } \equiv 1.781477937 \\
& \text { b4 } \equiv-1.821255978 \\
& \text { 七5 } \equiv 1.330274429 \\
& p \equiv .2316417 \\
& z(x) \equiv \frac{\exp \left[\frac{2}{2}\right]}{2.506628275} \\
& t(x) \equiv \frac{1}{1+p \cdot|x|}
\end{aligned}
$$

$\operatorname{NOR}(x) \equiv\left|(x>0)-z(x) \cdot\left[b 1 \cdot t(x)+b 2 \cdot t(x)^{2}+b 3^{2} t(x)^{3}+b 4 \cdot t(x)^{4}+b 5 \cdot t(x)^{5}\right]\right|$

$$
\begin{aligned}
& c 0 \equiv 2.51551 \% \\
& c 1 \equiv .002953 \\
& \tau 2 \equiv .010328 \\
& d 1 \equiv 1.432798 \\
& d 2 \equiv .189267 \\
& d 3 \equiv .001308
\end{aligned}
$$

$$
t(p)=\sqrt{\ln \left[\frac{1}{((p) . s)-p)^{2}}\right]}
$$


$\operatorname{INOF}(p) \equiv(-1)^{p<.5} \cdot\left[t(p)-\frac{c 0+c 1 \cdot t(p)+c 5 \cdot t(p)^{2}}{1+d 1 \cdot t(p)+d 2 \cdot t(p)^{2}+d 3 \cdot t(p)^{3}}\right]$

$n:=199$

$i:=0 \ldots n$

$\operatorname{ptp}{ }_{i}:=1.0 \cdot \exp \left[0.20 \cdot \operatorname{INUR}\left[\frac{i+r n d(1)}{n+1}\right]\right]$ 0

$\operatorname{dir}=1.0 \cdot \exp \left[0.15 \cdot \operatorname{INOF}\left[\frac{i+r n d(1)}{n+1}\right]\right] \quad 0$

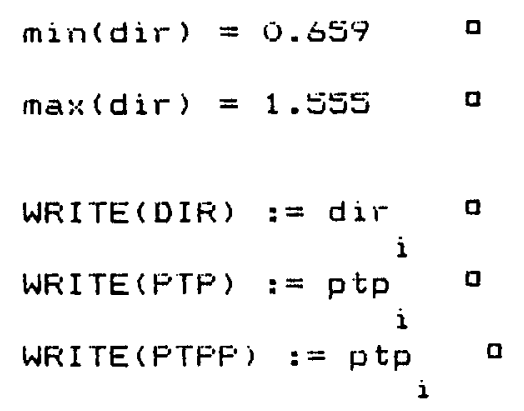

Fiandomly mi: both the direction and the two peak-to-peak arrays EXIT TO DOS and run CADMIX

dir $:=$ FEAD (DIR)

$\operatorname{ptp}^{i}:=$ FEAD (FTP)

$c:=0 \quad \ldots 4$

$\operatorname{ptpp}:=\operatorname{READ}(F T F F)$

\begin{tabular}{|c|c|c|}
\hline dir & $p t p$ & $p t p p$ \\
\hline$\frac{c}{1087}$ & $\frac{c}{1.033}$ & $\quad c$ \\
\hline 0.236 & 0.922 & 0.907 \\
\hline 1.189 & 1.013 & 1.643 \\
\hline 1.236 & 0.975 & 1.108 \\
\hline 1.194 & 1.026 & 1.346 \\
\hline
\end{tabular}




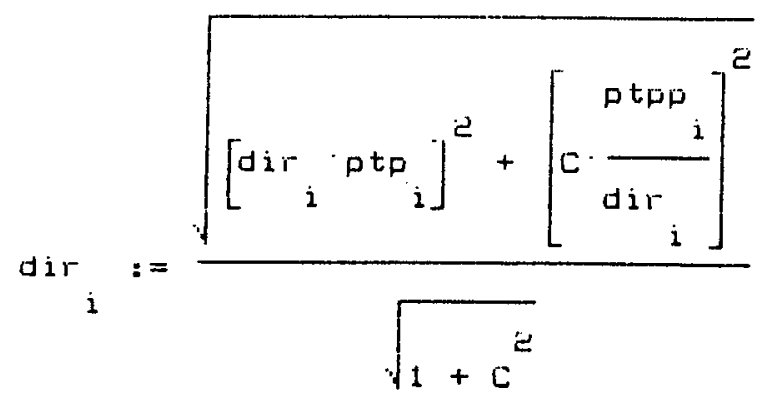

$$
\begin{aligned}
& \min (d i r)=0.459 \\
& \max (\text { dir })=1.835 \\
& \text { m : }=10 \\
& j:=0 . \ldots m \\
& \operatorname{spc}:=(\min (d i r)-.0001)+\frac{j}{m} \cdot(\max (d i r)-\min (d i r)+.0002) \\
& \text { freq : = hist (spc,dir) } \\
& k:=0 \ldots(m-1) \\
& \sum_{k} \operatorname{frea}_{k}=200 \\
& \operatorname{cum}_{0}:=0 \\
& p:=1 \ldots n \\
& \underset{p}{\text { cuin }}:=\text { cuin }_{p-1}+\operatorname{freq}_{p-1} \\
& \operatorname{cum}_{p}:=\frac{\text { cuin }}{n+1}
\end{aligned}
$$

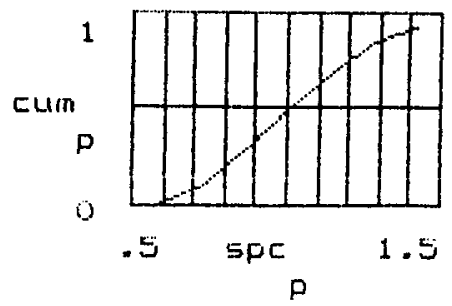


$s:=$ Ispline (spc. cum)

Find the mediain value:

$x:=1.0$

root (interp $(5, \operatorname{spc}$, cum, $x)-.5, x)=0.799$

Find the + 10 value:

ract (interp $(5,5 p c$, cum, $x)-.84134, x)=1.28$ s

\begin{tabular}{|c|c|c|}
\hline & cuin & SPC \\
\hline$-j$ & & \\
\hline 0 & 0 & 0.453 \\
\hline 1 & 0.01 & 0.596 \\
\hline 2 & 0.105 & 0.734 \\
\hline 3 & 0.295 & 0.971 \\
\hline 4 & 0.515 & 1.004 \\
\hline 5 & 0.69 & 1.147 \\
\hline 6 & 0.94 & 1.284 \\
\hline 7 & 0.925 & 1.422 \\
\hline 8 & (). 96 & 1.56 \\
\hline 9 & 0.29 & 1.697 \\
\hline 10 & 1 & 1.835 \\
\hline
\end{tabular}

Find the - $1 \sigma$ value:

raot (interp $(s$, sp, cum, $x)-.15866, x)=0.779$

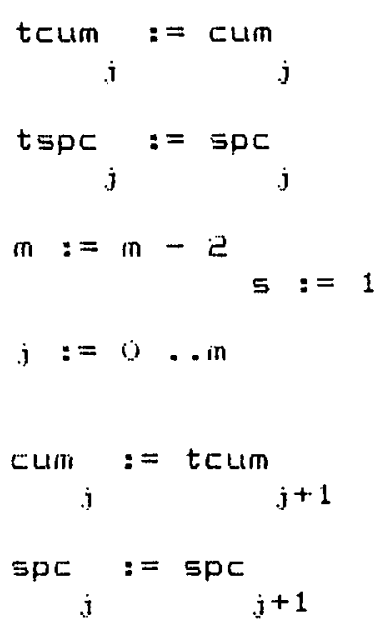

\begin{tabular}{|c|c|c|}
\hline & cum & spc \\
\hline & & \\
\hline 0 & 0.01 & 0.526 \\
\hline & 0.105 & 0.734 \\
\hline 2 & 0.295 & 0.971 \\
\hline 3 & 0.515 & 1.009 \\
\hline 4 & 0.67 & 1.147 \\
\hline 5 & 0.84 & 1.284 \\
\hline 6 & 0.925 & 1.4.2. \\
\hline 7 & 0.96 & 1.56 \\
\hline 8 & 0.94 & 1.622 \\
\hline
\end{tabular}


This program take the calculated probabilities cif failure and the associated peak arcund acceleration values arid performs a least squares fit in the loq-probability domain to obtain the "best fit" median and fo value.
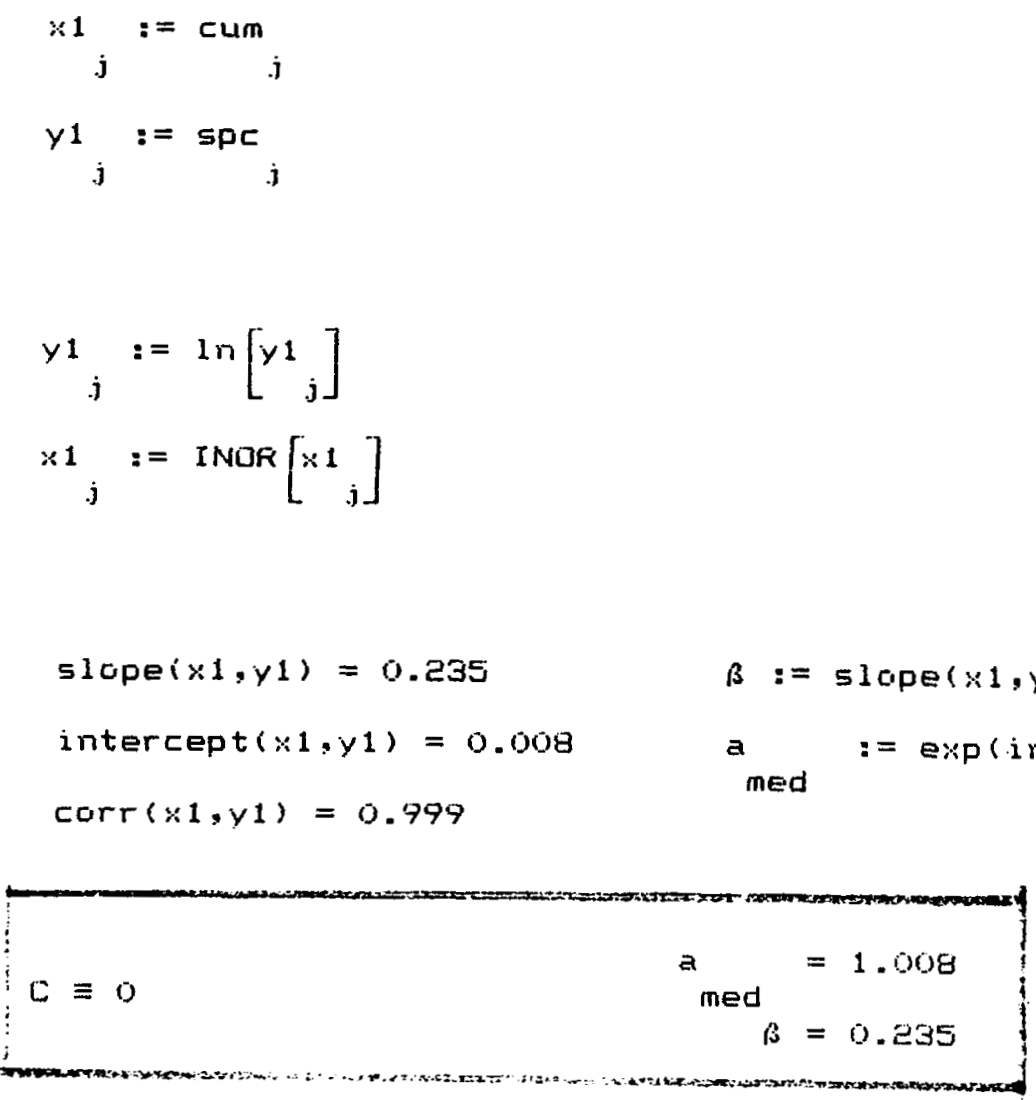

$21:=i n t e r c e p t\left(x 1, y_{1}\right)+\operatorname{slcpe}(x 1, y 1) \cdot \times 1$

$$
\begin{aligned}
& \max \left(y_{1}\right)=0.529 \\
& \min \left(y_{1}\right)=-0.517 \\
& \min (-1)=-0.539 \\
& \max (\times 1)=2.327 \\
& \min (\times 1)=-2.327
\end{aligned}
$$

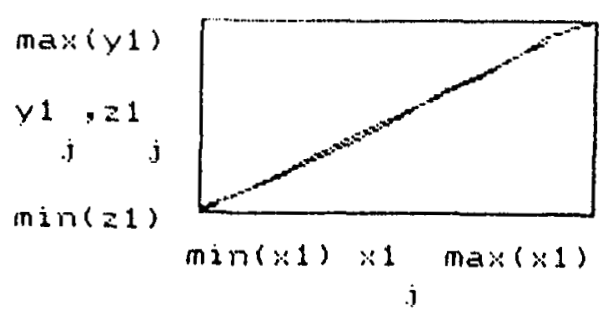




\begin{tabular}{|c|c|c|c|}
\hline \multicolumn{2}{|r|}{$\therefore 1$} & $y^{1}$ & $\geq 1$ \\
\hline$j$ & & & \\
\hline 0 & -2.327 & -0.517 & $-0,-39$ \\
\hline 1 & -1.254 & -0.31 & -0.287 \\
\hline 2 & -0.538 & $-0,138$ & -0.119 \\
\hline 3 & 0.038 & 0.009 & 0.012 \\
\hline 4 & 0.495 & 0.172 & 0.124 \\
\hline 5 & 0.994 & 0.25 & 0.241 \\
\hline 6 & 1.44 & 0.352 & 0.346 \\
\hline 2 & 1.751 & 0.444 & 0.412 \\
\hline 8 & 2.327 & 0.529 & 0.554 \\
\hline
\end{tabular}

Tabulate probabilities to compare calculated and best fit values:

$a_{j} \quad:=\exp \left[\begin{array}{r}y^{1} \\ \\ \end{array}\right]$

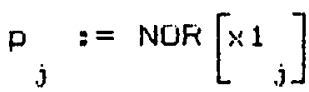

$P_{\text {best }}:=\operatorname{NOF}\left[\frac{\left[\ln \left[\frac{a}{a}\left[\begin{array}{l}a \\ \text { med }\end{array}\right]\right.\right.}{\beta}\right]$

a

\begin{tabular}{|c|}
\hline$j$ \\
0 \\
\hline 1 \\
\hline 2 \\
\hline 3 \\
\hline 4 \\
\hline 5 \\
\hline 6 \\
\hline 7 \\
\hline 8 \\
\hline
\end{tabular}
Feat: grcund accelerations

Frobability of failure values
Best fit probability of failure values

\begin{tabular}{|l|}
\hline 0 \\
\hline 0.01 \\
\hline 0.105 \\
\hline 0.295 \\
\hline 0.515 \\
0.69 \\
\hline 0.84 \\
\hline 0.925 \\
\hline 0.96 \\
\hline 0.97 \\
\hline
\end{tabular}

best

0.013

0.088

0.269

$0.500^{2}$

0.709

0.844

0.924

0.969 


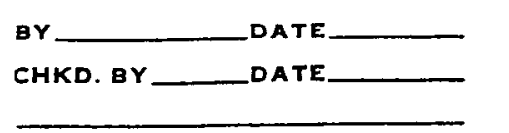

PROJECT

PAGE $\stackrel{h}{h}$ OF

$F=-100-40$ fore

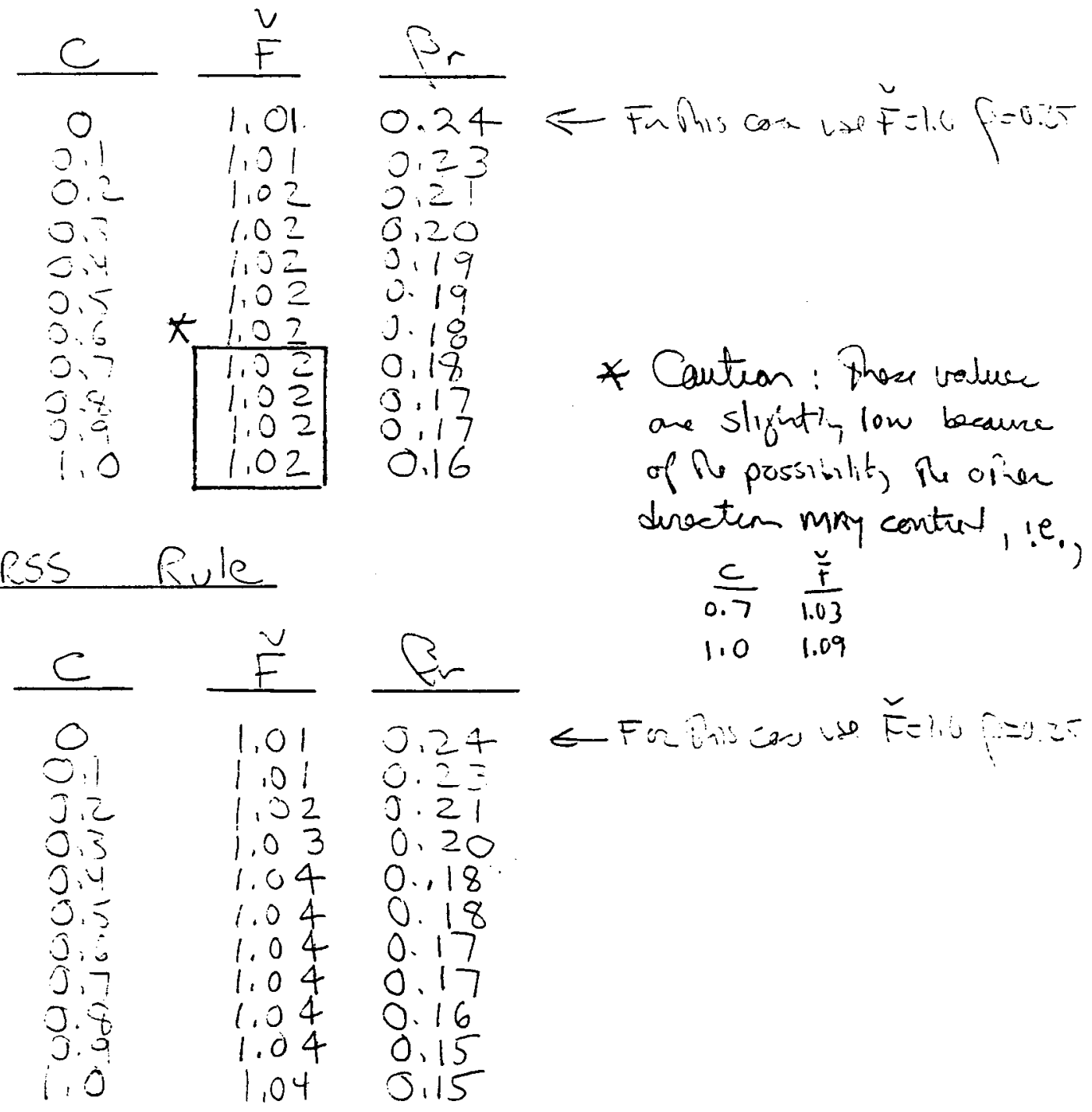

Fo Sis Rule

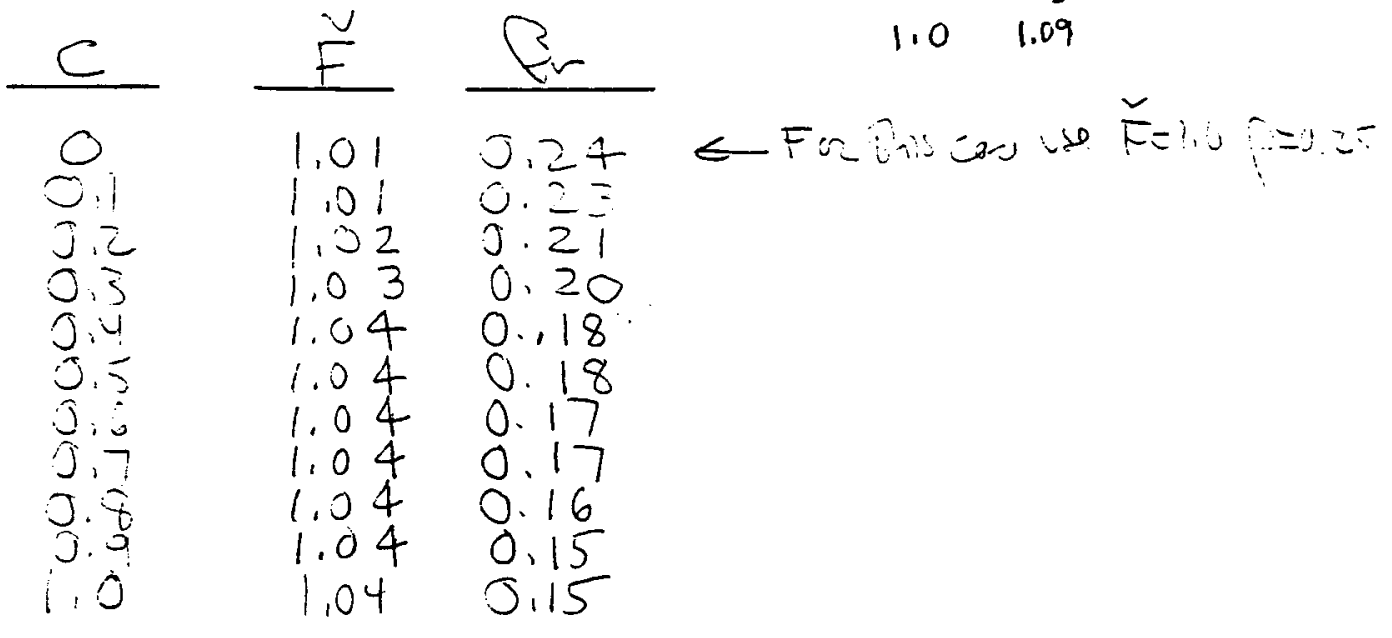

Note: There facture one trove case use a single

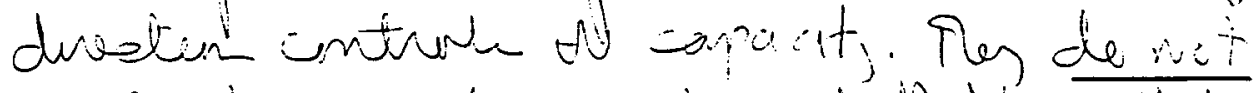
apply to case of capacity ontriblad by ald dive...

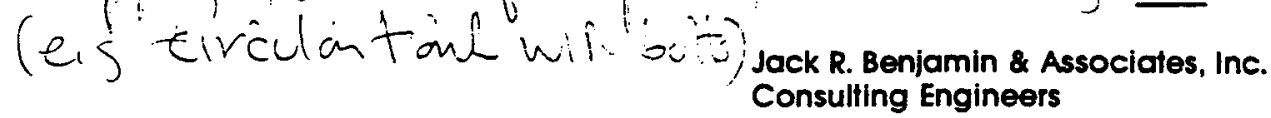

C-164 
Appenoijix B

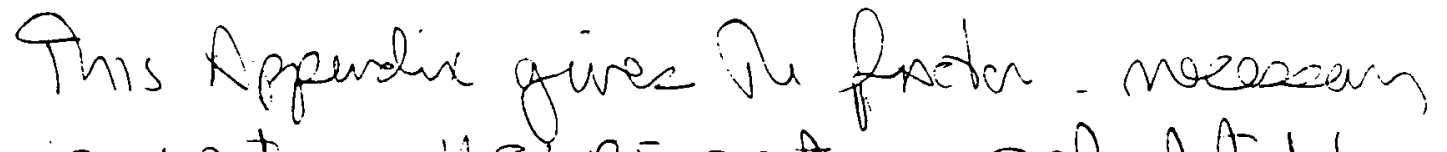
to comert a NCLPF spectum caleubletes bacad on Ru ossumping $\rightarrow$ lat the ground motion hoiracid mpuic are melean boil in ols serse of peak-tup pal varicbility and in fie sense of a seametric overage to tur hersentel compononts, den olvewoder, a HCLPF spectum calewbetes on thin basi woibs be compened to a real eartheriale by first areragun (ceometricall, in two herizonial Drestim spectar and Ren comparing it to i HCLPF speetur. Anbe fosereng name of

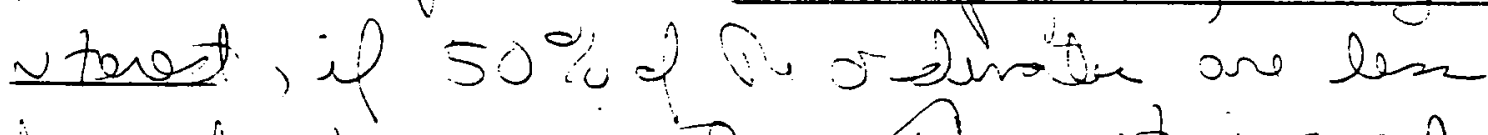
ham VIS XCLPF speetren, then it is corcluded at hu NCLPF haz not been exceoded.

To. convert to a $84 \%$ NEP marimun mporar spoetrum in vorvaluitse due to bor

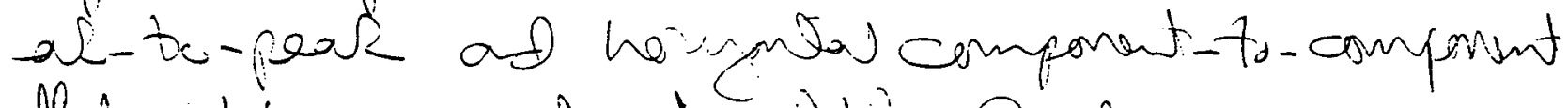

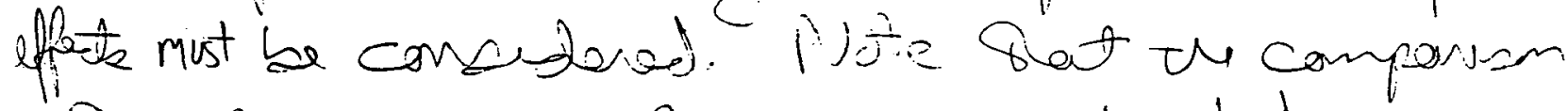

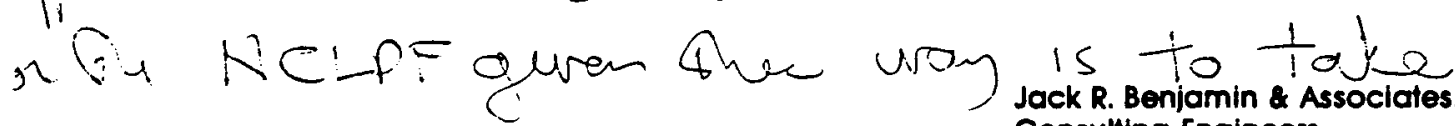

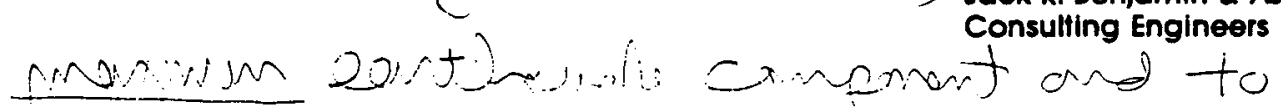

C-165 


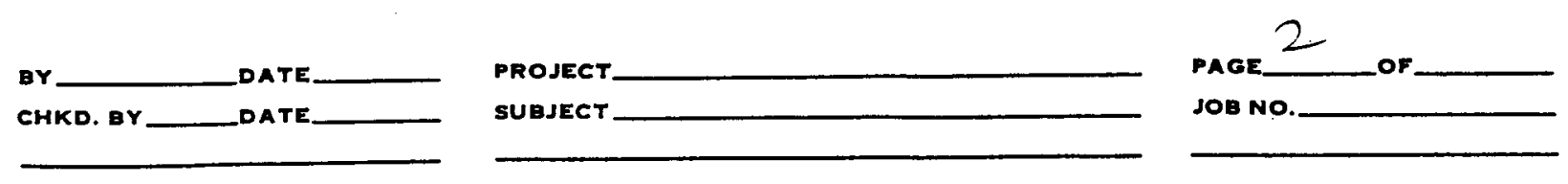

compare in cosvepsidin roporve speetrum to fine HCLF spetur en lio herenen

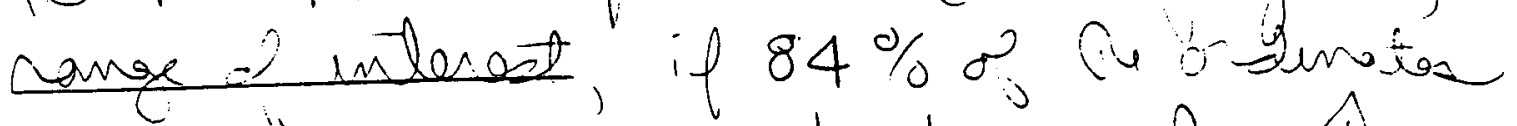

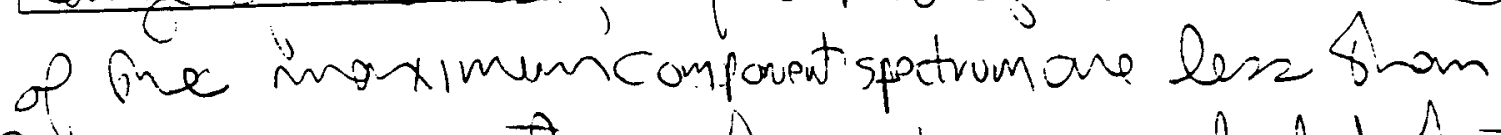
qu HCLPF spetium, then it is crneludel fi-at Fe NCLPF has not been excosoled.

MRTICXO prospan PEXk.com caleulater firsta deshitutis for ong he duetur-to-dicedin vavalubt, to find in medien and $\beta_{r}$ in de moximen component. This is simply

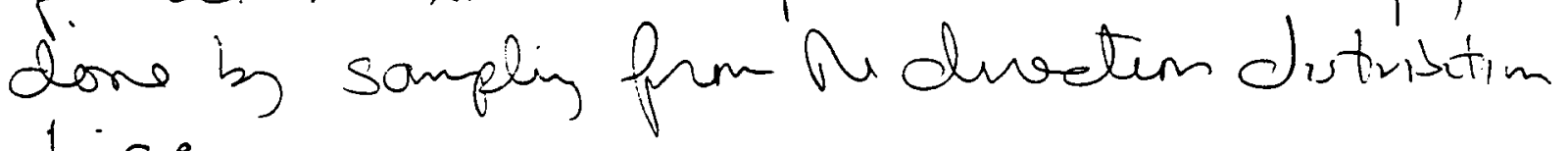
div:

$$
p\left(d_{1 r}\right) \underbrace{\beta_{d i r}=0.15}_{1.0}
$$

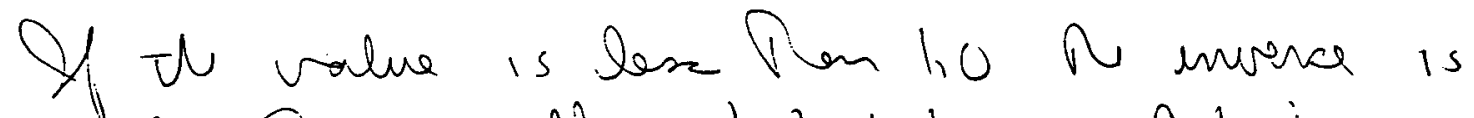
ised. Th reaulting distribitim is pit

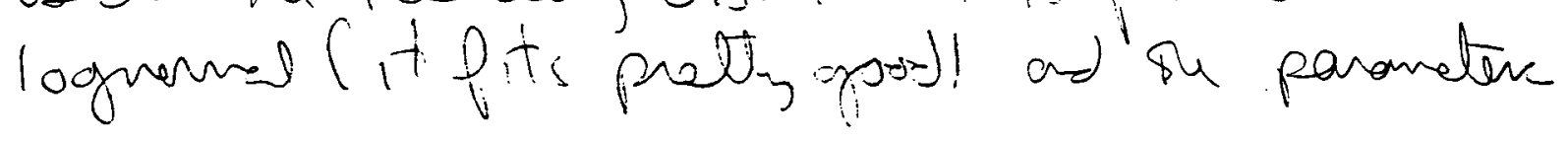
are:

$$
\begin{aligned}
& \because F=1,11 \\
& P_{r}=0,12
\end{aligned}
$$

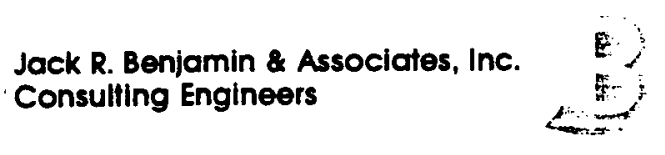

C-166 


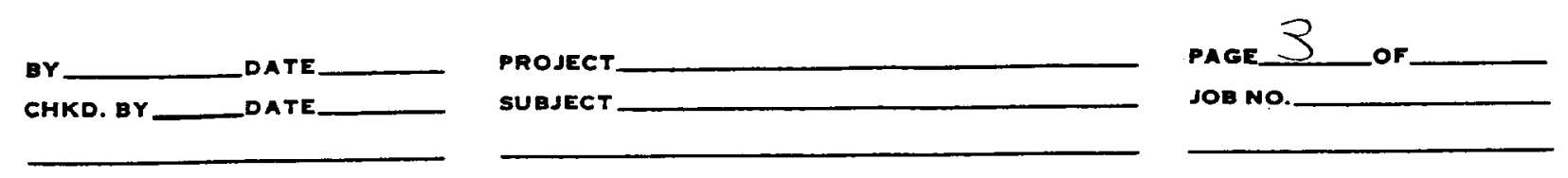

This wer dme ar a side step in use in the Tank analupir

Ple sazos) Progum PtrkfDir, MCD calcwite Qhe disibutin when bor dreeter-tordrisetern and peaf-to-feok vared inize ar present

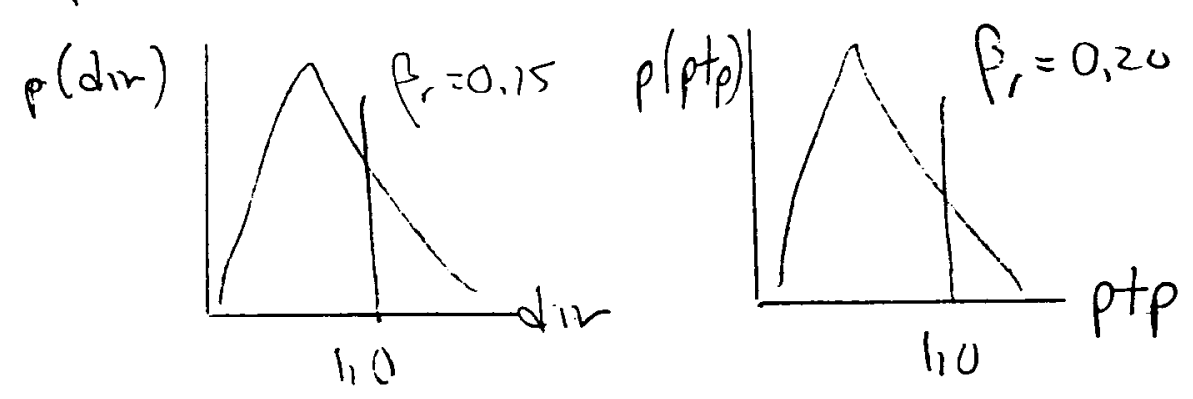

Qhe conbros parameten $M(d, r)$ etp hes a lognorna (goos) $f t$ ) distributin win parameters

$$
\begin{aligned}
& \mathscr{F}=1.11 \quad \text { whore: } M\left(d_{i r}\right)=d_{i v} \quad d i r>1 \\
& \beta=0.24 \\
& =1 / d r \quad \operatorname{dir}<1
\end{aligned}
$$

Thus, O6 84 NeP moximun componet pata is just: $F_{84, \text { max }}=1,11 e^{.24}=1,4$

Therepre to covsent a HCLPF spotim form a median level to a 84 NEP maximun componat. HCLPF spetrem, jus multiplo a former by 1,4 .

Jack R. Benjamin \& Assoctates, Inc.
Consulting Engineers

C-167 
MCAD FILES FEAK.COM

Tis urcoram calculates the distribution for the maximum of two horizarital zomponents where only the maximum component variability is included

JOFMAL DISTFIEUTION

$1 \equiv .319381530$

$\supseteq \equiv-.356563782$

$3 \equiv 1.781477937$

$\dot{+} \equiv-1.821255978$

$5 \equiv 1.330274429$

$\eta \equiv .2316419$

$$
(x) \equiv \frac{\exp \left[\frac{2}{2}\right]}{2.506628275}
$$$$
a R(x) \equiv\left|(x>0)-Z(x) \cdot\left[b 1 \cdot t(x)+b e^{\prime} t(x)^{2}+b 3 \cdot t(x)^{3}+b 4 \cdot t(x)^{4}+b 5 \cdot t(x)^{5}\right]\right|
$$

INUEFSE NOFMAL DISTRIEUTION

$$
\begin{aligned}
& .0 \equiv 2.515517 \\
& 1 \equiv .802853 \\
& : 2 \equiv .010328 \\
& : 1 \equiv 1.432788 \\
& i 2 \equiv .189269 \\
& i 3 \equiv .001308
\end{aligned}
$$$$
(p) \equiv \sqrt{\ln \left[\frac{1}{2(p)-.5)-p)^{2}}\right]}
$$ 


$$
\operatorname{NOR}(p) \equiv(-1)^{p<\cdot 5}\left[t(p)-\frac{c 0+c 1 \cdot t(p)+c 5 \cdot t(p)^{2}}{1+d 1 \cdot t(p)+45 \cdot t(p)^{2}+d 3 \cdot t(p)^{3}}\right]
$$

$$
\begin{aligned}
& 1:=199 \\
& :=0 \ldots n
\end{aligned}
$$$$
\operatorname{jir}:=1.0 \exp \left[0.15 \cdot \operatorname{INOK}\left[\frac{i+r n d(1)}{n+1}\right]\right]
$$

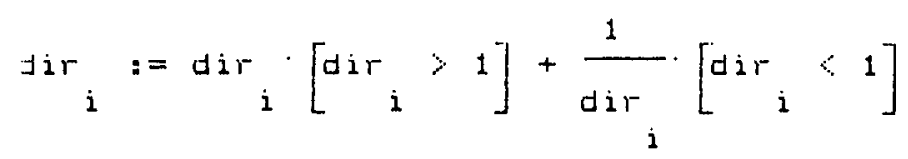

$$
\operatorname{nin}(d i r)=1.001
$$

$\operatorname{nax}(d i r)=1.534$

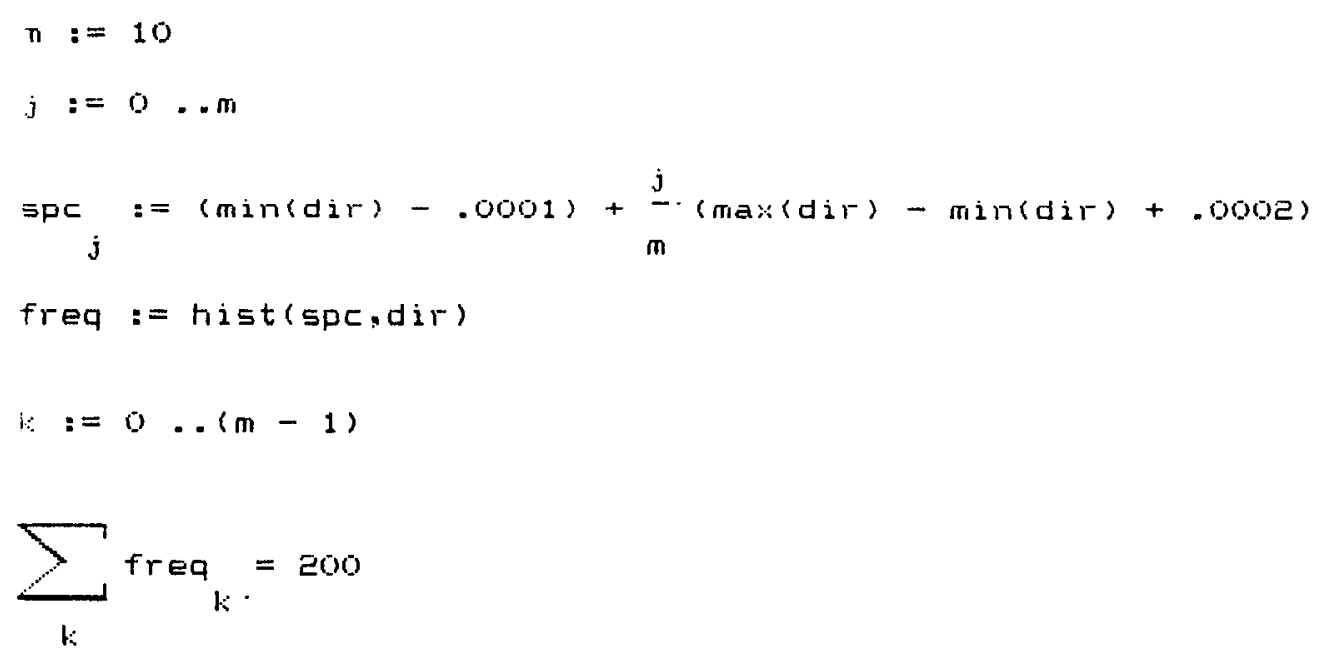




$$
\begin{aligned}
& { }_{0}^{[L m}:=0 \\
& \omega:=1 \ldots \mathrm{m} \\
& \operatorname{cum}_{p}:=\operatorname{cum}_{p-1}+\text { freq }_{p-1} \\
& \operatorname{cum}_{p}:=\frac{\frac{\operatorname{cum}}{n+1}}{n}
\end{aligned}
$$

\begin{tabular}{|c|c|c|}
\hline & cum & $5 P C$ \\
\hline$j$ & & \\
\hline 0 & 0 & 1.001 \\
\hline 1 & 0.275 & 1.054 \\
\hline e & 0.5 & 1.107 \\
\hline 3 & 0.68 & 1,161 \\
\hline 4 & 0.8 & 1.214 \\
\hline 5 & 0.985 & 1.267 \\
\hline 6 & 0.935 & 1.321 \\
\hline 7 & 0.97 & 1.374 \\
\hline 8 & 0.98 & 1.427 \\
\hline 9 & 0.99 & 1.48 \\
\hline 10 & 1 & 1.534 \\
\hline
\end{tabular}$$
s:=1 \text { spline (spc:cum) }
$$

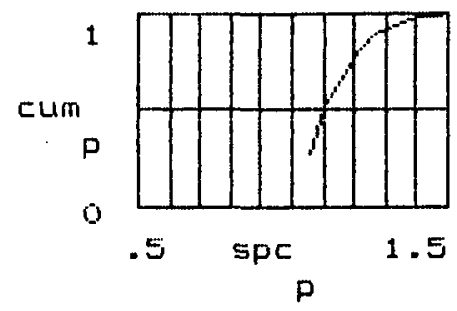

Find the median value:

$\because:=1.0$

Focit(interp $(s$, spe, cum,x) - .5,x) $=1.107$

Find the $+1 \sigma$ value:

vat(iriterp $(5, \operatorname{spc}$, cum,x) $-.84134, x)=1.237$

Find the - 10 value:

cot(interp $(5,5 p($, cum, $x)-.15866, x)=1.031$ 


$$
\begin{aligned}
& \operatorname{cum}_{j}:=\operatorname{cum}_{j} \\
& : \operatorname{spc}_{j}:=\operatorname{spc} c_{j} \\
& :=m-=1
\end{aligned}
$$

\begin{tabular}{|c|c|c|}
\hline & cum & SPC \\
\hline 0 & 0.275 & 1.054 \\
\hline 1 & 0.5 & 1.107 \\
\hline 2 & 0.68 & 1.161 \\
\hline 3 & 0.8 & 1.214 \\
\hline 4 & 0.885 & 1.262 \\
\hline 5 & 0.935 & 1.321 \\
\hline 6 & 0.97 & 1.374 \\
\hline 2 & 0.98 & 1.427 \\
\hline 8 & 0.99 & 1.48 \\
\hline
\end{tabular}

This program take the calculated probabilities of failure and the associated peak grcund acceleration values and performs a least squares fit in the log-probability domain to obtain the "best fit" median and $\beta$ value.

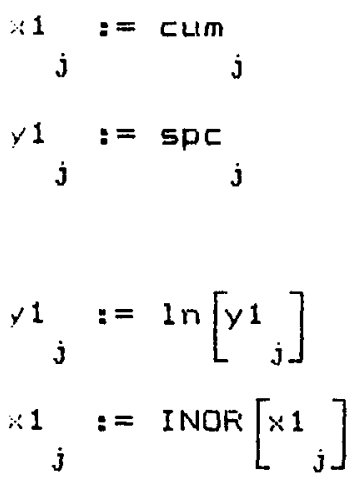



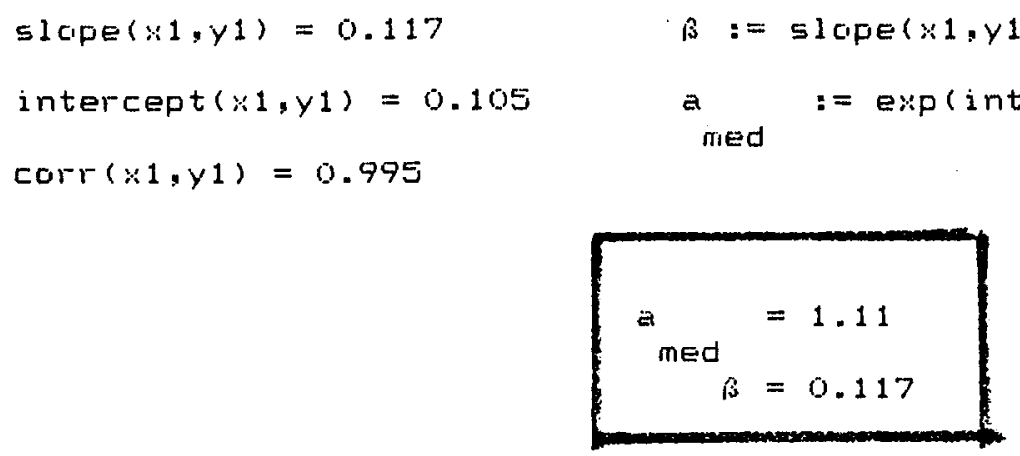

$\because 1_{j}:=$ intercept $(\times 1, y 1)+\operatorname{slcpe}(\times 1, y 1) \cdot \times 1$

$$
\begin{aligned}
& \operatorname{nax}\left(y_{1}\right)=0.392 \\
& \operatorname{nin}(y 1)=0.053 \\
& \operatorname{nin}(=1)=0.034 \\
& \operatorname{nax}(\times 1)=2.327 \\
& \operatorname{in}\left(x_{1}\right)=-1.514
\end{aligned}
$$

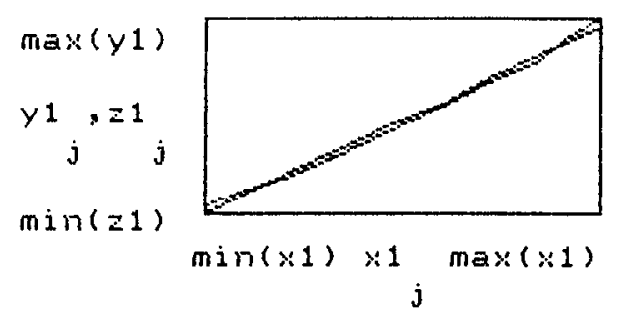

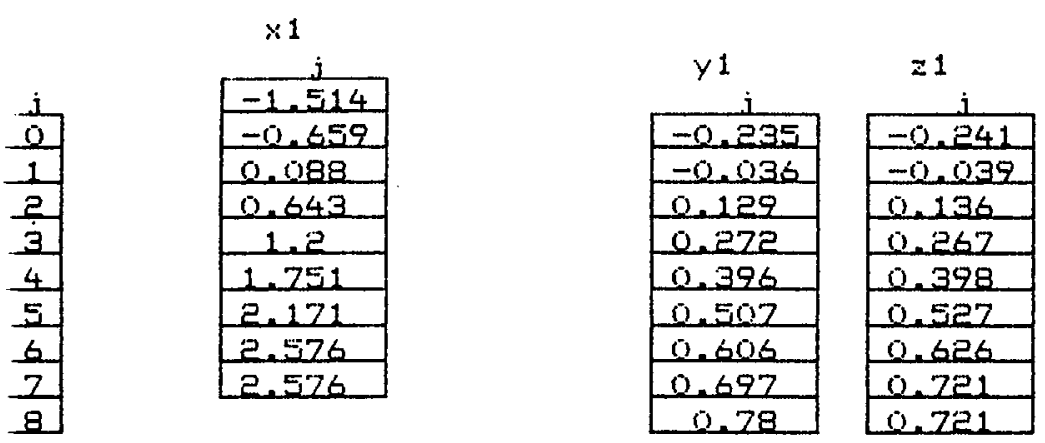

Tatulate protatilities to compare calculated and best fit values:

$$
\begin{aligned}
& :=\exp \left[\begin{array}{ll}
y_{1} & \\
& j
\end{array}\right] \\
& :=\operatorname{NOF}\left[\begin{array}{ll}
\times 1 & \\
& j
\end{array}\right]
\end{aligned}
$$

Feat grcund accelerations

Prcitability of failure values 


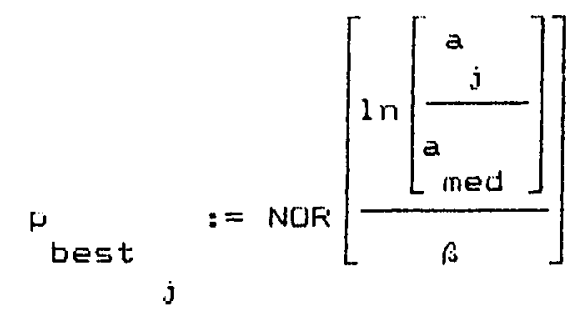

Best fit probability of failure values

a

\begin{tabular}{|l|}
\hline$j$ \\
\hline 0 \\
\hline 1 \\
\hline 2 \\
\hline 3 \\
\hline 4 \\
\hline 5 \\
\hline 6 \\
\hline 7 \\
\hline 8 \\
\hline 0.791 \\
\hline 1.138 \\
\hline 1.312 \\
\hline 1.486 \\
\hline 1.66 \\
\hline 1.833 \\
\hline 2.007 \\
\hline 2.181 \\
\hline
\end{tabular}

$p$

best

\begin{tabular}{r|}
0.068 \\
\hline 0.259 \\
\hline 0.524
\end{tabular}

0.524

0.747

0.884

0.252

0.982

0.993

0.998 


\section{MCAD FILES DIFiREAK.MCD}

This program calculates the distribution for the maximum of two horizontal components where both the peak-to-peak and maximum component variability are included

NOFMAL DISTFIEUTION

$$
\begin{aligned}
& =1 \equiv .317381530 \\
& د 2 \equiv-.356563782 \\
& =3 \equiv 1.781477937 \\
& 54 \equiv-1.821255978 \\
& =5 \equiv 1.330274429 \\
& P \equiv .2316419
\end{aligned}
$$$$
Z(x)=\frac{\exp \left[\frac{2 x}{2}\right]}{2.506628275}
$$$$
=(x) \equiv \frac{1}{1+p \cdot|x|}
$$$$
\operatorname{VOR}(x) \equiv\left|(x>0)-z(x) \cdot\left[b 1 \cdot t(x)+b 2 \cdot t(x)^{2}+b 3 \cdot t(x)^{3}+b 4 \cdot t(x)^{4}+b 5 \cdot t(x)^{5}\right]\right|
$$

INVERSE NOFMAL DISTFIBUTION

$$
\begin{aligned}
& =0 \equiv 2.515517 \\
& =1 \equiv .802853 \\
& : 2 \equiv .010328 \\
& 11 \equiv 1.432788 \\
& 12 \equiv .189267 \\
& : 3 \equiv .001308
\end{aligned}
$$

$$
(p) \equiv \sqrt{\ln \left[\frac{1}{((p) .5)-p)^{2}}\right]}
$$




$$
\begin{aligned}
& \operatorname{NOF}(p) \equiv(-1)^{p<.5} \cdot\left[t(p)-\frac{c 0+c 1 \cdot t(p)+c 2 \cdot t(p)^{2}}{1+d 1 \cdot t(p)+d)^{2} t(p)^{2}+d 3 \cdot t(p)^{3}}\right] \\
& :=199 \\
& :=0 \quad \ldots \\
& t_{i}:=1.0 \cdot \exp \left[0.20 \cdot \operatorname{INOF}\left[\frac{i+r n d(1)}{n+1}\right]\right] \\
& \operatorname{Jir}=1.0 \cdot \exp \left[0.15 \cdot \operatorname{INOR}\left[\frac{i+r n d(1)}{n+1}\right]\right]
\end{aligned}
$$

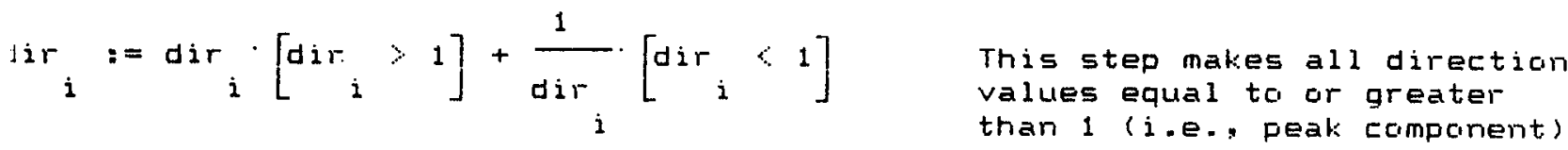

$$
\begin{aligned}
& \operatorname{lin}(d i r)=1.001 \\
& \text { iax }(d i r)=1.534 \\
& \text { JFITE(DIR) }:=\text { dir } \\
& \text { JRITE (PTF) }:=p t_{i}^{i}{ }_{i}
\end{aligned}
$$

\begin{tabular}{|c|c|}
\hline dir & $p t p$ \\
\hline$\frac{c}{1.24}$ & $\frac{c}{1.131}$ \\
\hline 1.01 & 0.903 \\
\hline 1.181 & 1.12 \\
\hline 1.17 & 0.8 \\
\hline 1.032 & 0.814 \\
\hline
\end{tabular}

Fandomly mix both the direction and the two peak-to-peak arrays EXIT TO DOS and run CADMIX

$$
\begin{aligned}
& \operatorname{jir}_{i}:=\operatorname{READ}(D I R) \\
& \operatorname{itP}_{i}:=\operatorname{READ}(\mathrm{FTF})
\end{aligned}
$$$$
\text { c }:=0 . .4
$$ 


$$
\begin{aligned}
& \operatorname{Jir}_{i}:=\operatorname{dir} \operatorname{ptp}_{i} \\
& n i n(d i r)=0.617 \\
& \operatorname{nax}\left(d_{i r}\right)=2.354 \\
& n:=10 \\
& j:=0 \ldots m \\
& \text { spe }:=(\min (d i r)-.0001)+\frac{j}{m}(\max (d i r)-\min (d i r)+.000 z) \\
& \text { FrEq := hist(spc,dir) } \\
& \therefore:=0 \ldots(m-1) \\
& \sum_{k} \text { freq }=200 \\
& \operatorname{um}_{0}:=0 \\
& j:=1 \ldots m \\
& \underset{p}{\operatorname{cum}}:=\operatorname{cum}_{p-1}+\underset{p-1}{\text { freq }} \\
& =\lim _{p}:=\frac{p}{n+1}
\end{aligned}
$$

$$
\equiv:=1 \text { spline(spc.cum) }
$$
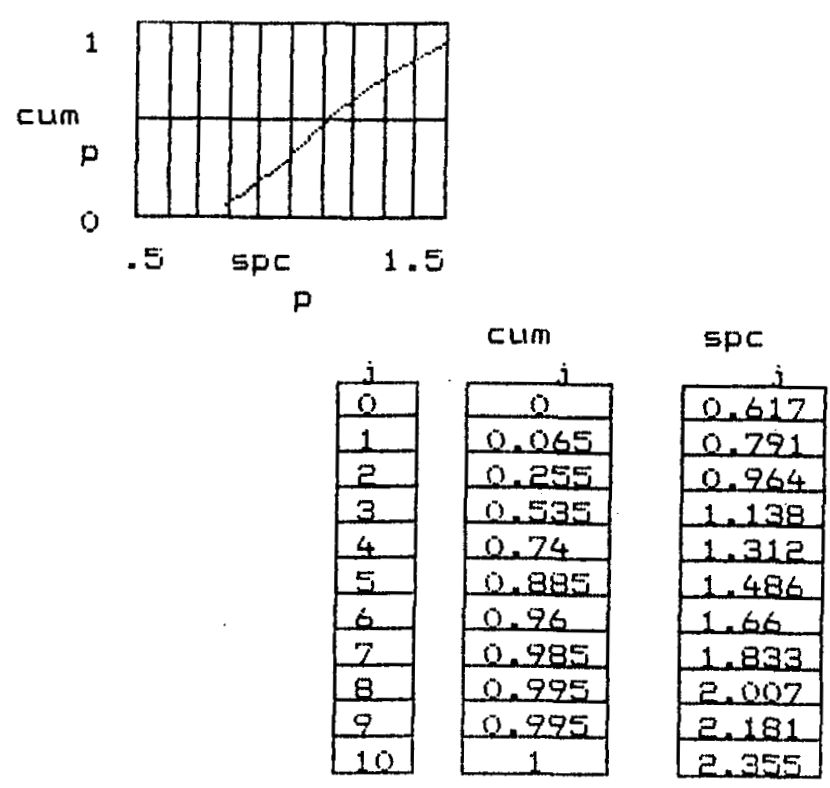


\section{Find the median value:}

$x:=1.0$

rout $($ interp $(5$, spc, (um, $x)-.5, x)=1.115$

Find the + io value:

rocitirterp $(s$, spc.cumi.x) - .84134,x)=1.425

$$
s=\frac{1}{2} \sin \frac{1.42)^{-}}{1892}=0.27
$$

Find the - 1o value:

roctinterp $(5.5 p($, cum, $x)-.15866, x)=0.992$

$\operatorname{tcum}_{\mathfrak{j}}:=\operatorname{cum}_{j}$

$\operatorname{tspc}_{j}:=\operatorname{spc}{ }_{j}$

$m:=m-2$

$5:=1$

$j:=0 \ldots m$

$\operatorname{cum}_{j}=$ tcum $_{j+1}$

$\operatorname{spc}:=\operatorname{spc}$

j $j+1$

\begin{tabular}{|c|c|c|}
\hline & cum & SFC \\
\hline & & $i$ \\
\hline () & 0.065 & 0.791 \\
\hline 1 & 0.255 & 0.964 \\
\hline 2 & 0.535 & 1.138 \\
\hline 3 & 0.74 & 1.312 \\
\hline 4 & 0.885 & 1.486 \\
\hline 5 & 0.96 & 1.66 \\
\hline 6 & 0.985 & 1.833 \\
\hline 7 & 0.995 & 2.007 \\
\hline 8 & 0.995 & 2.181 \\
\hline
\end{tabular}




\begin{tabular}{|c|c|c|c|}
\hline & $\because 1$ & y1 & $\geq 1$ \\
\hline$i$ & & $i$ & i \\
\hline 0 & $-1,514$ & -0.235 & -0.241 \\
\hline 1 & -0.652 & -0.036 & $-0,039$ \\
\hline 2 & 0.089 & 0.129 & 0.136 \\
\hline 3 & 0.643 & 0.272 & 0.267 \\
\hline 4 & 1.2 & 0.396 & 0.398 \\
\hline 5 & 1.251 & 0.507 & 0.522 \\
\hline 6 & 2.171 & 0.606 & 0.620 \\
\hline 7 & 2576 & 0.697 & 0.721 \\
\hline 8 & 2.576 & 0.28 & 0.721 \\
\hline
\end{tabular}

Tabulate probabilities to compare calculated and test fit values:

$a_{j}:=\operatorname{Exp}\left[\begin{array}{ll}Y 1 \\ & j\end{array}\right]$

$\partial_{j}:=\operatorname{NOF}\left[\begin{array}{ll}x & \\ & j\end{array}\right]$

$\operatorname{vist}_{\text {best }}:=\operatorname{NDF}\left[\frac{\left[\begin{array}{l}a \\ a \\ \text { med }\end{array}\right]}{\beta}\right]$
Feak grcund accelerations

Frotatility of failure values

Best fit probability of failure values

\begin{tabular}{|c|c|}
\hline i & ${ }^{a}$ \\
\hline 0 & 0.791 \\
\hline 1 & 0.964 \\
\hline 2 & 1.138 \\
\hline 3 & 1.312 \\
\hline 4 & 1.486 \\
\hline 5 & 1.6 \\
\hline 6 & 1.833 \\
\hline 7 & 2.007 \\
\hline 8 & 2. 181 \\
\hline
\end{tabular}

p

\begin{tabular}{|l|}
\hline 0.065 \\
\hline 0.255 \\
\hline 0.535 \\
\hline 0.74 \\
\hline 0.885 \\
\hline 0.76 \\
\hline 0.985 \\
\hline 0.295 \\
\hline 0.995 \\
\hline
\end{tabular}

p best

\begin{tabular}{|l|}
\hline 0.068 \\
\hline 0.259 \\
\hline 0.524 \\
\hline 0.742 \\
\hline 0.884 \\
\hline 0.252 \\
\hline 0.982 \\
\hline 0.993 \\
\hline 0.998 \\
\hline
\end{tabular}


Appendix $C$

This appendir deceriber a simple prosodur

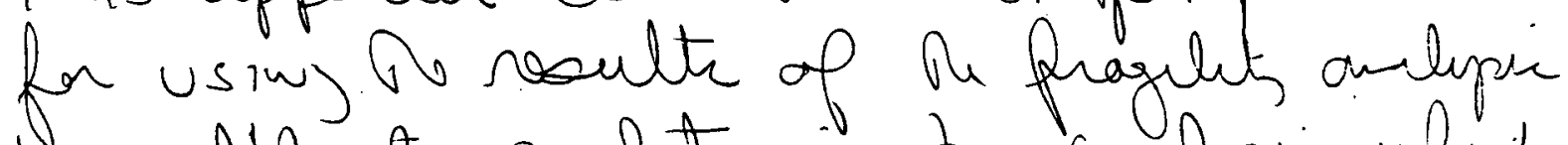
to calibrat a deterministic curalupir ulved prosdveer he same NCLPF on obtarres vsing ar fraglity appooch. This is a bachlit technique. "Carbage in gures gentrage bxck" The procodure simply sxys tat if yo have of resulte of a fragitity aralizeis yo can use fien to decid ulat cmserseten to put in eoch parameter (e.g.inespouse spechm NEP level, allowate stress, danping, ete.) in orden to pufom a singl determunistic analuper to produe Vis same NCLPF. This is a permal approacl for reconciling the CDFM and FA predures.

A proof that de proedure should wolk is given followin, be preaden stepe

Jack R. Benjamin \& Associates, Inc. Consulting Engineers

$C-180$ 
BY

CHKD. BY DATE

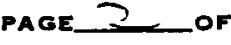

JOE NO.

PROCEOURE STEPS

Sh following stape are peformed to obtain are parameter values to perfom a singe determinete araluper to obtain the same NCLPF as calculated from he FA approded:

1 Calculate $1.65\left(\beta_{r}+\beta_{u}\right)$. This is Qu target conservatesm.

2. For edel of th respmes citegrier (e.s. strengih dvetility, isulding dompin, exupunat dompingete) assign a part dob tongit conservatem.

Note: guve ol most to Phore categorie that have in biggost catogory P's

3. For eas a re indiudual categry pents divide she portion of hie tanget cmeervation

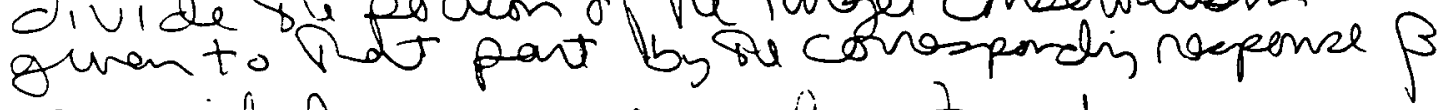
e.s. If to consouratim for strengch is assigint 0.33 and $n$ conespordin $\beta$ is 0.20 hem, $\gamma$ is jest:

$$
\gamma=\frac{0.33}{20}=1.65
$$

Jack R. Benjamin \& Associates, Inc. Consulting Engineers

C-181 


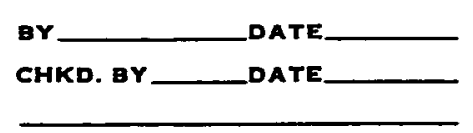

PROJECT.

PACE 3 OF

JOB NO.

4. Simply go to lese of the corresperdin perameter ad sebet he same leved of cmoenation guven by 0 re $\gamma^{\prime}$ s e.s for strenget, sebet te allowable strese at on $1.65 \sigma$ lerel, i.e

$$
\text { stren }=v^{J} e^{-1.65 \beta_{s} \sin x}
$$

5 Shen perform hi deterministrc aralypi using the caleulated parancter value

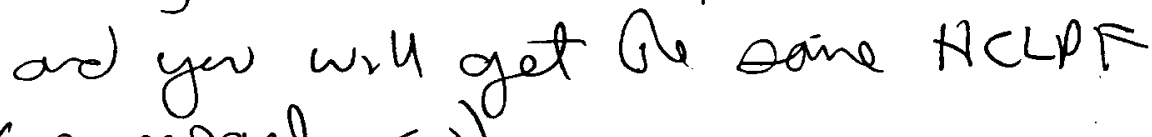
(or mearly so)

Jack R. Benjamin \& Associates, Inc. Consulting Engineers

C-182 
PROOF

Reaponse, $\mathbb{R}$

$$
\begin{aligned}
& \text { Parameters, } x_{i} \quad \Gamma^{\text {Vectornotation }} \\
& R=f\left(x_{1}, x_{2}, \ldots, x_{n}\right)=f(\underline{x}) \\
& \tilde{R} \cong f\left(\check{x}_{1}, \breve{x}_{2}, \ldots, \breve{x}_{N}\right)=f(\underline{\tilde{x}})
\end{aligned}
$$

Now cateulat the vesponse at some confideree level, i.e.

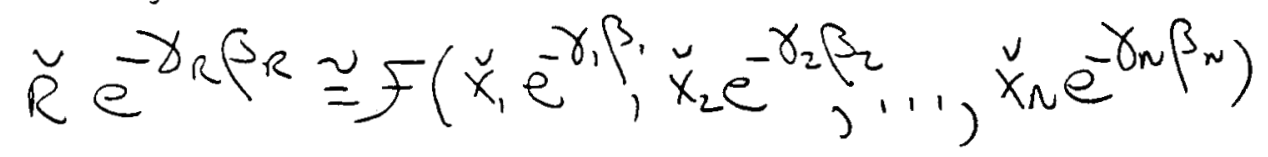

Te questem at Mis point is: what one be $\gamma_{i}$ 's?

Defue: $\gamma_{R} \beta_{R}=\gamma_{R_{1}} \beta_{R_{1}}+\gamma_{R_{2}} \beta_{R_{2}}+\cdots+\gamma_{R_{N}} \beta_{r_{N}}$

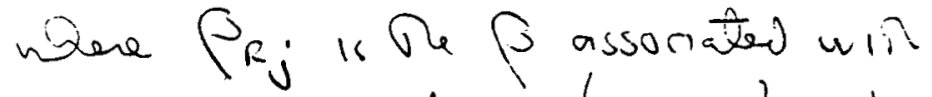
responce typrej (e.s. stranget and buildin $\nu_{R_{j}}$ is he associated level of onsenation

Note: Dt is clean dat frone is a lange number of combination of $\gamma_{R_{j}}$ Rat con sotis $f_{7}$ a defuntion

Jack R. Benjamin Associotes, Inc. Consulting Engineers

C-183 


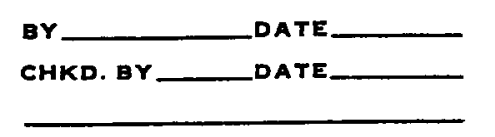

Cemonder: $\beta_{e}=\left[\sum f^{2} R_{j}\right]^{1 / 2}$

Now expardin (1) by subsoituting de defunter into or left side of egn 1 ad makin an apporimation to ive rieit side (typecalls went is done in isersmic PRA calealaterm).

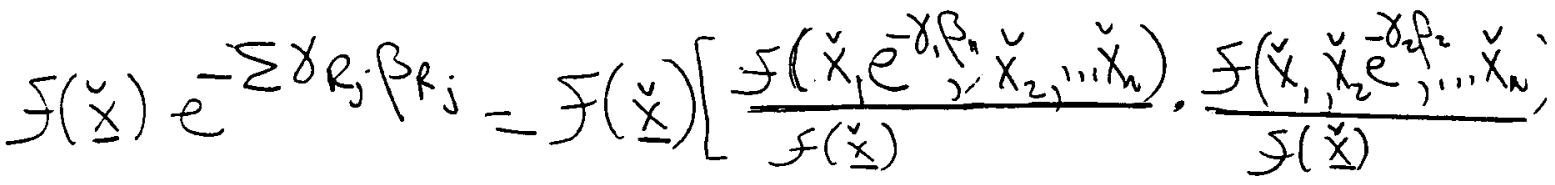

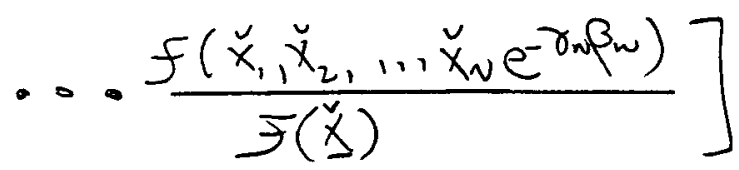

but:

$$
f\left(\tilde{x}_{1}, \tilde{x}_{i}, \ldots \dot{x}_{i} e^{-\gamma_{i}\left(\beta_{i}\right.}, \ldots, \tilde{x}_{N}\right) \cong f(\underline{x}) e^{-\gamma_{i} \beta_{i} R_{i}}
$$

heree (shis how gav get $\beta_{R_{i}}$ )

$$
\sum \gamma_{R_{j} \beta_{e_{j}}}=\sum \gamma_{i} \beta_{R_{i}}
$$

ore solution is simply $\gamma_{i}=\gamma_{R_{i}}$

Thus 16 defunch $\gamma_{R} \beta_{R}=\sum \gamma_{R} \beta_{\dot{R}_{i}}$ and usius $\gamma_{i}=\gamma_{R i}$ The prodede steps follow

Jack R. Benjamin \& Associates, Inc. Consulting Engineers

$\mathrm{C}-184$ 


\title{
An Approach to Comparing the CDFM and FA Methods
}

\author{
by John W. Reed
}

An approach is presented to compare and reconcile the results from the HCLPF calculations using the CDFM and FA methods. The purpose for performing a reconciliation is to identify where conservatism is placed in the CDFM analysis and to obtain a measure of influence of each of the basic parameters relative to the conservatism in the HCLPF capacity. The approach is based on the procedure that $\mathrm{J}$. W. Reed used to obtain backfit CDFM results from the FA analysis, which is documented earlier in this appendix.

The approach is based on two fundamental results. First, the number of standard deviations, $\gamma$, between the value of a basic parameter (e.g., damping, response spectrum input, or member strength) used in the CDFM analysis and the corresponding median value (in a multiplicative sense, since the underlying distribution is assumed to be lognormal) is the same $\boldsymbol{\gamma}$ conservatism in the HCLPF capacity due to that basic parameter.

Second, the following relationship is true when the HCLPF values from the CDFM and FA methods are the same:

$$
\sum \gamma_{\mathrm{i}} \beta_{\mathrm{CAP}} \mathrm{j}=1.65\left(\beta_{\mathrm{r}}+\beta_{\mathrm{u}}\right)
$$

where:

$\gamma_{j}$ : Number of standard deviations between the basic parameter $i$ and its median value (in a multiplicative sense, since the underlying distribution is lognormal).

BCAPj: the logarithmic standard deviation of the final capacity due to the variability in basic parameter $i$. 
$\beta_{r}, \beta_{\mathrm{U}}$ : The total randomness and uncertainty logarithmic standard deviations for the final capacity, respectively.

The procedure to reconciling the results from the CDFM and FA methods consist of the following steps:

1. Identify the equation which relates the basic parameters to the final capacity of the component. Note that in using this approach the reconciliation can be performed only if the same equation is used in both the CDFM and FA calculations.

2. Using a Taylor series expansion of the final capacity equation, and a second moment approximation, obtain the median and logarithmic standard deviation values of the final capacity in terms of the statistics of the basic parameters (i.e., median and $\beta$ ).

3. For each basic parameter value, $\operatorname{CDFM}_{j}$, assumed in the CDFM analysis, and the corresponding statistics for that basic parameter obtained in the FA analysis, calculate the $\gamma_{j}$ values as follows:

$$
\gamma_{i}=\left|\frac{1}{\beta_{i}} \ln \left(\frac{\operatorname{CDFM}_{i}}{\text { Median }_{j}}\right)\right|
$$

4. From the equation for the logarithmic standard deviation of the capacity, $\beta$ CAP, in terms of the basic parameter statistics, calculate $\beta$ CAPi which is the $\beta$ in capacity due to the variability in basic parameter $i$. This step can be performed in terms of the combined basic parameter variability or in terms of the separate randomness and uncertainty parts (i.e., $\beta_{r_{j}}$ and $\left.\beta_{u_{j}}\right)$. The only constraint is that the variability of all the basic parameters, either combined or separate, must be accounted for. 
5. As a check, $\sum \gamma_{j} \beta_{C A P i}$ should be essentially equal to $1.65\left(\beta_{r}\right.$ and $\beta_{\mathrm{u}}$ ) as described above (see equation 1 ). In general the two parts will not be exactly equal because the second moment approach is only approximate.

From these results, the $\boldsymbol{\gamma}_{\boldsymbol{i}}$ values indicate the level of conservatism assumed in the CDFM analysis for each of the basic parameters. The ratio $\gamma_{j} \beta_{\mathrm{CAPi}} / 1.65\left(\beta_{\mathrm{r}}+\beta_{\mathrm{u}}\right)$ is the fraction of the total conservatism contributed by the ith parameter value used in the CDFM method.

\section{Example}

The calculations by R. P. Kennedy for the Starting Air Tank are used as an example to demonstrate a reconciliation between the CDFM and FA approaches for calculating the HCLPF capacity (see Appendix A for Kennedy's calculations). The governing equation for the capacity of the Starting Air Tank follows:

$$
\mathrm{CAP}=\frac{28.8 \mathrm{Tu}+11.3}{38.2 \mathrm{AF}_{\mathrm{H}}+11.3 \mathrm{~F}_{\mathrm{V}}} \text { (units: } \mathrm{g} \text { ) }
$$

$$
\text { where } \quad \begin{aligned}
& \mathrm{Tu}=\text { Angle capacity (units: kips) } \\
& \mathrm{AF}_{H}=\text { Horizontal response factor (unitless) } \\
& \mathrm{F}_{\mathrm{V}}=\text { Combined vertical response factor (unitless) }
\end{aligned}
$$

Note that the constants in the equation contain the appropriate units so that the capacity is given in terms of acceleration (i.e., gravity units). Using a Taylor series expansion and the second moment approach, the median and logarithmic standard deviation for capacity in terms of the basic parameters follow: 
Median:

$$
\check{C A P}=\frac{28.8 \mathrm{Tu}+11.3}{38.2 \mathrm{AFH}_{\mathrm{H}}+11.3 \mathrm{FV}_{\mathrm{V}}}
$$

Logarithmic Standard Deviation (squared):

$$
\begin{aligned}
\beta_{C A P}^{2}= & \left(\frac{28.8 \check{T u}}{28.8 \check{T u}+11.3}\right)^{2} \beta_{T u}^{2}+\left(\frac{38.2 \check{A F}_{H}}{38.2 \check{A F_{H}+11.3 \check{F}_{V}}}\right)^{2} \beta_{A F_{H}}^{2} \\
& +\left(\frac{11.3 \check{F_{V}}}{38.2 \check{A F}_{H}+11.3 \check{F}_{V}}\right)^{2} \beta_{F_{V}}^{2}
\end{aligned}
$$

Using the fragility parameters obtained by Kennedy and the above

\begin{tabular}{|c|c|c|c|c|}
\hline Parameter & Median & $\beta_{r}$ & $\beta_{u}$ & $\beta_{C}$ \\
\hline $\begin{array}{c}\mathrm{Tu} \\
\mathrm{AF}_{\mathrm{H}} \\
\mathrm{F}_{\mathrm{V}}\end{array}$ & $\begin{array}{l}3.34 \\
2.51 \\
0.32\end{array}$ & $\begin{array}{c}- \\
0.22 \\
0.53\end{array}$ & $\begin{array}{l}0.16 \\
0.22 \\
0.19\end{array}$ & $\begin{array}{l}0.16 \\
0.31 \\
0.56\end{array}$ \\
\hline CAP & $1.08 \mathrm{~g}$ & 0.21 & 0.26 & 0.33 \\
\hline
\end{tabular}
equations the final results are summarized below for the fragility analysis.

Note that the capacity values differ slightly from Kennedy's results since he obtained the capacity values by direct simulation rather than the second moment method. The HCLPF capacities obtained by the two approaches are shown below.

Method HCLPF

$$
\begin{array}{cll}
\text { CDFM } & 0.48 & 1.08 \mathrm{e}^{-1.65(0.21+0.26)}
\end{array}
$$

Since the HCLPF values by the two approaches differ slightly the $\sum_{\gamma \beta}$ value must be adjusted before reconciling the results as follows: 


$$
1.08^{-\sum \gamma \beta}=0.48 \cdots>\sum \gamma \beta=0.81
$$

Table 1 gives the results of reconciling the CDFM and FA approaches. The first three columns give the basic parameters and the results Kennedy obtained in his analyses using the two approaches. The $\beta_{C}$ values are obtained by the SRSS combination of the $\beta_{r}$ and $\beta_{U}$ values. The fourth column gives logarithmic standard deviations of the final capacity from each of the basic parameters using equation 5 . Note that the three terms in the parentheses of equation 5 represent influence coefficients for the effects of the basic parameters on the variability of the Starting Air Tank capacity. Both $T u$ and $A_{H}$ (i.e., the angle capacity and horizontal earthquake effect, respectively) have potentially significant influence, but $F_{V}$ (i.e., the vertical earthquake effect) has little influence.

The fifth column gives the $\gamma$ values, which are the number of standard deviations (a measure of conservatism) assumed in the CDFM approach for each of the basic parameters. They come from the standard relationship for the lognormal distribution which relates the number of standard deviations. which a value is away from the median (in a multiplicative sense) knowing the median and $\beta$ (see equation 2).

The sixth column gives the ${ }^{\gamma}{ }_{\text {CAP }}$ value corresponding to each basic parameter using the results from columns four and five. Finally, the last column gives the probability level (which is a measure of the level of conservatism) assumed for each basic parameter used in the CDFM approach.

Based on the results in column six, most of the contribution to the conservatism in the HCLPF capacity using the CDFM approach comes from the angle capacity, Tu, and the horizontal response factor, AFH. Almost none comes from the vertical earthquake component. This last result is to be expected since the vertical component has little potential influence on the tank capacity. 
From the probability level results it is seen that the horizontal and vertical earthquake component values used in the CDFM analysis are only slightly conservative ( 0.50 would be median) while a very large factor of conservatism was introduced in the angle capacity value. Finally, Table 1 shows that the $\sum_{\gamma \beta}$ value calculated (i.e., 0.83 ) is close to the target value of 0.81 . This slight difference is expected since the second moment approach used is approximate. 


\section{Table 1 Peconciliation Between COFM and FA Approaches}

Basic Parameter CDFM Value FA Parameters $\beta_{\text {CAP }}-\gamma \quad \begin{gathered}\gamma \beta_{\text {CAP }} \\ \text { (fraction of total) Prob. Level }\end{gathered}$

$$
\gamma=\frac{1}{0.16} \ln \left(\frac{3.34}{1.79}\right)
$$

Angle Capacity, Tu

$$
\begin{aligned}
\text { Tu } & =3.34 \\
\beta_{\mathrm{C}} & =0.16
\end{aligned}
$$

$(0.89)(0.16)=0.14$

$$
\gamma=3.90
$$

$(0.14)(3.90)=\frac{0.55}{(.66)} \quad \Phi(3.90)=0.99995$

Horizontal Response

Factor, $\mathrm{AF}_{\mathrm{H}}$

$$
\begin{aligned}
& 3.33 \quad \begin{array}{llll}
\mathrm{AF}_{\mathrm{H}}=2.51 & (0.96)(0.31)=0.3 & \gamma=0.91 & (0.30)(0.91)=0.27 \\
\beta_{\mathrm{C}}=0.31 & & (.33)
\end{array} \quad \Phi(0.91)=0.82 \\
& \gamma=\frac{1}{0.56} \ln \left(\frac{0.44}{0.32}\right)
\end{aligned}
$$

Canbined Vertical $(1.11)(0.40) \quad F_{V}=0.32$

Response Factor, $F_{V}=0.44 \quad \beta_{C}=0.56$

$$
\gamma=\frac{1}{0.16} \ln \left(\frac{3.33}{2.51}\right)
$$

$(0.40)(0.56)=0.02$ 


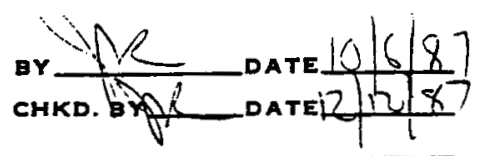

APPENDIX D

This appendix guree listivjs of the responce spectan used in the araluper

pares

2-4 Novizontol apoun response specha $(3,5,7$, as $10 \%$ dempin $)$

5-7 Norizuntal flon respenre spectra $(2,345,4,55$ or $7 \%$ comping) 8-11 Averaged Acr zontwo Plor response speta
$(2,3,5,4,5$, ond $7 \%$ dompin $)$

12-15 Vertea flon vesponse spectra $(2,3.5,4,5$ and $7 \%$ damping

Jack R. Benjamin \& Associates, Inc. Consulting Engineers

C-192 
MCAD FILES GSFECTFA. CFH

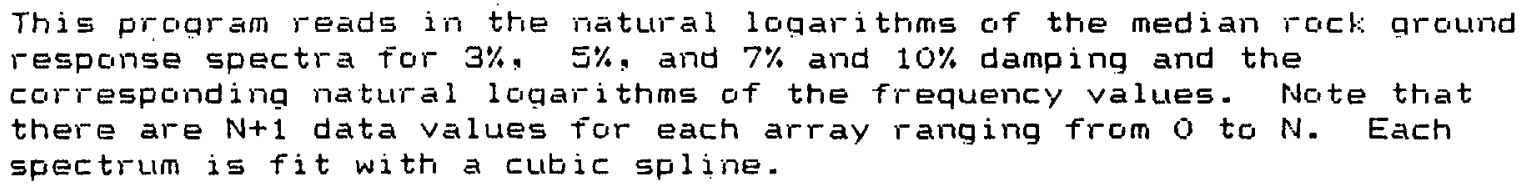

HORIZONTAL DIFECTION

$N:=40$

$i:=0 \ldots N$

Fiead in frequency values:

$X L F F 3:=$ FEAD $(X L F F 3)$

i

$X_{i}:=$ FEAD $(X L F F I S)$

$X L F R 7:=$ READ $(X L F F 7)$

i

XLFF:10 := READ $(X L F F 10)$

i

Fiead in spectral values:
FMEDFSFC. $3 \%$ cantains the Ins of the frequencies corresponding to the $3 \%$ Sa values

FMEDRSFC. $5 \%$ contains the lns of the frequencies correspanding to the $5 \%$ Sa values

FMEDFSFC. $7 \%$ contains the ins of the frequencies curresponding to the $7 \%$ Sa values

FMEDFSFC. $10 \%$ contains the Ins of the frequencies corresponding to the $10 \%$ Sa values

MEDFSFC. $3 \%$ contains the Ins of the $3 \%$ Sa values MEDFISFC. $5 \%$ contains the lns of the $5 \%$ Sa values MEDFSFC. $7 \%$ contains the ins of the $7 \%$ Sa values MEDFSFC. $10 \%$ contains the Ins of the $10 \%$ Sa vallues

S3:= cSF 1 ine (XLFR3,XSA3)

$55:=$ ᄃSF 1 ine (XLFR5, XSAS)

$57:=$ [SP 1 ine (XLFR7, XSA7)

$510:=\operatorname{csp} 1$ ine (XLFFi $10, \times$ SA10) 
r. $:=1 \ldots 1200$

$\exp \left[\right.$ interp $\left[55\right.$, XLFRS, XSAS, in $\left.\left.\left[\frac{k}{20}\right]\right]\right]$

10
Example 5\% damped ground respanse spectrum scaled to $1.0 g$ pga

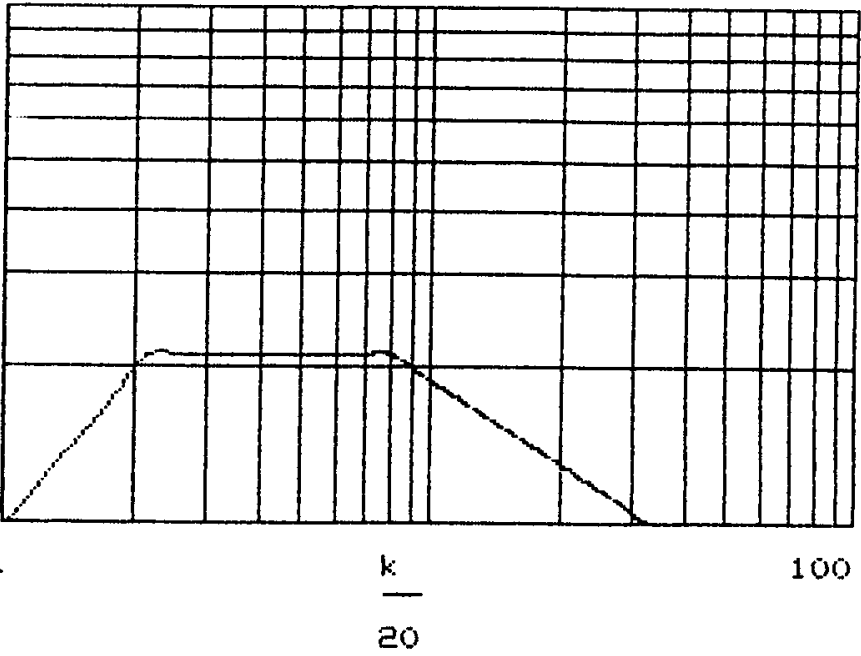

$j:=0 \quad . .40$

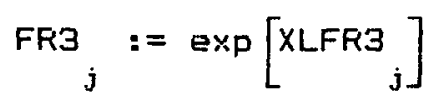

$\mathrm{SA}_{j}:=\exp \left[\mathrm{XSA3}_{j}\right]$

$\mathrm{FRS}_{j}:=\exp \left[\right.$ XLFRS $\left._{j}\right]$

$\operatorname{SAS}_{j}:=\exp \left[\begin{array}{ll}X_{j} & \\ & \\ & \end{array}\right]$

FR7 ${ }_{j}:=\exp \left[\begin{array}{rl}X L F R 7 \\ j\end{array}\right]$

SA7 $j_{j}=\exp \left[\begin{array}{ll}X S A 7 \\ \\ \end{array}\right]$

$F P_{j}:=\exp \left[\begin{array}{ll}X L F 10 \\ j\end{array}\right]$

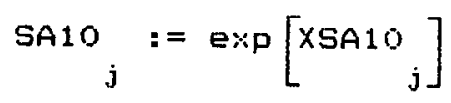




\begin{tabular}{|c|c|c|c|c|c|c|c|}
\hline FFI & SA3 & FF:S & SAS & FFT & SA7 & FFi10 & SA1O \\
\hline 0.3 & 0.322 & 0.3 & 0.29 & 0.3 & 0.266 & 0.3 & 0.24 \\
\hline 0.362 & 0.4 & 0.366 & 0.354 & 0.365 & 0.323 & 0.364 & 0.291 \\
\hline 0.449 & 0.489 & 0.446 & 0.431 & 0.444 & 0.393 & 0.441 & 0.353 \\
\hline 0.55 & 0.579 & 0.545 & 0.526 & 0.54 & 0.478 & 0.534 & 0.427 \\
\hline 0.673 & 0.733 & 0.664 & 0.642 & 0.657 & 0.582 & 0.548 & 0.518 \\
\hline 0.824 & 0.897 & 0.811 & 0.783 & 0.0 & 0.708 & 0.785 & 0.628 \\
\hline 1.008 & 1.098 & 0.999 & 0.255 & 0.973 & 0.861 & 0.252 & 0.761 \\
\hline 1.234 & 1.343 & 1.204 & 1.165 & 1.184 & 1.048 & 1.154 & 0.923 \\
\hline 1.51 & 1.644 & 1.472 & 1.422 & 1.44 & 1.275 & 1.399 & 1.119 \\
\hline 1.842 & 2.012 & 1.795 & 1.734 & 1.752 & 1.551 & 1.626 & 1.356 \\
\hline 2.263 & 2,463 & 2.19 & 2.116 & 2.131 & 1.887 & 2.056 & 1.644 \\
\hline 2.567 & 2.463 & 2.423 & 2.116 & 2.433 & 1.887 & 2.356 & 1.544 \\
\hline 2.913 & 2.463 & 2.838 & 2.116 & 2.777 & 1.887 & 2.698 & 1.644 \\
\hline 3.305 & 2463 & 3.23 & 2.116 & 3.17 & 1.882 & 3.091 & 1.644 \\
\hline 3.75 & 2.463 & 3.672 & 2.116 & 3.618 & 1.887 & 3.541 & 1.644 \\
\hline 4.254 & 2.463 & 4.186 & 2.116 & 4.129 & 1.887 & 4.056 & 1.644 \\
\hline 4.827 & 2.463 & 4.765 & 2. 116 & 4.713 & 1.887 & 4.646 & 1.644 \\
\hline 5.477 & 2.463 & 5.424 & 2.116 & 5.38 & 1.887 & 5.322 & 1,644 \\
\hline 6.214 & 2.463 & 1.124 & 2.116 & 6.141 & 1.887 & 6.097 & 1.644 \\
\hline 2.051 & 2.463 & 7.028 & 2.116 & 7.009 & 1.887 & 6.784 & 1.644 \\
\hline 8 & 2.463 & 8 & 2.116 & 8 & 1.887 & 8 & 1.644 \\
\hline 9.218 & 2.251 & 9.218 & 1.963 & 9.218 & 1.771 & 2.218 & 1.564 \\
\hline 10.621 & 2.057 & 10.621 & 1.821 & 10.621 & 1,662 & 10.621 & 1.489 \\
\hline 12.238 & 1.872 & 12.238 & 1.69 & 12.238 & 1.56 & 12.238 & 1.416 \\
\hline 14.101 & 1.717 & 14.101 & 1.568 & 14.101 & 1.464 & 14.101 & 1.348 \\
\hline 16.248 & 1.562 & 16.248 & 1.455 & 16.248 & 1.374 & 16.248 & 1.282 \\
\hline 18.722 & 1.434 & 18.722 & 1.347 & 18.722 & 1.289 & 18.722 & 1.22 \\
\hline 21.572 & 1.31 & 21 572 & 1.252 & 21.572 & 1.21 & 21.572 & 1.161 \\
\hline 24.856 & 1.198 & 24.856 & 1.162 & 24.856 & 1.135 & 24,856 & 1.105 \\
\hline 28.64 & 1.094 & 28.64 & 1.078 & 28.64 & 1.066 & 28.64 & 1.051 \\
\hline 33 & 1 & 33 & 1 & 33 & 1 & 33 & 1 \\
\hline 35.073 & 1 & 35.033 & 1 & 35.033 & 1 & 35.033 & 1 \\
\hline 37.191 & 1 & 37.191 & 1 & 37.191 & 1 & 37.191 & 1 \\
\hline 39.483 & 1 & 39.483 & 1 & 39.483 & 1 & 39.483 & 1 \\
\hline 41.915 & 1 & 42.915 & 1 & 41.715 & 1 & 41.215 & 1 \\
\hline 44.497 & 1 & 44.497 & 1 & 44.497 & 1 & 44.497 & 1 \\
\hline 47.237 & 1 & 47.239 & 1 & 47.239 & 1 & 47.239 & 1 \\
\hline 50.149 & 1 & 50.149 & 1 & 50.149 & 1 & 50.149 & 1 \\
\hline 53.239 & 1 & 53.238 & 1 & 53.238 & 1 & 53.238 & 1 \\
\hline 56.518 & 1 & 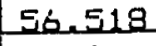 & 1 & 56.518 & 1 & 56.518 & 1 \\
\hline 80 & L & 60 & 1 & 60 & 1 & & 工 \\
\hline
\end{tabular}


MCAD FILES FGFECTFA. CKH

This prouram reads in the HOFIZONTAL flocir response spectra for e\%, $3.5 \%, 4 \%, 5 \%$ and $7 \%$ damping and the corresponding natural logarithms of the frequency values. Note that there are $N+1$ data values for each array ranging from o to $N$. Each spectrum is tit with a cutic spline.

HOFIZONTAL DIFECTION

$N:=45$

$i:=0 \ldots N$

$X L F R \quad:=F E A D(X L F F i)$

i

$X S A 2:=\operatorname{READ}(X S A E)$

HLFF.DAT contains the lns of the frequencies

HSAE.DAT contains the $2 \%$ Sa values

XSABS:= READ $(X S A B S)$

HSA3S.DAT contains the $3.5 \%$ Sa values

XSA4: $=$ FEAD $(X S A 4)$

HSA4.DAT cantains the $4 \%$ sa values

HSAS.DAT contains the $5 \%$ Sa values

$X S A 5:=\operatorname{FEAD}(X S A 5)$

HSA7.DAT contains the $7 \%$ Sa vallLes

$X S A 7:=$ FEEAD $(X S A 7)$ i

SE : = CSP I inE (XLFF, XSAE)

S35:= CSPline (XLFF, XSA35)

$54:=$ CSPI ine (XLFF, $X S A 4$ )

$55:=$ cSpline (XLFF, XSA5)

$57:=$ cspline $(X L F F, \times 5 A 7)$ 


$$
1::=1 \cdot .1200
$$

Example plat at $3.5 \%$ floor response spectrum

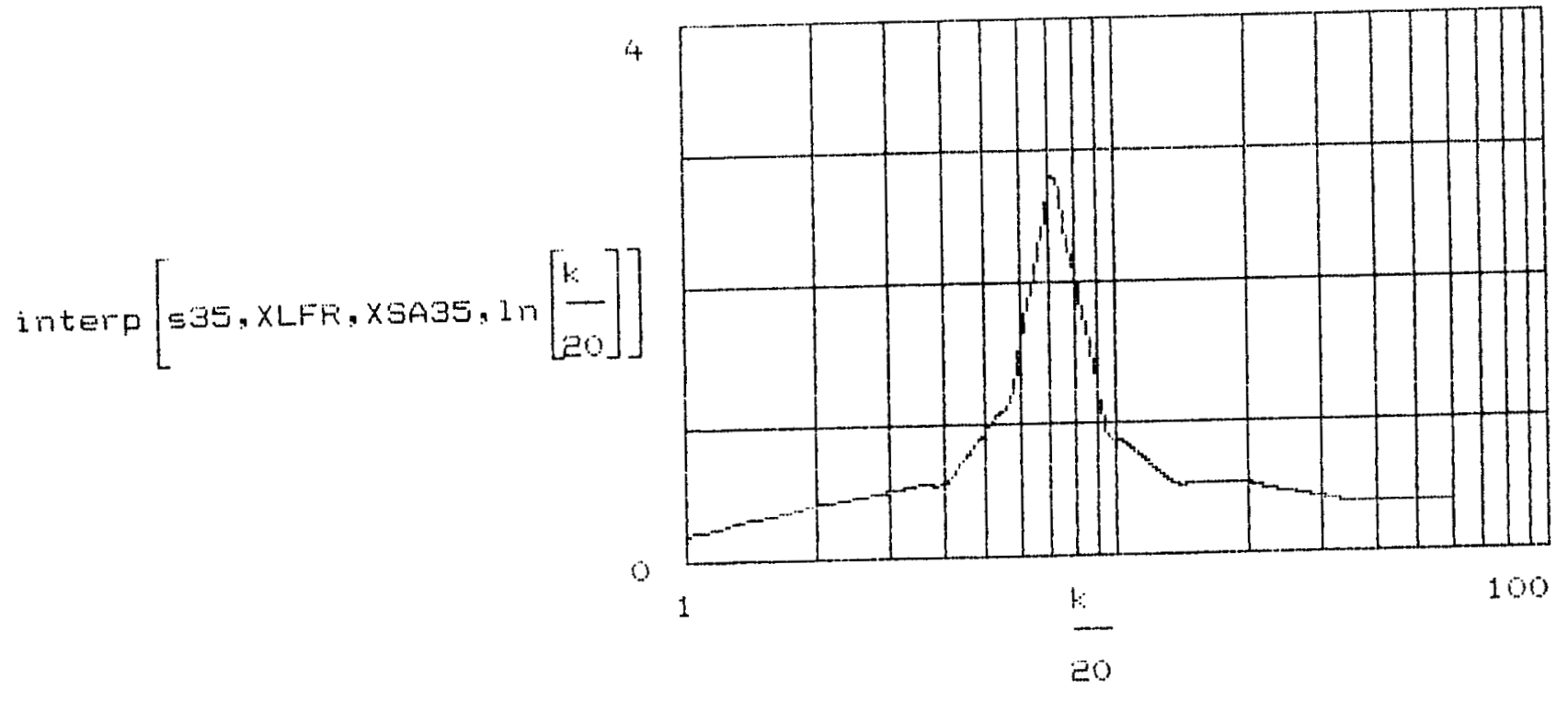

This plat took le secands to compute

$j:=0 \quad-.45$

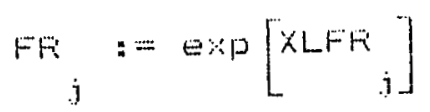




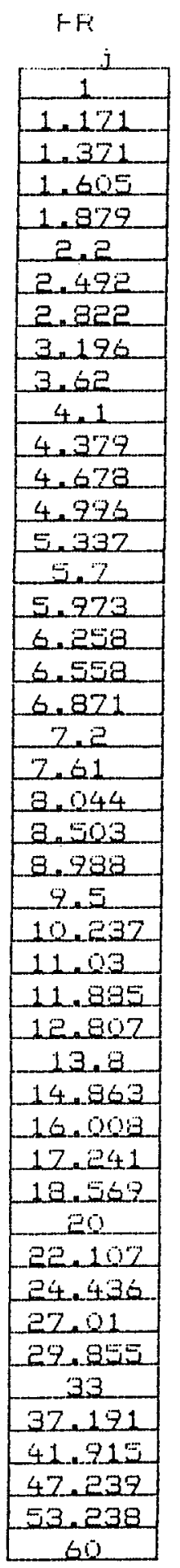

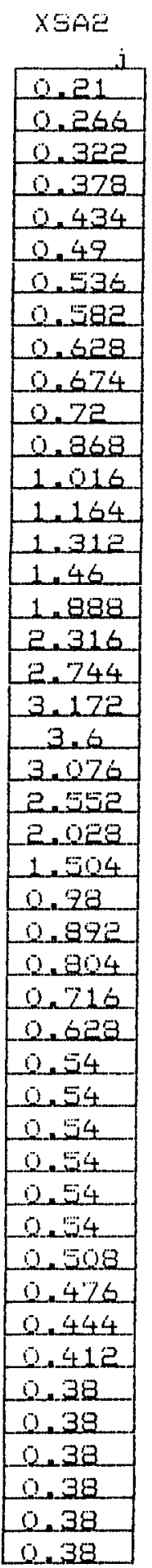

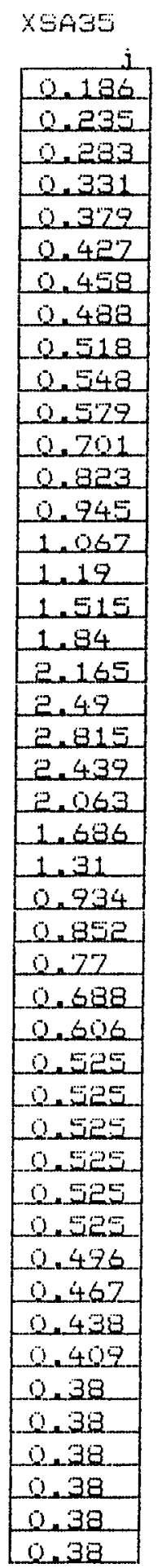

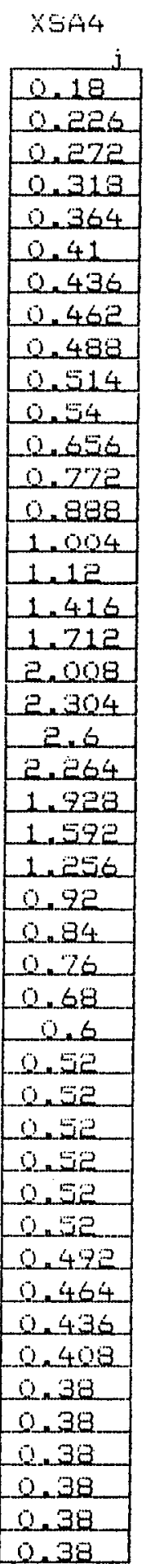

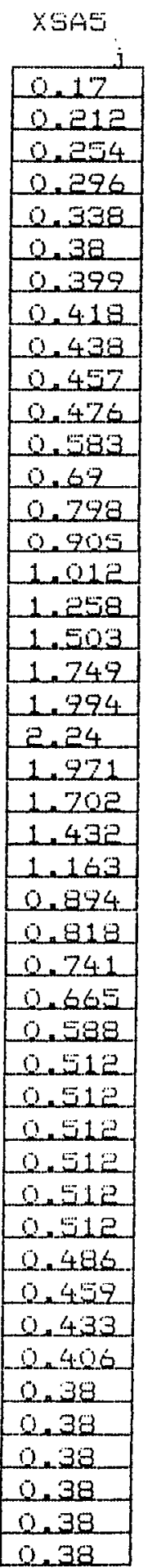

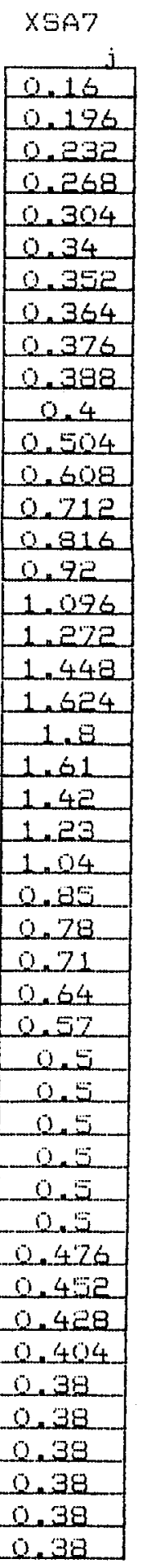


MCAD FILES AFHSFECT. AVE

This program reads in the averaged HOFizONTAL fluar response spectra for $2 \%, 3.5 \%, 4 \%, 5 \%$, and $7 \%$ damping (and the carresponding flour spectra) and the natural logarithms of the frequency values. Note that there are $N+1$ data values for each array ranging from 0 to $N$. Each spectrum is fit with a cubic spline.

HOFIIZONTAL DIFECTION

$N:=45$

$i:=0 \ldots N$

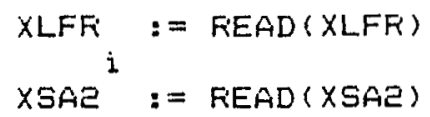

HLFR.DAT contains the Ins of the frequencies

XSA

XSA35:= READ (XSA35)

HSAE.DAT cantains the 2\% Sa values

$x^{i}:=$ FIEAD $(X S A 4)$

HSA3E.DAT contains the $3.5 \%$ Sa values

$X S A S^{i}:=\operatorname{FEAD}(X S A S)$

HSA4.DAT cantains the $4 \%$ Sa values

i

HSAS.DAT cantains the $5 \%$ Sa values

$\times S A 7:=\operatorname{FEAD}(X S A 7)$

HSA7.DAT contains the $7 \%$ Sa values

i

$535:=$ CSPline (XLFF: $\times$ SA35)

ASA2 : = READ (ASAC)

ASAE.DAT contains the AVEFAGED $2 \%$ Sa values

ASA35 : = READ (ASA35)

ASA35.DAT cuntains the AVEFAGED $3.5 \%$ Sa values

ASA4 ${ }^{i}:=\operatorname{FEAD}(A S A 4)$

ASA4.DAT cantains the AVEFAGED $4 \%$ Sa values

ASAS $^{1}:=$ FEAD (ASAS)

ASAS.DAT cuntains the AVERAGED $5 \%$ Sa values

ASA7 $7^{i}:=$ FEAD $(A S A 7)$

ASA7.DAT contains the AVERAGED $7 \%$ Sa values

a35:= CSpline (XLFF, ASA3S)

unave(t): $=\operatorname{interp}\left[535, x L F F, x \operatorname{SA} 35, \ln \left[\frac{k}{20}\right]\right]$

ave $(k):=$ interp $\left[\right.$ a35, XLFR, ASA35, In $\left.\left[\frac{k}{20}\right]\right]$ 
$k:=1 \ldots 1200$

Example plot of $3.5 \%$ flach response spectra

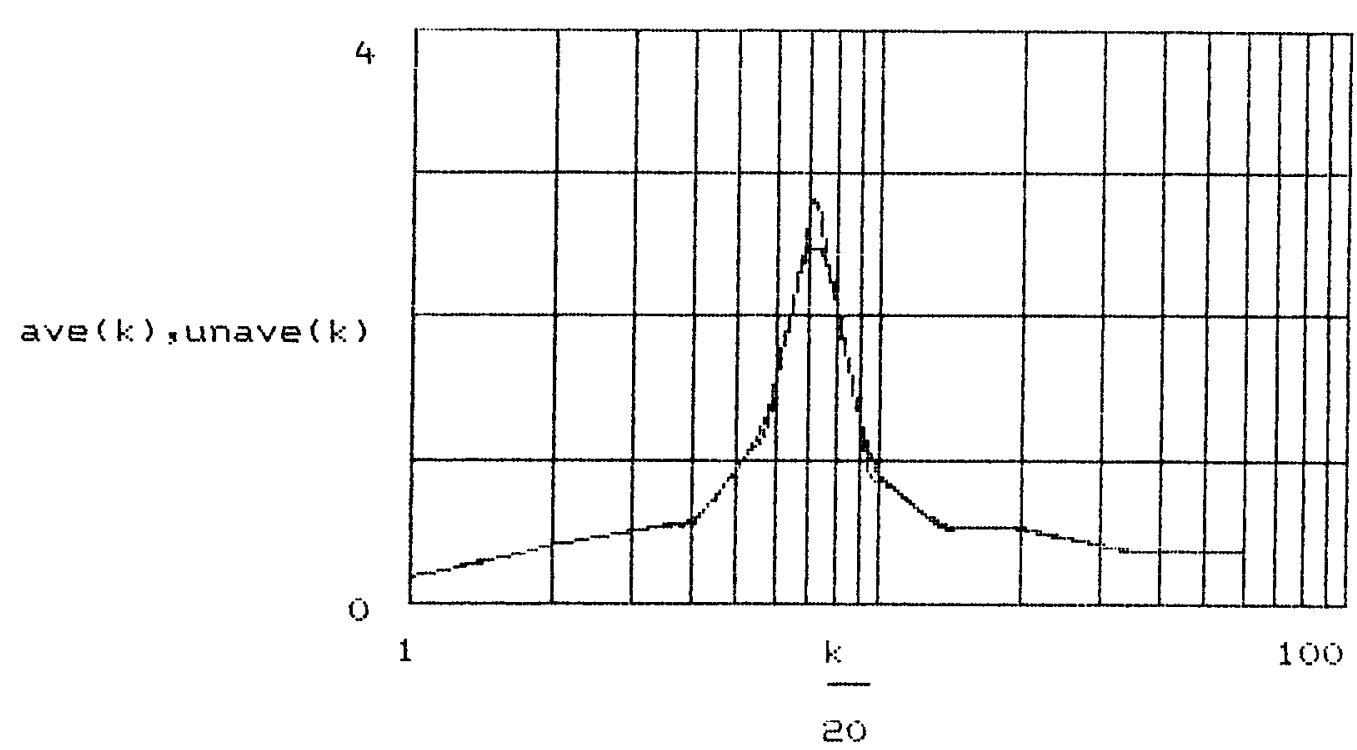

$j:=0 \quad .45$

$F F_{j}:=\exp \left[\begin{array}{rl}X F_{i} \\ j\end{array}\right]$ 


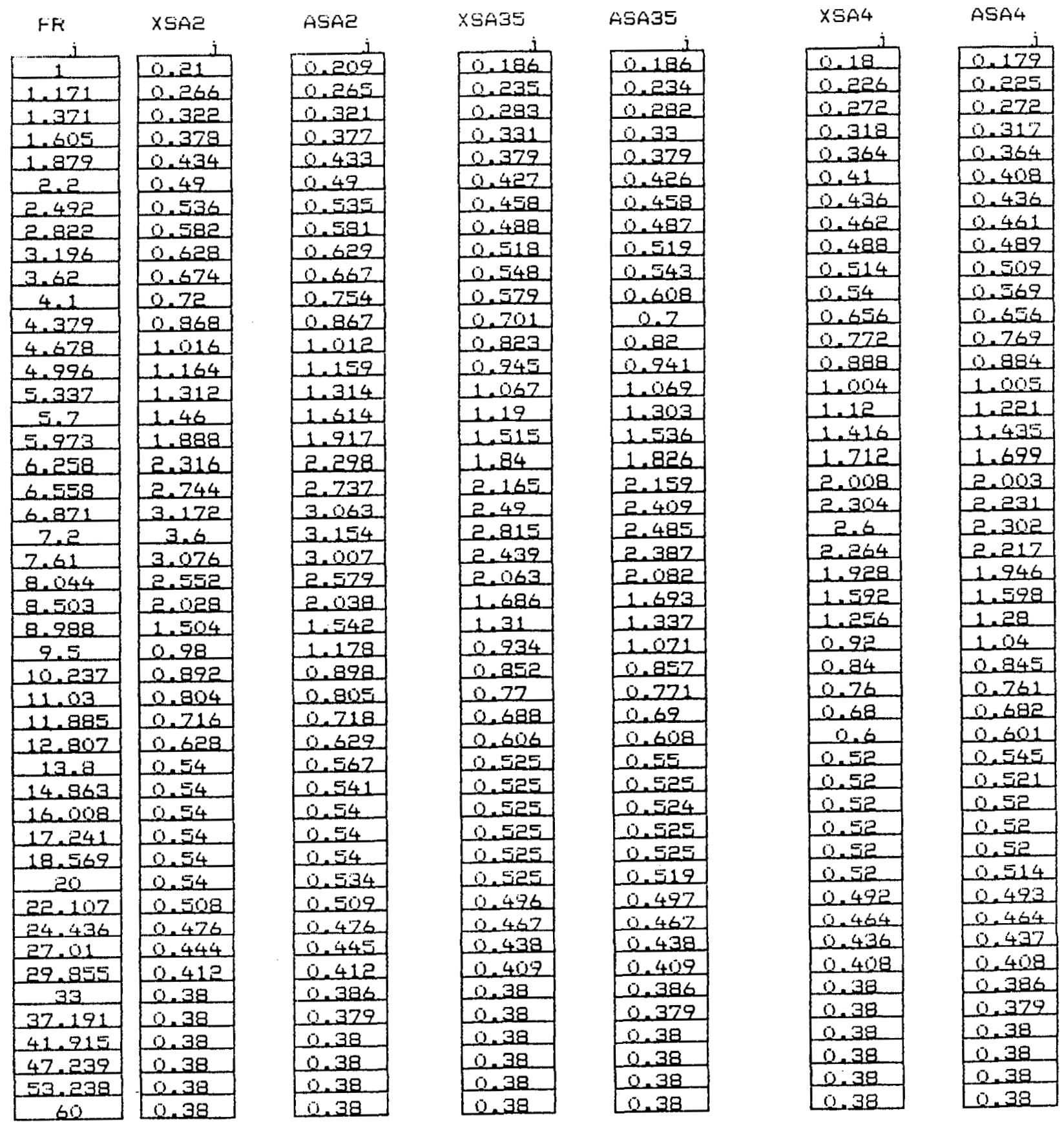




\begin{tabular}{|c|c|c|c|c|}
\hline $\mathrm{FF}^{\prime}$ & $\times 5 A 5$ & ASAS & XSA7 & ASA7 \\
\hline$j$ & & j & i & j \\
\hline 1 & 0.17 & 0.167 & 0.16 & 0.16 \\
\hline 1.171 & 0.212 & 0.212 & 0.196 & 0.126 \\
\hline 1.371 & 0.254 & 0.254 & 0.232 & 0.232 \\
\hline 1.605 & 0.296 & 0.275 & 0.268 & 0.268 \\
\hline 1.872 & 0.378 & 0.338 & 0.304 & 0.304 \\
\hline 2.2 & 0.38 & 0.378 & 0.34 & 0.338 \\
\hline 2.492 & 0.397 & 0.329 & 0.352 & 0.352 \\
\hline 2.822 & 0.418 & 0.418 & 0.364 & 0.363 \\
\hline 3.196 & 0.438 & 0.439 & 0.376 & 0.377 \\
\hline 3.62 & 0.457 & 0.452 & 0.388 & 0.383 \\
\hline 4.1 & 0.476 & 0.503 & 0.4 & 0.428 \\
\hline 4.379 & 0.583 & 0.583 & 0.504 & 0.504 \\
\hline 4.678 & 0.67 & 0.587 & 0.608 & 0.605 \\
\hline 4.296 & 0.798 & 0.794 & 0.712 & 0.209 \\
\hline 5.337 & 0.905 & 0.205 & 0.816 & 0.815 \\
\hline 5.7 & 1.012 & 1.092 & 0.92 & 0.967 \\
\hline 5.873 & 1.258 & 1.272 & 1.096 & 1.104 \\
\hline 6.258 & 1.503 & 1.497 & 1.272 & 1.265 \\
\hline 6.558 & 1.749 & 1.744 & 1.448 & 1.445 \\
\hline 6.871 & 1.994 & 1.935 & 1.324 & 1.581 \\
\hline 7.2 & 2.24 & 1.997 & 1.8 & 1.627 \\
\hline 2.61 & 1.771 & 1.933 & 1.61 & 1.583 \\
\hline 8.044 & 1.702 & 1.716 & 1.42 & 1.43 \\
\hline 8.503 & 1.432 & 1.438 & 1.23 & 1.234 \\
\hline 8. 988 & 1.163 & 1.182 & 1.04 & 1.053 \\
\hline 2.5 & 0.894 & 0.287 & 0.85 & 0.911 \\
\hline 10.237 & 0.818 & 0.821 & 0.78 & 0.783 \\
\hline 11.03 & 0.741 & 0.742 & 0.71 & 0.711 \\
\hline 11.885 & 0.665 & 0.667 & 0.64 & 0.642 \\
\hline 12.807 & 0.588 & 0.59 & 0.57 & 0.572 \\
\hline 13.8 & 0.512 & 0.536 & 0.5 & 0.522 \\
\hline 14.863 & 0.512 & 0.513 & 0.5 & 0.501 \\
\hline 16.008 & 0.512 & 0.512 & 0.5 & 0.5 \\
\hline 12.241 & 0.512 & 0.512 & 0.5 & 0.5 \\
\hline 18.567 & 0.512 & 0.512 & 0.5 & 0.5 \\
\hline 20 & 0.512 & 0.502 & 0.5 & 0.425 \\
\hline 22.107 & 0.486 & 0.487 & 0.470 & 0.477 \\
\hline 24.436 & 0.459 & 0.459 & 0.452 & 0.452 \\
\hline 27.01 & 0.433 & 0.433 & 0.428 & 0.420 \\
\hline 29.855 & 0.406 & 0.406 & 0.404 & 0.404 \\
\hline 33 & 0.38 & 0.385 & 0.30 & 0.385 \\
\hline 32.191 & 0.39 & 0.379 & 0.38 & 0.328 \\
\hline 41.915 & 0.38 & 0.38 & 0.38 & 0.39 \\
\hline 47.239 & 0.38 & 0.38 & 0.38 & 0.38 \\
\hline 53.238 & 0.38 & 0.38 & 0.38 & 0.38 \\
\hline 60 & 0.38 & 0.38 & 0.38 & 0.38 \\
\hline
\end{tabular}


MCAD FILES FSFECTFA.CKV

This prouram reads in the VefTICAL flacir respanse spectra for $2 \%$, $3.5 \%, 4 \%, 5 \%$ and $7 \%$ damping and the corresponding natural logarithins uf the frequency values. Note that there are $N+1$ data values for each array rangina from o to $N$. Each spectrum is fit with a cubic spline.

VEFTICAL DIFECTION

$N:=70$

i $:=0 \quad . . N$

$X L F F i:=$ FEAD $(X L F F$

ULFF.DAT contains the 1 ns of the firequencies

i

$\times S A 2:=F i E A D(X S A 2)$

VSAR.DAT contains the $2 \%$ Sa values

XSA35: = FEAD $(\times 5 A 35)$

i

$\times S A 4:=F E A D(X S A 4)$ i.

XSAS : = FEAD (XSAS)

i

XSA7 := FIEAD $(X S A 7)$ i

VSA3S.DAT cantains the $3.5 \%$ Sa values

VSA4.DAT contains the $4 \%$ Sa values

VSAS.DAT contains the $5 \%$ Sa values

vSA7.DAT contains the $7 \%$ Sa values

$S E:=$ : $5 P 1$ ine (XLFF: XSAC)

$535:=$ cspline(XLFFi, XSA35)

$54:=$ cEpline (XLFF: $X S A_{4}$ )

$55:=$ Cspline (XLFF, XSAS)

$57:=$ cspline (XLFF, XSA7) 
$t:=1 \ldots 1200$

Example plat of $3.5 \%$ flour response spectrum

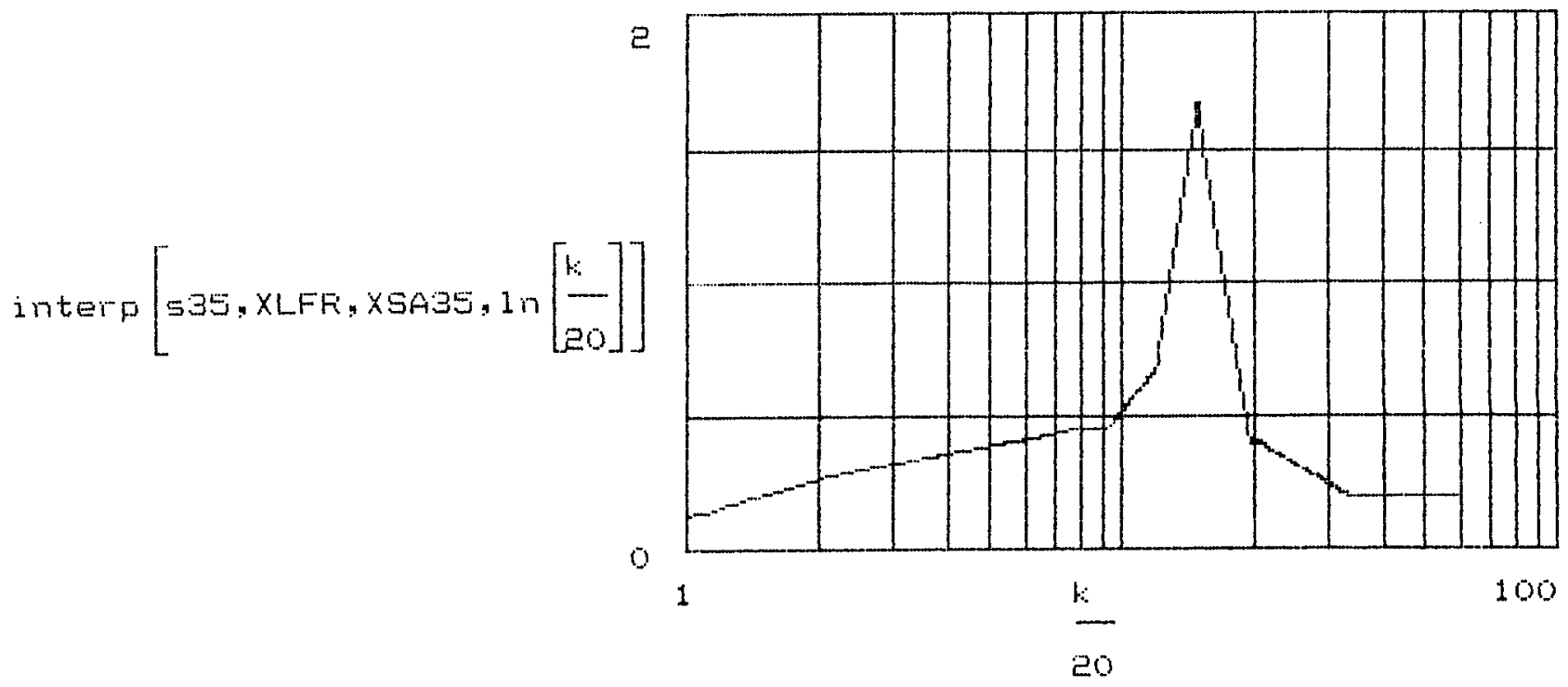

$$
\begin{aligned}
& j:=0 \ldots 50 \\
& F F_{j}:=\exp \left[\begin{array}{l}
X F_{F} \\
\end{array}\right]
\end{aligned}
$$




\begin{tabular}{|c|c|}
\hline $\mathrm{FF}$ & $\times S A E$ \\
\hline 1 & 0.14 \\
\hline 1.082 & 0.157 \\
\hline 1.171 & 0.178 \\
\hline 1.267 & 0.197 \\
\hline 1.371 & 0.216 \\
\hline 1.483 & 0.235 \\
\hline 1.605 & 0.254 \\
\hline 1.237 & 0.223 \\
\hline 1.879 & 0.292 \\
\hline 2.033 & 0.311 \\
\hline 2.2 & 0.33 \\
\hline 2.542 & 0.352 \\
\hline 2.954 & 0.384 \\
\hline 3.423 & 0.411 \\
\hline 3.966 & 0.438 \\
\hline 4.396 & 0.465 \\
\hline 5.325 & 0.472 \\
\hline 6.17 & 0.519 \\
\hline 2.15 & 0.546 \\
\hline 8.285 & 0.573 \\
\hline 9.6 & 0.6 \\
\hline 9.817 & 0.62 \\
\hline 10.038 & 0.64 \\
\hline 10.265 & 0.66 \\
\hline 10.476 & 0.58 \\
\hline 10.233 & 0.7 \\
\hline 10.775 & 0.72 \\
\hline 11.223 & 0.24 \\
\hline 11.476 & 0.76 \\
\hline 11.735 & 0.78 \\
\hline 12 & 0.8 \\
\hline 12.203 & 0.25 \\
\hline 12.531 & 1.1 \\
\hline 12.805 & 1.25 \\
\hline 13.085 & 1.4 \\
\hline 13.322 & 1.55 \\
\hline 13.564 & 1.7 \\
\hline 13.763 & 1.85 \\
\hline 14.267 & -2 \\
\hline 14.581 & 2.15 \\
\hline 14.9 & 2.3 \\
\hline 15.314 & 2. 114 \\
\hline 15.74 & 1.928 \\
\hline 16.172 & 1.742 \\
\hline 16.622 & 1.556 \\
\hline 17.099 & 1.37 \\
\hline 27.564 & 1.134 \\
\hline 18.052 & 0.928 \\
\hline 18.554 & 0,812 \\
\hline 12.07 & 0.626 \\
\hline 19.6 & 0.44 \\
\hline
\end{tabular}

\begin{tabular}{|l|}
\hline 0435 \\
\hline 0.124 \\
\hline 0.14 \\
\hline 0.156 \\
\hline 0.122 \\
\hline 0.187 \\
\hline 0.203 \\
\hline 0.219 \\
\hline 0.235 \\
\hline 0.251 \\
\hline 0.267 \\
\hline 0.283 \\
\hline 0.302 \\
\hline 0.321 \\
\hline 0.34 \\
\hline 0.359 \\
\hline 0.378 \\
\hline 0.397 \\
\hline 0.417 \\
\hline 0.436 \\
\hline 0.455 \\
\hline 0.474 \\
\hline 0.496 \\
\hline 0.518 \\
\hline 0.537 \\
\hline 0.561 \\
\hline 0.583 \\
\hline 0.604 \\
\hline 0.626 \\
\hline 0.648 \\
\hline 0.62 \\
\hline 0.672 \\
\hline 0.739 \\
\hline 0.886 \\
\hline 0.984 \\
\hline 1.081 \\
\hline 1.179 \\
\hline 1.276 \\
\hline 1.374 \\
\hline 1.471 \\
\hline 1.569 \\
\hline 1.665 \\
\hline 1.542 \\
\hline 1.418 \\
\hline 1.294 \\
\hline 1.169 \\
\hline 1.045 \\
\hline 0.921 \\
\hline 0.797 \\
\hline 0.673 \\
\hline 0.549 \\
\hline 0.425 \\
\hline
\end{tabular}

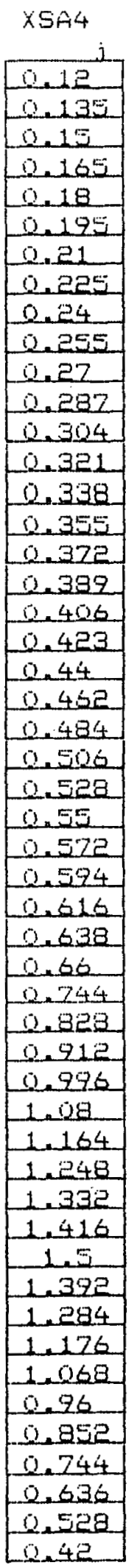

$X \subseteq A 7$

\begin{tabular}{|l|}
\hline 0.11 \\
\hline 0.120 \\
\hline 0.134 \\
\hline 0.146 \\
\hline 0.158 \\
\hline 0.17 \\
\hline 0.182 \\
\hline 0.124 \\
\hline 0.206 \\
\hline 0.218 \\
\hline 0.23 \\
\hline 0.239 \\
\hline 0.248 \\
\hline 0.257 \\
\hline 0.266 \\
\hline 0.275 \\
\hline 0.284 \\
\hline 0.293 \\
\hline 0.302 \\
\hline 0.311 \\
\hline 0.32 \\
\hline 0.34 \\
\hline 0.36 \\
\hline 0.39 \\
\hline 0.4 \\
\hline 0.42 \\
\hline 0.44 \\
\hline 0.46 \\
\hline 0.48 \\
\hline 0.2 \\
\hline 0.52 \\
\hline 0.560 \\
\hline 0.612 \\
\hline 0.658 \\
\hline 0.704 \\
\hline 0.25 \\
\hline 0.296 \\
\hline 0.842 \\
\hline 0.388 \\
\hline 0.934 \\
\hline 0.98 \\
\hline 0.922 \\
\hline 0.064 \\
\hline 0.806 \\
\hline 0.748 \\
\hline 0.09 \\
\hline 0.632 \\
\hline 0.574 \\
\hline 0.516 \\
\hline 0.49 \\
\hline
\end{tabular}


$j:=51 \ldots$

\begin{tabular}{|c|c|c|c|c|c|}
\hline$F R_{j}$ & XSAE & XSA35 & $X S A 4$ & XSAS & $X \subseteq A 7$ \\
\hline $\begin{array}{c}j \\
20.642 \\
\end{array}$ & 0.416 & 0.402 & 0.398 & $\begin{array}{r}j \\
0.391 \\
\end{array}$ & 0.38 \\
\hline 21.752 & 0.392 & 0.38 & 0.376 & 0.37 & 0.36 \\
\hline 22.716 & 0.368 & 0.357 & 0.354 & 0.348 & 0.34 \\
\hline 24.141 & 0.344 & 0.335 & 0.332 & 0.327 & 0.32 \\
\hline 25.432 & 0.32 & 0.312 & 0.31 & 0.306 & 0.3 \\
\hline 26.792 & 0.296 & 0.27 & 0.288 & 0.285 & 0.28 \\
\hline 28.225 & 0.272 & 0.267 & 0.266 & 0.264 & 0.26 \\
\hline 29.735 & 0.248 & 0.245 & 0.244 & 0.242 & 0.24 \\
\hline 31.325 & 0.224 & 0.222 & 0.222 & 0.221 & 0.22 \\
\hline 33 & 0.2 & 0.2 & 0.2 & 0.2 & 0.2 \\
\hline 35.033 & 0.2 & 0.2 & 0.2 & 0.2 & 0.2 \\
\hline 37.191 & 0.2 & 0.2 & 0.2 & 0.2 & 0.2 \\
\hline 39.483 & 0.2 & 0.2 & 0.2 & 0.2 & 0.2 \\
\hline 41.915 & 0.2 & 0.2 & 0.2 & 0.2 & 0.2 \\
\hline 44.497 & 0.2 & 0.2 & 0.2 & 0.2 & 0.2 \\
\hline 47.239 & 0.2 & 0.2 & 0.2 & 0.2 & 0.2 \\
\hline 50.149 & 0.2 & 0.2 & 0.2 & 0.2 & 0.2 \\
\hline 53.238 & 0.2 & 0.2 & 0.2 & 0.2 & 0.2 \\
\hline 56.518 & 0.2 & 0.2 & 0.2 & 0.2 & 0.2 \\
\hline 60 & 0.2 & 0.2 & 0.2 & 0.2 & 0.2 \\
\hline
\end{tabular}




\section{APPENDIX D}

JOHN W. STEVENSON 
Report on Development of High Confidence Low Probability of Fallure, HCLPF, Based on the Conservative Deterministic Fallure Margin, CDFM Method for Selected Mechanical and Electrical Equipment

\author{
Prepared for: \\ The Nuclear Systems Safety Progran \\ Lawrence Livermore National Laboratory \\ University of California \\ P. 0. Box 808 \\ Livermore, CA 94550 \\ Attn: R. C. Murray
}

Prepared by:

Stevenson and Associates

9217 Midwest Avenue

Cleveland, $\mathrm{OH} \quad 44725$

(216) $587-3805$ 
$87 C 1451$

O046G

\subsection{INTRODUCTION}

In this report High Confldence Low Probablilty of Fallure, HCLPF's estimates based on the Conservative Deterministic Fallure Margin, CDFM method are developed for the following mechanical and electrical components:

(1) Refueling Water Storage Tank (Vertical Mounted Flat Bottom Tank) Located on the ground assuming no soll structure interaction.

(2) Diesel Starting Air Tank (Vertical C1ip Angle Mounted Tank) Located on an Auxlliary Building Upper Floor

(3) Generic Motor Control Centers (Low Voltage) As Contained in the EPRI RP. No. -5223 pages D-34 to D-39

(a) Located on the ground assuming no soll structure interaction

(b) Located at an Auxllfary Buflding Upper Floor

(4) Component Cooling Heat Exchanger (Horizontal $\mathrm{HX}$ on Two Saddles) Located on an Auxtliary Building Upper Floor

(5) Sample Block Wall

Located on an Auxiliary Building Upper Floor

for which descriptions were transmitted by LLNL letter dated 4 August 1987.

For each of these five components, the limiting fallure mode(s) are identified and Initially the HCLPF seismic load capacity computed using the procedures contained in References 1 and 2 which are attached hereto as Appendix $A$. Subsequently HCLPF's were evaluated using the HCLPF estimating procedures recommended in Table 2.5 of the draft EPRI Margin Report (3) shown in Appendix $B$ to this report. The use of the Appendix B procedure, only significantly effects the HCLPF estimation for component $3 \mathrm{~b}$.

The selsmic load capacity for each component is initially expressed in terms of the limiting resultant seismic inertia load applied to the center of gravity of the components assuming the simultaneous application of two horizontal and one vertical components of earthquake. It is further assumed that resultant forces and moments developed from each direction of earthquake are combined on a SRSS basis and this resultant is combined absolutely with normal operating stresses to determine the limiting capacities. 
The seismic spectral capacities are converted to HCLPF's expressed in peak floor and peak ground acceleration values using the spectra[1] and information contained in Appendix $C$.

In addition to the HCLPF estimates presented herein best estimates ( 50 percent confidence and 50 percent probability of fallure) of fallure are presented in Section 8.0 of this report.

\subsection{EVALUATION OF THE VERTICAL MOUNTED TANK}

\subsection{Tank Geometry and Loading Data}

\subsubsection{Tank Geometry}

The tank overall geometry is shown in Figure 1a. Other geometric data are summarized as follows:

Anchor Bolts - 8-2" spaced at $45^{\circ}$ (typ.), 4'-0 Long

Anchor bolt Chairs - Detalls of the modiffed anchor bolt chairs are shown in Figure ib

\subsubsection{Materials}

Tank She 11, head and lugs are A240-304 Stainless Steel

Anchor bolts - ASTM A307 Ferretic Steel

\subsubsection{Design Data}

Operating pressure-0.25 psig

Operating temperature - Ambient

Design pressure-0.5 psig. 2" $\mathrm{H}_{2} \mathrm{O}$ vacuum

Design temperature-200 $\mathrm{F}$

Weight-76,500 ib empty, 2,385,000 ibs full[2]

Capacity-375,600 gal. full, 336,000 gal. usable.

Design Depth of Water, $37.0 \mathrm{ft}$.

\subsection{Tank Analysis}

The first activity in determining the COFM for the ground supported tank is to identify the lower bound failure mode(5) which are to be evaluated. Past experience in the design and evaluation of similar vertical tanks suggest the following two lower bound fatlure modes:

[1] It is noted that the Spectra provided are median spectra from NUREG/CR-0098 while the CDFM in accordance with Refs. 1 and 2 uses 84 percent NEP Spectra. For the purposes of this study the spectra provide in Appendix $C$ will be considered as 84 percentile NEP spectra.

[2] The weight full appears low, however this weight was assumed in this calculation since it is the same as the weight used by the panel

throughout this study. 
(1) Tension failure of anchor bolt or anchor bolt chairs

(2) Compression (buckling) fallure of tank wall

Following either of these two analytically determined fallure modes, a separation between the bottom and walls of the tank would be expected which would result in gross leakage of the tank.

The overturning moment for a nominal spectral acceleration of $1.0 \mathrm{~g}$ using the weight and geometry data shown in Figure la is determined as follows:

Overall Sefsmic Loads:

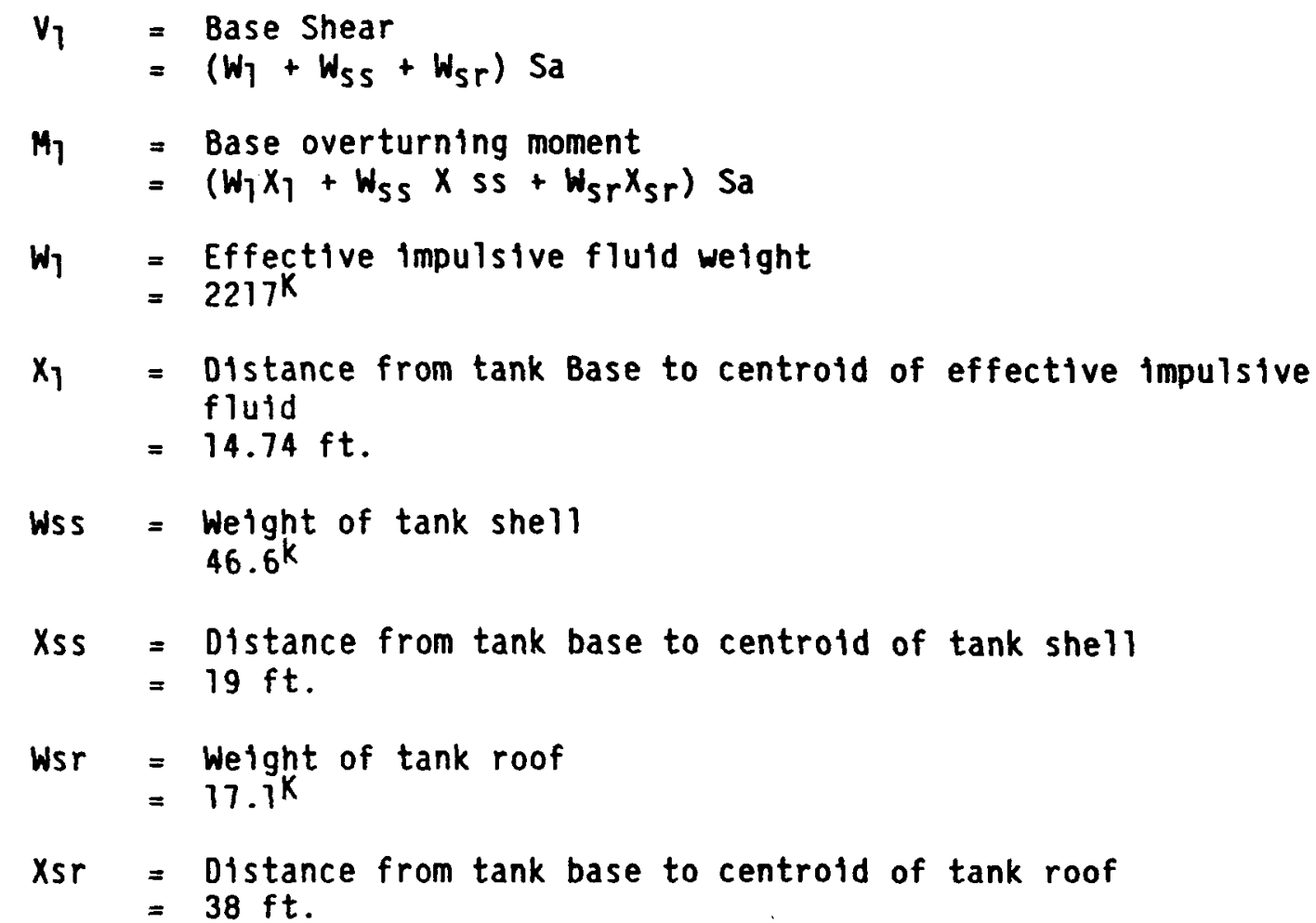

Base Shear for a Nominal 1.0g Lateral Acceleration

$v_{1}=(2217+46.6+17.1) \times 1.0$

$=2280$ Kips

Overturning moment for a Nominal $1.0 \mathrm{~g}$ Lateral Acceleration

$$
\begin{aligned}
M_{1} & =[2217(14.74)+46.6(19)+17.1(38)] \times 1.0 \times 12 \mathrm{in} / \mathrm{ft} \\
& =410.582 \mathrm{~K}-\mathrm{in}
\end{aligned}
$$

2.2.1 Evaluation of Anchor Bolt and Chair Capacities

Bolt Capacity: 
Actual yield strength capacity, $F_{y}$ of $2^{\prime \prime}$. A307 anchor bolts is determined

$$
F_{y}=S_{y} \times A Y f \times A_{b t}
$$

where:

$$
\begin{aligned}
& S_{y}=\text { Specified yield of material }=36 \mathrm{Kst} \\
& \text { AYf }=\text { Actual yield coefficlent }=1.05 \text { (assumed) } \\
& A_{b t}=\text { Area of threaded bolt } \\
& F_{y}=36 \mathrm{Kst} \times 1.05 \times 2.5 \mathrm{in}^{2}=94.5 \mathrm{Kips}
\end{aligned}
$$

\section{Chair Capacity:}

In defining this capacity the bolt chair capacity calculations performed in Reference 4 have been considered. The Reference 4 Calculation show the capacity of the anchor bolt chairs to be $67.7 \mathrm{Kips}$ each. However, I belleve the limiting value of $67.7 \mathrm{~K} /$ bolt chair can be increased by 10 percent to account for anticipated actual materlal properties of austenitic steel compared to the speciffed minimum yleld properties.

$$
67.7 \times 1.10=74.5 \mathrm{Kip} / \mathrm{bolt}
$$

Anchor capacities are therefore limited by chalrs behavior before modification.

Subsequent to the Reference 4 analysis, the bolt chairs on the tank were modifled to increase their capacities to $120 \mathrm{Kips}$ each as determine by Kennedy $(5)$. This increase in bolt chair capacity now means the bolt capacities of 94.5 Kips each now control anchorage design of the tank.

Overturning Moment Capacity of Bolts Based on Assumed Distribution Shown in Figure 2.

$$
\begin{aligned}
M_{u} & =F_{y} \text { bolt (lever arm to N.A.) } \\
M_{u} & =94.5 k \times(2 \times 46.3+2 \times 216+ \\
M_{u} & \quad 2 \times 385.7+1 \times 456) \\
M_{u} & =94.5 \times(92.6+432+771.4+456) \\
& =94.5 \times 1752 \mathrm{in} \\
& =765.564 \mathrm{~K}-\mathrm{in}
\end{aligned}
$$

\subsubsection{Buckling Capacity of Shell Due to Overturning Earthquake Moment}

Using the simple equation from Rourk(6) Table 35 (76), the critical buckling moment for the tank is determined.

$$
\begin{aligned}
& M_{C R}=\frac{0.72 E \mathrm{rt}^{2}}{\left(1-v^{2}\right)} \\
& M_{C R}=\frac{.72 \times 27.000 \times 240 \times(3 / 8)^{2}}{0.91} \\
& M_{C R}=720,989 \mathrm{~K}-\text { in }
\end{aligned}
$$


Obviously the overturning capacity is significantly larger than the bolt overturning capacity.

\subsubsection{Overturning Moment Mobilized by Weight of Fluid}

The moment moblilized to overcome dead weight of tank and liquid contents acts to counter the seismic overturning moment. In the limit, this dead weight moment is limited by the plastic moment capacity and membrane action of the base plate carrying the weight of water which lifts off the base as shown in Figure 3 .

The elastic section modulus, $Z$ of the $1 / 4^{\text {" }}$ base plate:

$$
\begin{aligned}
Z= & b h^{2} / 6=12^{\prime \prime} \times(1 / 4)^{2} / 6=0.125 \mathrm{fn}^{3} / \mathrm{ft} \text { of plate } \\
S_{y}= & 30 \mathrm{Ks} 1 ; S_{U}=75 \mathrm{KSI} \text { for SA } 240 \text { Type } 304 S S \text {. from Tables I-2.2 } \\
& \text { and I-3.2. ASME Section III, Div. } 1
\end{aligned}
$$

Elastic moment capacity of base plate:

$$
\mathrm{Me}=2 S_{\mathrm{y}}=.125 \times 30=3.75 \mathrm{k}-\text { in/ft of plate }
$$

Plastic moment capacity of Plate

$$
M_{p}=1.5 \times 3.75=5.63 \mathrm{k}-\text { in } / \mathrm{ft} \text { of plate }
$$

Plastic Moment Capacity of Uniformly load fixed end beams:

$$
\left.M_{p}=1 / 24 w\right]^{2}
$$

where:

$$
\begin{aligned}
w & =\text { pressure weight of water } \\
w & =62.41 \mathrm{~b} / \mathrm{ft}^{3} 37.0 \mathrm{ft}=2308.8 \mathrm{lbs} / \mathrm{ft}^{2} \\
w & =2308.8 / 12=192.4 \mathrm{lbs} / \mathrm{in} / \mathrm{ft} . \text { of } w a 11 \\
M & =w 1 \times 1
\end{aligned}
$$

$5.63 k-1 n=.192 \times 1^{2}$

$$
\begin{aligned}
1^{2} & =29.32 \mathrm{in}^{2} \\
1 & =5.42 \mathrm{in}
\end{aligned}
$$

$\mathrm{T}_{\mathrm{e}}=$ Holdown per inch of wall:

$$
T_{e}=192 / 12 \times 5.42=86.7 \text { Lbs/in of wall }
$$

Assume the membrane action of the plate increases the tensile capacity of the tank wall by 100 percent.

$$
\mathrm{T}_{\mathrm{e}}=173.4 \mathrm{~b} / \mathrm{b} \text { in of wall }
$$


Total Tension in Tank Wall

$173.4 \times 480$ in $\times \pi=261.48$ Kips

Assume Centroid of semi-circle is $2 / 5$ from centerline

$240 \times 2 / 5=96+216=312$ in

$261.4 \times 312^{\prime \prime}=81557 \mathrm{~K}-$ in

$(0.85 \times 261.4) \times(216-96)=26663 \mathrm{~K}-\mathrm{in}$

Total restoring dead weight moment

$81557+26663=108220 K-$ in

Total 0.T. moment capacity of the tank is the moment capacity of the bolts plus the stablizing effect of the weight of the fluid in the lift off region of the shell.

$$
\begin{aligned}
& 165,564+108220=273784 \mathrm{~K}-\text { in } \\
& (273784 / 410582) \times 1.0 \mathrm{~g}=0.667 \mathrm{~g} \text { lateral load capacity }
\end{aligned}
$$

\subsubsection{Determine Fundamental Impulse Frequency}

Fundamental Impulse Frequency:(4)

The horizontal impulsive response fundamental frequency is estimated using the Haroun \& Housner ( $H \& H$ ) method given in Ref. 7 . The $H$ \& $H$ coefficients are used since they are available for a variety of $h / R$ valves.

The $H$ \& $H$ frequency coefficients are developed for steel tanks of constant thickness fllled with water. For a tank of varying thickness, the frequency coefficient can be selected using an average thickness. A reasonable estimate of the effective tank thickness is $3 / 16^{\prime \prime}$ since this thickness is used over the top $60 \%$ of the tank shell where deformations and hydrodynamic pressures are the greatest.

$$
\begin{aligned}
\mathrm{h} & =\text { Shell thickness } \\
& =3 / 16^{\prime \prime} \\
H & =\text { Fluid Height } \\
& =37^{\prime \prime}-0 \\
& =444^{\prime \prime} \\
\mathbf{R} & =\text { Tank radius } \\
& =20^{\prime}-0^{\prime \prime} \\
& =240^{\prime \prime} \\
& =\text { She } 11 \text { dens } 1 \mathrm{ty} \\
& =4901 \mathrm{bs} / \mathrm{ft}^{3} \\
& 1728(386.4) \\
& =0.0007341 \mathrm{~b}-\sec ^{2} / \mathrm{in}^{4}
\end{aligned}
$$




$$
\begin{aligned}
E & =\text { She11 modulus of elasticity } \\
& =28 \times 10^{6} \mathrm{psi} \\
\frac{H}{R} & =\frac{444}{240} \\
& =1.85 \\
\frac{h}{R} & =\frac{0.1875}{240}=0.00078 \\
\omega_{f} H \quad 1 /(\mathrm{d} / E) & \simeq 0.08 \quad(\text { Fig. } 5 \text { of Ref. } 7) \\
\text { Circular natural frequency: } & =\frac{0.08}{444 \sqrt{\left(0.000734 / 28 \times 10^{6}\right)}} \\
\omega_{f} & =35.2=\omega_{f} \\
2 \pi f & =5.6 \mathrm{~Hz} \\
f \quad &
\end{aligned}
$$

\subsubsection{HCLPF Capacity of Tank}

Given a fundamental impulse frequence of $5.6 \mathrm{~Hz}$ for the tank and an assumed damping of 7 percent and neglecting sloshing gives an amplication factor to the ground from the spectrum given in Appendix $C$.

$$
\begin{aligned}
& 0.88 / .38=2.31 \\
& 0.667 / 2.31=.29 \mathrm{~g} \text { pga based on elastic response } \\
& \text { and }
\end{aligned}
$$

Given that ylelding in the bolt chairs would provide a small amount of additional global ductility in the resonant region of the tank, assume elastic response demand would be reduced by 10 percent.

This results in

$$
0.29 \mathrm{~g}+0.03 \mathrm{~g}=.32 \mathrm{~g} \mathrm{pga} \mathrm{HCLPF}
$$

for the Appendix $C$ spectra.

If the NUREG/CR 0098 Median Spectra for 7 percent damping is used in place of the Appendix B spectra and the tank is assumed ground mounted

$$
0.667 / 7.85=0.36 \mathrm{pga} \mathrm{HCLPF}
$$

\subsection{EVALUATION OF VERTICAL ANGLE CLIP MOUNTED TANK}

Given a vertical tank arrangement as shown in Figure 4. By inspection it appears that the limiting selsmic capacity of the tank will be in the clip angle anchorage of the tank's mounting ring.

\subsection{Determine Maximum Uplift Force on An Anchor}


Given the total welght of tank and mounting ring of tank shown in Figure 4 is 920 ibs. The distance to the c.g. of the tank from the base is 41.5 in. Total overturning selsmic moment for a nominal $1.0 \mathrm{~g}$ lateral load is

$$
920 \times 41.5=38180 \text { in-lbs. }
$$

less the restraining force of dead weight of the tank. Given that the vertical upward component of the earthquake is $2 / 3$ of horizontal only $1 / 3 \times$ $920=307$ lbs. acting down at the center of the tank would be avallable to offset the lateral overturning moment.

Taking moments about the assumed neutral axis of the tank which is taken as 2.4 inches from the edge of the mounting rings and neglecting the two inner clip angle supports as shown in Figure 4 .

Maximum Tension in Clip Angle

$$
\begin{aligned}
& {[38180-(307 \times 10.1)] / 22.6} \\
& (38180-3101) / 22.6=1552 \text { lbs }
\end{aligned}
$$

for a nominal $1.0 \mathrm{~g}$ lateral load.

\subsection{Determine the Clip Angle Anchor Capacity}

Given a A-36 angle and 3/4" diameter bolt hole as shown in Figure 5 , since the angle leg is welded on three sides, it can be assumed that vertical leg is fixed.

Maximum Moment in the Angle at the Bolt Hole

$$
\begin{aligned}
M & =\frac{T L}{2} \\
& =\frac{1552(1.625)}{2} \\
& =1261 \mathrm{lbs}
\end{aligned}
$$

Angle Section Modulus

$$
\begin{aligned}
& Z=\frac{2.25(0.25)^{2}}{6}=0.0234 \mathrm{in}^{3} \\
& S=\frac{1267}{0.0234}=53889 \mathrm{pst}
\end{aligned}
$$

The equivalent elastic moment stress capacity would be computed as follows:

$S_{e} \quad=S F \times F_{y} \times A Y C$

where:

$\mathrm{SF}=$ shape factor $=1.5$ 


$$
\begin{aligned}
& F_{y}=\text { Specified Minimum Yield }=36 \mathrm{Kst} \\
& \text { AYC }=\text { Actual Yield Coefficient }=1.05 \\
& \mathrm{~S}_{\mathrm{e}}=1.5 \times 36000 \times 1.05=56.7 \mathrm{Ks} 1
\end{aligned}
$$

Given that the maximum computed stress in the angle is 53889 psi from a net uplift of 1552 lbs for an earthquake lateral acceleration of $1.0 \mathrm{~g}$. Therefore the seismic capacity of the clip angle is $56.7 / 53.9=1.05 \mathrm{~g}$.

\subsection{Determine Fundamental Frequency of Atr Tank}

$$
\begin{aligned}
& I=\frac{\pi}{4}\left(12^{4}-11.625^{4}\right)=1942.3 \ln ^{4} \\
& E=29,000,000 \mathrm{psi}
\end{aligned}
$$

Total weight of tank and support collar $=920$ lbs. Unit wieght $w=921 / 91=10.11 \mathrm{lbs} / \mathrm{in}$

Assume Distributed Mass

From Ref. 6 - Table 36 (3)

$$
\begin{aligned}
f & =\frac{1}{2 \pi} \sqrt{\frac{12.4 E I g}{W L^{4}}} \\
f & =1 / 6.28 \sqrt{\frac{12.4 \times 29000000 \times 1942.3 \times 386}{10.11 \times 91^{4}}} \cong 99.3 \mathrm{~Hz}
\end{aligned}
$$

Estimate Stiffness Considering Only clip Angle as Support Restraint

$$
I=A d^{2}=0.5 \times 12^{2} \times 3=216 \mathrm{in}^{4}
$$

This results in a factor of approximately 9 decrease in stiffness and therefore approximately 3 in frequency. However, frequency still should be above $30 \mathrm{~Hz}$.

Therefore there should be no amplification of floor acceleration as a function of frequency.

\subsection{Estimate of HCLPF Capacity of the Air Tank}

The tank as evaluated considers only one horizontal component of earthquake. For rotationally symmetric components the second horizontal component would not change the component's earthquake capacity. For rectangular components in the limit the lateral capacity would be reduced in the ratio of $1 / 1.41$. For this component which is only sightiy rotationally unsymmetric considering the location of the clip angles in figure 4 use a ratio of $1 / 1.2$. The lateral load capacity of the tank is $1.05 / 1.2=0.88 \mathrm{~g}$ pfa HCLPF using the amplification in going from ground to floor acceleration for the Appendix $C$ spectrum yields.

$$
0.88 \times .18 / .38=0.42 \mathrm{pga} \mathrm{HCLPF}
$$


Yielding in the small clip angles would not add significantly to inelastic response tank.

\subsection{EVALUATION OF GENERIC MOTOR CONTROL CENTER}

To make this evaluation the GERS curve for MCC functioning during the E.Q. shown in Figure 1 page D-38 of the EPRI NP-5223 Report as shown in Append1X D was compared to the Appendix $C$ spectra for 5 percent damping. The GERS shows a spectra acceleration value of $7.5 \mathrm{~g}$. The RRS from Appendix $C$ shows a peak spectra acceleration of $2.2 \mathrm{~g}$ for 5 percent damping. Using the 0.8 demand factor from Table 2.5 from Appendix B; $2.2 \times 0.8=1.76 \mathrm{~g}$. The resultant HCLPF for the Appendix C spectrum:

$$
\begin{aligned}
& 1.5 / 1.76 \times .38=0.32 \mathrm{~g} \text { pfa HCLPF } \\
& 0.32 \times .18 / .38=.15 \mathrm{~g} \text { pga HCLPF }
\end{aligned}
$$

for the NUREG/0098 median spectrum at 5 percent damping

$$
1.5 / 2.1=.719 \mathrm{pga}
$$

\subsection{EVALUATION OF HORIZONTAL HX}

Given the horizontal heat exchanger shown in Figure 6 , it is assumed the shear load on the bolts at the fixed saddle will control the seismic load capacity of the HX. Given a total weight of $23.5 \mathrm{Kips}$ and a nominal lateral load coefficlent of $1.0 \mathrm{~g}$ applied to the $\mathrm{cg}$. of the $\mathrm{HX}$ the bolt shear reaction in the longitudinal direction would be $23.5 / 2 \mathrm{Kips}$ or $11.75 \mathrm{Kips} / \mathrm{bolt}$ and in the transverse direction $23.5 / 4$ or $5.88 \mathrm{~K} /$ bolt $\mathrm{K}$ ips load in each bolt.

The component shear stress in each bolt is determined:

$$
\begin{aligned}
& \text { Longitudinal }=11.75 / 0.601=19.55 \mathrm{Ksi} ; \text { Transverse }=5.88 / 0.601=9.78 \\
& \text { Ksi }
\end{aligned}
$$

Allowable bolt stress from ASME Section III-NF-1986 for Service Level D

$$
F_{v} \leq .42 S_{u}<.6 S_{y}
$$

where:

$$
\begin{aligned}
& S_{u}=\text { Specified Minimum Ultimate Strength }=60 \mathrm{Ksi} \\
& S_{y}=\text { Specified Minimum Yield Strength }=\text { (not defined) assume } 36 \\
& F_{v}=0.42 \times 60=25.2 \mathrm{Ksi} \text { allowable } \\
& F_{v}=0.6 \times 36=21.6 \mathrm{Ksi} \text { allowable (controls) }
\end{aligned}
$$

Resultant Shear Stress in Bolt for nominal $7.0 \mathrm{~g}$ lateral load

$$
\begin{aligned}
& f_{v}=\sqrt{(21.21)^{2}+(9.78)^{2}}=\sqrt{545.51} \\
& \text { Resultant Shear Stress: } \\
& f_{v}=23.36 \mathrm{Ksi}
\end{aligned}
$$


It should be noted that the current LRFD AISC Specification limits the allowable shear stress in the $A 307$ bolt based on the nominal area 0.601 in 2 to $16.1 \mathrm{Ks} 1$ for factored loads and the AISC N690 AISC Specification limits shear on nominal A307 bolt areas to $14.0 \mathrm{Kst}$ for a large earthquake loading. Since the allowable values from the AISC specification are signiflcantly lower than the ASME, the ASME code value is used without any adjustment for actual versus specified mintmum properties.

Resultant Applied Seismic Load:

$$
21.6 / 23.36 \times 1.0 \mathrm{~g}=0.925 \mathrm{~g}
$$

5.1 Compute Limiting Longitudinal Frequency of the Tank

$$
\begin{aligned}
& \begin{array}{ll}
f=1 / 2 \pi \quad \sqrt{K / M} \quad \begin{array}{l}
\text { WT } \\
\text { Mass }=23.50 \mathrm{~K}+\mathrm{ps} \\
\end{array} \quad 23.5 \mathrm{k} / 386-\mathrm{in} / \mathrm{sec}^{2}=0.0608 \mathrm{~K}-\mathrm{sec}^{2} / \mathrm{in}
\end{array} \\
& \text { weight }=23.50 \mathrm{~K} \quad \text { Horizontal Stiffness, - one saddle is slotted }
\end{aligned}
$$

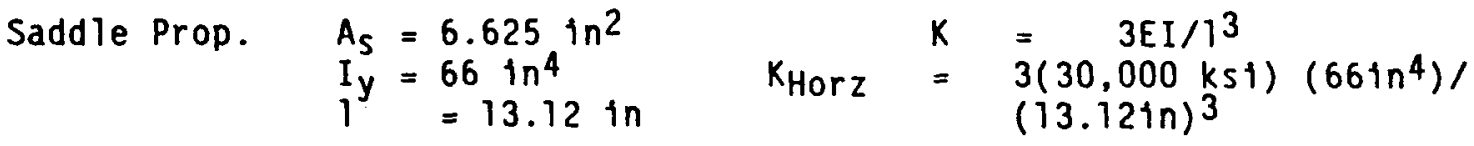

$$
\begin{aligned}
& =2630 \mathrm{~K} / \mathrm{in} \\
& f=1 / 2 \pi \sqrt{2630 \mathrm{k} / \text { in } / 0.0608 \mathrm{k} \cdot \sec ^{2} / \mathrm{in}}=33.3 \mathrm{~Hz}
\end{aligned}
$$

\subsection{Computation of HCLPF for the Horizontal HX}

Given that the $\mathrm{HX}$ has frequency response above $33 \mathrm{~Hz}$ for the 7 percent damping and the spectrum contained in Appendix $C$ is applicable:

$$
\begin{aligned}
& 0.93 \mathrm{~g} \text { pfa HCLPF } \\
& 0.93 \times .18 / .38=.44 \mathrm{~g} \text { pga HCLPF }
\end{aligned}
$$

Since shear failures tend to be non ductile, it is assumed that the component responds elastically.

\subsection{EVALUATION OF BLOCK WALL}

Assume a 12" thick reinforced concrete block wall 10 feet high can be analyzed as a one way vertical cantilever slab. Wall is reinforced by one layer of \#5 bar 0 16" center to center loçated in the center of the block with $f_{y}=60 \mathrm{ks} 1$ Assume compressive strength $f_{c}^{\prime}=3000$ ps 1 for Type $s$ mortar. 
From Table 4.3 of ACI $531-79$ for $12^{\prime \prime} \times 16^{\prime \prime}$ block

$$
\begin{aligned}
& w=111 \text { psf assuming grouted core } \\
& f_{m}=1700 \mathrm{psi} \text { masonry compressive strength } \\
& E_{m}=1000 \mathrm{f}_{\mathrm{m}}=1700,000 \mathrm{ps}, \mathrm{As}=0.31 \times 12 / 16=0.233 \mathrm{in} 2 / \mathrm{ft} \\
& \mathrm{Kd} \approx 1.5 \mathrm{in} ; \mathrm{d}=11.625 / 2=5.8 \mathrm{in} ; \mathrm{jd}=5.05 \mathrm{in} \\
& 1 \quad=10 \mathrm{ft} .
\end{aligned}
$$

6.1 Compute Bending Moment for a Nominal $1.0 \mathrm{~g}$ Lateral Load and Resultant Concrete and Steel Stresses

$$
\begin{aligned}
& M=1 / 2 \mathrm{w} 1^{2}=1 / 2 \times 111 \times 10^{2} \times 12 \mathrm{in} / \mathrm{ft}=66600 \mathrm{in}-1 \mathrm{bs} / \mathrm{ft} \\
& M=A_{s} f_{s} j d=0.233 \times 5.05 \mathrm{f}_{s}=66600 \mathrm{in}-1 \mathrm{bs}
\end{aligned}
$$

Tensile Stress in Reinforcement due to bending

$$
\begin{aligned}
f_{s} & =56.60 \mathrm{Kst} \\
f_{s} \text { all } & =60 \mathrm{Kst} \\
f_{s} / f_{s} \text { all } & =.94
\end{aligned}
$$

Compressive stress in concrete due to bending

$$
\begin{aligned}
& C=M / j d=66600 / 5.05=13788 \mathrm{lbs} \\
& f_{C}=13188 /(12 \times 1.5)=733 \mathrm{psi} \\
& f_{c^{a}} \text { all. }=0.72 \times 1700=1224 \mathrm{psi} \\
& f_{c} / f_{c} a 11=.60
\end{aligned}
$$

Tensile Stress in reinforcement controls design

Note: ACI 531-79 Coupled with SRP 3.8.4 Appendix A would give a slightly larger value for $f_{c}$ all.

\subsection{Compute Frequency of Wall}

Uncracked Moment of Inertia

$$
\begin{aligned}
I & =1 / 12 \times 16 \times(11.625)^{3}-1 / 12 \times 11.8 \times(8.63)^{3} \\
I & =1.33 \times 1571-632 \\
& =2089-632=1457 \\
I & =1457 / 1.33=1095 \mathrm{in}^{3} / \mathrm{ft} \text { of wall } \\
E_{m} & =1,700,000
\end{aligned}
$$

From Ref. 6.

$$
\begin{aligned}
f & =3.52 / 2 \pi \sqrt{E I g / w 1^{4}} \\
& =3.52 / 6.28 \sqrt{\left[1700000 \times 1095 \times 386 / 9.25 \mathrm{lbs} / \mathrm{in} \times(120)^{4} \mathrm{in}^{4}\right]}
\end{aligned}
$$




$$
=3.52 / 6.28 \sqrt{374.6}=10.8 \mathrm{~Hz}[1]
$$

\subsection{Computation of HCLPF for Block Wall}

Acceleration at c.g. of wall

$1.0 / .94=1.06$

Spectral Amplification for $10.8 \mathrm{~Hz}$ from Appendix C spectrum

$.75 / .38=1.97$

Use $0.8 \times$ spectral demand to account for inelastic response $0.8 \times 1.97=$ 1.58

$$
\begin{aligned}
& 1.06 / 7.58=0.67 \mathrm{~g} \text { pfa HCLPF } \\
& 0.67 \times .18 / .38=0.32 \mathrm{~g} \text { pga HCLPF }
\end{aligned}
$$

Note: In reinforced concrete shear walls changes in stiffness by a factor of 15 or more have been observed from first|cracking to fallure. If this same shift in stiffness before faliure were to occur in the block wall, the frequency would be reduced to $\simeq 3.0 \mathrm{~Hz}$. At this frequency for the Appendix $C$ Spectrum, the spectral amplification would be reduced to 1.0 and the effective pga HCLPF would be increased $0.32 \times 1.97=0.63 \mathrm{~g}$

\subsection{ESTIMATION OF BEST ESTIMATE RATHER THAN HCLPF Pga's}

In performing this evaluation, a computer program FRAGIL was used. This program is designed to take estimates of seismic capacities (HCLPF or otherwise) and apply estimated variability on both fallure probabllity as well as confidence as shown in Table 1 to determine the probablitity of fallure at the 95,50 and 5 percent probability and at the 95, 50, and 5 percent confidence levels. In this evaluation variablitity as to probability of fallure and confidence were estimated as coefficients of variation on both fallure and confidence. The results of these estimates are shown in Figures 7-13 of this report. However, in no case were the median estimates permitted to exceed $7.25 \times$ the calculated lateral load capacity at the center of gravity of the equipment.

\subsection{RESULTS AND CONCLUSION}

\subsection{HCLPF Estimation}

The following is a summary of the HCLPF determined in this evaluation.

[1] Note this is based on uncracked section actual frequency at or near fallure could be based on at least a partially cracked section. This calculation results in a conservative HCLPF estimate. 


\subsubsection{Vertical Flat Bottom Tank}

\section{Spectra Given NUREG/CR 0098 \\ in Appendix $C$ Median Ground Spectra \\ $0.32 \mathrm{~g} \mathrm{pga} \quad 0.36 \mathrm{~g} \mathrm{pga}$}

\subsubsection{Vertical Clip Angle Mounted Tank}

Floor Spectra Given

in Appendix $C$

$0.88 \mathrm{~g} \mathrm{pfa}$

$0.42 \mathrm{~g} \mathrm{pga}$

8.1.3 Generic Motor Control Center (Operating During EQ.)
a) Mounted on Floor
Floor Spectra Given
in Appendix $C$
$0.32 \mathrm{~g} \mathrm{pfa}$
$0.15 \mathrm{~g} \mathrm{pga}$

b) Mounted on Ground

NUREG/CR 0098 Median Ground Spectra

$.71 \mathrm{~g}$ pga

8.1.4 Horizontal $\mathrm{HX}$ on Two Saddles

Floor Spectra

Given in Appendix $C$

$0.93 \mathrm{~g} \mathrm{pfa}$

$0.44 \mathrm{~g} \mathrm{pga}$

\subsubsection{Block Wal1}

Floor Spectra

Given in Appendix $C$

$0.67 \mathrm{~g} \mathrm{pfa}$

$0.32 \mathrm{~g} \mathrm{pga[2]}$

\subsection{Best Estimate (50 Percent Probability) Peak Ground Acceleration} Evaluation of Fallure at the 50 Percent Confidence Levels

The following is a summary of the best estimate seismic peak ground acceleraion required to cause component fallure.

[2] See discussion in Section 6.3 concerning the change in wall stiffness and its effect on HCLPF estimation. 


\subsubsection{Vertical Flat Bottom Tank}

Floor Spectra Given

in Appendix $C$

(FIgure 7)

$1.25 \times .667=0.83 \mathrm{~g}$

$50 \%=1.10 \mathrm{~g}$

Use $0.83 \mathrm{~g}$ for median
NUREG/CR 0098

Median Ground Spectra

(Figure 8)

$1.25 \times 0.667=0.83 \mathrm{~g}$

$50 \%=1.20 \mathrm{~g}$

Use $0.83 \mathrm{~g}$ for median

\subsubsection{Vertical Clip Angle Mounted Tank}

Floor Spectra Given

in Appendix C (Figure 9)

$1.25 \times .88=1.10 \mathrm{~g}$

$50 \%=2.6 \mathrm{~g}$

Use $1.10 \mathrm{~g}$ for median

8.2.3 Generic Motor Control Center (Operating During E.Q.)

Floor Spectra Given

NUREG/CR 0098

in Appendix $C$

(Figure 10)

Median Ground Spectra

(Figure 11)

$1.25 \times 1.5 \mathrm{~g}=1.88 \mathrm{~g}$

$50 \%=4.0 \mathrm{~g}$

$1.25 \times 1.5=1.88 \mathrm{~g}$

Use $1.88 \mathrm{~g}$ for median

$50 \%=2.7 \mathrm{~g}$

Use $1.88 \mathrm{~g}$ for median

It should be noted that in developing the median estimate of fallure, I do not consider the GERS (Function during) curve shown in Figure 2 to Appendix $D$ of this report as a best estimate of functional fallure. Rather the curve shown is a lower bound estimate based on the test data shown. For this reason, I do not believe it is appropriate to base a median estimate of fallure on this

curve. Hence the median estimate contained in this report is higher than what would be expected using the GERS (Function during) curve only.

\subsubsection{Horizontal HX on Two Saddles}

Floor Spectra Given

in Appendix C (Figure 12)

$1.25 \times 0.925=1.15 \mathrm{~g}$

$50 \%=1.95 \mathrm{~g}$

Use $1.15 \mathrm{~g}$ for median

\subsubsection{Block Wall}

Floor Spectra Given

in Appendix C (Figure 13)

$1.25 \times 1.04=1.30 \mathrm{~g}$

$50 \%=2.10 \mathrm{~g}$

Use $1.30 \mathrm{~g}$ for median 
9.0 REFERENCES

(1) Kennedy, R.P., "Various Types of Reported Safety Margins and Their Uses," Section 2, Proceedings of EPRI/NRC Workshop on Nuclear Power Plant Reevaluation for Earthquakes Larger than SSE," Palo Alto, October 1984 .

(2) Prassinos, P.G. et. a 1. "Recommendations to the Nuc lear Regulatory Commission on Trial Guldelines for Selsmic Margin Reviews of Nuclear Power Plants," NUREG/CR-4482, March 1986.

(3) NTS Engineering and RPK Consulting; "Evaluation of Nuclear Power Plant Setsmic Margin," Tech. Paper No. 1551.05 Prepared for EPRI, Nov. 1986

(4) EQE, "Refueling Cavity Water Storage Tank - TK4" Seismic Margin Study, Apr 111987

(5) Kennedy, R.P. "Memorandum to HCLPF Panel - Modified Bolt Chairs for Tank TK-4", " 8 July 1987

(6) Roark, R.J. and Young, W.C. Formulas for Stress and Strain 5 th Ed. McGraw-H111, 1975.

(7) Haroun, M.A. and G.W. Housner, "Selsmic Design of Liquid Storage Tanks," Journal of the Technical Councils of ASCE, Vol. 107, No. TC1, 1981, PP 197-207 
Table 1 Estimates of Cov's of Equipment Seismic Margins as a Function of Location, Frequency and Damping

\begin{tabular}{|c|c|c|c|c|c|c|c|c|c|}
\hline \multirow{4}{*}{$\begin{array}{l}\text { EQUI IPMENT } \\
\text { MOUNTING }\end{array}$} & \multicolumn{9}{|c|}{ Cov (Percent) } \\
\hline & \multicolumn{8}{|c|}{ Faflure } & \multirow[t]{3}{*}{ Confidence } \\
\hline & \multicolumn{4}{|c|}{$\begin{array}{r}\text { Resonant } \\
2-15 \mathrm{~Hz} \\
\text { Damping } \\
\end{array}$} & \multicolumn{4}{|c|}{$\begin{array}{c}\text { Out of Resonance } \\
>15 \mathrm{~Hz} \\
<2 \mathrm{~Hz} \\
\text { Damping }\end{array}$} & \\
\hline & 3 & 5 & 7 & 10 & 3 & 5 & 7 & 10 & \\
\hline Floor $>40^{\circ}$ & 200 & 175 & 150 & 125 & 100 & 87 & 75 & 62 & 35 \\
\hline Floor $\leq 40^{\prime}$ & 150 & 125 & 110 & 90 & 85 & 60 & 50 & 45 & 25 \\
\hline Ground W/SSI & 100 & 85 & $75 \div$ & 65 & 60 & 55 & 45 & 40 & 15 \\
\hline Ground w/O/SSI & 90 & 80 & 70 & 60 & 55 & 50 & 40 & 40 & 10 \\
\hline
\end{tabular}




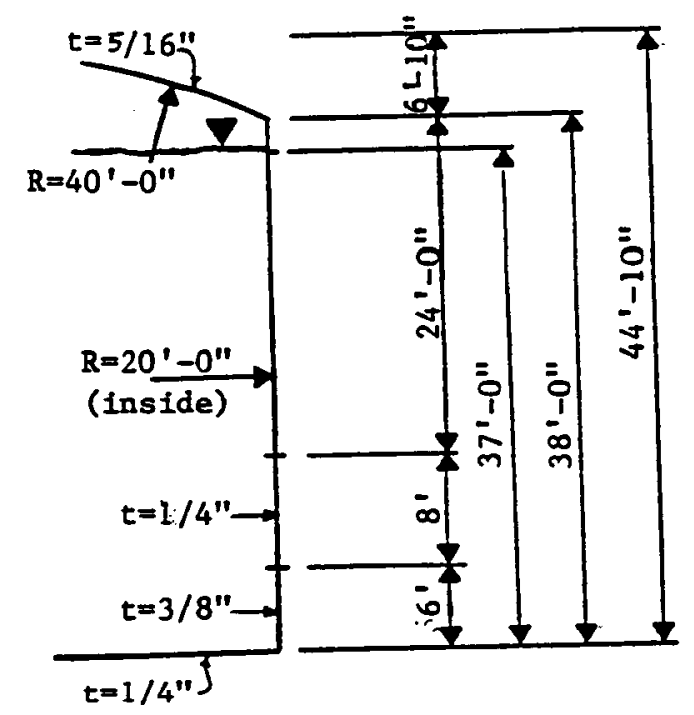

Figure la--Vertical Flat Bottom Tank Geometry
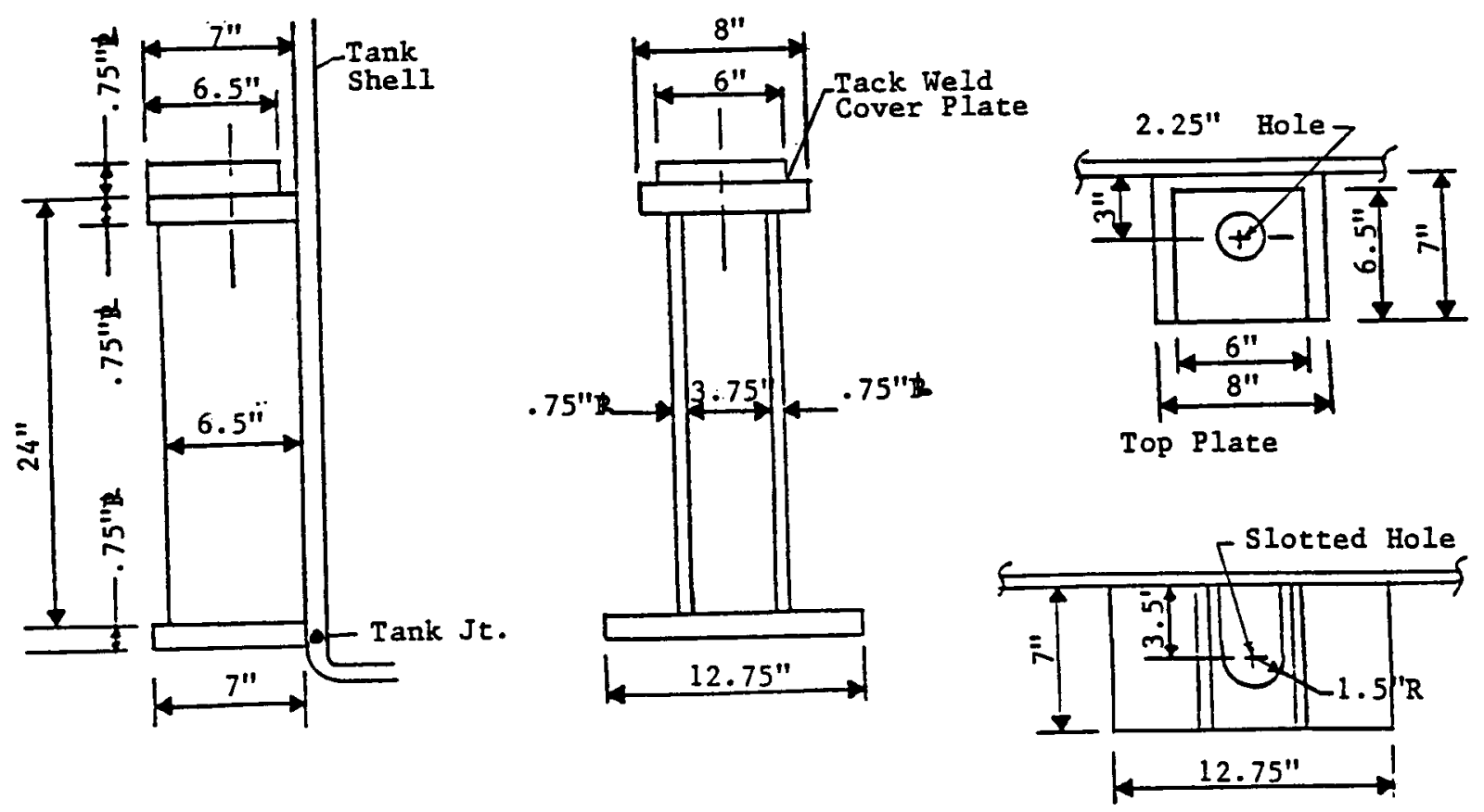

Figure 1b--Modified Bolt Chair for Flat Bottom Tanks 


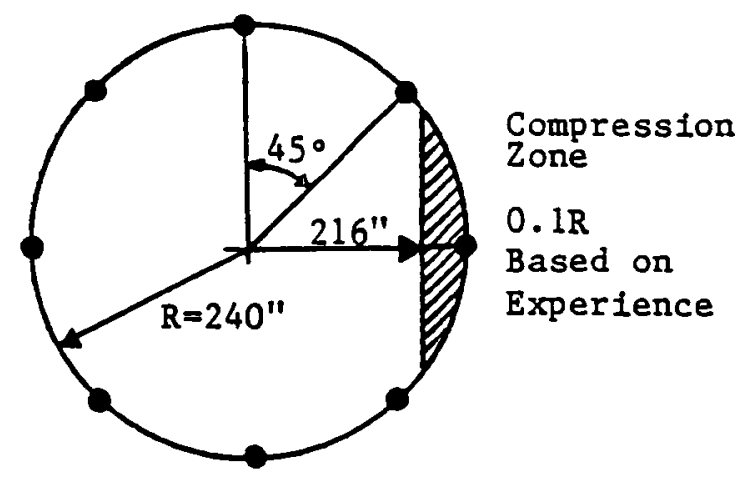

Figure 2--Overturning Diagram

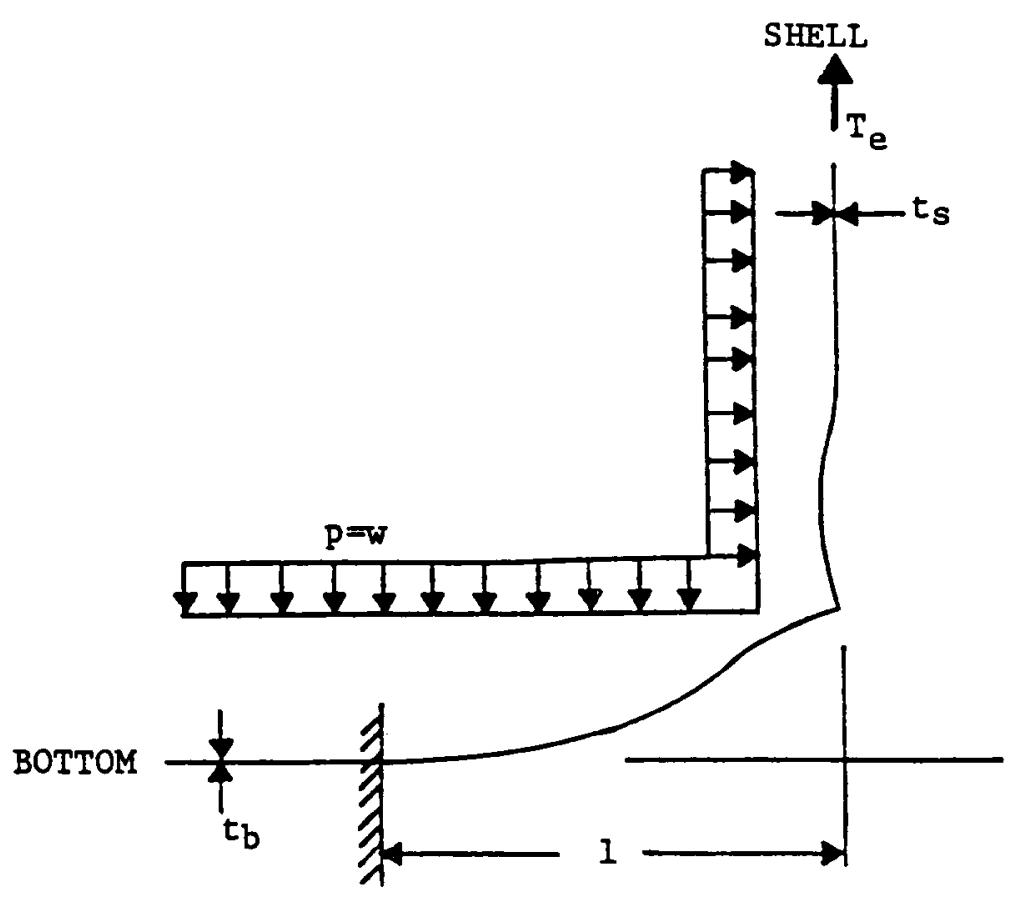

Figure 3--Schematic Illustration of Tank Bottom Behavior Near Tensile Lift-off Region of Tank Shell 

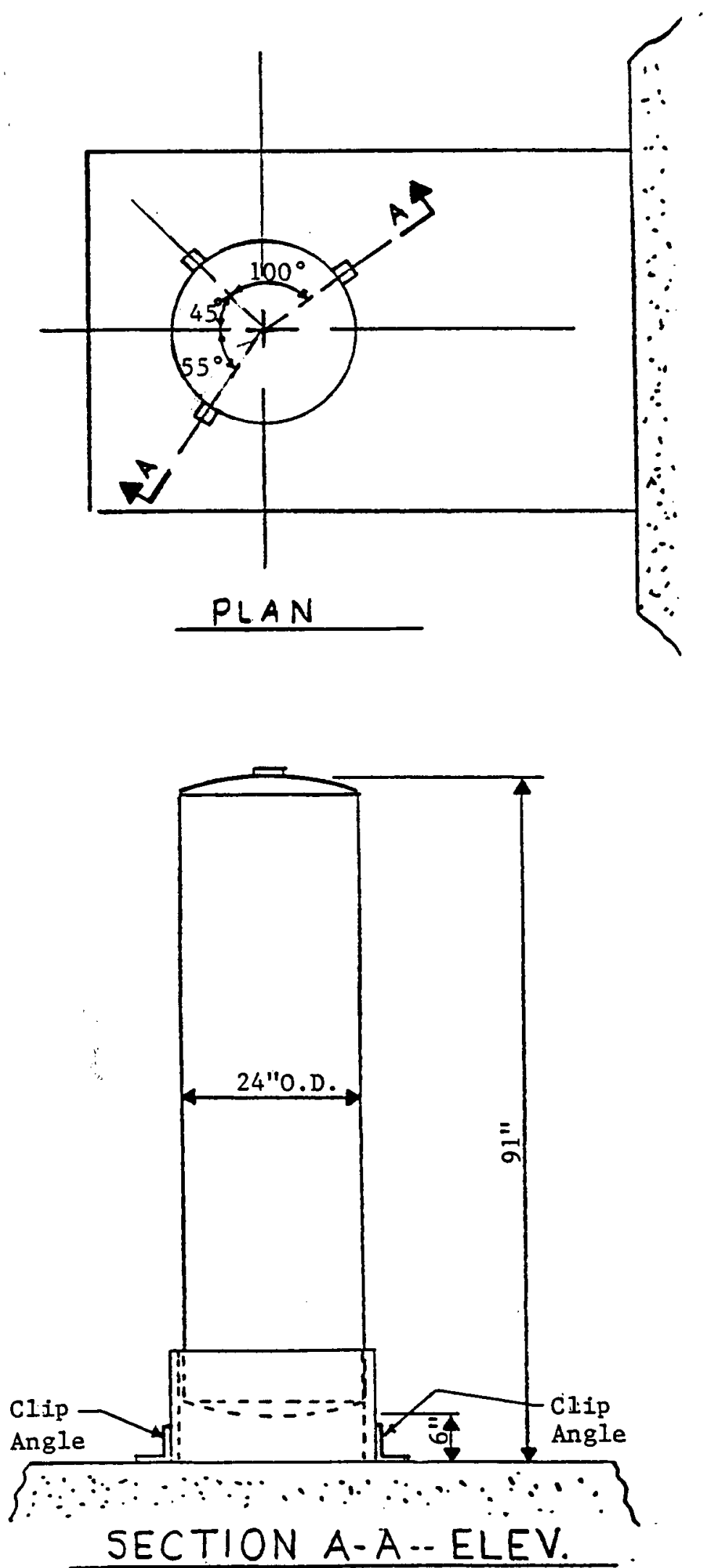

Figure 4--General Arrangement of Air Tank 


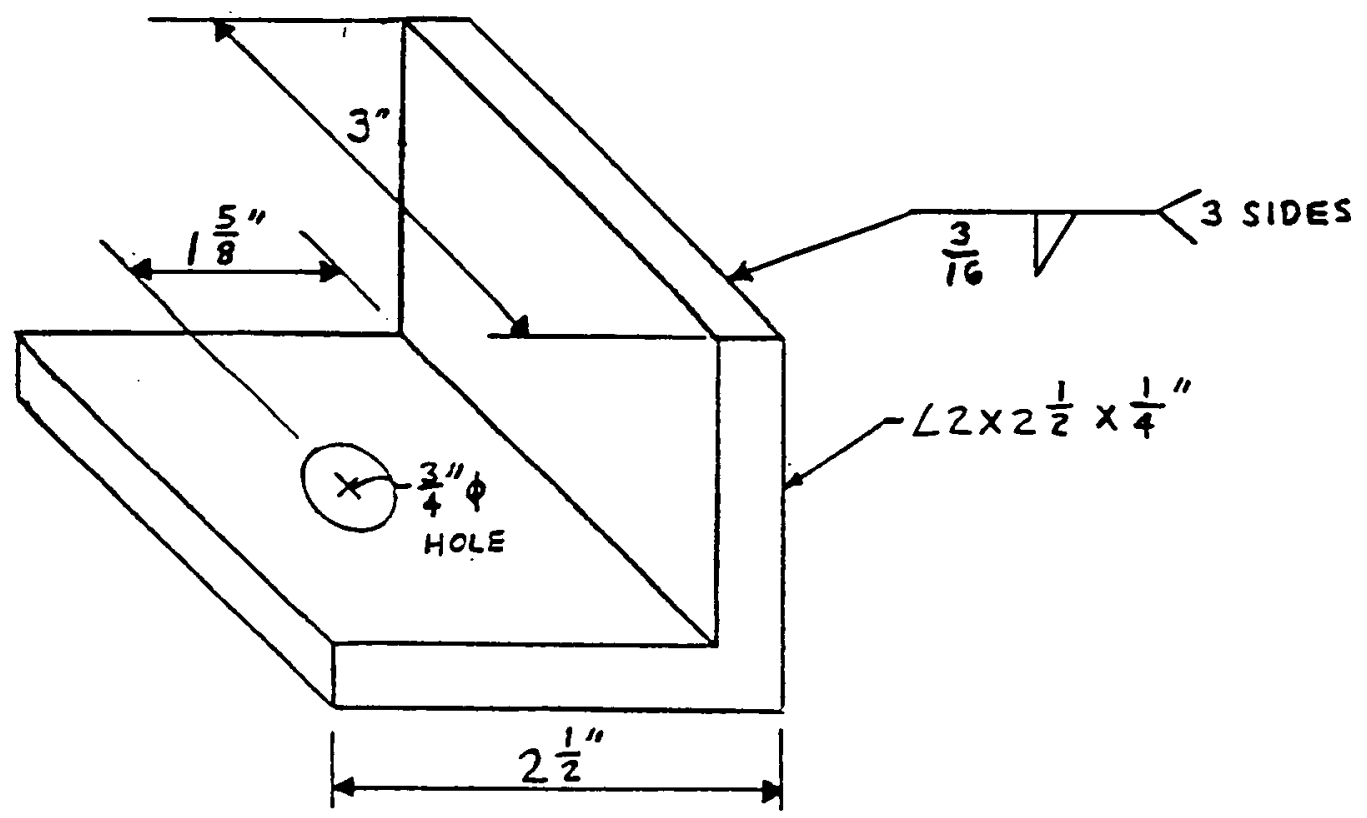

Figure 5--Clip Angle Detail 

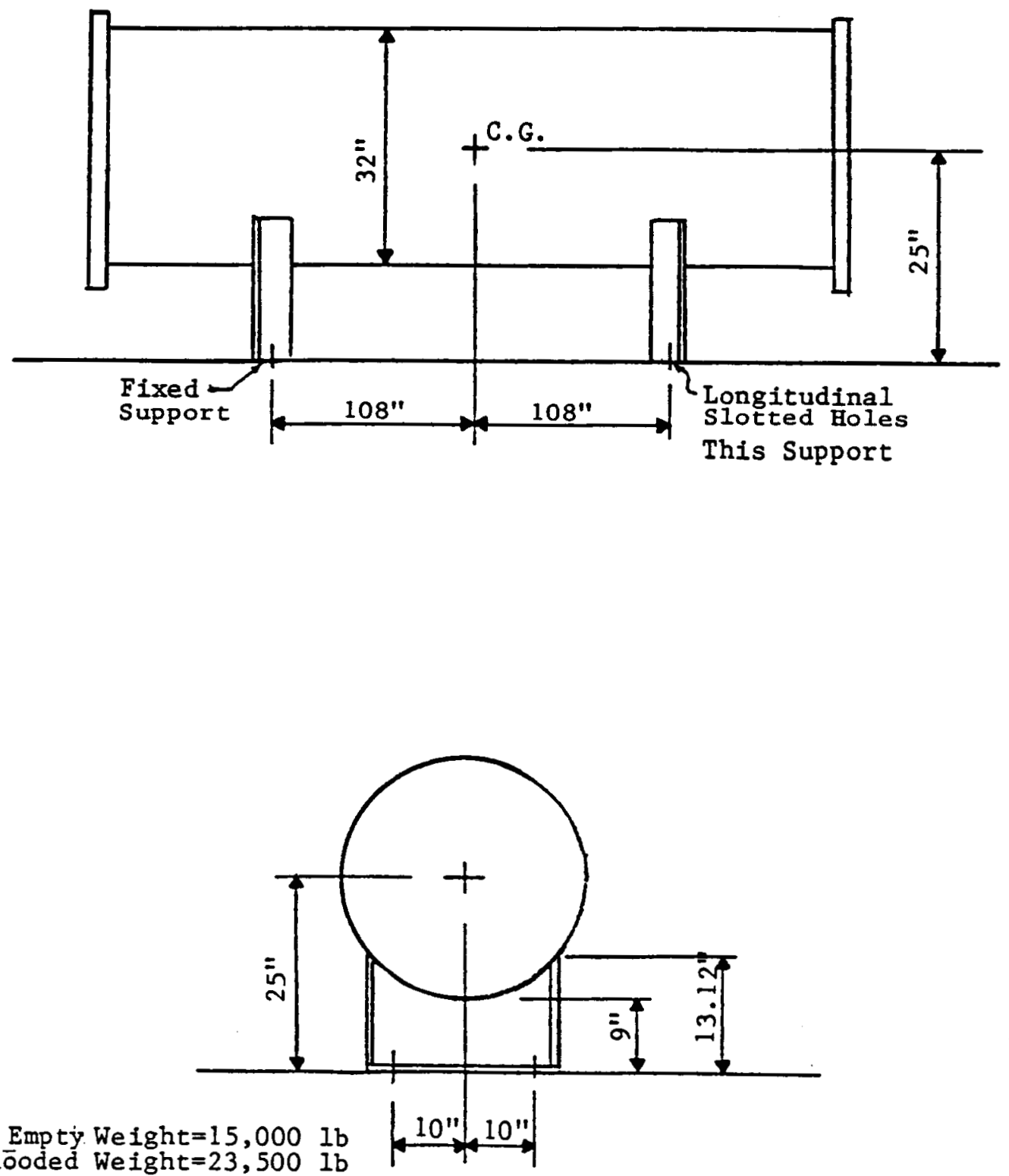

Empty. Weight $=15,000$ Flooded Weight $=23,500 \mathrm{Ib}$

Figure 6--General Arrangement of Horizontal Heat Exchanger 
Figure 7--HCLPF FRAGILITY CURUES FOR 95\%, 50\% AND 5\% CONFIDENCE LEUELS Component: Vertical Flat Bottom Tank HCLPF $=.32 \mathrm{~g}$

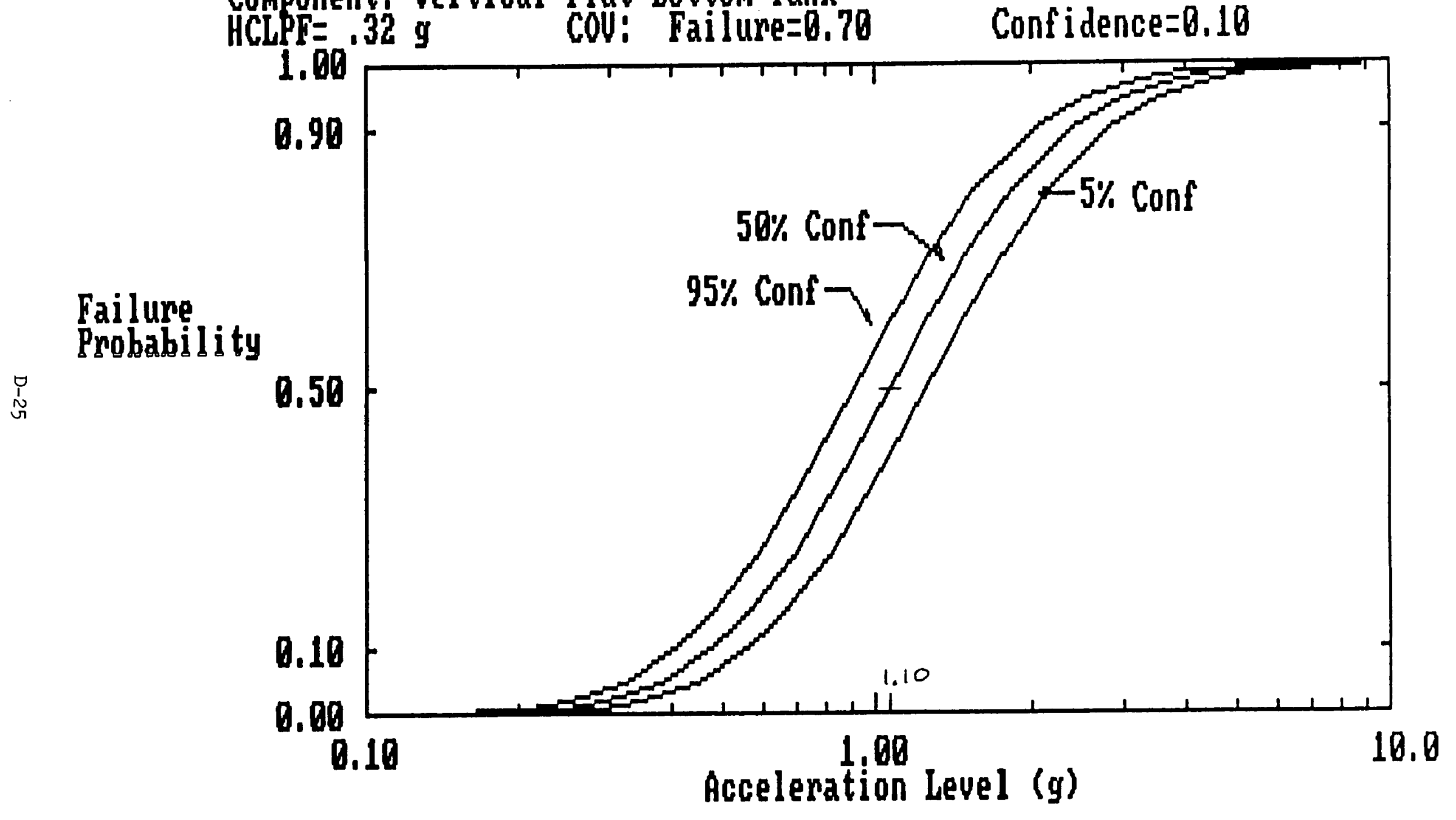


Figure 8--HCLPF FRAGILITY CURUES FOR 95\%, 50\% AND 5\% CONFIDENCE LEUELS Component: Uertical Flat Bot tom Tank

HCLPF $=.36 \mathrm{~g} \quad$ Cov: Failure $=0.70 \quad$ Conf idence $=0.10$

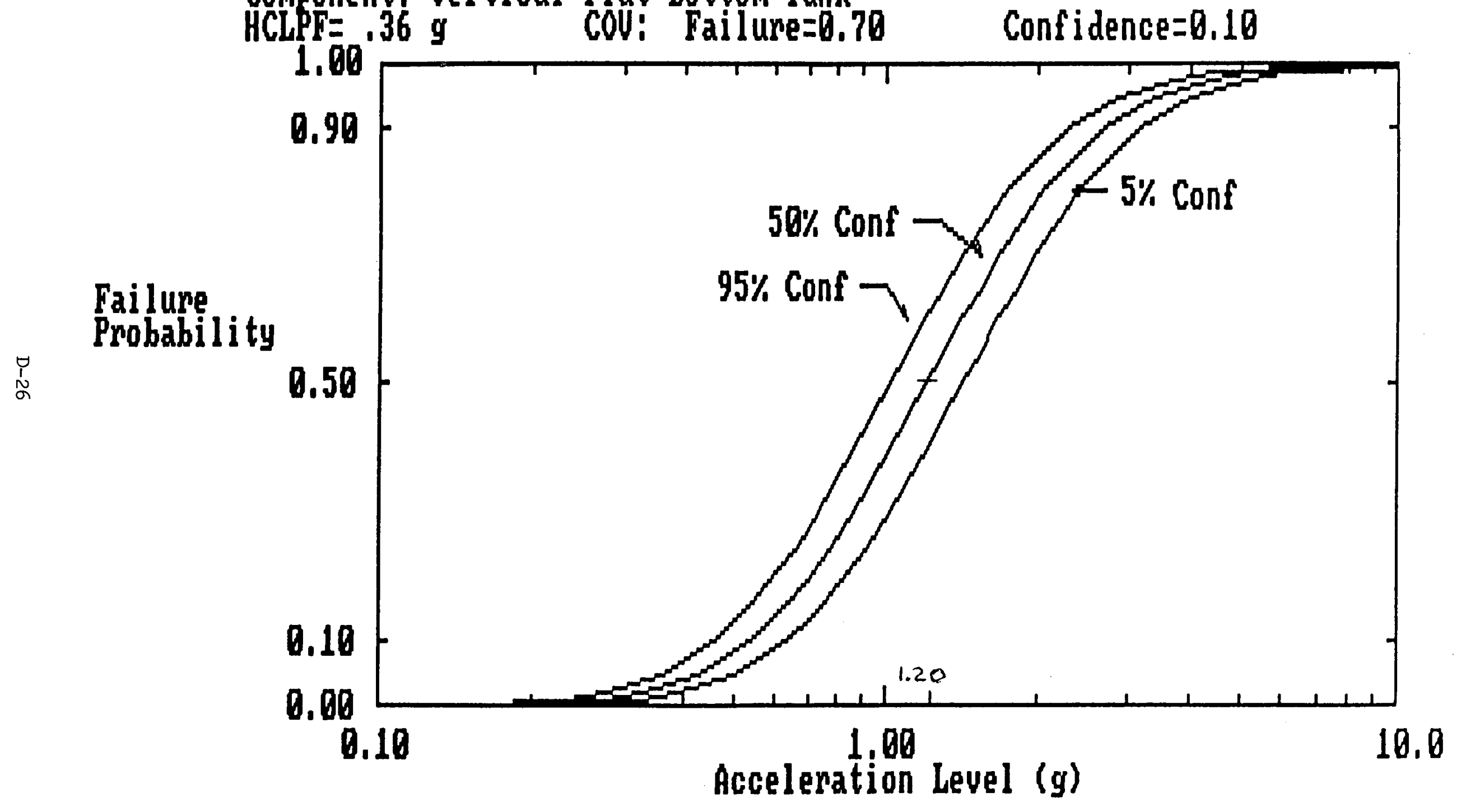


Figure 9--HCLPF FRAGILITY CURUES FOR 95\%, 50\% AND 5\% CONFIDENCE LEUELS Component: Vertical Clip Angle hounted Tank HCLPF $=.42 \mathrm{~g}$ Cov: Failure $=0.87$

Conf i dence $=0.35$

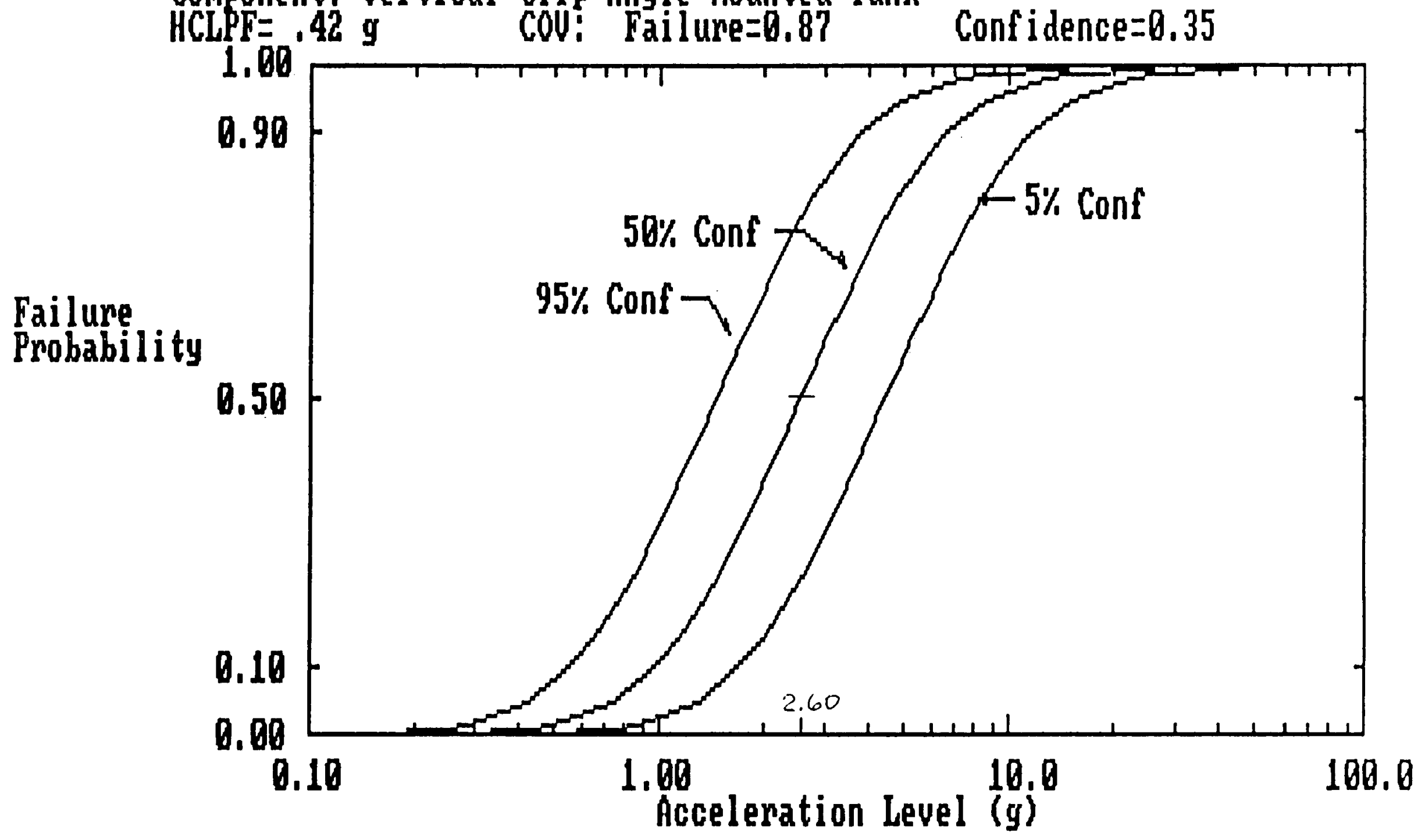


Figure 10-HCLPF FRAGILITY CURUES FOR 95\%, 50\% AND 5\% CONFIDENCE LEVELS Component: Generic Motor Control Center--Mounted on Floor
HCLPF $=32 \mathrm{~g} \quad$ cov: Failure $=1.75 \quad$ Conf idence $=0.35$

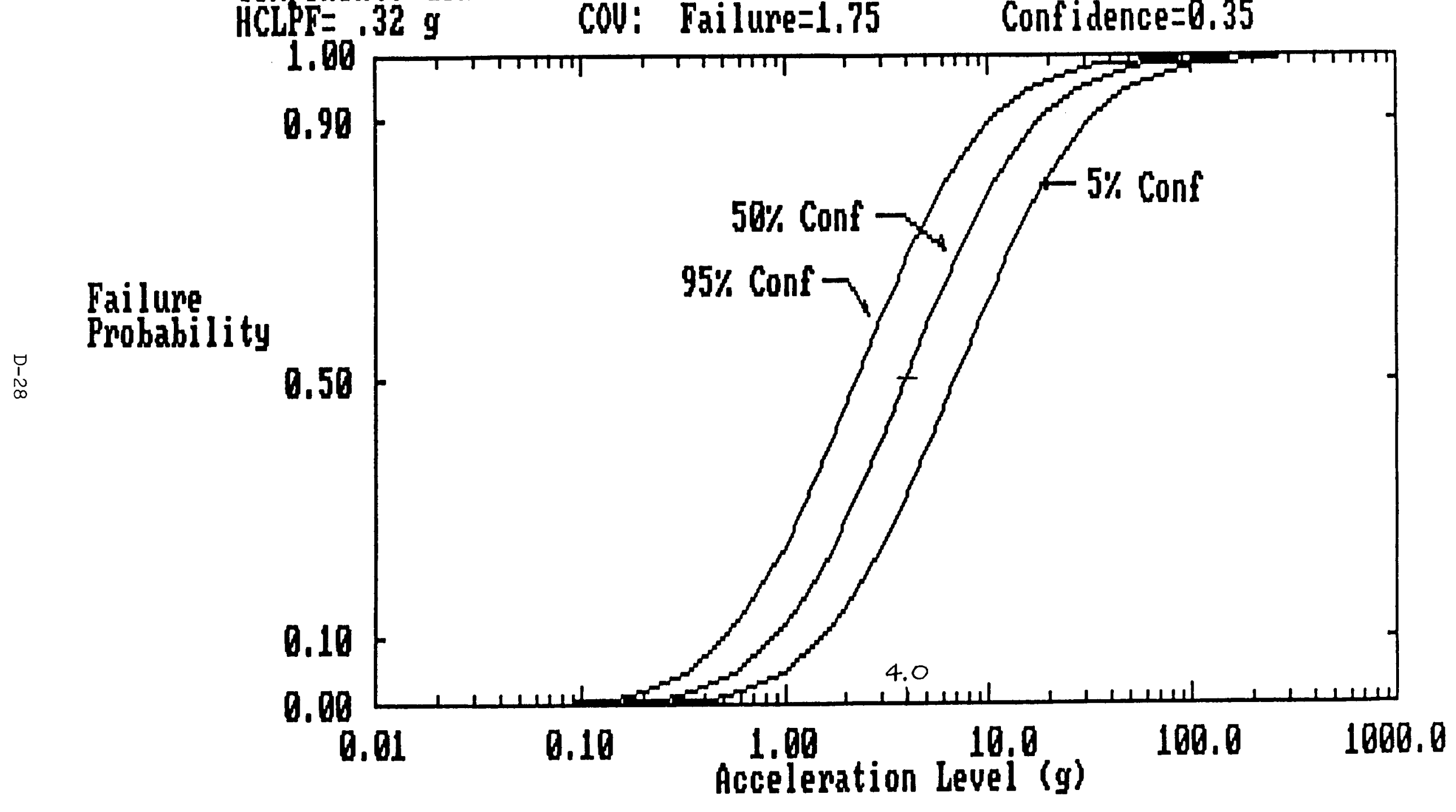


Figure 11-HCLPF FRAGILITY CURUES FOR 95\% 50\% AND 5\% CONFIDENCE LEUELS Component: Generic Motor Control Center--Mounted on Ground

HCLPF $=.71 \mathrm{~g} \quad$ COV: Failure $=0.80 \quad$ Conf idence $=0.10$

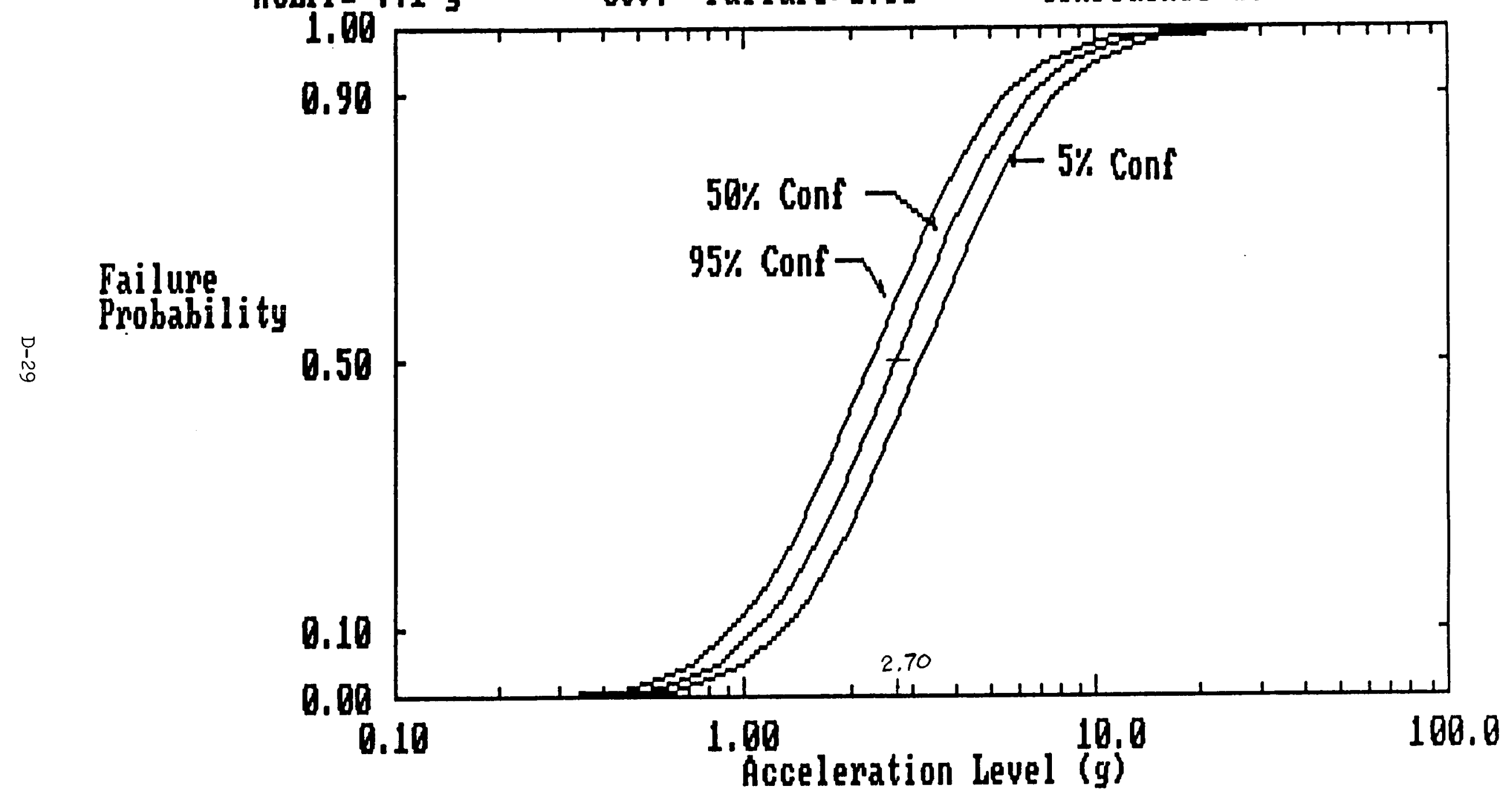


Figure 12-HCLPF FRAGILITY CURUES FOR 95\%, 50\% AND 5\% CONFIDENCE LEVELS Component: Horizontal HX on Two Saddles HCLPF $=.44 \mathrm{~g}$ Covi Failure $=0.60$ Conf idence $=0.35$

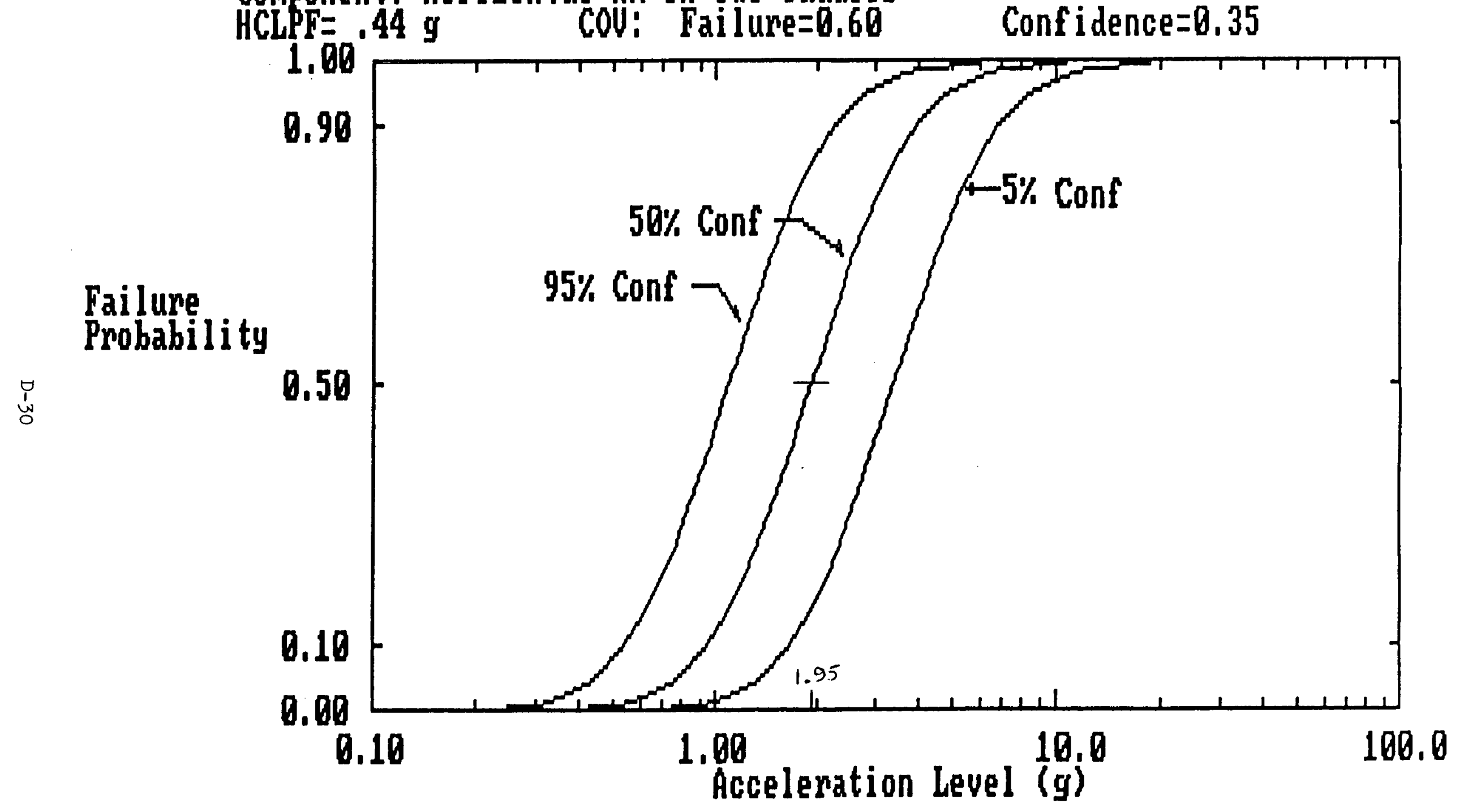


Figure 13-HCLPF FRAGILITY CURUES FOR 95\%, 50\% AND 5\% CONFIDENCE LEUELS Component: Block Wall

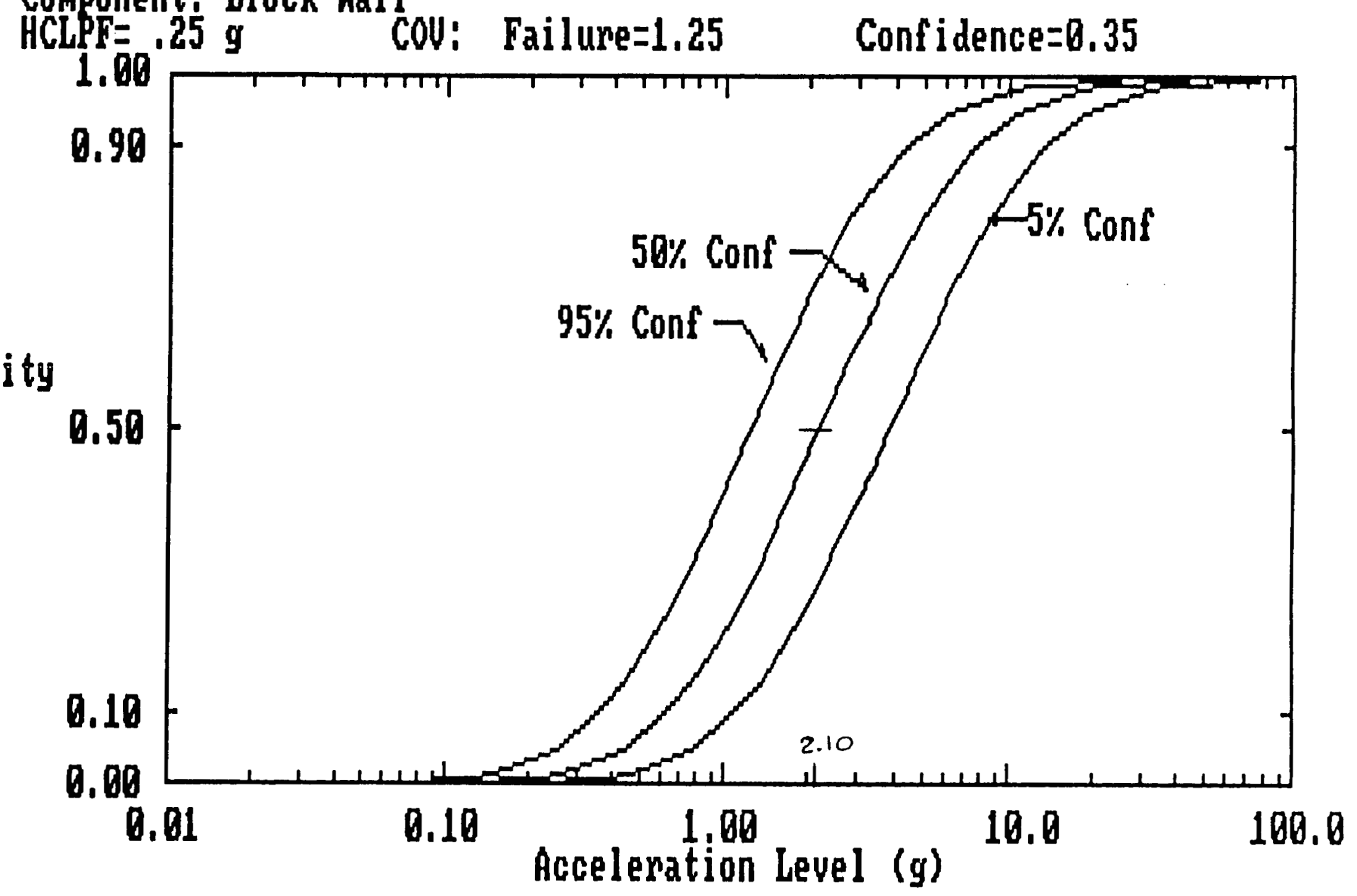


$87 C 7451$

0046G-9

Appendix A

Background on Development of COFM Method

for Determination of HCLPF's 
NUREG/CR-4482

UCID-20579

\section{Recommendations to the Nuclear Regulatory Commission on Trial Guidelines for Seismic Margin Reviews of Nuclear Power Plants}

\section{Draft Report for Comment}

Manuscript Completed: December 1985

Date Published: March 1986

Prepared by

P. G. Prassinos, M. K. Ravindra, and J. B. Savy, under the guidance and with the concurrence of the Expert Panel on the Quantification of Seismic Margins: R. J. Budnitz (Chairman), P. J. Amico, C. A. Cornell, W. J. Hall, R. P. Kennedy, J. W. Reed, and M. Shinozuka.

Lawrence Livermore National Laboratory

7000 East Avenue

Livermore, CA 94550

Prepared for

Division of Engineering Technology

Office of Nuclear Regulatory Research

U.S. Nuclear Regulatory Commission

Washington, D.C. 20555

NRC FIN No. A-0398 
The systems analyses performed to develop the Boolean expression should be fully documented. This documentation should include the analysis techniques and tools used, the method used to "prune" the fault trees and the justification, and a description of the basic events (their failure modes) that make up the Boolean expression

\subsection{Step 8 - Margin Evaluation of comonents and Plant}

Steps 7 and 8 are performed concurrently and with close interaction between the system and fragility analysts.

The components that require margin evaluation, called the "screened in" components, have been identified during the plant review and the two plant walkdowns. Design details and actual existing conditions have been recorded (as far as practical).

The objectives of the analysis in step 8 are:

$$
\begin{aligned}
& \text { - To estimate the HCLPF of these components } \\
& \text { - To estimate the HCLPF of the plant. }
\end{aligned}
$$

For each HCLPF evaluation, two alternative approaches are presented.

\subsubsection{Capacity of Components}

The concept of HCLPF is similar to the traditional notion of using codeminimum strengths and code-maximum loads in structural design codes. The specification of these minimums and maximums was done by code committees using past performance data, results of analysis and research, and collective expert judgments. They implicitly or explicitly recognize the uncertainties in loads and strengths. The capacity of a component calculated using these specifications was considered to be conservatively low. The HCLPF value calculated using the procedures described in this report has similar attributes: it is conservative, and it recognizes the uncertainties based on the panel's judgment.

There are two candidate approaches for calculating the HCLPF value of components: the Conservative Deterministic Failure Margin (CDFM) method proposed by Kennedy (Ref.7) and the fragility-analysis method. In the CDFM method, a set of deterministic rules (e.g., ground response spectra, damping, material strength, and ductilityl is prescribed; the capacity of the component determined using these rules gives a HCLPF value that may be more conservative than necessary. In the fragility-analysis method, the median ground acceleration capacity $A_{m}$ and the logarithmic standard deviations $B_{R}$ and $B_{U}$ for which there is less than a five percent probability of failure with 95 percent confidence. The randomness and uncertainty in the median capacity are assumed to be lognormally distributed. In the trial plant reviews, both these methods may require that seismic response analyses separate from the design analyses be performed. The fragility analyst must review the structural models used in the plant design to confirm the adequacy of these models and the appropriateness of scaling the responses. If scaling is not appropriate, the response analysis becomes a major effort in seismic margin reviews. In the CDFM method, values for a number of parameters (e.g., system ductility, 
damping, and response spectra) need to be selected. In the fragility-analysis method median values $B_{R}$ and $B_{U}$ need to ber estimated by the fragility analyst. There have not been enough studies done to compare the HCLPF estimated using these two candidate methods for different components. Additional comparison studies should be conducted to identify situations where both methods would yield comparable results and those where the results would widely differ. A review of such results would also lead to a "calibration" of the parameter values of either or both methods so that the two methods give essentially identical capacity estimates. The final goal of such studies would be to provide a set of deterministic rules in the CDFM method for calculating the HCLPF of screened in components. Until such research is done, it is recommended that both the candidate methods be used to calculate the HCLPF of components in trial plant reviews. The trial plant reviews should be viewed as providing further basis and guidance on research towards calibration of the two candidate methods.

\subsubsection{Conservative Deterministic Failure Margin (CDFM) Method}

In this method a failure margin is computed using conservative material and response parameters but taking credit for conservatively defined failure capacity and inelastic energy absorption capability of structures and components. The following parameter values have been proposed (Ref. 7) and might be more conservative than necessary:

Load Combination:

Ground Response Spectrum:

Damping :

Structural Model:

Soil-structure Interaction:

Material strength:

Static Capacity Equations:

System Ductility:

(Inelastic Energy

Absorption)

Flor Spectra Generation:
Normal + Earthquake Review Level

848 Non-Exceedence Probability Site-specific Spectrum

Depending on the earthquake review level, the following are the conservative estimates of the median values:

Structure: $\quad 7 \%$

Piping: $\quad 5 \%$

Cable trays: $15 \%$

Best-estimate - median

Envelope expected parameter variation

95\% exceedance actual strength

$84 \%$ exceedance by test data or code equation

Conservatively selected to be between 1.0 and 1.5. For shear wall structures, should not be less than 1.3 .

Median damping value for equipment

Frequency shifting of floor spectra rather than peak broadening. 
For structure/equipment qualified by analysis, the response of the equipment is calculated using the above structural and equipment response parameters. potential failure modes of the equipment are identified. The static inelastic capacities of the structure/equipment are estimated. If the capacity of the structure/equipment exceeds the calculated response for the load combination (Normal + Earthquake Review Level), it is assumed that the component has a HCLPF value exceeding the earthquake review level peak ground acceleration: For equipment qualified by test, the floor spectrum for median equipment damping is generated using the above conservative structural and/or equipment response parameters. If the floor spectral values throughout the equipment Erequency range of interest are less than generic equipment ruggedness spectrum (GERS) for the equipment (Ref. 18), it is assumed that the equipment has a BCLPF exceeding the earthquake review level PGA. So far, GERS has been developed for seven classes of equipment (i.e., motor-operated valves, motor control centers, switchgear, batteries and battery racks, inverters, battery chargers, and relays). For other equipment, one should use the highest spectral value for which similar equipment has been qualified as the capacity.

The GERS will be lower than the lowest observed failure level for the equipment (i.e., the GERS is the highest level for which the equipment did not fail). For equipment mounted on floors at higher elevations in the structure, the conservatisms in floor spectra generation and the conservatisms in structural parameters (i.e., damping and system ductility) yield HCLPF values that are considerably less than the median capacities. However, for equipment on grade that do not include significant response conservatism, use of GERS or experience data may not guarantee that there is no "cliff" in the capacity beyond the value of HCLPF (i.e., the component may fail suddenly when the peak ground acceleration exceeds the HCLPF value, instead of a gradual increase in the probability of failure increases). To avoid this problem, it is recommended that the capacity determined by experience data for grade level equipment be reduced by a factor. This factor may be determined during the trial plant reviews.

By a judicious selection of the values of different parameters, the CDFM method aims to produce a conservative estimate of the component's HCLPF. However, the CDFM method is less conservative than the procedures given in the Standard Review Plan (Ref. 9). The load combination specified is more liberal compared to the SRP requirements, i.e., no OBE load combination and no LOCA + review earthquake load combination in the CDFM method. The ground response spectrum is a $84 \%$ nonexceedence probability site-specific spectrum and is expected to be less conservative than the R.G. 1.60 spectrum. Similarly, the camping values proposed for the seismic margin review are more liberal than those specified in the standard Review Plan.

The basis for the selection of values of different parameters in the CDFM methods and how they contribute to the high confidence in the capacity that assures a low probability of failure should be studied. For example, the use of 848 nonexceedence-probability site-specific spectrum and conservative estimates of the median damping are expected to result in a computed capacity indicating a low probability of failure. The use of material strength at $95 \%$ exceedance value and 848 exceedance value for static capacity prediction equations is expected to contribute to the high confidence statement about the capacity. However, this approach cannot be used to determine the 
contributions of different parameters because the seismic capacity of a component is a nonlinear function of these parameters; the impact on capacity of any value of a single parameter depends not only on the significance of the parameter on the median capacity but also on the relative variabilities (i.e., randomness and uncertainties) of all the parameters. The CDFM method discussed here may be even more conservative than necessary. Until further research on calibration is performed (discussed earlier), the degree conservatism cannot be quantified.

\subsubsection{Fragility-Analysis Method}

One method of describing the fragility of a component is to express it in terms of three parameters (Ref. 19): median capacity Am' logarithmic standard deviations $\beta_{R^{\prime}}$ and $B_{U}$ representing, respectively, randomness in the capacity and uncertainty in the median value. (Fragility Handbook, Ref. 19) Rather than estimating the median capacity as a product of an overall median safety factor times the SSE pga for the plant (where the overall safety factor is a product of a number of factors representing the conservatisms at different stages of analysis and design), the median capacity is evaluated using median structural and equipment response parameters, median material properties, and ductility factors, median static capacity predictions, and realistic structural modeling and method of analysis. If the fragility analyst is convinced that the scaling of response is appropriate, the median seismic capacity may be estimated as the product of the overall median safety factor and the SSE pga.

The median response of the structure/equipment for the earthquake review level (REL) is calculated. The median capacity of the structure/equipment is estimated as the median static capacity multiplied by the median inelastic energy absorption capacity factor. The median ground acceleration capacity of the structure/element is approximately estimated as:

$$
A_{m}=\text { (REL) } \quad \begin{aligned}
& \text { Median Normal Design } \\
& \frac{\text { Capacity }- \text { Load Response }}{\text { Median Response caused by REL }}
\end{aligned}
$$

This is valid because the normal loads have low variability and the normal design loads are conservatively selected.

In lieu of explicitly determined $B_{R}$ and $B_{U}$, the ACLPF value for the structure/ equipment may be conservatively estimated by assuming $B_{R}+B_{U}=0.08$ and the lognormal model: (Ref. 10 and 12)

$$
\text { HCLPF } \approx 0.25 A_{\mathrm{m}}
$$

If the HCLPF value calculated as above does not exceed the earthquake review level, the analyst may revise the capacity by estimating $B_{R}$ and $B_{U}$ using plant-specific data and PRA methods (i.e., seismic fragilities). Another option, if this proves to be too conservative, is to revise the mediancapacity estimate by performing further studies such as nonlinear, inelastic static, or time history dynamic analyses. 
This section is an excerpt from

EPRI Report NP-4101-SR

Section 2

VARIOUS TYPES OF REPORTED SEISMIC MARGINS AND THEIR USES

\author{
R. P. Kennedy*
}

\title{
INTRODUCTION
}

Nuclear power plant structures and safety-related systems have been generally. designed conservatively for a safe shutdown earthquake (SSE) and more conservativel for a smaller operating basis earthquake (OBE). Depending upon the relative conservatism of the design criteria, either the SSE or the OBE will control the design. For plants with SSE levels less than $0.2 \mathrm{~g}$, often non-seismic loadings control the design.

In recent years, increasing knowledge in the geoscience field has led to a better understanding that, although highly unlikely, it is possible for the nuclear power plant to be subjected to earthquake ground motion greater than the ground motion for which the plant was designed. For this reason, interest has developed in demonstrating that nuclear plant structures and safety-related systems can safely withstand earthquake ground motion larger than their design earthquake ground motions (SSE and OBE). Within this paper, this larger-than-design earthquake ground motion will be called the seismic margin earthquake (SME) to distinguish it from the design earthquakes. The plant has already been designed. Therefore, for the SME the goal is not to design the plant. The goal is to determine the perform ance of already-designed structures, components, and systems when subjected to the SME. Different and generally more liberal criteria should be used when evaluating the performance of structures, components and systems for the SME than were used design. Retrofit, and redesign, should only be contemplated if one cannot show a seismic margin greater than unity for the SME using these more liberal criteria. In other words, the SME is not a design earthquake. It is not a replacement for the SSE and generally has nothing to do with design. The SME is a performancecheck earthquake.

*Senior Consultant, Structural Mechanics Associates, Newport Beach, California 
VARIOUS TYPES OF SEISMIC MARGIN

Existing literature contains a wide variety of highly dissimilar criteria for determining seismic margin. Therefore, one must be careful to distinguish what type of seismic margin is being reported. Seismic margins reported in the literature can generally be divided into the following four categories:

1. Desian Seismic Maroin (OM) - The seismic margin computed using US NRC Reg. Guides (R.G.), Standard Review Plans (SRP), Design Code Capacities and load combinations.

2. Code (Elastic-Computed) Seismic Margin (CM) - Margin computed using possibly less. stringent structural response parameters (such as damping) and less stringent load combination (normal plus seismic) but assuming essentially elastic behavior and capacities defined by code.

3. Conservative Deterministic Seismic Margin Against Failure (CDFM)A failure margin computed using conservative material and response parameters but taking credit for conservatively defined failure capacity and inelastic energy absorption capability of structures and components.

4. Probabilistic Seismic Margin Against Failure (PFM) - Mediancentered estimate of seismic margin which also displays uncertainties in the estimate.

Both the Design Margin (OM) and the Code Margin (CM) represent traditionally computed margins. As such, they are prescriptive and essentially non-controversial. However, in most cases, such margins are very conservative and are not a good measure of the failure capacity. For some SME problems, it might be adequate to determine the $D M$ or $C M$. Some examples of when the $O M$ or $C M$ might be adequate are:

a. Where the SSE design response spectrum was a Housner spectrum, one might be required to demonstrate margin for a R.G. 1.60 spectrum.

b. Where the SSE waS $0.12 \mathrm{~g}$, one might be required to demonstrate margin for an SME of $0.14 \mathrm{~g}$.

Both of these examples do not require one to push oneself to demonstrate substantial margin. In my experience, the DM or CM approaches are generally adequate to demonstrate margin for a SME less than about $0.15 \mathrm{~g}$ or a SME less than about 1.2 times the SSE. For more severe SME problems, it is generally necessary to consider failure margins.

The Conservative Deterministic Failure Margin (CDFM) is more controversial than either the DM or CM. ACtual failure capacities are highiy uncerzain. A COFM does not display the uncertainty. However, it does represent a reasonably conservative, 
but realistic, measure of the failure capacity of the structure or component. The Probabilistic Failure Marein (PFM) fully displays the uncertainty in failure capacity and represents the most complete and best descriptor of the failure margin. However, there will always be great uncertainty about uncertainty. Secondly, the PFM attempts to remove all or nearly all consarvatism. As such, the PFM will tend to be more judgmental and controversial than the CDFM.

Irrespective of which of the above four types of seismic margin (SM) is being computed, this margin is generally obtained by one of the following equations:

$$
\begin{aligned}
& S M_{1}=\frac{C}{D_{S M E}+D_{N S}} \\
& S M_{2}=\frac{C-D_{N S}}{D_{S M E}}
\end{aligned}
$$

where $C$ represents the capacity, $D_{S M E}$ represents the demand (loading) from the SME, and $D_{N S}$ represents the non-seismic demand (laoding) from ali non-seismic loads in the load combination. In my opinion, Equation (2) provides a better description of the seismic margin than does Equation (1). As an example, assume: $C=100$; $D_{S M E}=20 ; D_{N S}=60$. For this example, $S M_{1}=1.25$ and $S M_{2}=2.00$. The seismic margin $S_{2}$ represents the multiplier by which the SME can be factored before reaching capacity $\mathrm{C}$ while the margin $\mathrm{SH}_{1}$ does not truly provide a seismic margin but provides a margin for the entire load combination. The seismic margin $\mathrm{SM}_{2}$ will often be much larger than the margin $S_{1}$ for structures or components in which seismic is not the dominant loading. In these cases, $S M_{1}$ is misleadingly low.

\section{CANOIDATE CRITERIA FOR VARIOUS SEISMIC MARGINS}

Table 2-1 presents some recommended criteria for use in estimating each of the different types of seismic margin for structures. Essentially each of the deterministic seismic margins (DM, CM, and CDFM) uses conservatively biased criteria. Generally, it is suggested that parameters be set at about either the $84 \%$ or $95 \%$ exceedance probability or non-exceedance probability (NEP) levels depending upon the degree of conservatism desired. For normally distributed parameters, the 84\% NE? and the $84 \%$ exceedance probability values correspond to plus and minus one standard deviation from the mean. This level of conservatism is considered to represent a reasonable degree-of-conservatism for individual structural response parameters. Some capacity parameters should probably be more conservatively selected. The 95\% exceedance probability corresponds to minus 1.65 standard deviations from the mean 
for normaliy distributed parameters. Such a value envelopes essentially all capacity data except for extreme outliers which are likely to be suspect data.

The probabilistic failure margin (PFM) approach uses median-centered estimates with uncertainty bands for each parameter. It is suggested that these uncertainty bands should encompass about the central 90\% of all possible parameter values. Thus, the uncertainty bands should encompass from about the $5 \%$ to the $95 \%$ NEP range with extreme outliers again being ignored.

TABLE 2-1

CANDIDATE SEISMIC MARGIN CRITERIA FOR STRUCTURES

(Example for Reinforced Concrete Structure)

\begin{tabular}{|c|c|c|c|c|}
\hline DARAMETER & $\begin{array}{l}\text { aesicy } \\
\text { marein } \\
\text { on }\end{array}$ & $\begin{array}{c}\text { coors } \\
\text { marain } \\
\text { or }\end{array}$ & $\begin{array}{c}\text { COnsERvative } \\
\text { OETERHIMISTIS } \\
\text { FAtLURE MARGin } \\
\text { COFH }\end{array}$ & $\begin{array}{l}\text { ProsasiLISTIC } \\
\text { PALLURE MARGIN } \\
\text { PFM }\end{array}$ \\
\hline ERRTHQUAKE & $\begin{array}{l}\text { SSE } \\
\text { OAE }\end{array}$ & $\sin$ & sine & $\sin$ \\
\hline LONo esmetratron & $\operatorname{sen}$ & normal - sre & noparal - sine & repreal - sine \\
\hline SDECTRA & R.6. 1.60 & S4E NEP & 84: NEP & 5.-958 NEP \\
\hline sampting & $\begin{array}{l}\text { R.6. } 1.61 \\
\left(\begin{array}{c}68 \\
72:-985\end{array}\right)\end{array}$ & $\begin{array}{l}\text { sas exezEgunce } \\
(7:)\end{array}$ & $\begin{array}{l}\text { sez Exezsance } \\
(7 \%)\end{array}$ & $\begin{array}{l}3:-951 \text { xEP } \\
(5:-203)\end{array}$ \\
\hline $\begin{array}{l}\text { stauctures } \\
\text { roder }\end{array}$ & meotan & meOLAN & meotan & $\begin{array}{l}\text { : MEatan } \\
\text { URCERTALT }\end{array}$ \\
\hline $\begin{array}{l}\text { SotL- } \\
\text { smucruae. } \\
\text { intenaction- }\end{array}$ & $\begin{array}{l}\text { Envelope } \\
\text { Expected } \\
\text { PARAnETER } \\
\text { vaAtarton }\end{array}$ & $\begin{array}{l}\text { Emvelope } \\
\text { ExpECTED } \\
\text { PARATETER } \\
\text { VARTATION }\end{array}$ & $\begin{array}{l}\text { ENvELOPE } \\
\text { ExpECTED } \\
\text { DARAMETER } \\
\text { VARLATIOA }\end{array}$ & $\begin{array}{l}\text { Megian } \\
\text { uxcERTAInTy }\end{array}$ \\
\hline $\begin{array}{l}\text { materRLAL } \\
\text { SiREMGí }\end{array}$ & $\begin{array}{l}\text { gesten } \\
\text { strentent } \\
\text { (3000 pSt) }\end{array}$ & 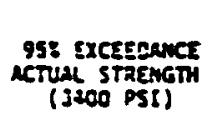 & $\begin{array}{l}\text { 95: ExceEqunCE } \\
\text { AcTuLL STREMETH } \\
\text { (J400 PSI) }\end{array}$ & 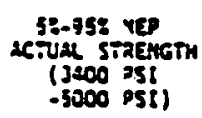 \\
\hline $\begin{array}{l}\text { starte } \\
\text { expacitry } \\
\text { enuarions }\end{array}$ & $\begin{array}{l}\text { coos capacitr } \\
\text { (ACI JiB) }\end{array}$ & $\begin{array}{l}\text { coos capactry } \\
\text { (גet jis) }\end{array}$ & 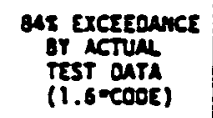 & 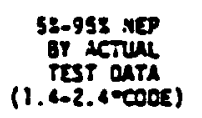 \\
\hline 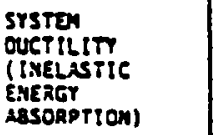 & $\begin{array}{l}\text { Iexoge } \\
(1.0)\end{array}$ & $\begin{array}{l}\text { IOTORE } \\
(1.0)\end{array}$ & $\begin{array}{c}\text { 95s ExcEEDAnce } \\
(1 . j)\end{array}$ & $\begin{array}{r}58-758.7 E P \\
(1.3-3.0)\end{array}$ \\
\hline
\end{tabular}


CONSERVATISM OF COMPUTED RESPONSE

Deterministic computed responses for a SME obtained using the parameters suggested in Table 2-1 and discussed in the previous sections will clearly be conservative when compared to median response results of a probabilistic margin review. In fact, one would have high confidence that there is greater than a $84 \%$ probability that actual responses to the SME would not exceed this deterministic computed response. Comparison of deterministic computed responses with probabilistic responses reported in several seismic PRAs indicate that the median response factor of safety for these deterministic computed responses range from a low of about 1.4 for a stiff concrete structure on rock to a high of about 3.0 for structures with significant SSI effects.

MATERIAL STRENGTH

For design, one generally uses conservatively biased design material strengths. For instance, concrete might have a design compressive strength of 3000 psi and one would have high confidence that this strength would be achieved or exceeded within 28 days after placement of the concrete. For seismic margin reviews to a SME, one should use conservatively biased actual material strengths which in the case of concrete take into account that concrete strength continues to increase with time beyond 28 days after placement. Material strengths used in deterministic seismic margin reviews for the SME should be sufficiently conservative that there is very little likelihood that actual strengths are less than those used in the margin review (approximately 95\%, excaedance probability strengths achieve this goal). For a PFM review, median material strengths plus the probable range of strengths should be used.

For a typical 3000 psi concrete design strength, one would likely expect that the 90\% bounds on actual strengths after two years would range from about 3400 psi to 5000 psi with a median of about 4200 psi. This full range should be considered in a PFM review while a deterministic margin review could use a conservative strength of 3400 psi in lieu of the design strength of 3000 psi.

STATIC STRENGTH OR CAPACITY EQUATIONS

Code equations for static strength or capacity are generally very conservatively biased. In cases where one is attempting to predict a failure margin (either CDFM or PFM) and when failure test data exists to demonstrate excessive conservatism in code equations for static strength or capacity, one should use actual failure test data in lieu of code equations to predict seismic margins for the SME. 


\section{INELASTIC ENERGY ABSORPTION CAPACITY}

Nearly all structures and components exhibit at least some ductility (i.e., ability to strain beyond the elastic limit) before failure. Because of the limited energy content and oscillatory nature of earthquake ground motion, this ductility is highly beneficial in increasing the seismic margin against failure for structures and components. The inelastic energy absorption, $F_{\mu}$, represents the ratio of the SME at which a certain system ductility $\mu$ is reached to the earthquake level for which failure would be predicted by linear elastic analysis. The additional seismic margin due to this inelastic energy absorption factor $F_{\mu}$ should be considered in any failure margin review. Ignoring this effect will lead to unrealistically low estimates of the failure margin. It is impossible to correlate performance of structures and equipment in past earthquake experience with capacities predicted by elastic analyses without considering the $F_{\mu}$ factor.

In a probabilistic failure margin (PFM) review, one should estimate the probable range on $F_{\mu}$. For instance, for a shear wall structure with highly non-uniform Demand/Capacity ratios throughout the structure (i.e., inelastic response is concentrated in localized regions), one might estimate the probable range for $F_{\mu}$ to be 1.3 to 3.0. This entire range should be used in a PFM review. For a conservative deterministic failure margin (CDFM) review, a conservative lower bound estimate on $F_{\mu}$ should be used. For this shear wall structure, such a conservative lower bound on $F_{\mu}$ might be 1.3. Actually, all but the most brittle structures and components will exhibit $F_{\mu}$ values of at least 1.3 so that values less than 1.3 should seldom be used for $F_{\mu}$ in a failure margin review.

\section{CONSERVATISM OF COMPUTED CAPACITY}

In a CDFM review, the capacity should be computed sufficiently conservatively so that if the computed response actualiy occurs, one has high confidence that the probability of failure is negligible. In other words, failure will occur if a severe unknown construction error exists or if the actual seismic response significantly exceeds the computed seismic response. Capacities computed following the guidelines in Table 2-1 and described in the previous sections for CDFM reviews are expected to achieve this goal.

Based upon comparison of capacities computed by the guidelines of Table 2-1 for CDF:i reviews with median probabilistic computed capacities reported in several seismic PRAs, it is estimated that the median probable capacities (50\% probability of failure) typicaliy lie between 1.4 and 3.0 times the CDFM computed lower bound 
capacities. Responses would have to be increased by factors of about 1.4 to 3.0 before failures would be expected.

\section{COMPARISON OF VARIOUS SEISMIC MARGINS}

Probabilistic Failure margins (PFM) are typically displayed by fragility curves such as that shown in Figure 2-1 for a typical $0.15 \mathrm{~g}$ SSE designed stiff shear wall structure founded on rock. These fragility curves illustrate that one has high confidence of low probability of failure for an SME less than 0.39 (i.e., 2 times the SSE) and has high confidence that the SME associated with a 50\% probability of failure lies between $0.5 \mathrm{~g}$ and $1.6 \mathrm{~g}$ with a median value of $0.9 \mathrm{~g}$. Discussion on the development of such fragility curves is contained in References 1 and 2 .

For this same structure, the SME associated with the Code Margin (CM) was only $0.16 \mathrm{~g}$ and the SME associated with a Conservative Deteministic Failure Margin (COFM) was $0.32 \mathrm{~g}$. Note that the CDFM value for the SME agrees closely with the high confidence of low probability of failure value of $0.3 \mathrm{~g}$ obtained from a PFM review. This close agreement between the CDFM value and the high confidence, low probability of failure value from a PFM review has been observed in a number of cases for which both margin reviews have been conducted. In other words, the CDFM criteria in Table 2-1 can probably be used to deterministically establish the lower bound on PFM fragility curves. In fact, one might prescriptively define

$$
\text { COFM } \approx 95 \% \text { confidence of less than } 5 \% \text { probability of failure }
$$

Ise of Equation (3) would provide a simpler and probably more consistent method of obtaining the high confidence $(=95 \%)$, low probability $(<5 \%)$ point on fragility curves than the separation of variables method described in References 1 and 2 . This point on the fragility curve would be deterministically determined using the CDFM criteria in Table 2-1. However, further validation of Equation (3) is needed.

A review of fragility curves presented in several seismic PRAs (Zion, Indian Point 2. Indian Point 3, Limerick, Millstone, Midland, and Seabrook) generaliy indicate a factor of 2.5 to 6.0 between the median (50\% failure) fragility value and the high confidence $(=95 \%)$, low probability $(<5 \%)$ point on the fragility curve. Thus, margins defined by the CDFM criteria would still contain substantial conservatism below median fragilities. 
level, the input to floor-mounted equipment should also be defined at the $84 \%$ NEP level. Use of $84 \%$ NE? floor spectra as input to equipment would provide the same level of response conservatism for equipment as exists for the structure. Unfortunately, the generation of $84 \%$ NEP floor spectra would require probabilistic structural response analyses which are more costly and have seldom been performed.

If 84\% NEP floor spectra were generated and used in a margin study, one would have to multiply such spectra by a scale factor for equipment qualified by testing. The CDFM procedure defined in Table 2-1 introduces considerable conservatism in estimating the capacity of equipment qualified by analysis for a given floor spectrum. This factor of conservatism from the median failure capacity is estimated to range from about 1.5 to more than 3 for equipment qualified by analysis. Thus, to introduce a similar conservatism for equipment qualified by testing would require a scale factor of about 2. In other words, for a CDFM review the floor response spectrum to be used for comparison with equipment qualification test response spectrum (TRS) should be:

$$
\text { TRS } \approx 2 \star(84 \% \text { NEP Floor Response Spectrum) }
$$

Again, it should be noted that within the current state-of-art one would not generally generate 84\% NEP floor spectra for a seismic margin review. Instead, one would likely use conservative broadened floor spectra generated using conservative deterministic structural response parameters similar to those described in Table 2-1. These conservative broadened floor spectra can be used directly to determine a CDFM for equipment qualified by testing. However, for equipment qualified by analysis some conservatism should be removed in a CDFM review. This reduction in conservatism can be accomplished by:

1. Using median or slightly greater than median damping values for computing equipment response.

2. Perform frequency shifting of floor spectra rather than frequency broadening to account for frequency uncertainty.

For a seismic margin review of equipment mounted on structures, one should generally use 5\% to $15 \%$ damped floor spectra as input to the equipment to partially compensate for the conservatism introduced in the generation of these spectra.

USE OF THE CONCEPT OF SEISMIC-INDUCED SCENARIOS IN SEISMIC MARGIN STUDIES

It is unnecessary to demonstrate seismic margin for all structures, components, and systems subjected to an SME. Instead, one should concentrate on the more likely 
$0046 G$

$87 C 7451$

This appendix is an excerpt from

EPRI Report NP-6041

\section{Appendix B}

Summary of CDFM Method Description From EPRI Report 
Table 2-5.

SUMMARY OF CONSERVATIVE DETERMINISTIC FAILURE MARGIN APPROACH

Load Combination:

Ground Response Spectrum:

Damping:

Structural Model:

So il-Structure-Interaction:

Material Strength:

Static Capacity Equations:

Inelastic Energy Absorption: (ductility)

In-Structure (Floor) Spectra Generation:

Source:
Nomal + SME

Conservatively specified (preferably $84 x$ NonExceedance Probability Site-Specif ic Spectrum, if Available)

Conservative estimate of medtan dawing

Best Estimate (Median) + Uncertainty Variation in Frequency

Best Estimate (Median) + Parameter Variation

Code Specified minimum strength or $95 \%$ exceedance actual strength if test data are avallable.

Code ultimate strength (ACI), maximum strength (AISC). Service Leve 10 (ASME), or functiona 1 ifmits. If test data are avaliable to demonstrate excessive conservatism of code equations, then use 84X exceedance of test data for capacity equation.

For non-brittle failure modes and linear analysis, use $80 \%$ of computed seismic stress in capacity evaluation to account for ductility benefits, or perform nonlinear analysis and go to $95 \%$ exceedance ductility levels.

Use frequency shifting rather than peak brodening to account for uncertainty plus use median damping. 
$87 C 1451$

0046G-10

Append $1 x \mathrm{C}$

Floor Spectra 


\section{FLOOR SPECTRA}

Attached are selected horizontal and vertical floor spectra at $2 \%, 4 \%$, and 7\% damping. They have been "debroadened", with some associated simplifications, from the broadened Maine Yankee turbine/service building spectra at EL 61'-0", E-W and vertical directions. A description of the dynamic model is contained on PP. 111 to 134 of Cygna's report (Appendix $C$ of the prior transmittal). $7 \%$ structure damping was used in the dynamic analysis. Ground motion input consisted of time-histories matching the median NUREG/CR-0098 spectra scaled to $0.18 \mathrm{~g}$ horizontal $\mathrm{PGA}$ and $0.12 \mathrm{~g}$ vertical $P G A$.

Unbroadened floor spectra at the desired equipment frequencies are not contained in the Dresden SEP report (only 0.5\% damping). Broadened horizontal spectra at $2 \%, 3 \%$, and $7 \%$ dampings are available. Also, vertical spectra are not contained. The Maine Yankee spectra were selected as the basis for the "debroadened" spectra for the following reasons:

- Vertical spectra are available.

- The fundamental horizontal frequency is comparable (7.5 $\mathrm{Hz}$ vs. $5 \mathrm{~Hz}$ )

- In-structure spectral accelerations at the peaks and high frequencies are about the same as for the Dresden spectra at upper elevations, for nearly equivalent ground input.

While some information is lost towards the higher frequencies in the debroadening, the same problem would exist with the broadened Dresden spectra. 


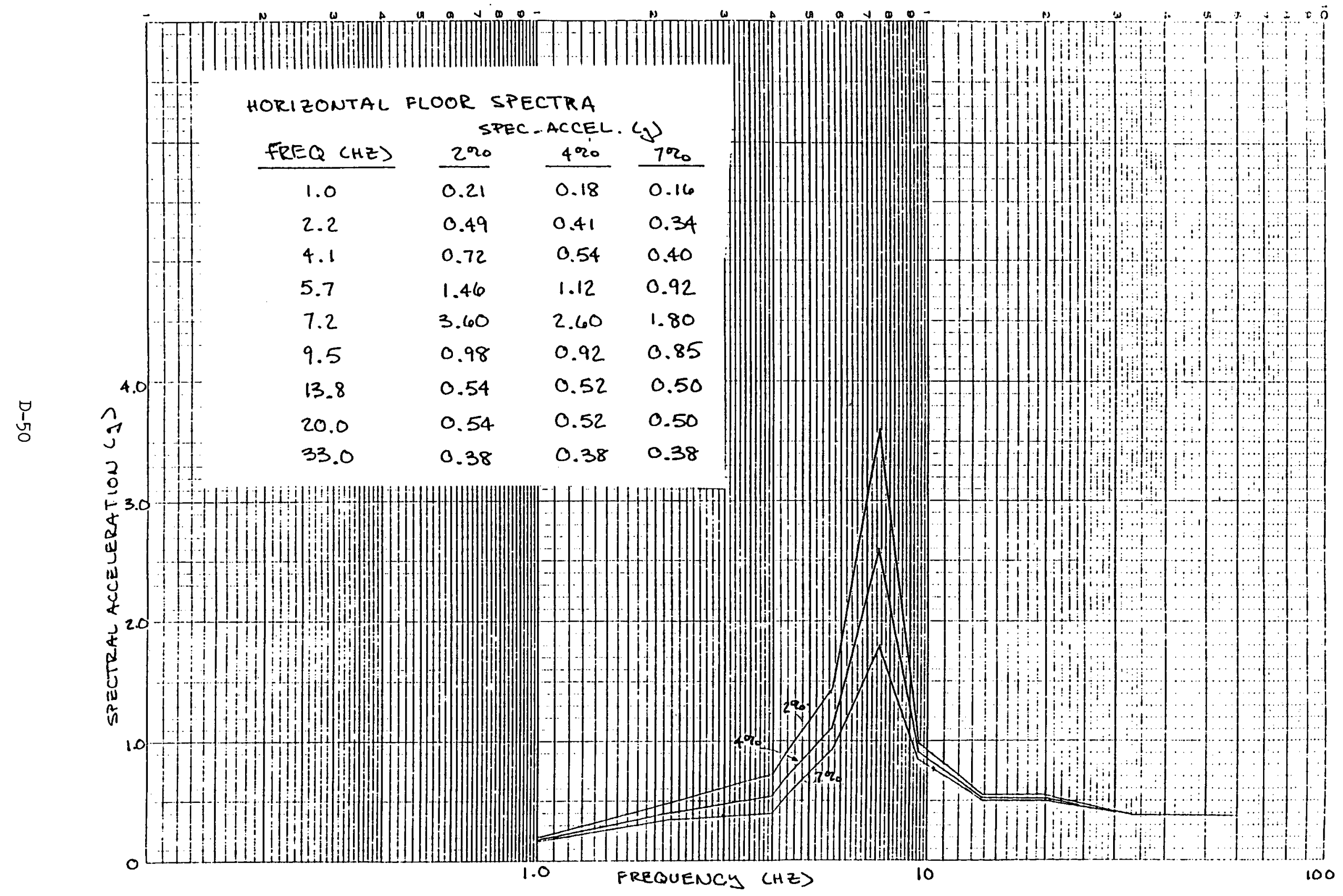




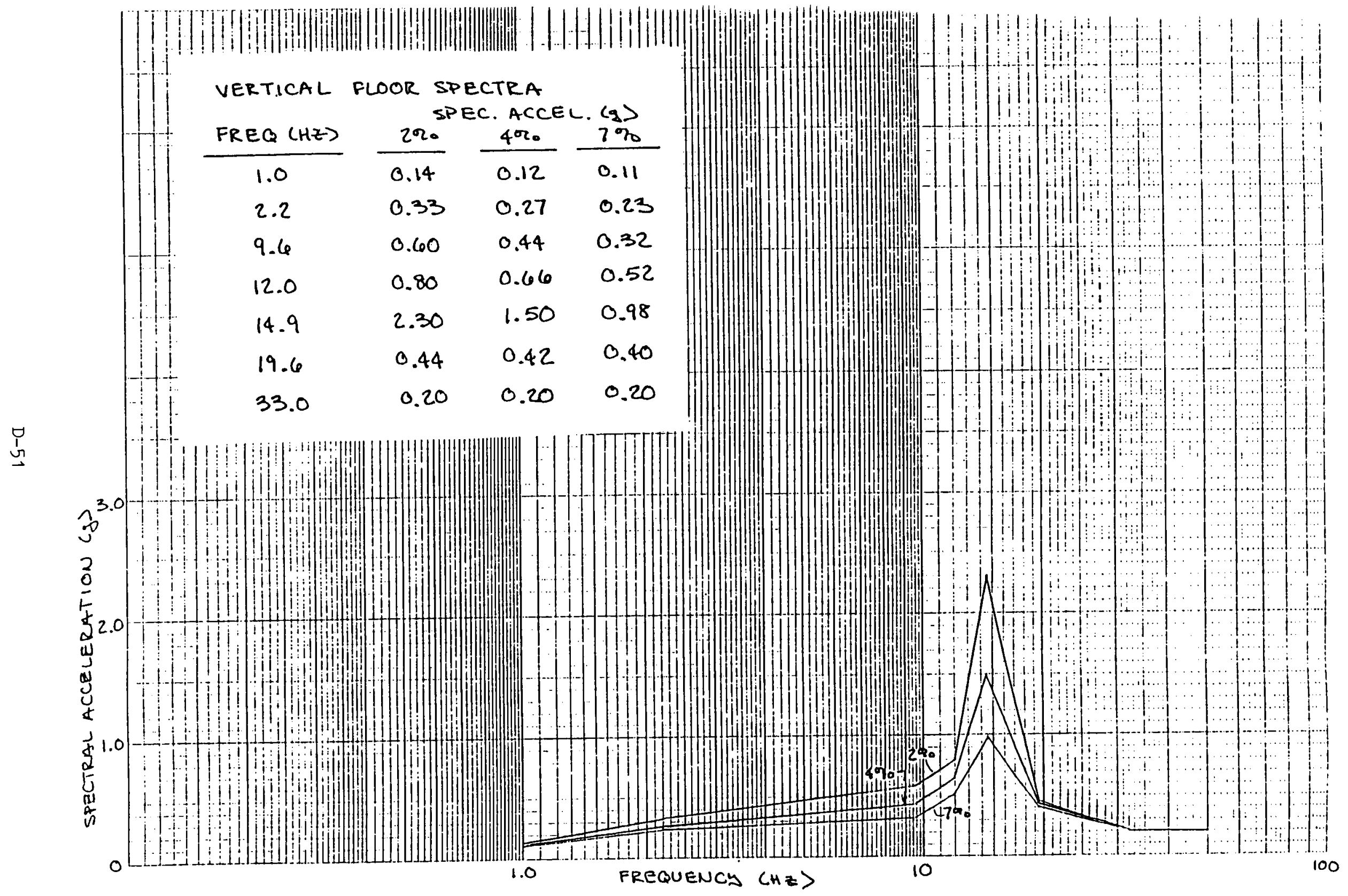


$87 C 7451$

0046G-11

Append 1X 0

Generic Selsmic Ruggedness of Power Plant Equipment 


\title{
Generic Seismic Ruggedness of Power Plant Equipment
}

\author{
NP-5223 \\ Research Project 1707-15
}

Final Report, May 1987

\author{
Prepared by \\ ANCO ENGINEERS. INC. \\ 9937 Jefferson Boulevard \\ Culver City, California 90232-3591 \\ Principal Investigators \\ C. 8. Smith \\ K. L. Merz
}

Prepared for

Electric Power Research Institute

3412 Hillview Avenue

Palo Alto, California 94304

EPRI Project Manager

G. E. Siter

Nulcear Plant Life Extension and Constructibility Program Nuclear Power Division 
GERS-MCC. 3

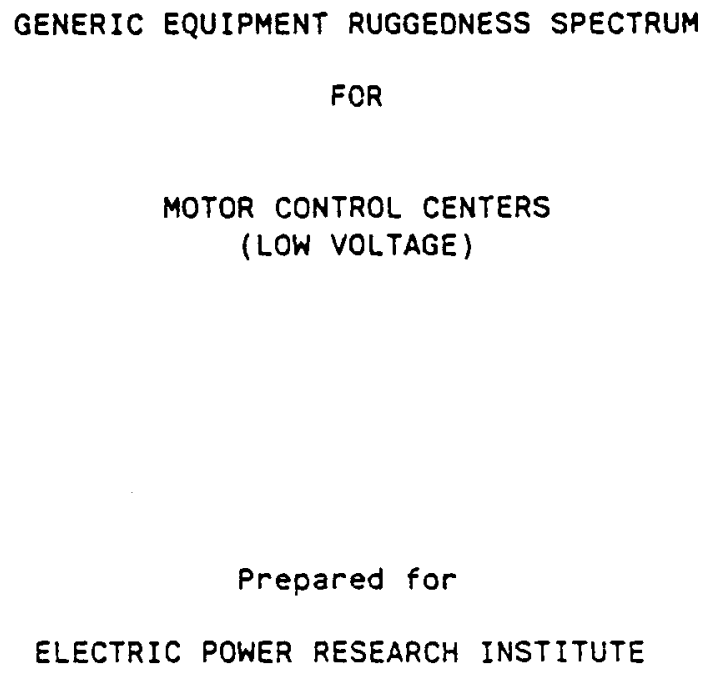


GERS-MCC. 3

$12 / 1 / 86$

\section{D INTROOUCTION}

A Generic Equipment Ruggeaness Specir'sm (GERS) for Mctor Control Centers (low voltage) is presented and discussed in the following sections.

\subsection{EQUIPMENT OESCRIPTION}

The equipment class covered by the GERS presented here is Low Voltage Motor Control Centers (MCC) which are steel enclosures containing various sizes of motor starters (contactors and control relays), circuit breakers, auxiliary relays, disconnect switches, control or distribution transformers, and panelboards. They may also have indicator lamps and meters. Cable or conduit entry can be from the bottom, top. or side. Units are low voitage rated at 600 VAC or 250 VDC. Typical lowvoltage NEMA nominal enclosure section sizes are 20 inches wide, 14 to 20 inches deep, and 90 inches high. They are fabricated of 14 gage or heavier steel sheets, framed with angles, and supported on channels at the bottom. The base channels are either integral with the MCC frame or are external members connected by internal bolts to the MCC frame. Multiple MCC sections may be grouped together to make widths to 120 inches or greater. The units must. be anchored at the base to a supporting structure. The validation of anchorage adequacy requires an independent evaluation. This equipment class covers virtually all low voltage MCCs used in power plants for critical motor control. The checklist given in section 5 can be used to screen for outliers.

\subsection{TEST DATA 8ASE}

The data base includes basic equipment descriptive information, test methods/ description, and test data covering a wide range of MCC for fifteen separate tests. The earliest test in the data base was conducted in 1974. Forty vertical MCC sections with weights ranging from 200 to 800 pounds (per section) from ten manurasturers which represent the range of units found in actual power plants are included in the data base. The units tested involved both single- and multi-section MCC units (up to six sections). Two of the tests included valid data; however, they do not meet the class inclusion rules. One of these tests had a top brace attachment (i.e., not entirely base-mounted), and the other MCC unit was housed in a nontypical (larger) enclosure. All units were mounted within NEMA-type metal enclosures with either welded or bolted anchorage. 
Twelve tests were performed with random, independent, biaxial input motions. One test used random, independent, triaxial input motion, while two additional tests utilized single-axis sine-beat inputs. The test results span the entire range of possible success and failure. Failure modes are relay chatter and minor structural base damage. In one test series involving four MCC sections, the equipment fragility limits were sought. In some cases, the tests were performed on Mccs in which artificially aged components had been installed, while in others, the components were new. Typical parameters which are monitored during testing include contact chatter and coil dropout. Tests are typically performed in a deenergized state and then repeated with the circuits energized. Hi-pot tests, under- and over-voltage relay functionality, and circuit breaker functionality are checked before and after the tests.

In general, the functionality of MCCs is limited by auxiliary relay and motor starter auxiliary contact chatter in the deenergized state. Thus, the issue of MCC function during a dynamic event is governed by the ruggedness of the relays present. It should be noted that all MCC units, dynamically tested, functioned in post-test operation, including those units that sustained minor base damage.

\subsection{GENERIC EQUIPMENT RUGGEDNESS SPECTRUM}

Figure 1 compares the GERS to the horizontal Test Response Spectra (TRS) (standardized to $5 \%$ damping) for all of the thirteen. TRS used to construct the GERS. Both the energized and the deenergized data from successful are compared in Figure 1. For this equipment class, dual GERS are proposed. The "function after" GERS accommodates the test data of several manufacturers over the frequency range of 1 to $33 \mathrm{~Hz}$. Also the "function after" GERS accommodates the tests where minor structural damage (not affecting function) occurred. The "function during" GERS conservatively accommodates the low bound of data base TRS for which relay chatter was noted in a wide spectrum of cabinet and relay or starter types as shown in Figure 2. The vertical TRS in all tests on which the GERS is based was approximately equal to the horizontal input motion. Thus, the GERS presented is valid for concurrent vertical and horizontal motion.

\subsection{CHECKLIST}

To apply this GERS to Motor Control Centers, the following criteria must be verified. 
- The MCC must be a low voltage unit with a floor-mounted NEMA-type enclosure with an average weight per verticai section that does not exceed 800 pounds (review of manufacturer's submittals is sufficient).

- The MCC must be base anchored and the installed anchorage must be evaluated (units which utilize a top brace attachment as part of the unit anchorage require a secarate evaluation in order to justify the "function after" GERS limit; however, the "function during" GERS inay be used directly with such units).

- The base anchorage must utilize the MCC base channels for attachment. Base anchorage details that induce significant bending of sheet are not acceptable.

- Cutouts in cabinet sheathing are less than 6-in. wide and 12-in. high in the lower half of the cabinet height.

- Alt door latches or screwdriver operated door fasteners must be secured.

- In order to utilize the "function during" Gers certain relays with low ruggedness must be excluded. Ali relays must have a GERS greater than $4.5 \mathrm{~g}$ within the amplified spectral region.

- Auxiliary contacts of contactors require a separate evaluation if they are used for interlocks or control signals. The "function during" GERS spectral levels nust be factored by O.fT to be applied to the auxiliary contacts of contactors. 0.67

- If the "function during" GERS limits for MCCs are exceeded by certain plant floor response spectra, a separate relay evaluation is required which accounts for specific relay ruggedness, relay location within an enclosure, and enclosure amplification. 

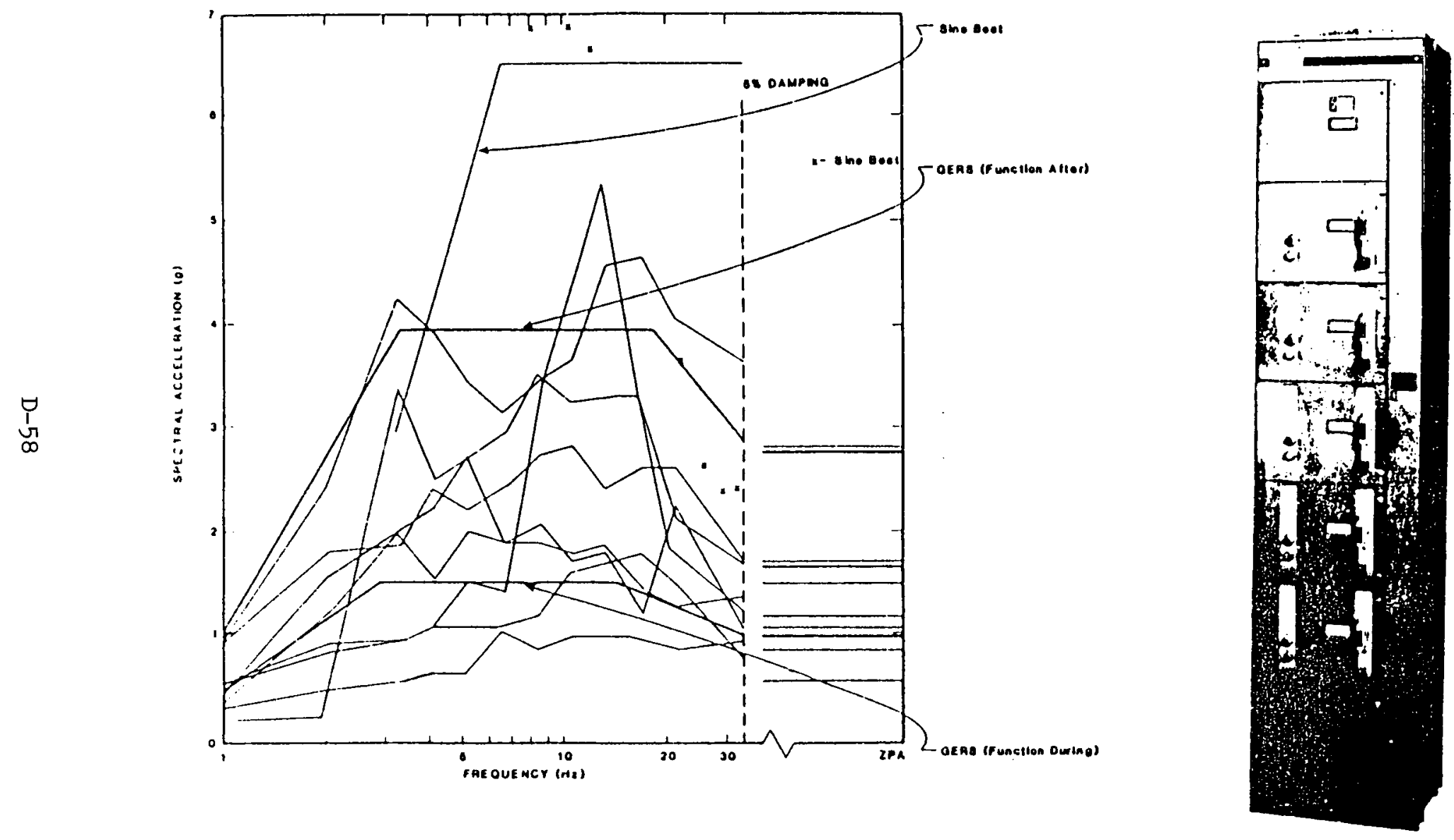

Figure 1. Comparison of GERS With Ruggedness Data: Function During and After for MCC 
$\operatorname{sRS}-\because C C .3$

$12,1 / b t$

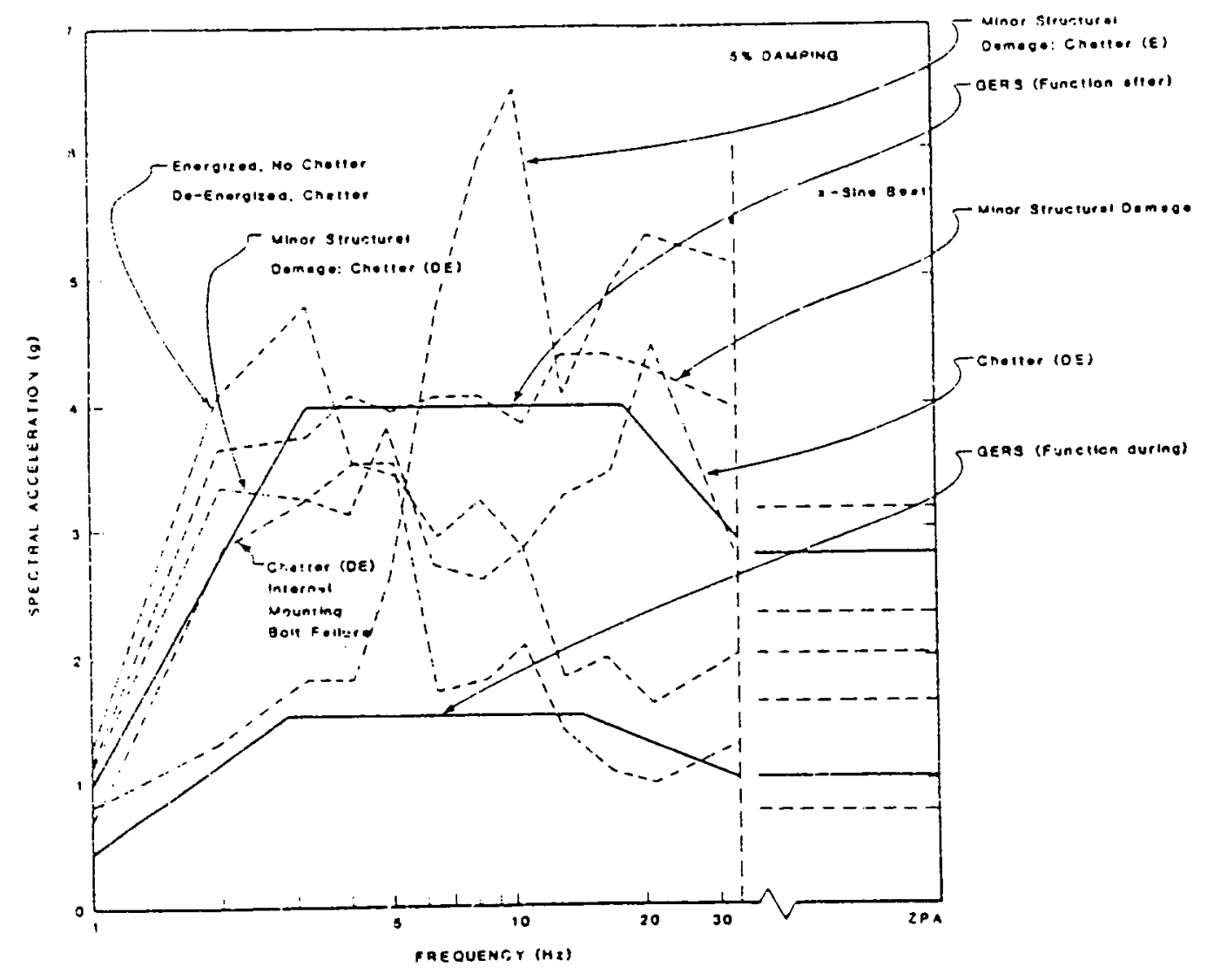

Figure 2. Comparison of GERS with failure data: function during and after for MCC. 
Table 1

Comparison of HCLPF Capacity Computations for Representative Components (FIrst Round Calculations)

\begin{tabular}{|c|c|c|c|c|}
\hline \multirow[b]{2}{*}{ Component } & \multicolumn{2}{|c|}{ HCLPF Capacity (g) } & \multirow{2}{*}{$\begin{array}{l}\text { Median } \\
\text { (g) }\end{array}$} & \multirow{2}{*}{$\begin{array}{l}\text { Capacily } \\
\text { Failure Mode }\end{array}$} \\
\hline & CDFM & FA & & \\
\hline \multicolumn{5}{|c|}{$\begin{array}{l}\text { Flat Bottom Storage Tank } \\
\text { (At Grade) }\end{array}$} \\
\hline $\begin{array}{l}\text { RPK } \\
\text { MKRPSH } \\
\text { JWR } \\
\text { JDS }\end{array}$ & $\begin{array}{r}0.29 \\
0.29 \\
\because \because 8 \\
0.3\end{array}$ & $\begin{array}{l}0.31 \\
0.26 \\
0.27 \\
-.\end{array}$ & $\begin{array}{l}0.67 \\
0.54 \\
0.53 \\
1.13\end{array}$ & $\begin{array}{l}\text { Combination of shell buckling } \\
\text { and anchor boll yields } \\
\text { Yield of anchor bolts }\end{array}$ \\
\hline
\end{tabular}

Auxiliary Contactor Chatter

(Function during GERS lock-in circuit potential)

a) Cabinet at Grade

$\begin{array}{lcccc}\text { RPK } & 0.54 & 0.59 & 1.26 & \text { Conlactor Chatter } \\ \text { MKRRDC } & 0.47 & 0.39 & 1.58 & \text { Contactor Chatter } \\ \text { JWR } & \because-. & 0.48 & 1.20 & \text { Conlactor Chatter } \\ \text { JDS } & 0.71 & -\because- & 1.88 & \text { Contactor Chatter }\end{array}$

b) Cabinet High-up

(Function during GERS lock-in circuil polential)

$\begin{array}{lcccc}\text { RPK } & 0.10 & 0.11 & 0.30 & \text { Contactor Chatter } \\ \text { MKRRDC } & 0.09 & 0.07 & 0.36 & \text { Contactor Chalter } \\ \text { JWR } & -\cdots . .12 & 0.11 & 0.43 & \text { Contactor Chalter } \\ \text { JDS } & 0.12 & -\cdots & 0.43 & \text { Contactor Chatter }\end{array}$

(used .87 knock-down factor)

(used .87 knock-down factor)

RPK Did calculations by CDFM (by EPRI methodology) first, then FA.

MKRADC/PSH Did calculations by FA first, then CDFM (by EPRI methodology)

JWR Tabulated values are from HCLPF Capacity calculations using input spectra as $84 \%$ NEP maximum horizontal direction.

JDS Did calculations by CDFM (by deterministic approach). 
Table 2

Comparison of HCLPF Capacity Computations for Representative Components

(Second Round Calculations)

\begin{tabular}{|c|c|c|c|c|}
\hline Component & $\begin{array}{c}\text { HCLPF } \\
\text { (g) }\end{array}$ & $\begin{array}{l}\text { Median } \\
\text { Capacity } \\
\text { (g) }\end{array}$ & $\begin{array}{l}\text { Capacily } \\
\text { Failure Mode }\end{array}$ & Comments/Remarks/Assumptions \\
\hline
\end{tabular}

Flat Bottom Storage Tank

(At Grade)

$\begin{array}{lll}\text { RPK } & 0.29 & 0.67 \\ \text { MKRPSH } & 0.29 & 0.54 \\ \text { JWR } & 0.28 & 0.55 \\ \text { JDS } & 0.32 & 0.83\end{array}$

Combination of shell buckling

and anchor bolt yields

Yield of anchor bolls and seperati... of base plate fincive wael plate

Auxiliary Contactor Chatter

(Function during GERS lock-in

circuit potential)

a) Cabinet at Grade

\section{RPK}

MKRRDC

JWA

JDS

b) Cabinet High-up

(Function during GERS lock-in

circuit potential)

RPK

MKRADC

JWR

JDS

0.11

0.09

0.11

0.15

\subsection{4}

0.47

0.48

0.71

1.26

1.58

1.20

1.88

See calculations (Appendix A) for further explanation.

0.30 Conlactor Chatter

0.36 Contactor Chatter

0.43 Conlactor Chatter

1.88* Contactor Chatter

Contactor Chatter (used .87 knock-down factor)

Contactor Challer

Contactor Challe

Contactor Chalter 
Table 2 (ContInued)

Comparlson of HCLPF Capaclty Computatlons for Representatlve Components

(Second Round Calculations)

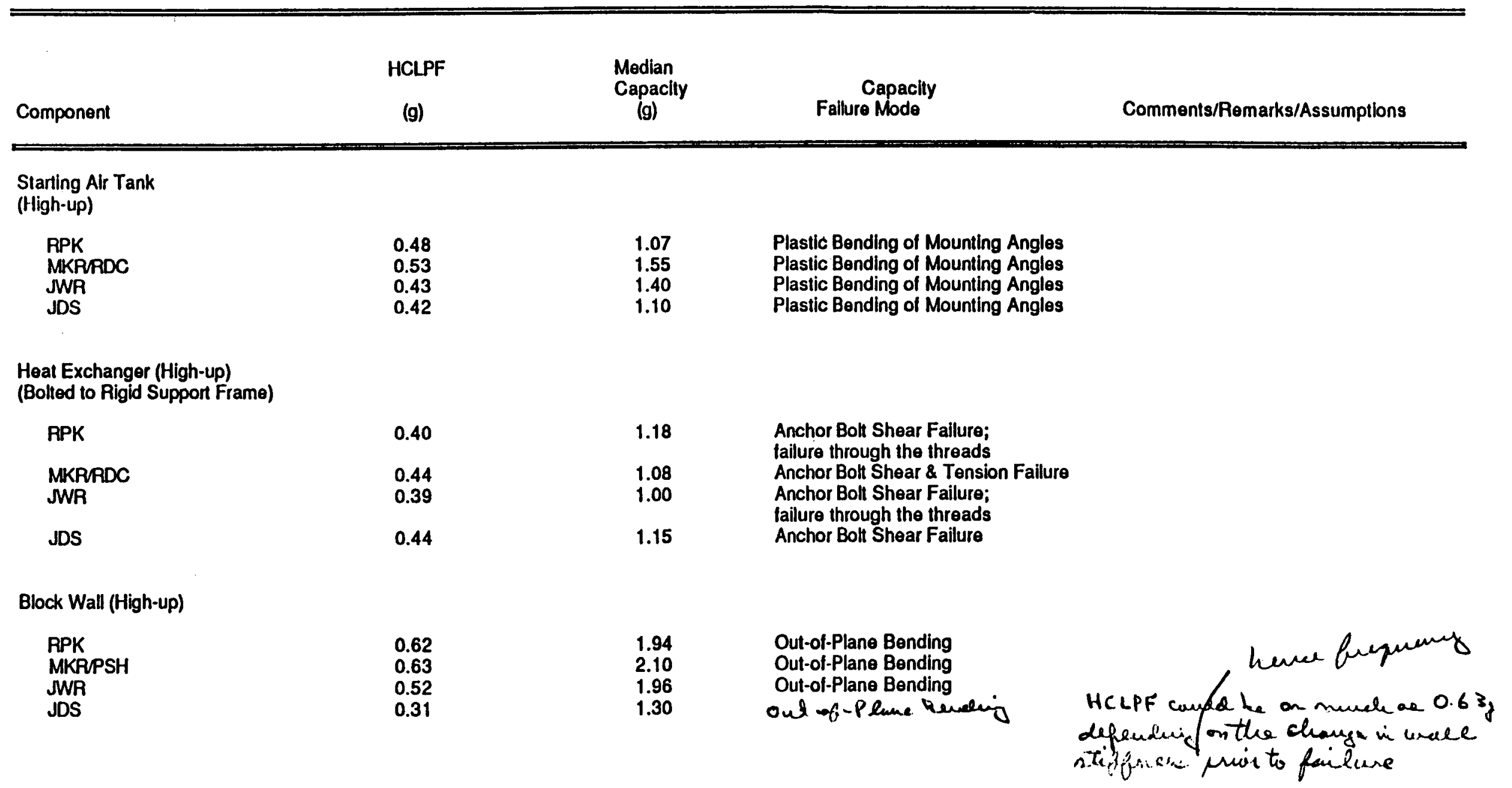




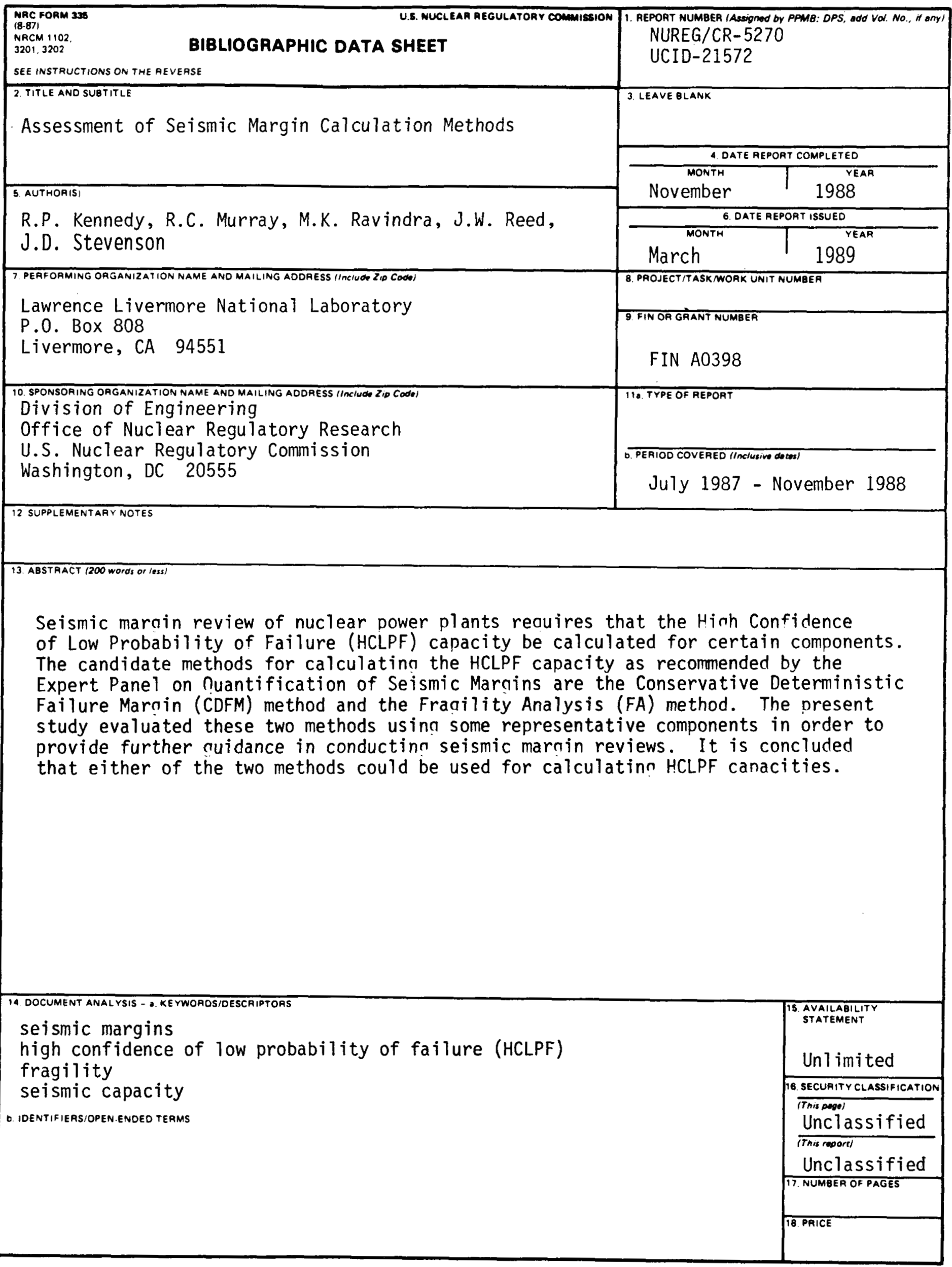

